\title{
Synthesis and \\ Characterisation of Metal \\ Chalcogenide Nanocrystals
}

\author{
By \\ John Harley Loveless Beal
}

\begin{abstract}
A thesis submitted to
Victoria University of Wellington in fulfilment of the requirements of the degree of
\end{abstract}

Doctor of Philosophy in Chemistry

Victoria University of Wellington 


\begin{abstract}
Nanomaterials are defined as materials which possess features with dimensions of less than $100 \mathrm{~nm}$. Nanocrystals are a subclass of nanomaterials, where the absolute dimensions of individual particles are within this range. Various effects become evident at such small scales, including notably: alterations in electronic structure and magnetic behaviour; and the predominance of surface chemistry. Consequently, the synthesis of nanocrystals with tailored properties via chemical methodology has become an area of some interest.
\end{abstract}

Metal chalcogenides form an important class of inorganic materials, which includes many technologically important semiconductors. Metal chalcogenides feature prominently among semiconductor nanocrystals synthesised to date, but the development of magnetic nanocrystals has focused primarily on metal, and metal oxide phases. Thus the aim of this project was the investigation and development of synthetic methodology for producing nanocrystals, focusing on the metal chalcogenides, with specific emphasis on magnetic metal chalcogenides (iron sulfides). Properties of nanocrystals and metal chalcogenides are discussed in Chapter 1.

As described in Chapter 2, metal chalcogenide nanocrystals were synthesised by high temperatures solution-phase reactions, and all samples were characterised by Transmission Electron Microscopy (TEM), Energy Dispersive X-ray Spectroscopy (EDS) and Electron Diffraction (ED). Powder X-ray Diffraction (XRD), Scanning Quantum Interference Device magnetometry (SQUID), Thermogravimetric analysis (TGA), Ultraviolet-visible (UV-vis) absorption and fluorescent emission spectroscopy were also used extensively.

CdSe nanocrystals with diameters $<10 \mathrm{~nm}$ are noted for their size-dependent absorption and emission in the visible region. As described in Chapter 3, an established synthesis was used to produce CdSe nanocrystals in order to explore the size-dependence of the optical properties of the nanocrystals, and to explore the possibility of transferring the nanocrystals to aqueous media.

As described in Chapter 4, high temperature reaction of iron salts and elemental sulfur in non-aqueous coordinating solvents was used to produce $\mathrm{Fe}_{1-x} \mathrm{~S}$ and $\mathrm{Fe}_{3} \mathrm{~S}_{4}$ 
nanocrystals. The factors affecting phase-selectivity, particle size and morphology were ascertained; and the magnetic properties of pure $\mathrm{Fe}_{1-x} \mathrm{~S}$, pure $\mathrm{Fe}_{3} \mathrm{~S}_{4}$ and mixtures of $\mathrm{Fe}_{1-x} \mathrm{~S}$ and $\mathrm{Fe}_{3} \mathrm{~S}_{4}$ were investigated.

As described in Chapter 5, thermal decomposition of iron salts in a coordinating solvent was used to synthesis iron metal or iron oxide intermediates, which could either be oxidised to iron oxide spinel; or sulfidised in situ to iron thiospinel $\left(\mathrm{Fe}_{3} \mathrm{~S}_{4}\right)$ nanocrystals. This approach proved to be a good source of small, monodisperse iron oxide spinel and iron thiospinel nanocrystals with the same average dimensions. The magnetic properties of the highly-researched iron oxide spinel nanocrystals were determined, and contrasted to those of the their far less investigated thioanalogues.

As described in Chapter 6, metal polysulfido complexes of the type $\left[\mathrm{M}(N-\mathrm{MeIm})_{x}\right] \mathrm{S}_{\mathrm{y}} / \mathrm{MS}_{y}(N \text {-MeIm })_{x}(\mathrm{M}=\mathrm{Fe}, \mathrm{Zn}, \mathrm{Mg} ; N$-MeIm $=N$-methylimidazole $)$ were synthesised from metal powders, elemental sulfur and $\mathrm{N}$-MeIm; then thermolysed in coordinating solvents to afford metal sulfide nanocrystals. Thus establishing a new general route for synthesis of metal sulfide nanocrystals from low-cost starting materials. 


\section{Acknowledgements}

I would firstly like to thank my supervisors Richard Tilley and Pablo Etchegoin for all their support over the course of my Ph.D. My sincere thanks also go to my colleagues in the Tilley research group, especially Teck Hock Lim, who was first my classmate, then my laboratory and officemate for the duration of our studies at VUW.

My thanks are also due to all the general staff of the School of Chemical and Physical Sciences, especially David Flynn, without whose careful and calm guidance on the electron microscopes this project would have come to nought. I would like to thank Kathryn Allan, Sarah Hoyte, Teresa Vaughan and Almas Zayya for all their companionship, help, advice and loaned glassware over the years. Special thanks also to Amane Shiohara, whose friendship during our Ph.D.s has meant a lot to me.

My deepest thanks to my family - my father and stepmother - Drs. Tim Beal and Kala Retna, who have been unstinting sources of support, for who knows better the trials and tribulations of the Ph.D. process than they?

I would also like to acknowledge the financial assistance of the MacDiarmid Institute for Advanced Materials and Nanotechnology, the Curtis-Gordon Research Scholarships, Victoria University of Wellington and the Royal Society of New Zealand. 


\section{Abbreviations, symbols and units}

\section{Chemical abbreviations}

$\begin{array}{ll}\text { acac } & \text { acetylacetonate } \\ \mathrm{Bu}^{t} & \text { tertiary butyl } \\ \mathrm{Ddtc} & \text { diethyldithiocarbamate } \\ \mathrm{Et} & \text { ethyl } \\ \mathrm{HDA} & \text { hexadecylamine } \\ \mathrm{Mer} & \text { 2-mercaptobenzothiazole } \\ \mathrm{MPA} & \text { mercaptopropionic acid } \\ N \text {-MeIm } & \text { N-methylimidazole } \\ \mathrm{OAc} & \text { acetate } \\ \mathrm{Ph} & \text { phenyl } \\ \text { Phen } & \text { phenanthroline } \\ \text { PMMA } & \text { polymethylmethacrylate } \\ \text { tetraglyme } & \text { tetraethylene glycol dimethyl ether } \\ \text { TMAH } & \text { tetramethylammonium hydroxide } \\ \text { TMEDA } & \text { tetramethylethylenediamine } \\ \text { TMS } & \text { trimethylsilyl } \\ \text { TOP } & \text { trioctylphosphine } \\ \text { TOPO } & \text { trioctylphosphine oxide }\end{array}$

\section{General abbreviations}

\begin{tabular}{ll} 
at $\%$ & Atomic percent \\
b.p. & Boiling point \\
bcc & Body-centred cubic \\
CCD & Charge-coupled device \\
cf. & confer \\
e.g. & exempli gratia \\
EDS & Energy Dispersive X-ray Spectroscopy \\
etc. & et cetera \\
et al. & et alia \\
FC & Field-cooled \\
fcc & Face-centred cubic \\
FFT & Fast Fourier Transform \\
FWHM & Full Width at Half Maximum \\
hcp & Hexagonal close-packed \\
HOMO & Highest Occupied Molecular Orbital \\
HPLC & High Pressure Liquid Chromatography \\
hrs & Hours \\
HRTEM & High Resolution Transmission Electron Microscopy \\
ICDD & International Centre for Diffraction Data \\
i.e. & id est \\
LUMO & Lowest Occupied Molecular Orbital \\
mins & Minutes \\
mmHg & Millimetres of mercury \\
m.p. & Melting point \\
MPMS & Magnetic Properties Measurement System \\
n/a & Not available / Not applicable \\
PDF & Powder Diffraction File \\
\hline
\end{tabular}




$\begin{array}{ll}\text { R value } & \text { Linear regression correlation constant } \\ \text { RIR } & \text { Relative Intensity Ratio } \\ \text { rpm } & \text { Revolutions per minute } \\ \text { SAED } & \text { Selected Area Electron Diffraction } \\ \text { SBW } & \text { Spectral Band Width } \\ \text { SEM } & \text { Scanning Electron Microscope } \\ \text { SI } & \text { Système International (d'Unités) } \\ \text { soln. } & \text { Solution } \\ \text { SQUID } & \text { Superconducting Quantum Interference Device } \\ \text { tech. } & \text { Technical grade } \\ \text { TEM } & \text { Transmission Electron Microscope } \\ \text { TGA } & \text { Thermogravimetric Analysis } \\ \text { UV } & \text { Ultraviolet } \\ \text { UV-vis } & \text { Ultraviolet-visible } \\ \text { vs. } & \text { versus } \\ \text { wt\% } & \text { Weight percent } \\ \text { XPS } & \text { X-ray Photoelectron Spectoscopy } \\ \text { XRD } & \text { X-ray diffraction } \\ \text { ZFC } & \text { Zero field-cooled }\end{array}$

\section{Crystallographic notation}

\begin{tabular}{|c|c|}
\hline$\AA$ & Ångström unit $\left(10^{-10} \mathrm{~m}\right)$ \\
\hline$a, b, c$ & $\begin{array}{l}\text { Unit cell parameters parallel to the } x, y \text {, and } z \text { axes of a crystal, } \\
\text { respectively }\end{array}$ \\
\hline$a^{*}, b^{*}, c^{*}$ & $\begin{array}{l}\text { Parameters of the reciprocal unit cell parallel to the } x^{*}, y^{*} \text {, and } z^{*} \\
\text { reciprocal axes, respectively }\end{array}$ \\
\hline$\alpha, \beta, \gamma$ & Angles between the pairs of unit cell edges $b c, c a$, and $a b$, respectively \\
\hline$\alpha *, \beta *, \gamma *$ & $\begin{array}{l}\text { Angles between the pairs of reciprocal unit cell edges } b^{*} c^{*}, c^{*} a^{*} \text {, and } \\
a^{*} b^{*} \text {, respectively }\end{array}$ \\
\hline$d$ & Interplanar spacing \\
\hline$d(h k l)$ & Interplanar spacing of the $(h k l)$ family of planes \\
\hline$d^{*}$ & Distance in reciprocal space \\
\hline$d^{*}(h k l)$ & Distance from the origin to the $h k l$ th reciprocal lattice point \\
\hline$\Delta d$ & Measured error in interplanar spacing \\
\hline$f_{j, \theta}$ & Atomic scattering factor \\
\hline$F(h k l)$ & Structure factor \\
\hline$(h k l),(h k i l)$ & $\begin{array}{l}\text { Miller, Miller-Bravais indices referring to the } x, y, z \text { and } x, y, u \text { and } z \\
\text { axes, respectively }\end{array}$ \\
\hline$\{h k l\}$ & Form of $(h k l)$ planes \\
\hline$h k l$ & $\begin{array}{l}\text { Reciprocal lattice point (or reflection) corresponding to the }(h k l) \text { family } \\
\text { of planes }\end{array}$ \\
\hline [UVW] & Zone or direction symbol \\
\hline$<\mathrm{UVW}>$ & Form of zone axes or directions \\
\hline$x, y, u, z$ & Crystallographic axes \\
\hline
\end{tabular}




\section{Units for Magnetic Properties}

S
$H$
$B$
$M$
$m$
$\chi$
$\chi$
$\mu$

$\begin{array}{ll}H & \text { Magnetic field strength } \\ B & \text { Magnetic induction } \\ M & \text { (Mass) magnetisation } \\ m & \text { Magnetic moment } \\ \chi & \text { (Volume) susceptibility } \\ \chi_{\rho} & \text { (Mass) susceptibility } \\ \mu & \text { Permeability }\end{array}$

Units (SI)

$\mathrm{A} \mathrm{m}^{-1}$

$\mathrm{T}$ (tesla)

$\mathrm{A} \mathrm{m}^{-2} \mathrm{~kg}^{-1}$

$\mathrm{A} \mathrm{m}^{2}$

dimensionless

$\mathrm{m}^{3} \mathrm{~kg}^{-1}$

$\mathrm{H} \mathrm{m}^{-1}$

\section{Symbols}

\begin{tabular}{ll} 
A & Absorbance \\
$a$ & Potential well width \\
$b$ & Interatomic spacing \\
$C$ & Concentration \\
$c$ & Speed of light \\
$D$ & Diffusion constant \\
$D_{h k l}$ & Crystallite length perpendicular to the $(h k l)$ planes \\
$E$ & Energy \\
$E_{g}$ & Band gap \\
$e$ & Charge of an electron \\
$\mathrm{G}$ & Reflection $h k l$ \\
$\mathbf{g}$ & Vector between 000 spot from direct beam and G \\
$H$ & Planck's constant \\
$H_{c}$ & Coercivity \\
$I$ & Intensity \\
$I_{(h k l)}$ & Intensity of the $h k l$ th reflection \\
$K$ & Magnetocrystalline anisotropy constant \\
& Scherrer constant \\
$L$ & Camera constant \\
$L_{(x)}$ & Langevin function \\
$l$ & Orbital angular quantum number \\
$M_{r}$ & Remanent magnetisation \\
$M_{s}$ & Saturation magnetisation \\
$m$ & Mass \\
$m_{l}$ & Magnetic quantum number \\
$m_{s}$ & Spin magnetic quantum number \\
$N_{v}$ & Number density \\
$P$ & Probability \\
$p$ & Momentum \\
$n$ & Principal quantum number \\
$R$ & Gas constant \\
$r$ & Radius \\
$\mathbf{S}$ & Relative intensity ratio \\
$S$ & Scattering vector \\
& Supersaturation \\
& Alpsolute) temperature \\
\hline &
\end{tabular}




$\begin{array}{ll}T_{\beta} & \text { Beta-transition temperature of NiAs-type } \mathrm{Fe}_{1-x} \mathrm{~S} \\ T_{\gamma} & \text { Gamma-transition temperature of NiAs-type } \mathrm{Fe}_{1-x} \mathrm{~S} \\ t & \text { Time } \\ U & \text { Potential energy } \\ \mathrm{V} & \text { Voltage } \\ V_{\mathrm{m}} & \text { Molar volume } \\ X & \text { Concentration (weight percent) } \\ x & x \text { coordinate } \\ \beta & \text { Semiangle of collection } \\ \gamma & \text { Surface tension } \\ \Delta f V & \text { Energy barrier } \\ \Delta G_{v} & \text { Gibbs function for crystallisation } \\ \eta & \text { Refractive index } \\ \theta & \text { Bragg angle } \\ \lambda & \text { Wavelength } \\ \lambda_{\max } & \text { Wavelength of absorption/emission maximum } \\ \mu & \text { Chemical potential } \\ \mu_{B} & \text { Reduced mass } \\ v_{0} & \text { Bohr magneton } \\ \sigma & \text { Attempt frequency factor } \\ \sigma_{f} & \text { Standard deviation } \\ \sigma_{p d} & \text { Ferrimagnetic contribution to magnetisation } \\ \Phi_{F} & \text { Para- and diamagnetic contribution to magnetisation } \\ \phi & \text { Fluorescence quantum yield } \\ \chi & \text { Incident angle } \\ \psi & \text { Root of spherical Bessel function } \\ \omega & \text { Wave function } \\ \hbar & \text { Scattering angle } \\ & \text { True angular breadth of reflection } h k l \\ & =h / 2 \pi \\ & \end{array}$




\section{Table of Contents}

Chapter 1 Introduction .............................................................. 1

1.1 Growth and stabilisation of colloidal nanocrystals ...................................... 4

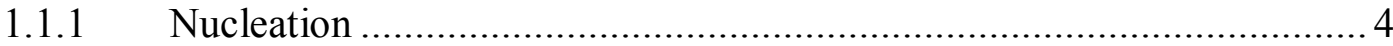

1.1.1.1 LaMer burst nucleation ............................................................. 4

1.1.1.2 Seeded-growth mechanism........................................................... 5

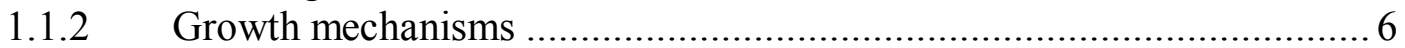

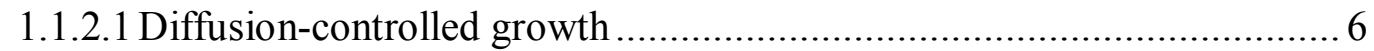

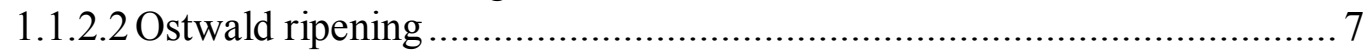

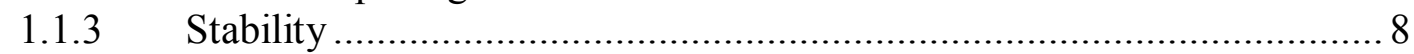

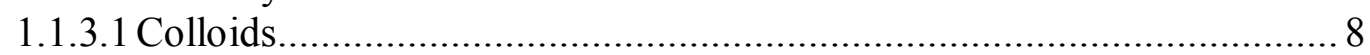

1.1.3.2 Charge and kinetic stabilisation of colloids....................................... 8

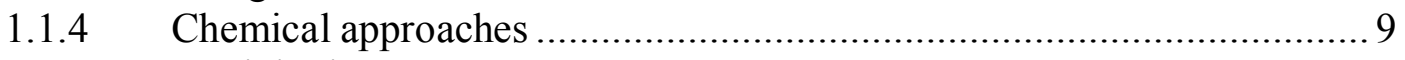

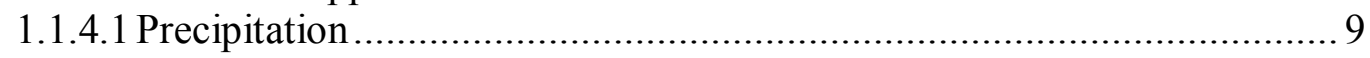

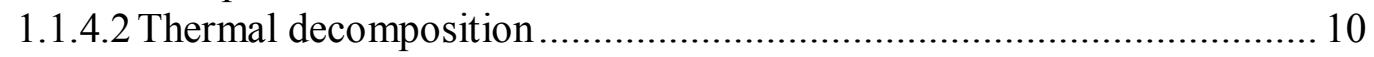

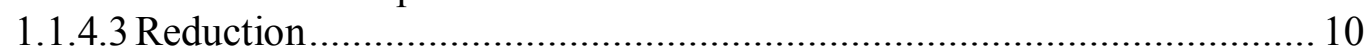

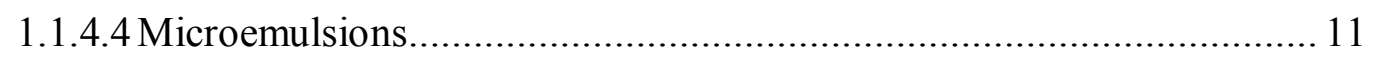

1.2 Physical properties of nanocrystals .................................................... 12

1.2.1 Electronic structure of low-dimensional semiconductors.................... 12

1.2.1.1 Electronic band structure ............................................................... 12

1.2.1.2 Quantum confinement (Particle-in-a-box theory) .............................. 13

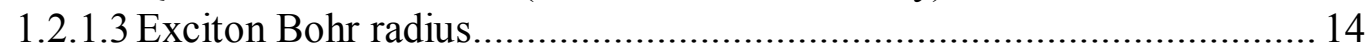

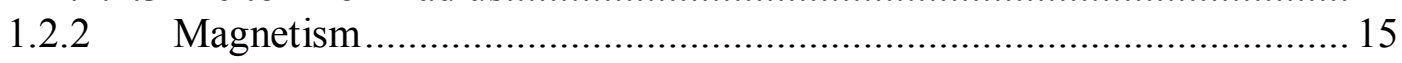

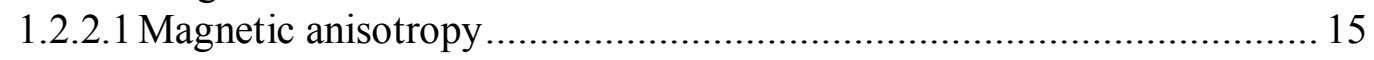

1.2.2.2 Magnetic domains and domain walls ............................................. 16

1.2.2.3 Magnetisation and demagnetisation processes ................................. 17

1.2.2.4 Single domain particles .............................................................. 18

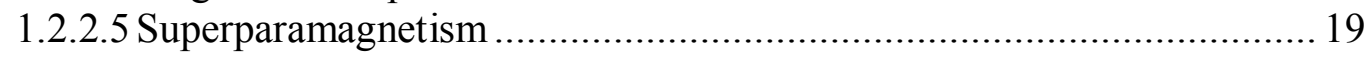

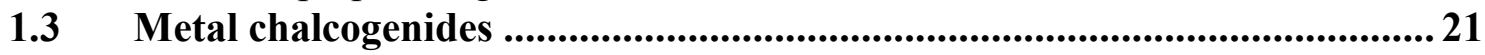

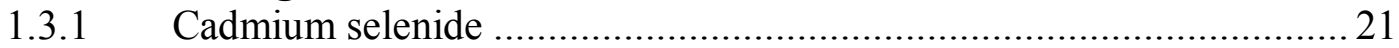

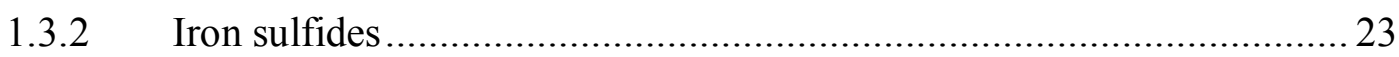

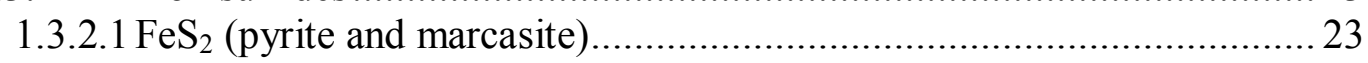

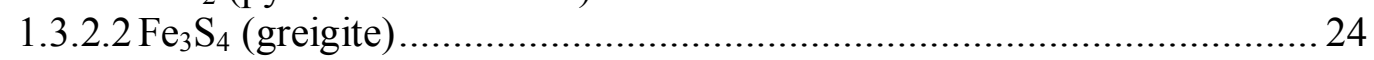

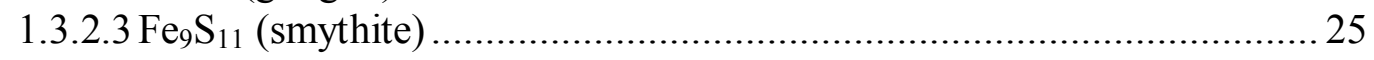

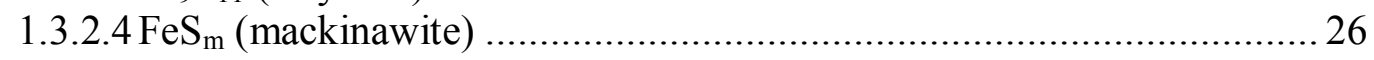

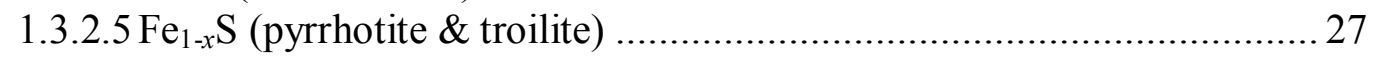

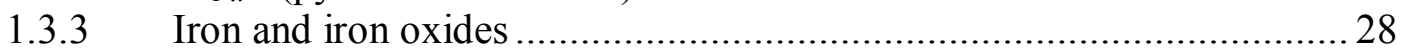

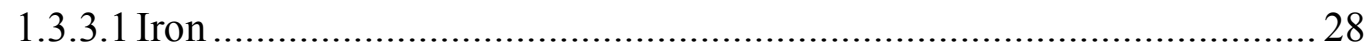

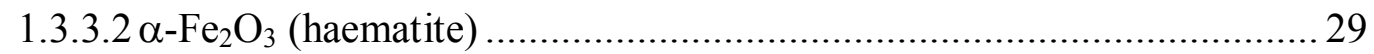

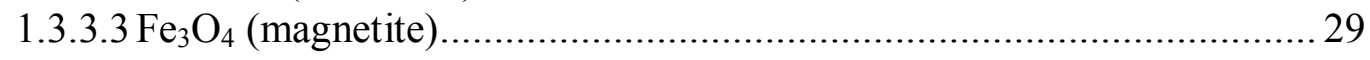

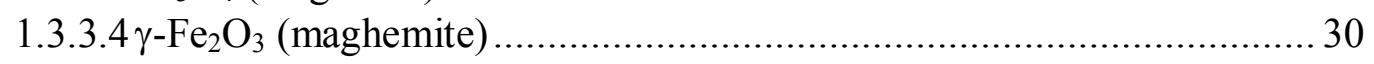

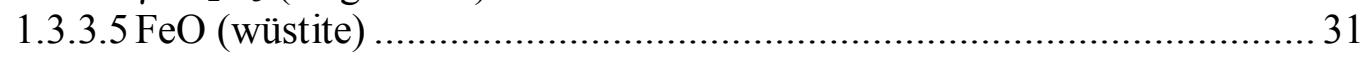

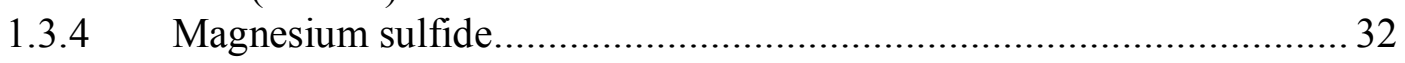

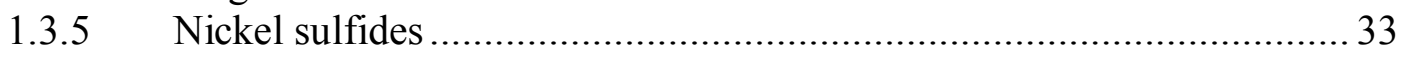

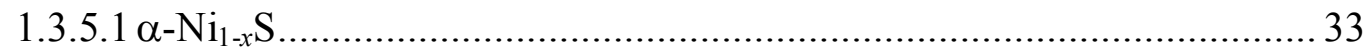

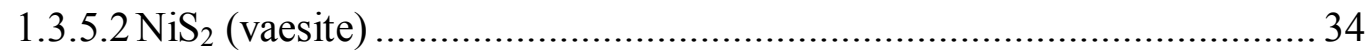

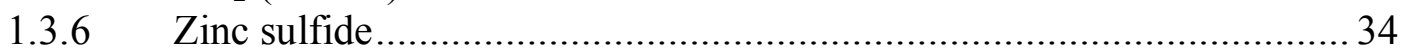

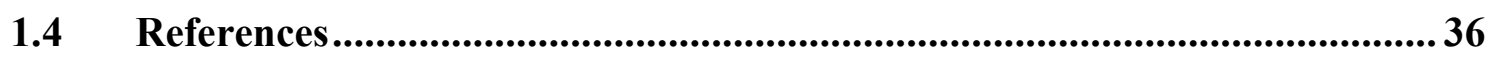




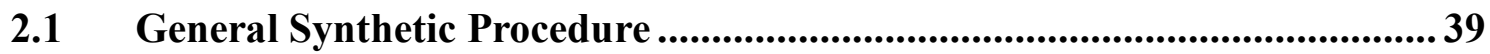

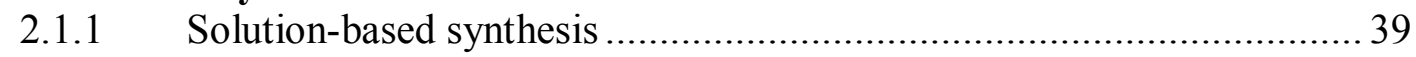

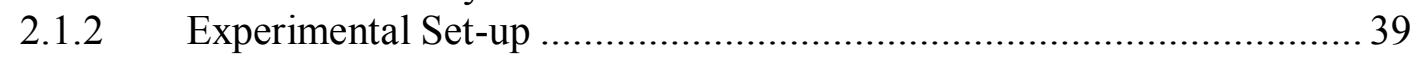

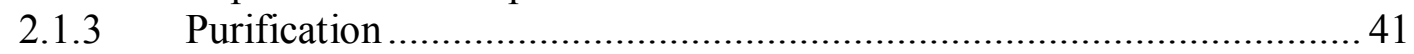

2.2 Characterisation Procedure .......................................................................... 42

2.2.1 Powder X-ray Diffraction (XRD) theory ......................................... 42

2.2.1.1 Diffraction of X-rays by a crystal ................................................ 42

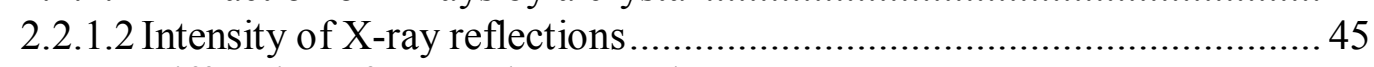

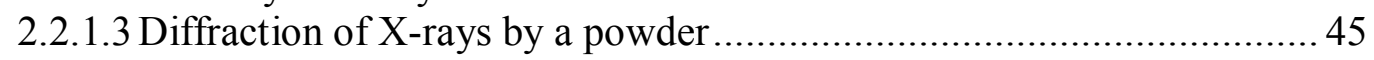

2.2.1.4 Line shape of X-ray reflections ................................................... 47

2.2.1.5 Information gathered from X-ray diffraction experiments................... 48

2.2.2 Acquisition and analysis of XRD patterns ...................................... 49

2.2.2.1 Instrumentation and sample preparation ....................................... 49

2.2.2.2 Qualitative phase analysis ........................................................ 51

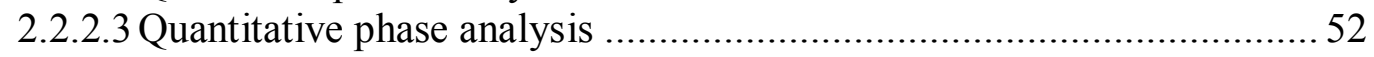

2.2.2.4 Semiquantitative analysis .............................................................. 52

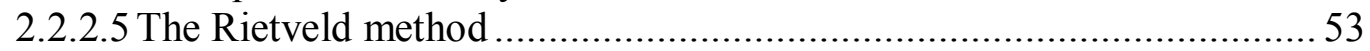

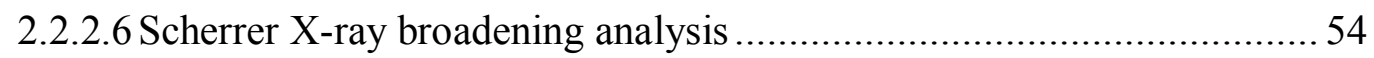

2.2.2.7 Simulation of diffraction patterns and crystal morphologies. ............... 55

2.2.3 Transmission Electron Microscopy (TEM) ....................................... 57

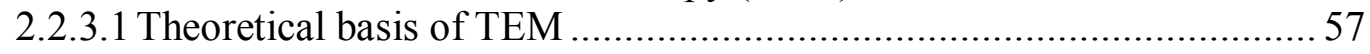

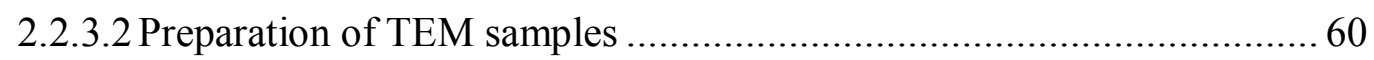

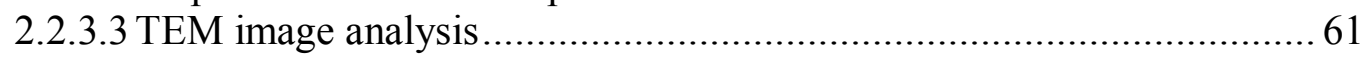

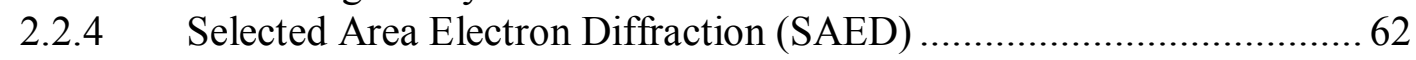

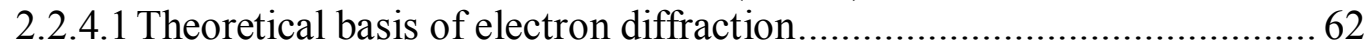

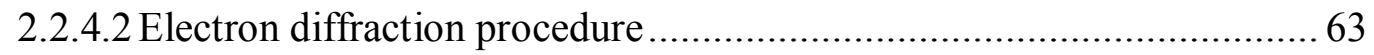

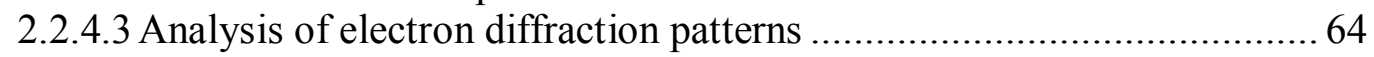

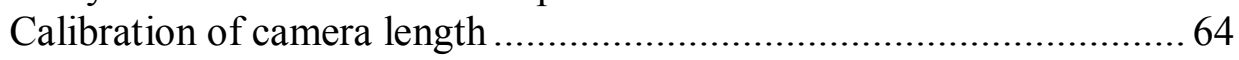

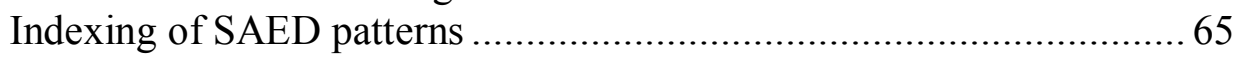

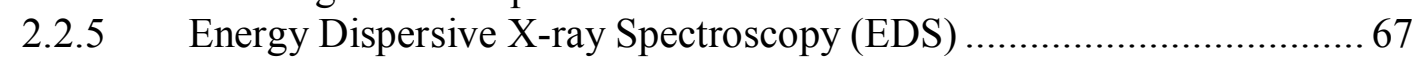

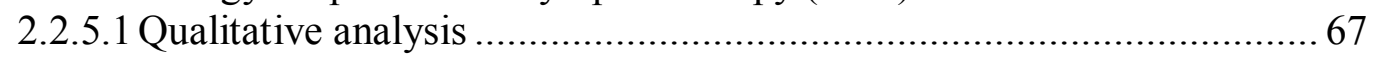

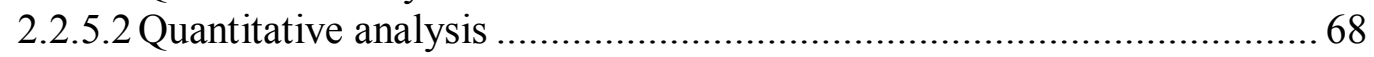

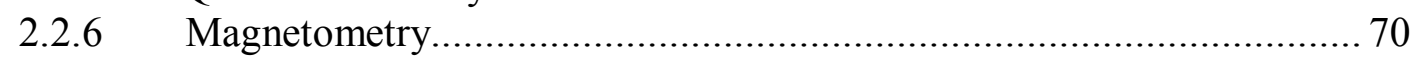

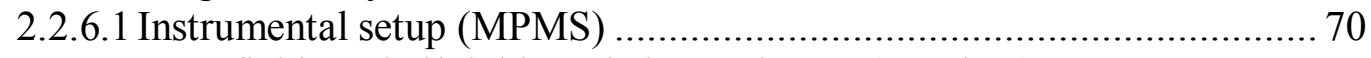

2.2.6.2 Zero-field-cooled/Field-cooled experiment $(Z F C / F C)$....................... 71

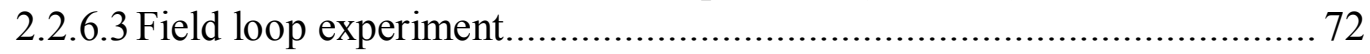

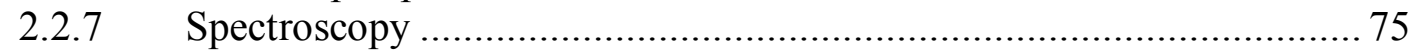

2.2.7.1 Ultraviolet-visible (UV-vis) spectroscopy ..................................... 75

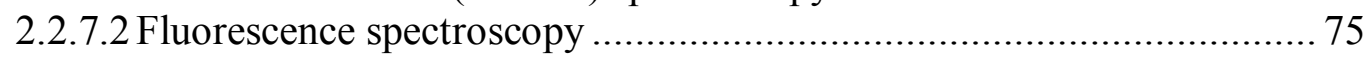

2.2.8 Determination of quantum yield.................................................. 76

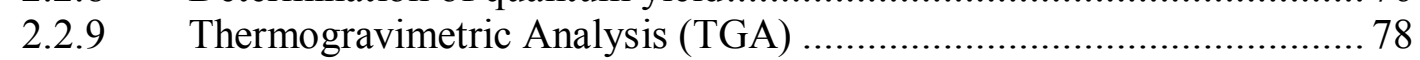

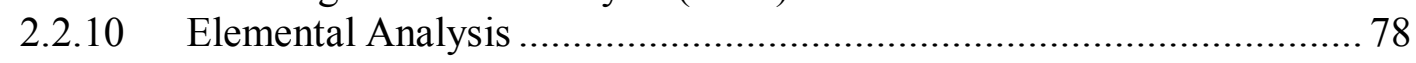

$2.3 \quad$ References ....................................................................................... 79 


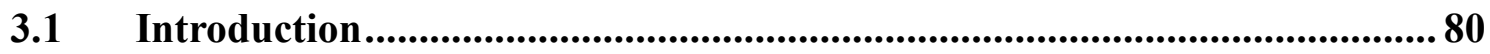

3.1.1 Quantum confinement in cadmium chalcogenide nanocrystals ............ 80

3.1.1.1 Synthesis of cadmium selenide nanocrystals..................................... 81

3.1.2 Capping of cadmium selenide nanocrystals with a wide-band-gap semiconductor......................................................................... 82

3.1.3 Solubilisation of cadmium selenide nanocrystals in aqueous media...... 84

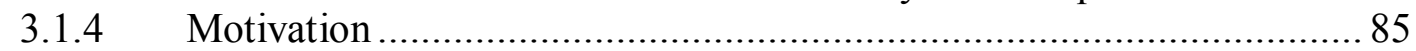

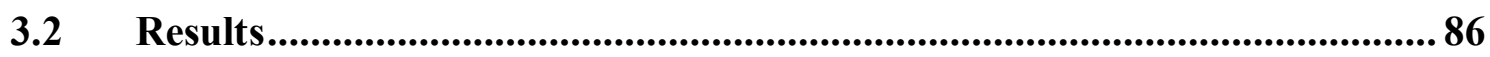

3.2.1 Evolution of cadmium selenide nanocrystal optical properties with

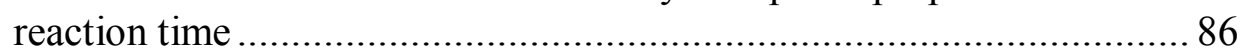

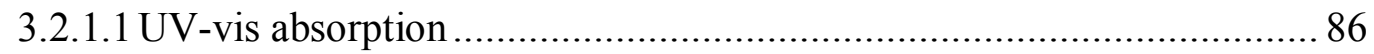

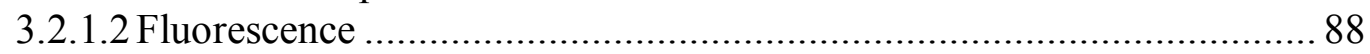

3.2.2 TEM examination of cadmium selenide nanocrystal samples ............... 90

3.2.3 Quantum yield of cadmium selenide nanocrystals .............................. 94

3.2.4 Capping of cadmium selenide nanocrystals with zinc sulfide ............. 101

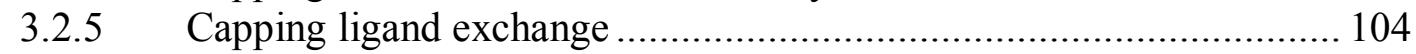

3.3 Discussion ............................................................................................. 106

3.3.1 Evolution of the optical properties of cadmium selenide nanocrystals 106

3.3.2 Quantum yield of cadmium selenide nanocrystals ........................... 108

3.3.3 Capping of cadmium selenide nanocrystals with zinc sulfide and capping ligand exchange .................................................................... 109

3.3.3.1 Capping of cadmium selenide nanocrystals with zinc sulfide............. 109

3.3.3.2 Capping ligand exchange ............................................................ 110

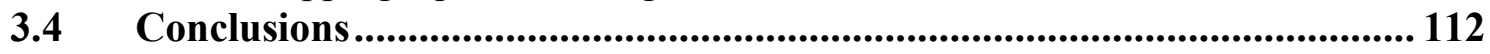

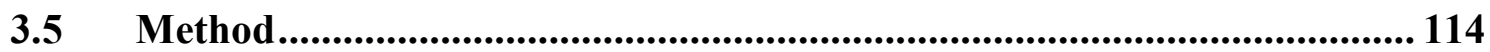

3.5.1 Synthesis of cadmium selenide nanocrystals ................................... 114

3.5.2 Measurement of optical properties of cadmium selenide or $\mathrm{CdSe} / \mathrm{ZnS}$

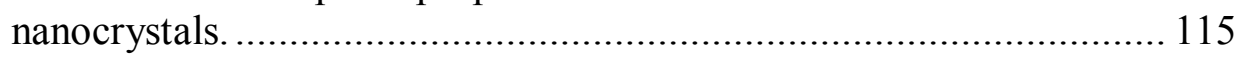

3.5.3 Quantum yield measurements ................................................ 115

3.5.3.1 Preparation of reference dye and cadmium selenide quantum dot

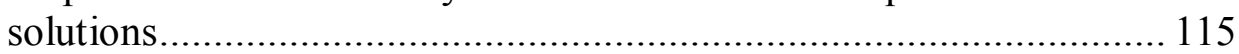

3.5.3.2 Optical measurements performed on cadmium selenide nanocrystal and

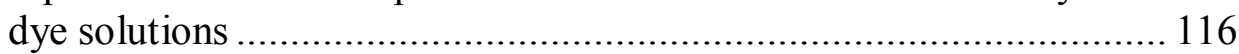

3.5.4 Capping of cadmium selenide nanocrystals with zinc sulfide ............. 116

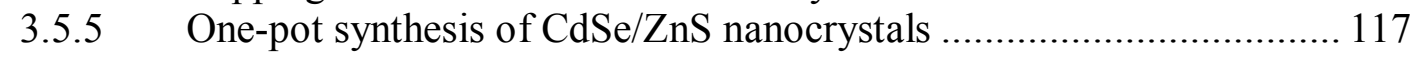

3.5.6 Capping ligand exchange reactions ................................................. 117

3.5.6.1 Capping of cadmium selenide nanocrystals with cysteine................. 117

3.5.6.2 Capping of cadmium selenide nanocrystals with mercaptopropionic acid

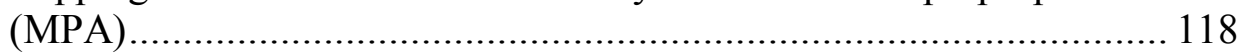

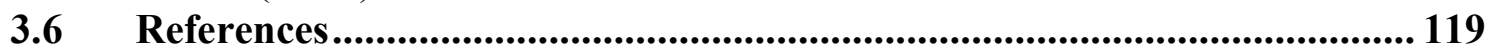




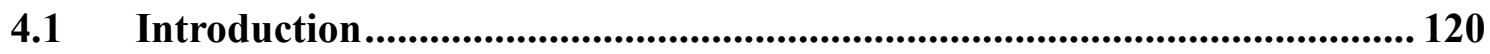

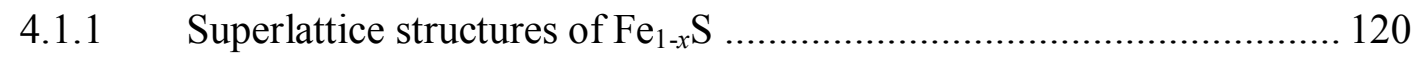

4.1.2 Formation and interconversion of iron sulfide phases......................... 122

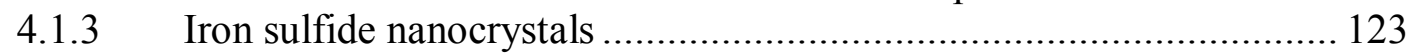

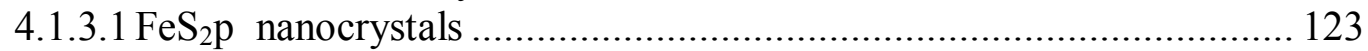

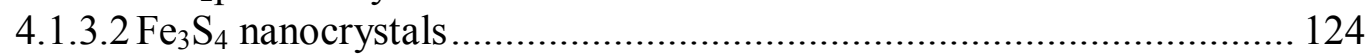

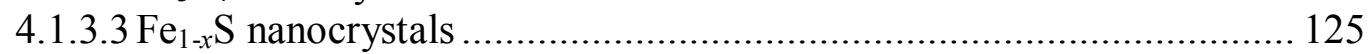

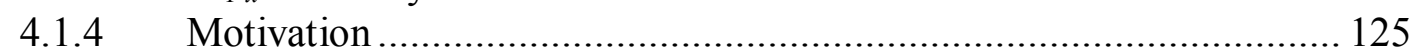

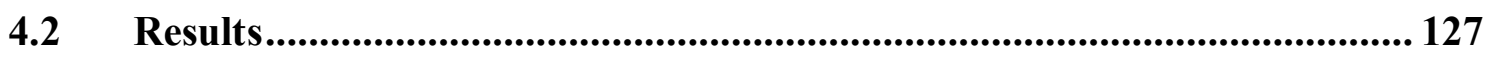

4.2.1 Quantification of iron sulfide nanocrystal phases by XRD ………..... 127

4.2.2 Reaction of $\mathrm{Fe}(\mathrm{acac})_{2}$ and sulfur in oleylamine ................................ 130

4.2.2.1 Reactions at temperatures below $200^{\circ} \mathrm{C}$.......................................... 130

4.2.2.2 Reactions at temperatures between 200 and $250^{\circ} \mathrm{C}$ for a duration of 30

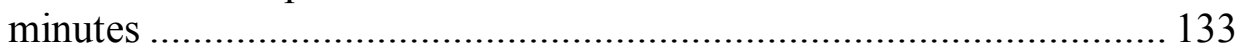

4.2.2.3 Reaction of $\mathrm{Fe}(\mathrm{acac})_{2}$ and sulfur in oleylamine at $200^{\circ} \mathrm{C}$ for $>30$ minutes

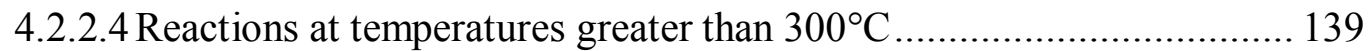

4.2.3 Reaction of $\mathrm{Fe}(\mathrm{acac})_{2}$ in oleylamine with injection of sulfur solution.. 144

4.2.4 Reaction of $\mathrm{FeCl}_{2}$ in oleylamine with injection of sulfur solution....... 148

4.2.5 Reaction of $\mathrm{Fe}(\mathrm{acac})_{2}$ and sulfur in hexadecylamine......................... 152

4.2.6 Addition of $\mathrm{FeCl}_{2}$ into solution of sulfur in oleylamine ..................... 155

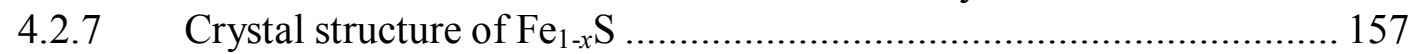

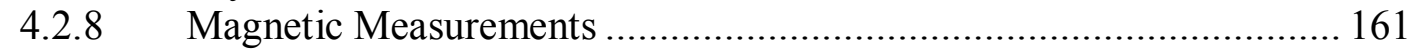

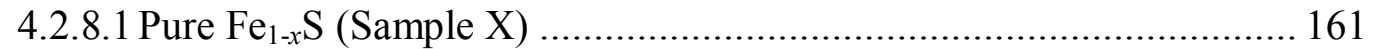

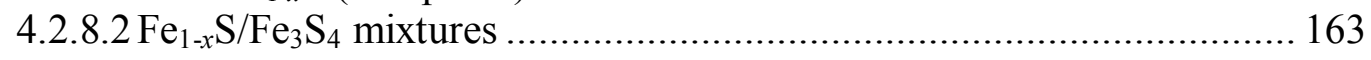

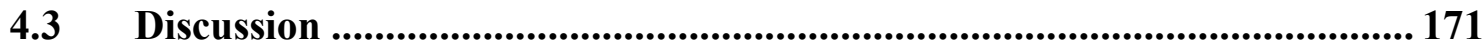

4.3.1 Synthesis of iron sulfide nanocrystals ……………….................... 171

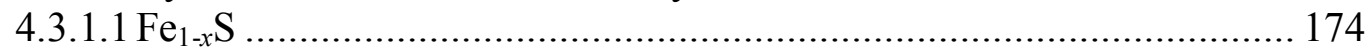

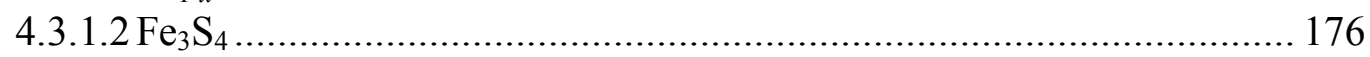

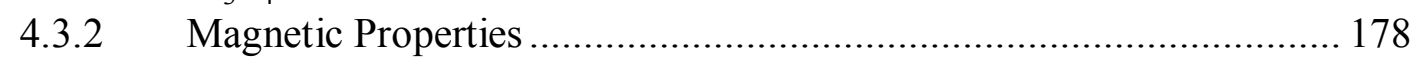

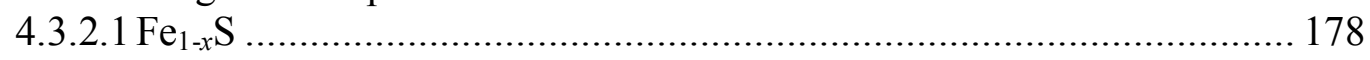

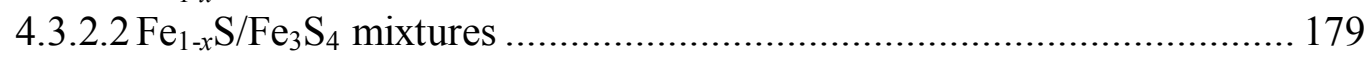

4.4 Conclusions..................................................................................................... 182

4.5 Method.......................................................................................................... 183

4.5.1 Reaction of $\mathrm{Fe}(\mathrm{acac})_{2}$ and sulfur in oleylamine .............................. 183

4.5.2 Reaction of $\mathrm{Fe}(\mathrm{acac})_{2}$ in oleylamine with injection of sulfur solution. 184

4.5.3 Reaction of $\mathrm{FeCl}_{2}$ in oleylamine with injection of sulfur solution....... 184

4.5.4 Reaction of $\mathrm{Fe}(\mathrm{acac})_{2}$ and sulfur in hexadecylamine......................... 185

4.5.5 Addition of $\mathrm{FeCl}_{2}$ into solution of sulfur in oleylamine ...................... 185

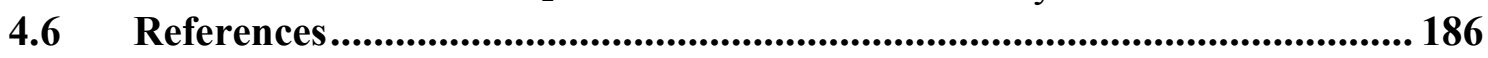




\section{Chapter 5 Sulfidisation or oxidation of iron/iron oxide}

\section{nanocrystals ...........................................................188}

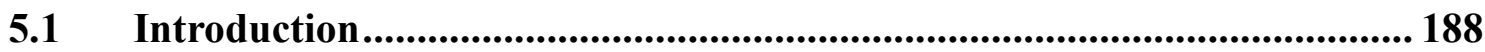

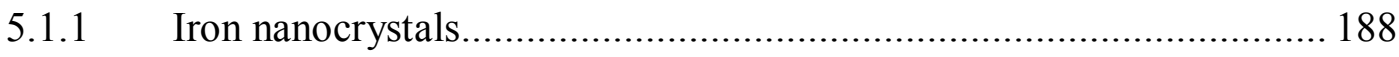

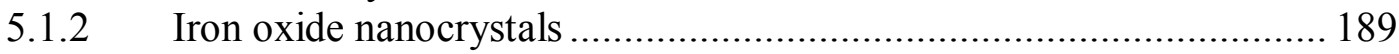

5.1.3 Distinguishing experimentally between $\mathrm{Fe}_{3} \mathrm{O}_{4}$ and $\gamma-\mathrm{Fe}_{2} \mathrm{O}_{3} \ldots \ldots \ldots \ldots . . . .191$

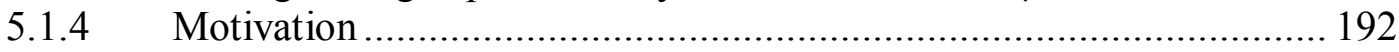

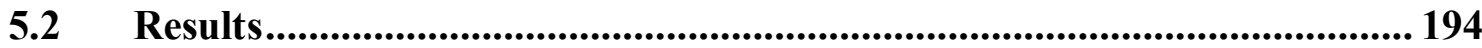

5.2.1 Synthesis of iron oxide nanocrystals .............................................. 194

5.2.1.1 Effect of reaction time and temperature ......................................... 194

5.2.1.2 Effect of replenishing iron precursor during reaction ....................... 199

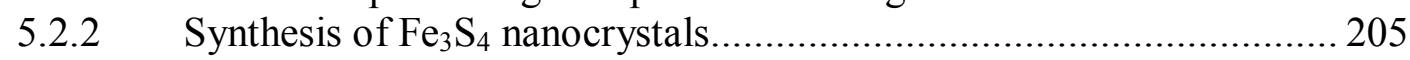

5.2.2.1 Sulfidisation of iron/iron oxide nanocrystals.................................. 205

5.2.2.2 Sulfidisation of iron/iron oxide nanocrystals followed by size-selective

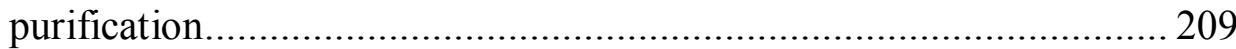

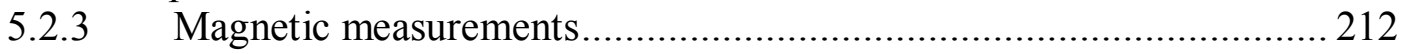

5.2.3.1 Iron oxide spinel nanocrystals (Sample XXIII) ............................. 212

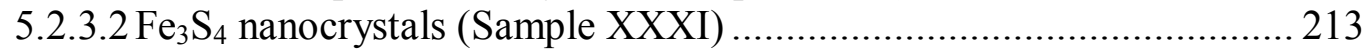

5.3 Discussion ........................................................................................... 215

5.3.1 Synthesis of iron oxide nanocrystals ........................................... 215

5.3.2 Synthesis of iron sulfide nanocrystals .......................................... 217

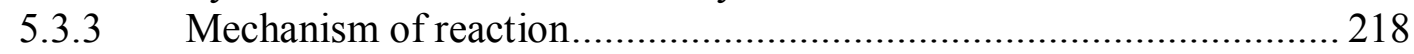

5.3.3.1 First proposed pathway $-\mathrm{Fe}_{3} \mathrm{O}_{4}$ as the intermediate ................................ 220

5.3.3.2 Second proposed pathway - elemental iron as the intermediate .......... 221

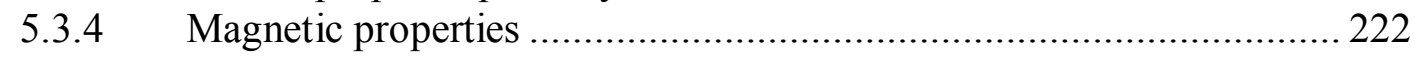

5.3.4.1 Iron oxide spinel nanocrystals (Sample XXIII) .............................. 222

5.3.4.2 $\mathrm{Fe}_{3} \mathrm{~S}_{4}$ nanocrystals (Sample XXXI) ........................................... 223

5.3.4.3 Comparision of $\mathrm{Fe}_{3} \mathrm{O}_{4}$ and $\mathrm{Fe}_{3} \mathrm{~S}_{4}$ nanocrystals .............................. 224

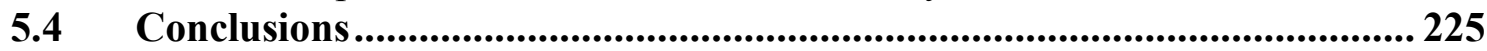

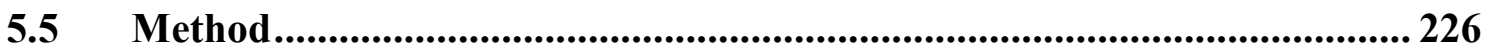

5.5.1 General synthesis of iron oxide nanocrystals .................................. 226

5.5.2 Synthesis of iron oxide nanocrystals involving replenishment of

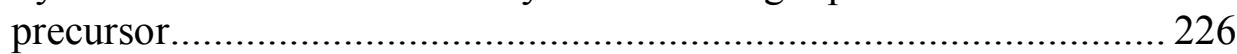

5.5.3 General synthesis of $\mathrm{Fe}_{3} \mathrm{~S}_{4}$ nanocrystals ....................................... 227

5.5.4 Synthesis of $\mathrm{Fe}_{3} \mathrm{~S}_{4}$ nanocrystals with rapid cooling …..................... 227

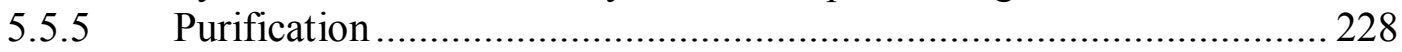

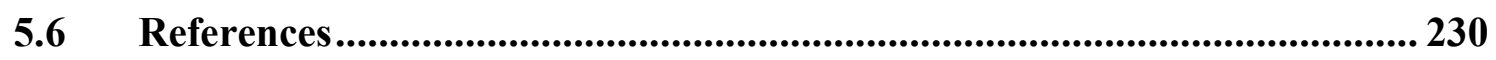




\section{Chapter 6 Single-source metal sulfide precursors for}

nanocrystal synthesis

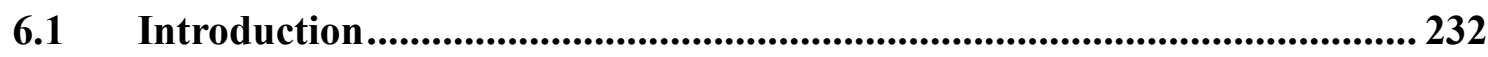

6.1.1 Single-source metal sulfide precursors .............................................. 232

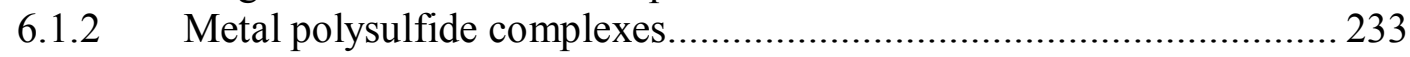

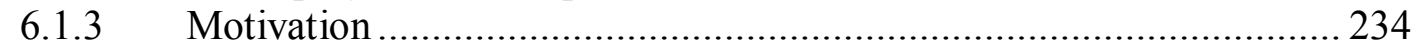

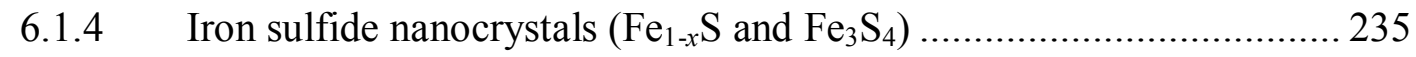

6.1.5 Zinc sulfide nanocrystals $(\mathrm{ZnS})$.................................................... 235

6.1.6 Nickel sulfide nanocrystals $\left(\mathrm{NiS}_{2}\right.$ and $\left.\alpha-\mathrm{Ni}_{1-x} \mathrm{~S}\right)$.............................2 235

$6.2 \quad$ Results.......................................................................................................... 237

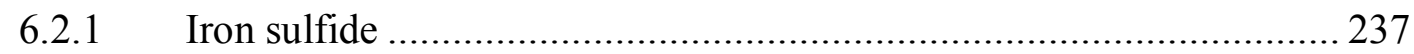

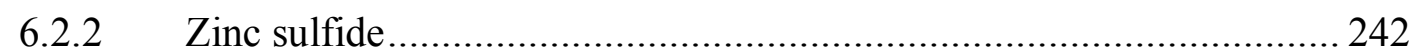

6.2.3 Magnesium sulfide/Magnesium oxide …………………………........ 251

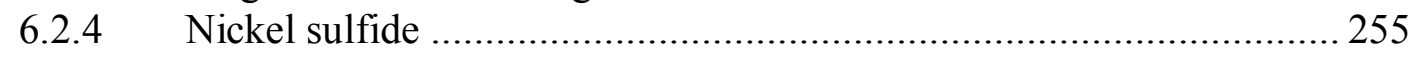

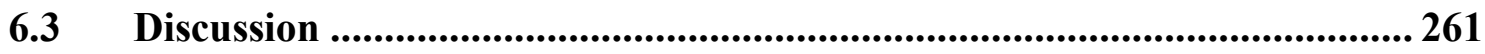

6.3.1 Formation of $N$-methylimidazolato metal polysulfide complexes .......261

6.3.1.1 Mechanism of complex formation ...................................................... 261

6.3.2 Decomposition of $N$-methylimidazolato metal polysulfide complexes 263

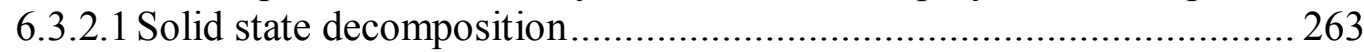

6.3.2.2 Decomposition in solution............................................................... 264

6.3.3 $\mathrm{N}$-methylimidazolato metal polysulfide precursors as single-source

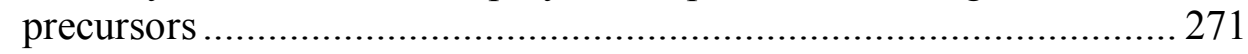

6.4 Conclusions................................................................................................ 272

6.5 Method............................................................................................................ 273

6.5.1 Single-source iron sulfide precursor - $\left[\mathrm{Fe}(N \text {-MeIm })_{6}\right] \mathrm{S}_{8} \ldots \ldots \ldots \ldots \ldots \ldots \ldots . . . . . . . . .273$

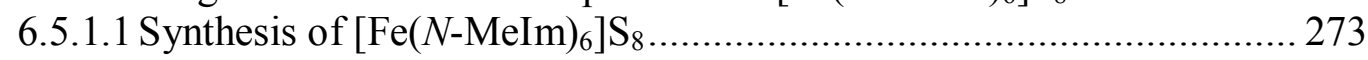

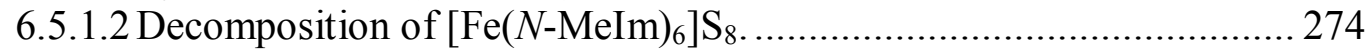

6.5.2 Single-source zinc sulfide precursor $-\mathrm{ZnS}_{6}(\mathrm{~N} \text {-MeIm })_{2} \ldots \ldots \ldots \ldots \ldots \ldots \ldots . . .275$

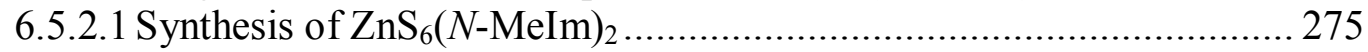

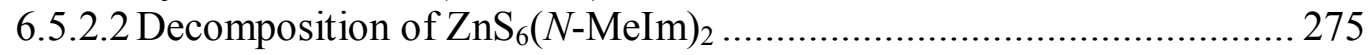

6.5.3 Single-source magnesium sulfide precursor - $\left[\mathrm{Mg}(N-\mathrm{MeIm})_{6}\right] \mathrm{S}_{8} \ldots \ldots .277$

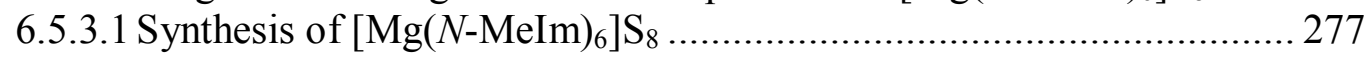

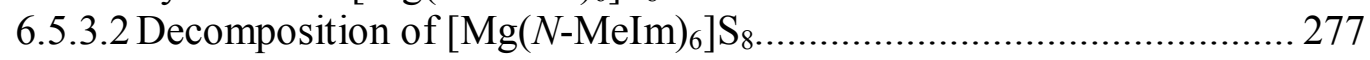

6.5.4 Single-source nickel sulfide precursor - $\left[\mathrm{Ni}(N-\mathrm{MeIm})_{6}\right] \mathrm{S}_{8} \ldots \ldots \ldots \ldots \ldots \ldots . .278$

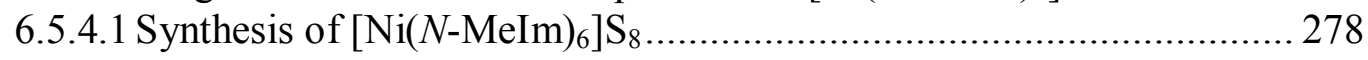

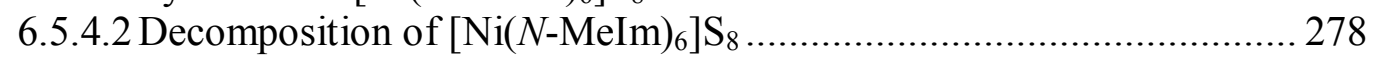

6.6 References.................................................................................................... 280 
7.1 Chapter 3 Synthesis of cadmium selenide nanocrystals........................... 282

7.2 Chapter 4 Synthesis of iron sulfide nanocrystals ..................................... 284

7.3 Chapter 5 Sulfidisation or oxidation of iron/iron oxide nanocrystals ....... 287

7.4 Chapter 6 Single-source metal sulfide precursors for nanocrystal synthesis ...... 290

7.5 Chapter 6 Single-source metal sulfide precursors for nanocrystal synthesis - future work ....................................................................................... 292

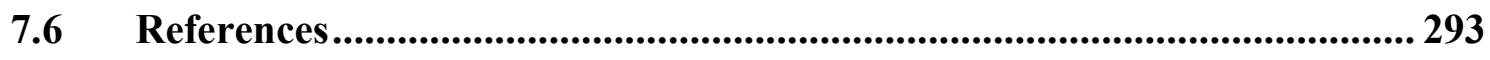




\section{Chapter 1 Introduction}

The past few decades have seen a rapid growth in interest in nanomaterials - materials with nanometre-sized features - spawned by the realisation that the surface, electronic, magnetic and other physical properties of materials are altered as their dimensions are reduced. The terms nanocrystal and nanoparticle are used somewhat interchangeably as catch-all terms for materials with all dimensions less than $100 \mathrm{~nm} .{ }^{1}$ This size boundary, although arbitrary, serves as a useful bench post for judging the size range within which size-dependent effects come to the fore.

This interest in the size-dependent properties of nanocrystals drove our research in the areas of synthesis and characterisation of metal chalcogenide nanocrystals. It was the aim of the project to develop synthetic techniques for metal chalcogenides, for which well-developed nanocrystal syntheses were not present in the literature, and to characterise the resulting samples with electron microscopy, diffraction techniques, optical spectroscopy and magnetic measurements as appropriate (see Chapter 2 Experimental Methods, for details of synthesis and characterisation techniques).

The many synthetic routes towards nanocrystals which have been investigated fall into the broad categories of physical and chemical techniques. The former term covers a variety of methodologies, such as laser ablation and glass annealing, ${ }^{2}$ which take place in the vapour and solid phases, respectively. While the latter term - in opposition to physical methods - embraces all methodologies based around the growth of nanocrystals as colloids in the solution phase (i.e. sols). ${ }^{1}$ Chemical methodology offers the benefits of simplicity, low cost, as well as the prospect of achieving a high degree of uniformity of size and shape. ${ }^{1}$ As it is chemical methodology that was used in this project, relevant aspects of theory concerning the growth and stabilisation of colloidal nanocrystal suspensions will be dealt with below (see section 1.1).

Among the physical and chemical properties affected by the restricted dimensions of nanocrystals are: the electronic structure and the magnetic domain structure. As these were the properties specifically investigated in this project, necessary theoretical discussions are included below: 
The response of the electronic structure of a semiconductor nanocrystal as its dimensions approach the exciton Bohr radius of the material is termed the quantum confinement effect, and leads to a strongly size-dependent band gap and hence a size-dependent optical absorption and emission profile. The theoretical basis of the quantum confinement effect will be reviewed in section 1.2.1.

As the dimensions of an ordered magnetic material are reduced, it passes from having multiple magnetic domains to being a single magnetic domain, and eventually to a situation where thermal energy is sufficient to overcome the barriers confining the magnetisation to a certain direction - a superparamagnetic state. This has consequences for various critical material magnetic parameters. The general theory of magnetism in materials, and specific aspects of magnetism in low-dimensional materials such as nanocrystals are reviewed below in section 1.2.2.

The general physical and chemical properties of the metal chalcognide phase systems encountered in this project are reviewed in section 1.3. These are namely: the cadmium selenide, iron sulfide, iron oxide, magnesium sulfide, nickel sulfide and zinc sulfide systems. More specific information is contained in the introduction sections of the relevant results chapters.

In Chapter 3, titled 'Synthesis of cadmium selenide nanocrystals', established literature methods for the synthesis of fluorescent cadmium selenide semiconductor nanocrystals were used as a starting point to develop skill in nanocrystal synthesis, purification and characterisation techniques. The size-dependent optical properties of cadmium selenide nanocrystals were investigated by correlating features of the absorption and emission spectra with particle size and size distribution data derived from TEM studies. In addition, the capping of the cadmium selenide nanocrystals with a layer of the wide-band gap semiconductor zinc sulfide, and the transfer of the nanocrystals from non-polar to polar media was undertaken. Finally, the quantum yield of the cadmium selenide nanocrystals was determined by comparison to fluorescent dye standards.

In Chapter 4, titled 'Synthesis of iron sulfide nanocrystals', the synthesis of iron sulfide nanocrystals from iron salts and elemental sulfur was investigated. Two crystalline iron sulfide phases $-\mathrm{Fe}_{1-x} \mathrm{~S}$ and $\mathrm{Fe}_{3} \mathrm{~S}_{4}$ - were encountered, and experimental parameters which affected the ratio of the two phases, crystalline morphology and particle size were 
investigated. The resulting samples, containing either pure $\mathrm{Fe}_{1-x} \mathrm{~S}$ or mixtures of $\mathrm{Fe}_{1-x} \mathrm{~S}$ and $\mathrm{Fe}_{3} \mathrm{~S}_{4}$ were subjected to magnetic measurements.

In Chapter 5, titled 'Sulfidisation or oxidation of iron/iron oxide nanocrystals', the work contained in Chapter 4 was extended in order to produce phase-pure samples of small $(<10 \mathrm{~nm})$ spherical $\mathrm{Fe}_{3} \mathrm{~S}_{4}$ nanocrystals. It was found that an induction time, where the iron salt was thermolysed in the absence of sulfur, led to the formation of monodisperse intermediate nanocrystals (postulated to be either elemental iron or $\mathrm{Fe}_{3} \mathrm{O}_{4}$ ), which could either be sulfidised to $\mathrm{Fe}_{3} \mathrm{~S}_{4}$, or oxidised by air to iron oxide spinel $\left(\mathrm{Fe}_{3} \mathrm{O}_{4} / \gamma-\mathrm{Fe}_{2} \mathrm{O}_{3}\right)$. The magnetic properties of samples of $\mathrm{Fe}_{3} \mathrm{~S}_{4}$ and iron oxide nanocrystals of similar average diameter and size distribution were then investigated.

In Chapter 6, titled 'Single-source metal sulfide precursors for nanocrystal synthesis', amine-stabilised metal polysulfide complexes were synthesised, and their use as single-source precursors for the synthesis of metal sulfide nanocrystals was investigated. Iron-, nickel- and zinc-containing complexes were successfully used to synthesis metal sulfide nanocrystals, however the magnesium-containing complex resulted in magnesium hydroxide particles. 


\subsection{Growth and stabilisation of colloidal nanocrystals}

Theories developed for the formation, growth, and stabilisation of micrometer-sized colloids, have been expanded to deal with nanocrystals during the past 20 years. Firstly, some these theory will be introduced, before the general categories of chemical nanocrystal syntheses and examples from the literature will be discussed.

\subsubsection{Nucleation}

Nuclei are aggregations of monomer building blocks, which have the potential to grow into larger particles. The formation of nuclei is termed nucleation; and may be homogeneous or heterogeneous in nature. Homogeneous nucleation involves the spontaneous formation of nuclei from monomers in solution - a process which faces a large kinetic barrier. Heterogeneous nucleation involves the formation of a nucleus on a pre-existing solid surface - a process which generally faces a lower activation energy barrier.

\subsubsection{LaMer burst nucleation}

While investigating the formation of sulfur suspensions, $\mathrm{LaMer}^{3}$ postulated the necessity of temporal separation of the nucleation and growth phases for the formation of monodisperse colloids. LaMer burst nucleation is described with reference to the LaMer plot shown below (Figure 1-1), which describes the evolution of supersaturation $(S)$ with time, where supersaturation is defined as the ratio between the monomer concentration in the bulk solution and the equilibrium monomer surface concentration.

During Phase I, the supersaturation lies somewhere below the critical supersaturation required for the kinetic barrier to homogenous nucleation to be surpassed and nucleation to occur. During Phase II some event increases the supersaturation of solution above the critical level, and the rate of nucleation increases to some finite level. In the case where further monomer is not supplied to replace that consumed in nucleation, the supersaturation will eventually fall back below the critical supersaturation, causing further nucleation to cease. Phase III is now entered, where monomer continues to be consumed solely by growth of existing particles until eventually equilibrium is reached $(S=1)$. Provided that Phase II (nucleation) is brief, and temporally distinct from Phase 
III (growth), all nuclei emerge from Phase II with a uniform size - a condition identified as a prerequisite for monodispersity of particles at the end of Phase III (growth).

LaMer burst nucleation theory led to the 'hot injection' method utilised in modern nanocrystal synthesis - where supersaturation is induced rapidly by injection of precursor into a preheated solution, such that the resulting burst of nucleation quickly consumes monomer and the supersaturation drops rapidly below the critical level. However, more recently, study of the nucleation and growth of iron oxide nanocrystals via the thermal decomposition of an $\mathrm{Fe}^{3+}$-oleate complex by Hyeon et al. ${ }^{4}$ suggested that even when nucleation is not induced explicitly by rapid injection of precursor into a hot solution, burst nucleation may occur. In the system studied by Hyeon et al., decomposition of the precursor to an active monomer species occurred as the temperature of the solution was increased, but nucleation of iron oxide nanocrystals did not occur until the induction temperature was reached, whereupon nucleation and growth occurred in a manner consistent with the LaMer mechanism.

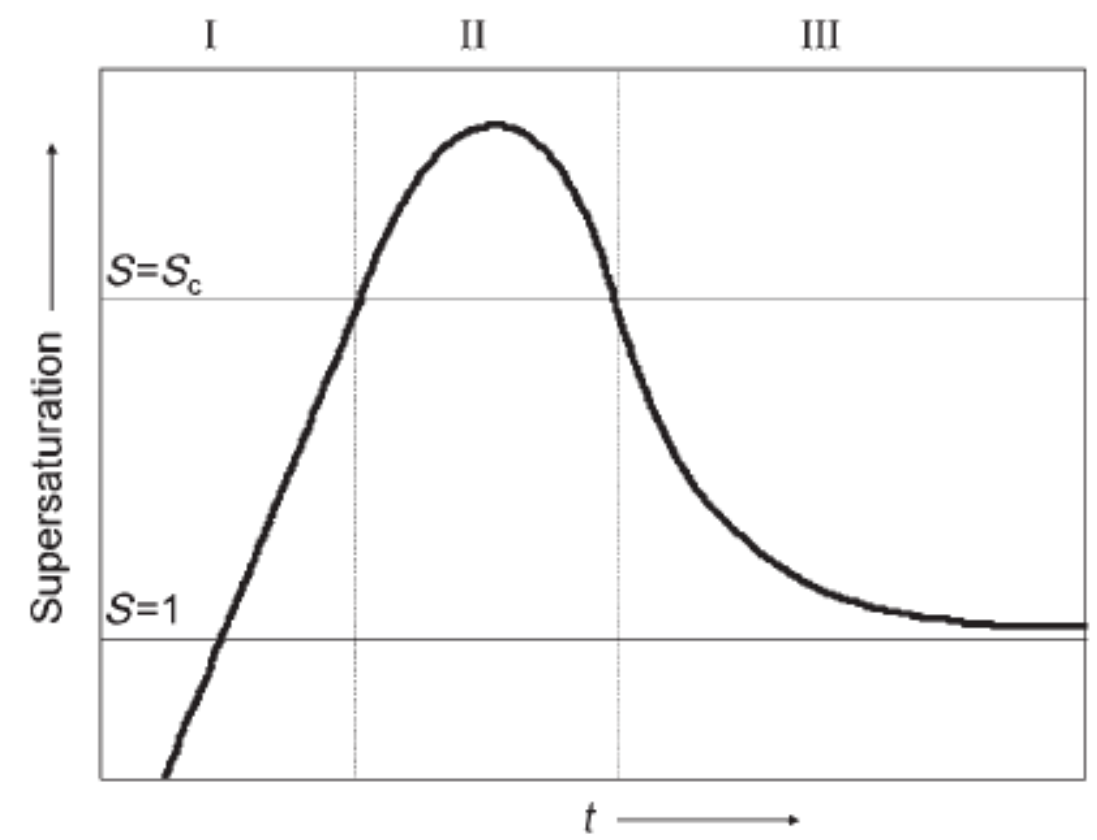

Figure 1-1 LaMer plot describing the change of supersaturation with time during the nucleation and growth of colloidal particles, from Hyeon et al. ${ }^{1}$ Where $S$ is supersaturation, and $S_{c}$ is the critical saturation.

\subsubsection{Seeded-growth mechanism}

An alternative approach is to rely on heterogeneous nucleation onto seed particles. If the concentration of monomer is maintained below the critical saturation, homogeneous 
nucleation is precluded, and growth will be directed solely on to the existing particles. This approach is often used to produce bimetallic, ${ }^{5,6}$ core/shell ${ }^{5,7}$ or highly anisotropic nanocrystals. ${ }^{5}$ In a sense, seeded growth forms the basis of cases where burst nucleation is followed by staggered further additions of monomer to allow continued growth of the existing particles.

\subsubsection{Growth mechanisms}

Nuclei below a certain critical radius, $r_{c}$, are thermodynamically unstable with respect to dissolution, thus over time nuclei smaller than $r_{c}$ tend to redissolve or grow larger than the critical radius by accumulating monomer. The critical radius depends on the balance of the surface energy of the nanocrystal and the supersaturation of the solution. This leads to expression (1), where $\gamma$ is the surface tension, $\Delta G_{v}$ is the Gibbs function for crystallisation, $V_{m}$ is the crystal molar volume, $R$ is the gas constant, $T$ is the absolute temperature and $S$ is the supersaturation.

$$
r_{c}=\frac{-2 \gamma}{\Delta G_{v}}=\frac{2 \gamma V_{m}}{R T \ln S}
$$

\subsubsection{Diffusion-controlled growth}

In the theory initially formulated by Reiss, ${ }^{8}$ the rate of growth of a particle is determined by the flux of monomer arriving at the surface - the diffusion of monomer to the surface being the rate limiting step, rather than reaction of monomer at the surface (reaction-controlled growth). Assuming the particles are separated such that their diffusion layers are undisturbed, the growth rate can be derived from Fick's first law, and represented (2), where $d r / d t$ is the rate of growth, $D$ is the diffusion coefficient, $r$ is the particle radius, and $C_{b u l k}$ and $C_{s}$ are the concentrations of monomer in the bulk solution and at the particle surface, respectively. ${ }^{1}$

$$
\frac{d r}{d t}=\frac{V_{m} D}{r}\left(C_{b u l k}-C_{s}\right)
$$

It can be seen from (2), that the growth rate of a particle is inversely proportional to its radius, hence large particles will grow more slowly than small particles. This inverse dependence on radius arises simply from the fact that the reaction rate is proportional to the surface area, which itself is proportional to the square of the radius; while the 
increase in volume scales with the third powder of the radius. This provides a mechanism whereby the radius distribution of an ensemble of growing particles cannot increase, and may decrease provided that the growth remains diffusion-controlled. ${ }^{1}$ This situation is frequently referred to in the literature as the focusing regime. ${ }^{9}$

\subsubsection{Ostwald ripening}

Diffusion-controlled growth is, however, insufficient to describe fully the growth of very small particles, as the growth rate is actually dependent both on the rate of precipitation and the rate of dissolution - which are affected by the chemical potential of the particles. The chemical potential of small particles is increased with respect to the bulk by the contribution of their surface energy. This is termed the Kelvin effect, and is shown in (3), where $\Delta \mu$, is the difference in chemical potential between the particle and the bulk. As this energy contribution is inversely proportional to radius, smaller particles have higher chemical potential - and as mentioned above are thermodynamically less stable than larger particles - and hence are driven to dissolve. This dissolution of smaller particles in the ensemble provides monomer for further growth of the larger particles thus leading to a broadening or defocusing of the size distribution, in a process which is termed Ostwald ripening. ${ }^{1}$

$$
\Delta \mu=\frac{2 \gamma V_{m}}{r}
$$

The degree of supersaturation is one of the primary growth control factors - controlling the critical radius, and whether diffusion-controlled or reaction-controlled growth is favoured. When supersaturation is high (for instance immediately following burst nucleation), the critical radius is small (see (1)) - hence most nuclei are larger than the critical radius and will grow. High supersaturation also fosters diffusion-controlled growth, which leads to a narrowing of the size distribution - with high supersaturation the system is in the focusing regime.

As the monomer concentration is diminished by the growth process, the supersaturation drops, decreasing the critical radius such that smaller particles in the ensemble may start to dissolve, while the larger particles continue to grow - the system enters the defocusing regime. Further additions of monomers will cause the system to re-enter a focusing regime, while increasing the average particle radius - thus forming the basis of the seeded growth mechanism. However, if the monomer concentration is not 
replenished, simulations suggest the average particle size and size distribution will tend towards an equilibrium value, which is largely independent of the initial nucleus size. ${ }^{10}$

\subsubsection{Stability}

As the stability of colloidal suspensions is an important consideration in the chemical synthesis, purification, characterisation, storage and use of nanocrystals; the theoretical basis and some practical considerations of colloidal stability and stabilisation will be discussed.

\subsubsection{Colloids}

A colloid is a dispersion of particles of a discontinuous phase throughout a continuous medium. As such, all solution-based chemical syntheses of nanocrystals involve colloidal suspensions (sols) during the nucleation and growth phases.

Colloids are inherently thermodynamically less stable than the separated bulk materials due to the relatively large surface energy contribution of the discontinuous phase (see section 1.1.2.2). In addition to this thermodynamic tendency away from small divided particles, attractive van der Waal interactions between the particles provide a direct driving force for colloidal particles to aggregate. ${ }^{11}$

Colloidal particles below a certain size threshold (depending on the density of the particles and the viscosity of the solvent) are able to remain suspended in solution over extended time periods as the gravitational force driving them to settle is counteracted by the viscous drag of the solvent and the random thermal motion of the particles. ${ }^{11,12}$ If particles aggregate in solution, they tend to settle more quickly due to the larger size of the aggregate. In order to prevent aggregation and eventual phase separation, an energy barrier which will counteract the attractive van der Waals forces is required.

\subsubsection{Charge and kinetic stabilisation of colloids}

The barrier which stabilises a colloid may be electrostatic or steric in nature, and may be due to the inherent properties of the particle or due to some species absorbed onto the interface between the discontinuous and continuous phases.

Solid surfaces may possess an innate charge due to a slight imbalance in the number of cations and anions at the surface (for an ionic solid), or because of the ionisation of 
surface groups. ${ }^{11}$ Alternatively, charged surfactants or polymers can be added to adhere to the particle surface. The charged surface exerts an electrostatic field, which is screened by a diffuse layer of ions in solution of opposite polarity - together this system is referred to as the electrical double layer. ${ }^{11}$ Electrostatic repulsion between the electrical double layers of colloidal particles counteracts the attractive van der Waals interactions, and provides a barrier to aggregation.

Alternatively, electrically neutral surfactants that contain (in general) both a polar head-group and long/branched non-polar alkyl chain can be used to provide a steric barrier to aggregation. Once adhered to the surface, the surfactant improves interactions with non-polar solvent and provides a physical barrier to aggregation.

\subsubsection{Chemical approaches}

The intense interest in producing nanocrystals over the past few decades, has resulted in a boom of published chemical syntheses of nanocrystals. The general categories into which syntheses fall will be discussed below, and some examples from the literature will be given.

\subsubsection{Precipitation}

Some of the first published syntheses of very small $(<5 \mathrm{~nm})$, colloidal semiconductor nanocrystals involved precipitation from dilute aqueous solutions of inorganic precursors - such as the synthesis of $3.5 \mathrm{~nm} \mathrm{CdS}$ nanocrystals either with, ${ }^{13}$ or without ${ }^{14}$ the use of stabilising agents. This precipitation approach has also been used to synthesise magnetic $\mathrm{Fe}_{3} \mathrm{O}_{4}{ }^{15}$ and $(\mathrm{Fe}, \mathrm{Co})_{3} \mathrm{O}_{4}$ nanocrystals, ${ }^{16}$ where aqueous solutions of $\mathrm{M}^{2+} / \mathrm{M}^{3+}$ salts are basified until the metal oxides precipitate.

The precipitation method has the strong benefits of involving simple execution, aqueous solvent, and cheaper precursors than other methodologies (e.g. organometallic precursors used in the thermal decomposition method). However, ripening or defocusing of the size distribution, and its concomitant alteration of the spectral properties was observed to proceed rapidly in unpassivated CdS nanocrystals in aqueous solution at room temperature. ${ }^{13,14}$ 


\subsubsection{Thermal decomposition}

In the thermal decomposition method, nucleation and growth occur at high temperature $\left(\sim 100-350^{\circ} \mathrm{C}\right)$, generally in non-aqueous, high-boiling point solvents (although in the hydrothermal method water is used as the solvent $>100^{\circ} \mathrm{C}$ in a high-pressure autoclave). ${ }^{17}$ The thermal decomposition method has the benefit of being highly amenable to the hot-injection method, where injection of precursor into preheated solvent induces burst nucleation. The use of such a high-temperature, hot injection approach was pioneered by Bawendi et al. ${ }^{18}$ for the synthesis of highly crystalline, monodisperse CdSe nanocrystals from the organometallic precursor dimethyl cadmium and trioctylphosphine selenide. There has been a subsequent trend towards less hazardous, less reactive precursors, ${ }^{19,20}$ but the thermal decomposition method has become extremely widespread. ${ }^{1}$

The benefits of the thermal decomposition method lie with the wide range of solvents, surfactants, and precursors; and the wide nucleation/growth temperature range available. The thermal decomposition method relies on control of the reaction kinetics, and the use of coordinating solvents to control the average size and size distribution of the nanocrystals produced.

\subsubsection{Reduction}

Reduction of metal salts to form elemental metals is arguably the oldest method of deliberately synthesising colloidal nanocrystals, starting with the preparation of gold colloids by Faraday in the $19^{\text {th }}$ Century. ${ }^{1}$ In the 1950s Turkevich et al. ${ }^{21}$ utilised a wide range of reducing agents to produce gold colloids, from white phosphorus to sodium citrate. The latter of which has become a well established reducing agent/stabiliser for noble metal nanocrystals.

More recently, hydride reducing agents such as sodium borahydride or superhydride (lithium triethylborohydride); or alcohol reducing agents (especially hexanediol) have been used to produce various transition metal nanocrystals (e.g. Co, Fe, FePt, CoPt). ${ }^{1}$ 


\subsubsection{Microemulsions}

Both the reduction approach to producing elemental metal nanocrystals, and the precipitation method of producing binary compounds are frequently modified by the use of microemulsions as 'microreactors' to restrict the dimensions of the resulting nanocrystals. ${ }^{1,22}$ Microemulsions are colloids involving two immiscible liquid phases most frequently water and a non-polar organic solvent - stabilised by a surfactant.

Precursors such as metal salts or reducing agents can be dissolved within the nanometre-sized droplets of the aqueous discontinuous phase. When microemulsions of two precursors are combined, collisions between droplets can induce mixing of the precursors while the total volume available for the growing particle remains strictly limited by the dimensions of the droplet. 


\subsection{Physical properties of nanocrystals}

Nanocrystals display a variety of size-dependent alterations in their chemical and physical properties compared to the bulk material. Among these are counted a preponderance of surface chemistry over chemistry of the bulk; an alteration and quantisation of electronic states; and an alteration of the magnetic domain structure and trend towards superparamagnetism in magnetic nanocrystals.

The size-tunable optical absorption and emission of semiconductor nanocrystals; and the magnetic properties of nanocrystals were focused on in this project, basic theory will now be introduced covering the areas of the electronic structure of semiconductor nanocrystals, and magnetism in solids.

\subsubsection{Electronic structure of low-dimensional semiconductors}

The size-tuneable electronic and optical properties of low-dimensional semiconductors, or quantum dots, have been one of the primary driving forces behind research into nanocrystals. The prospect of producing inorganic chromo- and fluorophores, whose absorption and emission profiles are altered by the particle size distribution and shape distribution, has excited much interest. The theoretical basis for the size-dependence of the electronic structure of quantum dots will now be discussed.

\subsubsection{Electronic band structure}

The electronic structure of nanocrystals can be viewed as an intermediate between the discrete energy levels of a molecule, and the broad continuum of energy states (band) which characterises a continuous solid. ${ }^{23}$ Nanocrystals of semiconducting materials feature an energy range for which no allowed states exist, analogous to the band gap of bulk semiconductors - although in the case of nanocrystals this band gap is highly dependent on the size of the nanocrystal. As in bulk semiconductors, electronic transitions across the band gap are responsible for the optical absorption and emission processes of the material, and as such the size-dependent optical properties of semiconductor nanocrystals are a consequence of the alteration of the band gap with nanocrystal size. ${ }^{2}$ 


\subsubsection{Quantum confinement (Particle-in-a-box theory)}

It has been demonstrated that in small semiconductor nanocrystals the optical absorption and emission maxima are significantly blue-shifted away from the values of the bulk semiconductor - the smaller the particle size the larger the blue-shift. As the average size of the nanocrystals increases, the absorption and emission maxima red-shift until they eventually converge with the values of the bulk. ${ }^{14,18}$ Hence, the band gap of small nanocrystals is larger than that of the bulk.

This originates in the quantum confinement, which is most simply modelled theoretically by treating the nanaocrystal as a three-dimensional potential energy well or box - with infinitely high walls, and considering the effects of the size of the box on the allowed electron energy states. The treatment for a three-dimensional potential well is ultimately extrapolated from the case of a one-dimensional potential well, which is outlined below:

The Schrödinger equation for a particle free to move in one dimension is given below in expression (4), where $\hbar$ is $h / 2 \pi$, and $h$ is Plank's constant, $m$ is the mass of the particle, $x$ is displacement in the $x$-direction, $\psi$ is the wavefunction, $U_{(\mathrm{x})}$ is the potential term, and $E$ is energy. The first term on the left hand side of the expression gives the kinetic energy of the particle, and the second term the potential energy. If boundary conditions are applied, such that the potential is zero within a well of length $a$, and infinite beyond it, only certain wave functions are suitable solutions for the Schrödinger equation - the energy states of the particle become quantised. The acceptable eigenvalues, or energy levels for the confined particle are then given by expression (5), where $n$ is the principal quantum number. A pictorial depiction of a one-dimensional energy well is shown below (Figure 1-2).

$$
\begin{aligned}
& -\frac{\hbar^{2}}{2 m} \frac{\partial^{2}}{\partial x^{2}} \psi(x)+U(x) \psi(x)=E \psi(x) \\
& E_{n}=\frac{\pi^{2} \hbar^{2}}{2 m a^{2}} n^{2}
\end{aligned}
$$




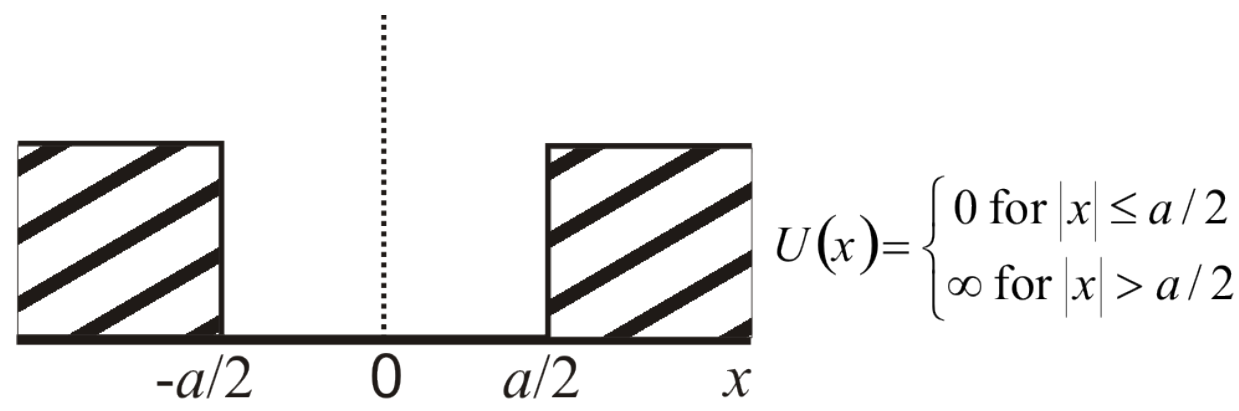

Figure 1-2 Pictorial depiction of a one-dimensional potential energy well.

\subsubsection{Exciton Bohr radius}

The diameter below which quantum confinement will begin to affect the electron energy states is dictated by the exciton Bohr radius of the material. An exciton is the electron-hole pair that results from the promotion of an electron from the valence band to the conduction band. An exciton has a characteristic average physical separation termed the exciton Bohr radius - the magnitude of which is a characteristic of the material. As the dimensions of a nanocrystal approach that of the exciton Bohr radius of the material, the energy states of the exciton become quantised, and strongly dependent on the particle dimensions (this occurs when the nanocrystal diameter is $\sim 2-3$ times the exciton Bohr radius). ${ }^{23}$ Thus the exciton Bohr radius is useful to judge the size of nanocrystal necessary for quantum confinement affects to come to the fore.

Expression (6) gives the eigenvalues of a system in the strong confinement regime, where $n$ and $l$ are the principal and angular momentum quantum numbers, respectively, $E_{g}$ is the band gap, $\mu$ is the reduced mass of the exciton, and $\chi$ is the root of the spherical Bessel function. Hence it can be seen that in the strong confinement regime the band gap of the material is modified from its bulk value by a term resulting from the quantum confinement of the exciton. ${ }^{2}$

$E_{n l}=E_{g}+\frac{\hbar^{2}}{2 \mu a^{2}} \chi_{n l}^{2}$

Electronic transitions in nanocrystals are typically described with the following notation: $n Q_{F}$ denotes the state of the hole wave function, where $n$ is the principal quantum number, $Q$ is the minimum orbital momentum $l$ value for the hole, and $F$ is the total momentum. $n Q_{e}$ denotes the state of the electron wave function, where $n$ is the principal quantum number, and $Q_{e}$ is the orbital momentum of the electron wave function. Hence the lowest energy transition is typically the $1 S_{3 / 2}-1 S_{e}$ transition. $^{24}$ 


\subsubsection{Magnetism}

Aspects of magnetism relevant to the behaviour of magnetic nanocrystals will be outlined below. Firstly, the defining relations for macroscopic magnetic properties will be given, followed by an introduction to the magnetic anisotropy (1.2.2.1), magnetic domains (1.2.2.2), magnetisation and demagnetisation processes (1.2.2.3) and the behaviour of single domain particles (1.2.2.4), including superparamagnetism (1.2.2.5). Units and symbols for magnetic quantities are given in Abbreviations (page v).

Magnetisation, $M$, is related to the applied magnetic field, $H$, by magnetic susceptibility, $\chi$ - as shown in expression (7).

$$
M=\chi H
$$

The magnetic flux density - or induced magnetisation - $B$, is proportional to the applied field in a vacuum, but within a material the magnetisation induced by the applied field makes a contribution to the magnetic flux density, as shown in (8), where $\mu_{0}$ is the magnetic permeability.

$$
B=\mu_{0}(H+M)
$$

\subsubsection{Magnetic anisotropy}

There are several sources of anisotropy in the magnetic properties of solids, including anisotropy due to sample shape; and the anisotropic effect of crystal structure on orientation of magnetisation - termed magnetocrystalline anisotropy.

Shape anisotropy originates in the effect of the surface poles on the magnetisation of the interior. Magnetic flux emerging with a component normal to the surface sets up "free poles" on the surface. The magnetic field between the north free poles and the south free poles may pass through the interior of the sample, where it opposes the magnetisation that first established the free poles. This demagnetising field set up by the surface "free poles" means it is easier to magnetise along the long axis than the short axis of an anisotropically shaped magnetic sample, as the long axis has relatively less exposure to the demagnetising field. ${ }^{25}$

Magnetocrystalline anisotropy is evident in the existence of preferred crystallographic orientations for magnetisation. For example, in b.c.c iron, the $<100>$ directions are more 
easily magnetised by an applied field than the $<111>$ directions. Hence for b.c.c iron, the $<100>$ directions are referred to as the easy axes, and the $<111>$ directions as the hard axes. Iron is an example of a material with cubic magnetocrystalline anisotropy, originating in the high symmetry of its cubic crystal structure. In addition to cubic anisotropy, there exists uniaxial magnetocrystalline anisotropy (which is typically the case in hexagonal crystal structures). In a uniaxial magnetic material there is a single axis parallel to the crystallographically unique axis (i.e. the $c$ axis) which may either be easy or hard, depending on the material and the temperature. The plane orthogonal to the axis is thus referred to either as the easy or hard plane. For example, in h.c.p. cobalt the [0001] direction is the easy axis, whereas the (0001) basal plane is hard.

Magnetocrystalline anisotropy originates in the anisotropy of the crystalline electric field, which itself originates in the anisotropic distribution of electron density inherent in directional chemical bonding. The orbital component of a local magnetic moment is obviously affected by the orientation and symmetry of the molecular or crystal orbitals, which creates a preferred orientation along which it lies. This preferred orientation is transmitted to the spin component of the local magnetic moment by spin-orbit coupling.

\subsubsection{Magnetic domains and domain walls}

Macroscopic samples of ferromagnetic or ferrimagnetic materials do not necessarily display a spontaneous magnetisation despite the presence of aligned local moments within the material. This is a consequence of the formation of magnetic domains regions of local alignment of magnetic moments. The magnetic moments of adjacent domains may cancel, such that no overall magnetisation results.

The formation of domains tends to confine the magnetic flux to within the sample, which in turn reduces the interaction between the flux and the free surface poles (demagnetisation energy, see section 1.2.2.1), thus providing an energetic driving force for domain formation. ${ }^{26}$ This is balanced against the energy cost of domain walls - the boundaries between magnetic domains. Figure 1-3 (a) shows a schematic diagram of a single magnetic domain, where magnetic flux is required to travel external to the sample. Figure 1-3 (b) shows a sample with magnetic domains arranged such that the magnetic flux is contained completely within the sample. 
(a)

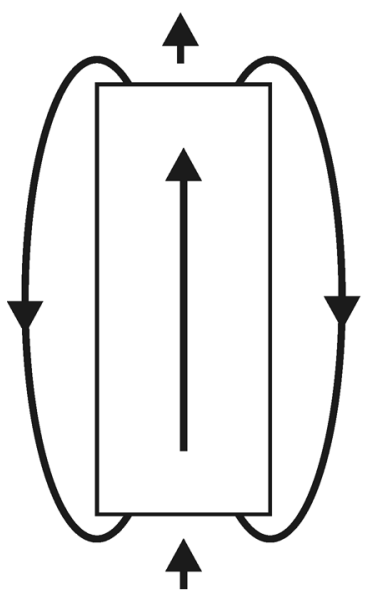

(b)

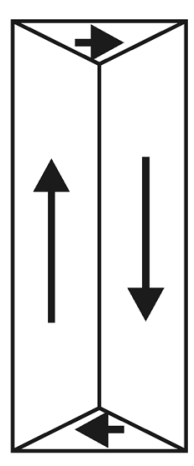

(c)

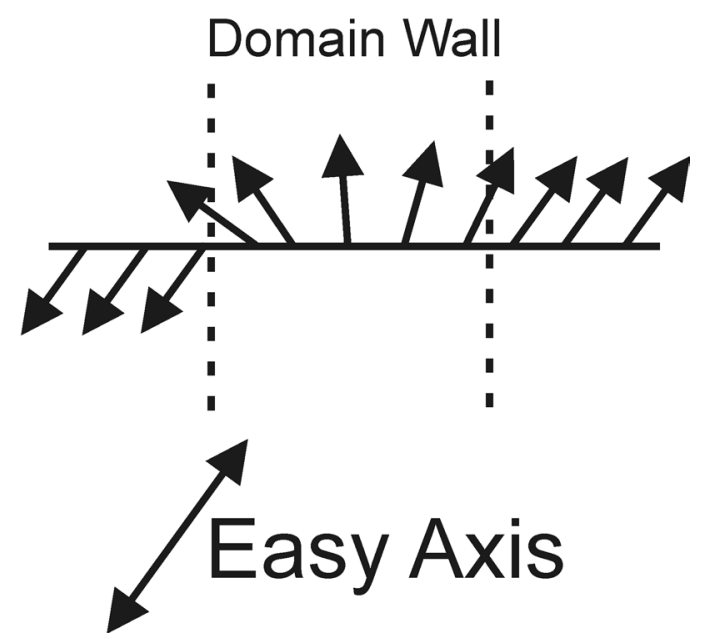

Figure 1-3 (a) Single magnetic domain (b) enclosed magnetic domains (c) $180^{\circ}$ domain wall.

Domain walls are areas within which local magnetic moments adopt orientations intermediate to the orientations of the local moments of the adjacent domains (see Figure 1-3 (c) for a schematic depiction of local moment orientations within a domain wall). In materials with uniaxial symmetry, domain walls are predominantly $180^{\circ}-$ with the magnetisation of adjacent domains orientated antiparallel to each other, but both aligned with the easy axis. Materials with cubic anisotropy commonly contain $90^{\circ}, 109^{\circ}$ and $71^{\circ}$ domain walls in addition to $180^{\circ}$ domain walls. In all cases, domain walls incur an energy cost, as they require local magnetic moments within the wall to overcome the magnetocrystalline anisotropy to adopt intermediate orientations away from the easy axis, and to be non-parallel to adjacent magnetic moments, which increases their exchange energy. ${ }^{27}$

\subsubsection{Magnetisation and demagnetisation processes}

When a magnetic field is applied to a domain-containing sample, a number of processes can occur. Magnetic domains with components of magnetisation aligned with the field can grow at the expense of domains not aligned with the field, through a process of domain wall motion - a process which is generally facile. Alignment with the applied field can also proceed via coherent rotation of domain magnetisation - a process which is generally less facile than domain wall motion, as it can require moving the magnetisation away from easy directions. Once total alignment of the sample has occurred through domain wall motion and coherent rotation, the sample has obtained a state of saturation, and will posses some saturation magnetisation, $M_{s}$ (see Figure 1-4). 
Upon removal of the applied field, domain magnetisations will quickly rotate back to the easy axes, reducing the magnetisation of the sample. However, domain walls can be trapped by magnetic defects such that the return of domain walls to their previous positions is hindered. Consequently the sample does not immediately return to a demagnetised state, but instead remains in a remanent state with some remanent magnetisation, $M_{r}$. Application of an applied field in the direction opposite to the initial field is then required to de-pin the domain walls, and return the sample to its previous demagnetised state - the magnitude of applied field necessary for this is referred to as the coercivity $\left(\mathrm{H}_{\mathrm{c}}\right)$. These quantities are typically measured in field loop experiments (see Chapter 2 section 2.2.6.2). A schematic diagram of the result of such an experiment is shown below (Figure 1-4), with saturation magnetisation, remanent magnetisation and coercivity marked.

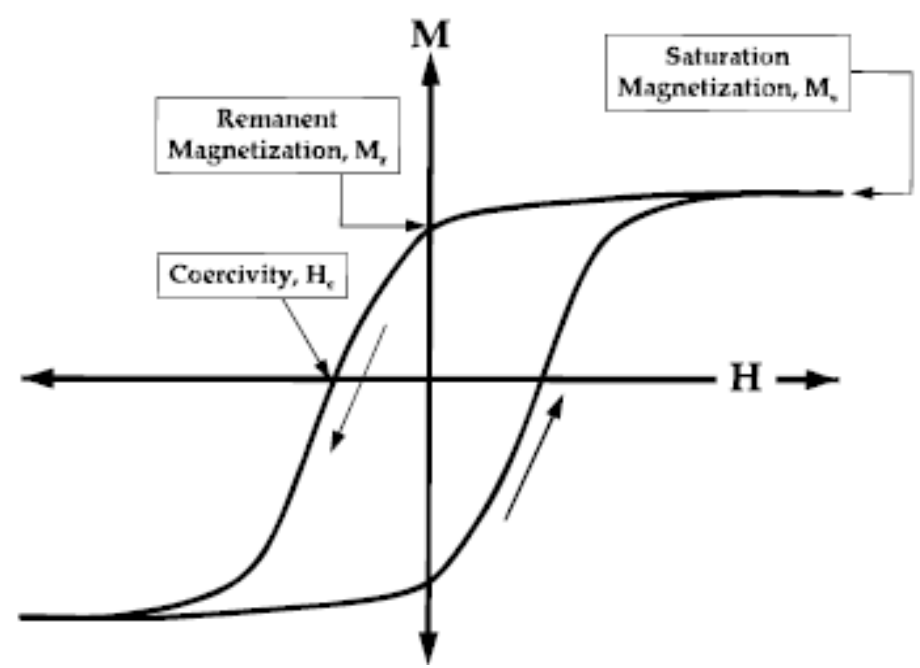

Figure 1-4 Field loop demonstrating hysteresis. The position of the experimentally obtained sample quantities of saturation magnetisation, $\mathrm{M}_{\mathrm{s}}$; remanent magnetisation, $\mathrm{M}_{\mathrm{r}}$; and coercivity, $\mathrm{H}_{\mathrm{c}}$, are marked.

\subsubsection{Single domain particles}

As the size of a sample of magnetic material is decreased, a critical size is reached below which it is no longer energetically favourable for the sample to consist of multiple magnetic domains, and the sample becomes a single domain particle. The critical size of a particle depends upon the balance of the increase of the exchange energy required for domain wall formation, and the decrease in the demagnetisation energy entailed by domain formation. As an example, the calculated critical radius for spherical iron particles is $15 \mathrm{~nm}$, and the critical minor semiaxis length for prolate ellipsoidal iron particles with a 1:10 aspect ratio is $60 \mathrm{~nm}^{27}$ 
The magnetisation of an ensemble of single domain particles by an applied field cannot proceed via domain wall motion, and the magnetisation process must rely instead solely on coherent and/or incoherent rotation of domain magnetisations. The coercivity of isolated single domain particles is therefore higher than multiple domain particles of the same material. This predicted trend has been confirmed experimentally, with coercivity of iron and cobalt powders increasing by an order of magnitude for a thousand-fold reduction in average particle size. ${ }^{27}$

\subsubsection{Superparamagnetism}

As the size of an isolated single domain particle is decreased further, a (temperature-dependent) size limit is reached, beyond which the direction of the domain magnetisation is no longer held fixed by shape or magnetocrystalline anisotropy. Thermal energy is now sufficient to cause the magnetisation to flip between stable orientations within the experimental timeframe, meaning that the sample relaxes out of the metastable remanent state to the more stable demagnetised state upon removal of an applied field. ${ }^{26}$ The sample is described as being in a superparamagnetic state, as each single domain particle now behaves in a manner analogous to a paramagnetic ion with massive spin. ${ }^{27}$

Thus the behaviour of ensembles of superparamagnetic particles are similar to paramagnetic samples, in as much as they display a lack of coercivity and remanent magnetisation, and their behaviour can be modelled by the classical Langevin equation (9), where $L_{(x)}$ is the Langevin function, $k$ is the Boltzmann constant and $T$ is the absolute temperature.

$$
M=N_{v} L(\mu H / k T)
$$

The probability per unit time that the magnetic moment of a particle will traverse the energy barrier between stable orientations is given by expression (10), where $P$ is the probability of a magnetic domain switching out of a metastable remanent state per unit time. This is dependent on the size of the energy barrier, $\Delta f V$, and the attempt frequency factor, $v_{0} \sim 10^{9} \mathrm{~s}^{-1} \cdot{ }^{26}$ If stability is defined as a flipping probability of less than $10 \%$ over a given time period, the critical radius is given by expression (11), where $r_{0}$ is the superparamagnetic critical radius and $K$ is the first-order magnetocrystalline anisotropy 
constant. If a magnetocrystalline anisotropy constant of $K=10^{5} \mathrm{~J} \mathrm{~m}^{-3}$ is assumed, the critical radius for a one year period, $r_{0}^{1 y r} \approx 7.3 \mathrm{~nm}$, whereas the critical radius for one second, $r_{0}^{1 s} \approx 6 \mathrm{~nm}$. This illustrates the strong size dependence of superparamagnetic behaviour. $^{26}$

$P=v_{0} \exp (\Delta f V / k T)$

$r_{0}^{1 y r} \approx\left(\frac{10 k T}{K}\right), r_{0}^{1 s} \approx\left(\frac{6 k T}{K}\right)$

For a given sample, and more useful parameter for judging the transition to a superparamagetic state may be the average blocking temperature $\left(T_{B}\right)$ - the temperature at which the majority of the sample crosses to a superparamagnetic state (as judged by the time scale of the experiment). The blocking temperature is readily determined experimentally, as it manifests itself as a discontinuity in the slope of a plot of magnetisation versus temperature in a zero-field cooled and field cooled experiment (see Chapter 2 section 2.2.6.2 for details). 


\subsection{Metal chalcogenides}

The general chemistry and physical properties of the metal chalcogenide phase systems encountered in this project are detailed below.

\subsubsection{Cadmium selenide}

Cadmium selenide is a greenish-brown or dark red crystalline solid. In common with the majority of the group 12 chalcogenides, it can adopt either the cubic zinc blende or hexagonal wurtzite crystal structures, although the wurtzite form is slightly more thermodynamically stable. ${ }^{28}$

In the wurtzite structure (space group $\mathrm{P}_{3} \mathrm{mc}$ ), selenium atoms form a hexagonal closepacked array, with the cadmium atoms tetrahedrally coordinated by selenium (see Figure 1-5 for crystal structure diagram). In the zinc blende structure (space group $F \overline{4} 3 m$ the selenium atoms form a cubic close-packed array, in which the cadmium atoms are also tetrahedrally coordinated (see Figure 1-6 for crystal structure diagram).

Cadmium selenide has a tendency to be slightly selenium deficient, ${ }^{29}$ and as such is usually an $n$-type semiconductor ${ }^{30}$ with a direct band gap of $1.840 \mathrm{eV}$ at $0 \mathrm{~K}$, and $1.74 \mathrm{eV}(712 \mathrm{~nm})$ at $300 \mathrm{~K} .{ }^{31}$ Semiconductors can absorb photons with energies greater than their band gaps, consequently bulk cadmium selenide absorbs throughout the UV and visible region of the spectrum (see for transmittance spectrum of bulk cadmium selenide). The exciton Bohr radius of cadmium selenide has been determined to be 5.6 $n$ n. $^{24}$ 

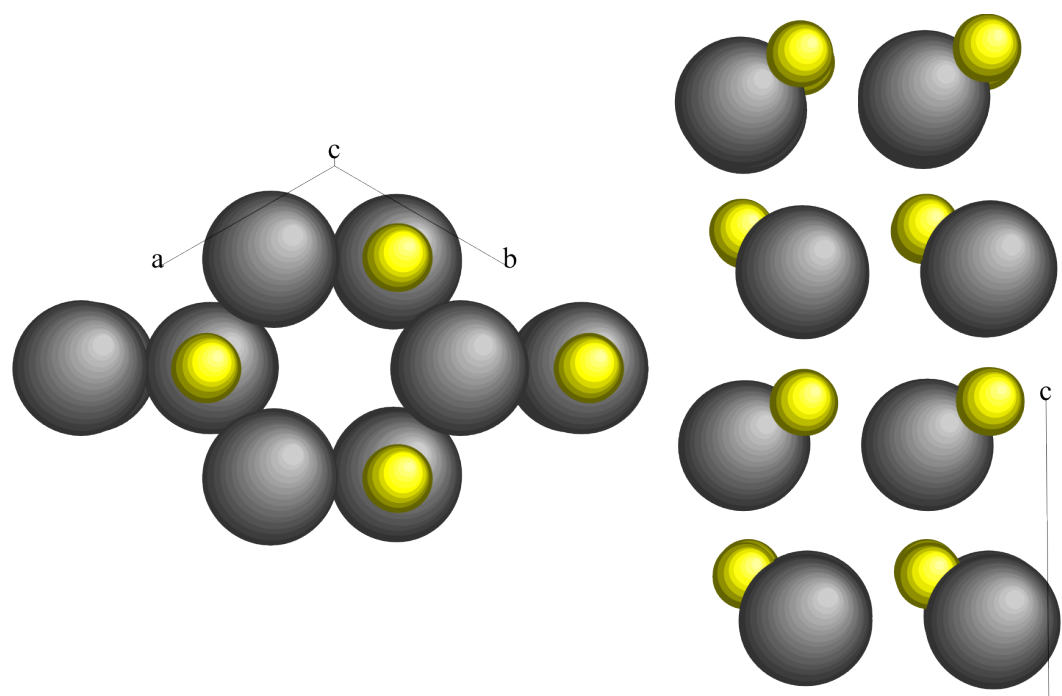

(O)

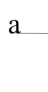

[010]
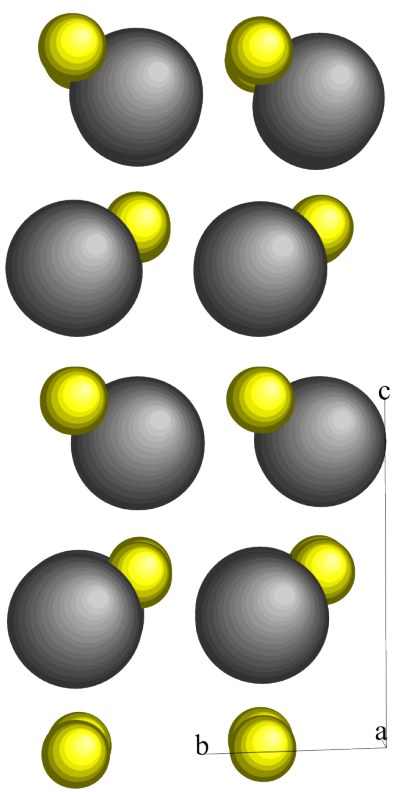

[100]

Figure 1-5 Crystal structure of hexagonal CdSe (wurtzite structure). Cadmium is shown in yellow and selenium is shown in grey. Atomic radii are shown at $75 \%$ scale for clarity.

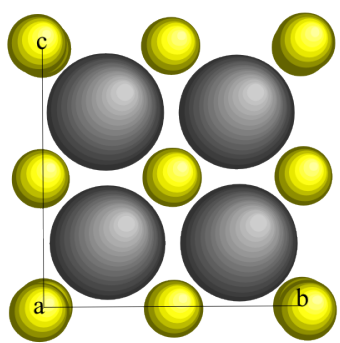

[100]

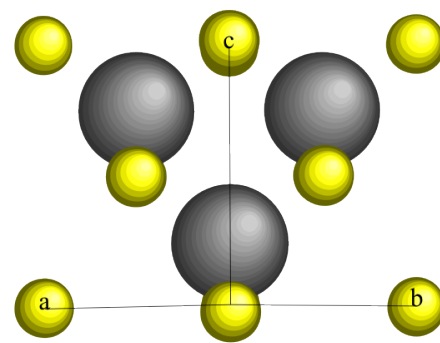

[110]
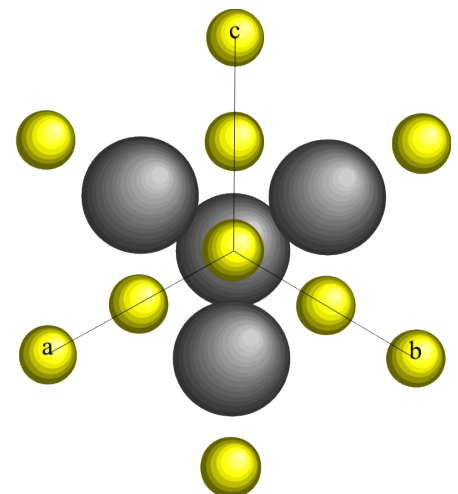

[111]

Figure 1-6 Crystal structure of cubic CdSe (zinc blende structure). Cadmium is shown in yellow and selenium is shown in grey. Atomic radii are shown at $75 \%$ scale for clarity.

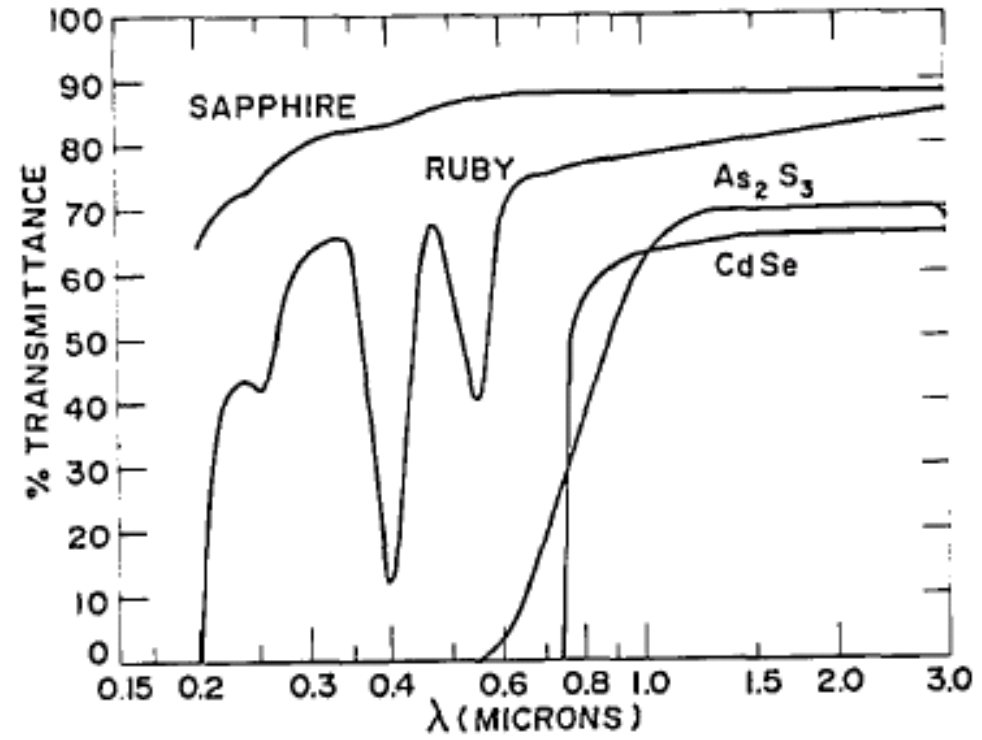

Figure 1-7 Transmittance spectrum for a $1.67 \mathrm{~mm}$ thick cadmium selenide single crystal from McCarthy. ${ }^{32}$ 


\subsubsection{Iron sulfides}

Iron and sulfur form a number of binary compounds, whose compositions extend from the two polymorphs of iron disulfide $\left(\mathrm{FeS}_{2} \mathrm{p}\right.$, pyrite and $\mathrm{FeS}_{2} \mathrm{~m}$, marcasite) through to $\mathrm{Fe}_{1-x} \mathrm{~S}$ (the pyrrhotite family). Many iron sulfide phases are geologically highly significant sulfur-bearing minerals, and the iron sulfides have been extensively studied for their role in geology, rock magnetism and the global biogeochemical sulfur cycle. ${ }^{33}$ The most important crystalline iron sulfide phases are introduced below:

\subsubsection{1 $\mathrm{FeS}_{2}$ (pyrite and marcasite)}

The two iron disulfide polymorphs $\mathrm{FeS}_{2} \mathrm{p}$ (pyrite), and the much rarer $\mathrm{FeS}_{2} \mathrm{~m}$ (marcasite), crystallise in the rock salt structure (cubic space group $P a \overline{3}$ ), and in the orthorhombic space group Pnnm, respectively (see Figure 1-8 and Figure 1-9 for crystal structure models of $\mathrm{FeS}_{2} \mathrm{p}$ and $\mathrm{FeS}_{2} \mathrm{~m}$, respectively). The short S-S distance in $\mathrm{FeS}_{2}$ (2.177 and $2.223 \AA$ in $\mathrm{FeS}_{2} \mathrm{p}$ and $\mathrm{FeS}_{2} \mathrm{~m}$, respectively, ${ }^{34}$ cf. $3.5 \AA$ in $\mathrm{Fe}_{3} \mathrm{~S}_{4},{ }^{35}$ where the sulfur atoms form a simple close-packed array) leads to the interpretation that sulfur is in the form of disulfide ions $\left(\mathrm{S}_{2}{ }^{2-}\right)$, and that consequently the iron is in the +2 oxidation state.

The octahedrally coordinated $d^{6} \mathrm{Fe}^{2+}$ is low-spin in both $\mathrm{FeS}_{2} \mathrm{p}$ and $\mathrm{FeS}_{2} \mathrm{~m}$, leading to the absence of ordered magnetism. Weak, temperature-independent Van Vleck paramagnetism is however observed. ${ }^{36}$ Both polymorphs have a lustrous, metallic appearance and are semiconductors, with indirect band gaps reported as $0.78 \mathrm{eV}$ $(1590 \mathrm{~nm})$ and $0.34 \mathrm{eV}(1220 \mathrm{~nm})$ at $298 \mathrm{~K}$, for $\mathrm{FeS}_{2} \mathrm{p}$ and $\mathrm{FeS}_{2} \mathrm{~m}$, respectively.

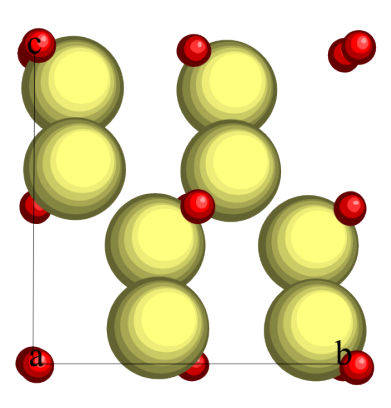

[100]

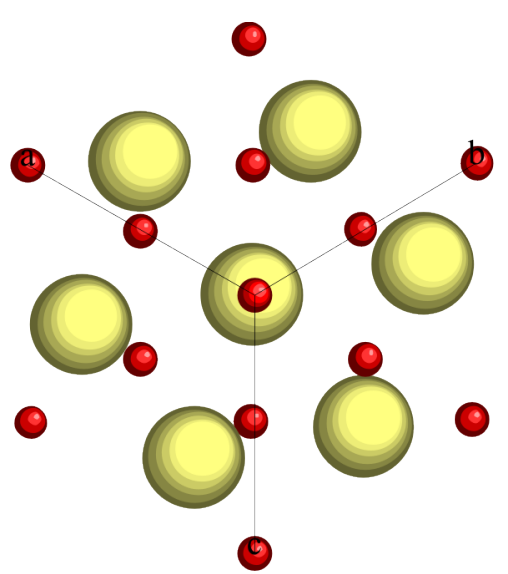

[111]

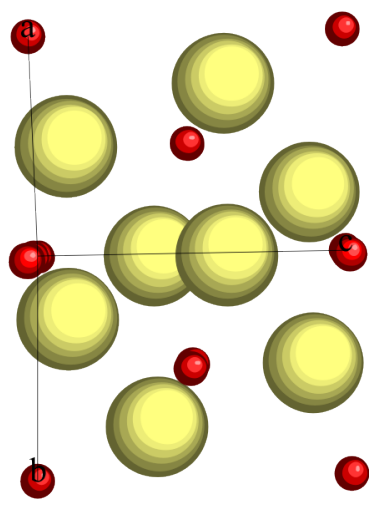

[110]

Figure 1-8 Crystal structure model of $\mathrm{FeS}_{2} \mathrm{p}$ (pyrite, space group $\mathrm{Pa} \overline{3}$ ). Iron is shown in red, sulfur is shown in yellow. Atomic radii are shown at $40 \%$ scale for clarity. 


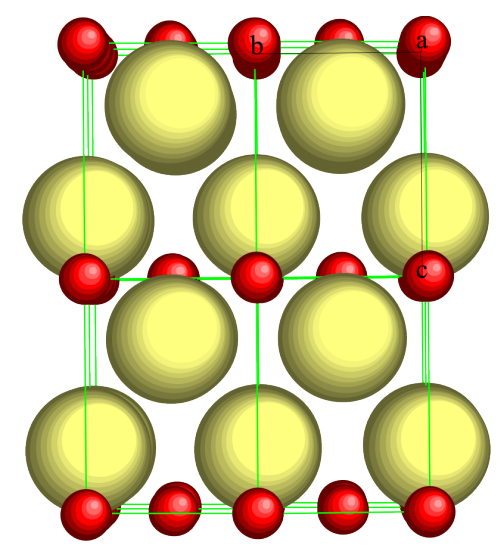

[100]

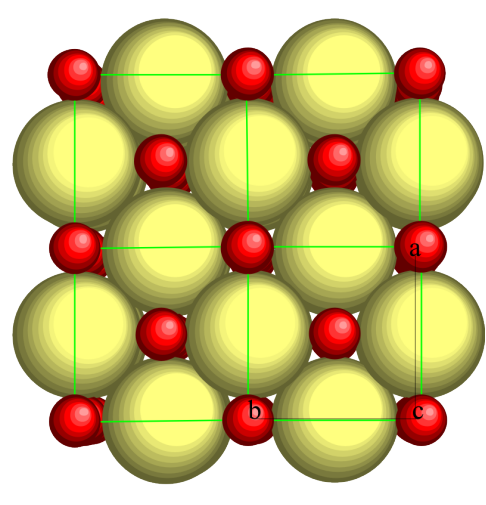

[001]

Figure 1-9 Crystal structure model of $\mathrm{FeS}_{2} \mathrm{~m}$ (marcasite, space group Pnnm). Iron is shown in red, sulfur is shown in yellow. Atomic radii are shown at $40 \%$ scale for clarity.

\subsubsection{2 $\mathrm{Fe}_{3} \mathrm{~S}_{4}$ (greigite)}

Black $\mathrm{Fe}_{3} \mathrm{~S}_{4}$ (greigite) crystallises in the spinel structure (space group $F d \overline{3} m$ ), and as such is the thio-analogue of magnetite $\left(\mathrm{Fe}_{3} \mathrm{O}_{4}\right)$ (see Figure 1-10 for crystal structure model of $\mathrm{Fe}_{3} \mathrm{~S}_{4}$ ). In contrast to the ubiquity of $\mathrm{Fe}_{3} \mathrm{O}_{4}$, the existence of $\mathrm{Fe}_{3} \mathrm{~S}_{4}$ was only confirmed in the 1960s. ${ }^{35}$ Since that time it has become a frequently identified iron-containing phase in sediments isolated from sulfate-reducing environments. ${ }^{33} \mathrm{Fe}_{3} \mathrm{~S}_{4}$ has been described as metastable, and has been observed to disproportionate to $\mathrm{Fe}_{1-x} \mathrm{~S}$ and elemental sulfur at $280^{\circ} \mathrm{C}$ under vacuum, ${ }^{37}$ or at $190^{\circ} \mathrm{C}$ in aqueous solution. ${ }^{38}$

Unlike its oxide analogue, where a high degree of ionicity can be assumed for iron oxygen bonds, $\mathrm{Fe}_{3} \mathrm{~S}_{4}$ can be expected to have a high degree of covalency in its bonding. This fact complicates any assignment of formal oxidation states to iron occupying tetrahedral and octahedral sites within the structure. Mössbauer spectroscopy measurements suggest that iron in tetrahedral sites is in the $\mathrm{Fe}^{3+}$ oxidation state - in line with expectations for an inverse spinel (cf. $\left.\mathrm{Fe}_{3} \mathrm{O}_{4}\right)$. However, instead of an equal mixture of $\mathrm{Fe}^{2+}$ and $\mathrm{Fe}^{3+}$ in the octahedral sites, it appears possible that only $\mathrm{Fe}^{2+}$ is present - in line with observations that octahedrally-coordinated iron in other sulfides is invariably $\mathrm{Fe}^{2+} \cdot{ }^{37}$ It was suggested by Spender et al., ${ }^{37}$ that this reduction of $\mathrm{Fe}^{3+}$ to $\mathrm{Fe}^{2+}$ in the octahedral sites might be enabled by the introduction of holes into the valence band. Another complicating factor is the non-stochiometry observed for this phase by various workers. ${ }^{33,37}$ Again iron vacancies are expected to be compensated for electronically by oxidation of $\mathrm{Fe}^{2+}$ to $\mathrm{Fe}^{3+}$. 
The observed magnetic moment for $\mathrm{Fe}_{3} \mathrm{~S}_{4}$ has been placed at $2.2 \mu_{\mathrm{B}} /$ formula unit, ${ }^{37}$ compared to the figure of $4.1 \mu_{\mathrm{B}} /$ formula unit for $\mathrm{Fe}_{3} \mathrm{O}_{4}$. As a moderate degree of electron delocalisation is expected, electron band models have been proposed to account for the ferrimagnetism and semimetallic conductivity. ${ }^{37}$ A Néel temperature of $333^{\circ} \mathrm{C}$ has been proposed for $\mathrm{Fe}_{3} \mathrm{~S}_{4}$ by extrapolation of a magnetisation versus temperature curve to zero field, however $\mathrm{Fe}_{3} \mathrm{~S}_{4}$ decomposed at these temperatures. ${ }^{37}$ Figures for the saturation magnetisation of $\mathrm{Fe}_{3} \mathrm{~S}_{4}$ are quoted in the range of $24^{(39)}-40^{(37)} \mathrm{A} \mathrm{m}^{-2} \mathrm{~kg}^{-1}$, but appear highly dependent on the grain size. The magnetic easy axes were determined to be the $<100>$ axes, at odds to $\mathrm{Fe}_{3} \mathrm{O}_{4}$ whose easy axes are the $<111>$ axes. ${ }^{40}$

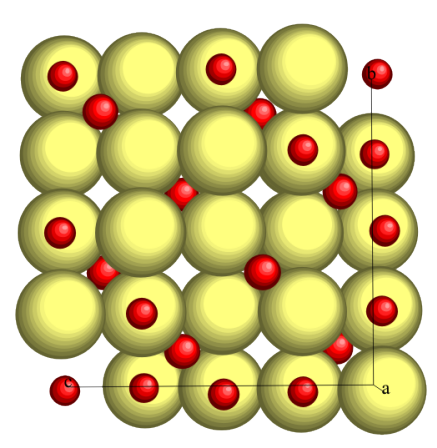

[100]

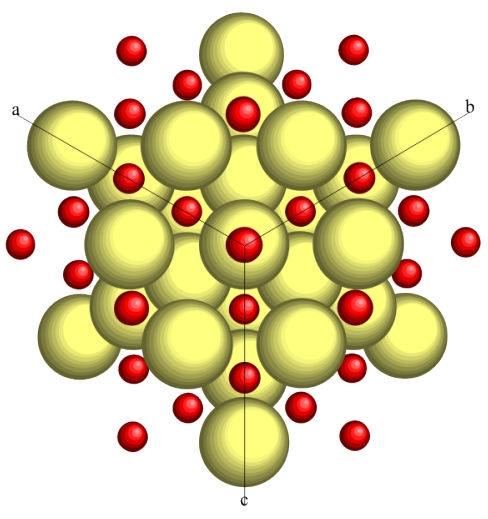

[111]

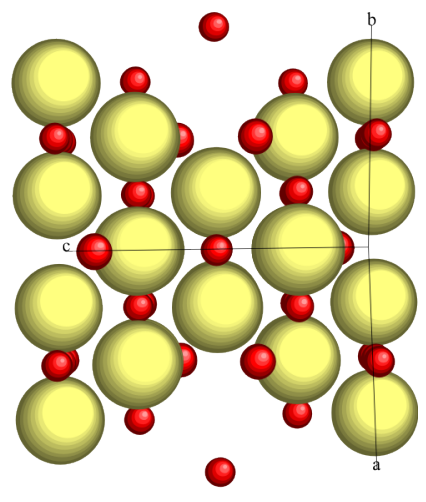

[110]

Figure 1-10 Crystal structure of $\mathrm{Fe}_{3} \mathrm{~S}_{4}$ (gregite, space group $F d \overline{3} m$ ). Iron is shown in red, sulfur is shown in yellow. Atomic radii are shown at $75 \%$ scale for clarity.

\subsubsection{3 $\quad \mathrm{Fe}_{9} \mathrm{~S}_{11}$ (smythite)}

$\mathrm{Fe}_{9} \mathrm{~S}_{11}$ crystallises in the rhobohedral space group $R \overline{3} \mathrm{~m}$. The phase was initially believed to have a composition of $\mathrm{Fe}_{3} \mathrm{~S}_{4},{ }^{41}$ and was hence considered a rhombohedral polymorph of cubic $\mathrm{Fe}_{3} \mathrm{~S}_{4}$ (greigite, see section 1.3.2.2). Upon reanalysis it was found to have the distinct composition: $\mathrm{Fe}_{9} \mathrm{~S}_{11}{ }^{42}$ Studies of the magnetic properties of $\mathrm{Fe}_{9} \mathrm{~S}_{11}$ are scant, but it is generally described as being "strongly magnetic", and the Mössbauer study by Makarov concluded that the magnetic ordering was ferromagnetic. ${ }^{43}$ The upper thermal stability limit of $\mathrm{Fe}_{9} \mathrm{~S}_{11}$ is approximately $75^{\circ} \mathrm{C} .{ }^{42} \mathrm{Fe}_{9} \mathrm{~S}_{11}$ is considered a metastable phase, which only forms if pathways to $\mathrm{FeS}_{\mathrm{m}}$ or $\mathrm{FeS}_{2} \mathrm{p}$ are blocked: synthetic $\mathrm{Fe}_{9} \mathrm{~S}_{11}$ has been formed only in aqueous reactions containing $\mathrm{FeCO}_{3}$ suspensions below $50^{\circ} \mathrm{C}$, where precipitation of $\mathrm{FeS}_{\mathrm{m}}$ is suppressed; or as an exsolution from rapidly quenched, $\mathrm{FeS}_{2}$ p-free $\mathrm{Fe}_{1-x} \mathrm{~S}$. ${ }^{44}$ 


\subsubsection{4 $\quad \mathrm{FeS}_{\mathrm{m}}$ (mackinawite)}

$\mathrm{FeS}_{\mathrm{m}}$ (mackinawite) is a stoichiometric iron monosulfide, which crystallises in a tetragonal layer structure (space group $P 4 / \mathrm{nnm}$ ), with a layer of iron atoms in a square-planar arrangement, tetrahedrally coordinated by sulfur atoms in an adjacent layer (see Figure 1-11 for a crystal structure model). The layers are stacked along the $c$ axis, and bound together solely by van der Waals interactions. ${ }^{33,45}$

Mössbauer spectroscopy on $\mathrm{FeS}_{\mathrm{m}}$ revealed that the $d^{6} \mathrm{Fe}^{2+}$ is low-spin, and that the material therefore has no spontaneous magnetic moment, ${ }^{46}$ and no magnetic ordering. ${ }^{47}$ The low-spin configuration, and short $\mathrm{Fe}-\mathrm{Fe}$ distance in $\mathrm{FeS}_{\mathrm{m}}(2.5976 \AA)^{48}$ have lead to the proposal that $\mathrm{FeS}_{\mathrm{m}}$ is a metallic conductor within the layers of the crystal structure, which is supported by DFT studies. ${ }^{49}$

Deposition of $\mathrm{FeS}_{\mathrm{m}}$ from aqueous solutions of $\mathrm{Fe}^{2+}$ and $\mathrm{S}^{2-}$ ions is rapid (starting within $10 \mathrm{~ms}$ of mixing), ${ }^{33}$ and the average particle size can be as low as $2 \mathrm{~nm}$ - hence freshly precipitated $\mathrm{FeS}_{\mathrm{m}}$ has often been referred to as amorphous (when judged solely by conventional powder XRD), though low-angle scattering shows this not to be the case. In aqueous systems at least, $\mathrm{FeS}_{\mathrm{m}}$ is the initially precipitated iron sulfide phase, which may subsequently converted to other iron sulfide phases (see below Chapter 4 section 4.1.2). Attempts to synthesise $\mathrm{FeS}_{\mathrm{m}}$ directly from the elements has thus far proved unsuccessful. $^{47}$

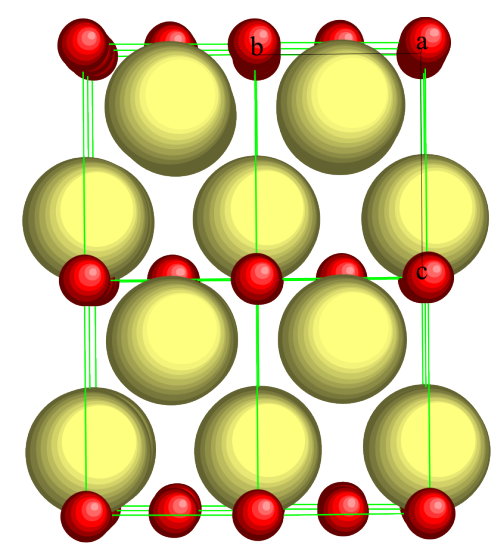

[100]

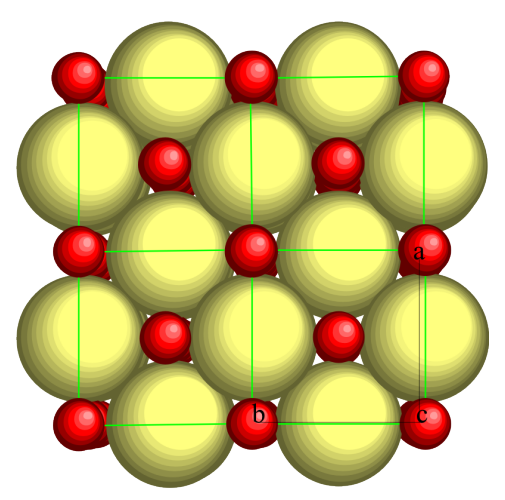

[001]

Figure 1-11 Crystal structure model of $\mathrm{FeS}_{\mathrm{m}}$ (mackinawite, space group $\mathrm{P} 4 / \mathrm{nmm}$ ). Iron is shown in red, and sulfur is shown in yellow. Atomic radii are shown at $75 \%$ scale for clarity. 


\subsubsection{5 $\quad \mathrm{Fe}_{1-x} \mathrm{~S}$ (pyrrhotite \& troilite)}

There exists a large number of iron sulfide phases based on the hexagonal close-packed NiAs-type structure, typically with an iron-deficient composition $-\mathrm{Fe}_{1-x} \mathrm{~S}$ - where $0<x<0.125{ }^{50}$ This group of common minerals are generally referred to as pyrrhotites. In the pure NiAs-type structure (1C), sulfur forms an hcp lattice, and $\mathrm{Fe}^{2+}$ occupies the octahedral holes, with any iron vacancies due to iron deficiency randomly distributed as a solid solution (see Figure 1-12 for crystal structure model). The stability field of the $1 \mathrm{C}$ structure does not extend down to room temperature at any composition, ${ }^{51}$ and the structure is unquenchable - at the transition temperature $\left(90-140^{\circ} \mathrm{C}\right)^{52}$ the $1 \mathrm{C}$ structure rapidly equilibrates to one or more of the vacancy-ordered structures discussed in Chapter 4 section $4.1 .1 .^{50}$ As the magnetic properties of $\mathrm{Fe}_{1-x} \mathrm{~S}$ are largely dependent on the composition and superlattice structure, these will be discussed later, in the aforementioned section.

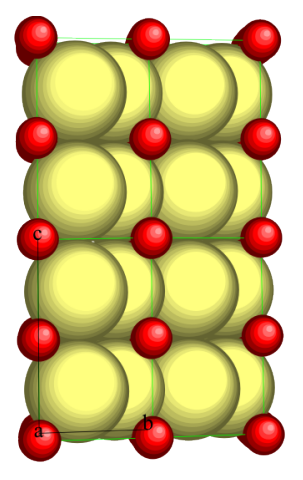

[100]

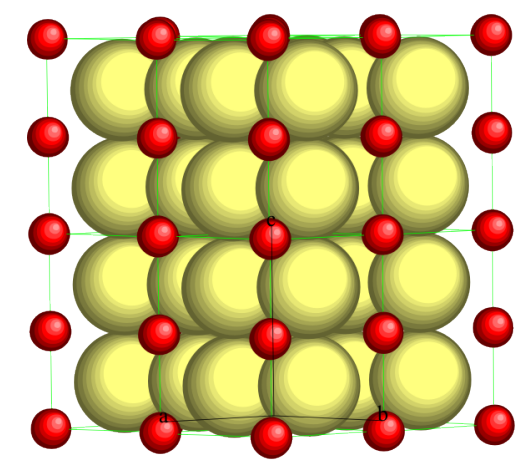

[110]

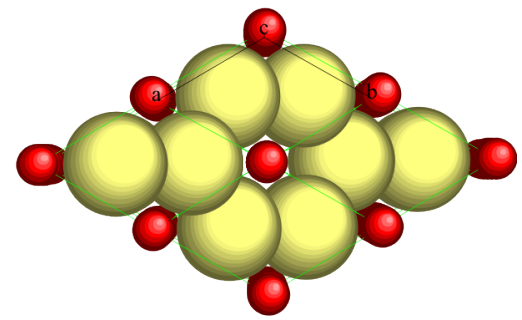

[001]

Figure 1-12 Crystal structure of NiAs-type $\mathrm{Fe}_{1-x} \mathrm{~S}$ (space group $P 6_{3} m c$ ). Iron is shown in red, sulfur is shown in yellow. Atomic radii are shown at $75 \%$ scale for clarity. 


\subsubsection{Iron and iron oxides}

Iron forms a variety of compounds with oxygen, with compositions ranging from $\mathrm{FeO}$ to $\mathrm{Fe}_{3} \mathrm{O}_{4}$.

\subsubsection{Iron}

At room temperature and ambient pressure iron possesses a bcc crystal structure $(\alpha-\mathrm{Fe}$, $\operatorname{Im} \overline{3} \mathrm{~m}$, see Figure 1-13 for crystal structure model), which converts to a fcc structure above $911^{\circ} \mathrm{C}(\gamma$-Fe, $F m \overline{3} m)$, then returns to a bcc structure $(\delta$-Fe, $\operatorname{Im} \overline{3} \mathrm{~m})$ between $1392^{\circ} \mathrm{C}$ and its melting point at $1536^{\circ} \mathrm{C} .^{53}$

Iron is a metallic conductor, with a resistivity of $9.7 \mu \Omega \mathrm{cm}^{-1}$ (at $20^{\circ} \mathrm{C}$ ) ${ }^{28}$ and is ferromagnetic below its Curie temperature of $770^{\circ} \mathrm{C} .{ }^{54}$ Iron has 8 valence electrons, slightly less than one of which is considered itinerant by transport measurements $\left(4 s^{0.95}\right)$; the remaining 7 are assigned to the relatively localised $3 d$ band $\left(3 d^{7.05}\right)$. Both spin-up and spin-down subbands are populated, resulting in an observed magnetic moment of $2.2 \mu_{\mathrm{B}} / \mathrm{Fe}^{54}$ Iron has a high saturation magnetisation of $1.7 \mathrm{MA} \mathrm{m}^{-1}$ $\left(217 \mathrm{~A} \mathrm{~m}^{2} \mathrm{~kg}_{-}{ }^{1}\right){ }^{54}$
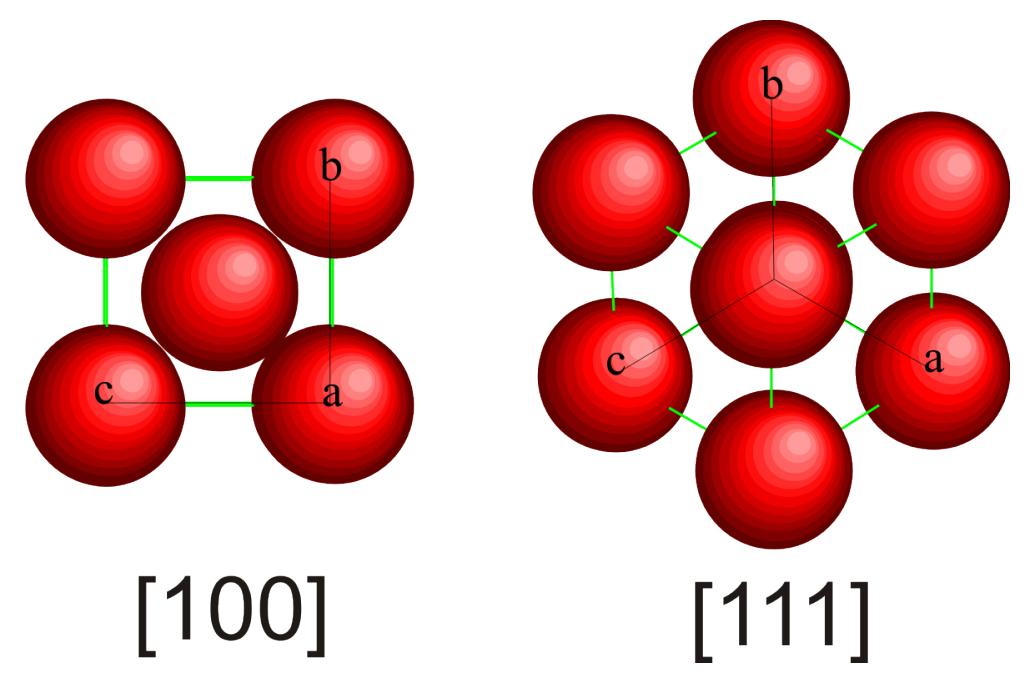

Figure 1-13 Crystal structure of $\alpha$-Fe (bcc, $\operatorname{Im} \overline{3} \mathrm{~m})$. 


\subsubsection{2 $\alpha-\mathrm{Fe}_{2} \mathrm{O}_{3}$ (haematite)}

$\alpha-\mathrm{Fe}_{2} \mathrm{O}_{3}$ (haematite) is a red compound, which crystallises in the $R \overline{3} c$ space group (see Figure 1-14 for crystal structure model). Like other iron oxides, superexchange leads to antiferromagnetic ordering in $\alpha-\mathrm{Fe}_{2} \mathrm{O}_{3}{ }^{54}$ Consequently, despite having a magnetic moment of $5 \mu_{\mathrm{B}}$ /formula unit, $\alpha-\mathrm{Fe}_{2} \mathrm{O}_{3}$ displays only very weak magnetisation $(\sim 0.36$ A $\mathrm{m}^{2} \mathrm{~kg}^{-1}$ ) above $-13^{\circ} \mathrm{C}$, due to a slight canting of the antiparallel spins. ${ }^{55}$

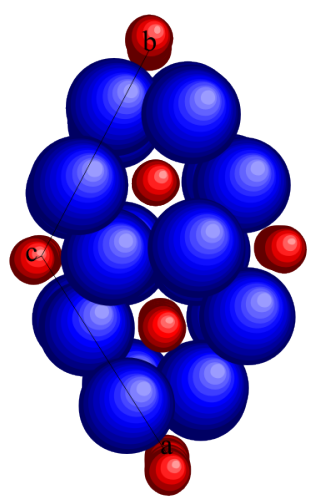

[001]

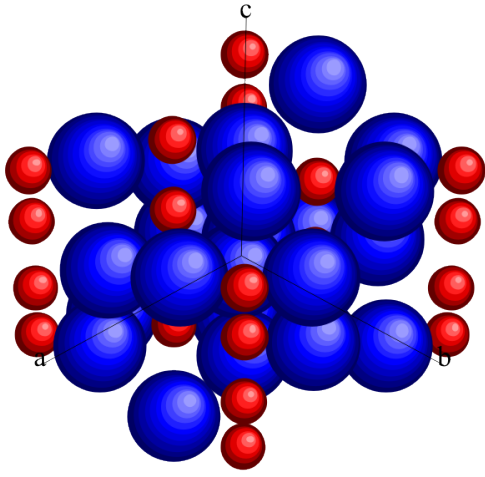

[111]

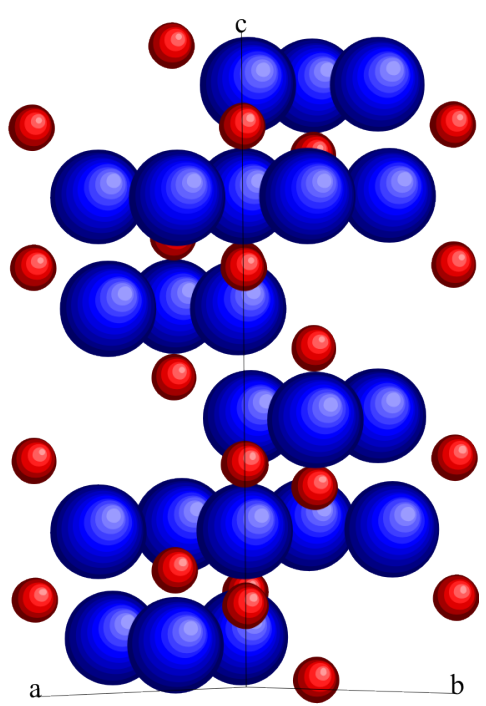

[110]

Figure 1-14 Crystal structure of $\alpha-\mathrm{Fe}_{2} \mathrm{O}_{3}(R \overline{3} c)$. Iron is shown in red, oxygen is shown in blue. Atomic radii shown at $75 \%$ scale for clarity.

\subsubsection{3 $\quad \mathrm{Fe}_{3} \mathrm{O}_{4}$ (magnetite)}

$\mathrm{Fe}_{3} \mathrm{O}_{4}$ (magnetite) is a black mixed $\mathrm{Fe}^{2+} / \mathrm{Fe}^{3+}$ oxide, with an inverse spinel structure (i.e. $\mathrm{Fe}^{\mathrm{III}}\left[\mathrm{Fe}^{\mathrm{II}} \mathrm{Fe}^{\mathrm{III}}\right] \mathrm{O}_{4}$, space group $F d \overline{3} m$ ), in which one in eight of the tetrahedral holes in the oxide fcc lattice are occupied by $\mathrm{Fe}^{3+}$ ions, and one in two of octahedral holes are occupied by $\mathrm{Fe}^{2+}$ and $\mathrm{Fe}^{3+}$ ions, in equal numbers (see Figure 1-15 for crystal structure model). At room temperature electron hopping between $\mathrm{Fe}^{2+}$ and $\mathrm{Fe}^{3+}$ ions in octahedral sites leads to moderate conductivity. ${ }^{56}$ Below the so-called Verwey transition point, which occurs at $120 \mathrm{~K}$, a distinct increase in resistivity is observed, which is attributed to localisation of $\mathrm{Fe}^{2+}$ and $\mathrm{Fe}^{3+}$ ions. Accompanying the increase in resistivity is a structural alteration from cubic to monoclinic symmetry, and the disappearance of magnetocrystalline anisotropy. ${ }^{57}$ Superexchange leads to antiferromagnetic coupling between each sublattice (tetrahedral and octahedral sites), resulting in an overall

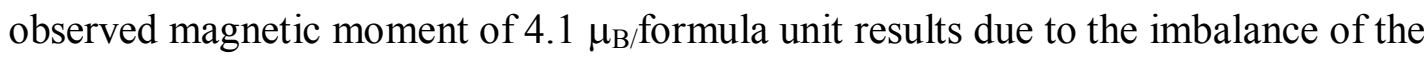


two sublattices (ferrimagnetism). The Néel temperature of the phase is $577^{\circ} \mathrm{C}$, and the saturation magnetisation of the bulk material is $84 \mathrm{~A} \mathrm{~m}^{2} \mathrm{~kg}^{-1} .54$

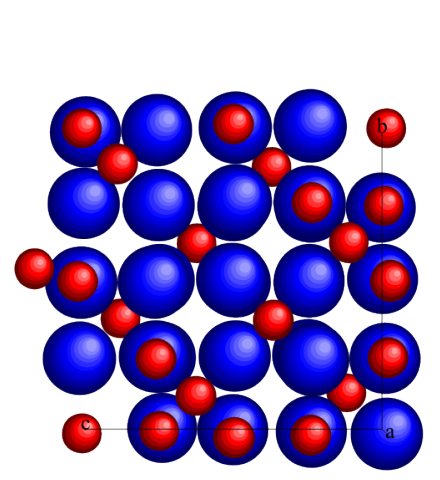

[100]

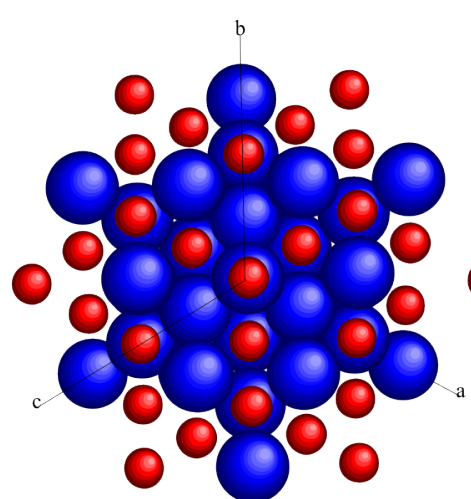

[111]

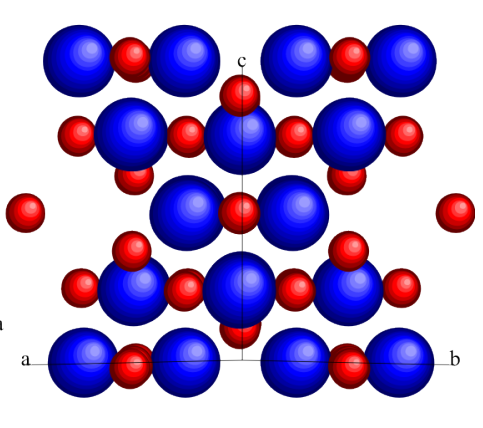

[110]

Figure 1-15 Crystal structure of $\mathrm{Fe}_{3} \mathrm{O}_{4}(F d \overline{3} m)$. Iron is shown in red, oxygen is shown in blue. Atomic radii shown at $75 \%$ scale for clarity.

\subsubsection{4 $\gamma-\mathrm{Fe}_{2} \mathrm{O}_{3}$ (maghemite)}

$\gamma-\mathrm{Fe}_{2} \mathrm{O}_{3}$ (maghemite) is a metastable brown solid with a defect spinel structure. Within

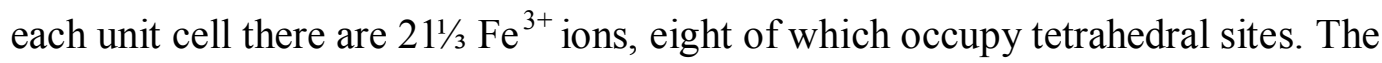
remainder are distributed randomly in the octahedral sites - i.e. there are $2 \frac{2}{3}$ cation vacancies distributed in octahedral sites. ${ }^{58}$ Ordering of the cation vacancies can result in either a primitive cubic base structure $\left(\mathrm{P}_{2} 32\right)$, or tetrahedral symmetry superlattice with a tripling of the cell in the $c$ direction $\left(\mathrm{C}=3 a, P 4_{3} 2{ }_{1} 2\right) .{ }^{59}$ Although formation of a solid solution of $\mathrm{Fe}_{3} \mathrm{O}_{4}-\gamma-\mathrm{Fe}_{2} \mathrm{O}_{3}$ has been observed also, where the fcc symmetry of the spinel was retained. ${ }^{58}$ Oxidation of $d^{6} \mathrm{Fe}^{2+}$ to $d^{5} \mathrm{Fe}^{3+}$ increases the observed magnetic moment to $5.0 \mu_{\mathrm{B}}$, however the saturation magnetisation at $74 \mathrm{~A} \mathrm{~m}^{2} \mathrm{~kg}^{-1}$ is slightly lower than that of $\mathrm{Fe}_{3} \mathrm{O}_{4} .{ }^{54}$ When heated under vacuum $\gamma-\mathrm{Fe}_{2} \mathrm{O}_{3}$ can be converted to $\mathrm{Fe}_{3} \mathrm{O}_{4}$, but when heated in air, it will convert to hep $\alpha-\mathrm{Fe}_{2} \mathrm{O}_{3}$. The crystal structure model for $\gamma-\mathrm{Fe}_{2} \mathrm{O}_{3}$ containing randomly located vacancies, where the $F d \overline{3} m$ space group is retained, is essentially identical to $\mathrm{Fe}_{3} \mathrm{O}_{4}$, and is shown above in Figure 1-15. Shown below is the crystal structure model for the primitive cubic $\left(P 4_{1} 32\right)$ alteration (Figure $1-16)$. 


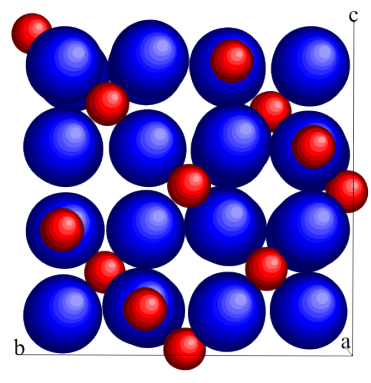

[100]

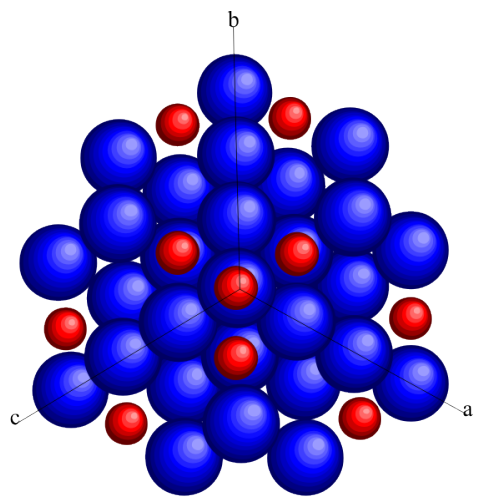

[111]

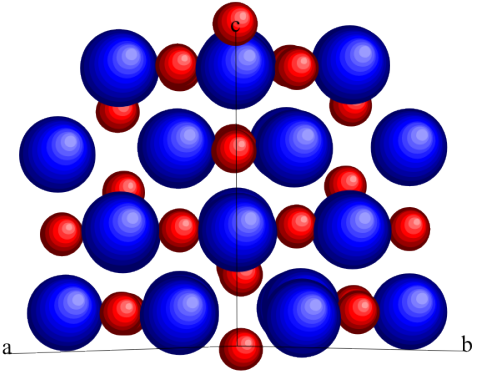

[110]

Figure 1-16 Crystal structure of primitive cubic $\gamma-\mathrm{Fe}_{2} \mathrm{O}_{3}\left(P 4_{2} 32\right)$. Iron is shown in red, oxygen in blue. Atomic radii are shown at $75 \%$ scale for clarity.

\subsubsection{FeO (wüstite)}

$\mathrm{FeO}$ (wüstite) is idealised as an iron(II) monoxide, but its non-stochiometry is well-known, ${ }^{60}$ and the bulk phase has a homogeneity range of $\mathrm{Fe}_{0.84} \mathrm{O}$ to $\mathrm{Fe}_{0.95} \mathrm{O}{ }^{28} \mathrm{Like}$ many other transition metal monoxides it crystallises with the rock salt structure (space group $F m \overline{3} m$, see Figure 1-17 for the crystal structure model), however iron vacancies have been found to cluster with iron $\mathrm{Fe}^{3+}$ ions occupying interstitial tetrahedral sites in the cubic oxide lattice. ${ }^{60}$ The magnetic moment is $4 \mu_{\mathrm{B}} /$ formula unit, reflecting the $d^{6}$ configuration of $\mathrm{Fe}^{2+}$, however the phase is antiferromagnetic below a Néel temperature of approximately $197 \mathrm{~K}\left(-76^{\circ} \mathrm{C}\right)$, and paramagnetic above and consequently does not support a saturation magnetisation at room temperature. ${ }^{25} \mathrm{FeO}$ is metastable, and is known to disproportionate to $\mathrm{Fe}$ and $\mathrm{Fe}_{3} \mathrm{O}_{4}$, or oxidise to $\mathrm{Fe}_{3} \mathrm{O}_{4}{ }^{28}$

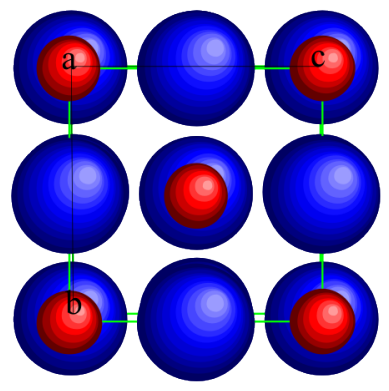

[100]

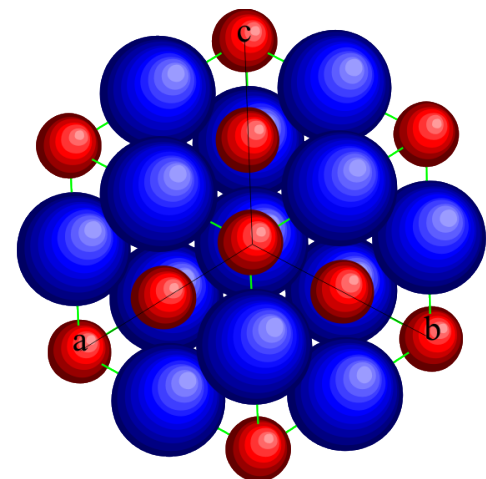

[111]

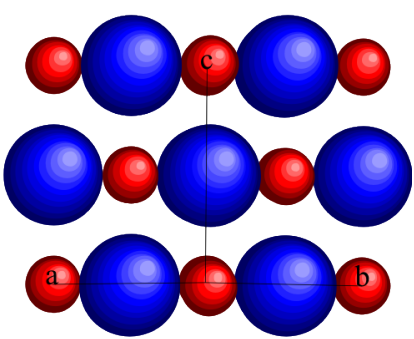

[110]

Figure 1-17 Crystal structure of FeO. Iron is shown in red, oxygen is shown in blue. Atomic radii shown at $75 \%$ scale for clarity. 


\subsubsection{Magnesium sulfide}

Magnesium sulfide in the bulk crystallises in the $F m \overline{3} m$ space group, with the rock salt structure (see Figure 1-18 for representation of crystal structure), although the zinc blende alteration has been prepared as a thin film. ${ }^{61}$ Magnesium sulfide has been of interest as a wide band gap semiconductor for device manufacture. The band gap of the rock salt structure has been calculated by pseudopotential density functional theory (DFT) to be $2.6 \mathrm{eV}$ and indirect. ${ }^{62}$ Whereas extrapolation of experimental measurements placed the direct band gap of the zinc blende alteration at $5.5 \mathrm{eV} .^{61}$

Magnesium sulfide appears to have been prepared in the nanocrystalline state only by high energy ball milling of $\mathrm{MgH}_{2}$ and elemental sulfur, to produce magnesium sulfide with an average crystallite diameter of $11 \mathrm{~nm}$, as judged by Scherrer line-broadening analysis. $^{63}$

A principle reason for this dearth of work may lie in the instability of magnesium sulfide with respect to hydrolysis by atmospheric moisture noted in the literature. ${ }^{64}$ The synthesis of Goo et al. ${ }^{63}$ mentioned above, was performed under an argon atmosphere, as were all characterisation procedures. Application of magnesium sulfide nanocrystals is hence likely to be severely limited by this instability.

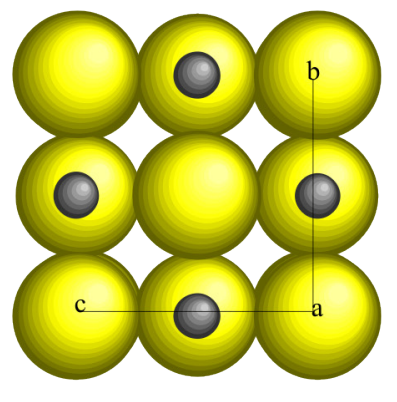

[100]

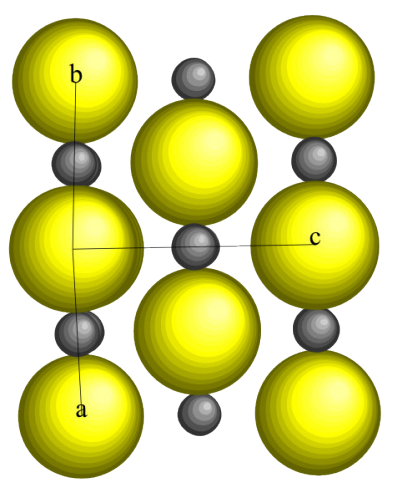

[110]

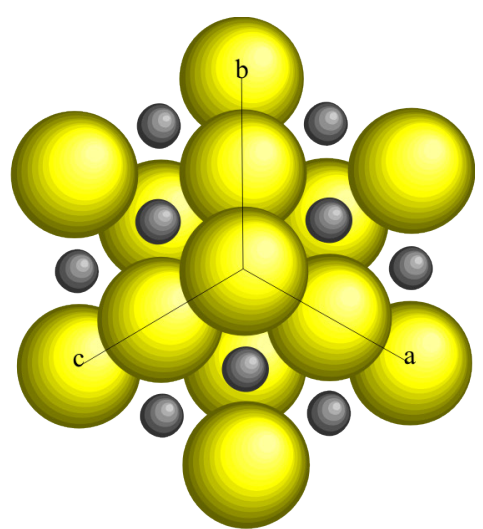

[111]

Figure 1-18 Crystal structure of magnesium sulfide $(F m \overline{3} m$, rock salt). Magnesium is in grey, and sulfur is in yellow. Atomic radii at $75 \%$ scale for clarity. 


\subsubsection{Nickel sulfides}

Nickel and sulfur form a variety of stoichiometric, and non-stoichiometric phases, ranging in composition from $\mathrm{Ni}_{3} \mathrm{~S}_{2}$ to $\mathrm{NiS}_{2}$. These include the polymorphs of $\mathrm{Ni}_{3} \mathrm{~S}_{2}$ : rhombohedral $\beta-\mathrm{Ni}_{3} \mathrm{~S}_{2}$ and cubic $\alpha-\mathrm{Ni}_{3} \mathrm{~S}_{2} ;$ orthorhombic $\mathrm{Ni}_{7} \mathrm{~S}_{6}$ and $\mathrm{Ni}_{9} \mathrm{~S}_{8}$; rhombohedral $\beta$-NiS; hexagonal $\alpha$-NiS (more properly $\alpha-\mathrm{Ni}_{1-x} \mathrm{~S}$ ); cubic spinel $\mathrm{Ni}_{3} \mathrm{~S}_{4}$ and cubic $\mathrm{NiS}_{2}$. The two nickel sulfide phases encountered in this project were $\alpha-\mathrm{Ni}_{1-x} \mathrm{~S}$ and $\mathrm{NiS}_{2}$. These two phases will now be introduced more fully. $\alpha-\mathrm{Ni}_{1-x} \mathrm{~S}$ and $\mathrm{NiS}_{2}$ are known carcinogens. $^{65}$

\subsubsection{1 $\alpha-\mathrm{Ni}_{1-x} \mathrm{~S}$}

$\alpha-\mathrm{Ni}_{1-x} \mathrm{~S}$ is the high temperature polymorph of NiS. The inversion temperature between the polymorphs varies from 379 to $282^{\circ} \mathrm{C}$, with decreasing nickel content; although $\alpha-\mathrm{Ni}_{1-x} \mathrm{~S}$ is quenchable as a metastable phase at room temperature. ${ }^{66}$ It ranges in composition from stoichiometric, to nickel deficient $\left(48 \mathrm{at} \% \mathrm{Ni}\right.$ at $356^{\circ} \mathrm{C}$, and $45 \mathrm{at} \%$ $\mathrm{Ni}$ at the eutectic of $\mathrm{Ni}_{1-x} \mathrm{~S}-\mathrm{NiS}{ }_{2}$ at $985^{\circ} \mathrm{C} . \alpha-\mathrm{Ni}_{1-x} \mathrm{~S}$ is isostructural with $\mathrm{Fe}_{1-x} \mathrm{~S}-$ crystallising in the hexagonal space group $\mathrm{P}_{3} m c$ with the NiAs-type structure (see Figure 1-19 for a representation of the crystal structure).

At room temperature $\alpha-\mathrm{Ni}_{1-x} \mathrm{~S}$ is a paramagnetic metallic conductor, ${ }^{67}$ but below $263 \mathrm{~K}$ transitions to an antiferromagnetic semiconductor, with magnetic moments (of $1.5 \mu_{\mathrm{B}}$ per formula unit) aligned ferromagnetically within a layer of nickel atoms, and antiferromagnetically between adjacent layers. ${ }^{68}$

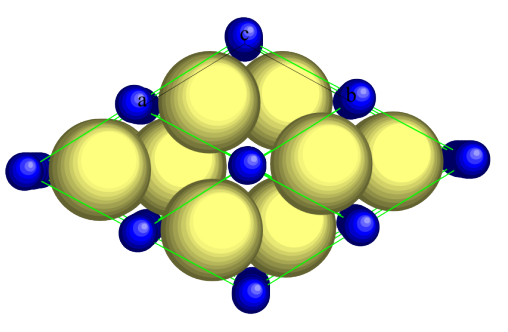

[001]

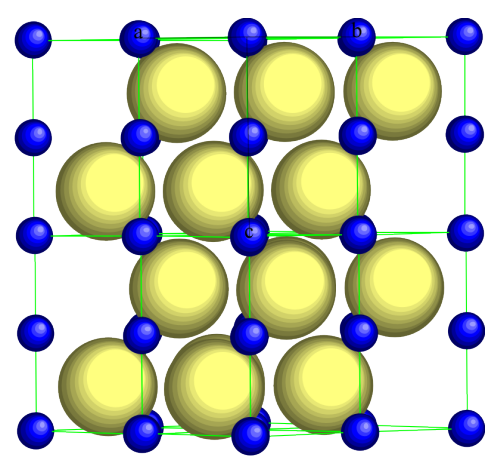

[110]

Figure 1-19 Crystal structure of $\alpha-\mathrm{Ni}_{1-x} \mathrm{~S}\left(P 6_{3} m c\right.$, NiAs-type). Nickel is shown in blue, and sulfur is shown in yellow. Atomic radii are shown at $75 \%$ for clarity. 


\subsubsection{2 $\mathrm{NiS}_{2}$ (vaesite)}

$\mathrm{NiS}_{2}$ is a stoichiometric phase, which crystallises in the cubic $P a \overline{3}$ space group, and is isostructural with $\mathrm{FeS}_{2}-\mathrm{p}$ (see section 1.3.2.1) - which can be viewed as being based on the rock salt structure, with fcc arrays of $\mathrm{Ni}^{2+}$ cations and $\mathrm{S}_{2}{ }^{2-}$ anions. $\mathrm{NiS}_{2}$ has a complex electronic and magnetic structure: at room temperature it is a paramagnetic insulator, which transitions to an antiferromagnetic insulator below $\mathrm{T}_{\mathrm{N}}$ of $<40 \mathrm{~K}^{69}$

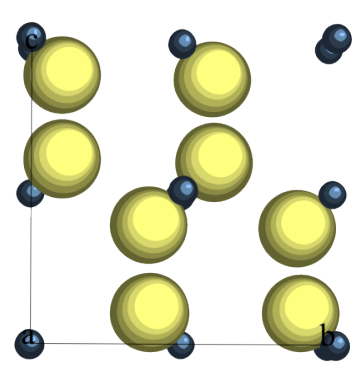

[100]

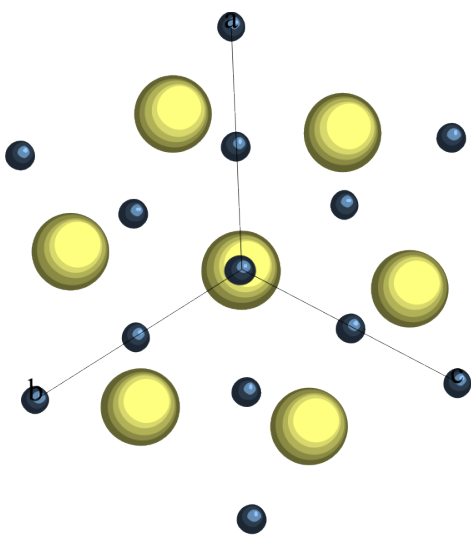

[111]

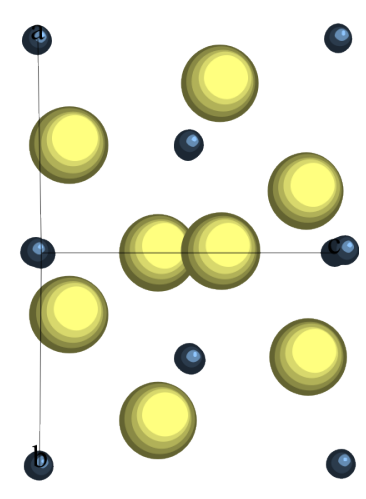

[110]

Figure 1-20 Crystal structure of $\mathrm{NiS}_{2}(\mathrm{~Pa} \overline{3}$, pyrite structure). Nickel is shown in blue, and sulfur is shown in yellow. Atomic radii are shown at $40 \%$ scale for clarity.

\subsubsection{Zinc sulfide}

Zinc sulfide has two polymorphs: zinc blende (sphalerite) and wurtzite; the names of which now serve as the names of the prototype structures, which are found in many AB-type compounds - especially the group 12 sulfides and oxides. ${ }^{28}$ Both structures feature tetrahedral coordination of the zinc atoms, and differ principally in the type of close packing found in the anion lattice - cubic in zinc blende, and hexagonal in wurtzite. See Figure 1-21 for a representation for the zinc blende structure, and Figure 1-22 for the wurtzite structure. Cubic zinc blende is the thermodynamically more stable polymorph at low temperatures, however the phase formed depends on temperature and other reaction conditions, such as sulfur concentration - hence it is common for a mixture of the two phases to be obtained..$^{70,71}$ 


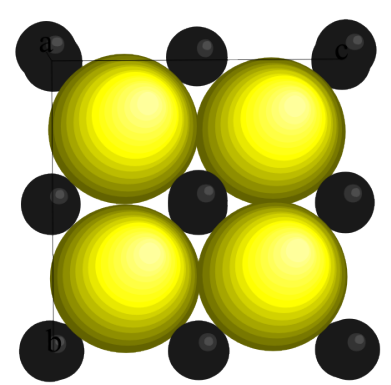

[100]

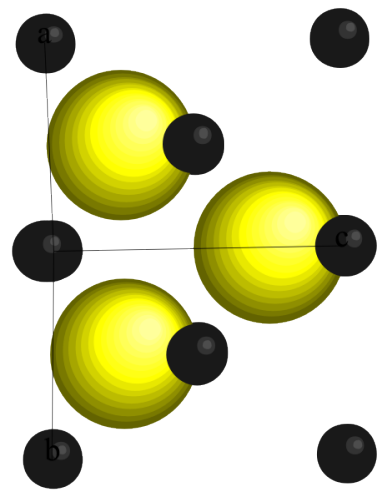

[110]
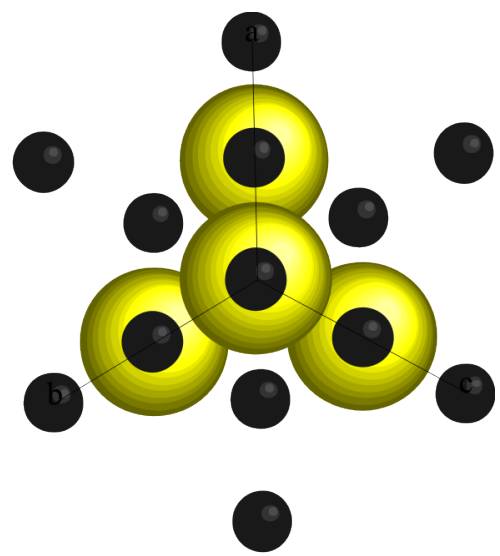

[111]

Figure 1-21 Crystal structure of zinc sulfide $(\overline{F 4} 3 m$, zinc blende). Zinc is shown in black, sulfur is shown in yellow. Atomic radii at $75 \%$ for clarity.

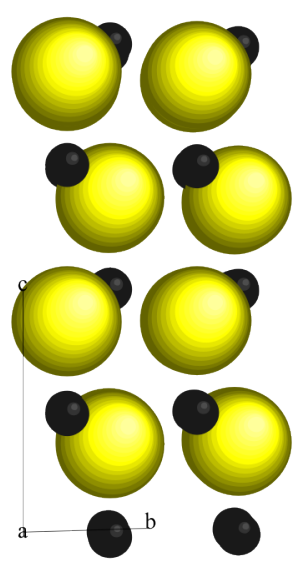

[100]

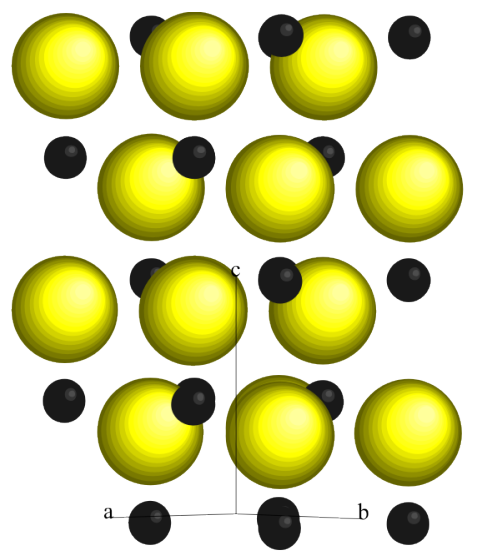

[110]

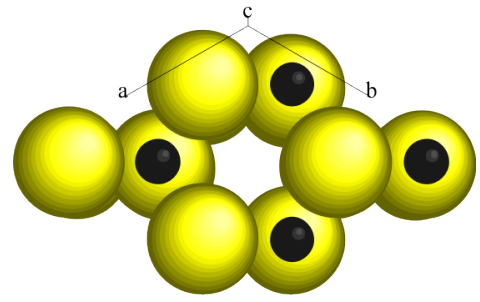

[001]

Figure 1-22 Crystal structure of zinc sulfide $\left(P 6_{3} m c\right.$, wurtzite). Zinc is shown in black, sulfur is shown in yellow. Atomic radii at $75 \%$ for clarity.

Pure zinc sulfide is white, and is commonly used as a pigment. Zinc sulfide doped with various transition metals serves as a phosphor for electron beams, X-rays or gamma rays; and hence is frequently used in cathode ray tubes, or in phosphorescent screens. ${ }^{28}$ Zinc sulfide is a wide band gap semiconductor $(\sim 3.8 \mathrm{eV}, 330 \mathrm{~nm}),{ }^{72}$ and as such has found much use as a passivating layer for narrow band gap materials, such as cadmium selenide. CdSe/ZnS core/shell nanocrystals have been widely researched over the past two decades, as the zinc sulfide capping has been found to increase the quantum yield of the cadmium selenide core $;^{73,74}$ eliminate or reduce trap-state emission $;{ }^{73}$ reduce photobleaching $;^{73}$ and decrease toxicity associated with the leaching of cadmium ions. ${ }^{75}$ 


\subsection{References}

(1) J. Park, J. Joo, S. G. Kwon, Y. Jang and T. Hyeon, Angew. Chem. Int. Ed., 2007, 46, 4630.

(2) S. V. Gaponenko, Optical Properties of Semiconductor Nanocrystals, Cambridge University Press, Cambridge, 1998.

(3) V. K. LaMer and R. H. Dinegar, J. Am. Chem. Soc., 1950, 72, 4847.

(4) S. G. Kwon, Y. Piao, J. Park, S. Angappane, Y. Jo, N.-M. Hwang, J.-G. Park and T. Hyeon, J. Am. Chem. Soc., 2007, 129, 12571.

(5) S. E. Habas, H. Lee, V. Radmilovic, G. J. Somorjai and P. Yang, Nature Mater., 2007, 6, 692.

(6) J. P. Wilcoxon and P. P. Provencio, J. Am. Chem. Soc., 2004, 126, 6402.

(7) A. R. Kortan, R. Hull, R. L. Opila, M. G. Bawendi, M. L. Steigerwald, P. J. Carroll and L. E. Brus, J. Am. Chem. Soc., 1990, 112, 1327.

(8) H. Reiss, J. Chem. Phys., 1951, 19, 482.

(9) X. Peng, J. Wickham and A. P. Alivisatos, J. Am. Chem. Soc., 1998, 120, 5343.

(10) D. V. Talapin, A. L. Rogach, M. Haase and H. Weller, J. Phys. Chem. B, 2001, 105, 12278.

(11) R. Hunter, J., Foundations of Colloid Science, Oxford University Press, Oxford, 2001.

(12) J. T. G. Overbeek, Chem. Aust., 1981, 48, 419.

(13) R. Rossetti, J. L. Ellison, J. M. Gibson and L. E. Brus, J. Chem. Phys., 1984, 80, 4464.

(14) R. Rossetti, S. Nakahara and L. E. Brus, J. Chem. Phys., 1983, 79, 1086.

(15) P. Berger, P. Enzel, N. B. Adelman, K. J. Beckman, D. J. Campbell, A. B. Ellis and G. C. Lisensky, J. Chem. Educ., 1999, 76, 943.

(16) J. A. Carrazana-García, M. A. Lopez-Quintela and J. R. Rey, IEEE Transactions on Magnetics, 1995, 31, 3126

(17) Y. Mao, T.-J. Park, F. Zhang, H. Zhou and S. S. Wong, Small, 2007, 3, 1122.

(18) C. B. Murray, D. J. Norris and M. G. Bawendi, J. Am. Chem. Soc., 1993, 115, 8706.

(19) L. Qu, Z. A. Peng and X. Peng, Nano Lett., 2001, 1, 333.

(20) I. Mekis, D. V. Talapin, A. Kornowski, M. Haase and H. Weller, J. Phys. Chem. $B, 2003, \mathbf{1 0 7}, 7454$.

(21) J. Turkevich, P. C. Stevenson and J. Hillier, Discussions of the Faraday Society, 1951, 11, 55.

(22) C. Petit, P. Lixon and M.-P. Pileni, J. Phys. Chem., 1993, 97, 12974.

(23) M. G. Bawendi, M. L. Steigerwald and L. E. Brus, Annu. Rev. Phys. Chem., 1990, 41, 477.

(24) A. I. Ekimov, F. Hache, M. C. Schanne-Klein, D. Ricard, C. Flytznis., A. Kudryavtsev, T. V. Yazeva, A. V. Rodina and A. L. Efros, J. Opt. Soc. Am. B: Opt. Phys., 1993, 10, 100.

(25) R. O'Handley, Modern Magnetic Material: Principles and Applications, John Wiley \& Sons, New York, 2000, 29-43.

(26) R. C. O'Handley, Modern Magnetic Materials: Principles and Applications, John Wiley \& Sons, New York, 2000, 306-307.

(27) A. H. Morrish, The Physical Principles of Magnetism, The Institute of Electrical and Electronics Engineers, New York, 2001, 340-344.

(28) N. N. Greenwood and A. Earnshaw, Chemistry of the Elements, ButterworthHeinemann, Oxford, 1984.

(29) A. Reisman, M. Berkenblit and M. Witzen, J. Phys. Chem., 1962, 66, 2210. 
(30) M. Itakura and H. Toyoda, Jpn. J. Appl. Phys., 1965, 4, 560.

(31) C. Kittel, Introduction to Solid State Physics, John Wiley New York, 1986.

(32) D. E. McCarthy, Appl. Opt., 1967, 6, 1896

(33) D. Rickard and G. W. I. Luther, Chem. Rev., 2007, 107, 514.

(34) G. Brostigen and A. Kjekhus, Acta Chem. Scand., 1969, 23, 2186.

(35) B. J. Skinner, R. C. Erd and F. S. Grimaldi, Am. Mineral., 1964, 49, 543.

(36) M. S. Jagadeesh and M. D. Seehra, Phys. Lett. A, 1980, 80, 59.

(37) M. R. Spender, J. M. D. Coey and A. H. Morrish, Can. J. Phys., 1972, 50, 2313.

(38) M. Uda, Z. Anorg. Allg. Chem., 1967, 350, 105.

(39) M. Uda, Am. Mineral., 1965, 50, 1487.

(40) S. Yamaguchi and H. Wada, J. Appl. Phys., 1969, 41, 1873.

(41) R. C. Erd, H. T. Evans and D. H. Richter, Am. Mineral., 1957, 42, 309.

(42) L. A. Taylor and K. L. Williams, Am. Mineral., 1972, 57, 1571.

(43) E. F. Makarov, A. S. Marfunin, A. R. Mkrtchyan, G. N. Nadzharyan, V. A. Povitskii and R. A. Stukan, Sov. Phys. Solid State, 1969, 11, 391.

(44) Y. Furukawa and H. L. Barnes, Geochim. Cosmochim. Acta, 1996, 60, 3581.

(45) M. Wolthers, S. J. Van der Gaast and D. Rickard, Am. Mineral., 2003, 88, 2007.

(46) D. J. Vaughan and M. S. Ridout, J. Inorg. Nucl. Chem., 1971, 33, 741.

(47) A. R. Lennie, K. E. R. England and D. J. Vaughan, Am. Mineral., 1995, 80, 960.

(48) A. R. Lennie, S. A. T. Redfern, P. F. Schofield and D. Vaughan, Mineral. Mag., 1995, 59, 677.

(49) A. J. Devey, R. Grau-Crespo and N. H. de Leeuw, J. Phys. Chem. C, 2008, 112, 10960.

(50) S. A. Kissin and S. D. Scott, Econ. Geol., 1982, 77, 1739.

(51) A. Sugaki and H. Shima, Sci. Rep. Tohoku Univ. 3rd Ser. Mineral. Petro. Econ. Geol., 1977, 13, 147.

(52) H. Wang and I. Salveson, Phase Transitions, 2005, 78, 547.

(53) W. Martienssen and H. Warlimont, eds., Springer Handbook of Condensed Matter and Materials Data, Springer, Berlin, 2005.

(54) R. C. O'Handley, Modern Magnetic Materials: Principles and Applications, John Wiley and Sons, New York, 2001, 99.

(55) R. Nathans, S. J. Pickart, H. A. Alperin and P. J. Brown, Physical Review A, 1964, 136, 1641.

(56) E. J. W. Verwey, Nature, 1939, 144, 327.

(57) F. Walz, J. Phys.: Condens. Matter, 2002, 14, R285.

(58) G. A. Waychunas, Review of Mineralogy, 1991, 25, 11.

(59) C. Greaves, J. Solid State Chem., 1983, 49, 325.

(60) H. Fjellvåg, F. Grønvold and S. Stølen, J. Solid State Chem., 1996, 124, 52.

(61) H. Okuyama, K. Nakano, T. Miyajima and K. Akimoto, Jpn. J. Appl. Phys., 1991, 30, L1620.

(62) S. Duman, S. Bağc1, H. M. Tütüncü and G. P. Srivastava, Phys. Rev. B, 2006, 73, 205201.

(63) N. H. Goo and M. Hirscher, J. Alloys Compd., 2005, 404-406, 503.

(64) E. Tiede, Ber. Dtsch. Chem. Ges., 1916, 49, 1745.

(65) G. Aylward and T. Findlay, S I Chemical Data, Jacaranda Wiley, Milton, 1994.

(66) G. Kullerud and R. A. Yund, J. Petrology, 1962, 3, 126.

(67) J. L. Horwood, L. G. Ripley, M. G. Townsend and R. J. Tremblay, J. Appl. Phys., 1971, 42, 1476.

(68) S. J. Gurman and J. B. Pendry, Surf. Sci., 1976, 57, 241.

(69) M. Matsuura, H. Hiraka, K. Yamada and Y. Endoh, J. Phys. Soc. Jpn., 2000, 69, 1503.

(70) S. D. Scott and H. L. Barnes, Geochim. Cosmochim. Acta, 1972, 36, 1275. 
(71) P. J. Thomas, P. Christian, S. Daniels, Y. Li, Y. S. Wang and P. O'Brien, Pure Appl. Chem., 2006, 78, 1651.

(72) D. V. Talapin, I. Mekis, S. Götzinger, A. Kornowski, O. Benson and H. Weller, J. Phys. Chem. B, 2004, 108, 18826.

(73) M. A. Hines and P. Guyot-Sionnest, J. Phys. Chem. B, 1996, 100, 468.

(74) B. O. Dabbousi, J. Rodriguez-Viejo, F. V. Mikulec, J. R. Heine, H. Mattoussi, R. Ober, K. F. Jensen and M. G. Bawendi, J. Phys. Chem. B, 1997, 101, 9463.

(75) A. M. Derfus, W. C. W. Chan and S. N. Bhatia, Nano Lett., 2004, 4, 11. 


\section{Chapter 2 Experimental Methods}

\subsection{General Synthetic Procedure}

Detailed experimental procedures are supplied in the method sections of each results chapter. A generalised description of the synthetic and purification procedures used in this project follows.

\subsubsection{Solution-based synthesis}

In this project, metal chalcogenide nanocrystals were synthesised exclusively by solution-based chemical methodology. Reactions were in general between a metal-containing precursor compound (e.g. iron(II) acetylacetonate, $\mathrm{Fe}(\mathrm{acac})_{2}$ ) and a chalcogen-containing precursor compound (e.g. trioctylphosphine selenide, Se(TOP) or elemental sulfur). Although in the case of the work presented in Chapter 6 (Single-source Metal Sulfide Precursors for Nanocrystal Synthesis), both the metal and chalcogenide source were within a single compound.

Coordinating solvents were used in order to restrict the size of the resulting nanocrystals. These solvents included long-chain primary amines (e.g. hexadecylamine, oleylamine) or alkylphospine oxides (trioctylphosphine oxide), which are generally thought to adhere to the nanocrystal surface after synthesis and purification and provide both dispersibility in non-polar solvents, and a steric barrier to aggregation. These solvents were also chosen for their high boiling points $\left(>300^{\circ} \mathrm{C}\right)$, as temperatures between $200-300^{\circ} \mathrm{C}$ proved necessary for most syntheses.

Nitrogen was used to provide an inert atmosphere during reactions in order to prevent the formation of oxides, and all solvents were saturated with nitrogen before use termed degassing. This was typically achieved by bubbling nitrogen through the solvent for 15 - 30 minutes before use, although in some cases solvents were distilled under vacuum and stored under nitrogen.

\subsubsection{Experimental Set-up}

A schematic of the basic experimental setup used in this project is shown below (Figure 2-1). Nanocrystal synthesis reactions were performed in a glass three-necked round 
bottom flask. The flask was fitted with a water condenser for cooling, and a glass pocket housing a K-type thermocouple, which extended into the liquid to enable monitoring of the solution temperature. The top of the condenser was connected via an adaptor to a dual nitrogen-vacuum manifold, such that a pressure-equalised nitrogen atmosphere could be maintained in the apparatus. The apparatus was either purged by flowing nitrogen through, or evacuated and filled with nitrogen prior to use.

The remaining neck of the flask was fitted with a ground glass stopper or rubber septum, and was used for the introduction of reactants and the removal of aliquots of reaction mixture. When a septum was not used, the nitrogen flow through the manifold was set, such that when the stopper was removed a vigorous flow of nitrogen down the condenser and out the neck blanketed the contents of the flask from the atmosphere. Hence transfers of material could be achieved while maintaining an inert atmosphere in the apparatus. A heating mantle with built-in magnetic stirrer was used to heat the flask, and reaction temperatures in excess of $300^{\circ} \mathrm{C}$ could be regularly reached and maintained. Glass-coated stir bars were used in conjunction with the magnetic stirrer, as reaction temperatures routinely exceeded the service temperature of Teflon.

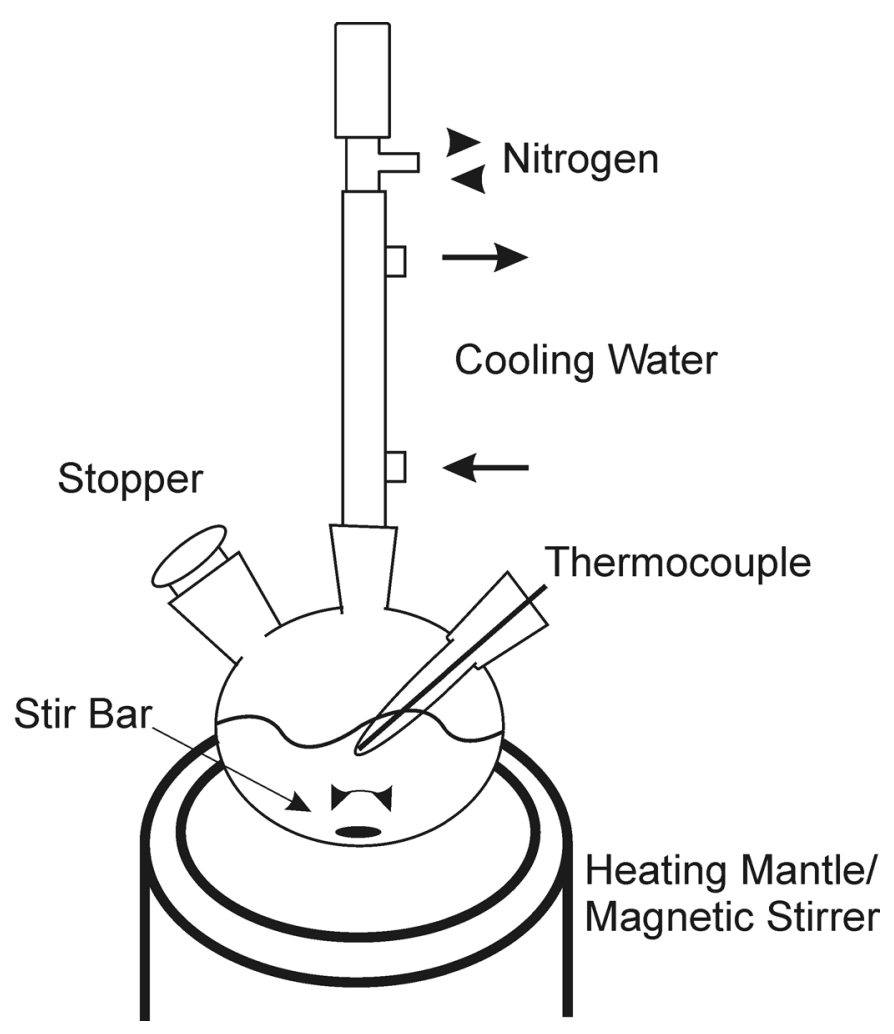

Figure 2-1 Schematic of the basic experimental setup for nanocrystal synthesis reactions. 
Preparation and manipulation of the metal complexes appearing in Chapter 6 were achieved using standard airless techniques. A dual nitrogen-vacuum manifold was used, with a vacuum pump capable of reaching pressures of $0.6 \mathrm{mmHg}(80 \mathrm{~Pa})$. Reactions were conducted in a Schlenk tube, which was evacuated and filled with nitrogen before use. Transfers of liquids were performed by syringe; and transfers of solid were performed rapidly to minimise exposure to the atmosphere - while the contents of the initial and receiving vessels were blanketed with a flow of nitrogen during transfer.

\subsubsection{Purification}

The non-volatility and high boiling point of the solvents used for reactions is disadvantageous for purification purposes. In order to remove the solvent and any remaining dissolved species, centrifugation was used to isolate the inorganic fraction. Typically, nanocrystal suspensions were first destabilised by adding a poor solvent (this was in most cases a relatively polar solvent, such as acetone). Unfavourable interactions between the non-polar surface ligands on the nanocrystals with a polar solvent lead to aggregation of particles, hastening precipitation. The turbidity of the suspension was used as a rough guide to the degree of aggregation - once a solution became turbid, a significant fraction of the suspended material could be precipitated rapidly by centrifugation.

Once a pellet had formed, the supernatant was decanted, and the pellet was washed with clean solvent (typically toluene) and agitated (typically a mixture of manual shaking and sonication). This process of destabilisation, centrifugation to a pellet, decanting of supernatant and resuspension in solvent was repeated several times as required. It was generally found that $2-3$ cycles would render a sample sufficiently pure from non-volatile organic materials for TEM analysis. 


\subsection{Characterisation Procedure}

\subsubsection{Powder X-ray Diffraction (XRD) theory}

Powder X-ray diffraction is a powerful tool for identifying crystalline phases, and is especially useful in the case of mixtures of phases. Diffraction techniques in general are complementary to elemental analysis, which is able to determine composition, but unable to establish chemical identity. In areas where multiple structural polymorphs, or a range of phases with similar compositions exist, diffraction evidence is especially critical to establish identity. Electron diffraction was also used extensively in this project to give localised information (see section 2.2.4), however powder XRD was preferred when a complex mixture of phases was present, and to give confirmation of the overall phase composition of the sample.

\subsubsection{Diffraction of X-rays by a crystal}

Electromagnetic radiation can be scattered by interaction with matter. When the electric field component of a photon impinges upon an electron, an alternating acceleration is imparted to the electron, which responds by emitting radiation - this can be viewed as an absorption and re-emission process that scatters radiation in all directions for a given scattering angle. ${ }^{1}$ Photons, which are scattered by the periodic electron density inherent to the ordered nature of a crystal, interfere with one another, giving rise to a diffraction pattern. Spacing between planes of atoms in crystals is on the order of the wavelength of X-ray radiation (in the order of $10^{-10} \mathrm{~m}$ ), leading to a strong scattering interaction for X-ray wavelengths.

The Laue equations stipulate the geometric requirements for constructive interference, and hence reinforcement, of X-rays scattered from an array of atoms. Constructive interference occurs when photons scattered from different centres remain in phase. This occurs in the case of a regularly-spaced one-dimensional array of atoms, when the difference in path travelled for photons scattered from adjacent centres is equal to an integer number of wavelengths, as is depicted schematically in Figure 2-1 and formalised in expression (1), where $b$ is the interatomic spacing; $\phi$ and $\psi$ are the incident and scattered angles, respectively; $\lambda$ is wavelength; and $k$ is an integer.

$$
b\left(\cos \psi_{2}-\cos \phi_{2}\right)=k \lambda
$$


Expression (1) can be converted to a vector format, as shown in expression (2), where b is the unit vector along the relevant axis, $\mathbf{s}$ is the vector of the scattered wave, $\mathbf{s}_{0}$ is the vector of the incident wave, and $\mathbf{S}$ is the scattering vector, which is defined in (3) and depicted in Figure 2-3. This can be generalised to an array in all three dimensions to give the three Laue equations shown as expression (4):

$\frac{\mathbf{b} \bullet\left(\mathbf{s}-\mathbf{s}_{0}\right)}{\lambda}=\mathbf{b} \bullet \mathbf{S}=k$

$\mathbf{S}=\left|\mathbf{s}-\mathbf{s}_{0}\right| / \lambda$

$\mathbf{a} \bullet \mathbf{S}=h$

$\mathbf{b} \bullet \mathbf{S}=k$

$\mathbf{c} \bullet \mathbf{S}=l$

When all three equations are satisfied simultaneously, the three dimensional array - or crystal - scatters in phase, and the scattered X-rays reinforce each other to emerge at a set orientation with respect to the incident beam. The X-rays scattered from the crystal at a particular angle are termed a reflection, and may be detected on photographic film or an X-ray detector (e.g. scintillation counter, proportional detector, or CCD area detector).

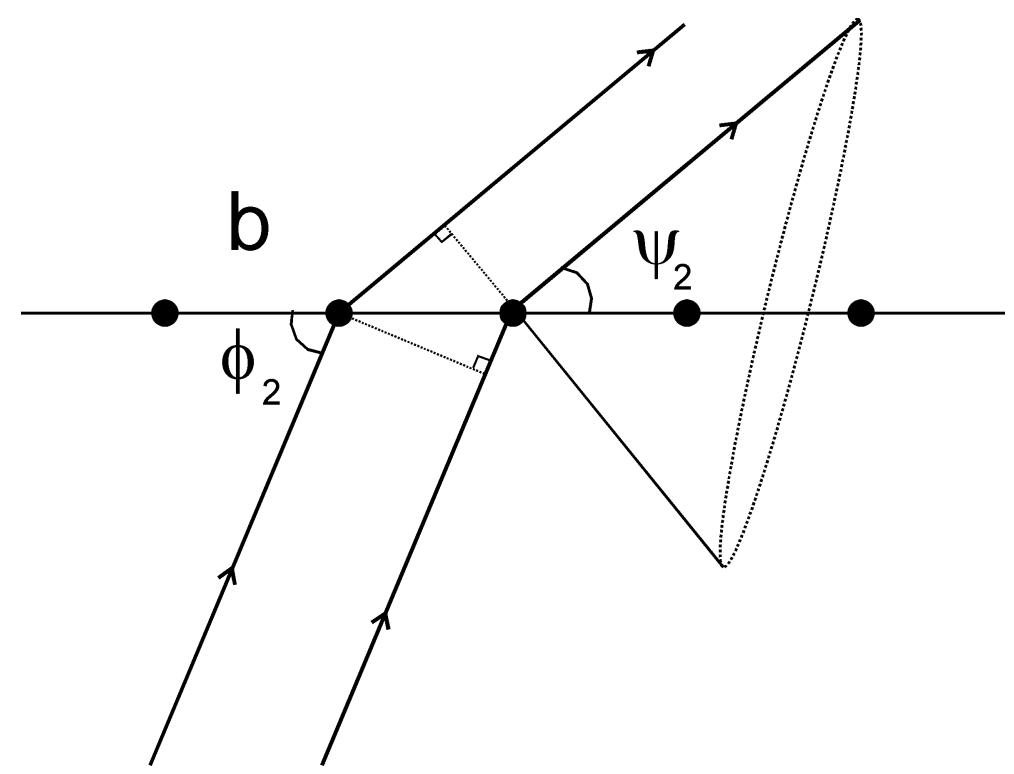

Figure 2-2 Schematic depicting scattering from a one-dimensional array. 


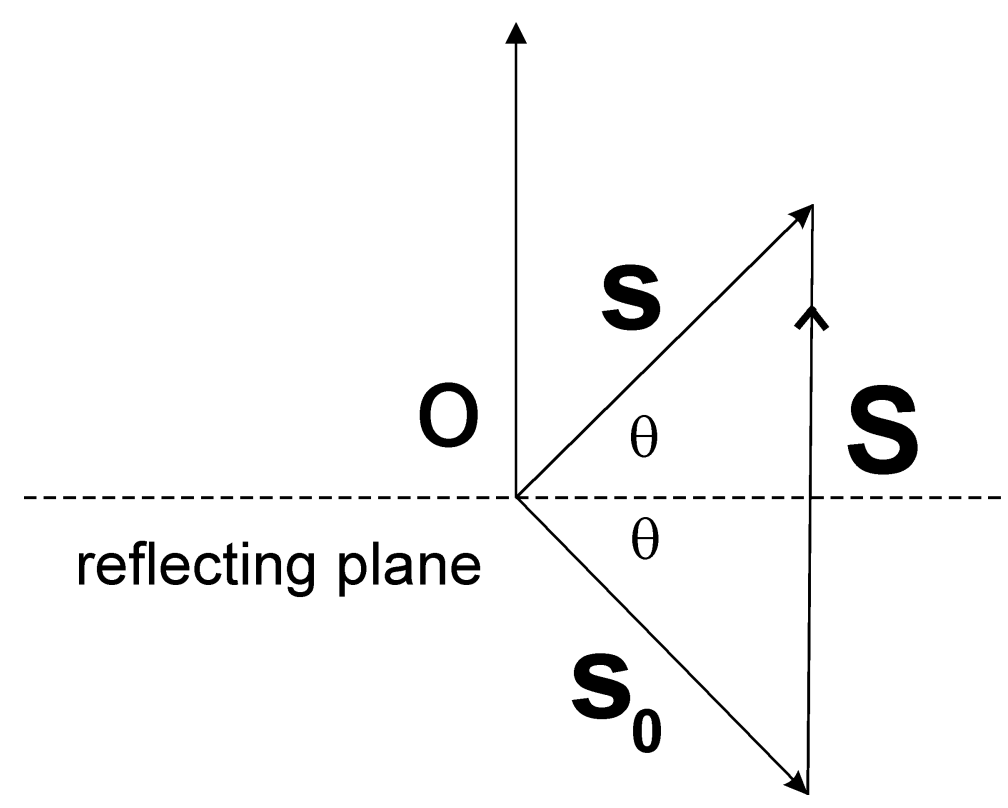

Figure 2-3 Definition of scattering vector.

An alternative method of conceptualising the scattering process is given by the Bragg model, which is derived from the Laue diffraction laws. As shown in Figure 2-4, two parallel rays are pictured as reflecting from neighbouring parallel planes. For the two rays to emerge in-phase, the extra path length travelled by the second ray must equal an integer number of wavelengths. This condition is only satisfied for a given interplanar spacing, $d_{h k l}$, and for a given wavelength, $\lambda$, by a particular angle, $\theta$, termed the Bragg angle. This leads to the Bragg equation (5), where $n$ is an integer. The diffraction angle $2 \theta$ - the angle between the incident and scattered beam - can be measured experimentally, and if monochromatic radiation source of known wavelength is used, the interplanar spacing, $d_{h k l}$, can be determined. Conventionally, for this purpose the value of $n$ is assumed to be unity.

$2 d_{(h k l)} \sin \theta=n \lambda$

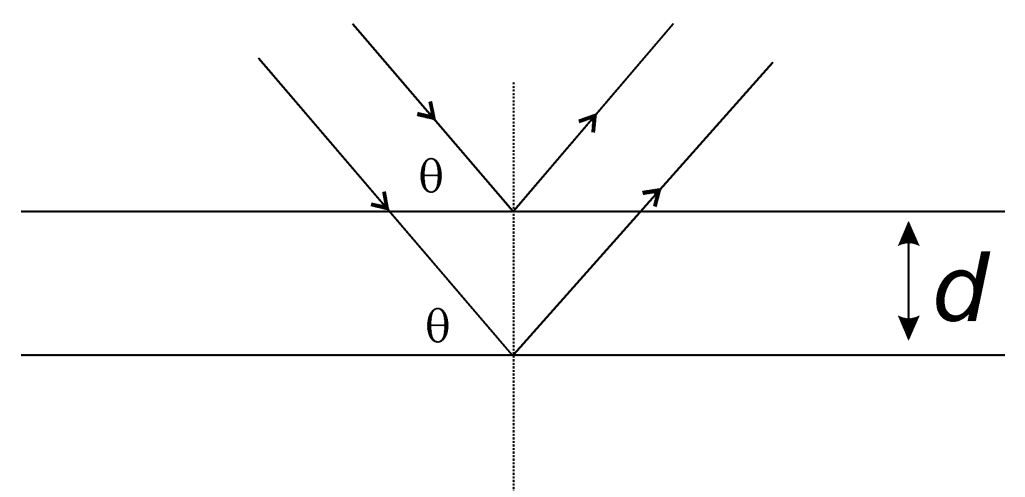

Figure 2-4 Bragg model of reflection of X-rays from a set of parallel planes. 


\subsubsection{Intensity of X-ray reflections}

The absolute intensity of X-ray reflections is dependent on experimental parameters, such as the intensity of the X-ray source, geometry of the diffractometer, integration time and the degree of absorption of the sample. Consequently, the intensity of reflections is normalised, and reported as a relative intensity.

The relative intensity of a reflection is governed by the structure factor shown in expression (6), where the structure factor, $F_{(h k l)}$, of a set of planes with indices, $h k l$, is the sum of scattering contributions from all $n$ atoms within the unit cell. The contribution of the $j$ th atom is the product of its atomic scattering factor $f_{j, \theta}$, and an exponential term containing its fractional coordinates within the unit cell.

$$
F(h k l)=\sum_{j=1}^{n} f_{j, \theta} \exp \left[\mathrm{i} 2 \pi\left(h x_{j}+k y_{j}+l z_{j}\right)\right]
$$

As can be seen, the relative intensities contain much information about the structure of a crystal, and indeed if steps are taken to solve the so-called phase problem of crystallography and a three-dimensional model of the electron density distribution of the crystal may be derived.

\subsubsection{Diffraction of X-rays by a powder}

For a single crystal with a given orientation to the incident X-ray beam, a set of planes with a characteristic interplanar spacing may satisfy the Bragg condition, leading to the observation of a set of symmetry-related reflections. In order to observe all possible reflections, the orientation of the crystal with respect to the incident beam must be systematically varied to bring all planes sequentially into diffraction conditions. Experimentally this can be achieved by rotating an orientated specimen crystal around three non-collinear axes, to collect progressively the entire diffraction pattern.

An alternative approach is to work with a powder of many small, randomly-orientated crystallites. The Bragg condition for all interplanar spacings will be satisfied by a sub-population within the sample, and all reflections will be observable simultaneously. As the crystallites are distributed randomly around the incident beam axis, instead of a set of discrete spots for each set of symmetry-related reflections, a cone of diffracted 
X-rays is produced (see Figure 2-5). Again, the diffracted X-rays may be detected on photographic film, or by an X-ray detector, and the angle between the generator of the cone and the incident beam measured to provide the Bragg angle.

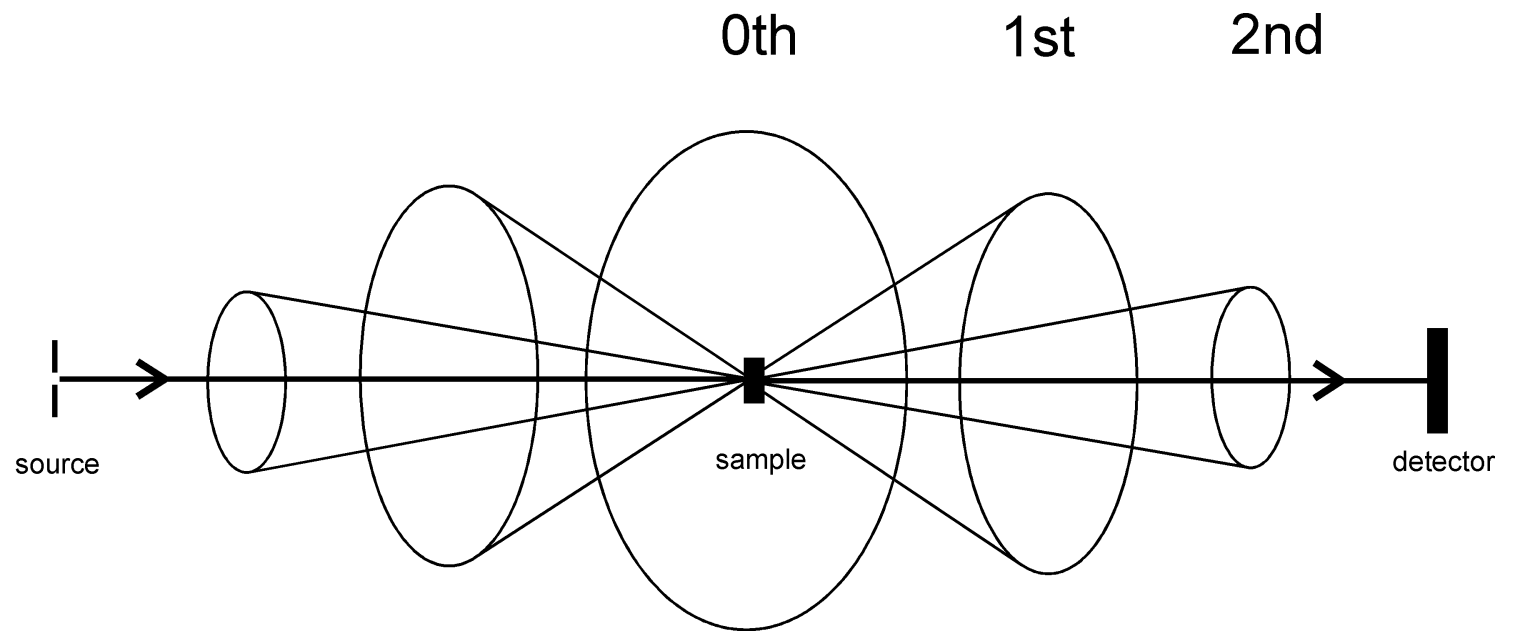

Figure 2-5 Schematic depicting diffraction from a powder, showing cones of diffracted X-rays for successive orders of diffraction. 


\subsubsection{Line shape of X-ray reflections}

Most modern powder X-ray diffraction work is performed with diffractometers, rather than photographic film-based cameras. The diffraction pattern is typically displayed as a line graph of intensity versus $2 \theta$. This intensity profile represents a one dimensional slice through the cones of diffracted X-rays illustrated in Figure 2-5. X-ray reflections are represented as peaks rising above the background. The line shape of X-ray diffraction peaks contains information about the average crystallite diameter. The width of a reflection $h k l$ (usually defined as the FWHM) can be related to the average diameter of the crystallites in the direction perpendicular to these planes, $D_{h k l}$, by the Scherrer equation shown in expression (7), where $K$ is a constant dependent on the crystallite shape, $\lambda$ is the wavelength of the radiation, $\omega$ is the pure diffraction breadth of the reflection, and $\theta$ is the Bragg angle. ${ }^{2}$

$D_{h k l}=\frac{K \lambda}{\omega \cos \theta}$

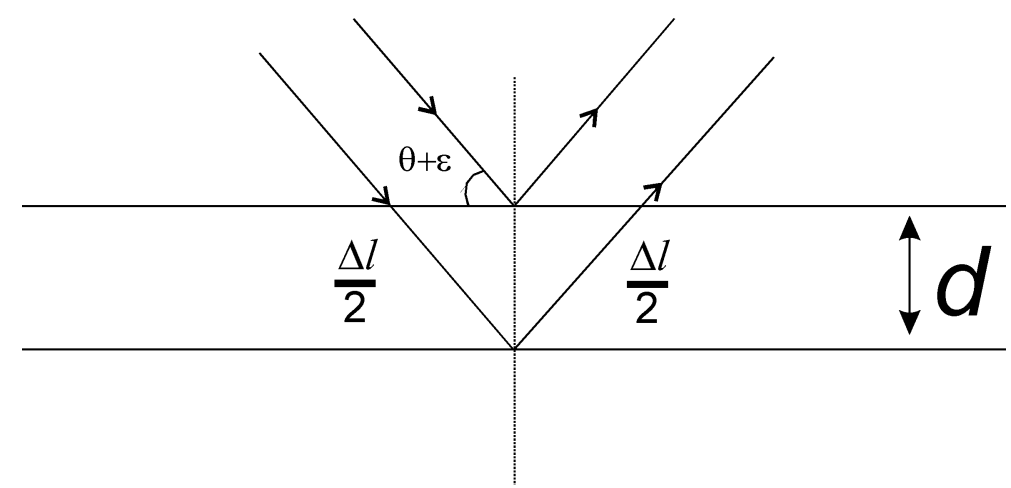

Figure 2-6 Bragg model of X-ray reflection for derivation of the Scherrer equation.

The Scherrer equation can be derived from the Bragg equation (see (5) for the Bragg equation, and refer to Figure 2-6 for an illustration of Bragg model applied to the Scherrer equation). The amplitude of diffracted X-rays is at a maximum when the additional path length travelled by X-rays incident on successive planes, $\Delta l$, equals an integer number of wavelengths. This can be generalised to cases where the angle differs from the Bragg angle by some amount $\varepsilon$ (see expression (8)). As the number of planes in the diffracting body decreases - and the thickness perpendicular to the planes decreases - the destructive interference at $(\theta+\varepsilon)$ is less complete, and X-rays are 
scattered over a greater angular range - which is observed as a broadening of the X-ray reflection line shape.

$$
\begin{aligned}
& \Delta l=2 d \sin \theta=n \lambda \\
& \Delta l=2 d \sin (\theta+\varepsilon)
\end{aligned}
$$

\subsubsection{Information gathered from X-ray diffraction experiments}

As has been discussed in the preceding sections, the angular dependence and relative intensity of X-ray diffraction from a single crystal, or powder of crystals contains much information about the internal structure of the crystal, and in principle can be used to develop a full model of this structure. However a much simpler use of this phase-specific diffraction information is as a finger printing tool to identify and quantify crystalline phases, and this is the purpose for which XRD was used in this project:

- The angles and relative intensities of diffraction peaks can be used for qualitative identification of multiple crystalline phases within a mixture.

- Various methodologies exist to quantify the relative amounts of the identified phases in the mixture based on the relative intensities of their superimposed individual diffraction patterns.

- The size-dependent line broadening effect described above (2.2.1.4) can be used to judge the average crystallite size of phases. 


\subsubsection{Acquisition and analysis of XRD patterns}

Instrumentation, sample preparation and XRD pattern acquisition parameters will be outlined in section 2.2.2.1. The procedure for qualitative identification of crystalline phases by XRD will be dealt with in section 2.2.2.2, and methodologies for quantitative phase analysis which were investigated are dealt with in section 2.2.2.3. The use of Scherrer X-ray line broadening analysis to calculate average crystallite size will be outlined in section 2.2.2.6. The use of software to simulate powder XRD patterns, crystal reciprocal lattices, single crystal SAED patterns and crystal morphologies will be discussed in section 2.2.2.7.

\subsubsection{Instrumentation and sample preparation}

A commercial Philips X'Pert X-ray powder diffractometer system was used with a copper anode glass X-ray source with a long fine focus. The X-ray source was typically operated at $40 \mathrm{kV}$ tension and $20 \mathrm{~mA}$ current. The beam-side optics included Soller slits to limit axial divergence of the beam, and a fixed-width $1^{\circ}$ divergence slit to limit the spread of the beam in the sample plane. The goniometer was operated in the Bragg-Brentano or parafocusing geometry - whereby the sample was rotated at half the rate of the detector, in order to retain a constant sample-to-receiver distance while scanning through $2 \theta{ }^{2}$ Secondary optics included a fixed-width $0.1 \mathrm{~mm}$ receiving slit, and further Soller slits to limit the width and vertical divergence of the diffracted beam; and fixed-width $1^{\circ}$ antiscatter slit to prevent the entry of X-rays scattered outside the sample. A curved graphite secondary monochromator was used to filter all but the desired $\mathrm{Cu} \mathrm{K} \alpha$ wavelength from the proportional detector. The use of a secondary monochromator was particularly important for samples containing iron, as $\mathrm{Cu} \mathrm{K}_{\alpha}$ radiation - with a wavelength of $1.5418 \AA$ - falls below the $\mathrm{K}$ absorption edge for iron $\left(1.7429 \AA^{(2)}\right)$, and can therefore excite fluorescence.

Samples were ground by hand in an agate mortar until fine enough to pass through a sieve. The powder was placed on a Perspex sample holder with a $10 \mathrm{~mm} \times 20 \mathrm{~mm} \times 0.2 \mathrm{~mm}$ indented area. The powder was spread to fill this volume, and smoothed with a glass cover slip to provide a flat surface flush with the Perspex. The Perspex of the holder produced an amorphous hump in the diffraction pattern between approximately $10-20^{\circ} 2 \theta$. Consequently high quality diffraction patterns were 
typically only recorded in the range $25-80^{\circ} 2 \theta$ if a preliminary scan indicated the absence of diffraction peaks in the $<20^{\circ} 2 \theta$ range. Typical scan parameters were a step size of $0.05^{\circ}$, and a dwell time of $5-16$ seconds, as necessary to provide a sufficient signal-to-noise ratio.

Diffraction patterns were collected using the proprietary PanAnalytical Data Collector software program and manipulated using the PanAnalytical Highscore software suite provided by the instrument manufacturer. The latter software was used for functions such as background determination, peak position determination, determination of peak width and relative intensity, and stripping of $\mathrm{Cu} \mathrm{K}_{\alpha 2}$ contribution:

The software used the iterative method of Sonneveld and Visser ${ }^{3}$ to determine the background of the diffraction pattern automatically. In this approach a 5\% sample of the data points are considered. The intensity of a given point is compared to the mean of its neighbours on either side. If the intensity exceeds the sum of the mean and a tolerance factor, it is replaced by the mean. Successive cycles leave a smooth function estimating the background, which is then subtracted from the raw diffraction pattern to produce a difference signal. Noise is estimated to lie within three standard deviations of the mean of a subset of data from the difference signal.

Peak positions were determined by the minimum second derivative method, whereby a polynomial function is fitted to the data, and the second derivative of the function is used to compile a second derivative map. Points of inflection (minima and maxima) in the second derivative map were evaluated as possible peaks in the profile, if the intensity at the position of the point of inflection exceeded the intensity estimated for the noise in the diffraction pattern. A Lorentz function is fitted to the peak position, in order to estimate the relative intensity and width of the peak. If the tip and base width are judged to fall within an acceptable range, the proposed peak position is accepted.

The $\mathrm{K}_{\alpha 2}$ contribution to the spectrum was estimated and subtracted using an algorithm based on the modified Rachinger ${ }^{4}$ and Ladell methods. ${ }^{5}$ The modified Rachinger method calculates the separation of the $\alpha 1-\alpha 2$ doublet, as dependent on the angle $2 \theta$. The Ladell method compensates for the difference in line shape in the $\alpha 1$ and $\alpha 2$ peaks by fitting the $\alpha 2$ peak with a series of angle-dependent subprofiles. As the relative intensity of the $\alpha 1-\alpha 2$ doublet is known from spectroscopic techniques (2:1 
$\alpha 1: \alpha 2$ ), once the separation and line profile of the $\alpha 2$ peak have been calculated, the $\alpha 2$ can be subtracted from the experimental diffraction pattern.

The goniometer of the diffractometer was calibrated using a silicon standard to ensure the correctness of the angular scale of the diffraction patterns. The observed angular positions of silicon diffraction peaks were compared to those recorded in reference file PDF \#27-1402 (Powder Diffraction File, see section 2.2.2.2) and a calibration curve was constructed (see Figure 2-7). The expression derived for the linear fit of the calibration curve data points was used to correct the systematic error in angular position.

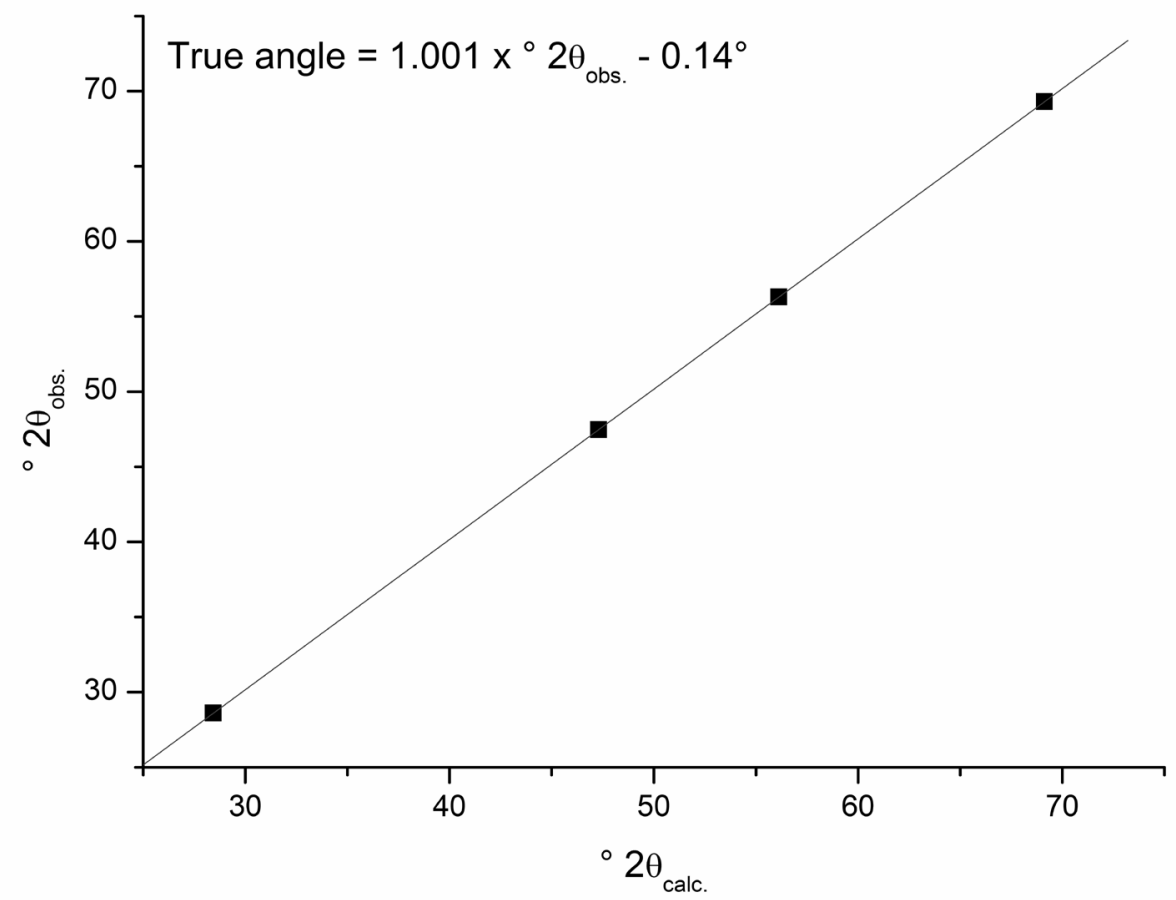

Figure 2-7 Calibration curve for goniometer angular scale derived from a silicon standard.

\subsubsection{Qualitative phase analysis}

After diffraction patterns were corrected for systematic error, and the peak positions and relative intensities were extracted in the manner described above, these data were compared to reference files from the PDF (Powder Diffraction File database published by the International Centre for Diffraction Data, ICDD) via the search and match facility of the X'pert Highscore software. When the diffraction pattern was significantly broadened by small crystallite size, or by a significant and irregularly-shaped background contribution, automatic identification was eschewed and comparison was made manually against PDF data files of likely candidates. 


\subsubsection{Quantitative phase analysis}

According to the adiabatic principle of X-ray diffraction, all crystalline phases within a mixture diffract to produce their characteristic pattern independent of the presence of any other phase - the pattern arising from each component of the mixture is superimposed with all others, and intensity of the diffraction peaks of one phase relative to those of any other phase is determined by the ratio of their respective mass absorption coefficients. $^{6}$

\subsubsection{Semiquantitative analysis}

The semiquantitative method of Chung, ${ }^{6-8}$ rather than relying directly on measured mass absorption coefficients, is based upon a comparison of Relative Intensity Ratio (RIR) values - the ratio between the intensity of the strongest peak of each phase in an equal-weight mixture of an analyte and $\alpha-\mathrm{Al}_{2} \mathrm{O}_{3}$ (corundum). Expression (9) is derived, where the concentration (weight percent), $X$, of a phase, $\alpha$, is related to the relative intensity, $I$, of its reflection, $h k l$, and the RIR value for the phase. The second term of the equation deals with the phase $\alpha$, as a component of a mixture composed of $n$ crystalline phases.

$$
X_{\alpha}=\frac{I_{(h k l) \alpha}}{R I R_{\alpha} I_{(h k l) \alpha}^{r e l}}\left[\frac{1}{\sum_{j=1}^{n}\left(I_{I(h k l)^{\prime} j} / R I R_{j} I_{(h k l)^{\prime} j}^{r e l}\right)}\right]
$$

This method has been developed into an automatic algorithm within the X'pert Highscore software suite, but can also be calculated manually from XRD data. General problems with this method include the recognised unreliability of some RIR values published within the PDF database. In addition, as RIR values have only been regularly published since the 1970s, they are absent from older files, which may however still be the primary reference file for some phases.

An additional limitation of the semiquantitative method includes the assumption of the absence of X-ray amorphous material within a sample - if amorphous material is in fact 
present, this leads to an over estimation of the absolute concentrations of the crystalline phases, although the relative concentrations of the crystalline phases remain unaffected. The semiquantitative method is relatively simple, and contains no compensation for sample-related effects such as the loss of peak intensity associated with the broadening of reflections by small average crystallite size. This is problematic, especially when dealing with nanocrystals, as size-related broadening can lead to the underestimation of the concentration of the phase(s) in the mixture with the smaller average crystallite $\operatorname{size}(s)$.

\subsubsection{The Rietveld method}

The Rietveld method ${ }^{9}$ of profile fitting was initially developed for neutron diffraction experiments, but was later generalised to X-ray diffraction. In the Rietveld method diffraction patterns are simulated on the basis of an initial crystal structure model, and then a number of parameters are refined by a regression algorithm to minimise the difference between the simulated and experimental patterns. ${ }^{10}$ The Rietveld method can be used to quantify the composition of a mixture, as long as crystal structure models can be provided for all crystalline components.

Quantitative analysis is performed on the basis of a refinement where refinable parameters include the Rietveld scale factor for each phase. The Rietveld method allows the determination of the relative concentrations of phases without recourse to experimental mass absorption coefficient values, as the scale factors are determined $a b$ initio from the relevant crystal structure models with the use of atomic scattering factors. The relative concentrations of phases are then treated as a variable in the refinement. Once the refinement has converged on a solution, the absolute concentrations of the phases can be calculated from expression (10), where $W_{p}$ is the weight percentage of phase $p, S$ is the Rietveld scale factor, $\mathrm{Z}$ is the number of formula units per unit cell, $M$ is the mass of the formula unit, and $V$ is the unit cell volume ${ }^{11}$

$$
W_{p}=\frac{S_{p}(Z M V)_{p}}{\sum_{i} S_{i}(Z M V)_{i}}
$$


The Rietveld method offers several advantages over the semiquantitative method, in that sample-related factors such as peak broadening, contribution of amorphous material and preferred orientation of crystallites can be modelled. This in principle allows for a more accurate quantitative solution in cases where such factors are present. However the principal advantage lies in the fact that published RIR values are not required to perform quantitative analysis using the Rietveld method.

Rietveld refinements were performed using the Rietica software package. ${ }^{12}$ Parameters for the crystal structure models for phases identified in the mixture were sourced from the original literature publications, or the PDF database. In general the structure parameters were fixed, and only instrumental parameters such as the universal scale factor and angular calibration error; and sample-related parameters such as the Rietveld scale factors, peak broadening and preferred orientation parameters were refined. Convergence of the model and experimental pattern was judged on the basis of the weighted R-factor.

\subsubsection{Scherrer X-ray broadening analysis}

The use of Scherrer X-ray broadening analysis has been validated for use on nanocrystals with diameters less than $10 \mathrm{~nm}$ by Weller et al. ${ }^{13}$ Their comparative study on monodisperse, spherical $\mathrm{CoPt}_{3}$ nanocrystals in the $5-8 \mathrm{~nm}$ size range found good agreement between Scherrer X-ray broadening analysis and other sizing techniques, such as TEM image analysis and Small Angle X-ray Scattering (SAXS).

Caveats included the necessity of choosing an appropriate value for the constant $K$, which is dependent on shape and size distribution. A value of 0.9 is considered appropriate for spherical particles. ${ }^{13}$ For some well-defined non-spherical geometries appropriate values of $K$ have been published, ${ }^{14}$ however in cases where the value of $K$ has not been derived for the specific geometry, or a range of crystal habits are present in the sample it is evident that caution must be taken with the accuracy of results from Scherrer X-ray broadening analysis. In cases where the size distribution of the sample is broad, the average crystallite size determined by Scherrer analysis is known to be skewed towards the larger size fraction. ${ }^{14}$ 
Data was extracted from diffraction patterns for use in Scherrer X-ray broadening analysis using the X'pert Highscore software suite: the background was estimated and subtracted; and the $\mathrm{K}_{\alpha 2}$ contribution to the diffraction pattern was stripped. Peaks in the diffraction pattern were located using the second-derivative minimum method, and were fitted with a Gaussian function in order to calculate their FWHMs and angular positions, $\theta$. The pure diffraction breadth, $\omega$, for each reflection $h k l$ was calculated by taking the difference between the measured peak FWHM and the FWHM of peaks from a silicon powder standard. The silicon powder standard was assumed to have an average crystallite size larger than $200 \mathrm{~nm}$, and therefore its peak widths were solely the result of instrumental broadening. ${ }^{2}$

The data were then inputted into the Scherrer equation (7), to calculate $D_{h k l}$ for each resolved reflection. For spherical particles, these were then averaged and the deviation calculated. For non-spherical particles this was not done, as the average crystallite size is anisotropic.

\subsubsection{Simulation of diffraction patterns and crystal morphologies.}

Schematic models of crystal structures were created using the CaRIne software package. ${ }^{15}$ Required inputs for the modelling of a structure were the space group, lattice parameters, and atomic coordinates. These were sourced either from the PDF file, or the original literature. The software produces a scale model of the unit cell, and positions the atoms at the specified coordinates in the asymmetric cell, and then performs the symmetry operations required by the space group to produce the entire unit cell. Atomic oxidation states and the corresponding radii are programmable parameters. The real-space crystal model can be rotated in three dimensions, and positioned to be viewed along specified crystallographic axes.

A weighted reciprocal lattice can be generated from the real space model by plotting all space group allowed reflections in reciprocal space. The allowed reflections are weighted by their structure factors, calculated automatically from the crystal structure model and atomic scattering factors using expression (6). The SingleCrystal extension of the CrystalMaker software package was used to simulate SAED patterns with a realistic appearance. Powder XRD patterns were simulated using CaRIne - the Bragg angle corresponding to the interplanar spacing of all space group allowed reflections is 
calculated and Gaussian functions are plotted with an intensity weighting calculated by the structure factors of the contributing reflections.

Crystal morphologies were simulated using the Shape software package. Required inputs for a phase were the lattice parameters, crystal system, point group, space group and surface energies. The lattice parameters, crystal system, point and space group were sourced from the primary literature or from the PDF database. Surface energies for the relevant forms were either sourced from computational simulations published in the literature, or were estimated by the Shape software package using the Donnay-Harker method, ${ }^{16}$ where the surface energy is assumed to be inversely proportional to the interplanar spacing of a form. 


\subsubsection{Transmission Electron Microscopy (TEM)}

Transmission Electron Microscopy (TEM), and the ancillary techniques of Electron Diffraction (ED, section 2.2.4) and Energy Dispersive X-ray Spectroscopy (EDS, section 2.2.5) were the primary characterisation techniques used in this project. These techniques combine as a powerful tool for determining a range of material parameters such as: sample morphology at high magnification, sample composition, and crystal structure. The theoretical basis and the operation of TEM is discussed below in section 2.2.3.1, followed by a description of the sample preparations procedures (section 2.2.3.2) and TEM image analysis procedures (2.2.3.3) used in this project.

\subsubsection{Theoretical basis of TEM}

The Rayleigh criterion establishes that the minimum distance resolvable by a microscope is approximately half of the wavelength of the radiation used to image the object. ${ }^{17}$ This is shown in expression (11), where the minimum resolvable distance, $\delta$, is equated with an expression containing the wavelength, $\lambda$, the refractive index of the medium, $\mu$, and the semiangle of collection, $\beta$. Thus the fundamental resolution limit of light microscopy is approximately $200 \mathrm{~nm}$. To circumvent this limitation, in electron microscopy a coherent electron beam is used in place of electromagnetic radiation to create a magnified image of an object.

The De Broglie relation establishes that particles of matter possess inherent wavelengths inversely proportional to their momentum, as shown in expression (12), where $h$ is Plank's constant, and $p$ is momentum. In the right hand term of expression (12) the momentum is substituted by an expression, where $m_{0}$ is the rest mass, $e$ the charge of an electron, $V$ is the voltage, and $c$ is the speed of light. Thus, if accelerated through a sufficient potential difference, electrons obtain wavelengths much smaller than electromagnetic radiation of the visible region of the spectrum. At an accelerating voltage of $200 \mathrm{kV}$ - as used in this project - electrons have a wavelength of $2.5 \mathrm{pm},{ }^{17}$ however the minimum resolvable distance is limited by instrumental factors to a point resolution of $\sim 200 \mathrm{pm}$. 
$\delta=\frac{0.61 \lambda}{\mu \sin \beta} \approx 0.5 \lambda$

$\lambda=\frac{h}{p}=\frac{h}{\left[2 m_{0} e V\left(\frac{1+e V}{2 m_{0} c}\right)\right]^{1 / 2}}$

A JEOL 2010 with a thermionic $\mathrm{LaB}_{6}$ electron source was used in this project (see Figure 2-8 for a schematic ray diagram). Resistive heating of the $\mathrm{LaB}_{6}$ crystal overcomes the work function of the crystal to provide free electrons, which are accelerated towards the earthed anode by the electrons by the selected potential difference. The electron beam is then manipulated by series of adjustable electromagnets, which serve as lenses. The electrons exiting the electron gun are condensed by the condenser lenses to a fine beam, whose dimensions are limited by the condenser aperture. The electron beam is focused by the objective lenses, whose pole pieces sit either side of the specimen plane. The divergence of the post-specimen beam is limited by the objective aperture, before it passes through the intermediate lens series, where the image of the sample is magnified before being projected by the projector lens onto the viewing plane. In the TEM used in this project, the viewing plane could be occupied alternatively by a phosphorescent viewing screen, a film camera, or a digital CCD camera. 


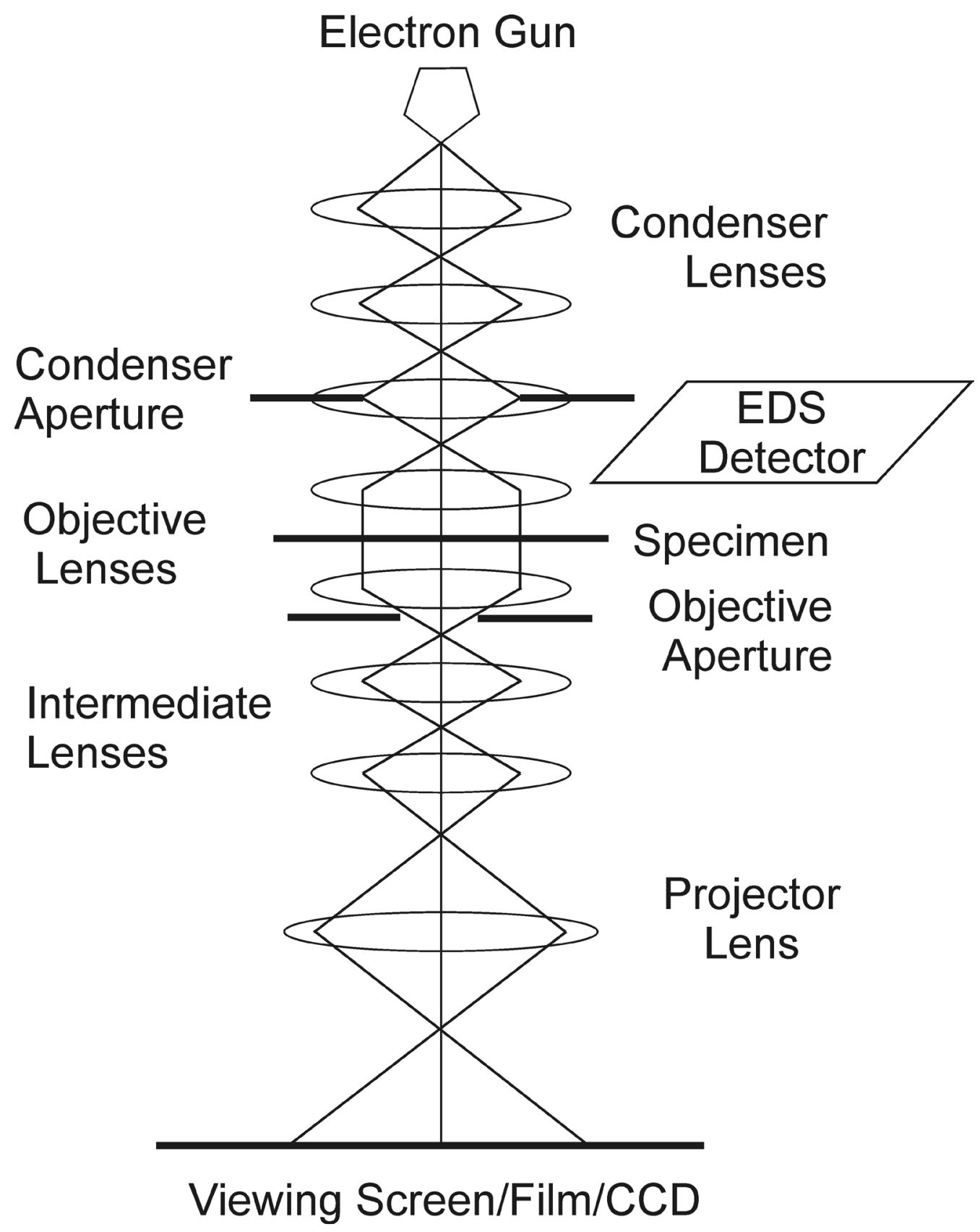

Figure 2-8 Schematic ray diagram of a TEM.

As the primary electron beam passes through the specimen, a number of processes occur, as summarised schematically below (Figure 2-9). Primary electrons may either be transmitted through the specimen without interacting (direct beam), or be scattered either elastically or inelastically. Both elastically and inelastically scattered electrons may be scattered in the forward or backward directions. The emission of X-rays from the specimen will be dealt with in section 2.2.5 (EDS). In TEM is the forward scattered electrons which provide the image - electrons are scattered to a greater or lesser degree depending on the thickness, and average atomic number of the material they pass through, leading to spatial variations in the intensity of the electron beam projected onto the viewing plane. 


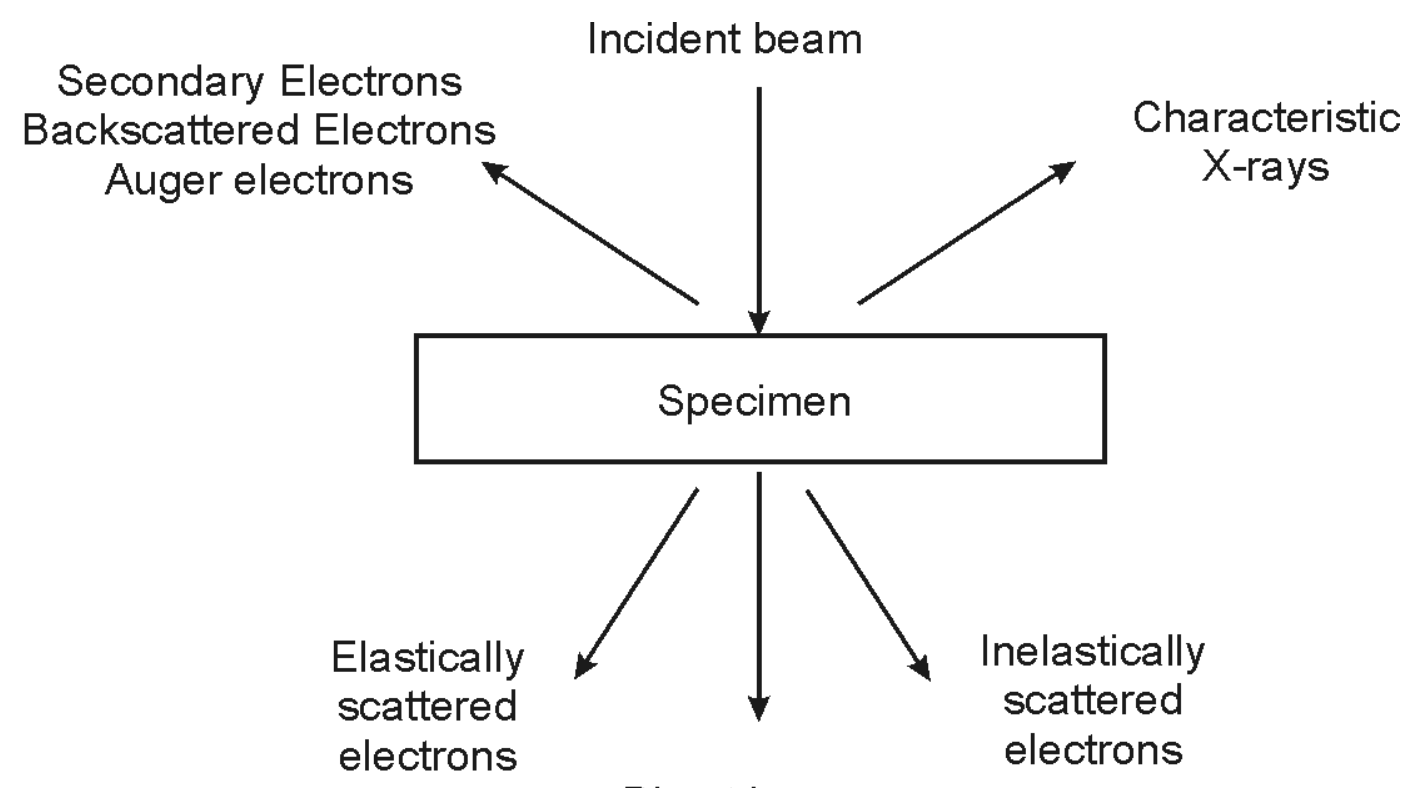

Direct beam

Figure 2-9 Interactions between the specimen and the electron beam.

\subsubsection{Preparation of TEM samples}

As the mean free path of electrons in a gas is low, the TEM column is operated at high vacuum to minimise the scattering of electrons outside of the sample. To preserve the instrumental vacuum during operation, it is important that material that can volatilise under high vacuum and electron beam irradiation is removed from the specimen during, purification, sample preparation and pump down. The purpose of the purification procedure described above in section 2.1.3 was largely to remove excess non-volatile organic material, and to suspend the sample in a volatile organic solvent, which evaporates quickly when deposited as a thin film.

As most samples in this project were colloidal suspensions, sample preparation typically involved placing a drop of dilute, well-dispersed toluene suspension on a 200-mesh amorphous carbon-coated copper TEM grid, blotting excess solvent away, and allowing the solvent to evaporate to leave the specimen as a thin film deposited across the grid. To prevent contamination of the sample once inside the TEM, an anticontamination device is used, whereby material volatilised from the sample under electron irradiation was condensed preferentially on a liquid nitrogen cooled finger present inside the column, rather than the onto the specimen grid. 


\subsubsection{TEM image analysis}

In addition to interpretation of real-space micrograph images, Fast Fourier Transforms (FFT) were performed on some images. An FFT transforms a function from a spatial domain to a frequency domain. In context of an image, features which posses a certain special repetition are transformed into a point corresponding to the frequency of repetition. The FFT of an image serves to highlight the presence of repeating features such as lattice fringes, as non-repeating features are transformed into a background. FFTs were performed using the Gatan Digital Micrograph software suite. 


\subsubsection{Selected Area Electron Diffraction (SAED)}

Electron Diffraction (ED) can be used to give information on the internal ordering crystalline material in a manner analogous to X-ray diffraction. Advantages of ED include the fact that a diffraction pattern can be obtained from a spatially defined area of the sample by the use of a Selected Area aperture. As this area can be imaged concurrently, the combination of ED and TEM imaging provides a large amount of localised information. The theoretical basis of ED will be outlined below in section 2.2.4.1. ED pattern acquisition and analysis procedures are discussed in sections 2.2.4.2 and 2.2.4.3, respectively.

\subsubsection{Theoretical basis of electron diffraction}

The process of electron diffraction is analogous to X-ray diffraction, in that electrons scattered through the Bragg angle by successive lattice planes interfere constructively, and are hence reinforced. In the microscope a beam diffracted by a set of lattice planes emerges from the underside of the specimen at a point - the angle by which the diffracted beam then diverges from the direct beam is equal to the Bragg angle. The diverged beams are collected by the objective lens, and refocused as spots at the back focal plane (see Figure 2-10 for schematic ray diagram). Hence the image formed at back focal plane is of the angular distribution of electron intensity (diffraction pattern), rather than the spatial distribution of electron intensity (the image of the specimen). 


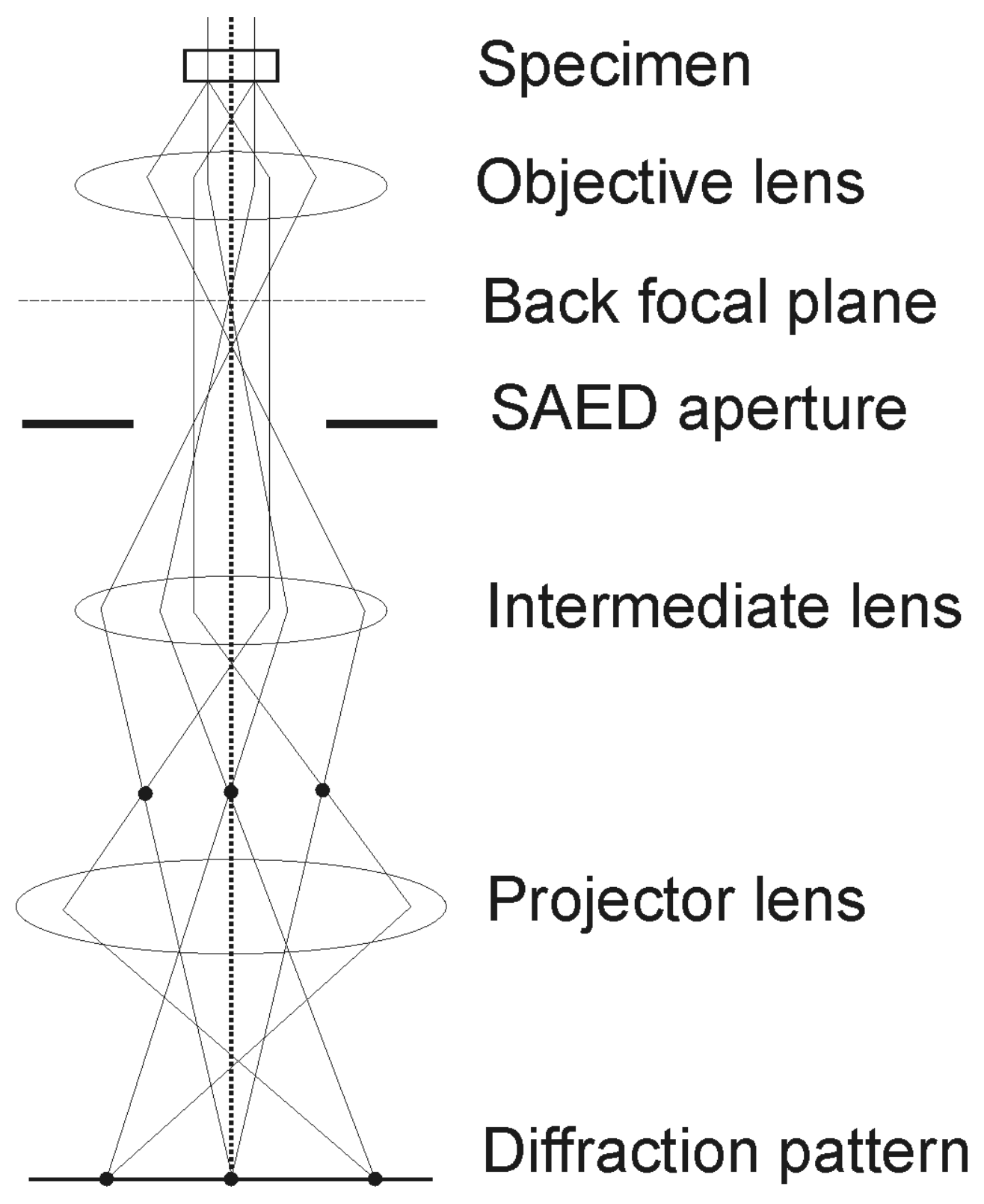

Figure 2-10 Schematic ray diagram of the electron diffraction process.

\subsubsection{Electron diffraction procedure}

To limit the area from which a diffraction pattern was collected, one of a series of selected area apertures of varying size was inserted into the electron beam below the specimen plane. The TEM was placed in diffraction mode, whereby the strength of the intermediate lens was altered automatically such that the back focal plane (diffraction pattern) rather than the image plane was magnified and projected onto the viewing plane. Diffraction patterns were recorded either on a Gatan digital CCD camera, or on Kodak SO-163 electron microscopy film plates, and developed with standard methodology. 


\subsubsection{Analysis of electron diffraction patterns}

Once the negative was developed, the distance between the central spot made by the direct beam, 000, and a given diffraction spot, G, was measured carefully from the negative with callipers and a ruler. To avoid errors associated with identifying the true centre of the diffraction pattern, it was preferred to measure and halve the distance between two symmetry-related diffraction spots, or the diameter of a diffraction ring from a polycrystalline sample. This distance, $D$, is related to the Bragg angle of the reflection and the magnification of the diffraction pattern - termed the camera length, $L$. Substituting $D$ and the camera length, $L$, into Braggs law (expression (5)) leads to expression (13), which directly relates the lattice spacing, $d_{h k l}$, to a term containing $D, L$ and the electron wavelength, $\lambda$. As the electron wavelength is difficult to measure with precision - and alters with voltage fluctuations in the microscope - the term $2 L \lambda$, called the camera constant, was derived by calibration from the diffraction pattern of a standard material.

$$
d_{h k l}=\frac{2 L \lambda}{D}
$$

\section{Calibration of camera length}

Camera constants were calibrated for camera lengths of 100, 150 and $200 \mathrm{~cm}$ using a sample of gold nanocrystals. An SAED pattern was recorded on film for each camera length. The patterns were indexed, and values of the camera constant were calculated for each reflection on the basis of $d_{h k l}$ values from PDF \#03-065-2870 (gold). The camera constant values were then plotted versus $D$, as shown below in Figure 2-11 for a camera length of $150 \mathrm{~cm}$. The uncertainty in the measurement of $D$ - estimated at \pm 0.5 - was determined to be the most significant contribution to the uncertainty of the calibration. The linear fit of the data points was weighted by the error bars in the $x$ and $y$ directions, and gave a standard deviation of 1.47 in the result of the linear expression. Hence, taking a level of confidence with $2 \sigma$, the uncertainty in value of the camera constant calculated from the linear expression was approximately 4\%. Combined with the measurement uncertainty of \pm 0.5 in the diameter of unknown rings, the uncertainty in calculated $d_{h k l}$ values was typically $\pm 0.1-0.2 \AA$. Good agreement has been attained for $d_{h k l}$ values calculated from SAED and XRD. 


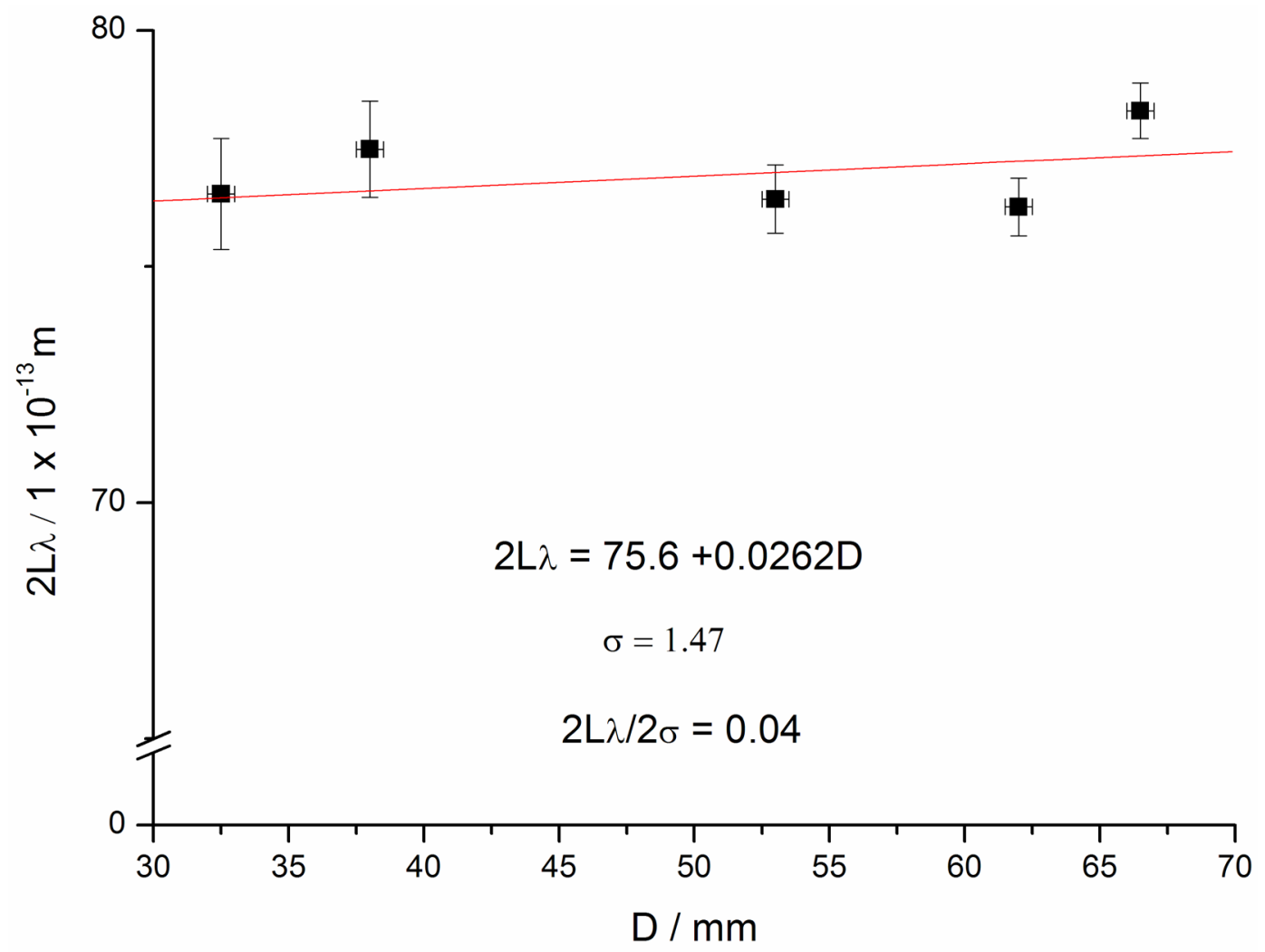

Figure 2-11 Camera constant values calculated for a gold standard with a camera length of $150 \mathrm{~cm}$.

\section{Indexing of SAED patterns}

Indexing of the ring patterns originating from polycrystalline samples was generally achieved by comparison of calculated $d_{h k l}$ values with reference files from the PDF database. The relative intensity of the reflections were generally estimated from the negative by eye, but if higher precision was required the negative was scanned, and the Scion Image software suite was used to generated a line density trace of a slice through the diffraction pattern.

Indexing of single crystal electron diffraction patterns is somewhat more involved than for polycrystalline samples, as in general only reflections from a single crystallographic zone are present. In some cases the graphical indexing method of Lewis ${ }^{18}$ was used. In this approach the distance, $D$, measured for a reflection is related to possible Miller indices, $h k l$, by a constant $A$, as shown in expression (14).

$$
D=A\left[h^{2}+k^{2}+l^{2}\right]^{1 / 2}
$$


To determine the unknown, $A,\left[h^{2}+k^{2}+l^{2}\right]^{1 / 2}$ is plotted on one axis, with distance on the other (see Figure 2-12 for an example based on the indexing of a diffraction pattern from $\mathrm{Fe}_{3} \mathrm{~S}_{4}$ ). Vertical lines are drawn for all values of $h k l$ allowed by the space group of the crystal, and horizontal lines are drawn for each measured value of $D$. Each intersection of a horizontal and vertical line represents a possible indexing solution for that reflection, and a line drawn from the origin with slope $A$, will simultaneously select solutions for all reflections, thereby giving the correct indexing of the whole diffraction pattern.

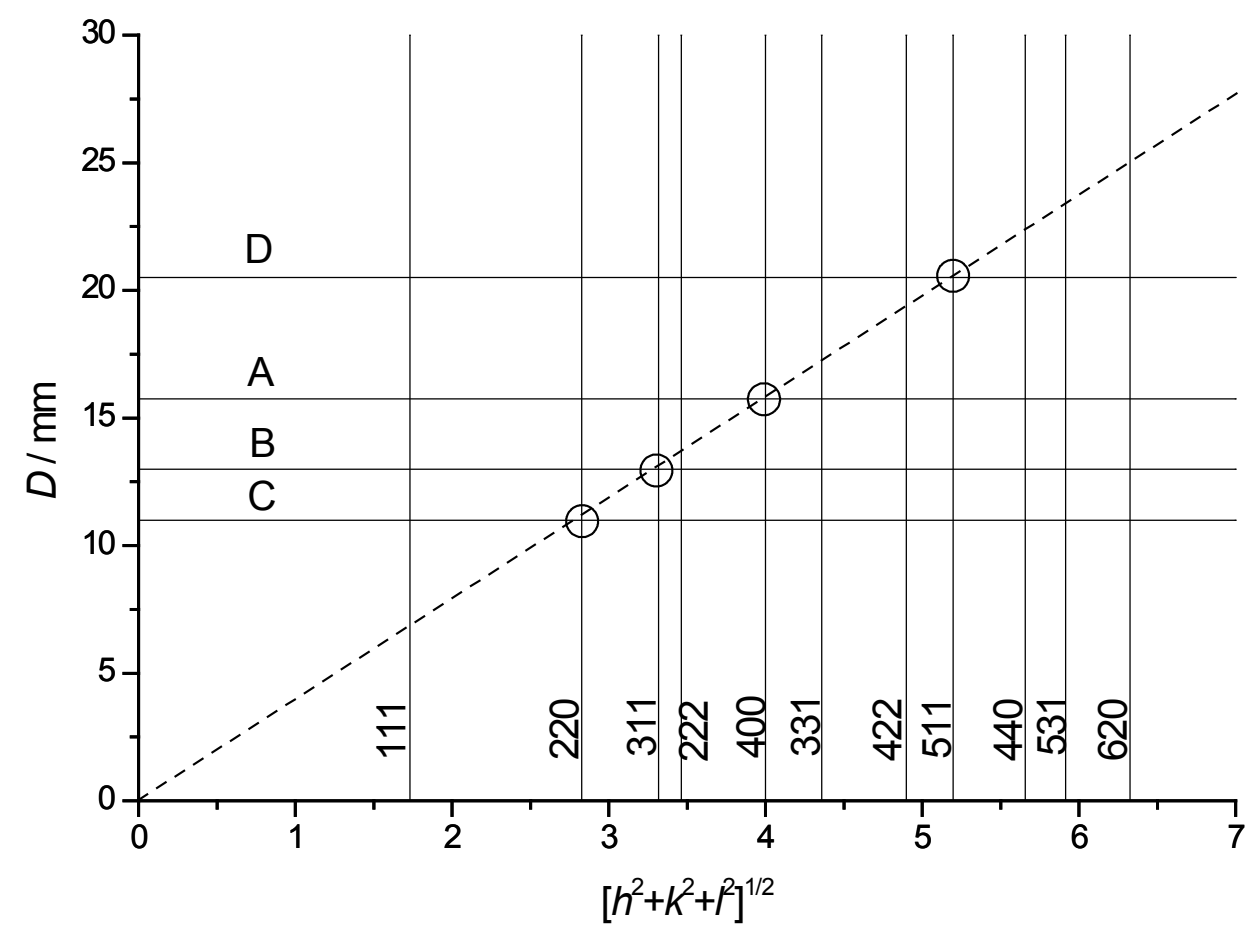

Figure 2-12 Plot of $D$ versus $\left[h^{2}+k^{2}+l^{2}\right]^{1 / 2}$ for an SAED pattern of $\mathrm{Fe}_{3} \mathrm{~S}_{4}$. 


\subsubsection{Energy Dispersive X-ray Spectroscopy (EDS)}

The interaction of high energy electrons and atoms may stimulate the emission of photons in the X-ray region of the spectrum. These X-rays fall into two broad categories - a broad spectrum of bremsstrahlung X-rays emitted when a primary electron is decelerated by interaction with the electric field of an atomic nucleus, and characteristic $\mathrm{X}$-rays of concise wavelengths emitted from material ionised when a core electron is ejected after collision with the high-energy primary electron.

Characteristic X-rays are named as such because they possess discrete energies corresponding to the electron transitions which occur as the stimulated ion relaxes from the excited state back to the ground state. As these transitions involve core electron states - rather than valance electrons - they are characteristic of the element, but relatively insensitive to its chemical state. Hence the X-ray photons emitted when the sample is ionised by the primary electron beam may be used to gain information on the elemental composition of the sample. This is achieved by inserting the detector of an Energy Dispersive X-ray Spectrometer (EDS) into the TEM column, in close proximity to the sample.

The detector consists of semiconductor diode with a central region of intrinsic semiconductor, sandwiched between an initial p-type region (termed the dead layer) and an n-type region. If an X-ray photon penetrates into the intrinsic region, it may transfer its energy to the semiconductor and generate electron-hole pairs, the number of which is proportional to the energy transferred. The electrons and holes are then separated by a bias voltage placed across the device, and measured as a charge pulse, whose magnitude is proportional to the energy of the X-ray photon which generated it. Sequential determination of the energy of incoming X-rays leads to a histogram of electron energy vs. count, hereafter referred to as an EDS spectrum. ${ }^{17}$

\subsubsection{Qualitative analysis}

EDS spectra were acquired using the JEOL Analysis Manager software suite, which automatically controlled acquisition parameters such as the energy range of the spectrum, the number of channels into which photon energies were divided, and the 
time constant - the time allowed for the processing of each charge pulse. Care was taken adjust the sample illumination conditions to ensure the count rate did not exceed the optimum range during a measurement, and the proportion of detector dead time the time the detector is deactivated after receiving charge pulses - did not exceed $50 \%$ of the total collection, which could have entailed a loss of resolution. ${ }^{17}$

Due to the thinness of most deposited nanocrystal samples, relatively little material may be illuminated during an EDS measurement, resulting in a relatively low count rate. Consequently the total number of counts registered in an EDS spectrum was frequently relatively low, however in most cases all peaks were clearly resolved from the background. Peaks were assigned automatically by the EDS processing software, although care was taken to validate these assignments by verifying that all expected members of a family were present - i.e. iron $\mathrm{K}_{\alpha}, \mathrm{K}_{\beta}$ and $\mathrm{L}_{\alpha}$ peaks were all detected when iron was present.

Detected X-rays may originate in the area of the sample illuminated by the primary electron beam (sample X-rays); areas of the sample outside the illuminated area which are stimulated by scattered electrons (spurious X-rays); and the microscope and/or EDS detector itself (system X-rays). EDS spectra run on a blank TEM grid revealed signals from copper, carbon and oxygen - constituents of the carbon-coated copper grid. These post-specimen system X-ray signals were detected in all EDS spectra. These system X-ray peaks in general did not overlap with sample peaks, although the almost invariable presence of an oxygen signal did preclude the quantification of oxygen in oxygen-containing samples. However no iron signals were detected in EDS spectra run on blank grids, indicating the lack of interference from system X-rays in the qualitative identification and quantification of iron in samples. A small silicon $\left(\mathrm{K}_{\alpha}\right)$ peak was detected in some spectra, this was attributed to internal silicon fluorescence - the emission of an X-ray photon from the silicon of the dead layer of the detector, which is then detected as it passes into the intrinsic region of the detector diode.

\subsubsection{Quantitative analysis}

The basis of quantitative X-ray microanalysis is as follows: to determine the concentration, $C_{i}$, of an element, $i$, the measured X-ray intensity from the sample, $I_{i}$, is taken in ratio to the measured X-ray intensity from a standard, $I_{(i)}$, of known composition, $C_{(i)}$. The sensitivity factor, $K$, has a contribution from the atomic number, 
$Z$; the absorption of X-rays within the sample, $A$; and the fluorescence of X-rays within the sample, $F$.

$$
\frac{C_{i}}{C_{(i)}}=[K] \frac{I_{i}}{I_{(i)}}
$$

In the Cliff-Lorimer ratio method, expression (15) is modified on the basis of two elements, A and B, in a binary system - each serving as the standard for the other. ${ }^{17}$ In addition, the thin film criterion is applied - the assumption is made that due to the thinness of the TEM sample, absorption and fluorescence effects may be ignored. This leads to expression (16), where the weight percents of the elements, $C_{A}$ and $C_{B}$, are related to the ratio of their respective measured X-ray intensities, $I_{A}$ and $I_{B}$, by the relevant Cliff-Lorimer factor, $k_{A B}$. Note that this expression can be extended to ternary and higher order systems as required.

$$
\frac{C_{A}}{C_{B}}=k_{A B} \frac{I_{A}}{I_{B}}
$$

Cliff-Lorimer factors for the relevant microscope conditions were determined upon initial installation of the microscope and EDS system. Quantisation was therefore performed automatically by the Analysis Station software suite, with reference to the installed standards library. 


\subsubsection{Magnetometry}

The instrumental setup and sample preparation; and various experiments used in this project to characterise the magnetic behaviour of samples are described below in sections 2.2.6.1 - 2.2.6.3, respectively.

\subsubsection{Instrumental setup (MPMS)}

A Quantum Design Magnetic Properties Measurement System (MPMS) was used to perform a variety of magnetic measurements. It is comprised of a variable-field superconducting magnet housing a temperature-controlled environment within its bore, and a Superconducting QUantum Interference Device (SQUID) detector (see Figure 2-13 for schematic diagram). The applied field can be swept between $+4.8 \mathrm{MA} \mathrm{m}^{-1}$ and $-4.8 \mathrm{MA} \mathrm{m}^{-1}$, and the temperature within the bore can be swept between 4 and $300 \mathrm{~K}$.

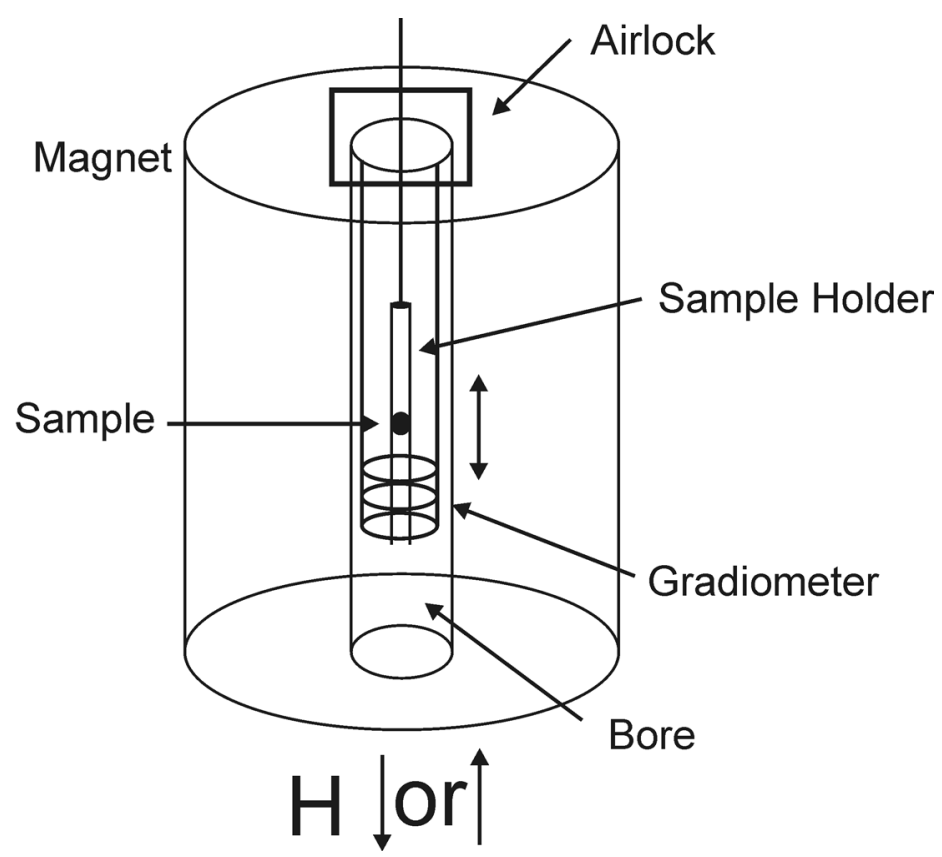

Figure 2-13 Schematic diagram of MPMS SQUID magnetometer.

Powdered samples were first desiccated under high vacuum overnight, weighed into an inverted gelatin capsule, and sealed. The gelatin capsule was suspended in a plastic straw, which was fastened to the sample rod, and inserted through an airlock into the helium-filled bore of the MPMS. A mechanical drive attached to the sample rod was used to move the sample vertically within the bore until it was centred within the SQUID detector coils. 
For a measurement, a field is applied and the magnetised sample is then drawn through the detector coils, inducing a magnetic flux in the detector coils. An additional field is applied by a feedback circuit to counteract this induced flux; and the voltage required to do so is used to calculate the magnetic moment of the magnetised sample via an automatic regression procedure. The magnetic moment of the sample was normalised to the sample mass, to provide a mass magnetisation of the sample.

The MPMS system uses a second-order gradiometer, based on a three coil system. The average signal of the outer two detector coils is subtracted from the signal of the inner coil. Hence, a homogenous object which extends beyond the detection coil throughout the measurement will produce no signal. The plastic straw used to mount the sample should therefore not contribute to the measured magnetic moment. The gelatin capsule containing the sample powder, however, could be expected to contribute to the signal. The average magnetic susceptibility of a gelatin capsule is on the order of $4 \times 10^{-10}$, (19) which is approximately three orders of magnitude smaller than that of the sample with the lowest susceptibility seen in this study. Therefore it was felt to be unnecessary to explicitly correct for the contribution of the probe and sample holder to the signal. Typically, a $6 \mathrm{~cm}$ scan length was used, over which there is a $1.4 \%$ variation in magnetic field strength. ${ }^{20}$ This is likely to be the most significant source of experimental error in hysteretic samples.

\subsubsection{Zero-field-cooled/Field-cooled experiment $(Z F C / F C)$}

Zero-field-cooled/Field-cooled (ZFC/FC) experiments measure the magnetisation of the sample as a function of temperature as the sample is first cooled with no applied field (ZFC), reheated, and then cooled again in the presence of an applied field. In a typical experiment: the sample was first cooled to $10 \mathrm{~K}$ with no applied field, a field of $2 \mathrm{kA}$ $\mathrm{m}^{-1}$ was applied and the sample was heated to $300 \mathrm{~K}$, then cooled back to $10 \mathrm{~K}$. During the heating and cooling sweeps, the magnetisation was measured at $15 \mathrm{~K}$ intervals. See Figure 2-14 below for an example of a $\mathrm{ZFC} / \mathrm{FC}$ plot. 


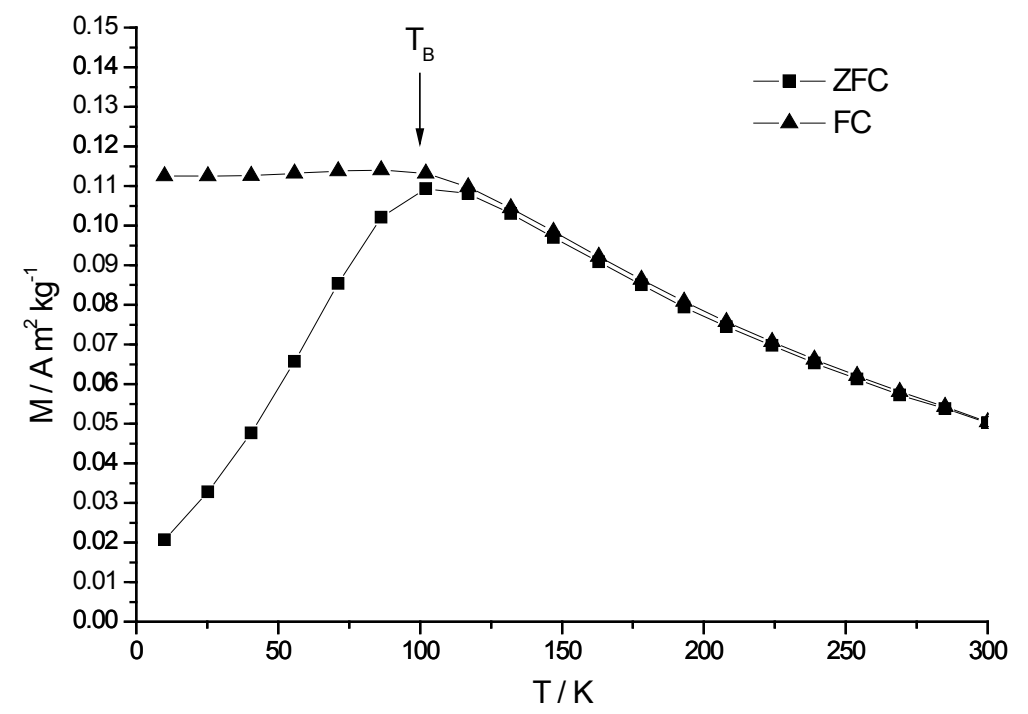

Figure 2-14 ZFC/FC curve.

\subsubsection{Field loop experiment}

A field loop experiment (alternatively known as a hysteresis loop), measures the induced magnetisation of the sample as a function of applied field strength. In a typical experiment, the field was varied between $+4.8 \mathrm{MA} \mathrm{m}^{-1}$ and $-4.8 \mathrm{MA} \mathrm{m}^{-1}$ - representing the maximum range of the instrument. The interval between data points was $1.8 \mathrm{MA} \mathrm{m}^{-1}$ at high field (>1.2 $\mathrm{MA} \mathrm{m}^{-1}$ ); approximately $0.17 \mathrm{MA} \mathrm{m}^{-1}$ in the range 0.08 1.8 $\mathrm{MA} \mathrm{m}^{-1}$, and $0.02 \mathrm{MA} \mathrm{m}^{-1}$ between 0 and $0.08 \mathrm{MA} \mathrm{m}^{-1}$. As the relation between applied field and induced magnetisation is more dynamic at low field strengths, a greater density of data points was required at low fields to provide sufficient detail. After the initial sweep from $+4.8 \mathrm{MA} \mathrm{m}^{-1}$ to $-4.8 \mathrm{MA} \mathrm{m}^{-1}$, the applied field was swept back to $+4.8 \mathrm{MA} \mathrm{m}^{-1}$ to supply the third and forth quadrants of the loop and a total of 52 data points. The result of four scans, at each field strength, was averaged for each data point.

The data was treated with methods detailed by von Dobeneck, ${ }^{21}$ which included a correction for the contribution of the probe and sample holder; interpolation and reduction of experimental data points; a centring correction; and a slope correction. It was felt that an explicit correction for the moment of the probe and sample holder was unnecessary in this case, because of features of the design of the MPMS instrument regarding the contribution of the sample holder, as mentioned above. Likewise, the interpolation procedure was deemed unnecessary, as the number of data points was small (52 data points vs. 600 - 1000 data points typically acquired by the alternating 
gradient force magnetometer used by von Dobeneck), ${ }^{21}$ they were distributed symmetrically with respect to the origin, and to high precision. The centring correction was made to ensure the loop was centre-symmetric. This was achieved numerically using (17), which has the effect of rotating each branch by $180^{\circ}$ around the origin and averaging it with the opposite branch, and vertically shifting the branches by half of their inter-tip distance to ensure exact closure of the loop at both ends.

$$
\begin{aligned}
& \sigma_{c e n}^{+}(H(i))=\frac{\sigma^{+}(H(i))-\sigma^{-}(H(-i))}{2} \\
& -\frac{\sigma^{+}\left(H_{m}\right)-\sigma^{-}\left(H_{m}\right)+\sigma^{+}\left(-H_{m}\right)-\sigma^{-}\left(-H_{m}\right)}{4}
\end{aligned}
$$

The slope correction was undertaken to evaluate the para- and diamagnetic components of the field loop, and so obtain correct values for parameters such as saturation magnetisation and coercivity. The procedure fitted the high-field region of the loop $\left(\geq 1.2 \times 10^{6} \mathrm{~A} \mathrm{~m}^{-1}\right)$ with the expression:

$$
M(H)=M_{s}+\chi_{p d} H-\frac{\alpha}{H}
$$

The total induced magnetisation $M(H)$ at applied field strength $H$, is the sum of the saturation magnetisation, $M_{s}$ (of the ferro-/ferrimagnetic component); the product of the combined para-/diamagnetic susceptibility, $\chi_{\mathrm{pd}}$, with $H$; and a term containing the constant $\alpha$. The software package Maple ${ }^{22}$ was used to apply Gauss-Jordan elimination to find a solution for (18). 


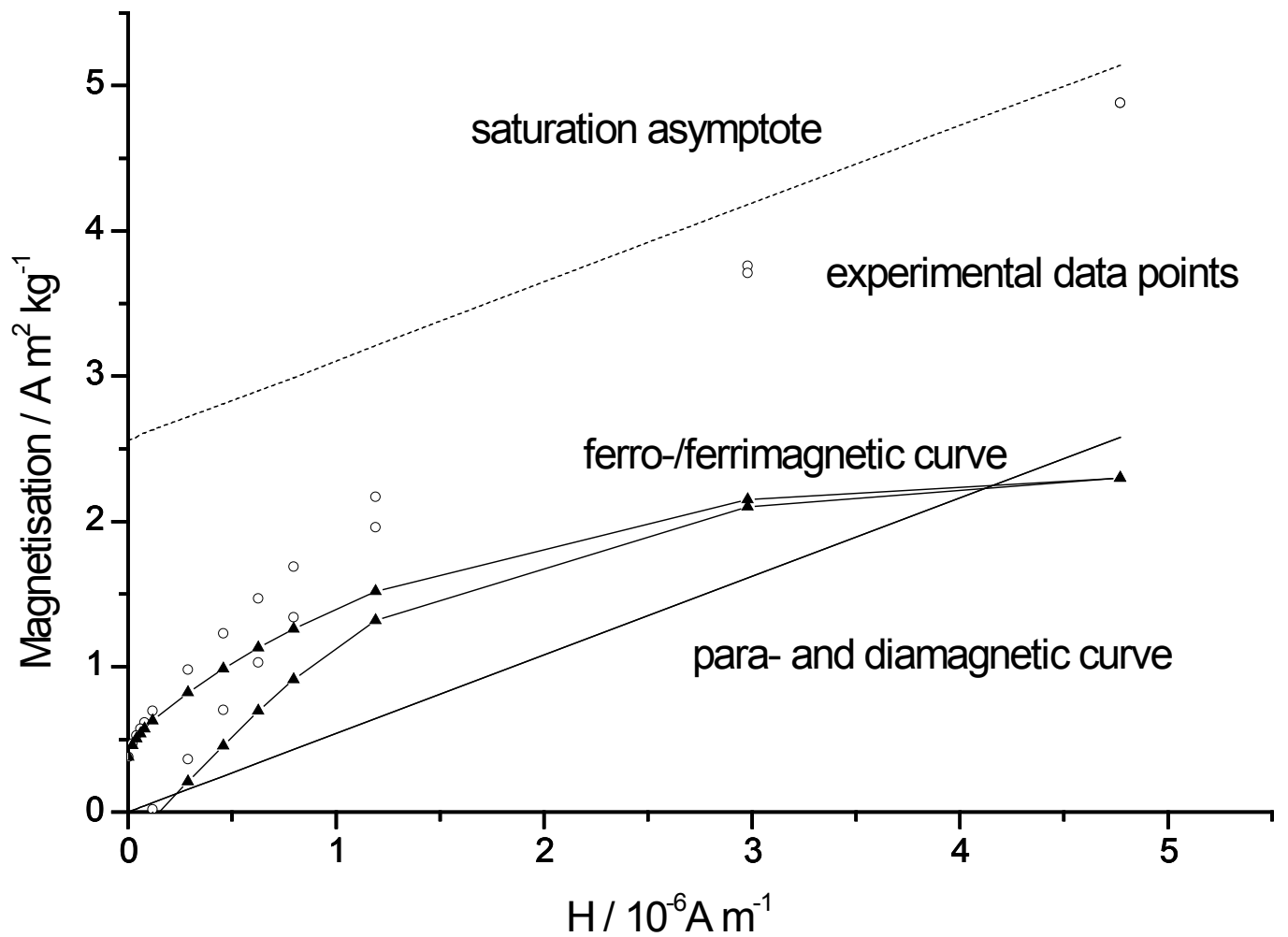

Figure 2-15 Slope correction applied to field loop.

Figure 2-15 shows the effect of the procedure in decomposing the symmetrisised experimental curve into a complex ferro-/ferrimagnetic and a linear para- and diamagnetic component. The decomposition provided a value for the saturation magnetisation (from expression (18); and the slope-corrected curve was used to derive other important magnetic parameters, such as the remanent magnetisation and coercivity - found from the interpolation of magnetisation to zero field, and interpolation of field to zero magnetisation, respectively. 


\subsubsection{Spectroscopy}

Absorption spectroscopy and fluorescence spectrometry were used in this project to gain information on the excitation and emission processes, respectively, which occurred in semiconductor nanocrystals with band gaps in the ultraviolet and visible regions of the spectrum.

\subsubsection{Ultraviolet-visible (UV-vis) spectroscopy}

A Varian Cary 100 double-beam spectrometer was used for UV-vis absorption measurements. This instrument utilises a $\mathrm{D}_{2}$ lamp as the UV source, and a quartz-iodine lamp as the visible source. A double beam chopper arrangement is used to ensure that the beams from the sample and reference cells are directed to the same spot on the photomultiplier, to eliminate any issues with position-dependent sensitivity. Furthermore, the diffraction grating is only moved to select a new wavelength during non-measurement phases during a scan, in order that the sample and reference cell receive exactly the same wavelength. Slit widths were set to achieve a Spectral Bandwidth (SBW) of $1 \mathrm{~nm}$.

Samples were prepared as dilute suspensions in a solvent with an appropriate spectral window for the range measured. A pair of $10 \mathrm{~mm}$ quartz cuvettes of matched absorbance were used to contain the sample, and a solvent blank. An initial baseline was first measured with a solvent blank in both sample and reference positions, before the actual sample was substituted in the sample position.

\subsubsection{Fluorescence spectroscopy}

The fundamental difference between a UV-vis absorption spectrometer and a fluorimeter is the collection angle - a fluorimeter uses a $90^{\circ}$ collection geometry to minimise the amount of light from the excitation source which reaches the detector, as it is the stimulated emission from the sample which is of interest. A Perkin-Elmer LS 55 fluorimeter pulsed xenon lamp source was used in this project. Typically slit widths of 5 $-10 \mathrm{~nm}$ were employed for both the excitation and emission monochromators. The solvent was checked for fluorescence prior to use, and samples were prepared as dilute suspensions to avoid light scattering. A four-sided sintered silica cuvette with a $10 \mathrm{~mm}$ path length was used to contain the samples. 


\subsubsection{Determination of quantum yield}

The fluorescence quantum yield, $\Phi_{\mathrm{F}}$, is the ratio between the number of photons absorbed to the number of photons emitted by a fluorophore, and is used as the measure of the efficiency of the fluorescence process. It is conventionally reported as a decimal or percentage. Determination of $\Phi_{\mathrm{F}}$ was conducted as laid out in the technical bulletin of Jobin Yvon Horiba Ltd., ${ }^{23}$ based on the comparative method of Williams et al. ${ }^{24}$

Dilute solutions with identical absorbance at a certain wavelength can be assumed to absorb an equal number of photons, hence the ratio of the integrated fluorescent intensities of the matched solutions is proportional to the ratio of the fluorescent quantum yields for that wavelength. If the absolute quantum yield of one solution is known, the quantum yield of the other can be calculated. A table of standard fluorescent dyes used in this project, their literature fluorescence quantum yield values, and solvents are shown below (Table 2-1).

Sample solutions with absorbance values of $0.02,0.04,0.06,0.08$, and 0.10 were prepared, and their emission measured with a fluorimeter. The integrated fluorescent intensity was plotted vs. absorbance, and a fit was made by linear regression (see Figure 2-16 for an example). The value of $\Phi_{\mathrm{F}}$ was calculated using expression (19), where the subscripts $X$ and $S T$ refer to an unknown and standard, respectively; Grad is the gradient of the function of integrated fluorescent intensity vs. absorbance; and $\eta$ is the refractive index of the solvent.

$$
\Phi_{X}=\Phi_{S T}\left(\frac{\operatorname{Grad}_{X}}{\operatorname{Grad}_{S T}}\right)\left(\frac{\eta_{X}^{2}}{\eta_{S T}^{2}}\right)
$$




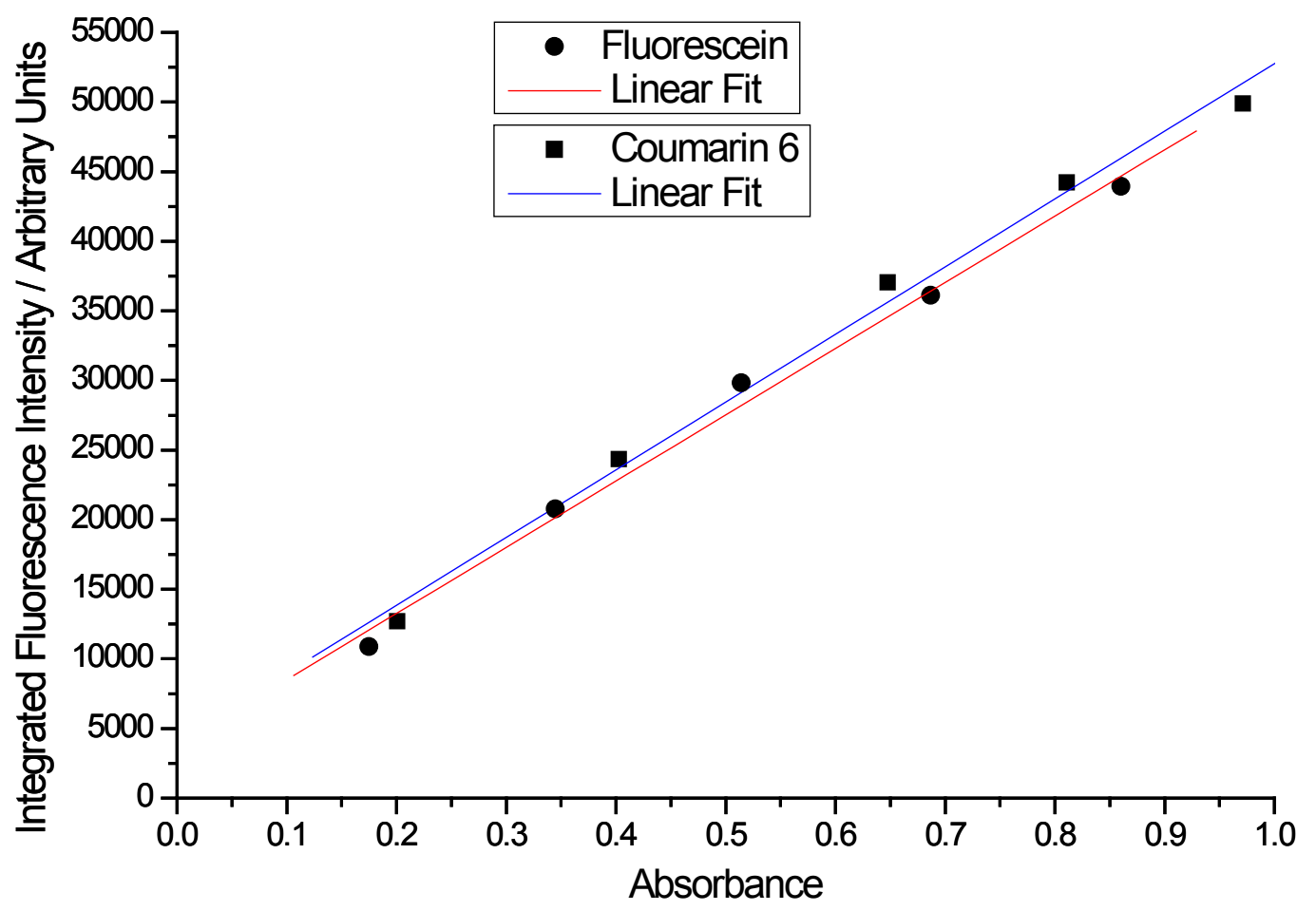

Figure 2-16 Integrated fluorescent intensity vs. absorbance for fluorescent dye standard solutions.

Two fluorescent dyes, of well established quantum yield, were used as standards. The quantum yield of each dye was first determined with respect to the other, and compared to the literature value to ensure the procedure was functioning satisfactorily. It should be noted that a variation of $\pm 10 \%$ between experimental and literature values of quantum yields is viewed as acceptable in the literature. ${ }^{23}$ The quantum yield of the unknown was then determined with respect to each standard dye, and the average was taken.

Table 2-1 Standard fluorescent dyes.

\begin{tabular}{|l|l|l|l|}
\hline Compound & Literature $\Phi_{\mathrm{F}}$ & $\begin{array}{l}\text { Emission } \\
\text { Range / nm }\end{array}$ & Solvent \\
\hline fluorescein & $0.79^{25}$ & $500-600$ & $0.1 \mathrm{M} \mathrm{NaOH}$ \\
\hline coumarin 6 & $0.78^{26}$ & $400-500$ & ethanol \\
\hline quinine sulphate & $0.54^{27}$ & $400-600$ & $0.1 \mathrm{H}_{2} \mathrm{SO}_{4}$ \\
\hline 9,10-diphenylanthracene & $0.90^{28}$ & $400-500$ & cyclohexane \\
\hline
\end{tabular}




\subsubsection{Thermogravimetric Analysis (TGA)}

Thermogravimetric analysis is used to monitor the weight of a sample with respect to temperature, to observe processes that involve mass changes. In this project a Shimadzu TGA-50 apparatus was used, with the sample contained in either a platinum or nickel sample pan. Gas flow through the furnace could be selected to be either nitrogen - to provide an inert atmosphere - or air to facilitate burn-out of combustible material. Typical gas flow rates were set between $25-50 \mathrm{~cm}^{3} \mathrm{~min}^{-1}$. The sensitivity of the balance was $1 \mu \mathrm{g}$, however the initial mass of samples was typically 5-10 mg, in order to minimise measurement error.

\subsubsection{Elemental Analysis}

Elemental analysis was conducted by the Campbell Microanalytical Laboratory at the University of Otago, Dunedin, New Zealand. 


\subsection{References}

(1) M. Ladd and R. Palmer, Structure Determination by X-ray Crystallography, Kluwer Academic/Plenum Publishers, New York, 2003.

(2) H. P. Klug and L. E. Alexander, X-ray Diffraction Procedures, John Wiley \& Sons, New York, 1954.

(3) E. J. Sonneveld and J. W. Visser, J. Appl. Crystallogr., 1975, 8, 1.

(4) R. Delhez and E. J. Mittemeijer, J. Appl. Crystallogr., 1975, 8, 609.

(5) J. Ladell, A. Zagofsky and S. Pearlman, J. Appl. Crystallogr., 1975, 8, 499.

(6) F. H. Chung, J. Appl. Crystallogr., 1974, 7, 526.

(7) F. H. Chung, J. Appl. Crystallogr., 1974, 7, 519.

(8) F. H. Chung, J. Appl. Crystallogr., 1975, 8, 17.

(9) H. M. Rietveld, J. Appl. Crystallogr., 1969, 2, 65.

(10) G. Will, Powder Diffraction, Springer-Verlag, Berlin, 2006.

(11) R. J. Hill and C. J. Howard, J. Appl. Crystallogr., 1987, 20, 467.

(12) B. A. Hunter, Powder Diffr., 1997, 22, 21.

(13) H. Borchert, E. N. Shevchenko, A. Robert, I. Mekis, A. Kornowski, G. Grübel and H. Weller, Langmuir, 2005, 21, 1931.

(14) J. I. Langford and A. J. C. Wilson, J. Appl. Crystallogr., 1978, 11, 102.

(15) C. Boudias and D. Monceau, Divergent S.A., Compiègne, 1998.

(16) J. D. H. Donnay, Am. Mineral., 1946, 31, 508.

(17) D. B. Williams and C. B. Carter, Transmission Electron Microscopy, Plenum Press, New York, 1996.

(18) W. F. Lewis, American Journal of Physics, 1978, 46, 429.

(19) Quantum Design San Diego, 2000.

(20) Quantum Design, San Diego, 1997.

(21) T. von Dobeneck, Geophys. J. Int., 1996, 124, 675.

(22) W. Maple, Waterloo Maple, Waterloo, 2008.

(23) Jobin Yvon Horiba, Stanmore, 1997.

(24) A. T. R. Williams, S. A. Winfield and J. N. Miller, Analyst, 1983, 108, 1067.

(25) J. Q. Umberger and V. K. LaMer, J. Am. Chem. Soc., 1945, 67, 1099.

(26) G. A. Reynolds and K. H. Drexhage, Opt. Commun., 1975, 13, 222.

(27) W. H. Melhuish, J. Phys. Chem., 1961, 65, 229.

(28) S. Hamai and F. Hirayama, J. Phys. Chem., 1983, 87, 83. 


\section{Chapter 3 Synthesis of cadmium selenide nanocrystals}

\subsection{Introduction}

Cadmium selenide nanocrystals are one of the most studied classes of semiconductor nanocrystals due to their strong and size-tunable visible absorption and emission profiles. Evidence of quantum confinement phenomena in cadmium chalcogenide nanocrystals is presented below, as well as literature syntheses of cadmium selenide nanocrystals; capping of cadmium selenide nanocrystals with wide band gap semiconductors; and the solubilisation of cadmium selenide nanocrystals in aqueous media. This is followed by the motivation for the work undertaken in this project.

\subsubsection{Quantum confinement in cadmium chalcogenide nanocrystals}

Evidence that the electronic structure of small semiconductor particles is altered by their limited dimensions - the so-called quantum confinement effect - was first gathered by observation of the shift in the wavelength of optical absorption and emission features of the particles with alteration of crystallite size.

Rossetti et al. ${ }^{1}$ first observed that the absorption spectra of dilute colloidal solutions of cadmium sulfide crystallites displayed a dependence on the size of the crystallites. The freshly prepared colloid had an average crystallite diameter of $4 \mathrm{~nm}$, which increased to over $10 \mathrm{~nm}$ upon aging. This growth in average diameter was accompanied by a red-shift in the absorption edge of approximately $40 \mathrm{~nm}$.

Such a size-dependence was also observed in the luminescence of $\mathrm{Cd}(\mathrm{S}, \mathrm{Se})$ crystallites embedded in borosilicate glass. ${ }^{2}$ Two samples were obtained containing crystallites of identical composition $\left(\mathrm{CdS}_{0.23} \mathrm{Se}_{0.73}\right)$ but different average diameters (10.6 $\mathrm{nm}$ and 9.4 $\mathrm{nm})$. The luminescence maximum of the smaller diameter sample was found to be blueshifted by approximately $15 \mathrm{~nm}$ with respect to the larger diameter sample; and the maxima in both samples were significantly shifted from that observed from bulk material contained in the samples.

Shortly thereafter, the absorption onset of small cadmium selenide colloids, prepared via an arrested precipitation method, was demonstrated to be blue-shifted by some 200 $\mathrm{nm}$ with respect to large, bulk-like cadmium selenide particles. ${ }^{3}$ In addition, the 
absorption spectrum of the smaller cadmium selenide particles contained a plateau at $380 \mathrm{~nm}$ (assigned to an excitonic transition), which was absent in the relatively featureless spectrum of the larger crystallites.

Bawendi et al. ${ }^{4}$ used a high-temperature, solution-phase synthesis involving organometallic precursors - followed by size-selective purification - to prepare samples with a progressively varied average crystallite diameter, and narrow size distribution. Both absorption and emission spectra displayed distinct size dependence: the absorption spectrum of for $1.2 \mathrm{~nm}$ crystallites showed distinct excitonic peaks, and was blue-shifted almost to the ultra-violet; whereas the absorption spectrum of $11.5 \mathrm{~nm}$ crystallites lacked distinct features, and had an absorption onset close to that of the bulk. The emission maximum of each sample tracked closely the wavelength of the first excitonic transition - assigned in cadmium selenide to the $1 S_{3 / 2}-1 S_{e}$ transition. ${ }^{5}$

In all of these cases, the semiconductor nanocrystals were shown to have a consistent crystal structure and composition throughout the size range, which was either identical or closely related to that of the bulk material, thus supporting the contention that only the restricted physical dimensions of the crystallite ensembles were responsible for alterations in the electronic structure.

\subsubsection{Synthesis of cadmium selenide nanocrystals}

Once it had been demonstrated that samples of semiconductor nanocrystals - such as cadmium selenide - could be prepared with a range of colours solely by varying the particle size distribution, interest grew in their potential applications as chromo- and/or fluorophores. Direct band gap semiconductor nanocrystals offer several advantages over traditional organic chromo- and fluorophores: ${ }^{6}$ The (absorbed and emitted) colour of the sample is finely tunable, and its alteration does not affect the general chemistry of the colloid. The emission profiles of semiconductor nanocrystals can be much narrower than those of organic dyes, allowing greater resolution of colour, while semiconductor nanocrystals have proved to be far more chemically and photochemically robust than organic dyes.

The arrested precipitation method of Rossetti ${ }^{1}$ occurs in an inverse micelle solution. Control of the particle size relies on manipulation of the water to surfactant ratio, in order to alter the size of the micelles, within which the nanocrystals are precipitated. 
The initial precipitation of cadmium chalcogenide from solution was however followed by a process of Ostwald ripening which was aggregated by the use of polar solvent ${ }^{1}$ thus it proved difficult to obtain a narrow size distribution which was stable over time using this method.

The high temperature approach of Bawendi et $a l .{ }^{4}$ relies on interrupting particle growth when an appropriate size is reached, by quenching the reaction by cooling. Coupled with post-synthesis size-selective precipitation, this approach yielded monodisperse samples with finely-controlled average particle size. This high temperature route offers the additional advantage of high-crystallinity, which improves the optical properties. ${ }^{4}$ The main disadvantage of the organometallic method lies in the use of highly toxic, pyrophoric reactants such as dimethylcadmium; necessitating the use of an inert atmosphere glovebox, and rendering scale-up difficult. ${ }^{7}$

The search for friendlier alternatives has included the use of alternative cadmium sources such as cadmium carbonates, carboxylates and oxide, which although still toxic, are much easier to work with. ${ }^{7}$ In addition, the initially published organometallic synthesis methods often used bis(trimethylsilyl)selenium $\left(\operatorname{Se}(\mathrm{TMS})_{2}\right)$ as the selenium source, however this was later generally replaced by a solution of trioctylphosphine selenide (Se(TOP)) in trioctylphosphine (TOP) ${ }^{4,8,9}$. While still air-sensitive, this has proved a convenient, and reactive selenium source.

Many solvent systems have been explored for synthesis, ranging from long-chain fatty acids $^{7}$ to commercial olive oil, ${ }^{10}$ however the most commonly used solvent system remains trioctylphosphine oxide (TOPO), often used with a co-solvent to prevent rapid solidification upon cooling. ${ }^{7}$

\subsubsection{Capping of cadmium selenide nanocrystals with a wide-band-gap semiconductor}

The large surface area to volume ratio of small nanocrystals entails a very substantial proportion of the atoms in the nanocrystal residing at the surface, hence the chemistry of the surface dominates that of the bulk. One consequence of this is that the electronic structure of semiconductor nanocrystals is very sensitive to the presence of surface defects. $^{11}$ 
Naturally occurring surface sites can act as electron or hole traps ${ }^{11}$ - once the electron and/or hole has entered the lower energy surface state (otherwise referred to as a trap state) several outcomes are possible, including radiative or non-radiative recombination, or some form of photochemistry:

- Trap states lie at energies within the band gap of the semiconductor - that is electron traps lie at energies below the conduction band, and hole traps at energies above the valance band. Radiative recombination of an electron-hole pair from a trap state will therefore produce a lower energy photon than recombination across the full band gap (band-edge recombination). The occurrence of significant trap state emission has been observed in semiconductor nanocrystals, ${ }^{3}$ which can prove problematic in that it 'pollutes' the emission spectrum, and reduces the purity of the emitted colour.

- Trap states in II-VI semiconductor nanocrystals have been observed to couple strongly to lattice phonons, ${ }^{11}$ increasing the chance of non-radiative recombination occurring. This in turn lowers the quantum yield of the nanocrystals (the ratio of photons absorbed to photons emitted).

- Thirdly, surface sites offer a location for interaction between conduction electrons or holes with the surrounding environment. More significant than the reduction of quantum yield associated with photochemical reactions, is the possibility of degradation of the nanocrystal - or photobleaching. ${ }^{12}$

To help circumvent these problems the nanocrystal can be coated with a shell of a high band gap material. This coating passivates the surface of the nanocrystal - the energy barrier for an electron-hole pair to move into the high band gap shell material restricts the excitons to the core, eliminating the pathway for photochemistry and non-radiative relaxation at the surface. ${ }^{13}$ Zinc sulfide is the most widely used inorganic passivating material. Like cadmium selenide, zinc sulfide can adopt either a wurtzite or zinc blende structure, with cell parameters close to those of cadmium selenide (wurtzite: $\mathrm{ZnS} a=$ $3.78, c=6.19 \AA$ cf. CdSe $a=4.30, c=7.01 \AA$; zinc blende: $\mathrm{ZnS} a=5.42 \AA \mathrm{cf}$. CdSe $a$ $=6.08 \AA$ ). With a band gap of $3.6 \mathrm{eV}$ at $300 \mathrm{~K}^{14}$ (cf. $1.74 \mathrm{eV}$ for CdSe) it has proved a good candidate for passivation of cadmium selenide. Reported quantum yields for $\mathrm{CdSe} / \mathrm{ZnS}$ nanocrystals have been as high as $50 \%{ }^{8}$ 
The application of an additional layer has an added benefit when CdSe nanocrystals are considered for biomedical applications. Research has indicated that photooxidation of CdSe nanocrystals under UV-irradiation can lead to leaching of cadmium ions into solution. ${ }^{12}$ This study also found that leaching of cadmium ions could be mitigated by the presence of an inert $\mathrm{ZnS}$ coating.

\subsubsection{Solubilisation of cadmium selenide nanocrystals in aqueous media}

The majority of the synthesis methods mentioned above in section 3.1.1.1 leave the nanocrystals coated in a layer of surfactant, which has the benefit of stabilising the nanocrystals from aggregation in colloidal solution. These surfactants (e.g. TOPO or fatty acids), however, typically only render the nanocrystals soluble in non-polar organic solvents. For various applications - most notably any biological or biomedical applications - solubility in aqueous media is essential. There have been several approaches employed for rendering cadmium selenide and other cadmium chalcogenide nanocrystals soluble in aqueous media; several of these are discussed below:

CdTe nanocrystals have been synthesised by an arrested precipitation method directly in an aqueous solution of L-cysteine, resulting in cysteine-stabilised nanocrystals. ${ }^{15}$ This method had the benefit of being a single synthetic step, and allowing conjugation of the capping molecules with a linker molecule by condensation of the amine moiety of the cysteine with an aldehyde.

For high temperature syntheses, which have the benefit of good control and high crystalline products, there is no alternative to the use of high boiling point solvents, many of which are non-polar. Substitution of the non-polar capping with a water-soluble coating - either organic or inorganic - must then be taken as a separate step:

$\mathrm{CdSe} / \mathrm{ZnS}$ nanocrystals have been coated with a layer of silica, which was subsequently functionalised. ${ }^{16}$ The presence of an additional barrier layer surrounding the cadmium selenide core proved beneficial in preventing the leaching of toxic cadmium ions, however this also increased the overall size of the composite particle, and the additional, involved synthetic procedure impacted negatively upon the yield of the synthesis. 
A simpler approach is to replace the surface capping on a cadmium selenide or $\mathrm{CdSe} / \mathrm{ZnS}$ nanocrystal with ligands which render the particles soluble in polar media. Ligands with a wide range of functionalities have been employed: Mercaptocarboxcylic acids, ${ }^{6,17,18}$ or mercaptoamino acids (e.g. cysteine) are common choices, but disulfides containing terminal amine functionalities have also been used. ${ }^{19}$ All of these studies postulate that the ligand is associated with the nanocrystal surface primarily through the thiol or disulfide moiety, either through electrostatic interaction or covalent bonding to the metal chalcogenide. These methods rely on the replacement ligand being both more nucleophilic than the original ligand, and having a higher affinity for the nanocrystal surface, in order that it can displace the original ligand (e.g. TOPO).

\subsubsection{Motivation}

In order to develop synthetic expertise, and gain experience with characterisation techniques pertinent to nanocrystals - especially TEM, SAED and EDS, which were to be used extensively throughout this project - it was decided to first work on a system which had been already investigated extensively and which could therefore be relied upon to produce samples amenable to characterisation. Cadmium selenide was chosen for this purpose because of its relative ease of synthesis, and good optical properties.

As detailed below in section 3.5, cadmium selenide nanocrystals were synthesised using an adapted literature method and the optical absorption and emission properties of a series of samples were correlated with the structural properties of the ensembles, as determined by TEM microscopy. In addition, capping of cadmium selenide with zinc sulphide; and the exchange of ligands to render the nanocrystals dispersible in polar media were attempted. 


\subsection{Results}

\subsubsection{Evolution of cadmium selenide nanocrystal optical properties with reaction time}

A rapid evolution of the optical absorption and emission of a reaction mixture of $\mathrm{Cd}(\mathrm{OAc})_{2}$ and $\mathrm{Se}(\mathrm{TOP})$ in $\mathrm{TOPO}$ at $300^{\circ} \mathrm{C}$ could be observed after the initiation of nucleation of cadmium selenide nanocrystals. Firstly, the absorption and emission profiles of the reaction mixture with respect to reaction time will be presented. The physical parameters of the nanocrystal ensembles as determined by TEM microscopy will then be correlated with the optical properties.

\subsubsection{UV-vis absorption}

When colourless $\mathrm{Se}(\mathrm{TOP})$ solution was injected into a colourless solution of $\mathrm{Cd}(\mathrm{OAc})_{2}$ in TOPO at $300^{\circ} \mathrm{C}$ (see 3.5.1 for details), the colour of solution was observed to change immediately to yellow, and then darken to orange, then red over a period of 20 minutes; and thereafter to black. These observations are supported by the UV-vis absorption spectra of purified aliquots of reaction mixture, which were withdrawn at intervals between 0.3 and 240 minutes after injection of Se(TOP) (see Figure 3-1 for $0.3-20$ minutes, Figure 3-2 for $60-240$ minutes).

The absorption onset (see Table 3-1) of the samples was observed to shift from an initial value of $550 \mathrm{~nm}$ immediately after injection, into the infrared after approximately one hour. Two absorption maxima are evident in each absorption spectrum, superimposed upon a sloping increase of absorption towards lower wavelengths. Like the absorption onset, these absorption maxima are observed to shift towards longer wavelengths with increasing reaction time; and to broaden and become less distinct. After 240 minutes both maxima appear as shoulders on the sloping absorption throughout the visible region of the spectrum.

The lowest energy absorption maximum was assigned to the $1 S_{3 / 2}-1 S_{e}$ transition, which has been determined to be the lowest energy excitonic transition in cadmium selenide nanocrystals. ${ }^{5}$ The identity of the transition or transitions leading to the higher energy absorption maximum was not determined, as the strength and energy of higher energy 


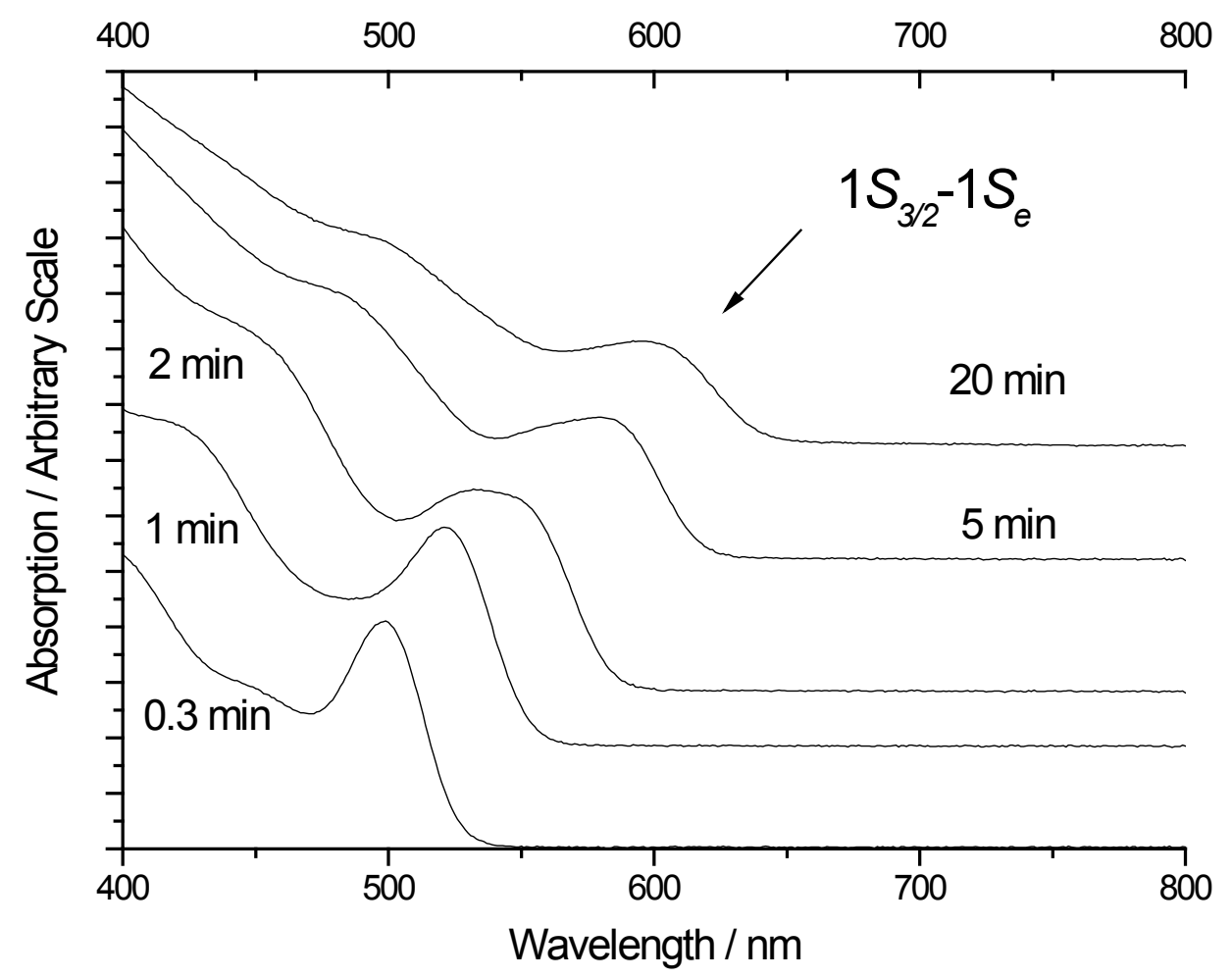

Figure 3-1 Absorption spectra of cadmium selenide nanocrystals (see 3.5.1 for synthesis) quenched after reaction times of $0.3-20$ minutes.

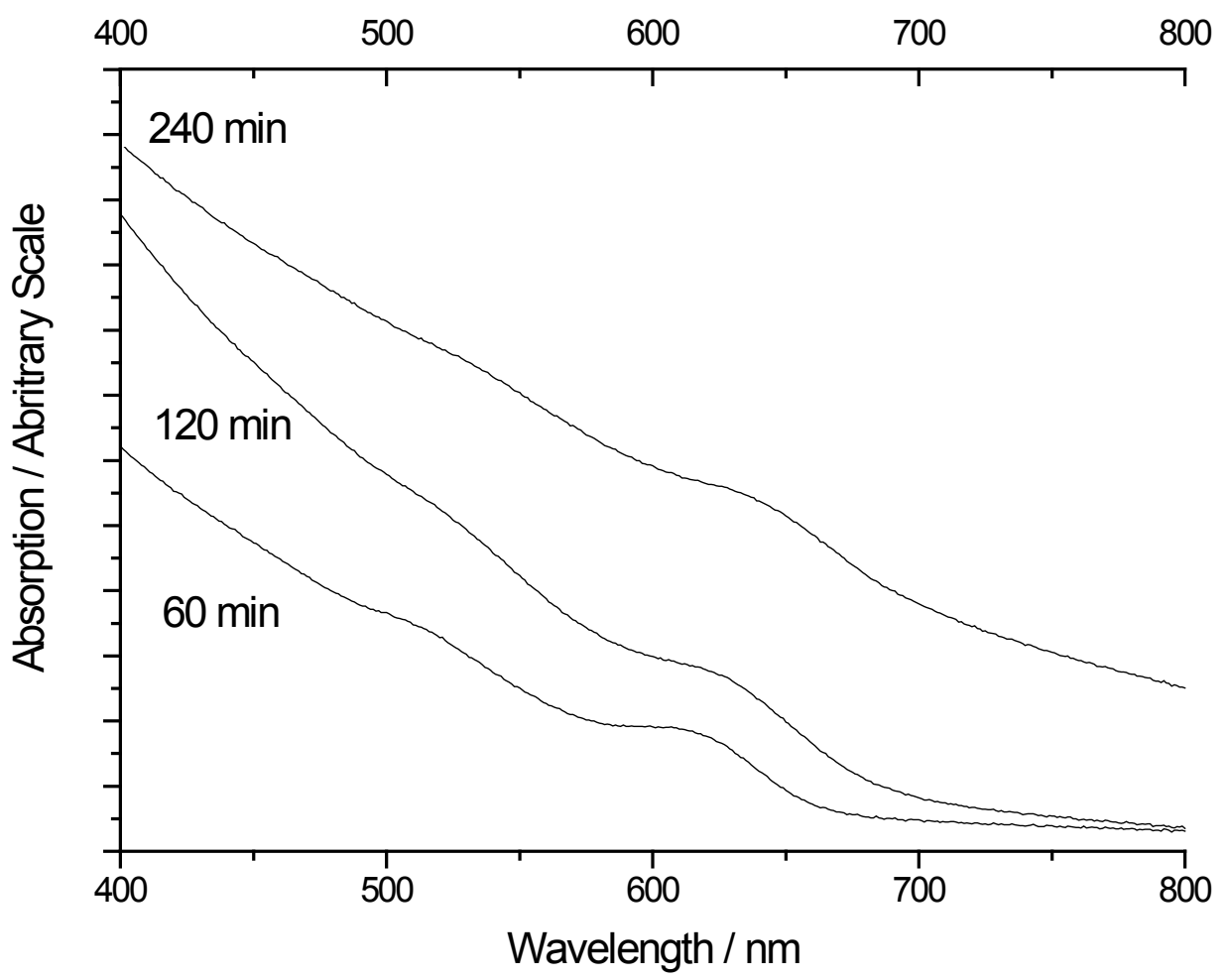

Figure 3-2 Absorption spectra of cadmium selenide nanocrystals (see 3.5.1 for synthesis) quenched after reaction times of $60-240$ minutes. 
Table 3-1 Absorption onset and position of lowest energy transition for cadmium selenide nanocrystal samples vs. reaction time. Determination of the average diameter of the cadmium selenide nanocrystals by TEM is detailed below (3.2.2).

\begin{tabular}{|c|c|c|c|}
\hline $\begin{array}{c}\text { Reaction } \\
\text { time } \\
/ \text { minutes }\end{array}$ & Absorption onset & $\begin{array}{c}\text { Position of } 1 S_{3 / 2}-1 S_{\mathrm{e}} \\
\text { transition } \\
/ \mathrm{nm}\end{array}$ & Average diameter \\
\hline 0.3 & 550 & 500 & $/ \mathrm{nm}$ \\
\hline 1 & 565 & 520 & $\mathrm{~nm} / \mathrm{a}$ \\
\hline 2 & 595 & 540 & $\mathrm{n} / \mathrm{a}$ \\
\hline 5 & 635 & 585 & $4.0 \pm 0.5$ \\
\hline 20 & 655 & 605 & $4.6 \pm 0.8$ \\
\hline 60 & 685 & 620 & $\mathrm{n} / \mathrm{a}$ \\
\hline 120 & $>700$ & 630 & $\mathrm{n} / \mathrm{a}$ \\
\hline 240 & $>700$ & 645 & $6.0 \pm 0.9$ \\
\hline
\end{tabular}

transitions in cadmium selenide nanocrystals has been found to depend strongly on diameter. ${ }^{5}$ The position of the $1 S_{3 / 2}-1 S_{e}$ transition is tabulated in Table 3-1, and was observed to shift from $500 \mathrm{~nm}$ at 0.3 minutes to $645 \mathrm{~nm}$ after 240 minutes reaction time.

\subsubsection{Fluorescence}

Each cadmium selenide nanocrystal sample displayed a single, sharp fluorescence emission peak (see Figure 3-3 for emission spectra). The position of the emission maximum tracked that of the $1 S_{3 / 2}-1 S_{e}$ transition absorption maximum (seeTable 3-2) shifting towards longer wavelengths with increasing reaction time. The Stokes shift (difference between the wavelength of the $1 S_{3 / 2}-1 S_{e}$ absorption maximum and the emission maximum) varied between $14-30 \mathrm{~nm}$, but it was evident that the emission maximum wavelength was correlated with the wavelength of the absorption maxima i.e. the energy of the fluorescence transition was not independent of the energy of the absorption transitions. This indicates that the fluorescence was band-edge emission, rather than trap state emission - which could be expected to remain relatively independent of alterations in the electronic structure of the nanocrystals.

The FWHM of the emission peaks increased from $37 \mathrm{~nm}$ for 0.3 minutes, to $53 \mathrm{~nm}$ after 240 minutes reaction time. This mirrored the broadening observed in the absorption spectra with increasing reaction time.

To summarise thus far, samples of cadmium selenide nanocrystals displayed an absorption onset and absorption maxima attributed to excitonic transitions, which broadened and shifted towards longer wavelengths with increasing reaction time. Band-edge emission was observed also to broaden and shift to longer wavelengths with 
increasing reaction time. This indicates an alteration of the electronic structure of the nanocrystal ensembles over time, which will now be correlated with the physical parameters of the nanocrystal ensembles as determined by TEM microscopy.

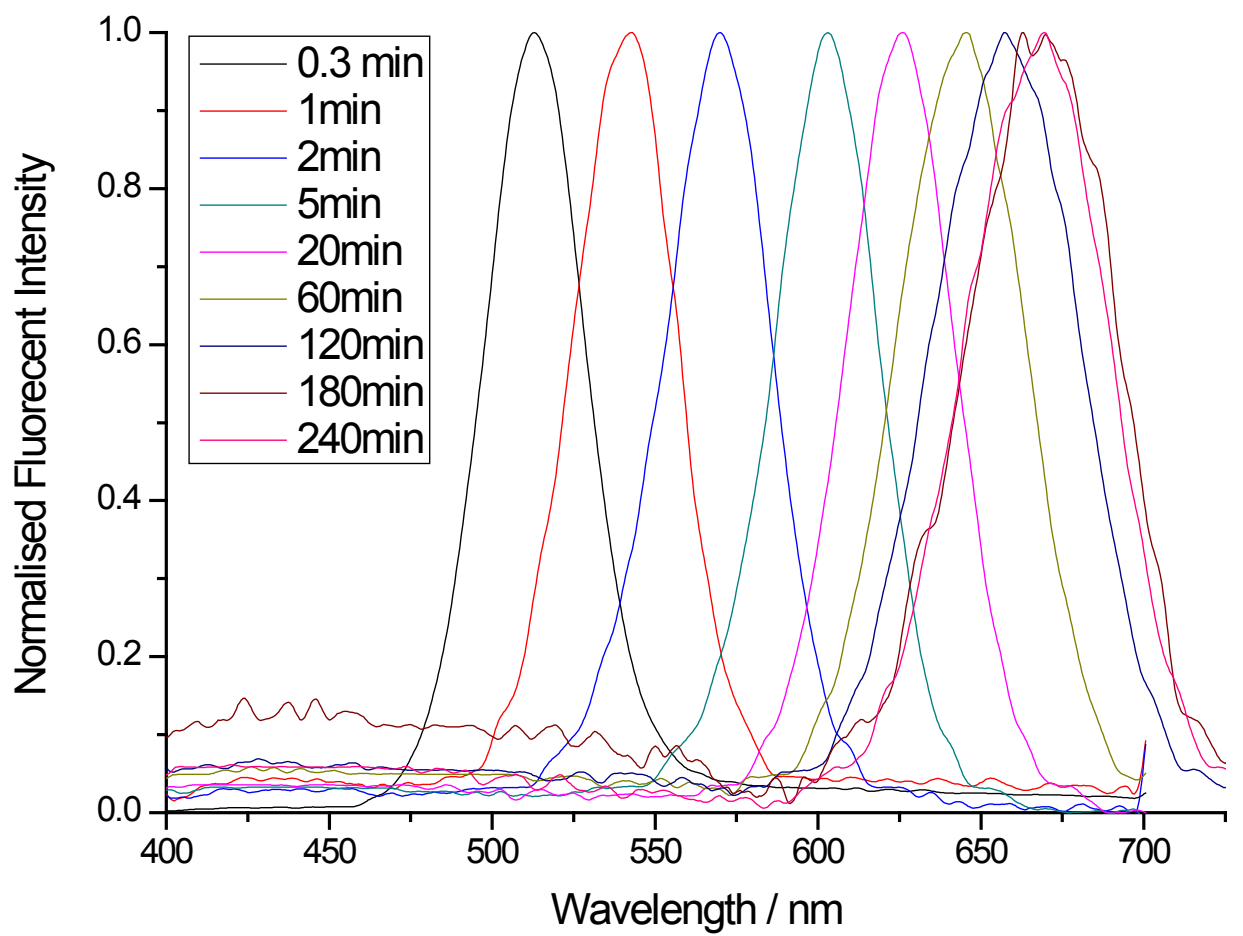

Figure 3-3 Fluorescence emission of cadmium selenide nanocrystals (see 3.5.1 for synthesis) quenched after reaction times of $0.3-240$ minutes. A constant excitation wavelength of $380 \mathrm{~nm}$ was used for all samples. Fluorescent intensity is normalised.

Table 3-2 Fluorescence maximum wavelength and FWHM of emission peak for cadmium selenide nanocrystal samples after reaction times of $0.3-240$ minutes. Determination of the average diameter of the cadmium selenide nanocrystals by TEM is detailed below (3.2.2).

\begin{tabular}{|c|c|c|c|c|}
\hline $\begin{array}{c}\text { Reaction } \\
\text { time } \\
/ \text { minutes }\end{array}$ & Fluorescence $\lambda_{\max }$ & FWHM & Stokes shift & $\begin{array}{c}\text { Average } \\
\text { diameter } \\
/ \mathrm{nm}\end{array}$ \\
\hline 0.3 & 514 & 37 & 14 & $2.8 \pm 0.4$ \\
\hline 1 & 542 & 38 & 22 & $\mathrm{n} / \mathrm{a}$ \\
\hline 2 & 570 & 40 & 30 & $\mathrm{n} / \mathrm{a}$ \\
\hline 5 & 603 & 37 & 18 & $4.0 \pm 0.5$ \\
\hline 20 & 625 & 39 & 30 & $4.6 \pm 0.8$ \\
\hline 60 & 646 & 48 & 26 & $\mathrm{n} / \mathrm{a}$ \\
\hline 120 & 657 & 54 & 27 & $\mathrm{n} / \mathrm{a}$ \\
\hline 240 & 670 & 53 & 25 & $6.0 \pm 0.9$ \\
\hline
\end{tabular}




\subsubsection{TEM examination of cadmium selenide nanocrystal samples}

Micrographs of cadmium selenide nanocrystals (see 3.5.1 for synthesis details) after 0.3, 5, 20, and 240 minutes reaction time are shown below in Figure 3-4. Average particle diameters and standard deviations, as determined from TEM micrographs, are displayed as histograms below in Figure 3-5. Insets in the micrographs show high resolution images of single nanocrystals displaying lattice fringes indicating they are single crystals.

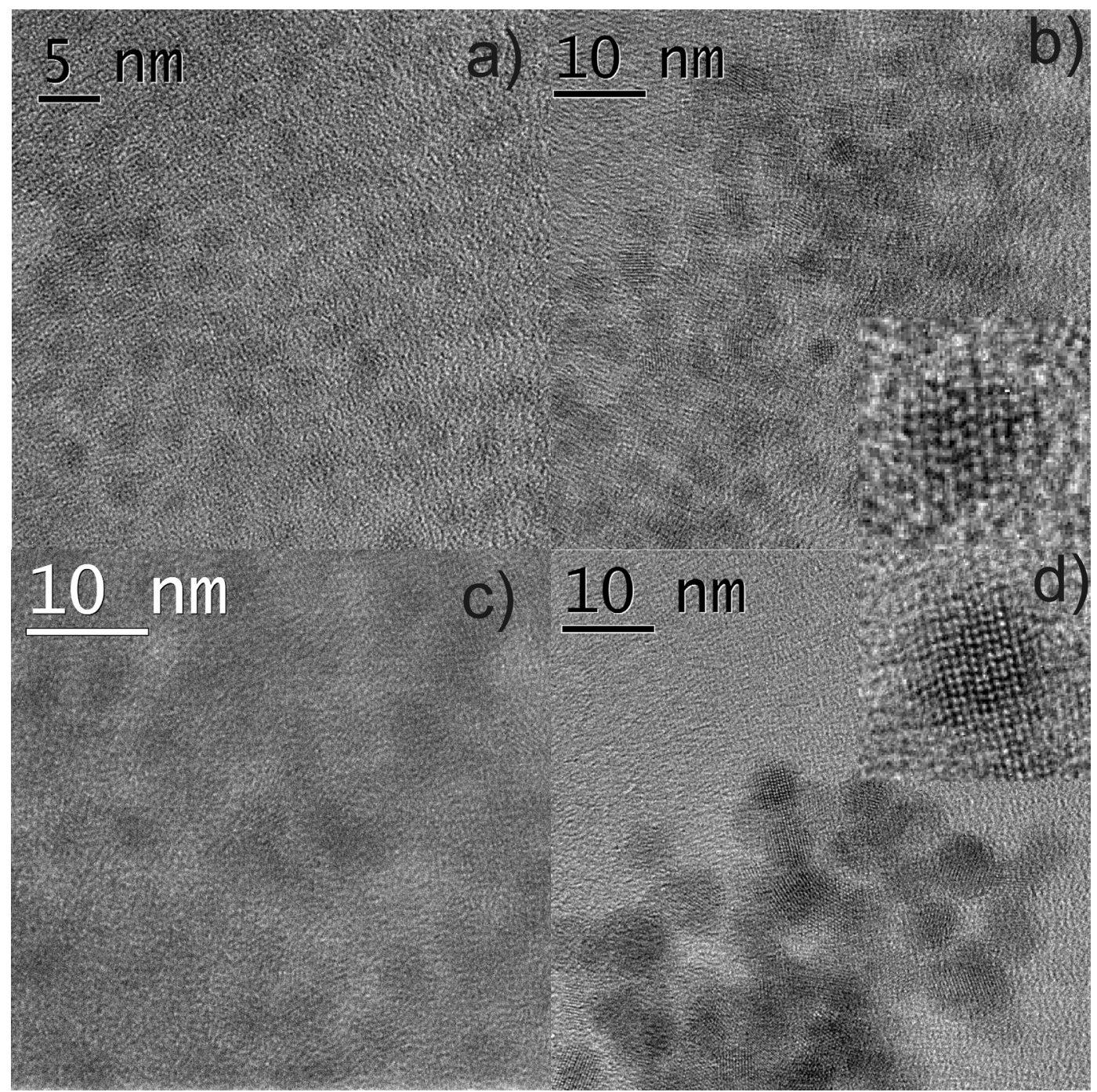

Figure 3-4 Cadmium selenide nanocrystals after reaction time of a) $0.3 \mathrm{~min}$ b) $5 \mathrm{~min}$ c) $20 \mathrm{~min}$ d) 240 min. Insets show high resolution images of single nanocrystals.

In the 0.3 minute sample, the nanocrystals formed appear spherical and have an average diameter slightly less than $3 \mathrm{~nm}$. After 5 minutes of reaction time the average diameter of the nanocrystals has increased to $4 \mathrm{~nm}$, and lattice fringes are evident in various nanocrystals indicating high crystallinity. The rate of increase in the average nanocrystal diameter slows after the first few minutes, and reaches and an average diameter of 4.6 
and $6.0 \mathrm{~nm}$ after 20 and 240 minutes of reaction time, respectively. Broadening of the nanocrystal size distribution occurs between 5 and 240 minutes, and although there is not a great increase on the relative standard deviation in nanocrystal diameters (from $12.5 \%$ at 5 minutes to $15 \%$ after 240 minutes ) - in absolute terms the standard deviation in nanocrystal diameter has increased to $0.9 \mathrm{~nm}$ by 240 minutes.
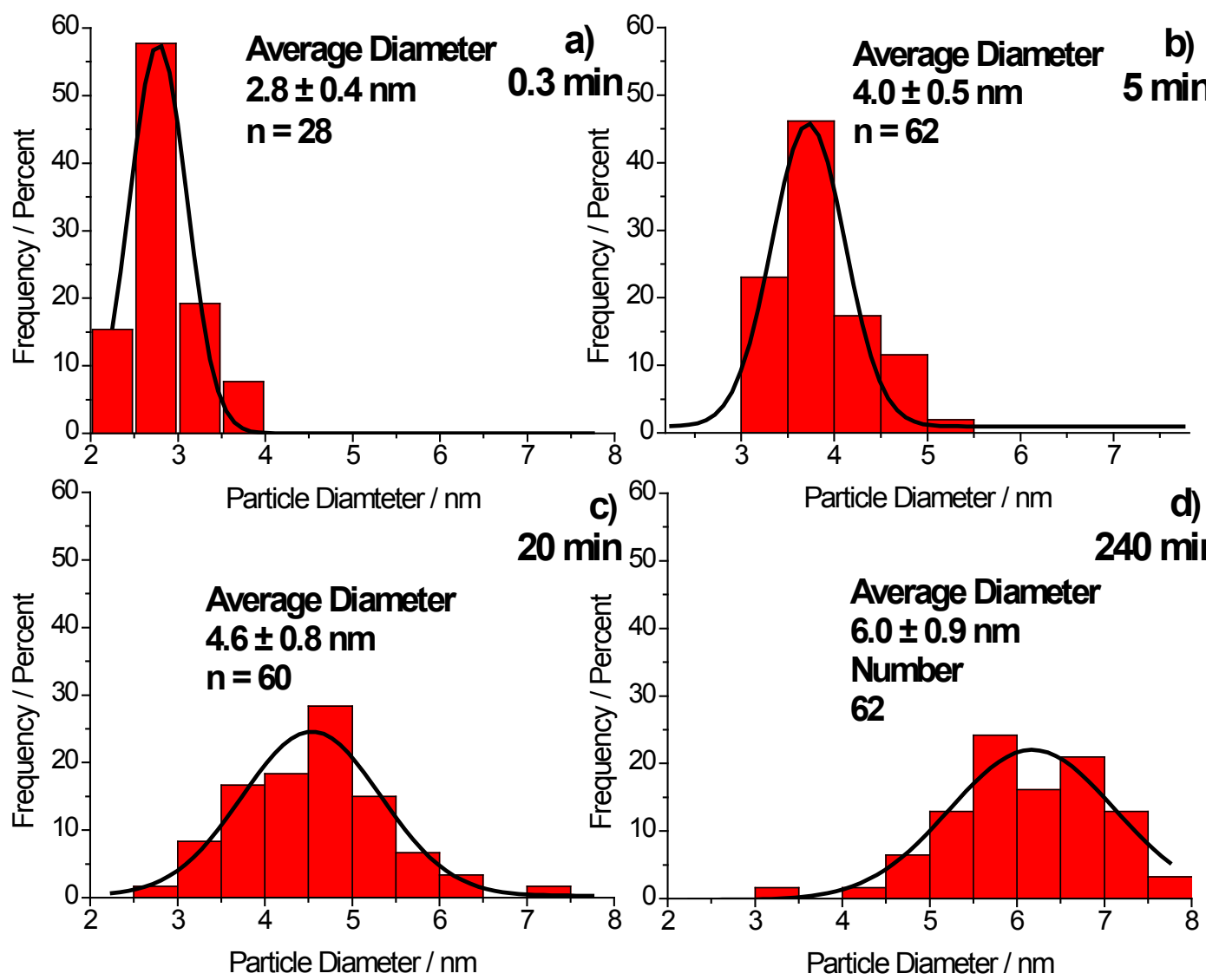

Figure 3-5 Histograms displaying the cadmium selenide nanocrystal size distribution as determined by TEM measurements after reaction times of a) $0.3 \mathrm{~min} \mathrm{b)} 5 \mathrm{~min}$ c) $20 \mathrm{~min}$ d) $240 \mathrm{~min}$.

The sample of nanocrystals withdrawn after 0.3 minutes reaction time did not diffract, presumably due to the extremely small size of the cadmium selenide nanocrystals $(<3$ $\mathrm{nm})$. However samples withdrawn at all other times diffracted, and a representative SAED pattern is displayed in Figure 3-6. The well-developed ring pattern was indexed to the hexagonal wurtzite polymorph of cadmium selenide (see Table 3-4 for reference data, and Table 3-3 for indexing parameters of the SAED pattern).

Table 3-3 Indexing parameters for SAED pattern of cadmium selenide nanocrystals.

\begin{tabular}{|c|c|c|c|}
\hline & Calculated $d$-spacing / & Proposed $h k l$ & Polymorph \\
\hline A & 3.5 & 100 & wurtzite \\
\hline & & 002 & wurtzite \\
\hline & & 101 & wurtzite \\
\hline / & Not Observed & 102 & wurtzite \\
\hline B & 2.2 & 110 & wurtzite \\
\hline C & 2.0 & 103 & wurtzite \\
\hline D & 1.8 & 112 & wurtzite \\
\hline
\end{tabular}




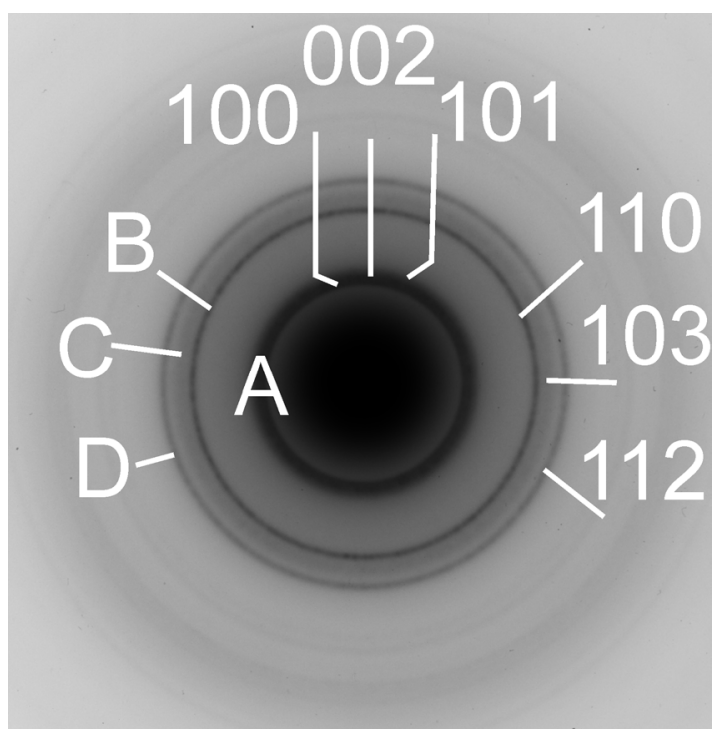

Figure 3-6 Representative SAED pattern for cadmium selenide nanocrystals (taken from the 240 minute sample). Pattern indexed to the hexagonal wurtzite polymorph of cadmium selenide.

Table 3-4 Reference details for the cubic zinc blende and hexagonal wurtzite polymorph of cadmium selenide.

\begin{tabular}{|c|c|c|l|c|c|c|}
\hline \multicolumn{2}{|c|}{$\begin{array}{c}\text { Cadmium selenide (zinc blende) } \\
\text { PDF \#04-003-5331 }\end{array}$} & \multicolumn{3}{c|}{$\begin{array}{c}\text { Cadmium selenide (wurtzite) } \\
\text { PDF \#04-007-9729 }\end{array}$} \\
\hline$h k l$ & $d / \AA$ & $\mathrm{I} / \%$ & & $h k l$ & $d / \AA$ & $\mathrm{I} / \%$ \\
\hline 111 & 3.20 & 9 & & 100 & 3.72 & 100 \\
\hline 200 & 2.77 & 100 & & 002 & 3.51 & 60 \\
\hline 220 & 1.96 & 61 & & 101 & 3.29 & 75 \\
\hline 311 & 1.67 & 3 & & 102 & 2.55 & 34 \\
\hline & & & & 110 & 2.15 & 72 \\
\hline & & & & 103 & 1.98 & 67 \\
\hline & & & & 200 & 1.86 & 11 \\
\hline & & & & 112 & 1.83 & 42 \\
\hline
\end{tabular}

EDS was used to monitor the composition of the cadmium selenide nanocrystal samples. In each sample phosphorus was detected in addition to cadmium and selenium - indicating that TOPO remained in the sample. The Cd:Se ratios of the samples are tabulated below (Table 3-5). The sample withdrawn after 0.3 minutes demonstrated a $\mathrm{Cd}$ :Se ratio of roughly 3:2, significantly in excess of what would be expected for stoichiometric cadmium selenide. This may have been due to the very weak signal from this sample. The remaining samples displayed Cd:Se ratios of approximately 1.2:1, which is outside the solubility range of cadmium in cadmium selenide in the bulk $(\mathrm{Cd} \text { :Se } 1.02: 1)^{20}$ - although similar $\mathrm{Cd}$ :Se ratios outside of the stability ranges of the bulk have been observed previously in nanocrystals. ${ }^{21}$ 
Table 3-5 Composition of cadmium selenide nanocrystals as determined by EDS.

\begin{tabular}{|c|c|c|c|}
\hline $\begin{array}{c}\text { Reaction Time } / \\
\text { Minutes }\end{array}$ & Cd / at $\%$ & Se / at $\%$ & Cd:Se \\
\hline 0.3 & 62 & 38 & $1.6: 1$ \\
\hline 5 & 55 & 45 & $1.2: 1$ \\
\hline 20 & 54 & 46 & $1.2: 1$ \\
\hline 240 & 53 & 47 & $1.1: 1$ \\
\hline
\end{tabular}




\subsubsection{Quantum yield of cadmium selenide nanocrystals}

In order to determine the fluorescence quantum yield of cadmium selenide nanocrystal samples, two fluorescent dyes (fluorescein and coumarin 6) of known quantum yield were used as references for the comparison procedure described in Chapter 2 section 2.2.8. Experimental details are given below, in 3.5.3. $460 \mathrm{~nm}$ was chosen as the excitation wavelength for this procedure, and hence the absorbance of all samples was assessed at this wavelength.

The absorption spectra of a dilution series of fluorescein solutions (see 3.5.3.1 for preparation) are shown in Figure 3-7. The absorbance at $460 \mathrm{~nm}$ for each member of the series is collated in Table 3-6. The fluorescence emission spectra for the series are shown in Figure 3-8, and the integrated fluorescence intensity of each sample is also collated in Table 3-6.

The absorption spectra of a dilution series of coumarin 6 solutions (see 3.5.3.1 for preparation) are shown in Figure 3-9. The absorbance at $460 \mathrm{~nm}$ for each member of the series is collated in Table 3-7. The fluorescence emission spectra for the series are shown in Figure 3-10, and the integrated fluorescent intensity of each of the samples is also collated in Table 3-7.

The integrated fluorescent intensity for each sample was plotted versus its absorbance at $460 \mathrm{~nm}$ (see Figure 3-11). A linear fit was made for the data points for each dye, using linear regression. The gradient of each fitted line, and its $\mathrm{R}$ value are collated in Table 3-8.

To ensure the validity of the procedure, the quantum yield of each of the two standard dyes was calculated based upon the literature value of the quantum yield of the other, using the expression shown in Chapter 2 section 2.2.8 equation (19). The resulting quantum yield values are tabulated below (Table 3-8), and were in each case within 6 percentage points of the literature value. 


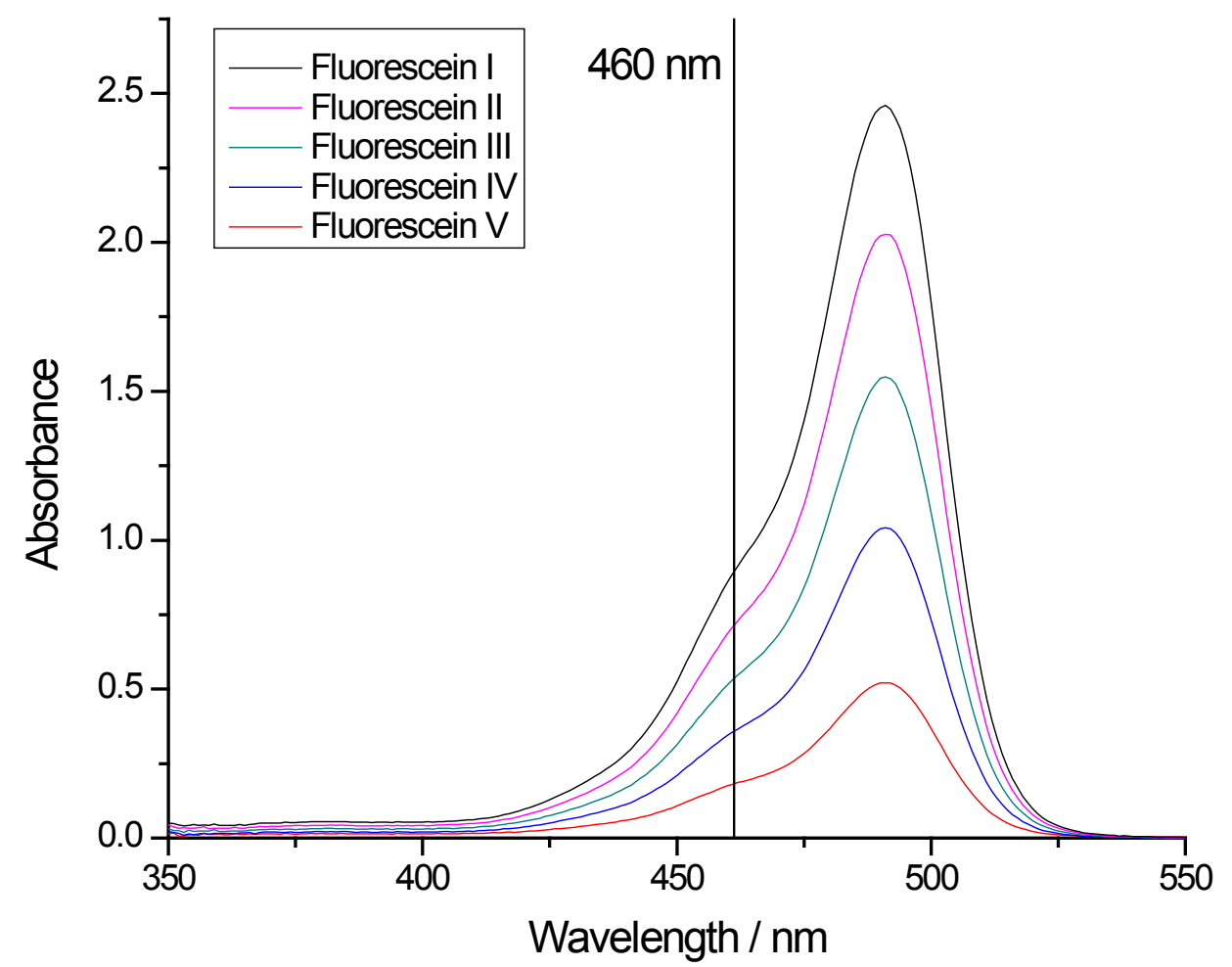

Figure 3-7 Absorption spectra of fluorescein standard dye dilution series.

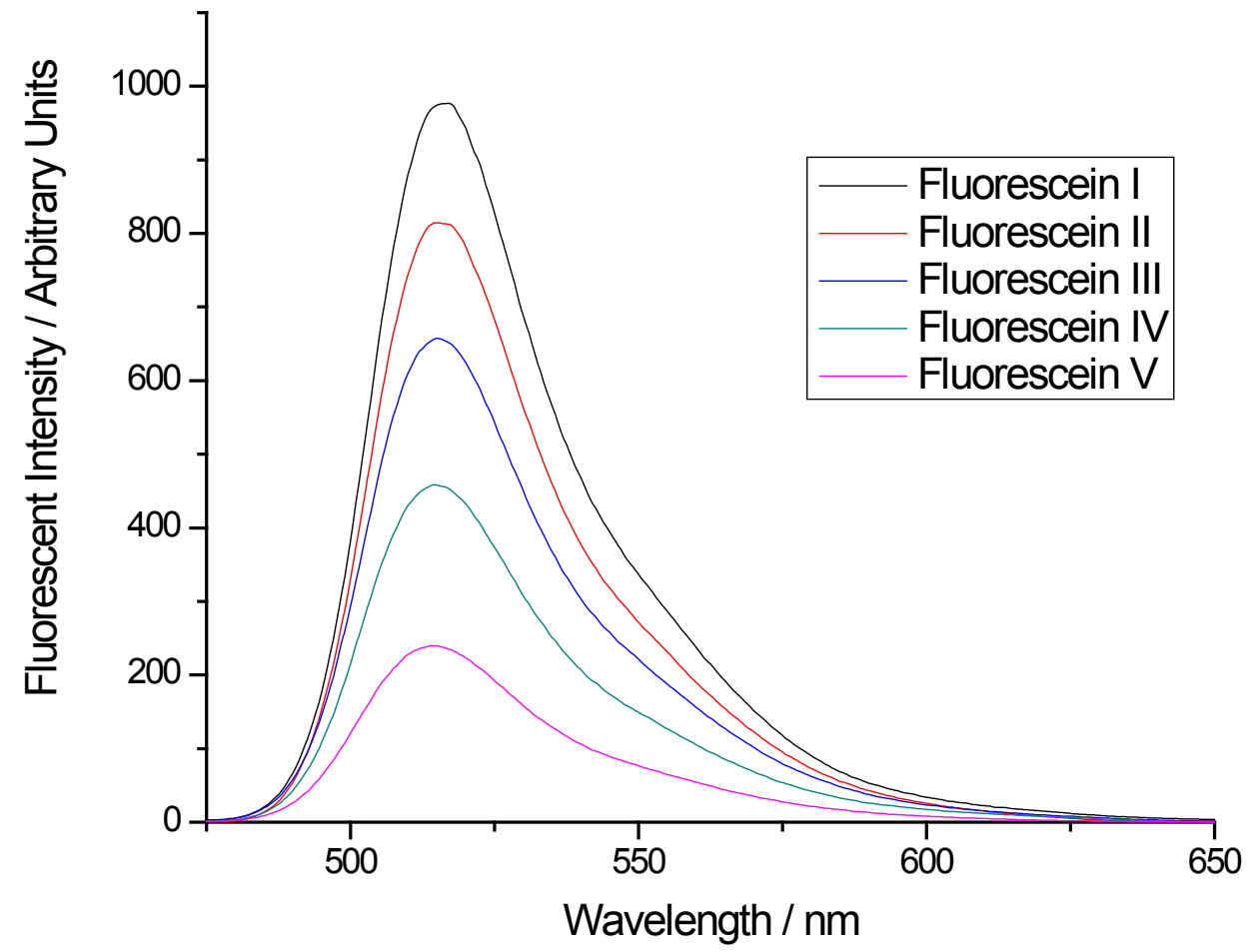

Figure 3-8 Fluorescence emission spectra of fluorescein standard dye dilution series.

Table 3-6 Parameters for fluorescein standard dye dilution series.

\begin{tabular}{|c|c|c|c|}
\hline & $\begin{array}{c}\text { Nominal } \\
\text { Concentration / } \\
\text { mol dm }\end{array}$ & $\begin{array}{c}\text { Absorbance at } \\
460 \mathrm{~nm}\end{array}$ & $\begin{array}{c}\text { Integrated Fluorescent } \\
\text { Intensity }(475-650 \mathrm{~nm}) / \\
\text { Arbitrary Units }\end{array}$ \\
\hline Fluorescein I & $1.5 \times 10^{-5}$ & 0.86 & 43978 \\
\hline Fluorescein II & $7.5 \times 10^{-6}$ & 0.69 & 36103 \\
\hline Fluorescein III & $5.9 \times 10^{-6}$ & 0.51 & 29826 \\
\hline Fluorescein IV & $4.4 \times 10^{-6}$ & 0.34 & 20753 \\
\hline Fluorescein V & $1.5 \times 10^{-6}$ & 0.18 & 10873 \\
\hline
\end{tabular}




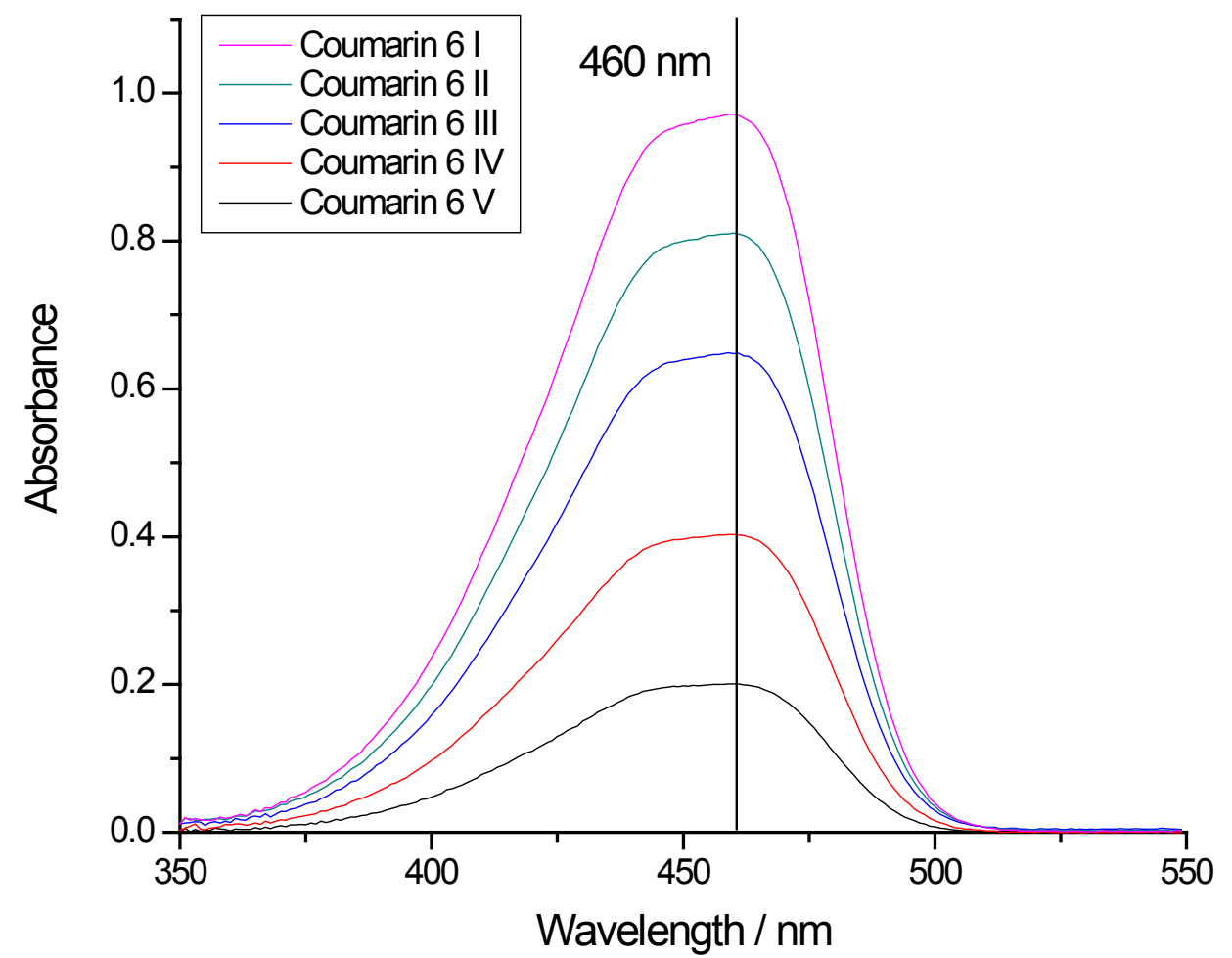

Figure 3-9 Absorption spectra of coumarin 6 standard dye dilution series.

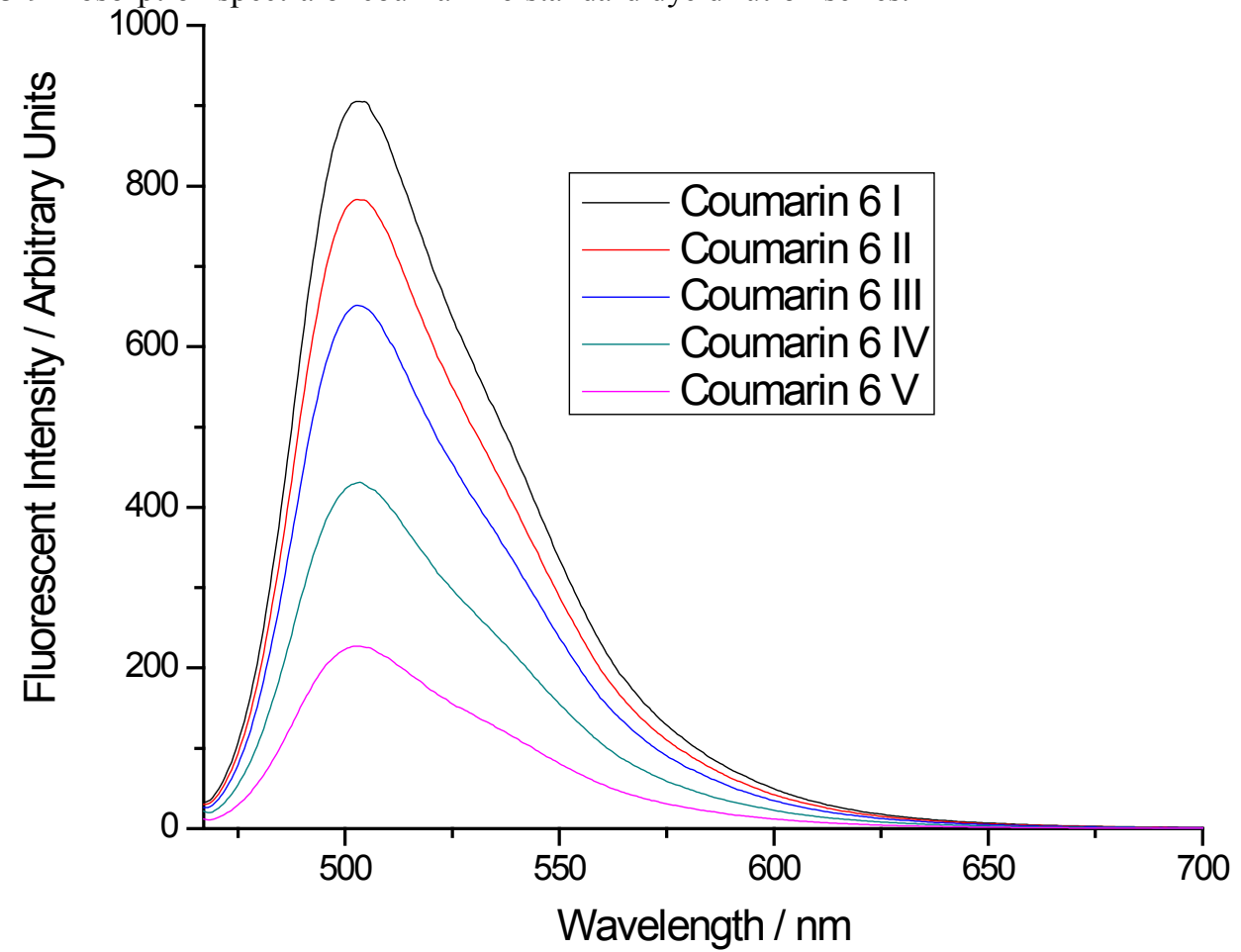

Figure 3-10 Fluorescence emission spectra of coumarin 6 standard dye dilution series.

Table 3-7 Parameters for coumarin 6 standard dye series.

\begin{tabular}{|c|c|c|c|}
\hline & $\begin{array}{c}\text { Nominal } \\
\text { Concentration } / \mathrm{mol} \\
\mathrm{dm}^{-3}\end{array}$ & $\begin{array}{c}\text { Absorbance at } \\
460 \mathrm{~nm}\end{array}$ & $\begin{array}{c}\text { Integrated Fluorescent } \\
\text { Intensity }(467-650 \mathrm{~nm}) / \\
\text { Arbitrary Units }\end{array}$ \\
\hline Coumarin 6 I & $2.3 \times 10^{-6}$ & 0.97 & 47879 \\
\hline Coumarin 6 II & $1.9 \times 10^{-6}$ & 0.81 & 44211 \\
\hline Coumarin 6 III & $1.5 \times 10^{-6}$ & 0.65 & 37041 \\
\hline Coumarin 6 IV & $9.4 \times 10^{-7}$ & 0.40 & 24334 \\
\hline Coumarin 6 V & $4.7 \times 10^{-7}$ & 0.20 & 12702 \\
\hline
\end{tabular}




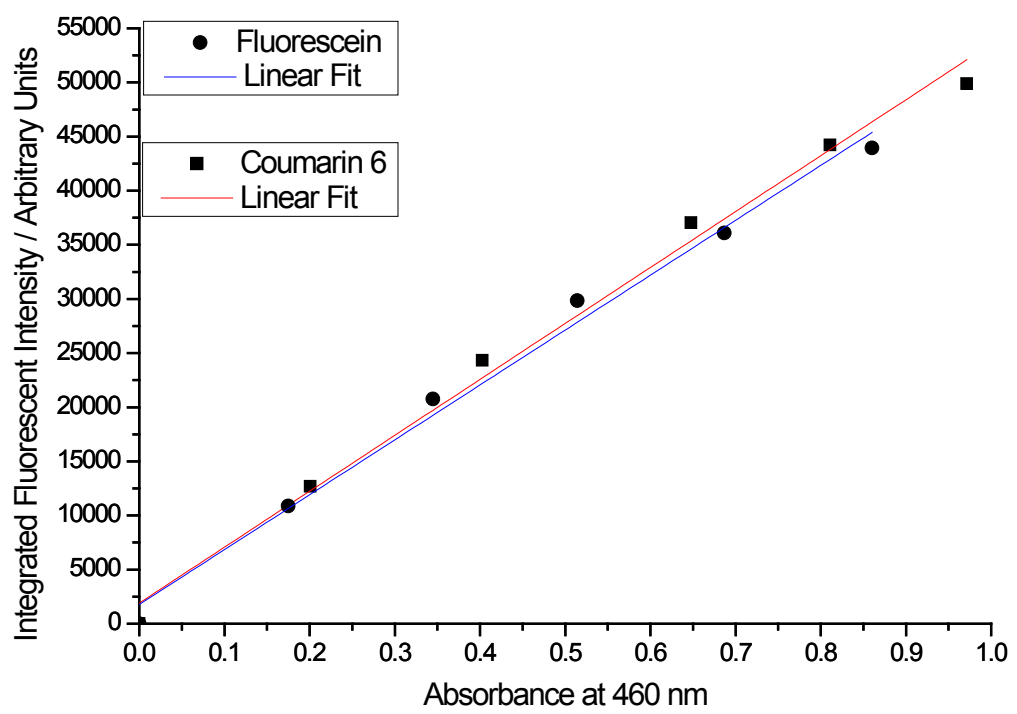

Figure 3-11 Integrated fluorescent intensity vs. absorbance at $460 \mathrm{~nm}$ for fluorescein and coumarin 6 dilution series.

Table 3-8 Quantum yield parameters for the reference dyes fluorescein and coumarin 6.

\begin{tabular}{|l|c|c|c|c|c|c|}
\hline & Solvent & $\begin{array}{c}\text { Refractive } \\
\text { index @ } \\
460 \mathrm{~nm}\end{array}$ & Literature $\Phi_{\mathrm{F}}$ & Gradient & $\mathrm{R}$ & $\begin{array}{c}\text { Calculated } \\
\Phi_{\mathrm{F}}\end{array}$ \\
\hline Fluorescein & $\begin{array}{c}0.1 \mathrm{M} \\
\mathrm{NaOH}\end{array}$ & 1.3434 & $0.79^{22}$ & 50700 & 0.989 & 0.74 \\
\hline Coumarin 6 & Ethanol & 1.3683 & $0.78^{23}$ & 51660 & 0.990 & 0.84 \\
\hline
\end{tabular}


A sample of cadmium selenide nanocrystals prepared as described in 3.5.1 was chosen as a representative sample, and a dilution series was prepared. The absorption spectra of the dilution series of cadmium nanocrystal solutions (see 3.5.3.1 for preparation) are shown in Figure 3-12. The absorbance at $460 \mathrm{~nm}$ of each member of the series is collated in Table 3-9. The fluorescence emission spectra for the series are shown in Figure 3-13, and the integrated fluorescent intensity of each of the samples is also collated in Table 3-9.

The integrated fluorescent intensity of each sample was plotted versus its absorbance at $460 \mathrm{~nm}$ (see Figure 3-14). A linear fit was made through the data points using linear regression. The gradient of the fitted line and its $\mathrm{R}$ value are collated in Table 3-10. The quantum yield $\left(\Phi_{\mathrm{F}}\right)$ of the cadmium selenide nanocrystal sample was calculated based on the literature quantum yield of each of the two reference dyes, and the result was averaged. 


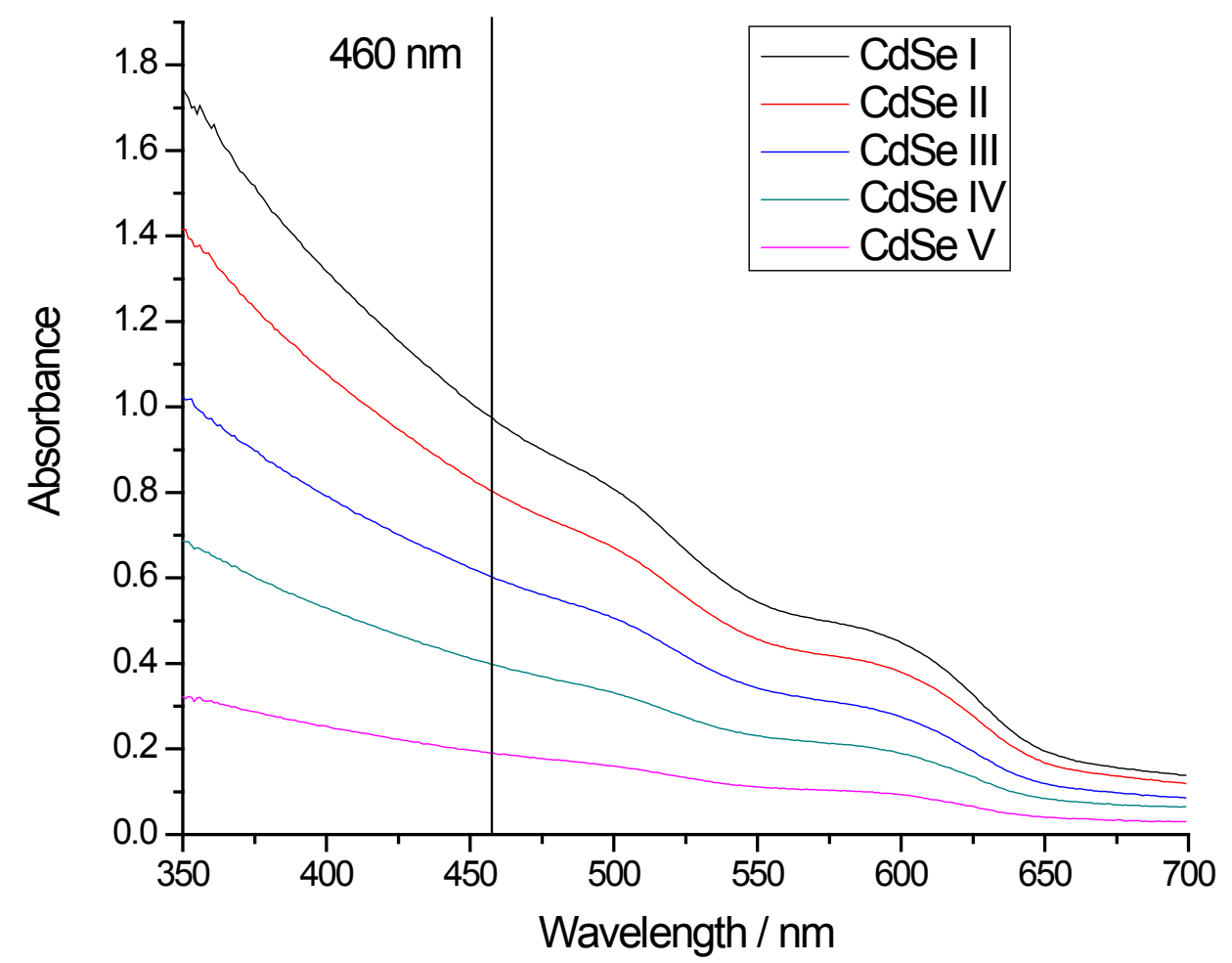

Figure 3-12 Absorption spectra of cadmium selenide nanocrystal dilution series.

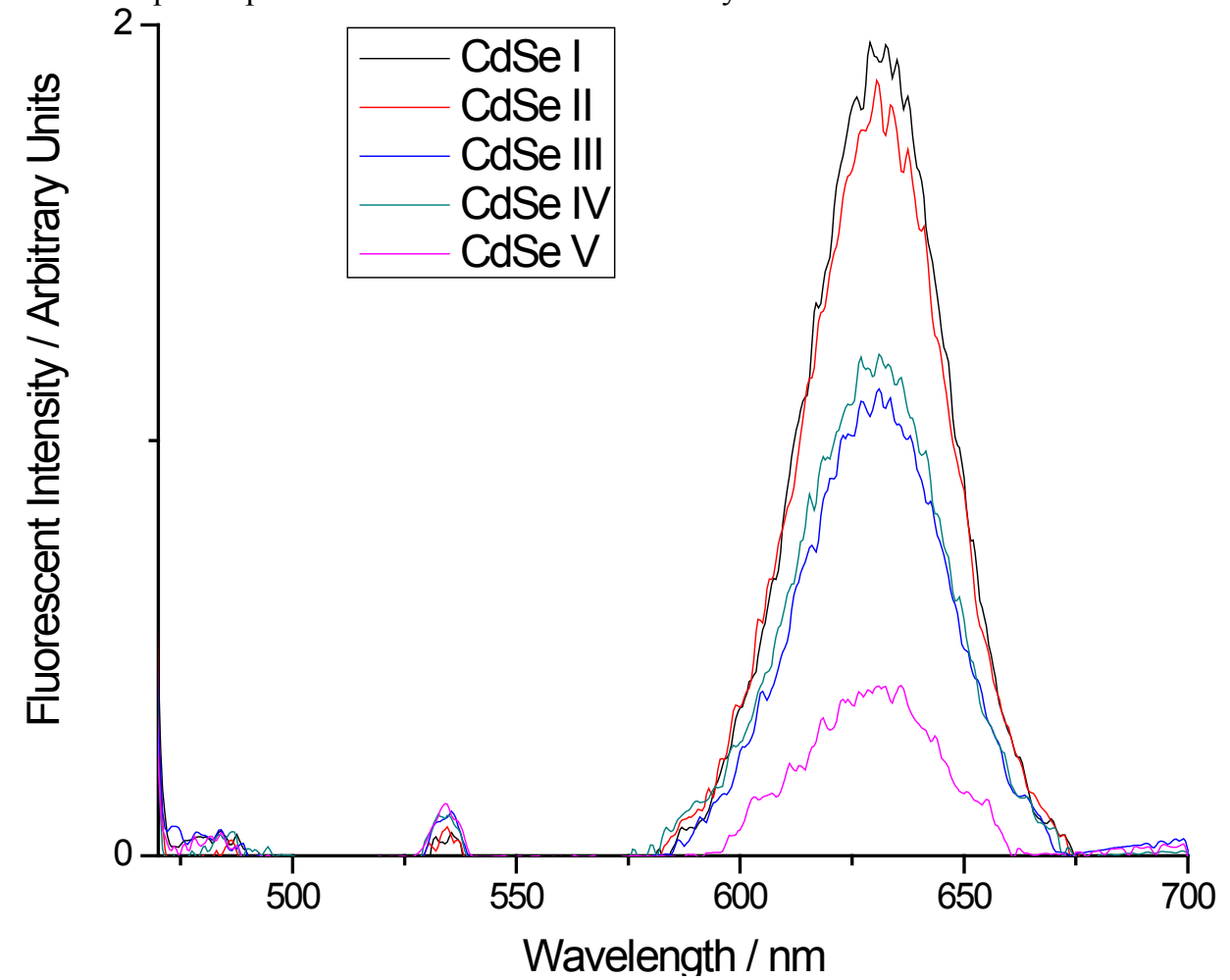

Figure 3-13 Fluorescence emission spectra of cadmium selenide nanocrystal dilution series.

Table 3-9 Parameters for cadmium selenide nanocrystal dilution series.

\begin{tabular}{|c|c|c|}
\hline & Absorbance at $460 \mathrm{~nm}$ & $\begin{array}{c}\text { Integrated Fluorescent } \\
\text { Intensity }(575-700 \mathrm{~nm}) / \\
\text { Arbitrary Units }\end{array}$ \\
\hline CdSe I & 0.96 & 75 \\
\hline CdSe II & 0.79 & 71 \\
\hline CdSe III & 0.60 & 45 \\
\hline CdSe IV & 0.39 & 50 \\
\hline CdSe V & 0.19 & 15 \\
\hline
\end{tabular}




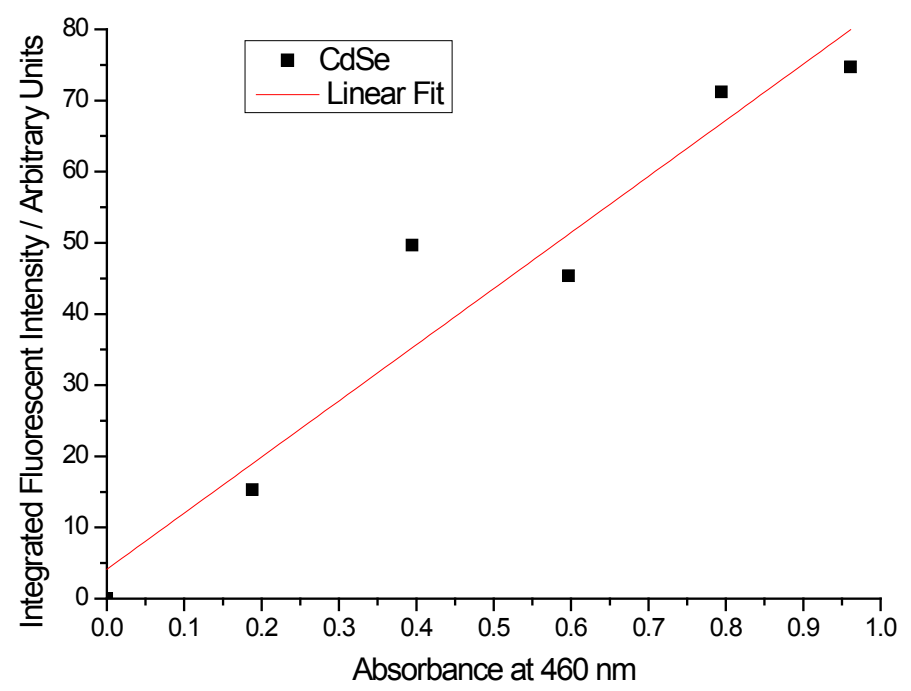

Figure 3-14 Integrated fluorescent intensity vs. absorbance for cadmium selenide nanocrystal dilution series.

Table 3-10 Quantum yield parameters for the CdSe nanocrystal sample.

\begin{tabular}{|c|c|c|c|c|c|}
\hline & Solvent & $\begin{array}{c}\text { Refractive } \\
\text { index @ } \\
460 \mathrm{~nm}\end{array}$ & $\begin{array}{c}\text { Gradient } \\
\text { see Figure } \\
3-14)\end{array}$ & $\mathrm{R}$ & $\begin{array}{c}\text { Calculated } \\
\Phi_{\mathrm{F}}\end{array}$ \\
\hline $\mathrm{CdSe}$ & Toluene & 1.514 & 79 & 0.911 & 0.002 \\
\hline
\end{tabular}




\subsubsection{Capping of cadmium selenide nanocrystals with zinc sulfide}

In order to passivate the cadmium selenide nanocrystals, it was attempted to deposit a

layer of zinc sulfide on surface of the nanocrystals. Firstly, cadmium selenide

nanocrystals which had been synthesised and purified as described in section 3.5.1 were

used in the procedure described in section 3.5.4. A micrograph of the pre-made

cadmium selenide nanocrystals before capping is shown in Figure 3-15 a), and a

micrograph from after the capping attempt is shown in Figure 3-15 b). The cadmium

selenide nanocrystals appeared small and spherical before the capping procedure, and there was no obvious change in appearance after the capping attempt, although low image quality made this difficult to discern.

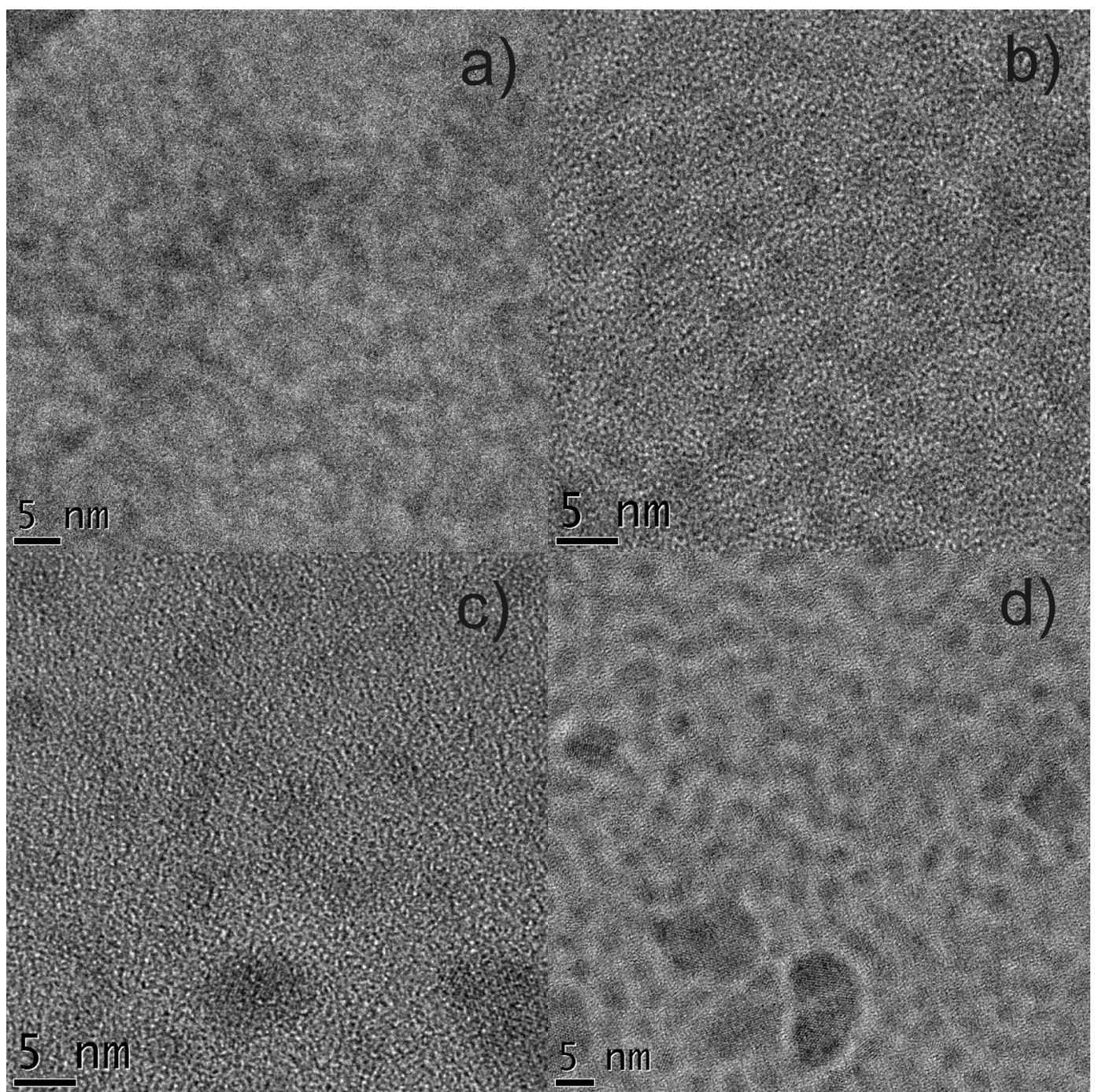

Figure 3-15 Micrographs of a) premade cadmium selenide nanocrystals b) cadmium selenide nanocrystals after zinc sulfide capping c) cadmium selenide nanocrystals from one-pot synthesis d) CdSe/ZnS nanocrystals from one-pot synthesis. 
The UV-vis absorption spectrum of the nanocrystals (Figure 3-16) shows a small red-shift of $7 \mathrm{~nm}$ in the position of $1 S_{3 / 2}-1 S_{e}$ absorption maximum ( $\lambda_{\max }$ shifts from 476 to $483 \mathrm{~nm}$ ) upon capping. Similar small red shifts have been observed previously upon the capping of cadmium selenide nanocrystals with zinc sulfide, ${ }^{9}$ though it is unclear in this case whether the shift is due to the effect of a zinc sulfide shell on the cadmium selenide nanocrystals, or due to particle growth that occurred during the capping procedure.

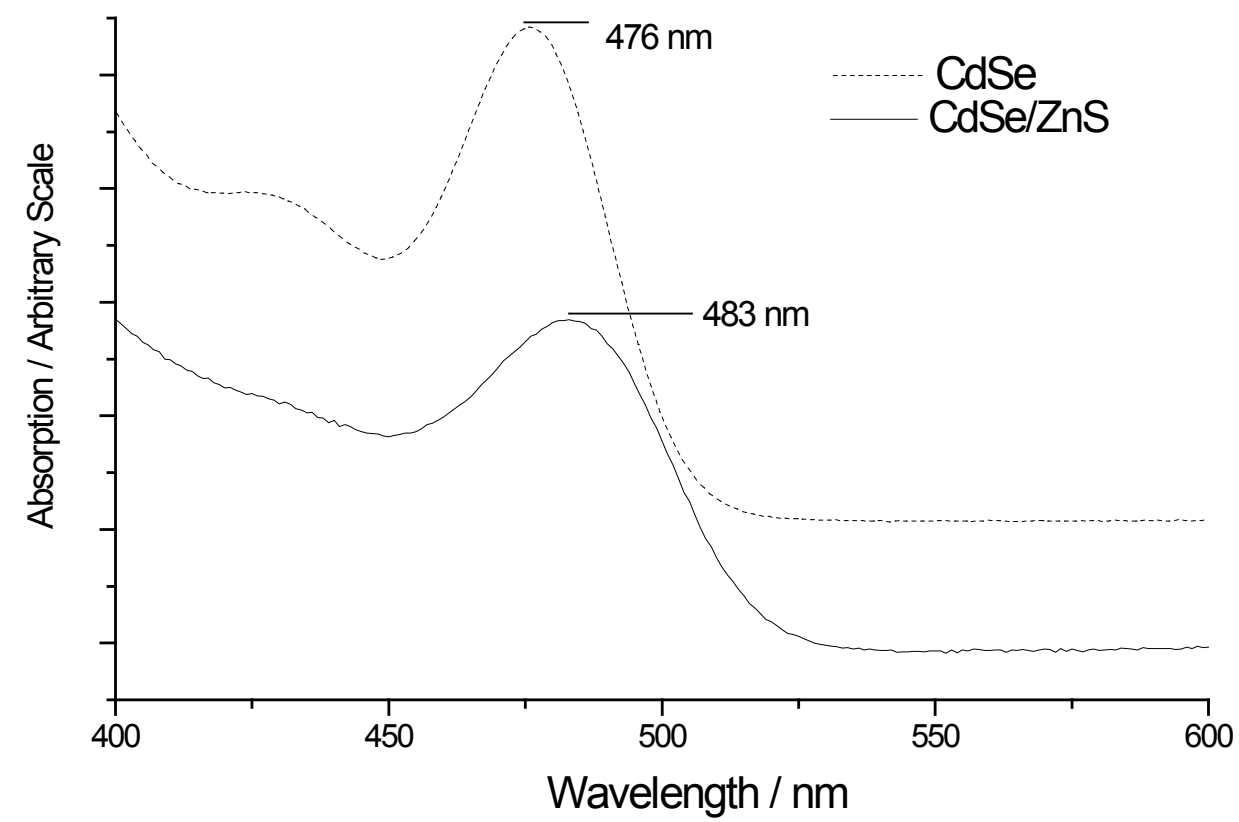

Figure 3-16 UV-vis absorption spectrum of cadmium selenide nanocrystals before and after capping with zinc sulfide.

EDS analysis on the sample after the capping attempt confirmed the presence of cadmium, selenium, zinc and sulfur in the sample (see Table 3-11). The Cd:Se ratio was not stoichiometric, and fell outside the range commonly seen in cadmium selenide nanocrystals (Cd:Se) $1.02 \pm 0.14) .{ }^{21}$ The $\mathrm{Zn}: \mathrm{S}$ ratio was far from stoichiometric, with zinc in greater than two times excess.

Table 3-11 Composition of cadmium selenide nanocrystal sample after capping attempt with zinc sulfide, as determined by EDS.

\begin{tabular}{|c|c|c|c|c|c|c|}
\hline & $\mathrm{Cd} / \mathrm{at} \%$ & $\mathrm{Se} / \mathrm{at} \%$ & $\mathrm{Cd}: \mathrm{Se}$ & $\mathrm{Zn} /$ at $\%$ & $\mathrm{~S} /$ at $\%$ & $\mathrm{Zn}: \mathrm{S}$ \\
\hline $\mathrm{CdSe} / \mathrm{ZnS}$ & 30 & 38 & $1: 1.2_{5}$ & 25 & 7 & $1: 0.3$ \\
\hline
\end{tabular}

A one-pot process was trialled to produce $\mathrm{CdSe} / \mathrm{ZnS}$ nanocrystals, as described in section 3.5.5. An aliquot of the cadmium selenide nanocrystal cores was taken before the addition of zinc sulfide. A micrograph of this purified aliquot of cadmium selenide nanocrystals is shown in Figure 3-15 c); and a micrograph from after the capping process is shown in Figure 3-15 d). The cadmium selenide nanocrystals produced in the 
first step are highly irregularly shaped, and with a wide size distribution. The micrograph from after the capping step shows large irregularly shaped particles in addition to a large number of smaller, monodisperse spherical particles.

UV-vis absorption spectra of the purified aliquot of cadmium selenide nanocrystals taken before the zinc sulfide capping stage; and of the final product are shown below in Figure 3-17. There is a small red-shift in the $1 S_{3 / 2}-1 S_{e}$ absorption maximum $\left(\lambda_{\max }\right.$ shifts from 526 to $535 \mathrm{~nm}$ ) after the capping step, similar to that observed above for the attempted capping of pre-made cadmium selenide nanocrystals.

EDS analysis of the sample after the capping stage (see Table 3-12) confirms the presence of cadmium, selenium, zinc and sulfur. However neither the cadmium and selenium; nor the zinc and sulfur are in a stoichiometric ratio, indicating the likely failure to produce $\mathrm{CdSe} / \mathrm{ZnS}$.

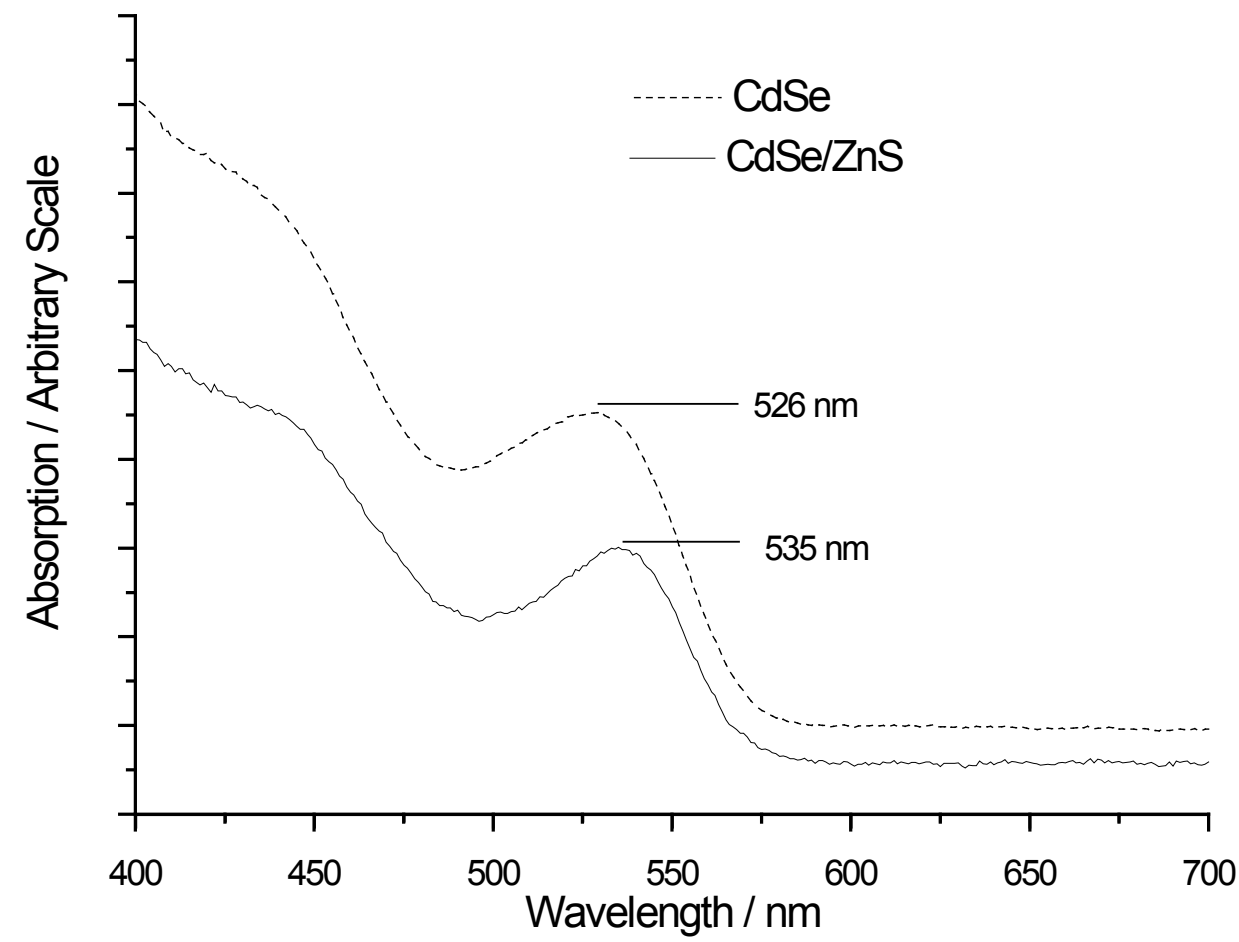

Figure 3-17 UV-vis absorption spectra of cadmium selenide nanocrystals before and after capping with zinc sulfide, produced in a one-pot process.

Table 3-12 Composition, as determined by EDS, of cadmium selenide nanocrystals produced in a one-pot process, before and after capping with zinc sulfide.

\begin{tabular}{|l|l|l|l|l|l|l|}
\hline & $\mathrm{Cd} / \mathrm{at} \%$ & $\mathrm{Se} / \mathrm{at} \%$ & $\mathrm{Cd}: \mathrm{Se}$ & $\mathrm{Zn} /$ at $\%$ & $\mathrm{~S} / \mathrm{at} \%$ & $\mathrm{Zn}: \mathrm{S}$ \\
\hline $\mathrm{CdSe} / \mathrm{ZnS}$ & 31 & 51 & $1: 1.6$ & 14 & 4 & $1: 0.3$ \\
\hline
\end{tabular}




\subsubsection{Capping ligand exchange}

Hydrophilic ligands were used to displace TOPO from cadmium selenide nanocrystals, in order to render the nanocrystals soluble in polar media. Cysteine and MPA were chosen for this approach as both contain a thiol functionality - which could be expected to show affinity for the metal chalcogenide surface - and a carboxylic acid functionality, which can ionise at biological $\mathrm{pH}$ and thereby stabilise the particles in a polar solvent. ${ }^{6}$

Samples of cadmium selenide synthesised in TOPO as described in section 3.5.1, had their TOPO coating replaced with cysteine, as described in section 3.5.6. The solubility of the nanocrystals in various solvents was then tested. Aliquots of the methanol solution from the capping ligand exchange reaction were combined with a precipitating solvent, and centrifuged in an attempt to induce precipitation of the particles. If precipitation occurred, the supernatant was decanted, and resuspension of the pellet in a resuspending solvent was attempted, with the aid of sonication.

The solubility of the as-synthesised TOPO-capped nanocrystals in various solvents is tabulated in Table 3-13, and the solubility of the cysteine-capped cadmium selenide nanocrystals is tabulated in Table 3-14. The as-synthesised particles could be precipitated by a moderately polar solvent, such as methanol, and could only be resuspended in a non-polar solvent, such as toluene. Conversely, the cysteine-capped particles could only be precipitated with the aid of a non-polar solvent, such as diethylether, and could be resuspended in water and methanol. There was no significant shift in the position of the $1 S_{3 / 2}-1 S_{e}$ absorption peak of cysteine-capped sample after capping ligand exchange (absorption spectrum not shown).

A similar procedure was repeated on TOPO-capped cadmium selenide nanocrystals using MPA, as described in section 3.5.6. The results for the MPA-capped particles are tabulated in Table 3-15, and can be contrasted with the results for a control sample tabulated in shown in Table 3-13. MPA-capped particles could only be precipitated with the aid of a non-polar solvent, and could then be resuspended easily in either methanol or water. There was no significant shift in the position of the $1 S_{3 / 2}-1 S_{e}$ absorption peak of the MPA-capped sample after capping ligand exchange (absorption spectrum not shown). 
Table 3-13 Solubility of as-synthesised cadmium selenide nanocrystals.

\begin{tabular}{|c|c|c|c|}
\hline $\begin{array}{c}\text { Precipitating } \\
\text { Solvent }\end{array}$ & Result & $\begin{array}{c}\text { Resuspending } \\
\text { Solvent }\end{array}$ & Result \\
\hline Diethylether & No precipitation & - & - \\
\hline Methanol & Precipitation & Methanol & No resuspension \\
\hline Methanol & Precipitation & Deionised Water & No Resuspension \\
\hline Methanol & Precipitation & Toluene & $\begin{array}{c}\text { Complete } \\
\text { Resuspension }\end{array}$ \\
\hline
\end{tabular}

Table 3-14 Solubility of cysteine-capped cadmium selenide nanocrystals.

\begin{tabular}{|c|c|c|c|}
\hline $\begin{array}{c}\text { Precipitating } \\
\text { Solvent }\end{array}$ & Result & $\begin{array}{c}\text { Resuspending } \\
\text { Solvent }\end{array}$ & Result \\
\hline None & No precipitation & - & - \\
\hline Ethanol & No precipitation & - & - \\
\hline Diethylether & Precipitation & Toluene & No resuspension \\
\hline Diethylether & Precipitation & Deionised water & $\begin{array}{c}\text { Moderate } \\
\text { resuspension }\end{array}$ \\
\hline Diethylether & Precipitation & Methanol & $\begin{array}{c}\text { Complete } \\
\text { Resuspension }\end{array}$ \\
\hline
\end{tabular}

Table 3-15 Solubility of MPA-capped cadmium selenide nanocrystals.

\begin{tabular}{|c|c|c|c|}
\hline $\begin{array}{c}\text { Precipitating } \\
\text { Solvent }\end{array}$ & Result & $\begin{array}{c}\text { Resuspending } \\
\text { Solvent }\end{array}$ & Result \\
\hline Methanol & No precipitation & - & - \\
\hline Ethanol & No precipitation & - & - \\
\hline Diethylether & Precipitation & Toluene & No resuspension \\
\hline Diethylether & Precipitation & Deionised water & $\begin{array}{c}\text { Complete } \\
\text { Resuspension }\end{array}$ \\
\hline Diethylether & Precipitation & Methanol & $\begin{array}{c}\text { Complete } \\
\text { Resuspension }\end{array}$ \\
\hline
\end{tabular}




\subsection{Discussion}

\subsubsection{Evolution of the optical properties of cadmium selenide nanocrystals}

Aliquots withdrawn at various points during the reaction of $\mathrm{Cd}(\mathrm{OAc})_{2}$ and $\mathrm{Se}(\mathrm{TOP})$ in TOPO at $300^{\circ} \mathrm{C}$ demonstrated a clear evolution with time of the optical properties of the cadmium selenide nanocrystals produced by the reaction. The absorption onset - which was $550 \mathrm{~nm}$ after 0.3 minutes - was initially blue-shifted far from its bulk value of approximately $710 \mathrm{~nm},{ }^{14,24}$ but shifted progressively towards the values of the bulk exceeding $700 \mathrm{~nm}$ after approximately 2 hours of reaction. Clear absorption maxima were evident in the absorption spectra, and the lowest energy absorption maximum was attributed to the $1 S_{3 / 2}-1 S_{e}$ excitonic transition. These excitonic absorption maxima which are not distinguishable in the bulk material (see Figure 1-8 for transmittance spectrum of bulk cadmium selenide) - also shifted towards longer wavelengths with increasing reaction time. In addition to shifting towards longer wavelength, the absorption maxima broadened and became less distinct with increasing reaction time, such that after 4 hours the absorption spectrum had become significantly similar to that of the bulk material.

TEM observations were made of the average diameter and size distribution of cadmium selenide nanocrystals produced at various times throughout the reaction. The average diameter of the nanocrystals increased from 2.8 to $6.0 \mathrm{~nm}$ during the 4 hours the reaction was monitored. Accompanying the increase in average diameter with increasing reaction time, the size distribution of the nanocrystals broadened. Although the relative standard deviation in nanocrystal diameters did not increase during the reaction ( $14 \%$ after 0 minutes and $15 \%$ after 240 minutes), in absolute terms the standard deviation increased from 0.5 to $0.9 \mathrm{~nm}$ during the reaction. This matches the behaviour expected in the burst nucleation model of nanocrystal growth - the rapid nucleation step produces a relatively monodisperse ensemble of nanocrystals. The early increase in average diameter is rapid, from $2.8 \mathrm{~nm}$ after 0.3 minutes to $4.0 \mathrm{~nm}$ after 5 minutes. This is likely due to a combination of high initial supersaturation and geometrical considerations - the increase in volume being inversely proportional to the diameter when growth is controlled by the surface area. The rate of increase of the average diameter falls as the average diameter increases, and as the initial supersaturation drops the ensemble enters the so-called defocusing regime, where Ostwald ripening leads to a broadening of the size distribution. 
The central postulate in the area of semiconductor nanocrystals is that alteration of the optical properties is dependent on the size and size distribution of the nanocrystals, rather than on alteration in the chemistry of the nanocrystals. SAED measurements on samples taken after various times during the reaction demonstrated that the nanocrystals produced were consistently of the hexagonal wurtzite polymorph of cadmium selenide. This is in line with literature precedent that high temperature syntheses of cadmium selenide produce predominantly the wurtzite polymorph. ${ }^{4}$ EDS measurements indicated a consistent, cadmium-rich composition for all reaction times sampled after 0 minutes. The composition of the 0.3 minute sample was deemed uncertain due to the low signal from the very small nanocrystals. Both of these points support that the contention that the evolution of the optical properties of cadmium selenide nanocrystals with reaction time is due to alterations in the average diameter and size distribution of the nanocrystals, rather than due to some fundamental alteration of the crystal chemistry.

The initial blue-shift of the absorption onset of the $<3 \mathrm{~nm}$ cadmium selenide nanocrystals compared to that of the bulk, is hypothesised to be due to quantum confinement effects on the electronic structure of the nanocrystals. The subsequent red-shift of the positions of the absorption onset and excitonic absorption maxima with increasing reaction time is therefore determined to be due to the observed increase in average diameter during the reaction. Fluorescence from the cadmium selenide nanocrystals in this project was observed to be 'band-edge', rather than originating from trap states. Therefore the red-shift of fluorescent emission maxima with reaction time is also due to the increase in average diameter.

The broadening of the absorption maxima with increasing reaction time may be partially due to the broadening expected from the reduced quantum confinement in larger diameter nanocrystals, but the observed broadening in size distribution of the nanocrystals with reaction time would also lead to a broadening of the absorption features. The FWHM of fluorescence peaks from cadmium selenide nanocrystals has been claimed to be a good indirect indicator of the size distribution of the nanocrystals, as it has been found to be essentially independent of the average nanocrystal size. ${ }^{25}$ Hence the increase in the FWHM of the fluorescence peaks observed in this project (from $37 \mathrm{~nm}$ after 0.3 minutes to $53 \mathrm{~nm}$ after 240 minutes) correlates with the observed increase in the absolute standard deviation of nanocrystal diameter from 0.4 to $0.9 \mathrm{~nm}$ during the reaction. 
The correlations determined in this project between the value of the average nanocrystal diameter and optical parameters such as position of the $1 S_{3 / 2}-1 S_{e}$ absorption maximum and emission maximum are summarised below in Table 3-16. The data from this work are compared to data published by Qu and Peng, ${ }^{25}$ and are found to agree within the limits of uncertainty in the average diameter.

Table 3-16 Summary of average nanocrystal diameters and optical properties, from this work and from that of Qu and Peng. ${ }^{25}$

\begin{tabular}{|c|c|c|c|c|c|c|}
\hline \multicolumn{5}{|c|}{ This work } & \multicolumn{3}{c|}{ Qu and Peng } \\
\hline $\begin{array}{c}\text { Reaction } \\
\text { Time / } \\
\text { min }\end{array}$ & $\begin{array}{c}\text { Average } \\
\text { diameter } \\
/ \mathrm{nm}\end{array}$ & $\begin{array}{c}\lambda_{\max } \text { of } \\
1 S_{3 / 2}-1 S_{e} / \\
\mathrm{nm}\end{array}$ & $\begin{array}{c}\text { Fluorescence } \\
\lambda_{\max } / \mathrm{nm}\end{array}$ & $\begin{array}{c}\text { UV-vis } \\
\lambda_{\max } / \\
\mathrm{nm}\end{array}$ & $\begin{array}{c}\text { Fluorescence } \\
\lambda_{\max } / \mathrm{nm}\end{array}$ & $\begin{array}{c}\text { Average } \\
\text { diameter } / \\
\mathrm{nm}\end{array}$ \\
\hline 0.3 & $2.8 \pm 0.4$ & 500 & 514 & 516 & 530 & 2.4 \\
\hline 5 & $4.0 \pm 0.5$ & 585 & 603 & 579 & 593 & 3.6 \\
\hline 20 & $4.6 \pm 0.8$ & 605 & 625 & 606 & 614 & 4.6 \\
\hline 240 & $6.0 \pm 0.9$ & 645 & 670 & \multicolumn{3}{|c|}{ No data given } \\
\hline
\end{tabular}

\subsubsection{Quantum yield of cadmium selenide nanocrystals}

The quantum yield determination procedure described in Chapter 2 section 2.2.8 was used to assess the fluorescence quantum yield of a representative cadmium selenide nanocrystal sample. The procedure involved comparing the emission of a series of samples of matched absorbance, including two reference dyes of known quantum yield (coumarin 6 and fluorescein). The two dyes were first cross-referenced to one another, confirming that the procedure was valid. The quantum yield calculated for the cadmium selenide nanocrystal sample was exceedingly low - at $0.2 \%$. This is smaller than the demonstrated precision of the determination procedure - although fluorescence was definitely detected from the cadmium selenide nanocrystal samples, the magnitude of the quantum yield cannot be stated with confidence.

There are several possible reasons for the apparently low quantum yield. The sample whose quantum yield was assessed, was observed to produce weak trap state emission at $525 \mathrm{~nm}$, in addition to the stronger band-edge emission at $630 \mathrm{~nm}$ (Figure 3-13). This indicates that the surface of the nanocrystals was poorly passivated - which can lead to 
non-radiative relaxation pathways ${ }^{11}$ - in addition to radiative the trap state emission and hence reduce the fluorescence quantum yield.

It has been observed that the fluorescence quantum efficiency of an ensemble of growing cadmium selenide nanocrystals reaches a maximum - termed the bright spot. ${ }^{25}$ The point at which the bright spot is reached is dependent on the reaction conditions, and a 5 - 10 times excess of selenium precursor was found to yield cadmium selenide nanocrystals with the highest fluorescence quantum yields, which was postulated to be due to the attainment of ideal surface rearrangement. ${ }^{25}$ Without optimisation to obtain this bright point, the fluorescence quantum yield of cadmium selenide nanocrystals quoted in the literature for the range above $600 \mathrm{~nm}$ is as low as a few percent. ${ }^{25}$ This suggests that the two-fold excess of selenium used in this project was insufficient to produce cadmium selenide nanocrystals of optimum fluorescence quantum yield, and the quantum yield we measured was not atypical.

\subsubsection{Capping of cadmium selenide nanocrystals with zinc sulfide and capping ligand exchange}

\subsubsection{Capping of cadmium selenide nanocrystals with zinc sulfide}

An attempt was made to deposit a layer of zinc sulfide on the surface of pre-made cadmium selenide nanocrystals (see sections 3.5.4 and 3.2.4 ), in order to provide better passivation of the surface, and improve the fluorescence quantum yield. Cadmium selenide nanocrystals were dissolved in a solution of zinc stearate in TOPO, and a solution of S(TOP) was injected. It was difficult to conclude whether this procedure had been successful in depositing zinc sulfide on the cadmium selenide nanocrystals, or whether homogeneous nucleation of zinc sulfide had occurred. The $1 S_{3 / 2}-S_{e}$ absorption maximum of the cadmium selenide nanocrystals displayed a slight red-shift $(7 \mathrm{~nm})$ from its original position after the capping procedure. This could either be due to the influence of a zinc sulfide coating or simply due to further growth of the cadmium selenide nanocrystals during the procedure (i.e. Ostwald ripening). ${ }^{8}$

EDS measurements indicated that cadmium, selenium, zinc and sulphur were all present in the sample after the capping procedure, however the $\mathrm{Zn}: \mathrm{S}$ ratio (1:0.3) was far from the expected stoichiometric ratio. The observation of the low solubility of zinc stearate 
at low temperatures, and the precipitation of a white solid after the reaction indicates that unreacted zinc stearate may have remained in the sample. The poor resolution of the micrographs of the cadmium selenide nanocrystals taken before and after the capping procedure (Figure 3-15 a) and b), respectively) make it impossible to conclude from TEM imaging whether the average size of the nanocrystals had increased - as would be expected if capping had been successful. No electron diffraction could be obtained from the sample - though this could either be due to the small size of the nanocrystals or the absence of crystalline material.

A one-pot, two step procedure was also trialled in an attempt to produce $\mathrm{CdSe} / \mathrm{ZnS}$ nanocrystals (see sections 3.5.5 and 3.2.4). $\mathrm{Cd}(\mathrm{OAc})_{2}$ and $\mathrm{Se}(\mathrm{TOP})$ were reacted in TOPO at $300^{\circ} \mathrm{C}$, the reaction mixture was cooled and zinc stearate was added, the reaction mixture was then reheated and $\mathrm{S}(\mathrm{TOP})$ was injected at $240^{\circ} \mathrm{C}$. An aliquot of the cadmium selenide nanocrystals taken before the addition of the zinc stearate displays clear excitonic transitions in the absorption spectrum (Figure 3-17), which were slightly red-shifted $(9 \mathrm{~nm})$ after the second part of the procedure. Micrographs of the sample before and after the addition of zinc sulfide precursors (Figure 3-15 c) and d), respectively) did not indicate whether zinc sulfide had successfully been deposited or not; although it was evident that the size distribution of the cadmium selenide nanocrystals was far broader than in previous stand-alone cadmium selenide syntheses. Once again EDS indicated the presence of cadmium, selenium, zinc and sulfur; although neither the Cd:Se, nor the $\mathrm{Zn}: \mathrm{S}$ ratio were appropriate for stoichiometric material indicating that the one-pot procedure was not suitable for producing $\mathrm{CdSe} / \mathrm{ZnS}$ nanocrystals.

As neither attempt to cap cadmium selenide nanocrystals with zinc sulfide was demonstratively successful, no quantum yield determination procedure was performed to compare the fluorescence quantum yield of these samples to the that of the representative cadmium selenide nanocrystal sample described earlier (section 3.1.1.1).

\subsubsection{Capping ligand exchange}

Both cysteine and MPA were used to displace TOPO from as-synthesised cadmium selenide nanocrystals, in order to render them dispersible in polar media. The as-synthesised cadmium selenide nanocrystals could be precipitated with the aid of 
polar solvents - such as methanol - and once precipitated, the precipitate could only be resuspended in non-polar solvents, such as toluene. Both cysteine- and MPA-capped cadmium selenide nanocrystals were observed to precipitate only when treated with a non-polar solvent (diethylether), and once precipitated, the samples could be resuspended in either methanol or water. MPA-capped samples were observed to be resuspended more completely in water than cysteine-capped samples. The degree of solubility of the cadmium selenide nanocrystals after capping ligand exchange was only tested qualitatively, and no testing of long-term colloid stability was performed. 


\subsection{Conclusions}

Cadmium selenide nanocrystals were successfully synthesised following a modified literature procedure, involving reaction of $\mathrm{Cd}(\mathrm{OAc})_{2}$ and $\mathrm{Se}(\mathrm{TOP})$ in $\mathrm{TOPO}$ at $300^{\circ} \mathrm{C}$. A distinct evolution of the absorption and emission spectra of the cadmium selenide nanocrystals was observed with increasing reaction time. The absorption spectra of the cadmium selenide nanocrystals displayed two distinct absorption maxima - the lower energy maximum was assigned to the $1 S_{3 / 2}-1 S_{e}$ excitonic transition. The position of the absorption onset was initially blue-shifted far from that of bulk cadmium selenide, but red-shifted until it reached that of the bulk after 4 hours of reaction time. The initially sharp absorption maxima also red-shifted, and broadened to become less distinct with increasing reaction time. Band-edge fluorescence was observed from the nanocrystals, and a broadening of the emission peaks was observed with increasing reaction time.

The average diameter of cadmium selenide nanocrystals produced by the reaction immediately following nucleation was $2.8 \mathrm{~nm}$. The average diameter increased to 6.0 nm over the course of 4 hours, and although the relative standard deviation in nanocrystal diameters remained static over this period (at $\sim 15 \%$ ), the absolute standard deviation increased from 0.5 to $0.9 \mathrm{~nm}$. The increase in average diameter was correlated to the progressive red-shift of the optical absorption and emission features towards that of the bulk material, and the broadening of the size distribution was correlated with the broadening of the optical absorption and emission features. This conclusion is in line with current theory, and numerical values for the correlation between the average cadmium selenide nanocrystal diameter and the related position of optical absorption and emission features match within uncetainty those published in the literature.

The fluorescence quantum yield of a representative sample of cadmium selenide nanocrystals was determined by comparison to two standard fluorescent dyes. The calculated result was $0.2 \%$, which was so small that the accuracy of this figure could not be justified by the precision of the measurements. The very low fluorescence quantum yield of the cadmium selenide nanocrystals synthesised in this work was found to be typical for a non-optimised synthesis in the relevant spectral window.

The deposition of a layer of zinc sulfide onto cadmium selenide nanocrystals was attempted, in order to improve their optical properties. As neither of the approaches 
tried was found to have demonstrably produced stoichiometric zinc sulfide, the quantum yield of the resulting nanocrystals was not tested.

Cysteine and MPA were used to displace TOPO from as-synthesised cadmium selenide nanocrystals, in order to render the nanocrystals dispersible in polar media. Both cysteine-capped and MPA-capped cadmium selenide nanocrystals were soluble in polar media, however MPA-capped nanocrystals proved to be more fully soluble in water than cysteine-capped nanocrystals. 


\subsection{Method}

Cadmium acetate hydrate $\left(\mathrm{Cd}(\mathrm{OAC})_{2} . x \mathrm{H}_{2} \mathrm{O}, 99.99+\%\right)$, coumarin $6(\geq 99 \%)$, cysteine (>98\%), fluorescein (free acid, standard grade for fluorescence), mercaptopropionic acid (MPA, >99\%), selenium (100 mesh, 99.99\%), trioctylphosphine oxide (TOPO, tech. grade 90\%), and zinc stearate (tech. grade) were purchased from Sigma Aldrich, and used as received. Trioctylphosphine (TOP, 90\%) was purchased from Sigma Aldrich, and remained packed under nitrogen. Sulfur (sublimed), and tetramethylammonium hydroxide (TMAH, 25\% soln. in water) were purchased from $\mathrm{BDH}$.

A $1 \mathrm{M}$ stock solution of Se in TOP (Se(TOP)) was prepared by purging a flask containing Se $(0.79 \mathrm{~g}, 10 \mathrm{mmol})$ with nitrogen, and injecting TOP $\left(10 \mathrm{~cm}^{3}\right)$. This was stirred until the grey selenium powder dissolved to give a colourless solution, which was stored under nitrogen.

\subsubsection{Synthesis of cadmium selenide nanocrystals}

TOPO (30 g) was placed in a flask, and heated to $150^{\circ} \mathrm{C}$ to melt. This liquid was degassed by bubbling nitrogen through for $30 \mathrm{~min} . \mathrm{Cd}(\mathrm{OAc})_{2} . x \mathrm{H}_{2} \mathrm{O}(0.2730 \mathrm{~g}, 1 \mathrm{mmol})$ was added, and stirred to dissolve, producing a colourless solution. The solution was heated to $300^{\circ} \mathrm{C}$, and $\mathrm{Se}$ (TOP) $\left(3.5 \mathrm{~cm}^{3}\right.$ of $1 \mathrm{M}$ soln., containing $\left.3.5 \mathrm{mmol} \mathrm{Se}\right)$ was injected quickly. The solution immediately changed from colourless to yellow, then to orange and red over a period of two minutes. After approximately 20 minutes the solution became black. The temperature was maintained at $300^{\circ} \mathrm{C}$ for the duration of the reaction.

Aliquots $\left(\sim 1 \mathrm{~cm}^{3}\right)$ were withdrawn immediately after injection $(\sim 0.3 \mathrm{~min})$, and at 1,2 , $5,20,60,120,180,240$ mins; and injected into methanol $\left(10 \mathrm{~cm}^{3}\right)$ to quench. The reaction mixture was cooled to $50^{\circ} \mathrm{C}$, and methanol $\left(10 \mathrm{~cm}^{3}\right)$ was injected to prevent the TOPO solidifying.

Samples were centrifuged at $14,000 \mathrm{rpm}$ for $5 \mathrm{~min}$ to collapse the particle suspension. The clear supernatant was discarded, and the precipitate was resuspended in toluene. To this was added an equal volume to methanol to destabilise the suspension, and 
centrifugation was repeated. After $2-3$ cycles the nanocrystals were suspended in toluene and kept as a solution.

\subsubsection{Measurement of optical properties of cadmium selenide or $\mathrm{CdSe} / \mathrm{ZnS}$ nanocrystals.}

UV-visible spectra were recorded on cadmium selenide or CdSe/ZnS nanocrystal solutions by adding further toluene to the purified nanocrystal solutions (as prepared in 3.5.1 - 3.5.5) to produce a dilute solution. Spectra were recorded in matched $10 \mathrm{~mm}$ path-length quartz cuvettes in a double beam spectrometer versus a toluene blank. Fluorescence spectra were recorded on the diluted purified cadmium selenide or $\mathrm{CdSe} / \mathrm{ZnS}$ solutions in a four-sided $10 \mathrm{~mm}$ path-length fused silica cuvette. Typical parameters were: an excitation wavelength of $380 \mathrm{~nm}$, an excitation slit width of $5 \mathrm{~nm}$; an emission slit width of $20 \mathrm{~nm}$, and a $1 \%$ transmission filter on the emission side to prevent saturation of the detector.

\subsubsection{Quantum yield measurements}

3.5.3.1 Preparation of reference dye and cadmium selenide quantum dot solutions A wavelength of $460 \mathrm{~nm}$ was chosen for the excitation wavelength in the quantum yield measurements, as this wavelength was capable of exciting the cadmium selenide nanocrystal sample, and both of the reference dyes (fluorescein and coumarin 6). Consequently, dilution series of the cadmium selenide nanocrystal sample and both reference dyes were prepared, in order to obtain solutions with an absorbance - at 460 $\mathrm{nm}$ - ranging from approximately $0.2-1.0$.

\section{Fluorescein reference solutions}

Fluorescein $\left(0.0163 \mathrm{~g}, 4.91 \times 10^{-5} \mathrm{~mol}\right)$ was dissolved in $0.1 \mathrm{M} \mathrm{NaOH}$ soln. $\left(1 \mathrm{dm}^{3}\right)$. The resulting solution was diluted quantitatively with $0.1 \mathrm{M} \mathrm{NaOH}$ soln. to provide a series of solutions with the following concentrations of fluorescein: $4.9 \times 10^{-5}, 1.5 \times$ $10^{-5}, 7.5 \times 10^{-5}, 6.0 \times 10^{-6}, 4.5 \times 10^{-6}, 1.5 \times 10^{-6} \mathrm{~mol} \mathrm{dm}^{-3}$.

\section{Coumarin 6 reference solutions}

Coumarin $6\left(0.0032 \mathrm{~g}, 9.14 \times 10^{-6}\right)$ was dissolved in ethanol $\left(1 \mathrm{dm}^{3}\right)$. The resulting solution was diluted quantitatively with ethanol to provide a series of solutions with the following concentrations of coumarin $6: 2.3 \times 10^{-6}, 1.9 \times 10^{-6}, 1.5 \times 10^{-6}, 9.4 \times 10^{-7}$, and $4.7 \times 10^{-7} \mathrm{~mol} \mathrm{dm}^{-3}$. 


\section{Cadmium selenide nanocrystal solutions}

A concentrated soln. of cadmium selenide nanocrystals in toluene was diluted with toluene. After a UV-visible spectrum was recorded (see below), the sample was diluted with further toluene, and the process repeated to produce a series of samples with an approximate absorbance at $460 \mathrm{~nm}$ of $0.1,0.08,0.06,0.04$ and 0.02 .

3.5.3.2 Optical measurements performed on cadmium selenide nanocrystal and dye solutions

UV-visible spectra for reference dyes and cadmium selenide nanocrystal solutions were recorded in a $100 \mathrm{~mm}$ path-length cell against a blank of the relevant solvent. A $1 \mathrm{~nm}$ Spectral Band Width (SBW) was used.

Fluorescence spectra for reference dyes and cadmium selenide nanocrystal solutions were recorded in a $10 \mathrm{~mm}$, four-sided fused silica cuvette. Emission and excitation slit widths of $10 \mathrm{~nm}$ and $5 \mathrm{~nm}$, respectively, were used. No filters were used. The excitation wavelength for all samples was $460 \mathrm{~nm}$, and the resulting spectra were measured in the range $467-700 \mathrm{~nm}$.

\subsubsection{Capping of cadmium selenide nanocrystals with zinc sulfide}

Sulfur (0.1202 g, $3.8 \mathrm{mmol})$ was placed in a flask, which was then purged with nitrogen. TOP $\left(6 \mathrm{~cm}^{3}\right)$ was injected, and stirred to produce a colourless solution containing S(TOP). Zinc stearate $(0.7037 \mathrm{~g}, 1.1 \mathrm{mmol})$ was loaded into a flask with TOPO (7 g), heated to $150^{\circ} \mathrm{C}$ to melt the TOPO. Stirring dissolved the zinc stearate to produce a slightly yellow solution, which was degassed by bubbling nitrogen through for $30 \mathrm{~min}$. The solution was cooled to $60^{\circ} \mathrm{C}$, and a solution of $2 \mathrm{~nm}$ cadmium selenide nanocrystals (as prepared in section 3.5 .1$)\left(0.95 \mathrm{~cm}^{3}\right.$ of toluene solution containing approximately $2 \times 10^{-7} \mathrm{~mol}$ of nanocrystals) was added, and the resulting solution heated to $200^{\circ} \mathrm{C}$. S(TOP) solution $\left(2 \mathrm{~cm}^{3}\right.$ of $0.6 \mathrm{M}$ soln. containing $1.3 \mathrm{mmol}$ sulfur $)$ was injected, and the solution was maintained at $200^{\circ} \mathrm{C}$ for $2 \mathrm{hrs}$, cooled to $60^{\circ} \mathrm{C}$, whereupon methanol $\left(5 \mathrm{~cm}^{3}\right)$ was injected to prevent solidification. No change occurred in the colour of the cadmium selenide nanocrystal solution during the reaction as judged by eye.

The reaction mixture was filtered to remove solid zinc stearate, and methanol $\left(20 \mathrm{~cm}^{3}\right)$ was added to destabilise the suspension. After centrifugation the supernatant was 
discarded, and the precipitate washed twice with toluene. The resulting yellow toluene solution was retained, and the precipitate discarded.

\subsubsection{One-pot synthesis of CdSe/ZnS nanocrystals}

Sulfur $(0.20 \mathrm{~g}, 6.2 \mathrm{mmol})$ was placed in a flask, which was then purged with nitrogen. TOP $\left(6 \mathrm{~cm}^{3}\right)$ was injected, and stirred to produce a colourless solution. $\mathrm{Cd}(\mathrm{OAc})_{2} \cdot x \mathrm{H}_{2} \mathrm{O}$ $(0.1376 \mathrm{~g}, 0.52 \mathrm{mmol})$ was placed in a flask with TOPO $(14 \mathrm{~g})$, and heated to $150^{\circ} \mathrm{C}$ to melt the TOPO. Stirring dissolved the $\mathrm{Cd}(\mathrm{OAc})_{2} \cdot x \mathrm{H}_{2} \mathrm{O}$ to produce a colourless solution, which was degassed by bubbling nitrogen through for $30 \mathrm{~min}$. The solution was heated to $300^{\circ} \mathrm{C}$, and $\mathrm{Se}(\mathrm{TOP})$ solution $\left(2 \mathrm{~cm}^{3}\right.$ of $1 \mathrm{M}$ soln., containing $2 \mathrm{mmol}$ selenium) was injected quickly. The solution changed from colourless to red within 2 minutes. The solution was cooled to $250^{\circ} \mathrm{C}$ for $20 \mathrm{~min}$, and then cooled to $60^{\circ} \mathrm{C}$. An aliquot $\left(\sim 1 \mathrm{~cm}^{3}\right)$ was removed and quenched in toluene $\left(10 \mathrm{~cm}^{3}\right)$.

Zinc stearate (1.294 g, $2.0 \mathrm{mmol})$ was added to the cadmium selenide nanocrystal solution, which was stirred until the zinc stearate dissolved into the red solution. The solution was heated to $150^{\circ} \mathrm{C}$ and degassed by bubbling nitrogen through for $15 \mathrm{~min}$, then heated to $240^{\circ} \mathrm{C} . \mathrm{S}$ (TOP) solution $\left(2 \mathrm{~cm}^{3}\right.$ of $1 \mathrm{M}$ soln. containing $2 \mathrm{mmol}$ sulfur) was injected, and the solution maintained at $190^{\circ} \mathrm{C}$ for $1 \mathrm{hr}$, before being cooled to $60^{\circ} \mathrm{C}$, whereupon methanol $\left(10 \mathrm{~cm}^{3}\right)$ was injected to prevent solidification.

The reaction mixture was centrifuged to collapse the suspension, the supernatant was discarded, and the precipitate resuspended in toluene. Methanol was added to destabilise the suspension, and centrifugation at $14,000 \mathrm{rpm}$ repeated. After $2-3$ cycles the nanocrystals were suspended in toluene and kept as a solution.

\subsubsection{Capping ligand exchange reactions}

\subsubsection{Capping of cadmium selenide nanocrystals with cysteine}

Cysteine (0.0225 g, $0.9 \mathrm{mmol})$ was dissolved in methanol $\left(15 \mathrm{~cm}^{3}\right.$, HPLC grade) basified to $\mathrm{pH} 13$ with TMAH solution. A solution of cadmium selenide nanocrystals (as prepared in 3.5.4) $\left(0.4 \mathrm{~cm}^{3}\right.$ of toluene solution of unknown concentration) was added. Initially two layers formed: a colourless methanol layer, and a yellow toluene 
layer, but stirring produced a monophasic yellow solution. The flask was flushed with nitrogen, and the solution was refluxed overnight. The solution was reduced to $1 / 4$ of its original volume by distillation under reduced pressure. Aliquots of the reaction mixture were combined with polar and non-polar solvents, and centrifuged to test the solubility of the capping ligand exchanged nanocrystals (see section 3.2.5 for results).

3.5.6.2 Capping of cadmium selenide nanocrystals with mercaptopropionic acid (MPA)

MPA $\left(20 \mathrm{~mm}^{3}\right)$ was added to methanol $\left(15 \mathrm{~cm}^{3}\right.$, HPLC grade) basified to $\mathrm{pH} 12$ with TMAH solution. A solution of cadmium selenide nanocrystals (as prepared in 3.5.5) (1 $\mathrm{cm}^{3}$ of toluene solution of unknown concentration) was added. The resulting orange solution was initially cloudy, but became clear upon stirring. The flask was flushed with nitrogen, and the solution was refluxed overnight. The solution was reduced to $1 / 2$ of its original volume by distillation under reduced pressure. Aliquots of the reaction mixture were combined with polar and non-polar solvents, and centrifuged to test the solubility of the capping ligand exchanged nanocrystals (see section 3.2.5 for results). 


\subsection{References}

(1) R. Rossetti, J. L. Ellison, J. M. Gibson and L. E. Brus, J. Chem. Phys., 1984, 80, 4464.

(2) J. Warnock and D. D. Awschalom, Phys. Rev. B, 1985, 32, 5529.

(3) N. Chestnoy, R. Hull and L. E. Brus, J. Chem. Phys., 1986, 85, 2237.

(4) C. B. Murray, D. J. Norris and M. G. Bawendi, J. Am. Chem. Soc., 1993, 115, 8706.

(5) A. I. Ekimov, F. Hache, M. C. Schanne-Klein, D. Ricard, C. Flytznis., A. Kudryavtsev, T. V. Yazeva, A. V. Rodina and A. L. Efros, J. Opt. Soc. Am. B: Opt. Phys., 1993, 10, 100.

(6) W. C. W. Chan and S. Nie, Science, 1998, 281, 2016.

(7) L. Qu, Z. A. Peng and X. Peng, Nano Lett., 2001, 1, 333.

(8) M. A. Hines and P. Guyot-Sionnest, J. Phys. Chem. B, 1996, 100, 468.

(9) B. O. Dabbousi, J. Rodriguez-Viejo, F. V. Mikulec, J. R. Heine, H. Mattoussi, R. Ober, K. F. Jensen and M. G. Bawendi, J. Phys. Chem. B, 1997, 101, 9463.

(10) S. Sapra, A. L. Rogach and J. Feldmann, J. Mater. Chem., 2006, 16, 3391.

(11) M. G. Bawendi, M. L. Steigerwald and L. E. Brus, Annu. Rev. Phys. Chem., 1990, 41, 477.

(12) A. M. Derfus, W. C. W. Chan and S. N. Bhatia, Nano Lett., 2004, 4, 11.

(13) M. J. Bruchez, M. Moronne, P. Gin, S. Weiss and A. P. Alivisatos, Science, 1998, 281, 2013.

(14) C. Kittel, Introduction to Solid State Physics, John Wiley New York, 1986.

(15) N. N. Mamedova, N. Kotov, A. L. Rogach and J. Studer, Nano Lett., 2001, 1, 281.

(16) D. Gerion, F. Pinuad, S. C. Williams, W. J. Parak, D. Zanchet, S. Weiss and A P. Alivisatos, J. Phys. Chem. B, 2001, 105, 8861.

(17) S. M. Simon, J. K. Jaiswal, H. Mattoussi and J. M. Mauro, Nat. Biotechnol., 2003, 21, 47.

(18) J. Aldana, A. Wang and X. Peng, J. Am. Chem. Soc., 2001, 123, 8844.

(19) A. Hoshino, K. Fujioka, T. Oku, M. Suga, Y. F. Sasaki, T. Ohta, M. Yasuhara, K. Suzuki and K. Yamamoto, Nano Lett., 2004, 4, 2163.

(20) A. Reisman, M. Berkenblit and M. Witzen, J. Phys. Chem., 1962, 66, 2210.

(21) J. E. Bowen Katari, V. L. Colvin and A. P. Alivisatos, J. Phys. Chem., 1994, 98, 4109.

(22) J. Q. Umberger and V. K. LaMer, J. Am. Chem. Soc., 1945, 67, 1099.

(23) G. A. Reynolds and K. H. Drexhage, Opt. Commun., 1975, 13, 222.

(24) D. E. McCarthy, Appl. Opt., 1967, 6, 1896

(25) L. Qu and X. Peng, J. Am. Chem. Soc., 2002, 124, 2049. 


\section{Chapter 4 Synthesis of iron sulfide nanocrytals}

\subsection{Introduction}

In this chapter the iron sulfide phase system was targeted in synthetic studies, and the resulting nanocrystal samples were characterised by TEM microscopy, XRD and magnetic measurements. The motivation behind the work conducted in this chapter will be discussed in more detail in section 4.1.4, after more details of the iron sulfide phase system, and the current state of knowledge regarding ironorrish sulfide nanocrystals are discussed below.

Some of the basic chemical and physical properties of various iron sulfide phases were outlined in Chapter 1 section (1.3.2). The topic of superlattice structures in $\mathrm{Fe}_{1-x} \mathrm{~S}$ will be introduced more thoroughly in section 4.1 .1 of this chapter, in preparation for the discussion of the crystal structure of $\mathrm{Fe}_{1-x} \mathrm{~S}$ crystallites synthesised in this project (section 4.2.7). The formation and interconversion pathways that have been established in the literature for iron sulfide phases will be reviewed in section 4.1.2, in preparation for the discussion of the formation of iron sulfide nanocrystals in this project (section 4.3.1). Finally, syntheses of iron sulfide nanocrystals from the literature will be reviewed in section 4.1.3 to provide a point of comparison for the iron sulfide nanocrystals synthesised in this project.

\subsubsection{Superlattice structures of $\mathrm{Fe}_{1-x} \mathrm{~S}$}

Outside of the stability region of the $1 \mathrm{C}$ structure of $\mathrm{Fe}_{1-x} \mathrm{~S}$ (the base NiAs-type unit cell with iron vacancies distributed as a solid solution), there exist a variety of vacancy-ordered superlattice structures, where the presence of regularly spaced iron vacancies expands the unit cell from the dimensions of the NiAs-type subcell $(A \approx 3.4440 \mathrm{C} \approx 5.8760 \AA)$ by some multiple. This enlarged supercell may retain the crystal symmetry of the parent structure (hexagonal system, space group $P 6_{3} m c$ ), or not

- leading to the fundamental division of the $\mathrm{Fe}_{1-x} \mathrm{~S}$ group into hexagonal and monoclinic regions (with compositions $0 \geq x>0.024$ and $0.024>x \geq 0.125$, respectively). ${ }^{1}$ The superlattice is generally identified by the factors relating the lattice parameters of the supercell to those of the subcell - for example the aforementioned pure NiAs-type structure is referred to as $1 \mathrm{C}$, as $a=1 \mathrm{~A}$ and $c=1 \mathrm{C}$, where lowercase letters refer to the 
lattice parameters of the supercell, and uppercase letters refer to the lattice parameters of the NiAs-type subcell.

The superstrutures of $\mathrm{Fe}_{1-x} \mathrm{~S}$, which are stable at room temperature and ambient pressure are mentioned below: $:^{2,3}$

- The stoichiometric end member of the $\mathrm{Fe}_{1-x} \mathrm{~S}$ series bears the mineral name troilite, and possesses the $2 \mathrm{C}$ structure $(a=\sqrt{3} \mathrm{~A}, c=2 \mathrm{C})$.

- Superstructures bearing at least pseudohexagonal symmetry are $6 \mathrm{C}\left(\mathrm{Fe}_{11} \mathrm{~S}_{12}, a=\right.$ $2 \mathrm{~A}, c=6 \mathrm{C}) ; 11 \mathrm{C}\left(\mathrm{Fe}_{10} \mathrm{~S}_{11}, a=2 \mathrm{~A}, c=11 \mathrm{C}\right)$; and 5C (Fe $\left.9 \mathrm{~S}_{10}, a=2 \mathrm{~A}, c=5 \mathrm{C}\right)$.

- The commonly cited monoclinic superstructure of $\mathrm{Fe}_{1-x} \mathrm{~S}$ is $4 \mathrm{C}\left(\mathrm{Fe}_{7} \mathrm{~S}_{8}, a=2 \mathrm{~A}, b\right.$ $=2 \sqrt{ } 3 \mathrm{~A} c=4 \mathrm{C})$.

In addition to the stable superstructures mentioned above, metastable superstructures, with non-integer values of the factors relating the supercell to subcell, have been obtained by quenching. ${ }^{2}$ These include the NC, NA and MC types:

- For the NC type, $a=2 \mathrm{~A}$ and $c=\mathrm{NC}(6.0>\mathrm{N}>3.0)$. Hence the aforementioned hexagonal superstructures: $5 \mathrm{C}, 6 \mathrm{C}$ and $11 \mathrm{C}$ are special cases of the $\mathrm{NC}$ type.

- For the NA type, $a=\mathrm{NA}$ and $c=3 \mathrm{C}(90>\mathrm{N}>40)$.

- For the MC type, $a=2 \mathrm{~A}$ and $c=\mathrm{MC}(4.0>\mathrm{M}>3.0)$.

Mechanisms by which the electronic neutrality of $\mathrm{Fe}_{1-x} \mathrm{~S}$ could be maintained include: formation of two localised $\mathrm{Fe}^{3+}$ ions for each $\mathrm{Fe}^{2+}$ vacancy; time-averaged non-integral charges on the iron ions; ${ }^{4}$ or formation of disulfide ions $\left(\mathrm{S}_{2}{ }^{2-}\right)$ within the structure. Support is given to the second possibility by the fact that $\mathrm{Fe}^{3+}$ was not observed in Mössbauer spectra of samples of $4 \mathrm{C} \mathrm{Fe}_{7} \mathrm{~S}_{8}$, indicating that $\mathrm{Fe}^{3+}$ cannot have a separate existence for longer than the period of the Mössbauer transition $\left(\sim 10^{-7} \mathrm{~s}\right){ }^{5}$ Fasiska $^{4}$ investigated this point while refining crystal structures of $\mathrm{Fe}_{1-x} \mathrm{~S}$, and found that the atomic radius of iron in $\mathrm{Fe}_{1-x} \mathrm{~S}$ fell between that expected for $\mathrm{Fe}^{2+}$ and $\mathrm{Fe}^{3+}(0.76$ and $0.64 \AA$, respectively). The average radius of iron varied with the composition of the phase, from $0.75 \AA$ in stoichiometric $\mathrm{FeS}$ (which matches the accepted figure for $\mathrm{Fe}^{2+}$ ) to $0.68 \AA$ in $\mathrm{Fe}_{7} \mathrm{~S}_{8}$. Thus supporting the hypothesis that in iron-deficient $\mathrm{Fe}_{1-x} \mathrm{~S}$ electronic neutrality is maintained by time-averaged non-integer charges on the cation. 
NiAs-type $\mathrm{Fe}_{1-x} \mathrm{~S}$ is noted for a number of magnetic ordering transitions. The base structure possesses antiferromagnetic ordering, with the magnetic moments of the iron atoms aligned parallel within the basal $(a b)$ plain, and antiparallel between adjacent layers. Below the $\alpha$-transition temperature $\left(\mathrm{T}_{\alpha}, 140-40^{\circ} \mathrm{C}\right.$ for $\left.50.0-48.0 \mathrm{at} \% \mathrm{Fe}\right){ }^{6}$ the antiferromagnetically coupled moments are aligned parallel to the $c$ axis, and move to lie within the basal plane above $\mathrm{T}_{\alpha}{ }^{7}$ The magnetic spin flip transition is coincident with transition from some vacancy-ordered superstructure below $\mathrm{T}_{\alpha}$ to a MnP-type structure. All NiAs-type phases show a relatively composition-independent transition $\left(\mathrm{T}_{\beta}\right)$ at $\sim 315^{\circ} \mathrm{C}$ to an apparent paramagnetic state - hence this is associated with the Néel temperature of the phase. ${ }^{6,8}$ The $\beta$-transition is accompanied by a first-order transition to the $1 \mathrm{C}$ solid solution structure. ${ }^{9}$

There is a trend towards higher susceptibilities with decreasing iron-content in $\mathrm{Fe}_{1-x} \mathrm{~S}$, as the introduction of iron vacancies leads to mismatch of the magnetic moments of filled and vacancy-containing layers. ${ }^{6}$ This trend is punctuated with decreases in the net magnetic moment for the $6 \mathrm{C}, 11 \mathrm{C}$ and $5 \mathrm{C}$ superlattices, which are antiferromagnetic due to the ordering of vacancy containing layers along the $c$ axis. ${ }^{9}$ In the intermediate composition range (47.0-48.0at \% Fe) a discontinuity in the susceptibility occurs at the so-called $\gamma$-transition. Above the transition temperature, $\mathrm{T}_{\gamma},\left(\sim 150-210^{\circ} \mathrm{C}\right.$ for $47.0-$ $48.0 \mathrm{at} \% \mathrm{Fe}$, respectively $)^{6}$ there is a marked increase in susceptibility, which is believed to originate in a randomisation in the distribution of iron vacancies.

\subsubsection{Formation and interconversion of iron sulfide phases}

The $\mathrm{FeS}_{m}-\mathrm{Fe}_{3} \mathrm{~S}_{4}-\mathrm{FeS}_{2}$ p pathway

As was mentioned in Chapter 1 section 1.3.2.4, FeSm is the initially precipitated iron sulfide phase in aqueous systems. Conversion of $\mathrm{FeS}_{\mathrm{m}}$ or $\mathrm{Fe}_{3} \mathrm{~S}_{4}$ to $\mathrm{FeS}_{2} \mathrm{p}$ in aqueous solution was previously thought to be solely via a dissolution process, however recent in situ synchrotron diffraction experiments on hydrothermally formed samples, indicated that $\mathrm{FeS}_{\mathrm{m}}$ is converted to $\mathrm{Fe}_{3} \mathrm{~S}_{4}$, as an intermediate, and thence to $\mathrm{FeS}_{2} \mathrm{p}$ in the solid state. ${ }^{10}$ The conversion of $\mathrm{FeS}_{\mathrm{m}}$ to $\mathrm{Fe}_{3} \mathrm{~S}_{4}$, as shown below in Scheme 4-1, requires the oxidation of two equivalents of $\mathrm{Fe}^{2+}$ to $\mathrm{Fe}^{3+}$, and the loss of one equivalent of $\mathrm{Fe}^{2+}$ (loss of iron, rather than gain of sulfur is the confirmed mechanism). Oxidation was hypothesised to occur at the surface via absorbed oxidants $\left(\mathrm{O}_{2}\right.$, polysulfides $)$, with 
migration of $\mathrm{Fe}^{3+}$ into the lattice and excess $\mathrm{Fe}^{2+}$ into solution. The following conversion of $\mathrm{Fe}_{3} \mathrm{~S}_{4}$ to $\mathrm{FeS}_{2} \mathrm{p}$ involves the further loss of one equivalent of $\mathrm{Fe}^{2+}$, reduction of two equivalents of $\mathrm{Fe}^{3+}$ to $\mathrm{Fe}^{2+}$, and oxidation of $\mathrm{S}^{2-}$ to $\mathrm{S}_{2}{ }^{2-}$. Again oxidation was hypothesised to occur at the surface via absorbed oxidants, while reduction of iron and cation migration occurred in the solid. ${ }^{10}$

$4 \mathrm{FeS} \stackrel{-2 \mathrm{e},-\mathrm{Fe}^{2+}}{\longrightarrow} \mathrm{Fe}_{3} S_{4} \stackrel{-2 \mathrm{e},-\mathrm{Fe}^{2+}}{\longrightarrow} 2 \mathrm{FeS}_{2} \mathrm{p}$

Scheme 4-1

Conversion of $\mathrm{FeS}_{m}, \mathrm{Fe}_{3} \mathrm{~S}_{4}, \mathrm{FeS}_{2} \mathrm{p} / \mathrm{m}, \mathrm{Fe}_{9} \mathrm{~S}_{11}$ to $\mathrm{Fe}_{1-\mathrm{x}} \mathrm{S}$.

If heated to a sufficiently high temperature, $\mathrm{FeS}_{\mathrm{m}}, \mathrm{Fe}_{3} \mathrm{~S}_{4}, \mathrm{FeS}_{2} \mathrm{p} / \mathrm{m}$ and $\mathrm{Fe}_{9} \mathrm{~S}_{11}$ will eventually convert to hexagonal $\mathrm{Fe}_{1-x} \mathrm{~S}$. As mentioned above, conversion of tetragonal $\mathrm{FeS}_{\mathrm{m}}$ to cubic close-packed $\mathrm{Fe}_{3} \mathrm{~S}_{4}$ has been demonstrated under hydrothermal conditions at temperatures between 100 and $200^{\circ} \mathrm{C}$. Subsequent conversion to either $\mathrm{FeS}_{2} \mathrm{p}^{10}$ or $\mathrm{Fe}_{1-x} \mathrm{~S}^{11}$ has been observed, with the conditions distinguishing between the two pathways unclear. Like the conversion of $\mathrm{Fe}_{3} \mathrm{~S}_{4}$ to $\mathrm{FeS}_{2} \mathrm{p}$ in the $\mathrm{FeS}_{\mathrm{m}}-\mathrm{Fe}_{3} \mathrm{~S}_{4}-\mathrm{FeS}_{2} \mathrm{p}$ pathway, conversion of $\mathrm{Fe}_{3} \mathrm{~S}_{4}$ to $\mathrm{Fe}_{1-x} \mathrm{~S}$ is believed to occur in the solid state, rather than by a dissolution process. ${ }^{11}$

$\mathrm{FeS}_{2} \mathrm{p}$ and $\mathrm{FeS}_{2} \mathrm{~m}$ can both be converted to $\mathrm{Fe}_{1-x} \mathrm{~S}$ in a solid-state process, by heating under vacuum or a range of atmospheres $\left(\mathrm{He}, \mathrm{H}_{2}, \mathrm{CO}\right)$ above $350-400^{\circ} \mathrm{C}$, accompanied by loss of sulfur, ${ }^{12}$ whereas $\mathrm{Fe}_{9} \mathrm{~S}_{11}$ has been noted to decompose to $\mathrm{FeS}_{2} \mathrm{p}$ and $\mathrm{Fe}_{1-x} \mathrm{~S}$ upon heating. ${ }^{13}$

\subsubsection{Iron sulfide nanocrystals}

Although many natural or synthetic samples of various iron sulfide phases have been found to have small crystallite sizes, there has been relatively little work directed at deliberately controlling particle sizes during synthesis, or characterising particle sizes and morphologies. Those studies where there has been a deliberate effort to control the particle size, or the size/morphology of crystals has been found to significantly affect the properties, are discussed below:

\subsubsection{1 $\mathrm{FeS}_{2} \mathrm{p}$ nanocrystals}

Spherical $\mathrm{FeS}_{2}$ p nanocrystals, $2-8 \mathrm{~nm}$ in diameter, were synthesised from reaction of an iron halide salt and sodium sulfide in inverse micelles. ${ }^{14}$ The particles displayed sharp excitonic bands in the UV-vis spectrum, as well as a size-dependent absorption 
onset ( $\sim 600$ and $\sim 790 \mathrm{~nm}$ for 2 and $3.5 \mathrm{~nm}$ particles, respectively), indicative of quantum confinement. The band gap remained indirect at all particle sizes.

Chaudhuri et al. ${ }^{15}$ and Qian et al. ${ }^{16}$ both reported the synthesis of $\mathrm{FeS}_{2} \mathrm{p}$ nanowires from iron nitrate and sodium sulfide in ethylenediamine under solvothermal conditions. As the average diameter $(60-80 \mathrm{~nm})$ is much greater than the Bohr radius in $\mathrm{FeS}_{2} \mathrm{p}(\sim 1.3$ $\mathrm{nm}),{ }^{14}$ quantum confinement is not anticipated. Further characterisation of the materials was not presented in the paper.

$20-30 \mathrm{~nm} \mathrm{FeS}_{2} \mathrm{p}$ particles were formed by high energy ball milling of bulk $\mathrm{FeS}_{2} \mathrm{p}$ or iron and sulfur for $48-72$ hours, and the resulting particles had an irregular morphology. Mössbauer spectroscopy showed no alteration in the magnetic structure of the $20-30 \mathrm{~nm} \mathrm{FeS}_{2} \mathrm{p}$ nanocrystals compared to the bulk material. ${ }^{17}$

\subsubsection{2 $\quad \mathrm{Fe}_{3} \mathrm{~S}_{4}$ nanocrystals}

Naturally occurring $\mathrm{Fe}_{3} \mathrm{~S}_{4}$ has been noted for its relatively small grain size, ${ }^{18}$ as have synthetic $\mathrm{Fe}_{3} \mathrm{~S}_{4}$ samples, whose grain size is often even smaller than natural samples, ranging from $<150$ to $400 \mathrm{~nm} .{ }^{19}$ Small average particle size $(9-14 \mathrm{~nm})$ was noted by Spender $e t a l .{ }^{20}$ for $\mathrm{Fe}_{3} \mathrm{~S}_{4}$ synthesised by hydrothermal methodology from $\left(\mathrm{NH}_{4}\right)_{2} \mathrm{Fe}\left(\mathrm{SO}_{4}\right)_{2}$ and $\mathrm{Na}_{2} \mathrm{~S}$. This was attributed as the reason for a superparamagnetic component in magnetic measurements. Average particle sizes were judged from Scherrer X-ray broadening, and no information on particle morphology was presented.

Recent syntheses of $\mathrm{Fe}_{3} \mathrm{~S}_{4}$ have employed either a hydrothermal ${ }^{21}$ or solvothermal (toluene $)^{22}$ process, producing highly aggregated acicular crystals and irregularly shaped $\mathrm{Fe}_{3} \mathrm{~S}_{4}$ particles with an average diameter of $30 \mathrm{~nm}$, respectively. A hydrothermal synthesis conducted in an applied magnetic field produced coherently aggregated $\mathrm{Fe}_{3} \mathrm{~S}_{4}$ microrods, among a variety of other morphologies. ${ }^{23}$ Intriguingly, $50 \mathrm{~nm}$ particles with a core of $\mathrm{FeS}_{2} \mathrm{p}$ and a shell of $\mathrm{Fe}_{3} \mathrm{~S}_{4}$ were reported as the product of a hydrothermal reaction by Yamaguchi and $\mathrm{Wada}^{24}$ in 1970 - therefore possibly representing one of the first published syntheses of what would today be referred to as core-shell nanocrystals. The only currently published synthesis of small, well-dispersed spherical $\mathrm{Fe}_{3} \mathrm{~S}_{4}$ nanocrystals is from the decomposition of the single-source precursor bis(tetra- $n$ butylammonium) tetrakis[benezenethiolato- $\mu_{3}$-sulfidoiron] $\left(\left[\mathrm{NBu}_{4}\right]_{2}\left[\mathrm{Fe}_{4} \mathrm{~S}_{4}(\mathrm{SPh})_{4}\right]\right)$ at $200^{\circ} \mathrm{C}$ in dodecylamine for $12-16$ hours. ${ }^{25}$ 


\subsubsection{3 $\quad \mathrm{Fe}_{1-x} \mathrm{~S}$ nanocrystals}

$\mathrm{Fe}_{1-x} \mathrm{~S}$ nanowires were produced by the annealing of a FeS-ethylenediamine complex at $200-300^{\circ} \mathrm{C},{ }^{26}$ and large, highly faceted $\mathrm{Fe}_{1-x} \mathrm{~S}$ crystallites were synthesised by the decomposition of Fe(Ddtc) $)_{2}(\mathrm{Phen})$ and $\mathrm{Fe}(\mathrm{Ddtc})_{3}$ (Ddtc = diethyldithiocarbamate; Phen $=1,10$-phenanthroline) single source precursors in oleylamine at $260-320^{\circ} \mathrm{C} .{ }^{27}$ The most recent synthesis of $\mathrm{Fe}_{1-x} \mathrm{~S}$ nanocrystals (more specifically $\mathrm{Fe}_{7} \mathrm{~S}_{8}$ ) is from the decomposition of the single-source precursor $\left[\mathrm{NBu}_{4}\right]_{2}\left[\mathrm{Fe}_{4} \mathrm{~S}_{4}(\mathrm{SPh})_{4}\right]$ in octylamine at $180^{\circ} \mathrm{C}$ for $12-16$ hours, which reportedly produced spherical $6 \mathrm{~nm} 4 \mathrm{C} \mathrm{Fe}_{7} \mathrm{~S}_{8}$ nanocrystals. ${ }^{25}$ This last synthesis is notable as the only report so far of $\mathrm{Fe}_{1-x} \mathrm{~S}$ nanocrystals in such a small size regime.

\subsubsection{Motivation}

Given that the iron sulfide phase system presents such a variety of compositions, crystal structures, electronic and magnetic properties - the system presents an attractive, if complex target for synthetic studies. Syntheses of iron sulfide nanocrystals are currently less developed than for many other metal chalcogenide systems - notably iron oxide although the rate of publication has picked up in recent years (see above).

Substantial amounts of work have been conducted on the synthesis of iron sulfide phases by the use of hydrothermal or solvothermal methods. ${ }^{15,16,22}$ Traditionally the target has been the reproduction or simulation of geological processes in order to gain insight into the formation of iron sulfide phases in nature. ${ }^{28}$ More recently the investigations have deliberately aimed to produce uniform iron sulfide nanocrystals of small size.

Our point of departure in this project was to use an ambient pressure, bench-top experimental set-up instead of a sealed bomb, as we felt that this would enable more experimental control and would lead to more uniform nanocrystal samples. We used the high-boiling point, coordinating alkylamine solvents (oleylamine or hexadecylamine (HDA)) in place of the water or toluene solutions favoured in previous studies, as this allowed us to achieve the high temperature necessary to produce crystallite iron sulfide nanocrystals at ambient pressure. 
The metalorganic coordination complex iron(II) acetylacetonate ( $\left.\mathrm{Fe}(\mathrm{acac})_{2}\right)$ was the initial choice for iron source, as metal acetylacetonate salts have proven records as precursors for inorganic metal phases, decomposing cleanly to give volatile organic byproducts. ${ }^{29}$ Previous syntheses of iron sulfide nanocrystals had relied on polar inorganic iron salts, such as $\mathrm{FeSO}_{4}$, and sulfide salts such as $\mathrm{Na}_{2} \mathrm{~S}^{(16)}$. However, given the moderately non-polar nature of our solvents, and the high solubility and reactivity of elemental sulfur in amine solvents ${ }^{30,31}$ we decided to use elemental sulfur as the sulfur source in all reactions.

As exploratory reactions of $\mathrm{Fe}(\mathrm{acac})_{2}$ with elemental sulfur proved successful in affording iron sulfide phases, we proceeded to examine the effect of temperature, reaction time, concentration of precursors, method of combination of reactants, and the Fe:S ratio of the precursors on the resulting iron sulfide nanocrystals. 


\subsection{Results}

As many of these reactions produced a mixture of iron sulfide phases - typically $\mathrm{Fe}_{1-x} \mathrm{~S}$ and $\mathrm{Fe}_{3} \mathrm{~S}_{4}$ - firstly the attempts to quantification of phase mixtures of nanocrystals by XRD will be discussed in section 4.2.1. Then the results of reactions to produce iron sulfide nanocrystals will be presented in sections 4.2.2- 4.2.6. Finally, the crystal structure of the $\mathrm{Fe}_{1-x} \mathrm{~S}$ crystallites produced in this chapter will be discussed (section 4.2.7), and the results of magnetic measurements conducted on a sample of pure $\mathrm{Fe}_{1-x} \mathrm{~S}$ (section 4.2.8.1) and mixture of $\mathrm{Fe}_{1-x} \mathrm{~S}$ and $\mathrm{Fe}_{3} \mathrm{~S}_{4}$ of varying composition and crystal morphology (section 4.2.8.2) will be presented.

Reactions where $\mathrm{Fe}(\mathrm{acac})_{2}$ and elemental sulfur were combined in oleylamine at room temperature, then heated to a certain reaction temperature are presented in section 4.2.2. These reactions are further subdivided into a number of categories, based upon their results. The conditions under which the iron sulfide nanocrystals were nucleated where controlled in a number of experiments using the 'hot injection' method, whereby either a sulfur solution was injected into a preheated iron-containing solution (sections 4.2.3 and 4.2.4), or the iron precursor was added to a preheated sulfur solution (section 4.2.6). The reaction of $\mathrm{Fe}(\mathrm{acac})_{2}$ and elemental sulfur in HDA is presented in section 4.2.5.

\subsubsection{Quantification of iron sulfide nanocrystal phases by XRD}

Two methods for the quantification of phase mixtures by XRD were outlined in Chapter 2 section 2.2.2.4-5: the semiquantitative method and the Rietveld method. Both of these were trialled for the quantification of phases in mixtures of iron sulfide nanocrystals. However neither method was found to give reliable results.

The semiquantitative method relies on RIR (Relative Intensity Ratios) values for each phase in the mixture to be analysed, in order to avoid the need for explicitly measured mass absorption coefficients, or a calibration curve constructed of known mixtures of the phases. ${ }^{32}$ Published RIR values were available in the PDF database for $\mathrm{Fe}_{1-x} \mathrm{~S}$ and $\mathrm{Fe}_{3} \mathrm{~S}_{4}$, however several values were available for each phase, and these were spread over a significant range. As there was no consensus on what the 'correct' RIR value was for each phase, it was decided to eschew the use of semiquantitative analysis, as the results would be strongly dependent on the RIR values chosen. 
It was attempted to measure RIR values for $\mathrm{Fe}_{1-x} \mathrm{~S}$ and $\mathrm{Fe}_{3} \mathrm{~S}_{4}$ experimentally in order to circumvent the unreliability of the published values. However, although pure samples of $\mathrm{Fe}_{1-x} \mathrm{~S}$ could be prepared (Samples VIII - X, section 4.2.2.4), samples of $\mathrm{Fe}_{3} \mathrm{~S}_{4}$ produced in this chapter were either contaminated with $\mathrm{Fe}_{1-x} \mathrm{~S}$, or size-related peak broadening rendered the samples unsuitable for use as standards. This inability to prepare pure $\mathrm{Fe}_{3} \mathrm{~S}_{4}$ also prevented the construction of a calibration curve of known mixtures of $\mathrm{Fe}_{1-x} \mathrm{~S}$ and $\mathrm{Fe}_{3} \mathrm{~S}_{4}$.

The Rietveld method was investigated for the quantification of mixtures of $\mathrm{Fe}_{3} \mathrm{~S}_{4}$ and $\mathrm{Fe}_{1-x} \mathrm{~S}$ nanocrystals. As this method is based upon the ab initio generation of simulated profiles, which are refined to match the experimental profile, it was hoped that the need for pure samples of the two phases would be avoided - as the necessary crystal structure parameters were readily available in the PDF database. The Rietica software package was used to perform refinements on samples synthesised in this chapter. Although a satisfactory convergence was achieved for a sample of pure $\mathrm{Fe}_{1-x} \mathrm{~S}$ with minimal size-related peak broadening, satisfactory refinements could not be achieved for samples containing $\mathrm{Fe}_{3} \mathrm{~S}_{4}$.

This is believed to be due to a number of sample-related factors. While in most cases the size-related peak broadening in $\mathrm{Fe}_{3} \mathrm{~S}_{4}$ was believed to be isotropic, as the observed morphology of the nanocrystals was spherical, in one case $\mathrm{Fe}_{3} \mathrm{~S}_{4}$ was observed to have a sheet-like morphology (Sample VII, section 4.2.2.3). This sample represented the purest sample of $\mathrm{Fe}_{3} \mathrm{~S}_{4}$ produced, however the crystallite morphology led to serious preferred orientation and anisotropic peak broadening effects, which could not be modelled adequately. In other samples containing $\mathrm{Fe}_{3} \mathrm{~S}_{4}$, the diffraction patterns were either broadened too greatly for a satisfactory fit to be obtained, or the contribution of amorphous material to the diffraction pattern background made it impossible to simultaneously match the relative intensities of $\mathrm{Fe}_{1-x} \mathrm{~S}$ and $\mathrm{Fe}_{3} \mathrm{~S}_{4}$ reflections - a requirement for confidence to be placed in the result of quantification by the Rietveld method.

As neither the semiquantitative, nor the Rietveld method succeeded in providing reliable quantification of phase mixtures in this case, XRD in this project was used predominantly qualitatively. Ideally, the ratio of the intensity of a reflection from one phase to the intensity of a reflection from another phase is proportional to the relative 
abundance of the two phases. ${ }^{33}$ Therefore the intensity ratio of the 400 reflection of $\mathrm{Fe}_{3} \mathrm{~S}_{4}$ and the 102 reflection of $\mathrm{Fe}_{1-x} \mathrm{~S}$ (denoted $\mathrm{I}\left(\mathrm{Fe}_{3} \mathrm{~S}_{4}\right)(400): \mathrm{I}\left(\mathrm{Fe}_{1-x} \mathrm{~S}\right)(102)$, or abbreviated to $\mathrm{I}_{400}: \mathrm{I}_{102}$ ) was used to give a rough indication of the relative abundances of the two phases in a mixture, rather than an absolute measure. Caveats include that size-related peak broadening reduces peak intensity, and therefore also affects peak intensity ratios. 


\subsubsection{Reaction of $\mathrm{Fe}(\mathrm{acac})_{2}$ and sulfur in oleylamine}

Reactions of $\mathrm{Fe}(\mathrm{acac})_{2}$ and sulfur were undertaken as detailed in section 4.5.1, varying the ratio of $\mathrm{Fe}(\mathrm{acac})_{2}$ to sulfur between 1:1 and 1:2.75, varying the concentration of the precursors between 0.01 and $0.1 \mathrm{~mol} \mathrm{dm}^{-3}$, varying the reaction temperature between 140 and $330^{\circ} \mathrm{C}$, and varying the duration of reaction between 30 and 240 minutes. The results of these experiments divide into four regimes, based upon their results, as detailed below:

\subsubsection{Reactions at temperatures below $200^{\circ} \mathrm{C}$}

Experimental parameters for the reaction of $\mathrm{Fe}(\mathrm{acac})_{2}$ and sulfur in oleylamine below $200^{\circ} \mathrm{C}$ are tabulated below (Table $\left.4-1\right)$. The ratio of $\mathrm{Fe}(\mathrm{acac})_{2}: \mathrm{S}$ was varied between 1:1 and $1: 2$ to ascertain whether this ratio affected the phase produced.

A TEM micrograph typical of Sample I and II is shown below (Figure 4-1 (a)). The samples consisted of very large stacks of thin sheets, with poorly defined, irregular edges. An SAED pattern of one such aggregate is shown in Figure 4-1 (b). The pattern contains three discernable, broad rings superimposed on a diffuse background indicating that the material is only poorly crystalline.

Table 4-1 Experimental parameters for the reaction of $\mathrm{Fe}(\mathrm{acac})_{2}$ and sulfur in oleylamine below $200^{\circ} \mathrm{C}$.

\begin{tabular}{|c|c|c|c|c|c|}
\hline Sample & $\mathrm{Fe}(\mathrm{acac})_{2}: \mathrm{S}$ & {$\left[\mathrm{Fe}(\mathrm{acac})_{2}\right]$} & $\begin{array}{c}\text { Reaction } \\
\text { Temp. } \\
/{ }^{\circ} \mathrm{C}\end{array}$ & $\begin{array}{c}\text { Reaction } \\
\text { Time } \\
/ \mathrm{min}\end{array}$ & Result \\
\hline I & $1: 1$ & 0.1 & 140 & 60 & $\begin{array}{c}\text { Poorly crystalline } \\
\text { iron sulfide/iron } \\
\text { oxide with unreact. } \\
\text { sulfur }\end{array}$ \\
\hline II & $1: 2$ & 0.1 & 140 & 60 & $\begin{array}{c}\text { Poorly crystalline } \\
\text { iron sulfide/iron } \\
\text { oxide with unreact. } \\
\text { sulfur }\end{array}$ \\
\hline
\end{tabular}




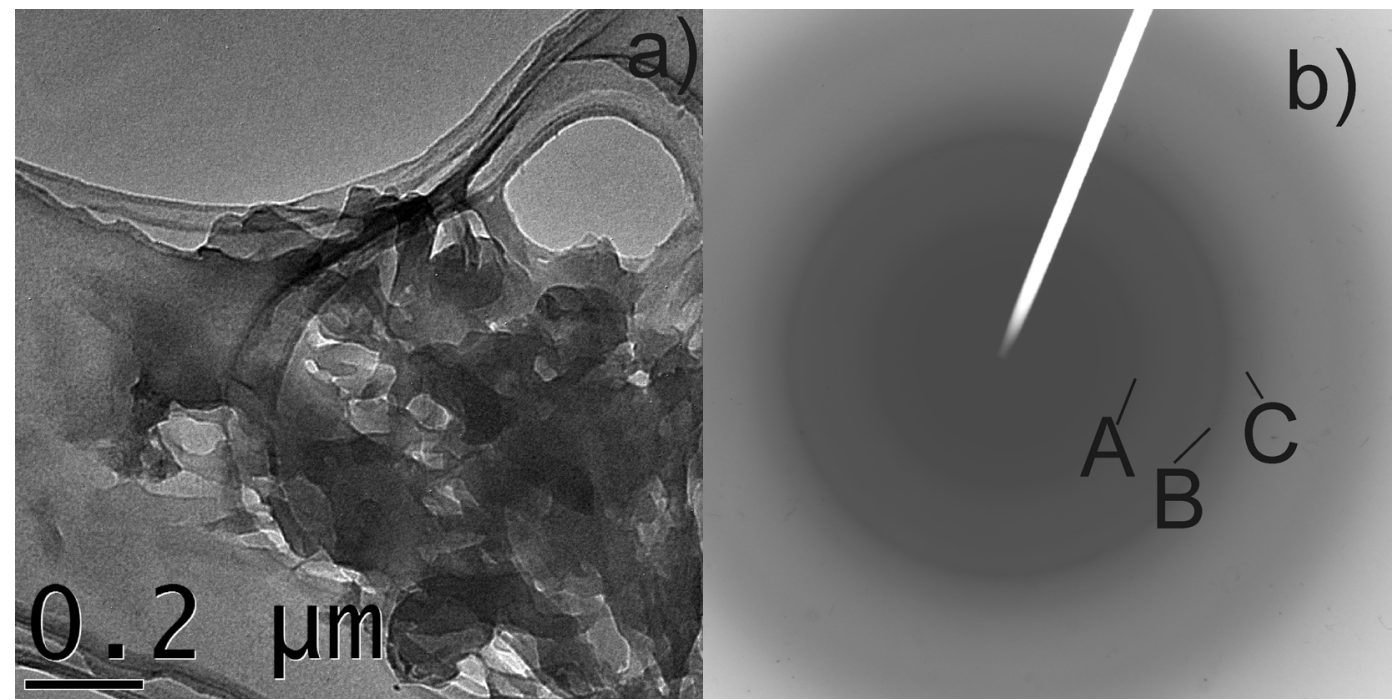

Figure 4-1 a) Micrograph of Sample II b) SAED pattern from of area shown in micrograph.

EDS results for Samples I and II confirmed the presence of iron, sulfur and oxygen in both samples. In Sample I the ratio of Fe:S was 7:3 - this excess of iron with respect to sulfur indicated the likely presence of iron oxides in addition to any iron sulfide that may have been present. In Sample II the measured ratio of Fe:S was highly variable with respect to location on the TEM grid, but sulfur was always in excess of one equivalent with respect to iron. As an initial ratio of $\mathrm{Fe}(\mathrm{acac})_{2}: \mathrm{S} 1: 2$ was used in this experiment, this suggested the possibility of unreacted sulfur remaining in the sample, however the presence of excess sulfur cannot be used to preclude the presence of iron oxides in the sample.

The products of both reactions were jet black powders, which were also judged to be poorly crystalline by XRD (XRD patterns of Samples I and II are shown below in Figure 4-2, along with an experimental XRD pattern of elemental sulfur for reference). Peaks within the range $10-25^{\circ} 2 \theta$ had good angular correspondence with those observed for elemental sulfur. Increasing the initial amount of sulfur by decreasing the $\mathrm{Fe}(\mathrm{acac})_{2}: \mathrm{S}$ ratio from 1:1 (Sample I) to 1:2 (Sample II) increased the relative intensity of these peaks, indicating that they do in fact correspond to unreacted elemental sulfur.

Within the range $25-60^{\circ} 2 \theta$ there were several very broad peaks at positions appropriate for several iron sulfide and oxide phases. Due to the breadth of the peaks it was impossible to confirm the presence of an iron sulfide phase. There was, in addition, a strong peak at $8^{\circ} 2 \theta$ in both samples, which could not be related to either sulfur or to the reference pattern for any iron sulfide or iron oxide phase. This peak remains unassigned. 
It was concluded that the reaction temperature of $140^{\circ} \mathrm{C}$ used in these experiments was insufficient to produce crystalline material within the 1 hour duration used. Although SAED and XRD provided tenuous evidence of the formation of iron sulfide and/or iron oxide phases, it was evident that excess unreacted sulfur remained after the reaction.

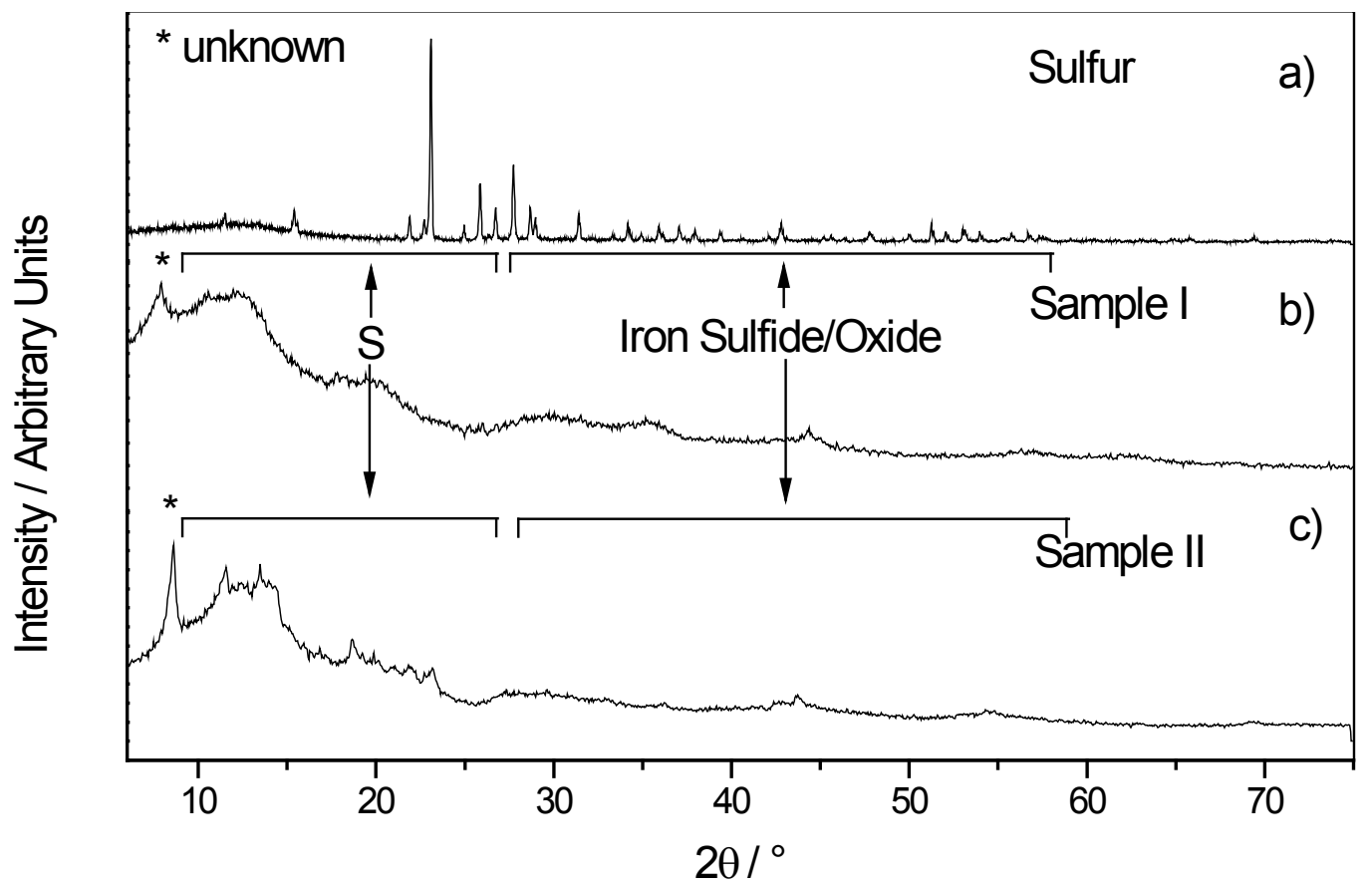

Figure 4-2 XRD patterns of a) elemental sulfur b) Sample I c) Sample II. 
4.2.2.2 Reactions at temperatures between 200 and $250^{\circ} \mathrm{C}$ for a duration of 30 minutes

The reaction temperature for reactions of $\mathrm{Fe}(\mathrm{acac})_{2}$ and elemental sulfur in oleylamine were increased to between 200 and $250^{\circ} \mathrm{C}$, in order to improve the crystallinity of the products. Experimental parameters are tabulated below (Table 4-2). It was found that a reaction temperature above $200^{\circ} \mathrm{C}$ produced a mixture of crystalline iron sulfide phases within a 30 minute reaction duration. Therefore the ratio of $\mathrm{Fe}(\mathrm{acac})_{2}: \mathrm{S}$ was varied from $1: 2$ to $1: 10$, to ascertain whether the amount of sulfur affected the phase of the iron sulfide products. Purified products from these reactions were in general black powders, however Sample VI - synthesised with an initial Fe(acac) $)_{2}$ :S ratio of 1:10 - was a viscous black paste, which was unsuitable for preparation as an XRD sample.

In contrast to the samples synthesised at $140^{\circ} \mathrm{C}$ (Samples I \& II), Samples III - VI (synthesised at $200-220^{\circ} \mathrm{C}$ ) displayed sharper, more well-defined peaks in their XRD patterns (see Figure 4-4). These could be assigned to a mixture of the phases $\mathrm{Fe}_{1-x} \mathrm{~S}$ and $\mathrm{Fe}_{3} \mathrm{~S}_{4}$. Peaks in the range $26-29^{\circ} 2 \theta$ indicated the continued presence of unreacted sulfur. The relative intensities of the sulfur peaks increased as the ratio of $\mathrm{Fe}(\mathrm{acac})_{2}: \mathrm{S}$ decreased from 1:2 (Sample III) to 1:2.75(Sample V).

Table 4-2 Experimental parameters for reactions of $\mathrm{Fe}(\mathrm{acac})_{2}$ and sulfur in oleylamine between 200 and $250^{\circ} \mathrm{C}$ for 30 minutes.

\begin{tabular}{|c|c|c|c|c|c|c|}
\hline Sample & $\mathrm{Fe}(\mathrm{acac})_{2}: \mathrm{S}$ & $\begin{array}{l}{\left[\mathrm{Fe}(\text { acac })_{2}\right]} \\
/ \mathrm{mol} \mathrm{dm}^{-3}\end{array}$ & $\begin{array}{c}\text { Reaction } \\
\text { Temp. } \\
/{ }^{\circ} \mathrm{C}\end{array}$ & $\begin{array}{c}\text { Reaction } \\
\text { Time / } \\
\text { min }\end{array}$ & Result & $\begin{array}{l}D_{h k l} \\
/ \mathrm{nm}\end{array}$ \\
\hline \multirow{3}{*}{ III } & \multirow{3}{*}{$1: 2$} & \multirow{3}{*}{0.05} & \multirow{3}{*}{220} & \multirow{3}{*}{30} & $\mathrm{Fe}_{3} \mathrm{~S}_{4}$ & $31 \pm 7$ \\
\hline & & & & & $\mathrm{Fe}_{1-x} \mathrm{~S}$ & 37 \\
\hline & & & & & Unreact. sulfur & 1 \\
\hline \multirow{3}{*}{ IV } & \multirow{3}{*}{$1: 1.5$} & \multirow{3}{*}{0.05} & \multirow{3}{*}{200} & \multirow{3}{*}{30} & $\mathrm{Fe}_{3} \mathrm{~S}_{4}$ & $26 \pm 5$ \\
\hline & & & & & $\mathrm{Fe}_{1-x} \mathrm{~S}$ & $25 \pm 1$ \\
\hline & & & & & Unreact. sulfur & 1 \\
\hline \multirow{3}{*}{ V } & \multirow{3}{*}{$1: 2.75$} & \multirow{3}{*}{0.05} & \multirow{3}{*}{220} & \multirow{3}{*}{30} & $\mathrm{Fe}_{3} \mathrm{~S}_{4}$ & $51 \pm 15$ \\
\hline & & & & & $\mathrm{Fe}_{1-x} \mathrm{~S}$ & / \\
\hline & & & & & Unreact. sulfur & 1 \\
\hline VI & $1: 10$ & 0.05 & 220 & 30 & Uncharacterised & / \\
\hline
\end{tabular}




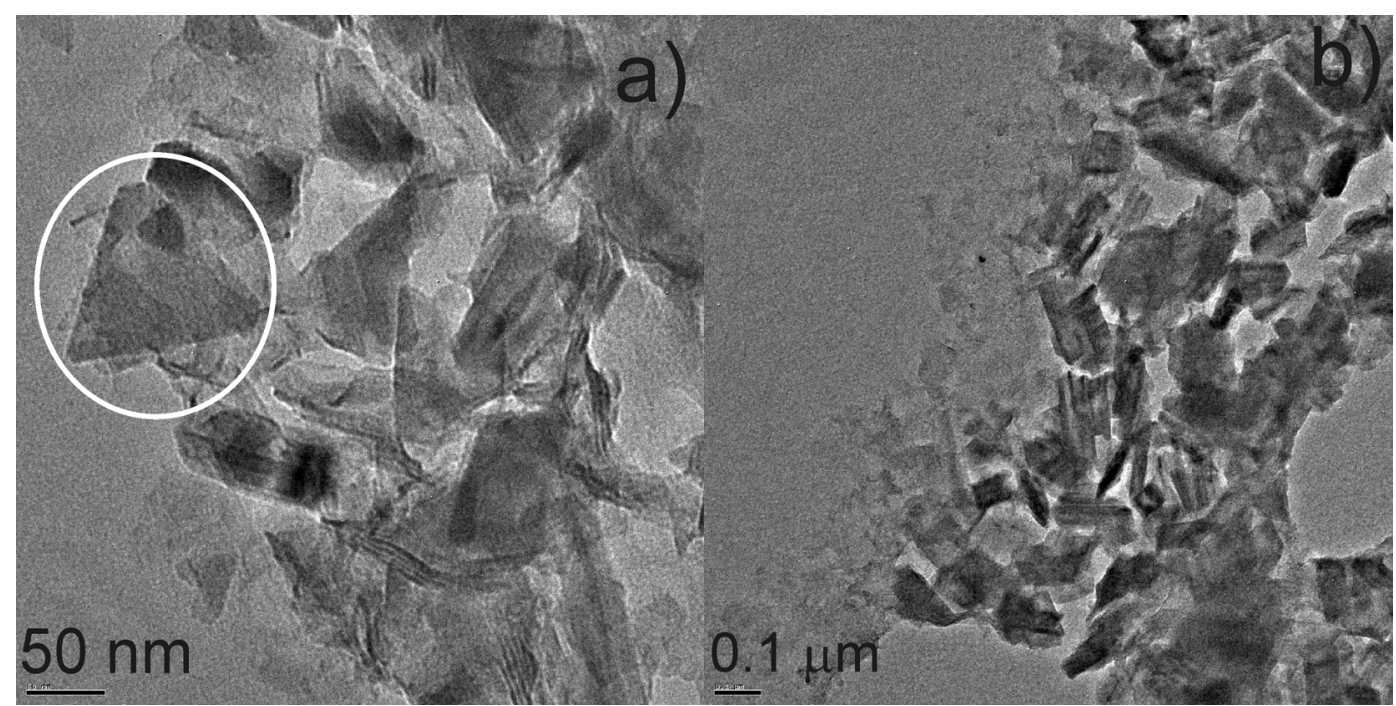

Figure 4-3 a) Micrograph of Sample III b) micrograph of Sample IV.

The $\mathrm{I}_{400}: \mathrm{I}_{200}$ ratio decreased from 2.7 for Sample III to 1.7 for Sample IV (indicating a relative increase in the proportion of $\left.\mathrm{Fe}_{1-x} \mathrm{~S}\right)$, as the ratio of $\mathrm{Fe}(\mathrm{acac})_{2}: \mathrm{S}$ was increased from $1: 2$ to $2: 3$. However Sample V, with a Fe(acac) $)_{2}$ S ratio of $1: 2.75$ contained only traces of $\mathrm{Fe}_{1-x} \mathrm{~S}$, hence these experiments did not indicate any definite trend between the ratio of $\mathrm{Fe}(\mathrm{acac})_{2}: \mathrm{S}$ and the ratio of $\mathrm{Fe}_{3} \mathrm{~S}_{4}: \mathrm{Fe}_{1-x} \mathrm{~S}$ produced. It was evident that excess sulfur remained in the samples after purification. This mixture of phases complicated EDS analysis, as results varied strongly from area to area within a TEM sample grid, as each area contained a mixture of iron sulfide phases and elemental sulfur.

TEM micrographs of Samples III and IV are shown below in Figure 4-3 (a) and (b), respectively. Both micrographs illustrate a mixture of faceted polyhedral crystals interspersed with extended sheets of irregularly shaped, low contrast material. The variety of crystal habits includes an apparent triangular prismatic morphology (see circled area of Figure 4-3 (a)) which is associated with $\mathrm{Fe}_{3} \mathrm{~S}_{4}$ (see section 4.2.4), and oblong crystallite morphology which is associated with $\mathrm{Fe}_{1-x} \mathrm{~S}$ (see section 4.3.1.1). It could not be determined whether the sheet-like material enveloping the highly faceted crystallites was an iron sulfide phase or the excess elemental sulfur known to remain in the samples.

The average crystallite size determined by Scherrer analysis (results are tabulated in Table 4-2, and calculations are tabulated in A2-1, see appendix 2) is similar for both $\mathrm{Fe}_{3} \mathrm{~S}_{4}$ and $\mathrm{Fe}_{1-x} \mathrm{~S}$ in samples III and IV, at $\sim 25$ and $\sim 30 \mathrm{~nm}$, respectively. In Sample V, the average crystallite size for $\mathrm{Fe}_{3} \mathrm{~S}_{4}$ is larger at $50 \mathrm{~nm}$ than in Samples III \& IV. These results correspond moderately well with the crystallite sizes observed by TEM. 


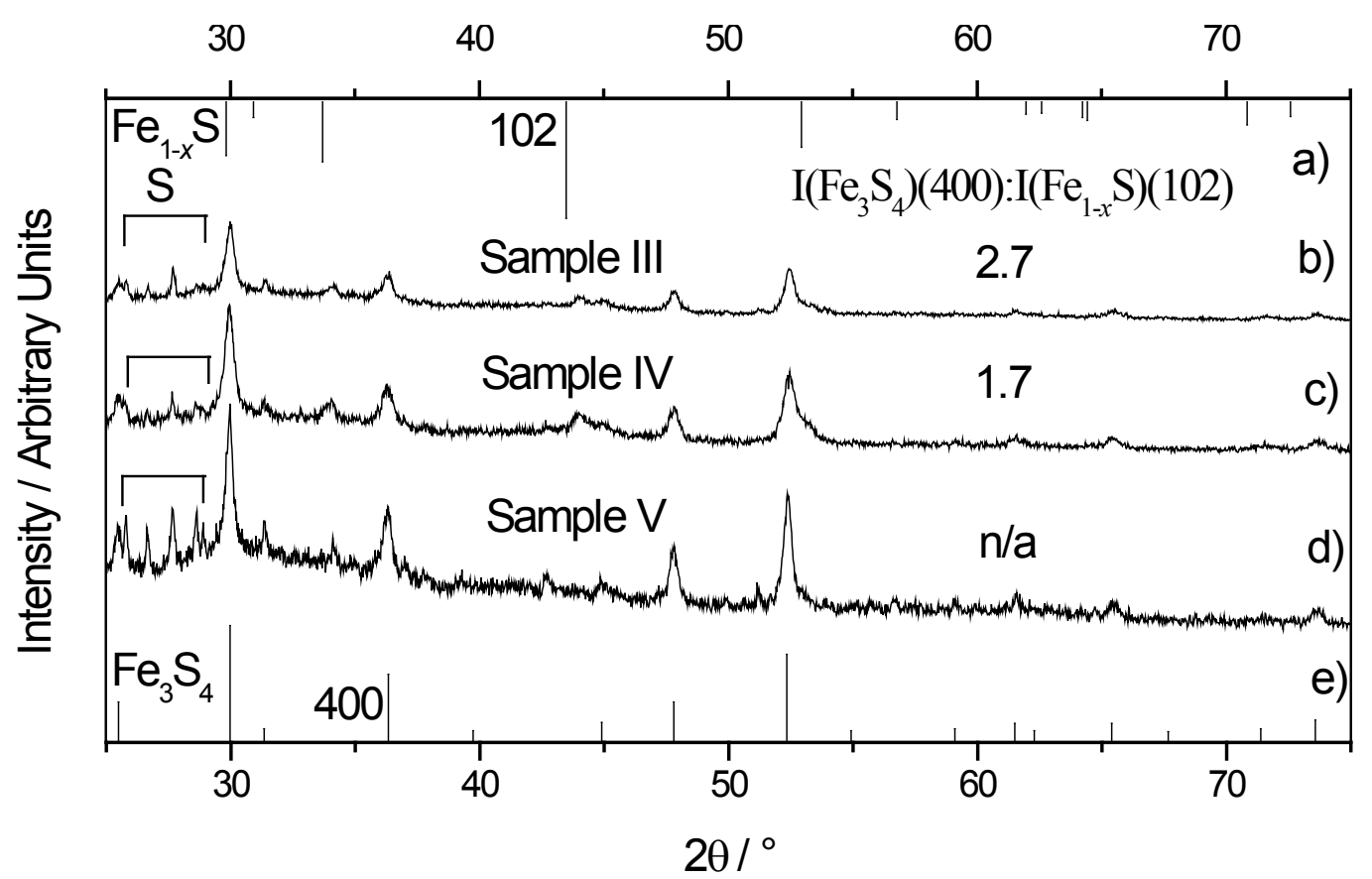

Figure 4-4 XRD patterns of a) $\mathrm{Fe}_{1-x} \mathrm{~S}$ reference b) Sample III c) Sample IV d) Sample V and e) $\mathrm{Fe}_{3} \mathrm{~S}_{4}$ reference. 
4.2.2.3 Reaction of $\mathrm{Fe}(\mathrm{acac})_{2}$ and sulfur in oleylamine at $200^{\circ} \mathrm{C}$ for $>30$ minutes

Increasing the reaction temperature from $140^{\circ} \mathrm{C}$ (section 4.2.2.1) to $200-220^{\circ} \mathrm{C}$

(4.2.2.2) had led to the production of crystalline iron sulfide phases within 30 minutes of reaction duration. A reaction was conducted at $200^{\circ} \mathrm{C}$ for a longer duration than had been used previously (240 minutes) in order to increase the crystallinity of the product, while remaining below the decomposition temperature of $\mathrm{Fe}_{3} \mathrm{~S}_{4}\left(\sim 200^{\circ} \mathrm{C}\right){ }^{11}$

Experimental are tabulated below (Table 4-3).

An XRD pattern of Sample VII is shown below (see Figure 4-5). The peaks are relatively broad, but are clearly defined, indicating a high degree of crystallinity. The presence of both $\mathrm{Fe}_{3} \mathrm{~S}_{4}$ and $\mathrm{Fe}_{1-x} \mathrm{~S}$ was confirmed by XRD, and there was no excess unreacted sulfur evident in the XRD pattern (the initial ratio of $\mathrm{Fe}(\mathrm{acac})_{2}: \mathrm{S}$ was $1: 1$ ). The ratio $\mathrm{I}_{400}: \mathrm{I}_{200}$ ratio was 0.8 , as compared with 2.7 and 1.7 for Samples III \& IV, respectively.

A TEM micrograph of Sample VII is shown in Figure 4-6 (a). The sample consisted of thin sheets, which were roughly rectangular in shape. The side lengths of the sheets varied from $\sim 50-400 \mathrm{~nm}$. In contrast to the sheet-like material seen in reactions conducted below $200^{\circ} \mathrm{C}$ (section 4.2.2.1), the sheets were not aggregated, and formed a single layer on the TEM grid. An HRTEM micrograph of a single sheet is shown in Figure 4-6 (c), displaying lattice fringes with a spacing of $0.25 \mathrm{~nm}$, which matches the spacing of the (400) planes of $\mathrm{Fe}_{3} \mathrm{~S}_{4}$. The sheet was confirmed as $\mathrm{Fe}_{3} \mathrm{~S}_{4}$ by an SAED pattern indexed to the [001] zone axis of $\mathrm{Fe}_{3} \mathrm{~S}_{4}$ (see Figure 4-6 (d)). The SAED pattern of an ensemble of sheets (Figure 4-6 (b)) was also indexed solely to $\mathrm{Fe}_{3} \mathrm{~S}_{4}$ (see A1-1 for indexing parameters).

Table 4-3 Experimental parameters the reaction of $\mathrm{Fe}(\mathrm{acac})_{2}$ and sulfur in oleylamine at $200^{\circ} \mathrm{C}$ for $>30$ minutes.

\begin{tabular}{|c|c|c|c|c|c|c|}
\hline Sample & $\mathrm{Fe}(\mathrm{acac})_{2}: \mathrm{S}$ & $\begin{array}{c}{\left[\mathrm{Fe}(\mathrm{acac})_{2}\right]} \\
/ \mathrm{mol} \mathrm{dm}^{-3}\end{array}$ & $\begin{array}{c}\text { Reaction } \\
\text { Temp. } \\
/{ }^{\circ} \mathrm{C}\end{array}$ & $\begin{array}{c}\text { Reaction } \\
\text { Time } \\
/ \mathrm{min}\end{array}$ & Result & $D_{h k l}$ \\
\hline VII & $1: 1$ & 0.1 & 200 & 240 & $\begin{array}{c}\mathrm{Fe}_{3} \mathrm{~S}_{4} \\
\text { sheets }\end{array}$ & $18 \pm 2$ \\
\cline { 5 - 7 } & & & & $\mathrm{Fe}_{1-x} \mathrm{~S}$ & $42 \pm 12$ \\
\hline
\end{tabular}




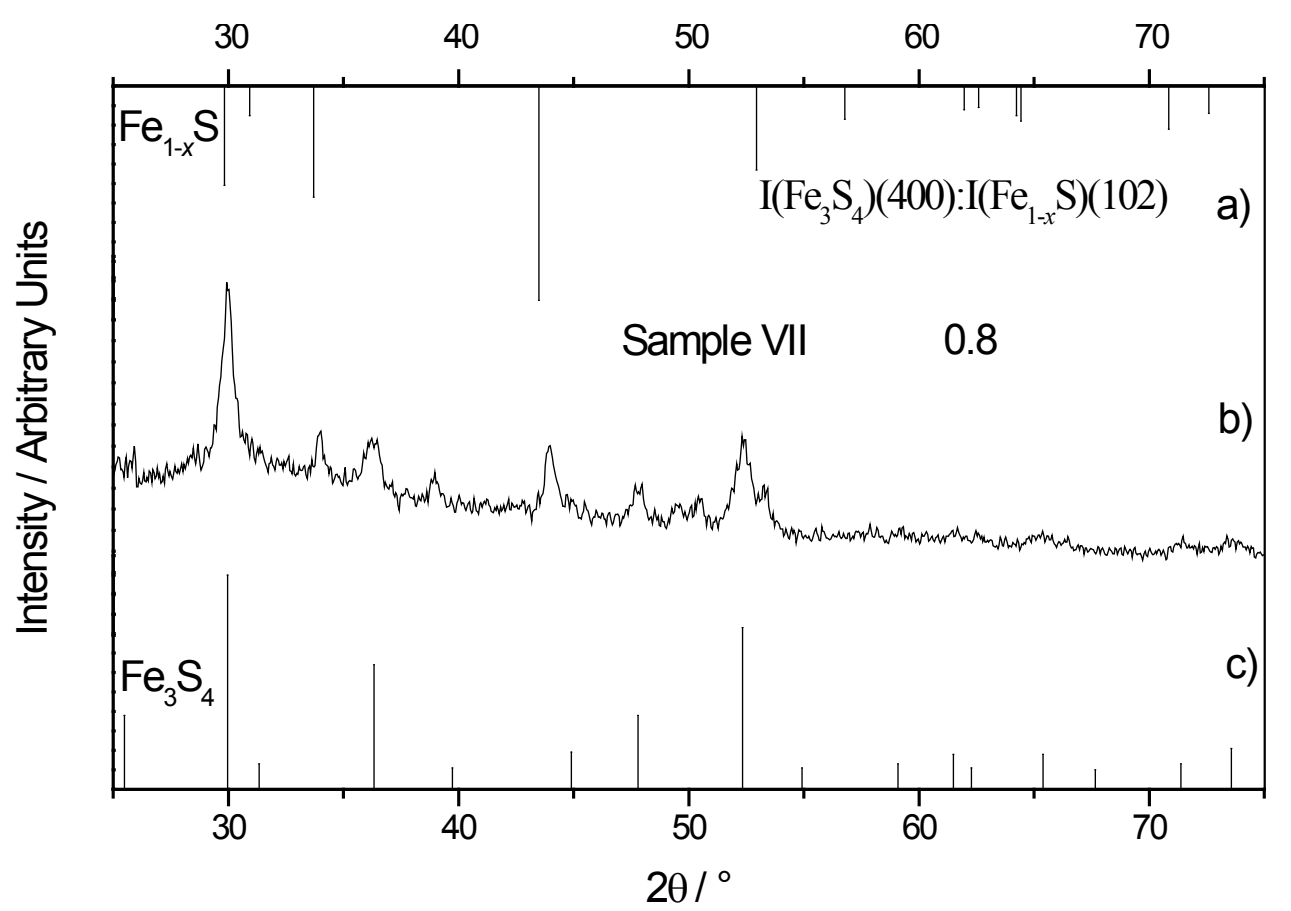

Figure 4-5 XRD patterns of a) $\mathrm{Fe}_{1-x} \mathrm{~S}$ reference b) Sample VII c) $\mathrm{Fe}_{3} \mathrm{~S}_{4}$ reference.

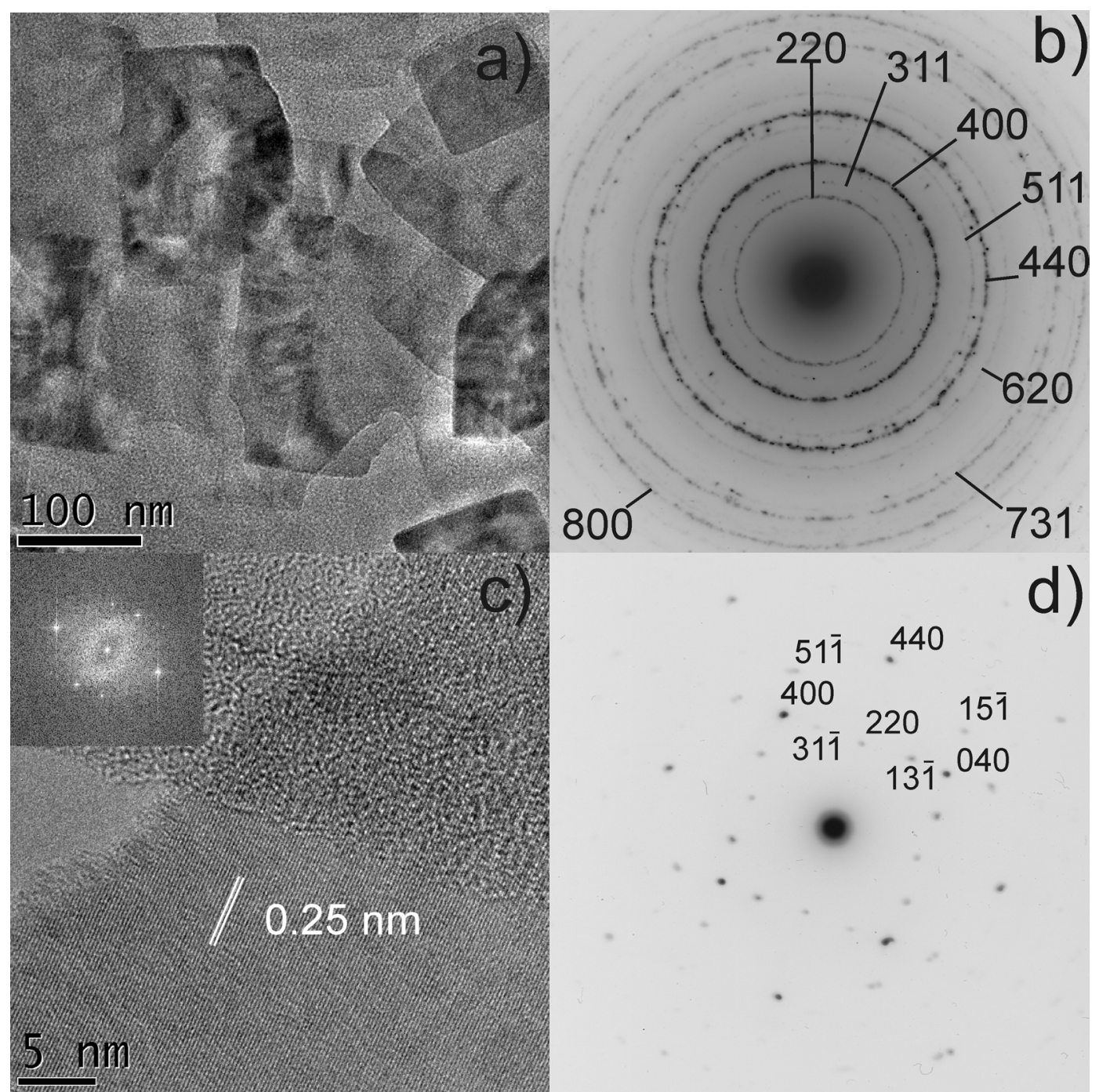

Figure 4-6 a) Micrographs of Sample VII b) SAED pattern of Sample VII, indexed to $\mathrm{Fe}_{3} \mathrm{~S}_{4}$

c) HRTEM micrograph of single sheet, with lattice spacing of $0.25 \mathrm{~nm}$ inset FFT of image d) SAED pattern of sheet imaged in (c), indexed to the [001] zone axis of $\mathrm{Fe}_{3} \mathrm{~S}_{4}$. 
The average crystallite size for both $\mathrm{Fe}_{3} \mathrm{~S}_{4}$ and $\mathrm{Fe}_{1-x} \mathrm{~S}$ phases was calculated using Scherrer analysis (results are tabulated in Table 4-3, calculations are tabulated in A2-2) The calculated average crystallite size for $\mathrm{Fe}_{3} \mathrm{~S}_{4}$ of $18 \mathrm{~nm}$ is low when compared to the side lengths of the sheets imaged above (Figure 4-6 (a). Given that HRTEM imaging indicated that the sheets are single-crystalline, the average crystallite size derived from the Scherrer equation appears to be some average of the relatively small thickness of the sheets and the much larger side lengths of the sheets. 


\subsubsection{Reactions at temperatures greater than $300^{\circ} \mathrm{C}$}

The reaction temperature for the reaction of $\mathrm{Fe}(\mathrm{acac})_{2}$ and sulfur in oleylamine was raised further to above $300^{\circ} \mathrm{C}$ in order to examine the effect on the phases produced. Reaction durations of 30 minutes were used, in line with the duration found sufficient in section 4.2.2.2 to produce crystalline material. The ratio of $\mathrm{Fe}(\mathrm{acac})_{2}: \mathrm{S}$ was held constant at 1:1, as results from section 4.2.2.2 indicated that this ratio did not affect the phases produced, and excess sulfur remained after purification to contaminate the iron sulfide. Concentrations of the precursors were varied between 0.01 and $0.1 \mathrm{~mol} \mathrm{dm}^{-3}$, in order to ascertain the effect on crystallite size. Experimental parameters are tabulated below (Table 4-4).

The products of these reactions were hard pellets, which were ground into jet black powders. XRD patterns are shown below for Samples IX and X (Figure 4-7). Insufficient material was isolated to prepare an XRD sample for Sample VIII. XRD shows that Samples IX and $\mathrm{X}$ are both pure, well crystallised $\mathrm{Fe}_{1-x} \mathrm{~S}$, with no indication of the presence of amorphous material. No reflections were observed in these diffraction patterns, which could not be indexed to the NiAs-type structure.

The composition of the sample as determined by repeated EDS measurement was Fe:S 48:52, or $\mathrm{Fe}_{12} \mathrm{~S}_{13}$ immediately after synthesis - corresponding to a mildly iron deficient $\mathrm{Fe}_{1-x} \mathrm{~S}$ phase. After one year the ratio had changed to Fe:S 50:50. This cannot indicate the gain of iron to form stoichiometric $\mathrm{Fe}_{1-x} \mathrm{~S}$, rather this must indicate that surface oxidation has taken place, resulting in the loss of sulfur from the sample.

Table 4-4 Experimental parameters for reactions of $\mathrm{Fe}(\mathrm{acac})_{2}$ and sulfur in oleylamine above $300^{\circ} \mathrm{C}$.

\begin{tabular}{|c|c|c|c|c|c|c|}
\hline Sample & $\mathrm{Fe}(\mathrm{acac})_{2}: \mathrm{S}$ & $\begin{array}{l}{\left[\mathrm{Fe}(\operatorname{acac})_{2}\right]} \\
/ \mathrm{mol} \mathrm{dm}^{-3}\end{array}$ & $\begin{array}{l}\text { Reaction } \\
\text { Temp. } \\
/{ }^{\circ} \mathrm{C}\end{array}$ & $\begin{array}{l}\text { Reaction } \\
\text { Time } \\
\text { / min }\end{array}$ & Result & $\begin{array}{l}D_{h k l} \\
/ \mathrm{nm}\end{array}$ \\
\hline VIII & $1: 1$ & 0.01 & 300 & 30 & $\begin{array}{c}\text { Faceted } \\
\mathrm{Fe}_{1-x} \mathrm{~S} \\
\text { crystallites } \\
\end{array}$ & I \\
\hline IX & $1: 1$ & 0.05 & 300 & 30 & $\begin{array}{c}\text { Faceted } \\
\mathrm{Fe}_{1-x} \mathrm{~S} \\
\text { crystallites }\end{array}$ & $84 \pm 66$ \\
\hline X & $1: 1$ & 0.1 & 310 & 30 & $\begin{array}{c}\text { Faceted } \\
\mathrm{Fe}_{1-x} \mathrm{~S} \\
\text { crystallites }\end{array}$ & $55 \pm 38$ \\
\hline
\end{tabular}




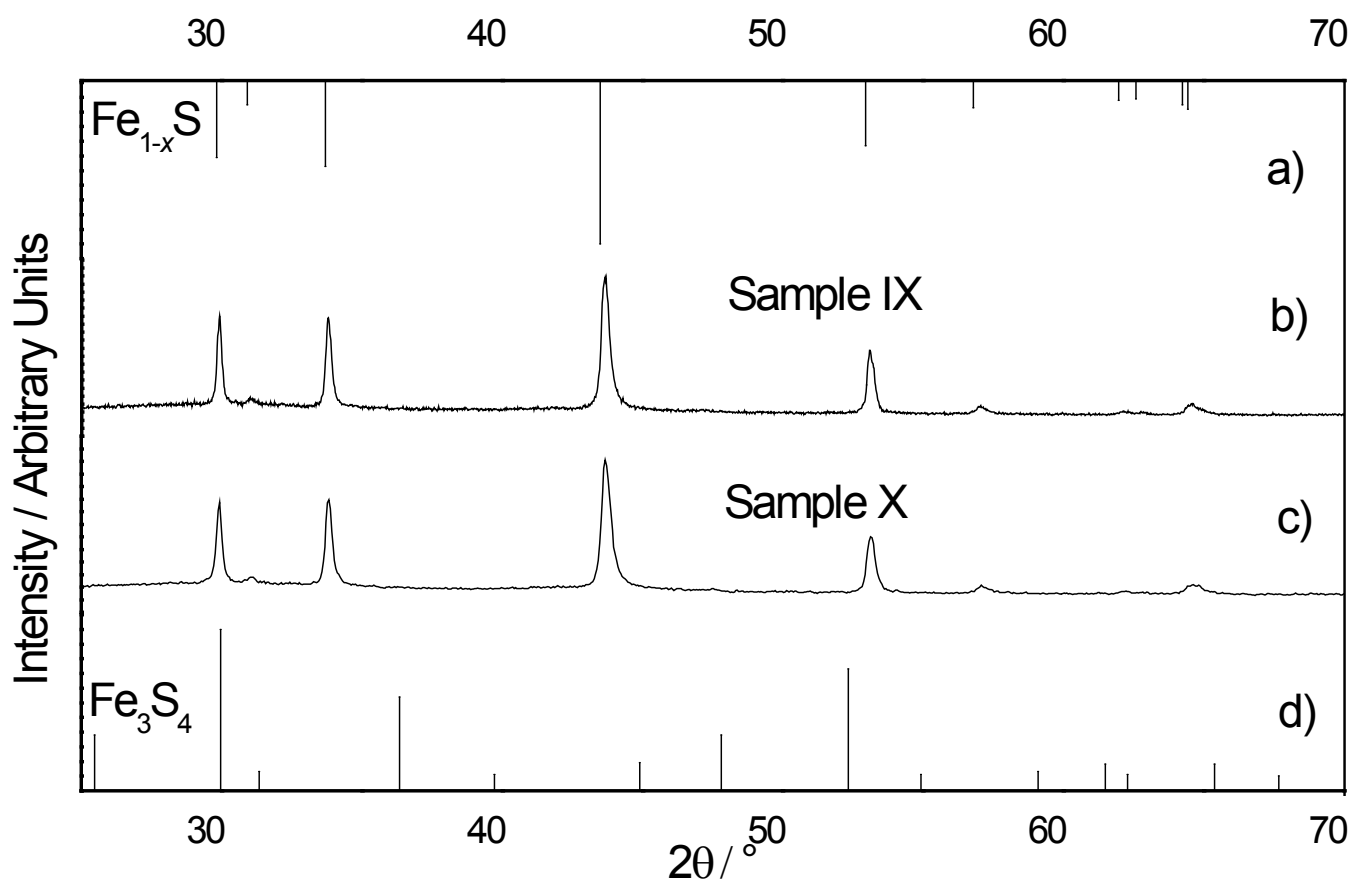

Figure 4-7 XRD patterns of a) $\mathrm{Fe}_{1-x} \mathrm{~S}$ reference b) Sample IX c) Sample X d) $\mathrm{Fe}_{3} \mathrm{~S}_{4}$ reference.

Micrographs and corresponding SAED patterns are shown below for Samples VIII - X (Figure 4-8). All three samples consisted of moderately large, highly faceted crystals of a variety of morphologies ranging from truncated triangular prisms to needles. The SAED pattern for each sample was indexed solely to NiAs-type $\mathrm{Fe}_{1-x} \mathrm{~S}$ (see A1-3 for indexing parameters); although the SAED pattern for Sample VIII (Figure 4-8 (b)) contained additional low-angle reflections, possibly related to $\mathrm{Fe}_{1-x} \mathrm{~S}$ superlattice structures (see section 4.2.7). This SAED pattern was recorded with a larger camera length than the others $(\mathrm{L}=200 \mathrm{~cm}$, $\mathrm{cf} . \mathrm{L}=150 \mathrm{~cm})$, thus these low-angle reflections may be present for the other samples, but lost in the saturated area surrounding the central spot. Each SAED pattern was comprised of spotty rings, indicating a large crystallite size for the polycrystalline samples.

Average crystallite size was calculated for Samples IX and X using Scherrer analysis (results are tabulated in Table 4-4, calculations are tabulated in A2-3). The mean for each sample taken over all reflections is 84 and $55 \mathrm{~nm}$ for Sample IX and X, respectively. These values correlate well with TEM observations. The large spread of $D_{h k l}$ values was expected, as the crystal habits were observed by TEM to be highly anisotropic. 


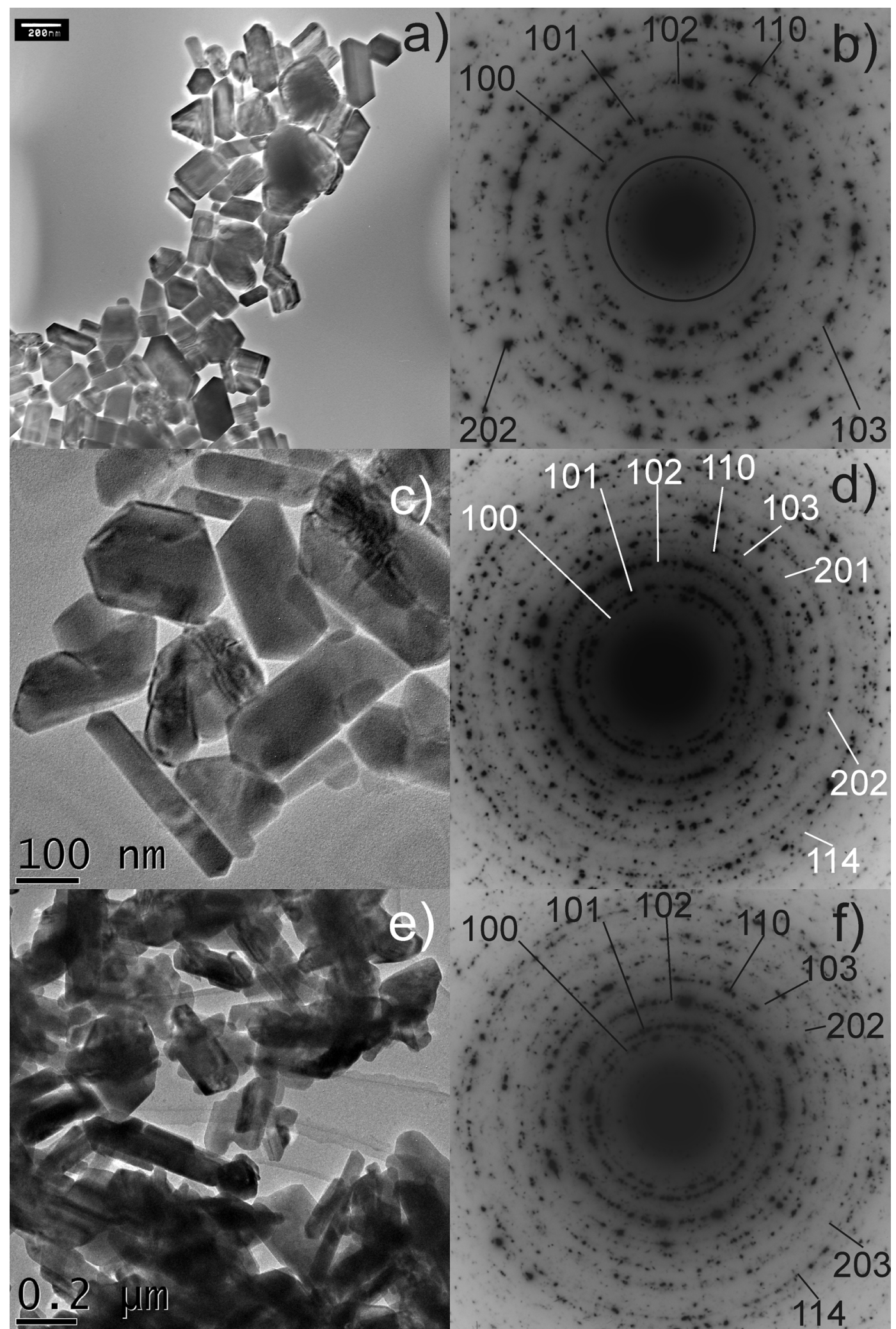

Figure 4-8 a) Micrograph of Sample VIII b) SAED pattern $(\mathrm{L}=200 \mathrm{~nm})$ of Sample VIII, indexed to $\mathrm{Fe}_{1-\mathrm{x}} \mathrm{S}$. Note the circled central region contains a number of reflections corresponding to large $d$-spacings. c) Micrograph of Sample IX d) SAED pattern of Sample IX, indexed to $\mathrm{Fe}_{1-x} \mathrm{~S}$

e) Micrograph of Sample X f) SAED pattern of Sample X, indexed to $\mathrm{Fe}_{1-x} \mathrm{~S}$. 
To verify that the initial $\mathrm{Fe}(\mathrm{acac})_{2}: \mathrm{S}$ ratio does not affect the ratio the abundance of iron sulfide for reaction temperatures above $300^{\circ} \mathrm{C}$, an experiment was conducted with $\mathrm{Fe}(\mathrm{acac})_{2}: \mathrm{S}$ of 1:2.75. Experimental parameters are tabulated below (Table 4-5).

An XRD pattern of Sample XI is shown below (Figure 4-10). The sample is predominantly $\mathrm{Fe}_{1-x} \mathrm{~S}$, with a small amount of $\mathrm{Fe}_{3} \mathrm{~S}_{4}$, as reflected in the small $\mathrm{I}_{400}: \mathrm{I}_{102}$ ratio of 0.1 . This is marginally higher than for Samples (VIII - X), however it cannot be concluded that the $\mathrm{Fe}(\mathrm{acac})_{2}: \mathrm{S}$ has a significant effect on the phase ratio of the products. There are no peaks in the range $26-29^{\circ} 2 \theta$ to indicate the presence of unreacted elemental sulfur, although a $\mathrm{Fe}(\mathrm{acac})_{2}: \mathrm{S}$ ratio of $1: 2.75$ was used.

A micrograph of Sample XI is shown below (Figure 4-9), displaying the highly faceted morphologies seen for $\mathrm{Fe}_{1-x} \mathrm{~S}$ in previous samples. Scherrer analysis was performed (results are tabulated in Table 4-5, calculations are tabulated in A2-4) - at $59 \mathrm{~nm} \mathrm{Fe}_{1-x} \mathrm{~S}$ in the sample has a larger average crystallite size than $\mathrm{Fe}_{3} \mathrm{~S}_{4}$ at $16 \mathrm{~nm}$. In addition $D_{h k l}$ values vary among the various reflections for $\mathrm{Fe}_{1-x} \mathrm{~S}$, indicating anisotropic morphology.

Figure 4-9 a) Micrograph of Sample XI

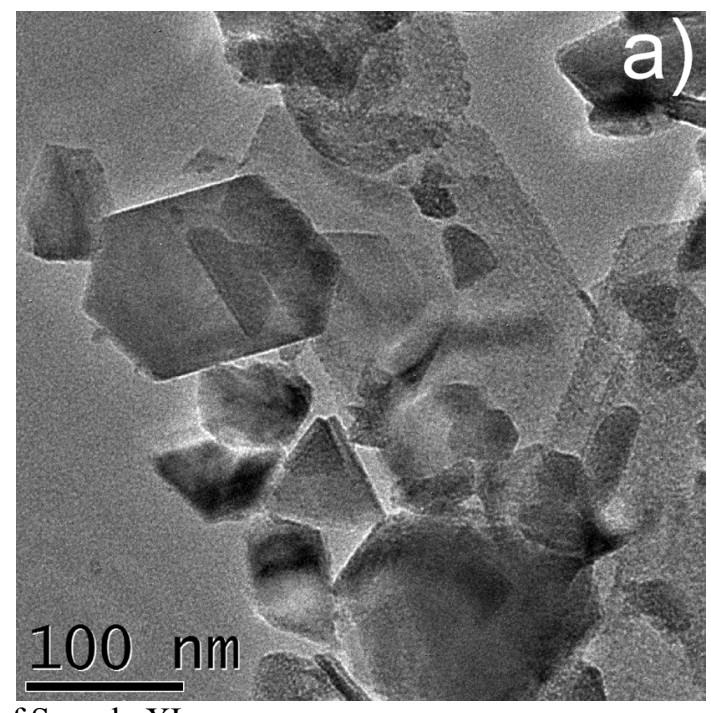

Table 4-5 Experimental parameters for reactions of $\mathrm{Fe}(\mathrm{acac})_{2}$ and sulfur in oleylamine above $300^{\circ} \mathrm{C}$.

\begin{tabular}{|c|c|c|c|c|c|c|}
\hline Sample & $\mathrm{Fe}(\mathrm{acac})_{2}: \mathrm{S}$ & {$\left[\mathrm{Fe}(\mathrm{acac})_{2}\right]$} & $\begin{array}{c}\text { Reaction } \\
\text { Temp. } \\
/{ }^{\circ} \mathrm{C}\end{array}$ & $\begin{array}{c}\text { Reaction } \\
\text { Time } \\
/ \mathrm{min}\end{array}$ & Result & $D_{h k l}$ \\
\hline $\mathrm{XI}$ & $1: 2.75$ & 0.05 & 300 & 30 & $\mathrm{~nm}$ \\
\cline { 3 - 6 } & & & & $\mathrm{Fe}_{3} \mathrm{~S}_{4}$ & $16 \pm 1$ \\
\hline
\end{tabular}




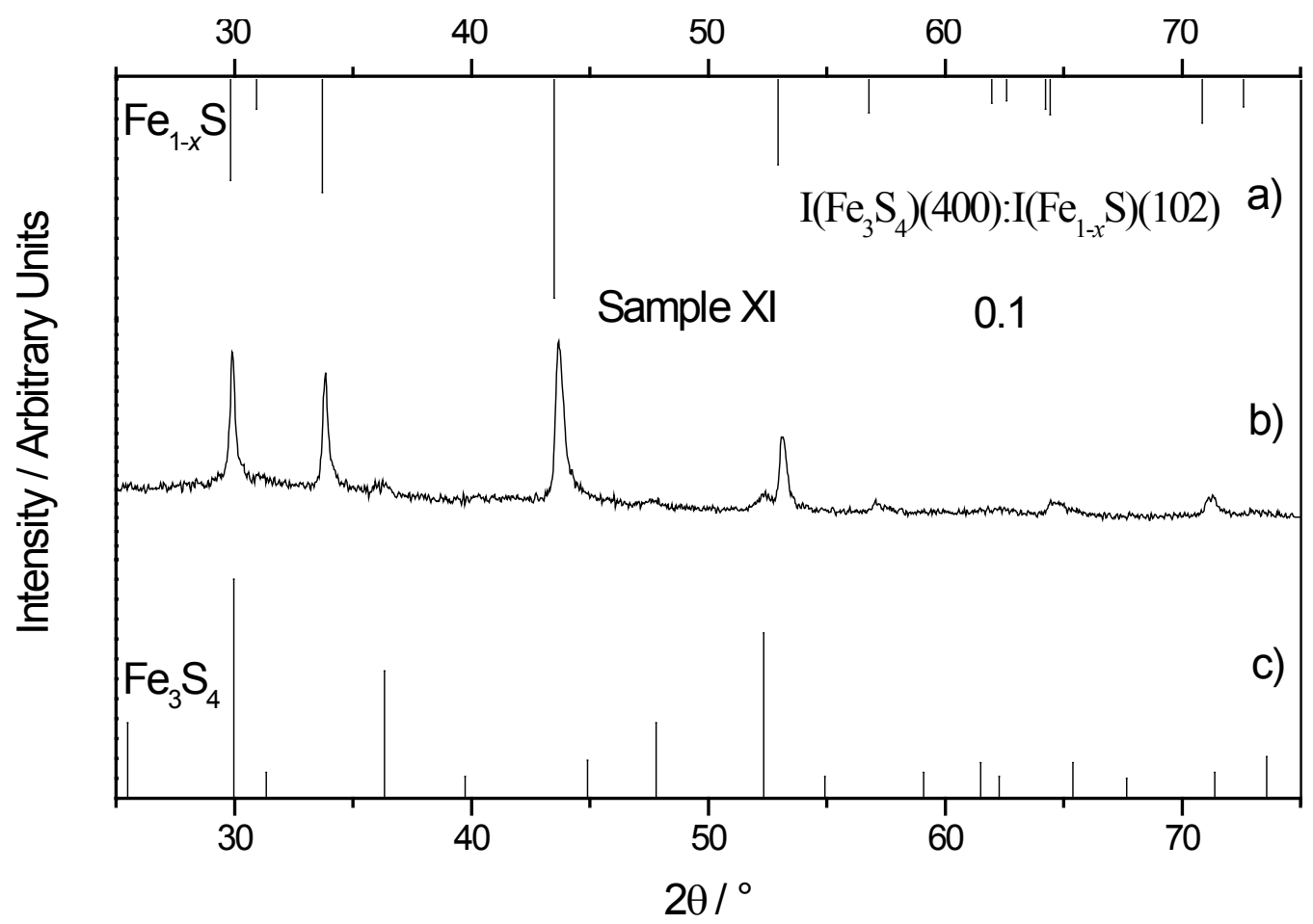

Figure 4-10 XRD pattern of a) $\mathrm{Fe}_{1-x} \mathrm{~S}$ reference b) Sample XI c) $\mathrm{Fe}_{3} \mathrm{~S}_{4}$ reference. 


\subsubsection{Reaction of $\mathrm{Fe}(\mathrm{acac})_{2}$ in oleylamine with injection of sulfur solution}

As a relatively large average particle size was evident in previous samples, the effect of rapidly inducing nucleation by injection of a sulfur solution into a preheated solution containing $\mathrm{Fe}(\mathrm{acac})_{2}$ followed by rapid cooling, was investigated as a method of limiting particle growth. This experimental procedure is detailed in section 4.5.2, and experimental parameters are tabulated below (Table 4-6). The effect of varying the period between injection of sulfur and initiation of cooling from 10 to 0 minutes was examined, as well as the effect of varying the $\mathrm{Fe}(\mathrm{acac})_{2}: \mathrm{S}$ ratio from 1:1 to 1:2.75.

XRD patterns for Samples XII - XIV are shown below (Figure 4-11). Sample XII, with 10 minutes of reaction at $290^{\circ} \mathrm{C}$ before cooling, displayed moderate crystallinity, judging by the sharpness of the peaks. Sample XIII and Sample XIV were cooled immediately after injection of sulfur into the $\mathrm{Fe}(\mathrm{acac})_{2}$-containing solution at $280^{\circ} \mathrm{C}$. The XRD pattern of Sample XIII displayed broad peaks, while that of Sample XIV, which was synthesised with a $\mathrm{Fe}(\mathrm{acac})_{2}$ :S ratio of $1: 2.75$, showed only very broad diffuse peaks. The presence of excess of unreacted sulfur in Sample XIV appeared to have increased the amorphous contribution to the XRD pattern.

Table 4-6 Experimental parameters reactions of $\mathrm{Fe}(\mathrm{acac})_{2}$ in oleylamine with injection of sulfur soln.

\begin{tabular}{|c|c|c|c|c|c|c|}
\hline Sample & $\mathrm{Fe}(\mathrm{acac})_{2}: \mathrm{S}$ & {$\left[\mathrm{Fe}(\mathrm{acac})_{2}\right]$} & $\begin{array}{c}\text { Reaction } \\
\text { Temp. } \\
/{ }^{\circ} \mathrm{C}\end{array}$ & $\begin{array}{c}\text { Reaction } \\
\text { Time } \\
/ \mathrm{min}\end{array}$ & Result & $D_{h k l}$ \\
\hline XII & $1: 1$ & 0.05 & 290 & 10 & ${\mathrm{Trace} \mathrm{Fe}_{3} \mathrm{~S}_{4}}$ & 5 \\
\hline XIII & $1: 1$ & 0.05 & 280 & 0 & $\mathrm{Fe}_{1-x} \mathrm{~S}$ & $43 \pm 15$ \\
\cline { 5 - 7 } & $1: 2.75$ & 0.05 & 280 & 0 & $\mathrm{Fe}_{3} \mathrm{~S}_{4}$ & $6 \pm 3$ \\
\hline XIV & $1: 3 \mathrm{Fe}_{1-x} \mathrm{~S}$ & $26 \pm 12$ \\
\hline
\end{tabular}




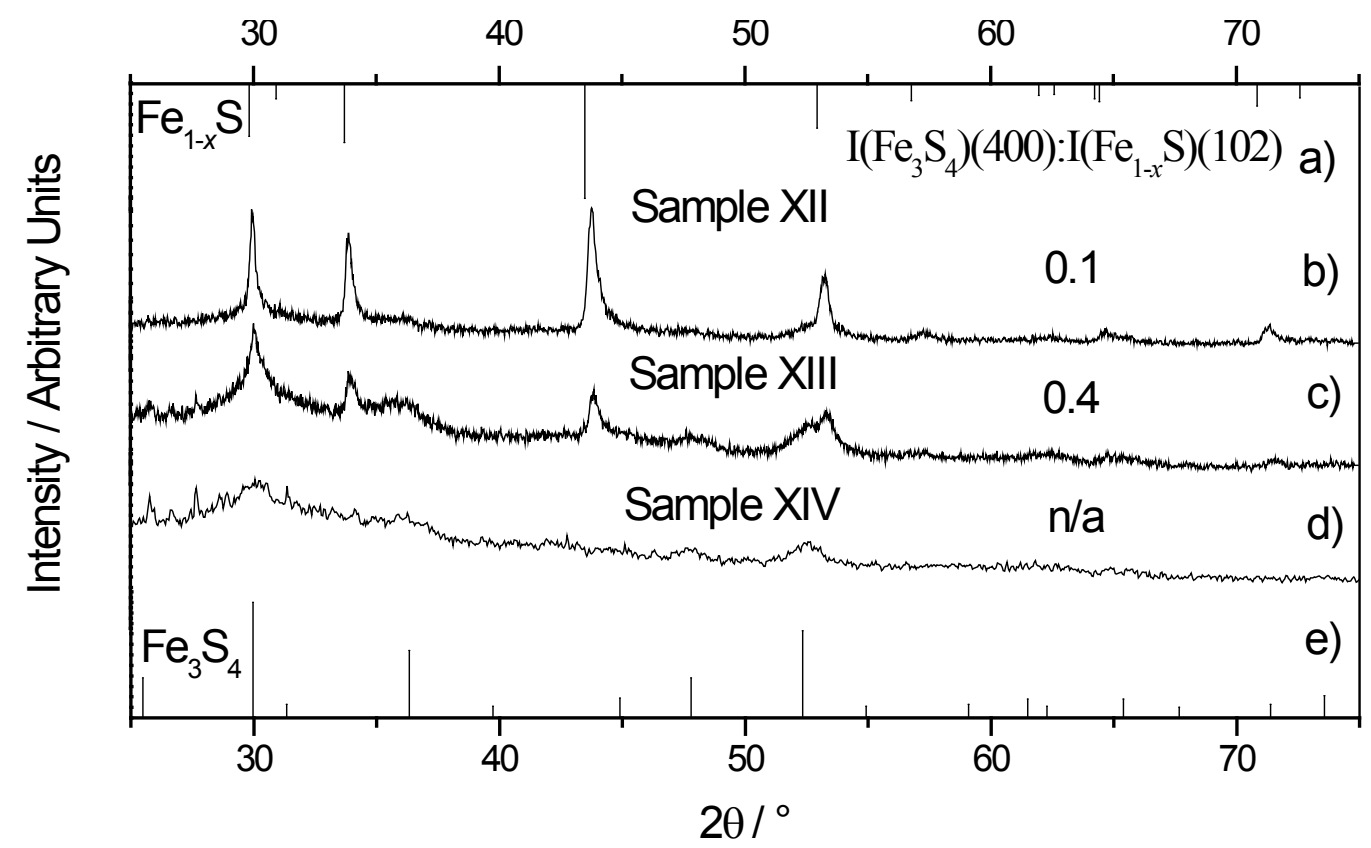

Figure 4-11 XRD patterns of a) reference $\mathrm{Fe}_{1-x} \mathrm{~S}$ b) Sample XII c) Sample XIII d) Sample XIV e) reference $\mathrm{Fe}_{3} \mathrm{~S}_{4}$.

Sample XII was predominantly $\mathrm{Fe}_{1-x} \mathrm{~S}$ with trace amounts of $\mathrm{Fe}_{3} \mathrm{~S}_{4}$, as reflected in the very low $\mathrm{I}_{400}: \mathrm{I}_{102}$ ratio of 0.1 , equal to that Sample XI, which was produced by reaction at $300^{\circ} \mathrm{C}$ for 30 minutes. Sample XIII contained a higher proportion of $\mathrm{Fe}_{3} \mathrm{~S}_{4}$, with an $\mathrm{I}_{400}: \mathrm{I}_{102}$ of 0.4 . This is significantly lower than that for samples produced by long reaction times at $200^{\circ} \mathrm{C}$, but higher than any other samples produced at $280-300^{\circ} \mathrm{C}$. Sample XIV appeared to be predominantly $\mathrm{Fe}_{3} \mathrm{~S}_{4}$, but the poor quality of the XRD pattern for this sample makes this difficult to support. This evidence suggests that $\mathrm{Fe}_{3} \mathrm{~S}_{4}$ is produced if the reaction duration at temperatures above its decomposition temperature $\left(\sim 200^{\circ} \mathrm{C}\right)$ is brief - that is if the solution is cooled immediately after nucleation. Conversely, if a temperature of $\sim 300^{\circ} \mathrm{C}$ is maintained for even 10 minutes, the proportion of $\mathrm{Fe}_{3} \mathrm{~S}_{4}$ produced is small.

Micrographs and SAED patterns for Samples XII - XIV are shown below (Figure 4-12), and indexing parameters of the SAED patterns are tabulated in A1-4. The micrograph of Sample XII (Figure 4-12 (a)) shows a mixture of $\sim 100 \mathrm{~nm}$ faceted crystals, of similar appearance to $\mathrm{Fe}_{1-x} \mathrm{~S}$ crystallites seen in previous samples. Also visible in this micrograph are areas of small, polydisperse, roughly spherical particles. The SAED pattern of this area contained contributions from both $\mathrm{Fe}_{3} \mathrm{~S}_{4}$ and $\mathrm{Fe}_{1-x} \mathrm{~S}$. The $\mathrm{Fe}_{3} \mathrm{~S}_{4}$ reflections were in the form of homogeneous rings, while the $\mathrm{Fe}_{1-x} \mathrm{~S}$ reflections were spotty, with only partially developed rings. This indicates that the $\mathrm{Fe}_{1-x} \mathrm{~S}$ crystallites are larger, and formed a less randomly oriented powder than the $\mathrm{Fe}_{3} \mathrm{~S}_{4}$ 
crystallites. The micrograph of Sample XIII shown in Figure 4-12 c) shows an area containing only small $(<20 \mathrm{~nm})$, irregularly shaped, polydisperse particles. The corresponding SAED pattern only contains reflections from $\mathrm{Fe}_{3} \mathrm{~S}_{4}$, thus confirming that the smaller particles visible in the micrographs are $\mathrm{Fe}_{3} \mathrm{~S}_{4}$.

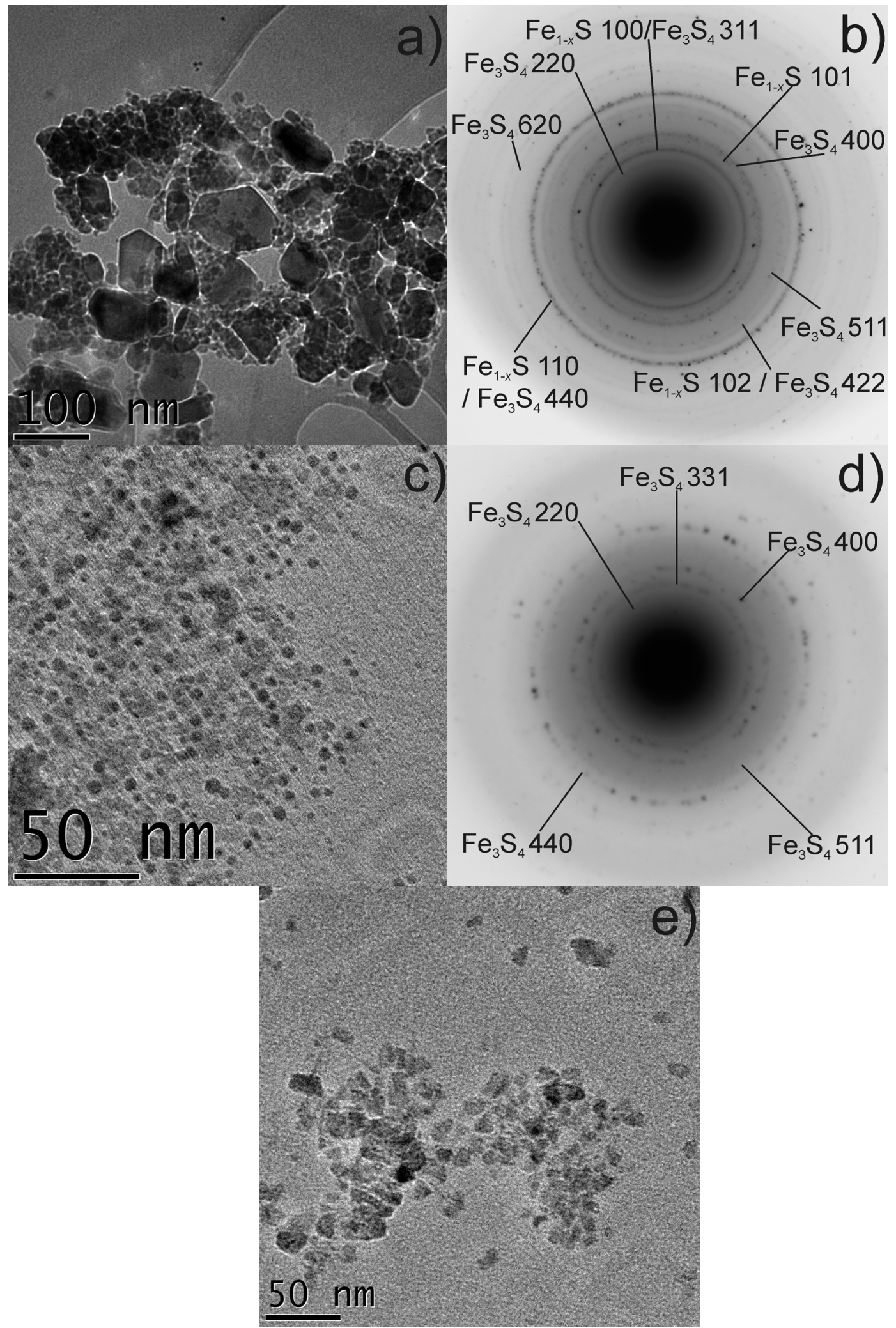

Figure 4-12 a) Micrograph of Sample XII b) SAED pattern of Sample XII, indexed to $\mathrm{Fe}_{1-x} \mathrm{~S}$ and $\mathrm{Fe}_{3} \mathrm{~S}_{4}$ c) Micrograph of Sample XIII d) SAED pattern of Sample XIII e) micrograph of Sample XIV. 
Scherrer analysis was performed for Samples XII - XIV (results are tabulated in Table 4-6, calculations are tabulated in A2-5). The average crystallite size of $\mathrm{Fe}_{1-x} \mathrm{~S}$ varied from $26 \mathrm{~nm}$ for Sample XIII to $43 \mathrm{~nm}$ for Sample XI. These results were in line with the crystallite sizes observed by TEM. The average crystallite size of $\mathrm{Fe}_{3} \mathrm{~S}_{4}$ was calculated to be $5-6 \mathrm{~nm}$ in both samples. This provides further confirmation that the $\sim 5 \mathrm{~nm}$ nanocrystals seen in Samples XII - XIV are $\mathrm{Fe}_{3} \mathrm{~S}_{4}$.

EDS results in indicated a composition of Fe:S ratio of 50:50 for Sample XII, which is in line with XRD results which show the sample to be essentially pure $\mathrm{Fe}_{1-x} \mathrm{~S}$. The ratio of Fe:S varied with location in Sample XIII, which was shown by XRD to comprise a mixture of $\mathrm{Fe}_{1-x} \mathrm{~S}$ and $\mathrm{Fe}_{3} \mathrm{~S}_{4}$. One area in Sample XIII had a Fe:S ratio of 39:61, which is within the range expected for a mixture of $\mathrm{Fe}_{3} \mathrm{~S}_{4}$ and $\mathrm{Fe}_{1-x} \mathrm{~S}$. Another area in Sample XIII had a Fe:S ratio of 53:47 - slightly more iron rich than would be expected for pure $\mathrm{Fe}_{1-x} \mathrm{~S}$. 


\subsubsection{Reaction of $\mathrm{FeCl}_{2}$ in oleylamine with injection of sulfur solution}

As above in section 4.2.3, the formation of iron sulfides was initiated by injecting sulfur solution into a preheated solution of the iron source, as detailed in section 4.5.3. To investigate the effect of iron source on the products formed, $\mathrm{FeCl}_{2}$ was substituted for $\mathrm{Fe}(\mathrm{acac})_{2}$. The experimental parameters are tabulated below (Table 4-7).

An XRD pattern of Sample XV is shown below (Figure 4-13). The XRD pattern is well developed, and was indexed to $\mathrm{Fe}_{1-x} \mathrm{~S}$ and $\mathrm{Fe}_{3} \mathrm{~S}_{4}$. The ratio $\mathrm{I}_{400}: \mathrm{I}_{102}$ was 0.2 , higher than for reactions conducted at $290-300^{\circ} \mathrm{C}$ for $10-30$ minutes (i.e. Sample XII and XI, respectively; both with $\mathrm{I}_{400}: \mathrm{I}_{102}=0.1$ ), indicating the presence of slightly more $\mathrm{Fe}_{3} \mathrm{~S}_{4}$ than samples produced at those temperatures.

Micrographs and an SAED pattern of Sample XV are shown below (Figure 4-14) (indexing parameters for the SAED pattern are tabulated in A1-5). The low magnification micrograph (Figure 4-14 (a)) shows an area of moderately large ( 20 $\mathrm{nm}$ ), faceted crystals, including several examples with a triangular or truncated triangular appearance. In addition to these particles, there are some smaller $(\sim<10 \mathrm{~nm})$, roughly spherical particles visible. The SAED pattern of Sample XV was indexed to a mixture of $\mathrm{Fe}_{1-x} \mathrm{~S}$ and $\mathrm{Fe}_{3} \mathrm{~S}_{4}$. Once again reflections from $\mathrm{Fe}_{3} \mathrm{~S}_{4}$ were less spotty than those of $\mathrm{Fe}_{1-x} \mathrm{~S}$, and formed more homogenous rings, again indicating that $\mathrm{Fe}_{3} \mathrm{~S}_{4}$ had a smaller crystallite size than $\mathrm{Fe}_{1-x} \mathrm{~S}$. EDS results for Sample XV gave a Fe:S ratio of 50:50, appropriate for $\mathrm{Fe}_{1-x} \mathrm{~S}$.

Table 4-7 Experimental parameters for reaction of $\mathrm{FeCl}_{2}$ in oleylamine with injection of sulfur soln.

\begin{tabular}{|c|c|c|c|c|c|c|}
\hline Sample & $\begin{array}{c}\mathrm{FeCl}_{2}: \\
\mathrm{S}\end{array}$ & {$\left[\mathrm{FeCl}_{2}\right]$} & $\begin{array}{c}\text { Reaction } \\
\text { Temp. } \\
/{ }^{\circ} \mathrm{C}\end{array}$ & $\begin{array}{c}\text { Reaction } \\
\text { Time } \\
/ \mathrm{min}\end{array}$ & Result & $D_{h k l}$ \\
\hline $\mathrm{XV}$ & $1: 1$ & 0.05 & 280 & 0 & $\mathrm{Fe}_{3} \mathrm{~S}_{4}$ & $20 \pm 3$ \\
\cline { 5 - 7 } & & & $\mathrm{Fe}_{1-x} \mathrm{~S}$ & $51 \pm 23$ \\
\hline
\end{tabular}




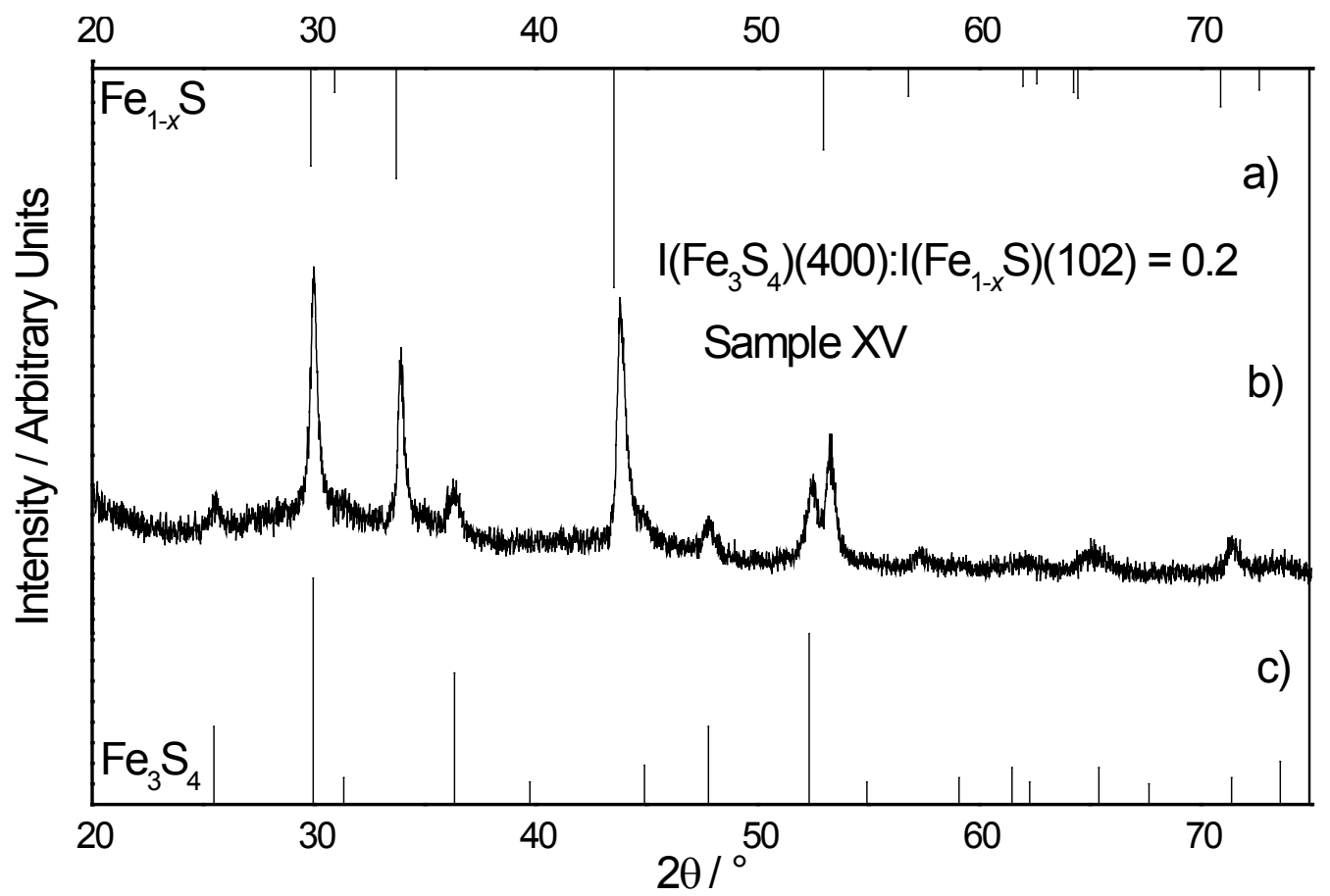

Figure 4-13 XRD pattern of a) reference $\mathrm{Fe}_{1-x} \mathrm{~S}$ b) Sample XV c) reference $\mathrm{Fe}_{3} \mathrm{~S}_{4}$.

A high resolution micrograph (Figure 4-14 (c)) of one of the crystals of triangular appearance displays two sets of lattice fringes. An FFT of the image is shown in Figure 4-14 (d) and displays apparent six-fold symmetry. The lattice spacing measured from the micrograph for both sets of fringes was $0.36 \mathrm{~nm}(3.6 \AA)$ - which matches that of the (220) planes of $\mathrm{Fe}_{3} \mathrm{~S}_{4}(3.5 \AA)$. The angle between the sets of lattice fringes was measured to be $120^{\circ}$, and the angle between one set of fringes and the edge of the crystallite was measured to be $35^{\circ}$. The [111] zone of $\mathrm{Fe}_{3} \mathrm{~S}_{4}$ contains the $\{220\}$ form of planes, and has $3 m$ point symmetry - which gives the appearance of six-fold rotational symmetry. The measured real space angle of $120^{\circ}$ between the sets of lattice fringes matches the expected reciprocal space angle of $60^{\circ}$ between the planes of the $\{220\}$ form, and the angle between the fringes and the edge matches that between the (220) and (111) planes.

Hence the crystal was determined to be $\mathrm{Fe}_{3} \mathrm{~S}_{4}$ oriented with its [111] zone axis parallel to the electron beam, and bounded by $\{111\}$ facets. A simulation of the [111] net of the $\mathrm{Fe}_{3} \mathrm{~S}_{4}$ reciprocal lattice is given below in Figure 4-14 (e) for comparison with the FFT of the image. The crystal has the appearance of triangular prismatic morphology, however this crystal form is not thermodynamically allowed for a crystal of the cubic system. ${ }^{34}$ A twinned octahedron morphology (a reflection twin of two octahedra with a (111) composition surface) is common for spinel $\left(\mathrm{MgAl}_{2} \mathrm{O}_{4}\right),{ }^{34}$ and other phases of the $m \overline{3} m$ point group, such as diamond. If viewed as a projection down the $[\overline{111}]$ zone, a 
twinned octahedron would appear as an equilateral triangle. A simulation of the morphology of a twinned octahedron of $\mathrm{Fe}_{3} \mathrm{~S}_{4}$ is shown below, both as viewed directly

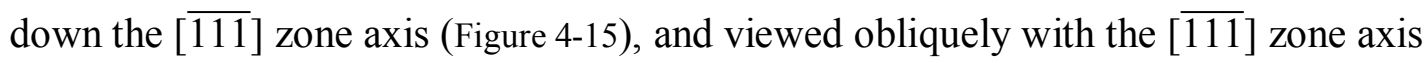
marked by a line.

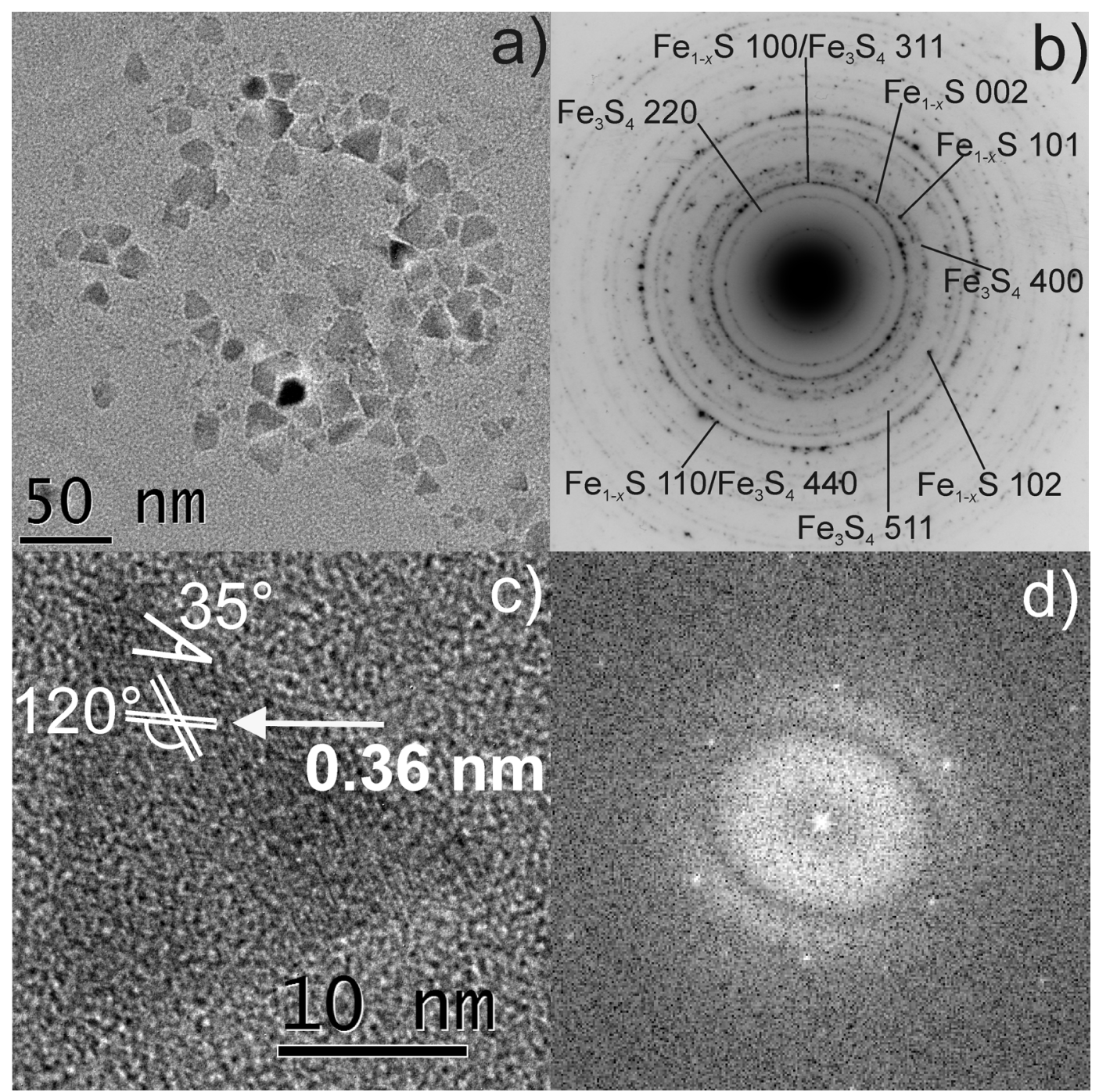

Figure 4-14 a) Micrograph of Sample XV b) SAED pattern of Sample XV, indexed to $\mathrm{Fe}_{1-x} \mathrm{~S}$ and $\mathrm{Fe}_{3} \mathrm{~S}_{4}$ c) high resolution micrograph of single crystal from Sample XV d) FFT of (c) 


\section{[111]}

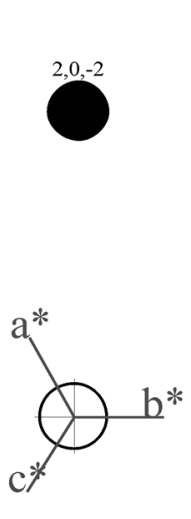

a)

b)

$0^{2.20}$
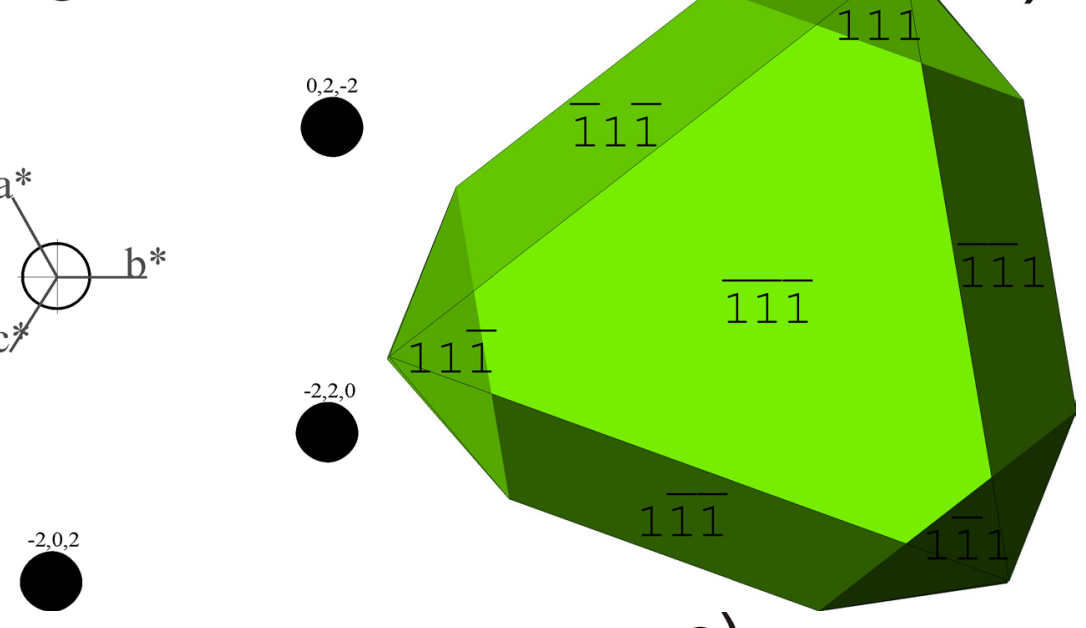

c)

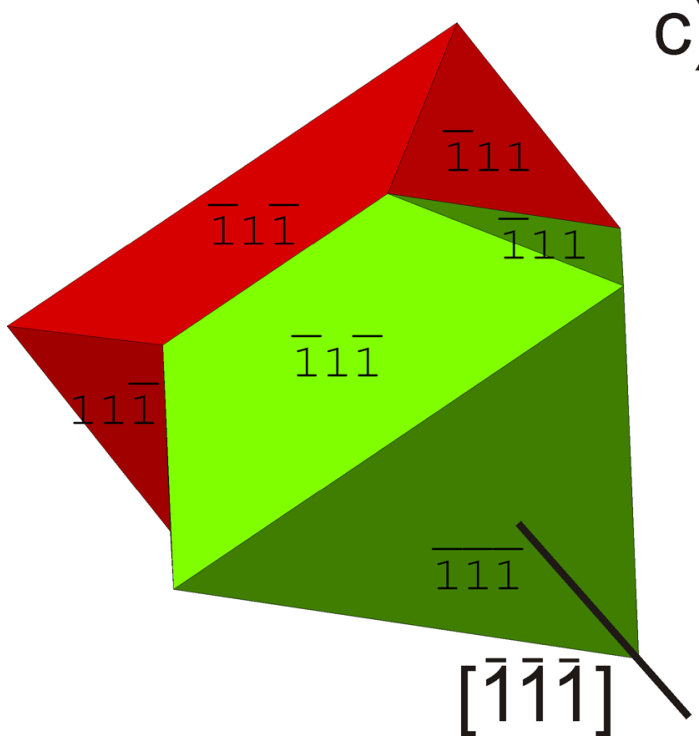

Figure 4-15 a) simulation of the [111] net of the $\mathrm{Fe}_{3} \mathrm{~S}_{4}$ reciprocal lattice

b) simulation of the crystal habit of a twinned octagon (reflection twin with a (111) composition surface), viewed along the [ $\overline{111}]$ zone and

c) viewed obliquely, with the $[\overline{111}]$ zone axis marked by a line.

Scherrer analysis was performed on Sample XV (results are tabulated in Table 4-7, calculations are tabulated in A2-6). The average crystallite size of $\mathrm{Fe}_{1-x} \mathrm{~S}$ at $51 \mathrm{~nm}$ was larger than that of $\mathrm{Fe}_{3} \mathrm{~S}_{4}$ at $20 \mathrm{~nm}$, although the average size of $\mathrm{Fe}_{3} \mathrm{~S}_{4}$ in Sample XV (synthesised from $\mathrm{FeCl}_{2}$ ) is significantly larger than the $5-6 \mathrm{~nm}$ seen in samples synthesised from $\mathrm{Fe}(\mathrm{acac})_{2}$ (Samples XII - XIV). The average size for $\mathrm{Fe}_{3} \mathrm{~S}_{4}$ derived from Scherrer analysis matches well the dimensions of the tetrahedral $\mathrm{Fe}_{3} \mathrm{~S}_{4}$ crystal shown above (Figure 4-14 (c)). 


\subsubsection{Reaction of $\mathrm{Fe}(\mathrm{acac})_{2}$ and sulfur in hexadecylamine}

To ascertain whether solvent affected the size or phase of products, HDA was used instead of oleylamine, as detailed in section 4.5.4. As previous experiments using oleylamine as the solvent indicated that the $\mathrm{Fe}(\mathrm{acac})_{2}: \mathrm{S}$ ratio had no effect on the resulting phase, the ratio was kept constant at 1:1 to avoid an excess of sulfur (see sections 4.2.2.2 and 4.2.2.4). Two reaction temperatures $\left(200\right.$ and $\left.300^{\circ} \mathrm{C}\right)$ were used. All experimental parameters are tabulated below (Table 4-8).

XRD patterns of Samples XVI and XVII are shown below (Figure 4-16). Sample XVI, which was produced at $200^{\circ} \mathrm{C}$ was found to be predominantly $\mathrm{Fe}_{3} \mathrm{~S}_{4}$, with only a trace of $\mathrm{Fe}_{1-x} \mathrm{~S}$ evident in the XRD pattern. The peaks in the XRD were broad, and the amorphous contribution to the background was significant. This indicates that Sample XVI, which was reacted at $200^{\circ} \mathrm{C}$ for 120 minutes, was not as crystalline as Sample VII, which was reacted at $200^{\circ} \mathrm{C}$ for 240 minutes (in oleylamine). In addition, there are peaks in the range $26-29^{\circ} \mathrm{C}$ which do not correspond to $\mathrm{Fe}_{3} \mathrm{~S}_{4}$. These are believed to be from unreacted sulfur, or HDA which was not removed during purification.

Sample XVII, which was produced at $300^{\circ} \mathrm{C}$, was found to be predominantly $\mathrm{Fe}_{1-x} \mathrm{~S}$, with a trace of $\mathrm{Fe}_{3} \mathrm{~S}_{4}$. The $\mathrm{I}_{400}: \mathrm{I}_{102}$ ratio is 0.1 , the same as all other samples synthesised at $300^{\circ} \mathrm{C}$ for $>10$ minutes. The XRD pattern of Sample XVII contains sharp peaks, and little background, indicating good crystallinity.

Table 4-8 Reaction parameters for reaction of $\mathrm{Fe}(\mathrm{acac})_{2}$ and sulfur in HDA.

\begin{tabular}{|c|c|c|c|c|c|c|}
\hline Sample & $\mathrm{Fe}(\mathrm{acac})_{2}: \mathrm{S}$ & $\begin{array}{l}{\left[\mathrm{Fe}(\mathrm{acac})_{2}\right]} \\
/ \mathrm{mol} \mathrm{dm}^{-3}\end{array}$ & $\begin{array}{c}\text { Reaction } \\
\text { Temp. } \\
/{ }^{\circ} \mathrm{C}\end{array}$ & $\begin{array}{c}\text { Reaction } \\
\text { Time } \\
\text { / min }\end{array}$ & Result & $\begin{array}{l}D_{h k l} \\
/ \mathrm{nm}\end{array}$ \\
\hline \multirow{3}{*}{ XVI } & \multirow{3}{*}{$1: 1$} & \multirow{3}{*}{0.05} & \multirow{3}{*}{200} & \multirow{3}{*}{120} & $\mathrm{Fe}_{3} \mathrm{~S}_{4}$ & $29 \pm 14$ \\
\hline & & & & & & \\
\hline & & & & & $\begin{array}{l}\text { Trace } \\
\mathrm{Fe}_{1-x} \mathrm{~S}\end{array}$ & 48 \\
\hline \multirow{2}{*}{ XVII } & \multirow{2}{*}{$1: 1$} & \multirow{2}{*}{0.05} & \multirow{2}{*}{300} & \multirow{2}{*}{30} & $\begin{array}{l}\text { Trace } \\
\mathrm{Fe}_{3} \mathrm{~S}_{4}\end{array}$ & I \\
\hline & & & & & $\mathrm{Fe}_{1-\mathrm{x}} \mathrm{S}$ & $115 \pm 37$ \\
\hline
\end{tabular}




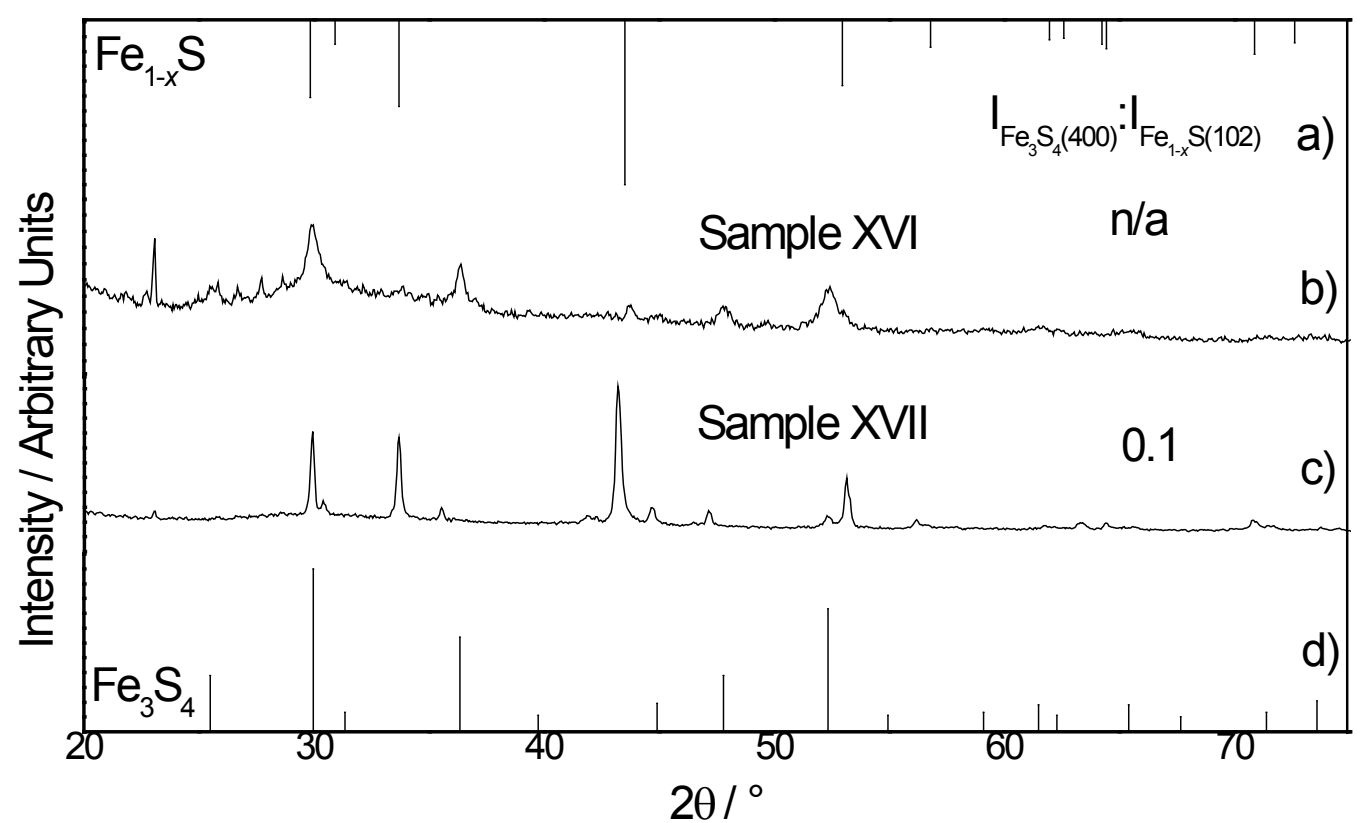

Figure 4-16 XRD patterns of a) reference $\mathrm{Fe}_{1-x} \mathrm{~S}$ b) Sample XVI c) Sample XVII d) reference $\mathrm{Fe}_{3} \mathrm{~S}_{4}$.

A micrograph and SAED pattern of Sample XVI are shown below (Figure 4-17), and indexing parameters for the SAED pattern are tabulated in A1-6. The micrograph of Sample XVI showed it to consist of irregularly shaped, sheet-like material - in contrast to the well defined rectangular $\mathrm{Fe}_{3} \mathrm{~S}_{4}$ sheets of Sample VII, which was reacted for 240 minutes at $200^{\circ} \mathrm{C}$. Three large, faceted crystals typically identified with the $\mathrm{Fe}_{1-x} \mathrm{~S}$ phase in previous samples, are evident in the circled area of the micrograph. The SAED pattern of the area is a well developed ring pattern, which was indexed to $\mathrm{Fe}_{3} \mathrm{~S}_{4}$. A single systematic row of reflections from a $\mathrm{Fe}_{1-x} \mathrm{~S}$ crystal are labelled in the SAED pattern. In conclusion, the sample consists of irregularly shaped polycrystalline $\mathrm{Fe}_{3} \mathrm{~S}_{4}$ with some amount of faceted $\mathrm{Fe}_{1-x} \mathrm{~S}$ crystallites.

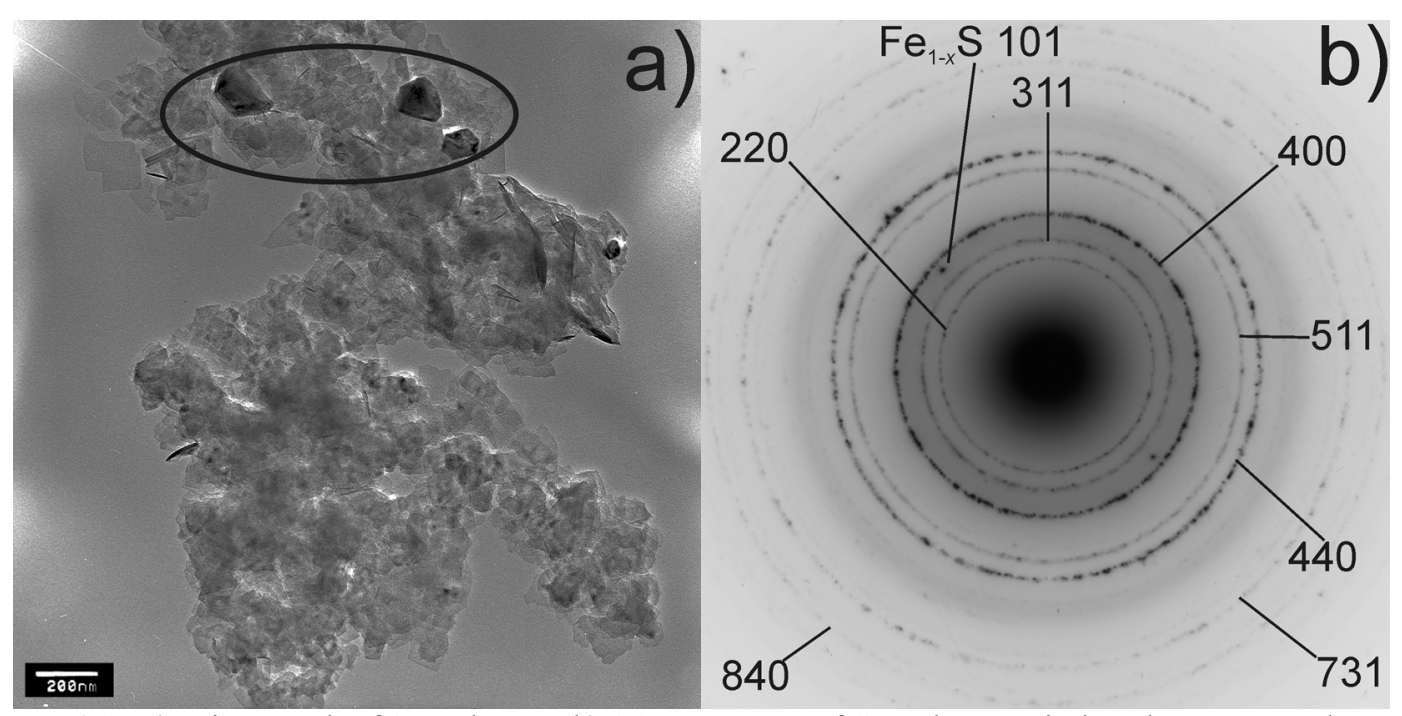

Figure 4-17 a) Micrograph of Sample XVI b) SAED pattern of Sample XVI, indexed to $\mathrm{Fe}_{3} \mathrm{~S}_{4}$ and containing a systematic row of reflections from a single $\mathrm{Fe}_{1-x} \mathrm{~S}$ crystallite. 
Scherrer analysis was performed on Samples XVI \& XVII (results are tabulated in Table 4-8, calculations are tabulated in A2-7). The average crystallite size of $\mathrm{Fe}_{1-x} \mathrm{~S}$ is larger than $\mathrm{Fe}_{3} \mathrm{~S}_{4}$ in both samples. At $29 \mathrm{~nm}$, the average crystallite size of $\mathrm{Fe}_{3} \mathrm{~S}_{4}$ is similar to other long duration reactions with oleylamine as the solvent, such as Sample VII. 


\subsubsection{Addition of $\mathrm{FeCl}_{2}$ into solution of sulfur in oleylamine}

As the average crystallite size of the $\mathrm{Fe}_{1-x} \mathrm{~S}$ phase was found to be consistently large throughout previous experiments (see sections 4.2.2.2 and 4.2.2.4), quenching experiments were used to investigate the rate of growth of $\mathrm{Fe}_{1-x} \mathrm{~S}$ crystallites, as detailed in section 4.5.5. $\mathrm{FeCl}_{2}$ dissolved in tetraglyme was used as the iron source, as a solution of higher concentration could be achieved than was possible using $\mathrm{Fe}(\mathrm{acac})_{2}$. The $\mathrm{FeCl}_{2}$ solution was injected into the preheated solution of sulfur in oleylamine, and aliquots were withdrawn and quenched by injection through a septum into cold, degassed acetone stored under a nitrogen atmosphere in order to prevent oxidation. All experimental parameters are tabulated below (Table 4-9).

Micrographs and SAED patterns for aliquots withdrawn after 0.5 , 5, and 30 minutes are shown below (Figure 4-18) (indexing parameters for SAED patterns of the 0.5 and 5 minute aliquots are tabulated in A1-7 and A1-8, respectively). After 0.5 minutes the sample consisted of irregularly shaped, sheet-like material. The SAED pattern was poorly developed, indicating low crystallinity in the sample. The SAED pattern was tentatively indexed to $\mathrm{FeS}_{2}(\mathrm{p})$. After 5 minutes that sample contained a large number of large (>50 nm) faceted crystallites, although there was still some sheet-like material enveloping the faceted crystallites. The SAED pattern is indexed to $\mathrm{Fe}_{1-x} \mathrm{~S}$, and the sharp, well developed rings indicate that the sample was highly crystalline. After 30 minutes, most of the sheet-like material was gone, leaving the faceted $\mathrm{Fe}_{1-x} \mathrm{~S}$ crystals as the major constituent. EDS results for aliquots taken at 5 and 30 minutes give compositions of Fe:S 48:52 - 50:50 - appropriate for $\mathrm{Fe}_{1-x} \mathrm{~S}$, which was identified as the crystalline product in these samples.

This experiment suggests that $\mathrm{FeS}_{2} \mathrm{p}$ may be an intermediate in the iron sulfide formation reaction. However it is evident that $\mathrm{FeS}_{2} \mathrm{p}$ does not remain present for more than a few minutes at a reaction temperature of $260^{\circ} \mathrm{C}$. In addition, it was evident from this quenching experiment that the large crystallite size and anisotropic crystal habit of $\mathrm{Fe}_{1-x} \mathrm{~S}$ is established within 5 minutes of the initiation of the reaction. Therefore the use of very short reaction times will not suffice to produce small $\mathrm{Fe}_{1-x} \mathrm{~S}$ crystallites. 
Table 4-9 Experimental parameters for the addition of $\mathrm{FeCl}_{2}$ into solution of sulfur in oleylamine.

\begin{tabular}{|c|c|c|c|c|c|}
\hline Sample & $\mathrm{FeCl}_{2}: \mathrm{S}$ & {$\left[\mathrm{FeCl}_{2}\right]$} & $\begin{array}{c}\text { Reaction } \\
\text { Temp. } \\
/{ }^{\circ} \mathrm{C}\end{array}$ & $\begin{array}{c}\text { Reaction } \\
\text { Time } \\
/ \text { min }\end{array}$ & Result \\
\hline XVIII & $1: 1$ & 0.01 & 260 & 0.5 & Sheet-like FeS $_{2} \mathrm{p}$ \\
\hline & & & & 5 & $\mathrm{Fe}_{1-x} \mathrm{~S}$ \\
\hline & & & & 30 & $\mathrm{Fe}_{1-x} \mathrm{~S}$ \\
\hline
\end{tabular}
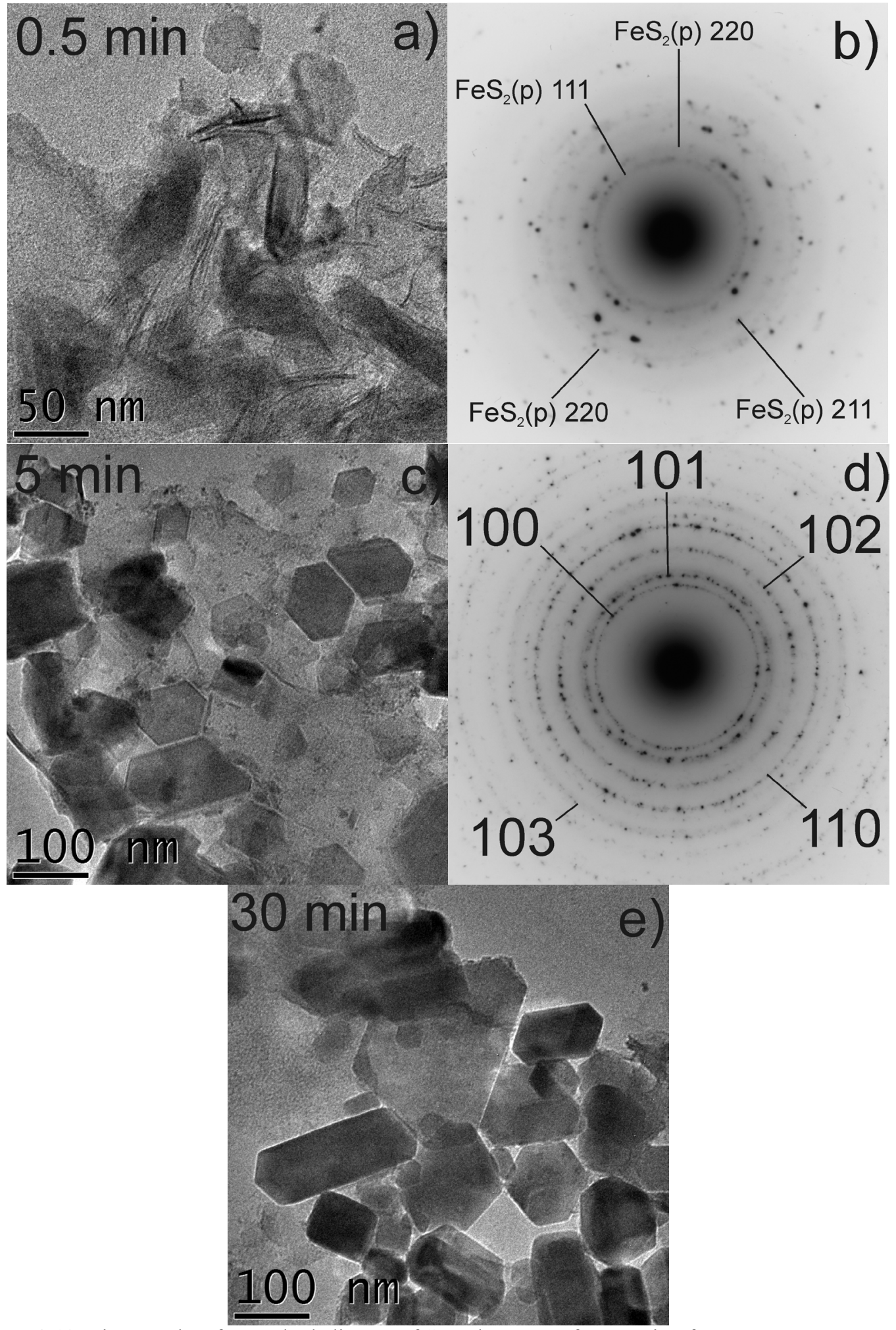

Figure 4-18 Micrographs of quenched aliquots of Sample XVIII after reaction for

a) 0.5 minutes c) 5 minutes e) 30 minutes;

b) and c) SAED patterns after 0 and 5 minutes, indexed to $\mathrm{FeS}_{2}(\mathrm{p})$ and $\mathrm{Fe}_{1-x} \mathrm{~S}$, respectively. 


\subsubsection{Crystal structure of $\mathrm{Fe}_{1-x} \mathrm{~S}$}

As mentioned above in Chapter 1 section 1.3.2.5, the base NiAs-type structure of $\mathrm{Fe}_{1-x} \mathrm{~S}$ (1C) is not stable below $90-100^{\circ} \mathrm{C}$, and at room temperature $\mathrm{Fe}_{1-x} \mathrm{~S}$ can be expected to have adopted some form of ordered vacancy superlattice. Attempts were made to identify the composition and structure of $\mathrm{Fe}_{1-x} \mathrm{~S}$ phase particles synthesised in this project. Sample X was used for this purpose, as it represented a sample of pure, highly crystalline $\mathrm{Fe}_{1-x} \mathrm{~S}$.

The unit cell parameters of a non-stoichiometric material vary linearly with its composition, without any change in the crystal symmetry of the compound (Vegard's law). ${ }^{35}$ Applying this principle, Yund and Hall determined an empirical expression for the composition of $\mathrm{Fe}_{1-x} \mathrm{~S}$ in terms of atomic percent iron, $Y$, based upon the $d$-spacing of the 102 reflection $\left(d_{102}\right):^{1}$

$Y=45.212+72.86\left(d_{102}-2.0400\right)+311.5\left(d_{102}-2.0400\right)^{2}$

The composition of Sample X was calculated from this expression; after the value of $d_{102}$ had been determined from XRD patterns which had been corrected against a silicon calibrant (see Chapter 2 section 2.2.2.1). Sample X was found to have a composition of 48 percent iron, corresponding to a composition of $\mathrm{Fe}_{12} \mathrm{~S}_{13}$. This matches well with the composition as determined by EDS measurements taken at the time the XRD pattern was recorded (Fe:S 48:52, $\sim \mathrm{Fe}_{12} \mathrm{~S}_{13}$ ).

Powder XRD of Sample X did not indicate any splitting of the 102 reflection of the NiAs-type structure (at $2.06 \AA, 44^{\circ} 2 \theta$ : see Figure 4-7 (c)) into a doublet of the 408 and $\overline{4} 08$ reflections of monoclinic $4 \mathrm{C} \mathrm{Fe}_{1-x} \mathrm{~S}\left(\mathrm{Fe}_{7} \mathrm{~S}_{8}\right)$, indicating that the sample retains hexagonal symmetry. ${ }^{36}$ This fits with the determined composition, which is more iron-rich than would be the case for monoclinic $4 \mathrm{C} \mathrm{Fe} \mathrm{1}_{-x} \mathrm{~S}$.

An SAED pattern of a single crystal from Sample $X$ is shown below (Figure 4-19), and contains both sublattice (NiAs-type reflections) and superlattice reflections. Figure 4-19 (a) shows the indexing of the NiAs-type reflections, which led to the assignment of the pattern to the [110] zone of $\mathrm{Fe}_{1-x} \mathrm{~S}$. Figure 4-19 (b) shows in italics the indices of those reflections which are kinematically-forbidden due to the $c$-glide of the $P 6_{3} m c$ space group of NiAs-type $\mathrm{Fe}_{1-x} \mathrm{~S}(00 l(l \neq 2 \mathrm{n}))$. Due to dynamical diffraction effects, 
kinematically-forbidden reflections are still in general observed in electron diffraction experiements. ${ }^{37}$ In addition to these allowed and kinematically-forbidden refection, rows of weak superlattice reflections are visible between the central spot and the $11 \mathrm{l}$ systematic row. The spacing of these superlattice reflections in directions parallel to the $c^{*}$ axis - as indicated in Figure 4-19 (b) - suggests a doubling of the lattice parameter $c$.

Spacing of superlattice reflection rows parallel to the $[110]^{*}$ direction indicates a fourfold increase in the $a$ and $b$ lattice parameters. However a 4A 2C type superlattice has not been observed previously in $\mathrm{Fe}_{1-x} \mathrm{~S} .{ }^{38} \mathrm{~A}$ model constructed on the basis of a $4 \mathrm{~A}$ $2 \mathrm{C}$ supercell with a composition of $\mathrm{Fe}_{59} \mathrm{~S}_{64}$ yielded a simulated [110] zone SAED pattern (see Figure 4-20) demonstrating features observed in the experimental pattern. However, as full determination of the $3 \mathrm{D}$ distribution of vacancies requires SAED patterns from at least two zones perpendicular to the $c$ axis (e.g. [100], [010] or [110]), ${ }^{39}$ the model cannot be properly tested using a single SAED pattern. As tilting the same crystallite between the aforementioned zones would require a special high-tilt goniometer, it is outside the capability of the TEM used in this project, and sufficient data could not be gathered to test the model.

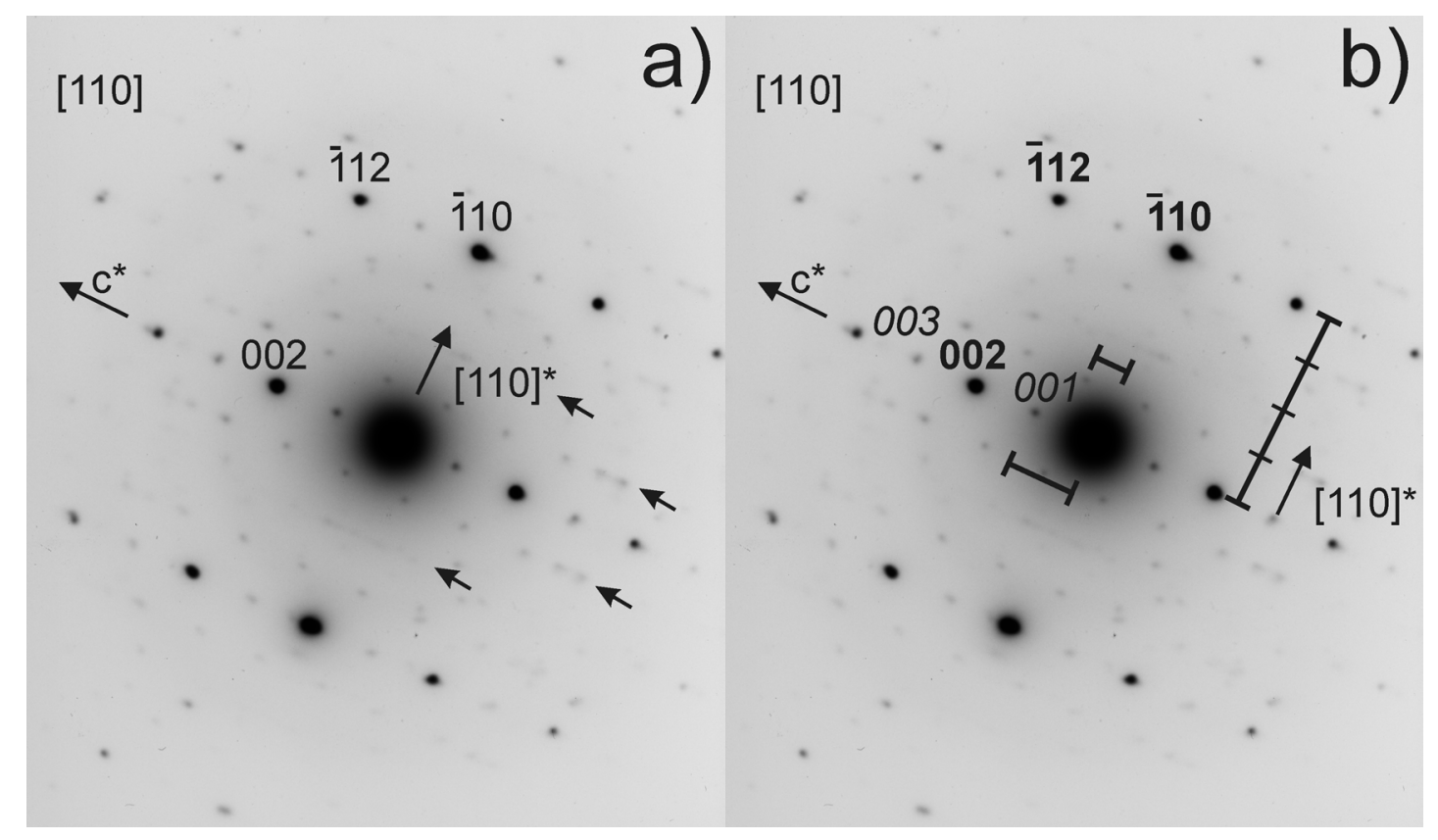

Figure 4-19 a) SAED pattern of $\mathrm{Fe}_{1-x} \mathrm{~S}$ from Sample $\mathrm{X}$, indexed to the [110] zone. Indices based on NiAs-type structure. Rows of weak superlattice reflections are indicated by arrows.

b) SAED pattern of $\mathrm{Fe}_{1-x} \mathrm{~S}$ : allowed substructure reflections indexed in bold, kinematically-forbidden reflections indexed in italics. Weak superlattice reflections showing an apparent doubling of the $c$ axis, and a fourfold increase parallel to the $[110]^{*}$ are indicated. 


\section{[110]}

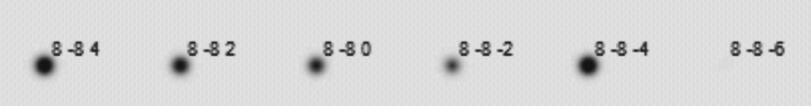

$\uparrow[110]^{*}$
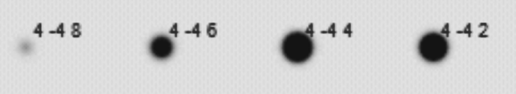

$\mathrm{C}^{4-40}$
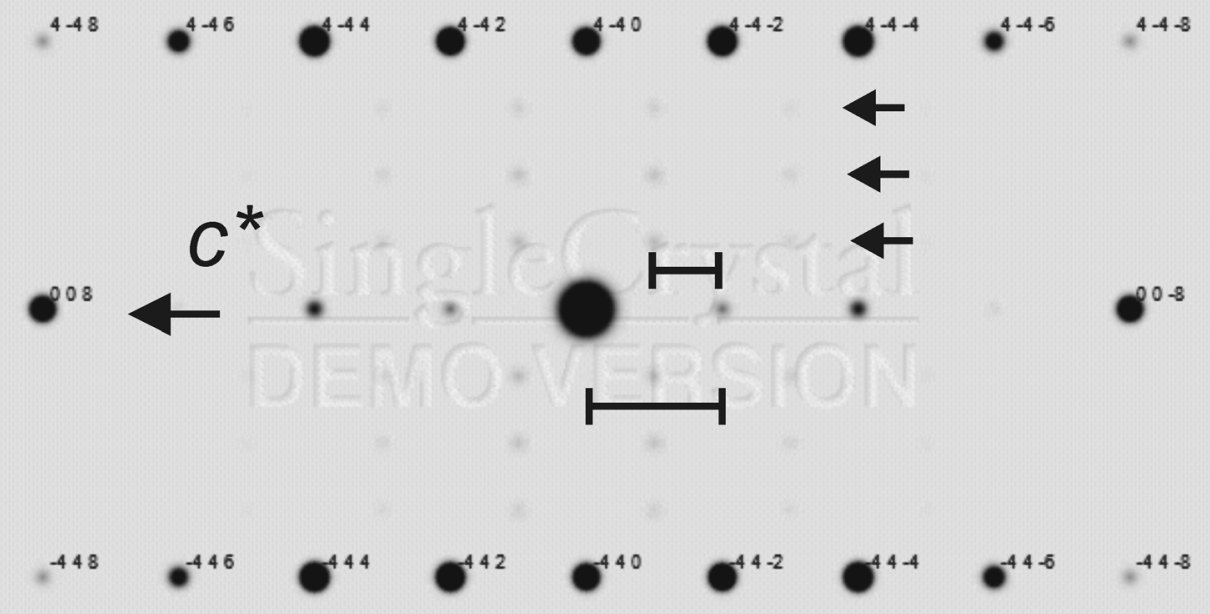

$-886$
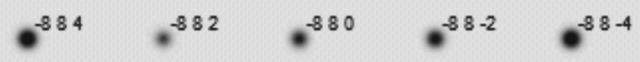

Figure 4-20 Simulated [110] zone SAED pattern for a hypothetical 4A $2 \mathrm{C} \mathrm{Fe}_{1-x} \mathrm{~S}$ superlattice. Indexing here refers to the supercell dimensions.

Superlattice reflections were not evident in the XRD pattern of Sample X (see Figure 4-7 (c)), when recorded on an amorphous PMMA holder which contributed significantly to the background. A sample was synthesised using the same parameters as Sample X, in order to obtain more material to perform further XRD experiments with. An XRD pattern was measured using a low-back ground holder, in order to reveal any weak superlattice reflections which had been buried by the contribution of the PMMA holder (note that as $\mathrm{Co} \mathrm{K}_{\alpha}$ radiation was used in this experiment reflections appear at different $2 \theta$ values than all other experiments, which were performed using $\mathrm{Cu} \mathrm{K}_{\alpha}$ radiation). A simulated XRD pattern (using Co $\mathrm{K}_{\alpha}$ radiation) for the $2 \mathrm{C}$ structure of NiAs-type $\mathrm{Fe}_{1-x} \mathrm{~S}$ is shown below with the experimental pattern (Figure 4-21 (a) and (b), respectively). There is a good correspondence between the non-NiAs-type reflections (marked with an asterisk) in the simulated and experimental patterns. 


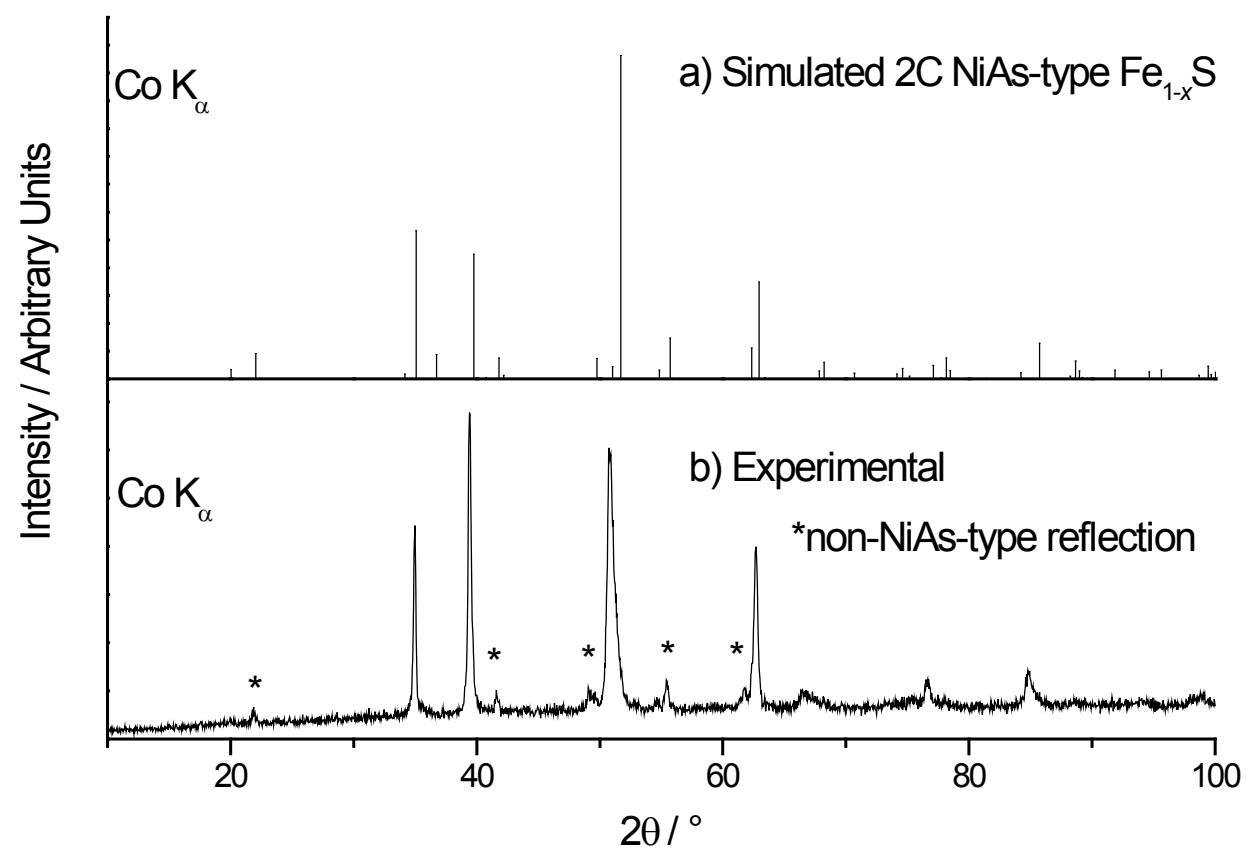

Figure 4-21 a) Simulated diffraction pattern for $2 \mathrm{C}$ NiAs-type $\mathrm{Fe}_{1-x} \mathrm{~S}$ b) experimental diffraction pattern for $\mathrm{Fe}_{1-x} \mathrm{~S}$ using low background holder and $\mathrm{Co} \mathrm{K}_{\alpha}$ radiation. Reflections not attributable to the base NiAs-type substructure are marked with an asterisk. 


\subsubsection{Magnetic Measurements}

In order to characterise the magnetic properties of the synthesised iron sulfide nanocrystals, magnetic measurements were performed on a number of samples. Details of magnetic experiments are outlined in Chapter 2 section 2.2.6. Measurements were conducted on a sample of pure $\mathrm{Fe}_{1-x} \mathrm{~S}$ (Sample $\mathrm{X}$ ), and on samples with varying proportions and morphologies of $\mathrm{Fe}_{3} \mathrm{~S}_{4}$ crystallites (Sample VII, containing $\mathrm{Fe}_{3} \mathrm{~S}_{4}$ sheets; and Sample XIII containing $6 \mathrm{~nm}$ spherical $\mathrm{Fe}_{3} \mathrm{~S}_{4}$ nanocrystals).

\subsubsection{Pure $\mathrm{Fe}_{1-x} \mathrm{~S}$ (Sample X)}

A ZFC/FC plot for Sample X (pure $\mathrm{Fe}_{1-x} \mathrm{~S}$ ) is shown below (Figure 4-22). The ZFC and FC traces are non-coincident for all measured temperatures $(10-300 \mathrm{~K})$, indicating that the sample demonstrates magnetic irreversibility throughout this temperature range. No magnetic transitions were evident within the measured temperature range.

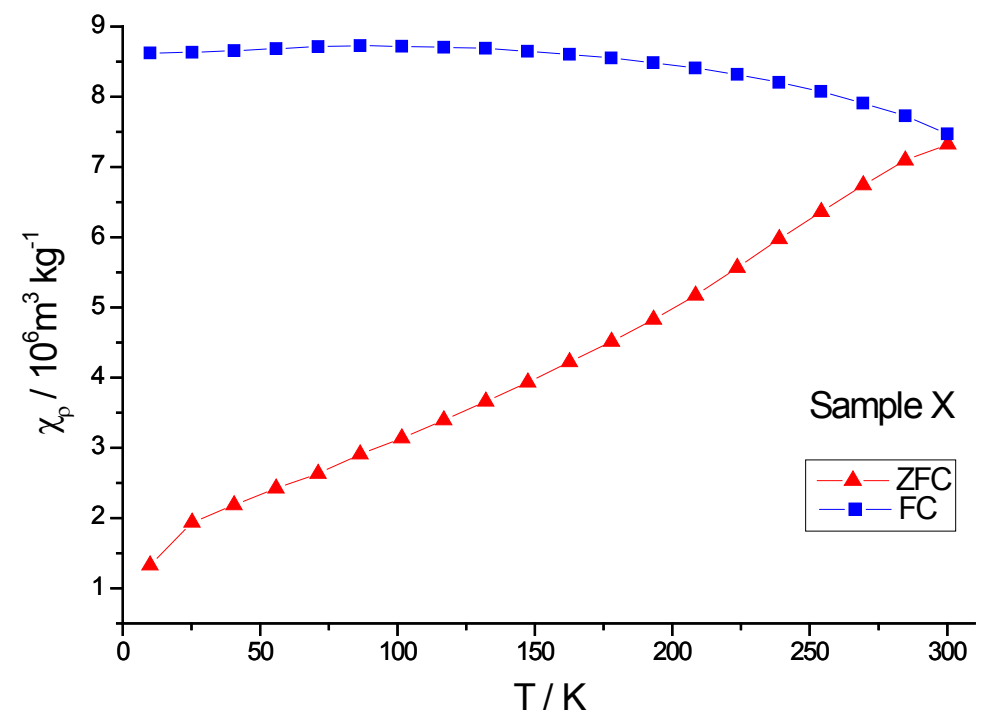

Figure 4-22 ZFC/FC plot of Sample X (pure $\mathrm{Fe}_{1-x} \mathrm{~S}$ ). $\mathrm{H}=1990 \mathrm{~A} \mathrm{~m}^{-1}$.

Field loops for Sample X at 10 and $300 \mathrm{~K}$ are shown below (Figure 4-23 and Figure 4-24, respectively). Magnetic parameters derived from the field loops are tabulated in Table 4-10. The field loops have a strong diagonal slope, and the sample is incompletely saturated even at the maximum applied field of $4.8 \times 10^{6} \mathrm{~A} \mathrm{~m}^{-1}$. The decomposition procedure described in Chapter 2 section 2.2.6.3 was used to estimate an approximate saturation magnetisation (the processed field loop is shown in Figure 4-23 (b)). At both 10 and $300 \mathrm{~K}$ the sample displays coercivity, and the field loops have a constricted or wasp-waisted appearance. 
Table 4-10 Magnetic parameters for Sample $\mathrm{X}$ (pure $\mathrm{Fe}_{1-x} \mathrm{~S}$ ).

\begin{tabular}{|c|c|c|c|c|c|}
\hline & $\begin{array}{c}\text { Temperature } \\
/ \mathrm{K}\end{array}$ & $M_{s} / \mathrm{A} \mathrm{m}^{2} \mathrm{~kg}^{-1}$ & $M_{r} / \mathrm{A} \mathrm{m}^{2} \mathrm{~kg}^{-1}$ & $M_{\mathrm{r}} / M_{s}$ & $H_{c} / \mathrm{kA} \mathrm{m}^{-1}$ \\
\hline Sample X & 10 & 1.5 & 0.6 & 0.40 & 294 \\
\hline & 300 & 2.6 & 0.4 & 0.15 & 112 \\
\hline
\end{tabular}

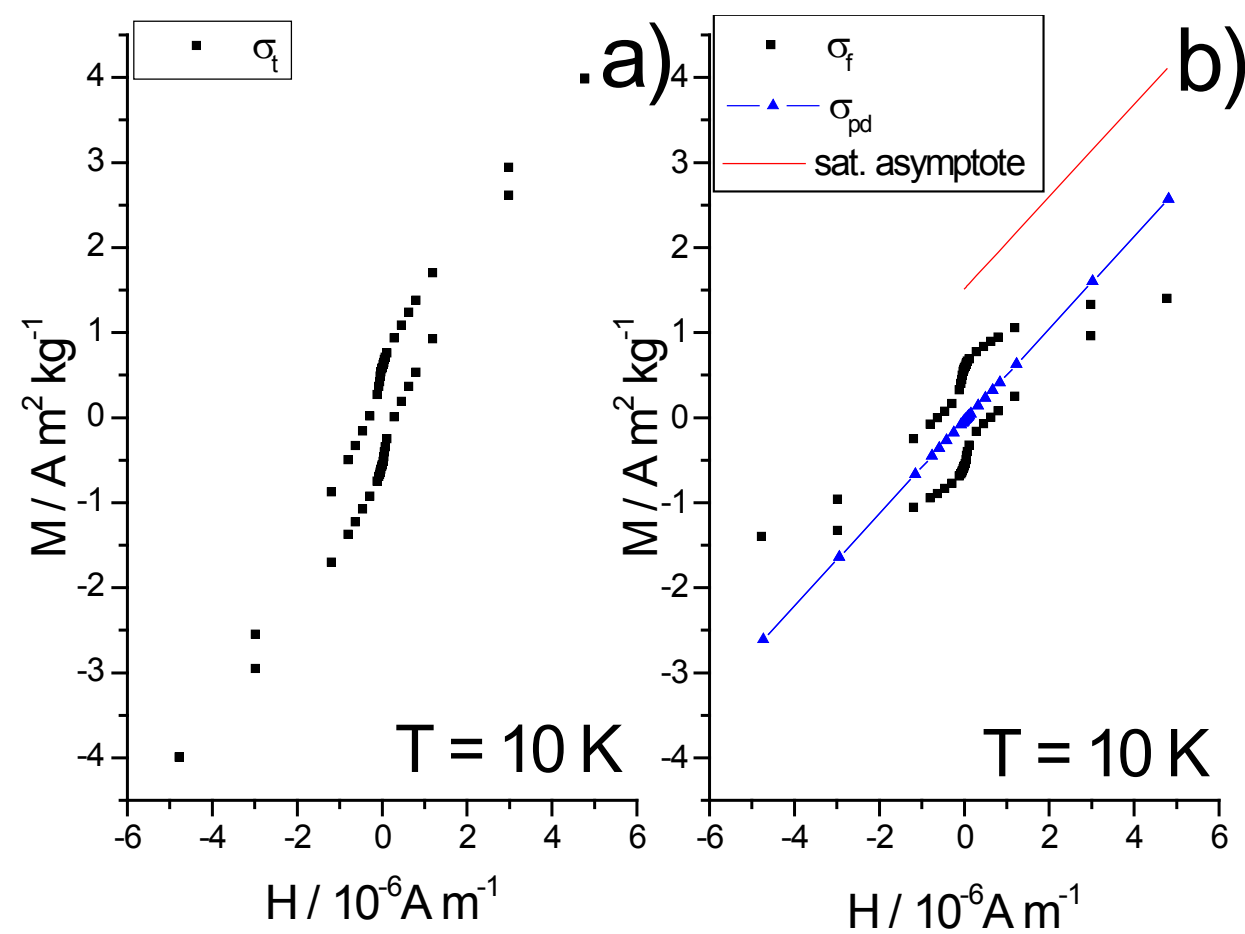

Figure 4-23 Field loop of Sample $\mathrm{X}, \mathrm{T}=10 \mathrm{~K}$ : a) raw data b) processed data.
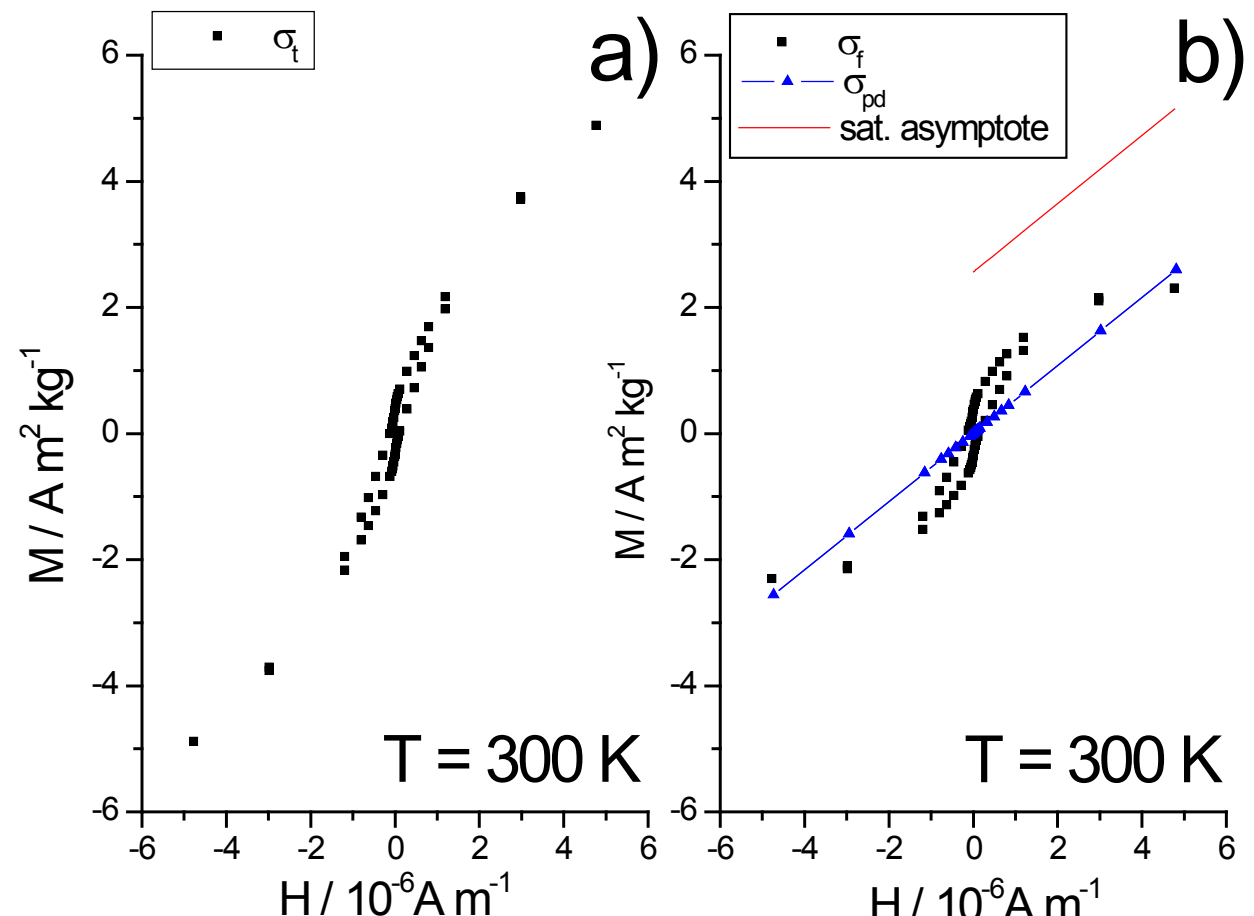

$\mathrm{H} / 10^{-6} \mathrm{~A} \mathrm{~m}^{-1}$

Figure 4-24 Field loop of Sample $X, T=300 \mathrm{~K}$ : a) raw data b) processed data. 


\subsubsection{2 $\quad \mathrm{Fe}_{1-x} \mathrm{~S} / \mathrm{Fe}_{3} \mathrm{~S}_{4}$ mixtures}

Sample VII contained mainly $\mathrm{Fe}_{3} \mathrm{~S}_{4}$ sheets; however $\mathrm{Fe}_{1-x} \mathrm{~S}$ was also identified in the XRD pattern. A ZFC/FC plot for Sample VII is shown below (Figure 4-25). The ZFC and $\mathrm{FC}$ traces are non-coincident for the measured temperature range $10-300 \mathrm{~K}$, indicating magnetic irreversibility throughout this temperature range. There is a sharp increase in the susceptibility in the ZFC trace between 10 and $25 \mathrm{~K}$, however without more data points within that temperature range it is difficult to determine whether there is a definite magnetic transition occurring in this temperature range. The susceptibility of Sample VII is notably higher than Sample X (pure $\mathrm{Fe}_{1-x} \mathrm{~S}$ ), due to the presence of ferrimagnetic $\mathrm{Fe}_{3} \mathrm{~S}_{4}$.

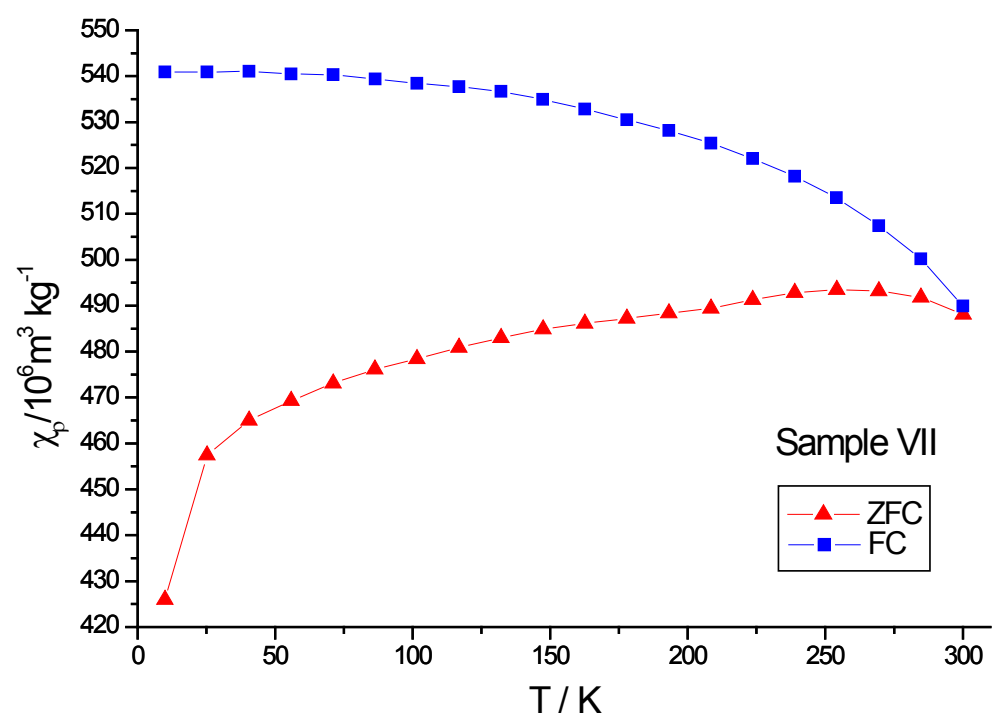

Figure 4-25 ZFC/FC plot of Sample VII. H=1990 A m ${ }^{-1}$.

Field loops for Sample VII recorded at 10 and $300 \mathrm{~K}$ are shown below (Figure 4-26 and Figure 4-27, respectively), and magnetic parameters derived from the field loops are tabulated below (Table 4-11). At both 10 and $300 \mathrm{~K}$ Sample VII displays very low coercivity, and the field loops are essentially closed at high field (forward and backward quadrants of the loop are coincident). In contrast to Sample X, Sample VII is essentially saturated with applied fields of 3- $4.8 \times 10^{6} \mathrm{~A} \mathrm{~m}^{-1}$. At $16.9 \mathrm{~A} \mathrm{~m}^{2} \mathrm{~kg}^{-1}$ (at $300 \mathrm{~K}$ ) the saturation magnetisation of Sample VII is substantially larger than that of Sample X (pure $\mathrm{Fe}_{1-x} \mathrm{~S}$ ), reflecting the far higher susceptibility of ferrimagnetic $\mathrm{Fe}_{3} \mathrm{~S}_{4}$ compared to antiferromagnetic $\mathrm{Fe}_{1-x} \mathrm{~S}$. 
Table 4-11 Magnetic parameters for Sample VII.

\begin{tabular}{|c|c|c|c|c|c|}
\hline & $\begin{array}{c}\text { Temperature } \\
/ \mathrm{K}\end{array}$ & $M_{s} / \mathrm{A} \mathrm{m}^{2} \mathrm{~kg}^{-1}$ & $M_{r} / \mathrm{A} \mathrm{m}^{2} \mathrm{~kg}^{-1}$ & $M_{\mathrm{r}} / M_{s}$ & $H_{c} / \mathrm{kA} \mathrm{m}^{-1}$ \\
\hline $\begin{array}{c}\text { Sample } \\
\text { VII }\end{array}$ & 10 & 16.9 & 8.2 & 0.48 & 28 \\
\hline & 300 & 14.3 & 5.2 & 0.36 & 15 \\
\hline
\end{tabular}

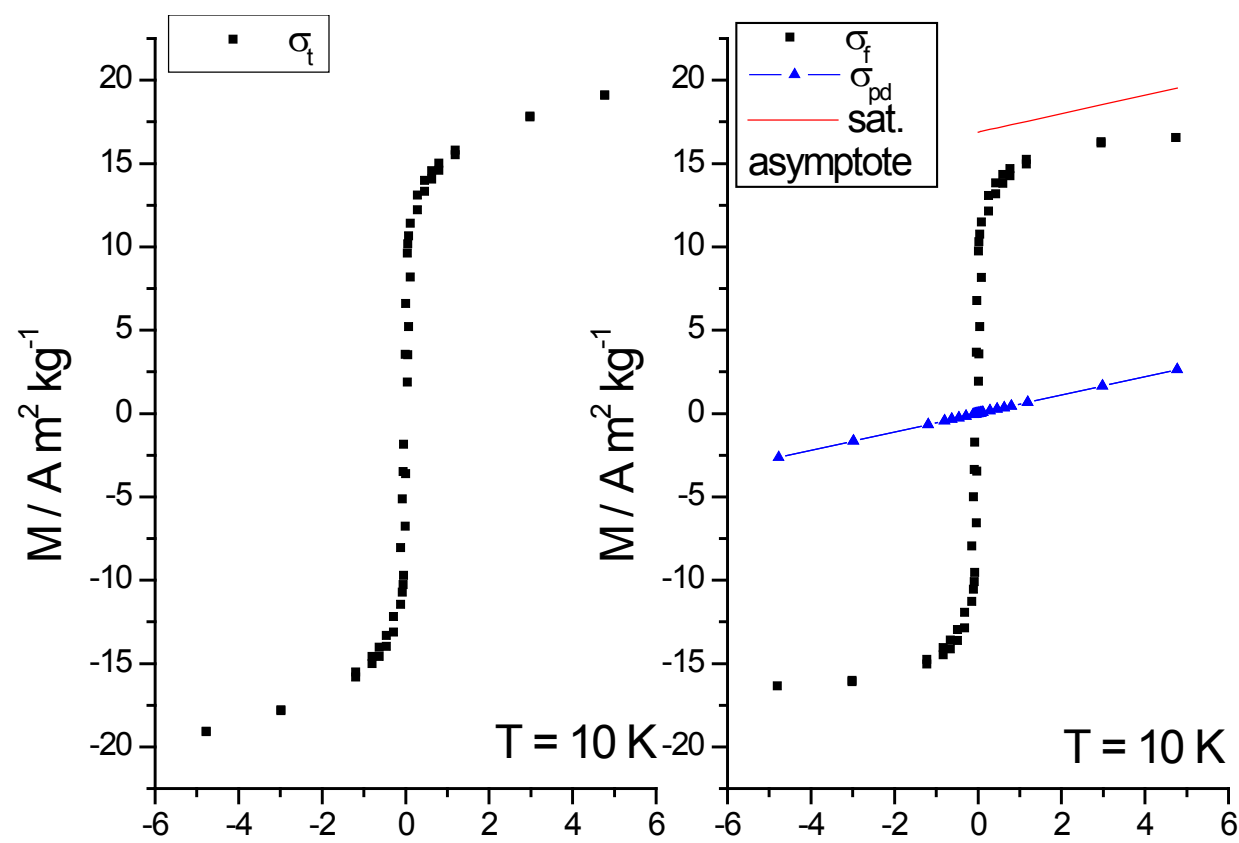

$$
\mathrm{H} / 10^{-6} \mathrm{~A} \mathrm{~m}^{-1} \quad \mathrm{H} / 10^{-6} \mathrm{~A} \mathrm{~m}^{-1}
$$

Figure 4-26 Field Loop of Sample VII, $\mathrm{T}=10 \mathrm{~K}$ : a) raw data b) processed data.

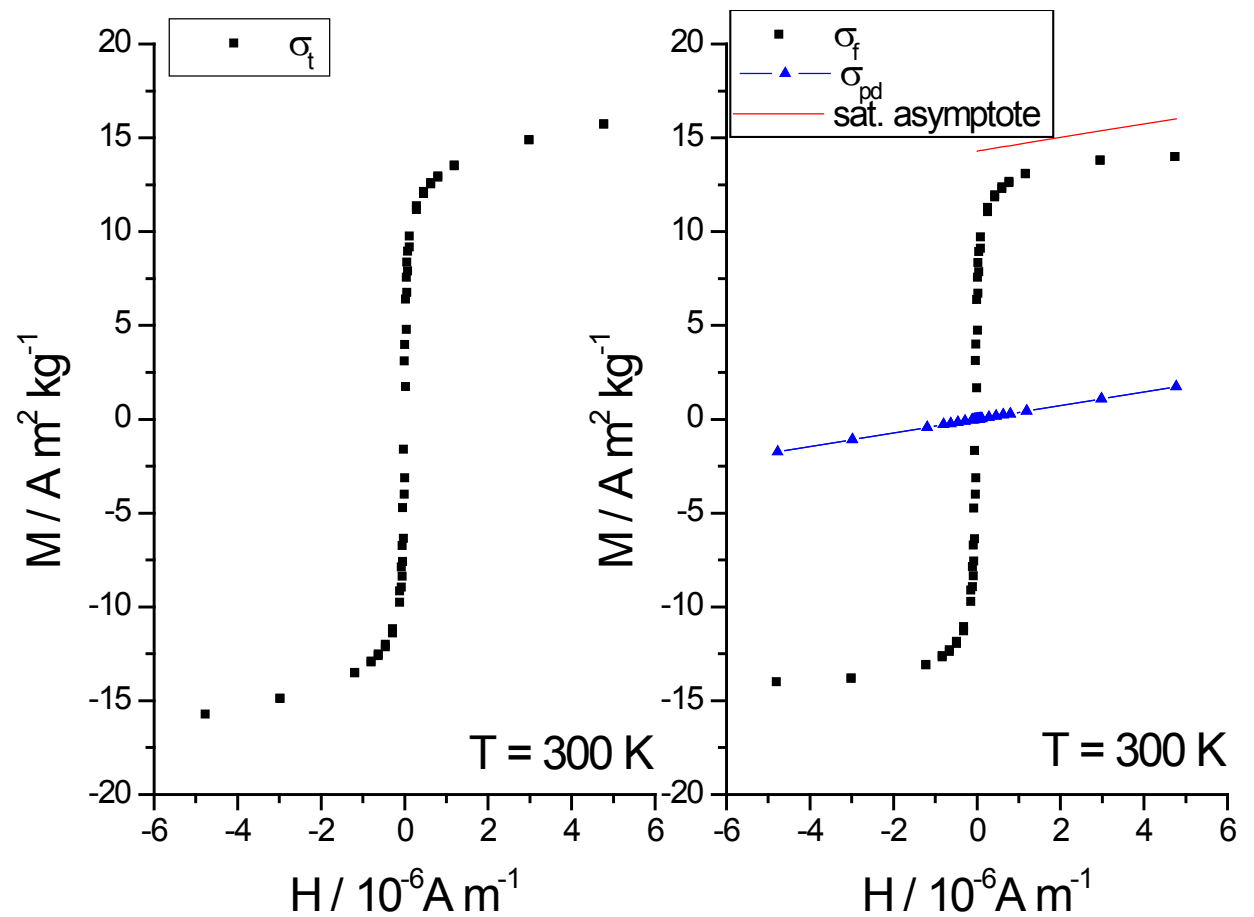

Figure 4-27 Field Loop of Sample VII, $\mathrm{T}=300 \mathrm{~K}$ : a) raw data b) processed data. 
Sample XII was comprised predominantly of $\mathrm{Fe}_{1-x} \mathrm{~S}$, with a trace of $5 \mathrm{~nm} \mathrm{Fe} \mathrm{S}_{4}$ nanocrystals. A ZFC/FC plot is shown below (Figure 4-28). The ZFC and FC traces are non-coincident between 10 and $270 \mathrm{~K}$, but coincide above this temperature. Both the ZFC and FC curve demonstrate fluctuations. The experiment was repeated with the same result, to ensure these fluctuations were not due to measurement error. The fluctuations suggest contributions from a range of sub-populations within the sample presumably due to the mixture of phases and crystallite sizes present.

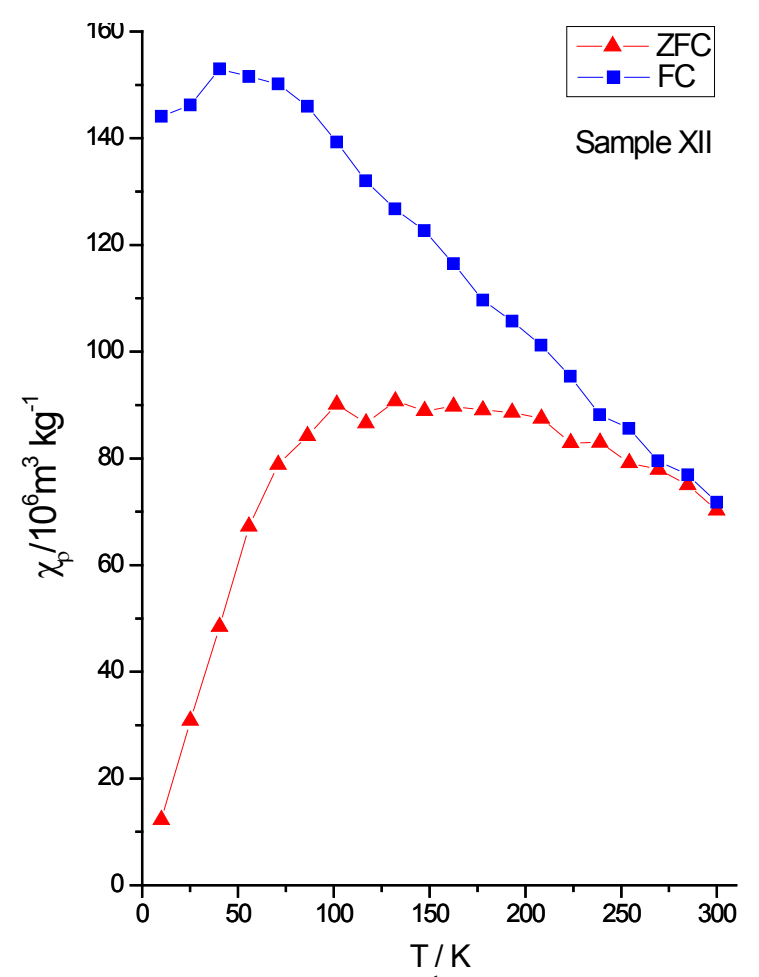

Figure 4-28 ZFC/FC plot of Sample XII. H=1990 A m ${ }^{-1}$.

Field loops for Sample XII at 10, 200 and $300 \mathrm{~K}$ are shown below (Figure 4-29, Figure 4-30 and Figure 4-31, respectively), and magnetic parameters derived from the field loops are tabulated in Table 4-12. Field loops of Sample XII are wasp-waisted, but with far lower coercivities than Sample X (pure $\mathrm{Fe}_{1-x} \mathrm{~S}$ ). The coervicity of the sample drops between 10 and $200 \mathrm{~K}$, and at $300 \mathrm{~K}$ hysteresis is barely observable. In combination with the coincidence of the ZFC and FC traces at this temperature, the absence of coercivity in the field loop indicates a substantial superparamagnetic contribution at room temperature. The field loops are incompletely saturated at the maximum applied field of $4.8 \times 10^{6} \mathrm{~A} \mathrm{~m}^{-1}$ - this may be due either to the difficulty of saturating $\mathrm{Fe}_{1-x} \mathrm{~S}$, or to presence of superparamagnetism in the $\mathrm{Fe}_{3} \mathrm{~S}_{4}$ fraction. At $4.6 \mathrm{~A} \mathrm{~m}^{2} \mathrm{~kg}^{-1}$ the saturation magnetisation of Sample XII is intermediate to that of Sample X (pure $\mathrm{Fe}_{1-x} \mathrm{~S}$ ) and Sample VII $\left(\mathrm{Fe}_{3} \mathrm{~S}_{4}\right)$. 


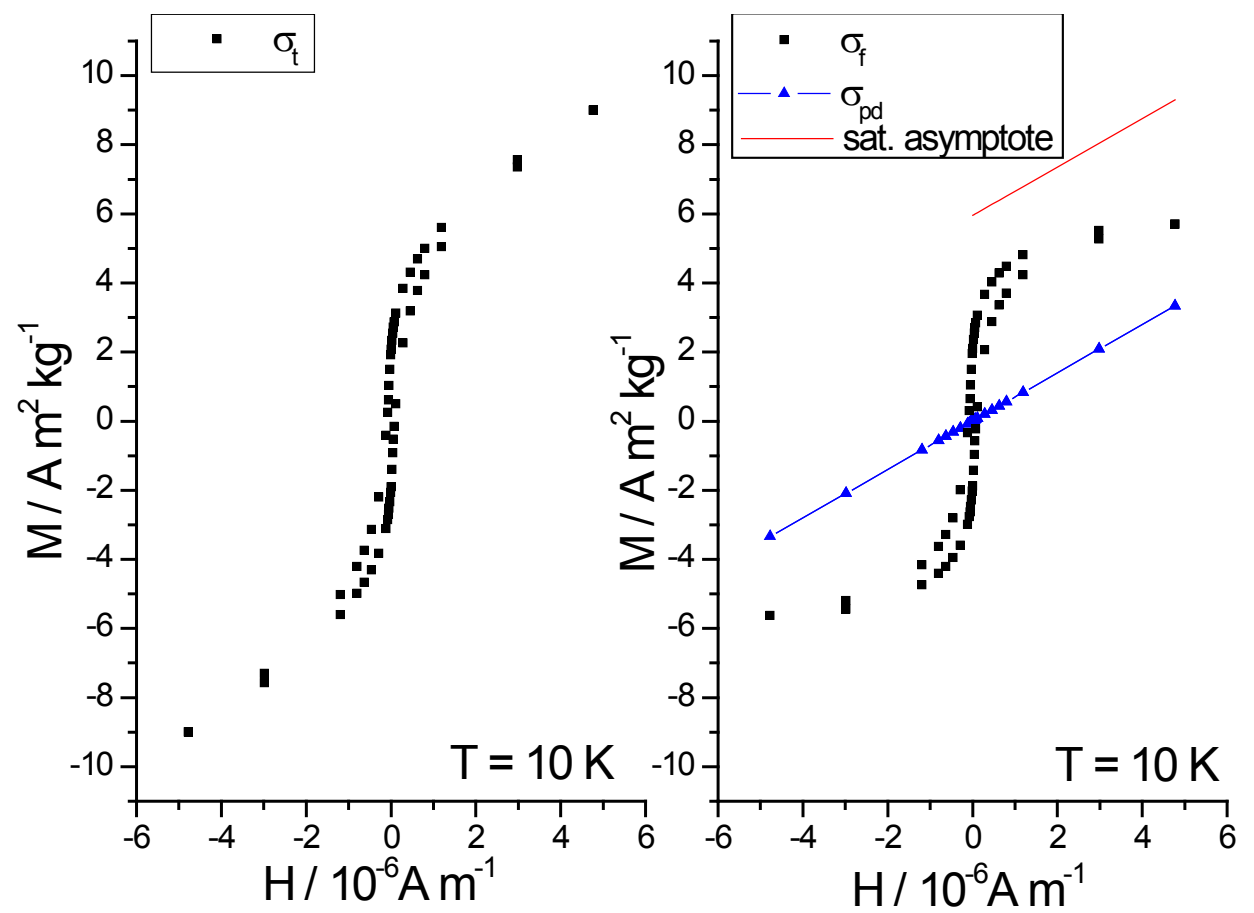

Figure 4-29 Field loop of Sample XII, $\mathrm{T}=10 \mathrm{~K}$ : a) raw data b) processed data.

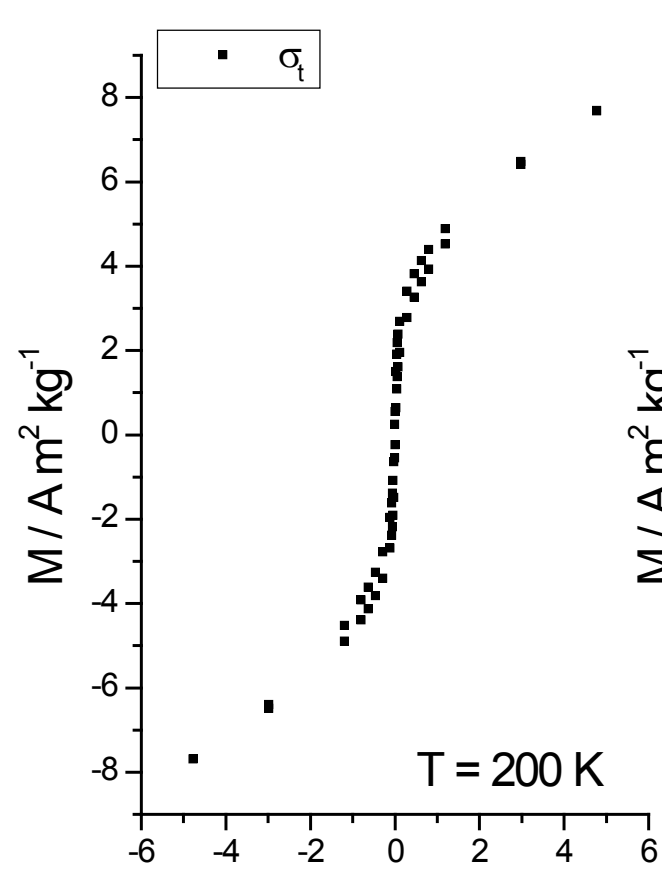

$\mathrm{H} / 10^{-6} \mathrm{~A} \mathrm{~m}^{-1}$

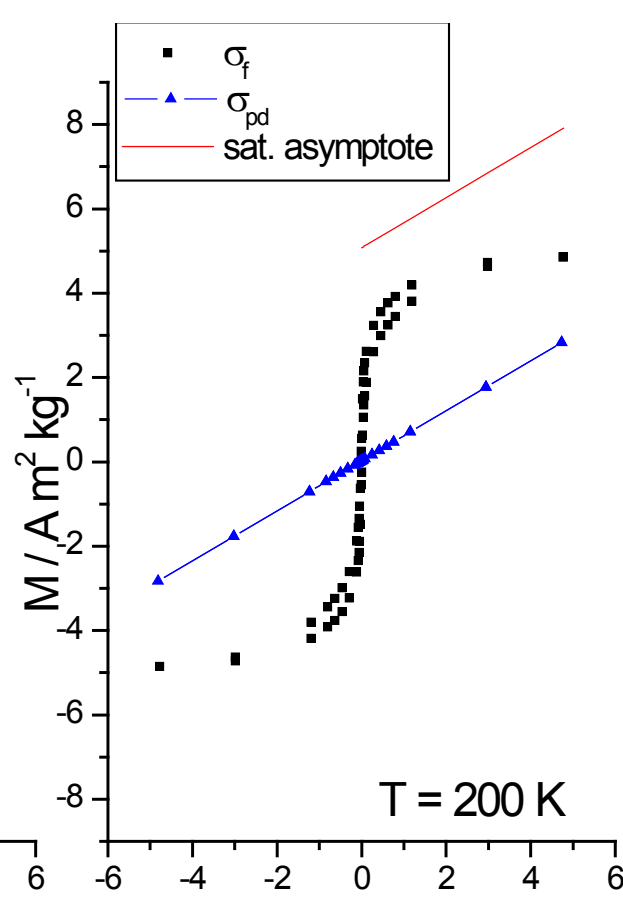

$\mathrm{H} / 10^{-6} \mathrm{~A} \mathrm{~m}^{-1}$

Figure 4-30 Field loop of Sample XII, $\mathrm{T}=200 \mathrm{~K}$ : a) raw data b) processed data. 


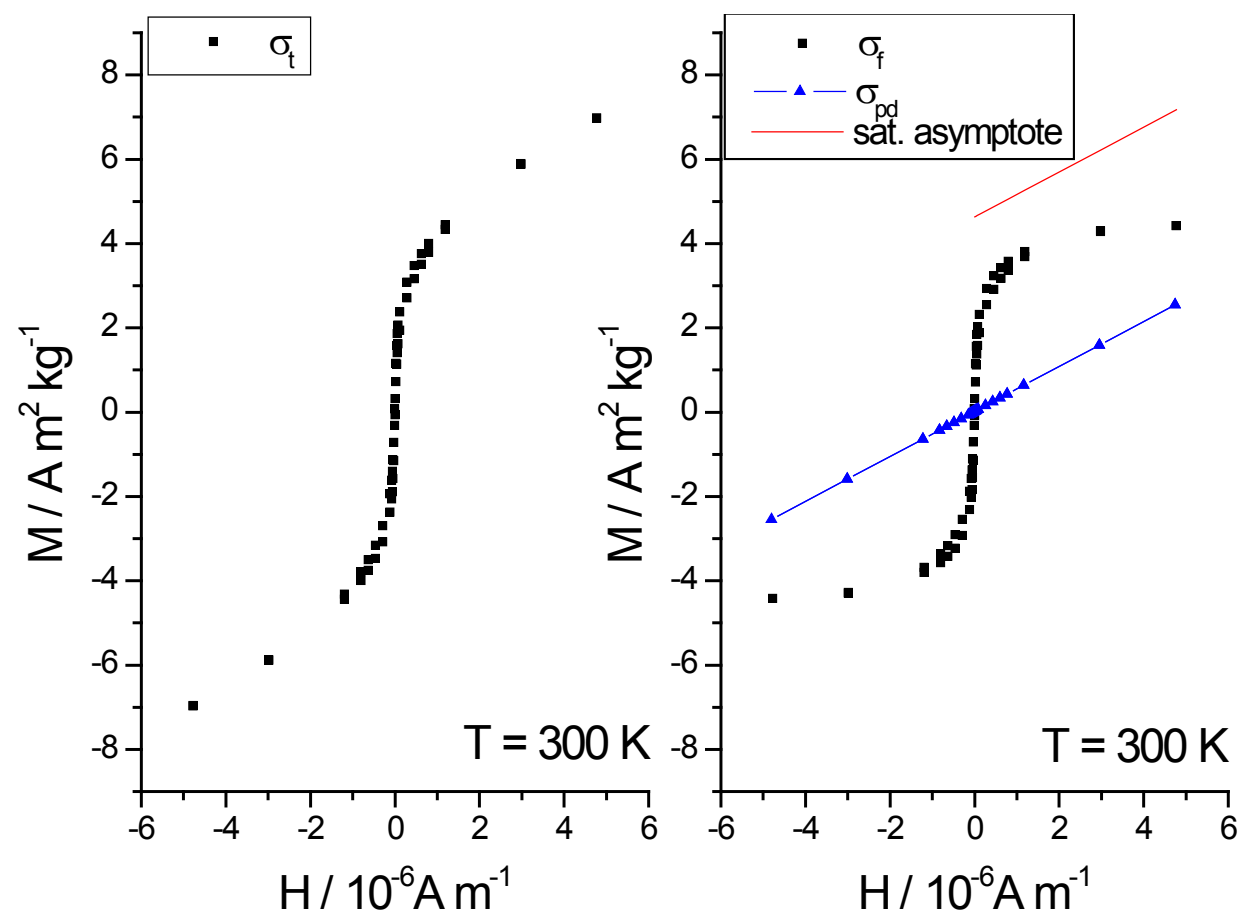

Figure 4-31 Field loop of Sample XII, $\mathrm{T}=300 \mathrm{~K}$ : a) raw data b) processed data.

Table 4-12 Magnetic parameters of Sample XII.

\begin{tabular}{|c|c|c|c|c|c|}
\hline & $\begin{array}{c}\text { Temperature } \\
/ \mathrm{K}\end{array}$ & $M_{s} / \mathrm{A} \mathrm{m}^{2} \mathrm{~kg}^{-1}$ & $M_{r} / \mathrm{A} \mathrm{m}^{2} \mathrm{~kg}^{-1}$ & $M_{\mathrm{r}} / M_{s}$ & $H_{c} / \mathrm{kA} \mathrm{m}^{-1}$ \\
\hline $\begin{array}{c}\text { Sample } \\
\text { XII }\end{array}$ & 10 & 6.0 & 2.0 & 0.33 & 92 \\
\hline & 200 & 5.1 & 0.4 & 0.08 & 7 \\
\hline & 300 & 4.6 & 0.2 & 0.04 & 4 \\
\hline
\end{tabular}

Sample XIII was comprised of large $\mathrm{Fe}_{1-x} \mathrm{~S}$ crystallites and $6 \mathrm{~nm} \mathrm{Fe} \mathrm{S}_{4}$ nanocrystals, and contained approximately four times as much $\mathrm{Fe}_{3} \mathrm{~S}_{4}$ as Sample XII. A ZFC/FC plot for Sample XIII is shown below (Figure 4-32). The ZFC and FC plots displayed a distinct magnetic transition occurring at approximately $75 \mathrm{~K}$, and coincided above a temperature of approximately $200 \mathrm{~K}$. Some fluctuations were still evident in the FC trace, indicating that subpopulations were still present. The susceptibilities demonstrated for Sample XIII were far higher than Samples X (pure $\left.\mathrm{Fe}_{1-x} \mathrm{~S}\right)$ and Sample XII $\left(\mathrm{Fe}_{1-x} \mathrm{~S}\right.$ with small amount of $5 \mathrm{~nm} \mathrm{Fe} \mathrm{S}_{4}$ nanocrystals), but lower than Sample VII ( $\mathrm{Fe}_{3} \mathrm{~S}_{4}$ sheets). 


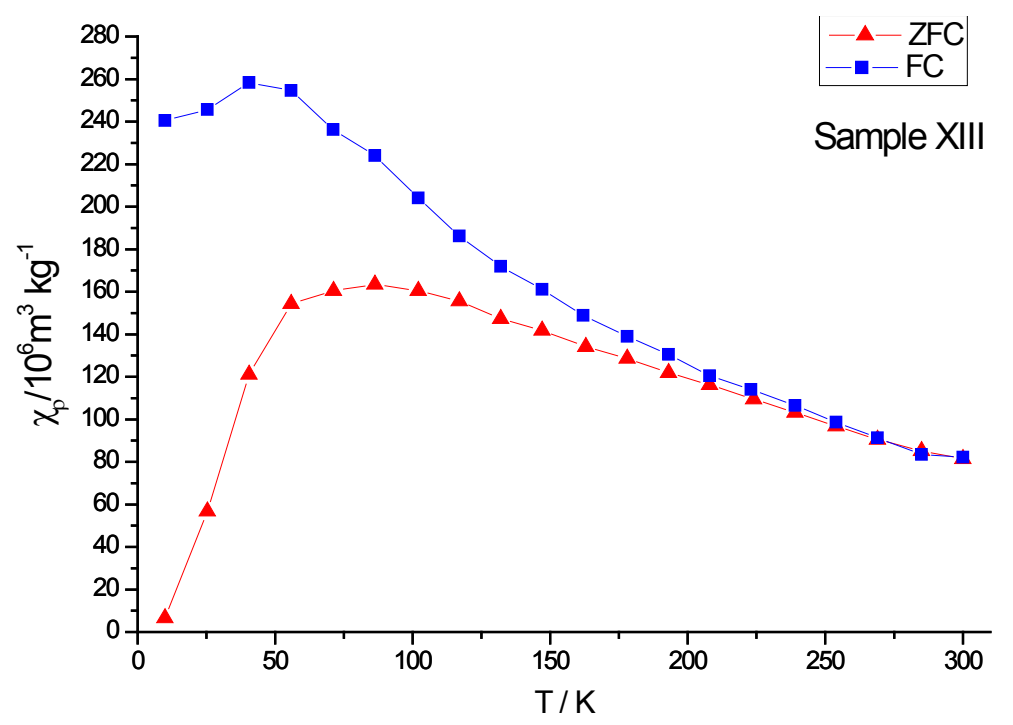

Figure 4-32 ZFC/FC plot of Sample XIII. $\mathrm{H}=1990 \mathrm{~A} \mathrm{~m}^{-1}$.

Field loops for Sample XIII at 10, 200 and $300 \mathrm{~K}$ are shown below (Figure 4-33, Figure 4-34 and Figure 4-35, respectively) and magnetic parameters determined from the field loops are tabulated in Table 4-13. The field loop at $10 \mathrm{~K}$ displays low coercivity (52 kA $\mathrm{m}^{-1}$ ), and is slightly wasp-waisted. Field loops at 200 and $300 \mathrm{~K}$ do not demonstrate hysteresis (i.e. there is no coercivity), and the line shape for both is as expected for a (super)paramagnetic sample. These observations are in line with the transition noted in the $\mathrm{ZFC} / \mathrm{FC}$ plot for the sample, with a transition from magnetically irreversible behaviour at low temperature, and completely magnetically reversible behaviour above $\sim 200 \mathrm{~K}$. A (super)paramagnetic sample is more difficult to saturate, ${ }^{40}$ and Sample XIII was clearly incompletely saturated at the maximum available field of $4.8 \times 10^{6} \mathrm{~A} \mathrm{~m}^{-1}$. Despite this difficulty, processing of the data was used to estimate an approximate saturation magnetisation for the sample. At $8.0 \mathrm{~A} \mathrm{~m}^{2} \mathrm{~kg}^{-1}$ (at $300 \mathrm{~K}$ ), this saturation magnetisation is greater than Sample XII ( $\mathrm{Fe}_{1-x} \mathrm{~S}$ with small amount of $5 \mathrm{~nm} \mathrm{Fe} \mathrm{S}_{4}$ nanocrystals) and Sample X (pure $\mathrm{Fe}_{1-x} \mathrm{~S}$ ), but only half as large as Sample VII (large $\mathrm{Fe}_{3} \mathrm{~S}_{4}$ sheets). 


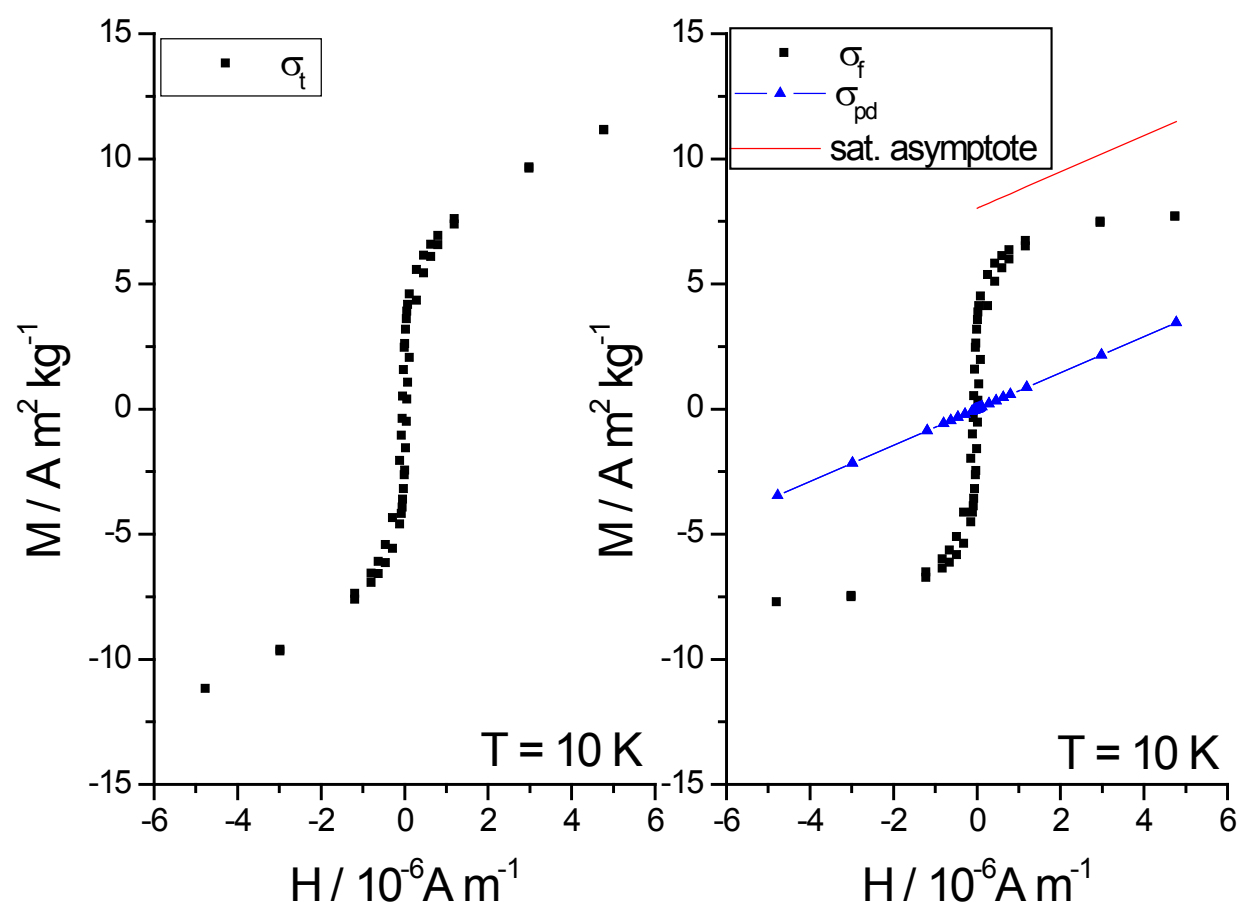

Figure 4-33 Field loop of Sample XIII, $\mathrm{T}=10 \mathrm{~K}$ : a) raw data b) processed data.

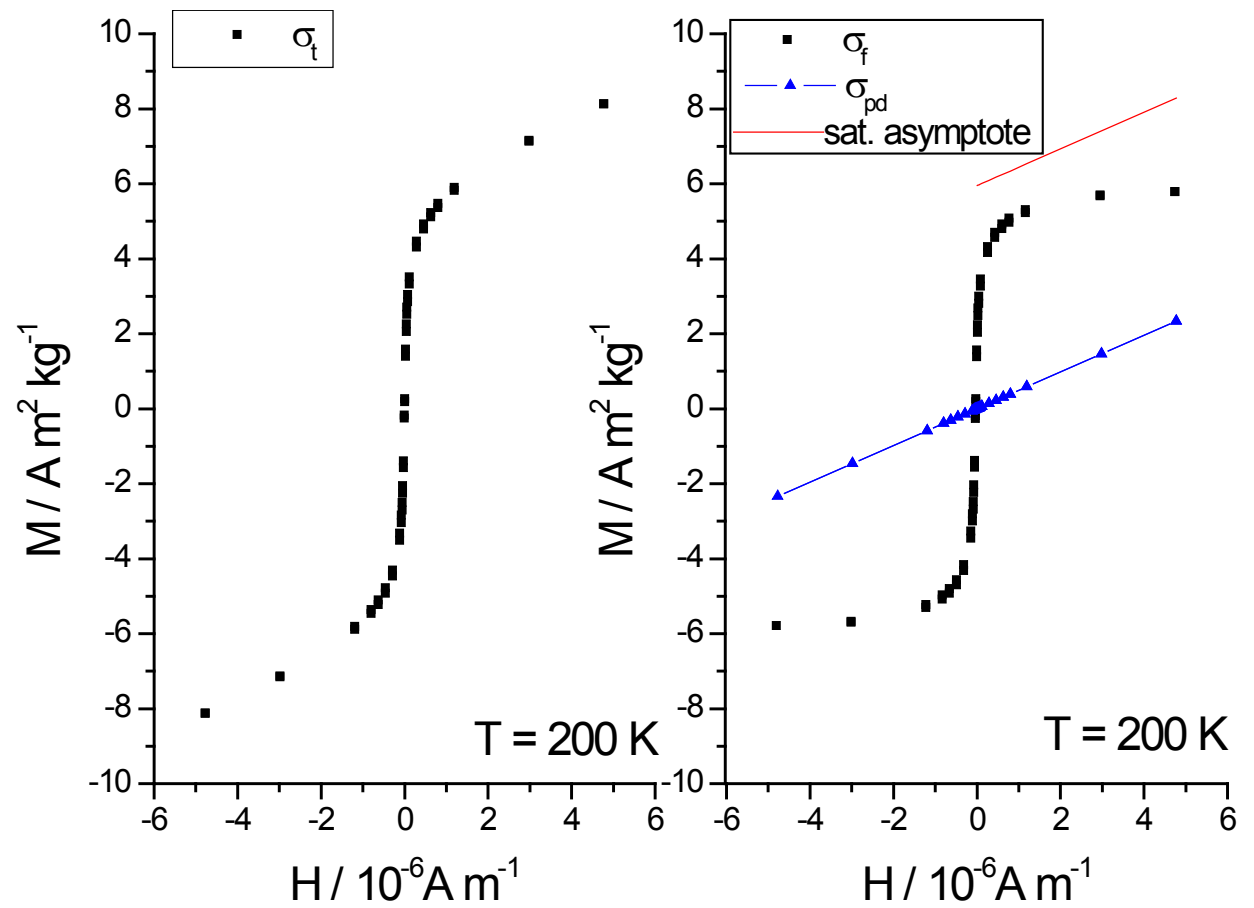

Figure 4-34 Field loop of Sample XIII, $\mathrm{T}=200 \mathrm{~K}$ : a) raw data b) processed data. 


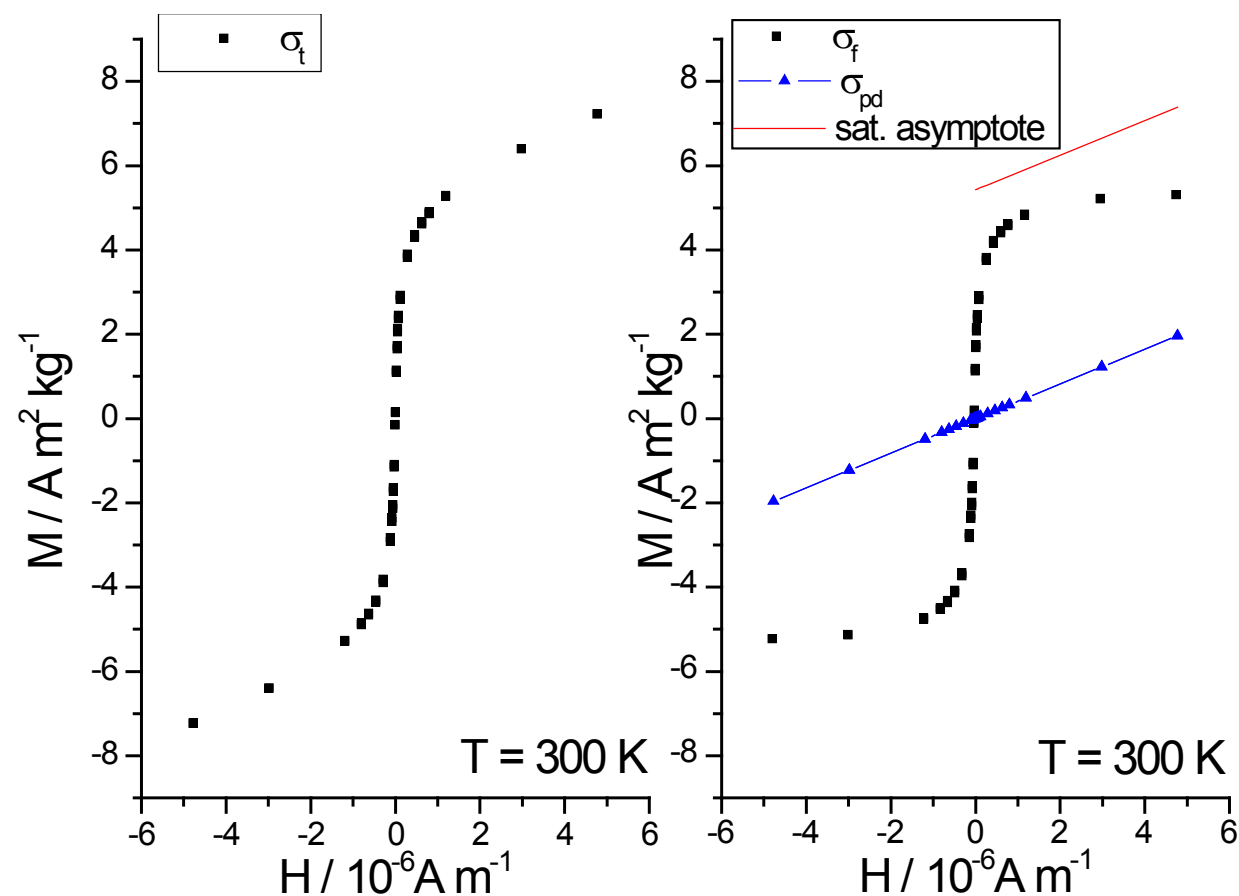

Figure 4-35 Field loop of Sample XIII, $\mathrm{T}=300 \mathrm{~K}$ : a) raw data b) processed data.

Table 4-13 Magnetic parameters of Sample XIII.

\begin{tabular}{|c|c|c|c|c|c|}
\hline & $\begin{array}{c}\text { Temperature } \\
/ \mathrm{K}\end{array}$ & $M_{s} / \mathrm{A} \mathrm{m}^{2} \mathrm{~kg}^{-1}$ & $M_{r} / \mathrm{A} \mathrm{m}^{2} \mathrm{~kg}^{-1}$ & $M_{\mathrm{r}} / M_{s}$ & $H_{c} / \mathrm{kA} \mathrm{m}^{-1}$ \\
\hline $\begin{array}{c}\text { Sample } \\
\text { XIII }\end{array}$ & 10 & 8.0 & 2.5 & 0.31 & 52 \\
\hline & 200 & 6.0 & $/$ & $/$ & $/$ \\
\hline & 300 & 5.4 & $/$ & $/$ & $/$ \\
\hline
\end{tabular}




\subsection{Discussion}

\subsubsection{Synthesis of iron sulfide nanocrystals}

\section{Effect of temperature and reaction duration}

The reaction of $\mathrm{Fe}(\mathrm{acac})_{2}$ and elemental sulfur below $200^{\circ} \mathrm{C}$ produced only poorly crystalline material, which could not be fully characterised. Increasing the duration of the reaction of $\mathrm{Fe}(\mathrm{acac})_{2}$ and elemental sulfur at $200^{\circ} \mathrm{C}$ to 4 hours produced a sample containing crystalline $\mathrm{Fe}_{3} \mathrm{~S}_{4}$ sheets and large faceted $\mathrm{Fe}_{1-x} \mathrm{~S}$ crystallites. Increasing the reaction temperature above $200^{\circ} \mathrm{C}$ favoured the $\mathrm{Fe}_{1-x} \mathrm{~S}$ phase over $\mathrm{Fe}_{3} \mathrm{~S}_{4}$ - reaction of $\mathrm{Fe}(\mathrm{acac})_{2}$ and elemental sulfur at $300^{\circ} \mathrm{C}$ for 30 minutes produced pure $\mathrm{Fe}_{1-x} \mathrm{~S}$.

\section{Effect of $\mathrm{Fe}(\mathrm{acac})_{2}: \mathrm{S}$ ratio}

Varying the ratio of $\mathrm{Fe}(\mathrm{acac})_{2}: \mathrm{S}$ did not affect the ratio of $\mathrm{Fe}_{3} \mathrm{~S}_{4}: \mathrm{Fe}_{1-x} \mathrm{~S}$ produced. Although quantification of the ratio of $\mathrm{Fe}_{3} \mathrm{~S}_{4}: \mathrm{Fe}_{1-x} \mathrm{~S}$ was difficult (see section 4.2.1), it is apparent that varying the ratio of $\mathrm{Fe}(\mathrm{acac})_{2}: \mathrm{S}$ from $1: 2$ to $1: 10$ for reactions at $\sim 220^{\circ} \mathrm{C}$ for 30 minutes (section 4.2.2.2) did not systematically affect the ratio of $\mathrm{Fe}_{3} \mathrm{~S}_{4}: \mathrm{Fe}_{1-x} \mathrm{~S}$ produced. All the aforementioned experiments favoured $\mathrm{Fe}_{3} \mathrm{~S}_{4}$ over $\mathrm{Fe}_{1-x} \mathrm{~S}$, whereas reaction of a $1: 2.75$ mixture of $\mathrm{Fe}(\mathrm{acac})_{2}: \mathrm{S}$ at $300^{\circ} \mathrm{C}$ for 30 minutes (Sample XI) produced $\mathrm{Fe}_{1-x} \mathrm{~S}$ with only a trace of $\mathrm{Fe}_{3} \mathrm{~S}_{4}$. This indicates that reaction temperature is the prime factor determining the ratio of $\mathrm{Fe}_{3} \mathrm{~S}_{4}: \mathrm{Fe}_{1-x} \mathrm{~S}$ for all synthetically practical $\mathrm{Fe}(\mathrm{acac})_{2}: \mathrm{S}$ ratios. Given that the excess sulfur was found to remain after purification (see section 4.2.2.2), it became apparent that using a ratio of $\mathrm{Fe}(\mathrm{acac})_{2}: \mathrm{S}$ greater than the 1:1.33 ratio required to produce $\mathrm{Fe}_{3} \mathrm{~S}_{4}$ was unnecessary and detrimental.

\section{Effect of reactant concentrations}

The $\mathrm{Fe}_{1-x} \mathrm{~S}$ crystallites produced by reaction of $\mathrm{Fe}(\mathrm{acac})_{2}$ and elemental sulfur at $300^{\circ} \mathrm{C}$ had average dimensions in excess of $50 \mathrm{~nm}$. To investigate the effect of precursor concentration on particle size, the precursor concentrations were varied between 0.01 and $0.1 \mathrm{~mol} \mathrm{dm}^{-3}$. There was a small apparent drop in the average crystallite dimension (as determined by Scherrer broadening analysis) from 84 to $55 \mathrm{~nm}$ when the precursor concentration was increased from 0.05 to $0.1 \mathrm{~mol} \mathrm{dm}^{-3}$ (Samples IX and X, respectively). However, as the crystallite size distribution was still quite wide in all cases, attention was turned to altering the nucleation conditions to attempt to control the crystallite size and size distribution. 


\section{Effect of nucleation conditions}

Rapid injection of one precursor into a preheated solution of the other was trialled as a means to control the particle size distribution, as burst nucleation theory suggests that temporal separation of the nucleation and growth phases leads to a narrow size distribution. The injection of sulfur solution into $\mathrm{Fe}(\mathrm{acac})_{2}$ in oleylamine at $290^{\circ} \mathrm{C}$ with cooling after 10 minutes produced $\mathrm{Fe}_{1-x} \mathrm{~S}$ crystallites with the average size of $43 \mathrm{~nm}$ (Sample XII), which was smaller than produced by reaction under similar conditions for 30 minutes (84 $\mathrm{nm}$ for Sample IX). However, more significant than this effect was the introduction to the sample of small $(\sim 5 \mathrm{~nm})$ spherical $\mathrm{Fe}_{3} \mathrm{~S}_{4}$ nanocrystals. The yield of $\mathrm{Fe}_{3} \mathrm{~S}_{4}$ nanocrystals was increased by cooling the reaction immediately after injection of the sulfur solution (Sample XIII), indicating that the small $\mathrm{Fe}_{3} \mathrm{~S}_{4}$ nanocrystals were not stable for more than 10 minutes at $280^{\circ} \mathrm{C}$.

It was evident from observations of colour change that $\mathrm{Fe}(\mathrm{acac})_{2}$ began to decompose when heated to around $90^{\circ} \mathrm{C}-$ far below the injection temperatures of $280-290^{\circ} \mathrm{C}$ which were used - therefore nucleation of some unknown phase (elemental iron or iron oxide) had occurred over a prolonged period in advance of the introduction of sulfur, and nucleation and growth could not be presumed to have occurred separately. To avoid this problem, $\mathrm{FeCl}_{2}$ was added to a solution of sulfur in oleylamine at $260^{\circ} \mathrm{C}$ and aliquots were removed and quenched (Sample XVIII). In this experiment large, poorly crystalline sheets were present in the aliquot withdrawn immediately after injection, and large $\mathrm{Fe}_{1-x} \mathrm{~S}$ crystallites were present in the aliquot withdrawn after 5 minutes. Evidently $\mathrm{Fe}_{1-x} \mathrm{~S}$ crystallites grow to a relatively large size very rapidly after initiation of reaction - thus severely diminishing the prospect of controlling the particle size and size distribution of the $\mathrm{Fe}_{1-x} \mathrm{~S}$ phase through altering the duration of reaction.

\section{Effect of iron source}

The effect of different precursors was investigated by substituting $\mathrm{FeCl}_{2}$ for $\mathrm{Fe}(\mathrm{acac})_{2}$. Sulfur solution was injected into a solution of $\mathrm{FeCl}_{2}$ in oleylamine at $280^{\circ} \mathrm{C}$ and immediately cooled (Sample XV). Like the analogous experiment using Fe(acac) (Sample XIII), a mixture of $\mathrm{Fe}_{1-x} \mathrm{~S}$ and $\mathrm{Fe}_{3} \mathrm{~S}_{4}$ was produced, however the average particle size of both phases was significantly larger $\left(50 \mathrm{~nm}\right.$ for $\mathrm{Fe}_{1-x} \mathrm{~S}$ and $20 \mathrm{~nm}$ for $\mathrm{Fe}_{3} \mathrm{~S}_{4}$ for Sample XV cf. $26 \mathrm{~nm}$ for $\mathrm{Fe}_{1-x} \mathrm{~S}$ and $6 \mathrm{~nm}$ for $\mathrm{Fe}_{3} \mathrm{~S}_{4}$ for Sample XIII). Thus it appears that $\mathrm{FeCl}_{2}$ favours more rapid growth of crystallites than $\mathrm{Fe}(\mathrm{acac})_{2}$. 


\section{Effect of solvent}

The effect of solvent was investigated by substituting HDA for oleylamine in a number of experiments. Reaction of $\mathrm{Fe}(\mathrm{acac})_{2}$ and elemental sulfur in $\mathrm{HDA}$ at $200^{\circ} \mathrm{C}$ for 2 hours produced $\mathrm{Fe}_{3} \mathrm{~S}_{4}$ (Sample XVI), whereas reaction at $300^{\circ} \mathrm{C}$ for 30 minutes produced $\mathrm{Fe}_{1-x} \mathrm{~S}$ (Sample XVII). The $\mathrm{Fe}_{3} \mathrm{~S}_{4}$ produced in HDA was irregularly shaped, unlike the well-defined $\mathrm{Fe}_{3} \mathrm{~S}_{4}$ sheets produced in oleylamine after 4 hours at $200^{\circ} \mathrm{C}$ (Sample VII). The $\mathrm{Fe}_{1-x} \mathrm{~S}$ crystallites produced in HDA had a larger average size $(115 \mathrm{~nm})$ than those produced under analogous conditions in oleylamine (84 $\mathrm{nm}$ for Sample IX).

\section{Summary}

To summarise thus far, temperature was found to be the most important parameter determining the outcome of reactions of an iron salt and elemental sulfur: $\mathrm{Fe}_{1-x} \mathrm{~S}$ forms rapidly between $250-300^{\circ} \mathrm{C}$, and pure $\mathrm{Fe}_{1-x} \mathrm{~S}$ can be produced by a reaction temperature of $300^{\circ} \mathrm{C}$ for a duration of 30 minutes. As the growth of $\mathrm{Fe}_{1-x} \mathrm{~S}$ was observed to be very rapid in oleylamine at this temperature, the particle size and size distribution cannot be controlled by separating the nucleation and growth phases and limiting the duration of the growth phase - an equilibrium size and size distribution quickly establishes itself. Reducing the temperature favours $\mathrm{Fe}_{3} \mathrm{~S}_{4}$, however long reaction times are necessary to produce crystalline material, and while crystalline $\mathrm{Fe}_{3} \mathrm{~S}_{4}$ sheets can be produced by reaction at $200^{\circ} \mathrm{C}$ for 4 hours, large $\mathrm{Fe}_{1-x} \mathrm{~S}$ crystallites are produced as well. Limited reaction times at intermediate temperatures produce mixtures of large $\mathrm{Fe}_{1-x} \mathrm{~S}$ crystallites and small $\mathrm{Fe}_{3} \mathrm{~S}_{4}$ nanocrystals, whose size appears consistent with burst nucleation.

Our observations of the temperature dependence of iron sulfide phase formation correlate well with those in the literature: Uda produced pure $\mathrm{Fe}_{3} \mathrm{~S}_{4}$ by hydrothermal reaction of $\left(\mathrm{NH}_{4}\right)_{2} \mathrm{Fe}\left(\mathrm{SO}_{4}\right)_{2} \cdot 6 \mathrm{H}_{2} \mathrm{O}$ and $\mathrm{H}_{2} \mathrm{~S}$ at $190^{\circ} \mathrm{C}$ for one hour followed by quenching. ${ }^{11,41} \mathrm{He}$ found that reaction at $180^{\circ} \mathrm{C}$ for one hour produced $\mathrm{Fe}_{3} \mathrm{~S}_{4}$ and $\mathrm{FeS}_{\mathrm{m}}$, whereas reaction at $210^{\circ} \mathrm{C}$ for one hour produced $\mathrm{Fe}_{1-x} \mathrm{~S}$. Difficulties producing pure, crystalline $\mathrm{Fe}_{3} \mathrm{~S}_{4}$ because of this temperature dependence have been voiced frequently in the literature. ${ }^{18,20,41}$ 


\subsubsection{1 $\quad \mathrm{Fe}_{1-x} \mathrm{~S}$}

A variety of highly faceted morphologies were evident for the $\mathrm{Fe}_{1-x} \mathrm{~S}$ crystallites synthesised in this project. Most crystallites were hexagonal plates, although elongated prismatic crystallites were also present (see Figure 4-8 (a) - (e)). These observed morphologies mirror those seen in hydrothermally grown $\mathrm{Fe}_{1-x} \mathrm{~S},{ }^{42}$ and more recently, submicron $\mathrm{Fe}_{1-x} \mathrm{~S}$ crystallites synthesised by the decomposition of $\mathrm{Fe}(\mathrm{Ddtc})_{2}$ (Phen) and $\mathrm{Fe}(\mathrm{Ddtc})_{3}$ single source precursors in oleylamine at $260-320^{\circ} \mathrm{C} .{ }^{27}$ Simulated equilibrium crystal morphologies for $\mathrm{Fe}_{1-x} \mathrm{~S}$ are presented below, based on either the Donnay-Harker method (Figure 4-36 (a)), or the morphology of $\mathrm{Fe}_{1-x} \mathrm{~S}$ as given by Bishop $^{34}$ (Figure 4-36 (b)). In both cases, dominant forms include the hexagonal prism $\{10 \overline{1} 0\}$ form and the pinacoidal $\{0001\}$ form. The variety of hexagonal plate morphologies seen in $\mathrm{Fe}_{1-x} \mathrm{~S}$ can be derived from these equilibrium morphologies by assuming variable growth rates for different faces of the $\{10 \overline{1} 0\}$ form distort the morphology away from that of a regular hexagon.

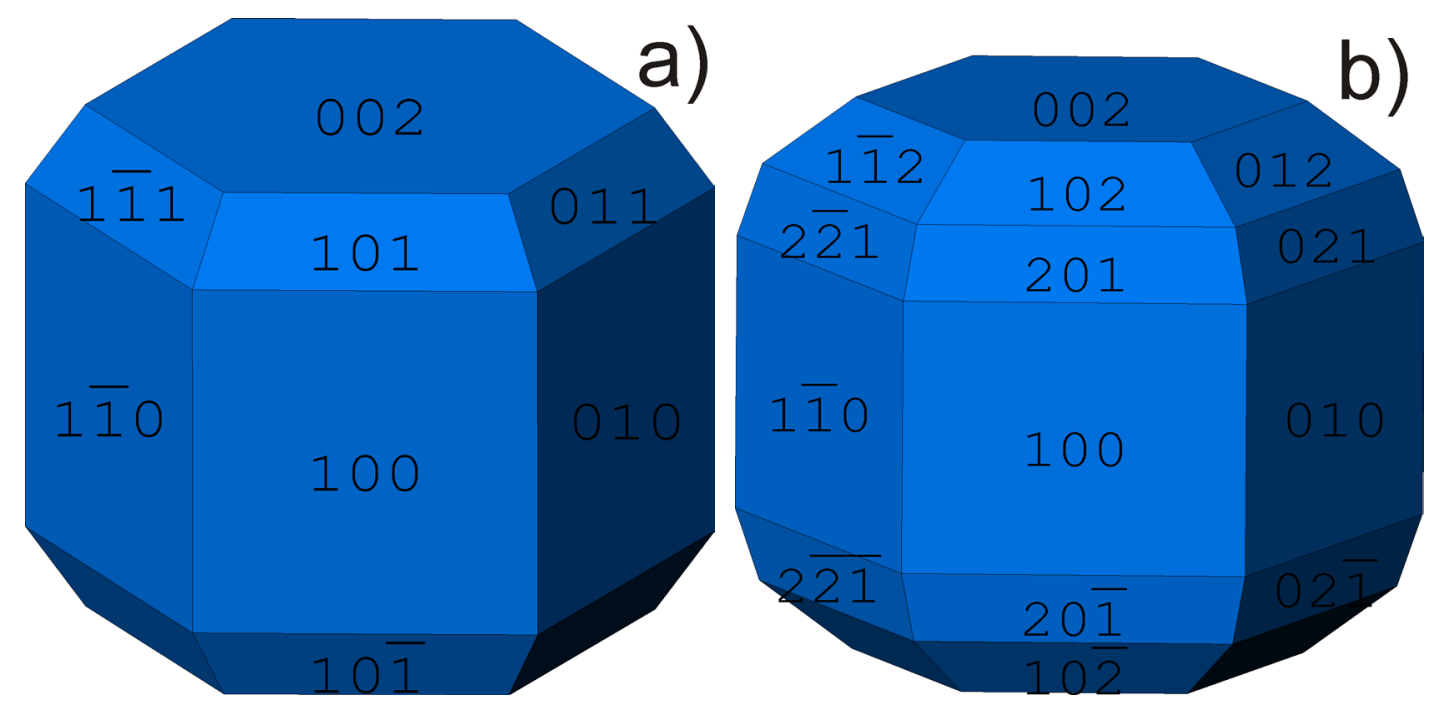

Figure 4-36 Simulated crystal morphology for $\mathrm{Fe}_{1-x} \mathrm{~S}: \mathrm{a}$ ) based on the Donnay-Harker method b) after Bishop. $^{34}$

The size range of $\mathrm{Fe}_{1-x} \mathrm{~S}$ crystallites seen in this project (average diameters determined by Scherrer broadening varied from $25-115 \mathrm{~nm}$, while actual crystallite dimensions observed by TEM were up to several hundred nanometres) are similar to that observed by Gao. ${ }^{27}$

The superstructures of $\mathrm{Fe}_{1-x} \mathrm{~S}$ observed most frequently in nature are hexagonal 5C, 6C, $11 \mathrm{C}$ and monoclinic $4 \mathrm{C} .^{3}$ Near stoichiometric hexagonal $2 \mathrm{C}$ is found in nature 
predominantly in non-terrestrial sources (meteorites and lunar rocks). ${ }^{43}$ Synthetic studies have produced all the aforementioned superstructures, as well as the NC, NA and MC types. ${ }^{2}$ XRD performed on a freshly synthesised sample on a low background holder matched $2 \mathrm{C} \mathrm{Fe}_{1-x} \mathrm{~S}$. Whereas SAED measurements on a sample two years after synthesis (Sample X) yielded superlattice reflections which were not consistent with any of the known superlattice structures (see section 4.2.7). It is conceivable that Sample $\mathrm{X}$ was initially of the $2 \mathrm{C}$ type immediately after synthesis, and that there has been some subsequent change in the superlattice structure over the period of two years as has been observed previously. ${ }^{44}$ Superlattice reflections in the XRD of Sample X may have been obscured by the background contribution of the holder used. Given these difficulties it is not possible to definitively assign a superstructure to Sample X, which was used in magnetic measurements as an example of pure $\mathrm{Fe}_{1-x} \mathrm{~S}$. In any case, these magnetic measurements, which were performed on freshly synthesised Sample X, indicate that it is antiferromagnetic, as detailed below in section 4.3.2.1. This serves to exclude the presence of ferrimagnetic monoclinic $4 \mathrm{C} \mathrm{Fe}_{1-x} \mathrm{~S}$, which is in agreement with all diffraction and composition data. 


\subsubsection{2 $\quad \mathrm{Fe}_{3} \mathrm{~S}_{4}$}

A variety of sizes and morphologies have been observed in the $\mathrm{Fe}_{3} \mathrm{~S}_{4}$ nanocrystals synthesised in this work: namely thin rectangular sheets, $\sim 6 \mathrm{~nm}$ spherical nanocrystals and $\sim 20 \mathrm{~nm}$ apparently triangular prismatic nanocrystals. The dimensions of these materials fall within the lower end of the size regime reported in the literature $\left(2.5^{(25}\right.$ $\left.400^{(19)} \mathrm{nm}\right)$. However little information is available in the literature on the morphology of synthetic $\mathrm{Fe}_{3} \mathrm{~S}_{4}$, as most studies quoted solely the average particle size determined by Scherrer broadening analysis, ${ }^{20}$ without the use of direct imaging techniques.

Rectangular $\mathrm{Fe}_{3} \mathrm{~S}_{4}$ sheets have not been reported in the literature, although disk-like forms have been reported for $\mathrm{Fe}_{3} \mathrm{~S}_{4}$ synthesised at $200^{\circ} \mathrm{C} .{ }^{45}$ Tabular habits are however frequently observed in $\mathrm{FeS}_{\mathrm{m}},{ }^{46,47}$ with elongation in directions perpendicular to the $c$ axis (see Figure 4-37 for the simulated equilibrium morphology of $\mathrm{FeS}_{\mathrm{m}}$ ). Solid state conversion of the tetragonal layer structure $\mathrm{FeS}_{\mathrm{m}}$ to cubic close-packed $\mathrm{Fe}_{3} \mathrm{~S}_{4}$ has been observed, ${ }^{48}$ and the orientated aggregation of $\mathrm{FeS}_{\mathrm{m}}$ particles, followed by conversion to $\mathrm{Fe}_{3} \mathrm{~S}_{4}$ is proposed as a route for $\mathrm{Fe}_{3} \mathrm{~S}_{4}$ formation. ${ }^{10}$ Given that $\mathrm{FeS}_{\mathrm{m}}$ is identified as the first iron sulfide phase to precipitate in aqueous solution, ${ }^{28}$ it seems likely that the poorly crystalline layered material observed in Samples I and II, produced at $140^{\circ} \mathrm{C}$, was also $\mathrm{FeS}_{\mathrm{m}}$. Therefore it is proposed that the observed tabular morphology of the $\mathrm{Fe}_{3} \mathrm{~S}_{4}$ sheets is a remnant from initially nucleated $\mathrm{FeS}_{\mathrm{m}}$ crystals, which have subsequently been converted to $\mathrm{Fe}_{3} \mathrm{~S}_{4}$ through a solid state reaction occurring at $200^{\circ} \mathrm{C}$.

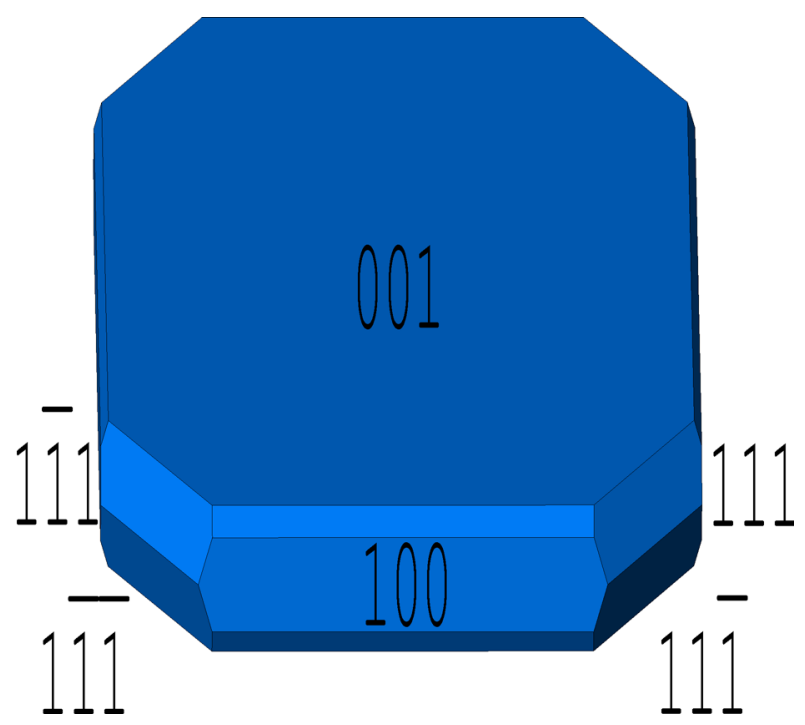

Figure 4-37 Simulated crystal morphology for $\mathrm{FeS}_{\mathrm{m}}$, based on the surface energies calculated by DFT. After Devey. 
The formation of twinned octahedral is common for $\mathrm{MgAl}_{2} \mathrm{O}_{4}$ (the parent of the spinel class), ${ }^{34}$ but has not been reported for $\mathrm{Fe}_{3} \mathrm{~S}_{4}$. Twinned octahedra of $\mathrm{Fe}_{3} \mathrm{~S}_{4}$ were predominantly observed in experiments where $\mathrm{FeCl}_{2}$ were used as the iron source, but were also observed in a sample where Fe(acac) $)_{2}$ was used (Sample III) (see Figure 4-3 (a)). Therefore it is apparent that the formation of twinned octahedra of $\mathrm{Fe}_{3} \mathrm{~S}_{4}$ was not solely an effect of the iron source, and that the more frequent observation in samples synthesised from $\mathrm{FeCl}_{2}$ is likely due to the larger average particle size obtained with $\mathrm{FeCl}_{2}$, which allowed an innate tendency for twinned octahedra to be observed. Whereas $\mathrm{Fe}_{3} \mathrm{~S}_{4}$ particles produced by rapid nucleation using $\mathrm{Fe}(\mathrm{acac})_{2}$ as the iron source had an average size of $5-6 \mathrm{~nm}-$ a size regime in which facet formation is suppressed.

Spherical $\mathrm{Fe}_{3} \mathrm{~S}_{4}$ have been synthesised by injection of a solution of $\left[\mathrm{NBu}_{4}\right]_{2}\left[\mathrm{Fe}_{4} \mathrm{~S}_{4}(\mathrm{SPh})_{4}\right]$ into dodecylamine. ${ }^{25}$ It was found that increasing the injection temperature from 200 to $230^{\circ} \mathrm{C}$ decreased the average diameter of the resulting nanocrystals from 4.5 to $2.5 \mathrm{~nm}$. It was proposed that this was due to burst nucleation, with higher temperatures increasing the number of nuclei produced and leading to more rapid exhaustion of monomer, and therefore smaller average diameter. It appears likely that burst nucleation is also responsible for the formation of small spherical $\mathrm{Fe}_{3} \mathrm{~S}_{4}$ nanocrystals in this project, as they were only observed in samples where iron sulfide formation was initiated by injection of sulfur solution, and the reaction was then promptly cooled (Samples XII - XIII). The difficulties in producing these small $\mathrm{Fe}_{3} \mathrm{~S}_{4}$ nanocrystals separately from $\mathrm{Fe}_{1-x} \mathrm{~S}$ in this system led to the work in Chapter 5 on the sulfidisation of iron particles. 


\subsubsection{Magnetic Properties}

\subsubsection{1 $\quad \mathrm{Fe}_{1-x} \mathrm{~S}$}

Sample X (pure $\mathrm{Fe}_{1-x} \mathrm{~S}$ ) demonstrated no magnetic transitions between 10 and $300 \mathrm{~K}$. This included the absence of the reported decrease in remanence of monoclinic $\mathrm{Fe}_{7} \mathrm{~S}_{8}$ (4C type $\mathrm{Fe}_{1-x} \mathrm{~S}$ ) at $30-34 \mathrm{~K}$, which was attributed to the transition from a multidomain to single- or pseudosingle domain magnetic structure. ${ }^{49}$ This is in line with the fact that the composition of Sample X (Fe 48at $\%$, S 52at $\% ; \mathrm{Fe}_{59} \mathrm{~S}_{64}$ ) clearly places it in the composition range of hexagonal $\mathrm{Fe}_{1-x} \mathrm{~S}$, and $\mathrm{XRD}$ indicates it retained the hexagonal symmetry of the NiAs-type structure.

Randomly orientated samples of $\mathrm{Fe}_{1-x} \mathrm{~S}$ are known to be difficult to saturate due to their large uniaxial magnetocrystalline anisotropy $\left(\sim 2.6 \times 10^{5} \mathrm{~J} \mathrm{~m}^{-3}\right){ }^{50}$ Applied fields as large as $12 \times 10^{6} \mathrm{~A} \mathrm{~m}^{-1}(15 \mathrm{~T})$ are required to overcome the magnetocrystalline anisotropy and rotate the spontaneous magnetisation out of the basal plane to align with the applied field. ${ }^{36}$ As some of the samples were incompletely saturated at the largest applied field available in the magnetometer used $\left(4.8 \times 10^{6} \mathrm{~A} \mathrm{~m}^{-1}\right)$, approximate saturation magnetisations were extracted from the high field data using the data decomposition procedure outlined in Chapter 2 section 2.2.6.3. The increase in approximate saturation magnetisation with increasing temperature observed in field loop experiments on Sample X, from $1.5 \mathrm{~A} \mathrm{~m}^{2} \mathrm{~kg}^{-1}$ at $10 \mathrm{~K}$ to $2.6 \mathrm{~A} \mathrm{~m}^{2} \mathrm{~kg}^{-1}$ at $300 \mathrm{~K}$, is behaviour characteristic of an antiferromagnet, where thermal energy promotes a mismatch between the magnetic sublattices, and increases the spontaneous magnetisation. ${ }^{51,52}$

The magnitude of the approximate saturation magnetisation of Sample $\mathrm{X}$ is comparable to the room temperature saturation magnetisation of $4.9 \mathrm{~A} \mathrm{~m}^{2} \mathrm{~kg}^{-1}$ reported for $<5 \mu \mathrm{m}$ natural $\mathrm{Fe}_{1-x} \mathrm{~S}$ crystallites of a similar composition ( $\mathrm{Fe} 47.41 \%$, S 52.25\%) by Dekkers. ${ }^{36}$ Dekkers reported a trend towards increasing saturation magnetisation with decreasing crystallite size of the aforementioned natural $\mathrm{Fe}_{1-x} \mathrm{~S}$, from $7.9 \mathrm{~A} \mathrm{~m}^{2} \mathrm{~kg}^{-1}$ for the $150-$ $250 \mu \mathrm{m}$ fraction to a maximum of $11.8 \mathrm{~A} \mathrm{~m}^{2} \mathrm{~kg}^{-1}$ for the $20-25 \mu \mathrm{m}$ fraction, after which the saturation magnetisation fell to its minimum at $4.9 \mathrm{~A} \mathrm{~m}^{2} \mathrm{~kg}^{-1}$ for the $<5 \mu \mathrm{m}$ fraction. ${ }^{36}$ However the author was unable to explain this trend. 
The coercivity of Sample X at $300 \mathrm{~K}$ was $112 \mathrm{kA} \mathrm{m}^{-1}$, higher than the coercivity of 57 $\mathrm{kA} \mathrm{m}^{-1}$ reported for the $<5 \mu \mathrm{m}$ fraction of natural $\mathrm{Fe}_{1-x} \mathrm{~S}$ by Dekkers, ${ }^{36}$ though this fits with the trend reported by Dekkers towards higher coercivity with decreasing grain size. Soffe $^{53}$ determined from direct domain observations that the transition between multidomain and single-domain behaviour occurs at an average particle diameter of 1.6 $\mu \mathrm{m}$, indicating that Sample $\mathrm{X}$ consists of single-domain particles. The coercivity of single-domain particles is higher than multidomain particles, as low coercivity magnetisation processes such as domain wall motion are not possible, and high coercivity mechanisms such as coherent magnetisation rotations dominate. ${ }^{40}$

The field loops of Sample X where distinctly wasp-waisted. Wasp-waisted field loops result from the weighted contributions of components of widely differing coercivities. ${ }^{54}$ Given that Sample X was determined to be pure $\mathrm{Fe}_{1-x} \mathrm{~S}$ by XRD and SAED, and substantial amounts of a second phase must be present to induce a wasp-waisted field loop, ${ }^{54}$ this suggests that the range of coercivities must arise from inhomogeneity in the size of $\mathrm{Fe}_{1-x} \mathrm{~S}$ crystallites in the sample, rather than the presence of a second magnetic phase.

\subsubsection{2 $\quad \mathrm{Fe}_{1-x} \mathrm{~S} / \mathrm{Fe}_{3} \mathrm{~S}_{4}$ mixtures}

Sample VII consisted of large $\mathrm{Fe}_{3} \mathrm{~S}_{4}$ sheets and $\mathrm{Fe}_{1-x} \mathrm{~S}$ crystallites. There were no magnetic transitions in the range $10-300 \mathrm{~K}$. It was previously determined that the $\mathrm{Fe}_{1-x} \mathrm{~S}$ synthesised in this project does not display the transition at $30-34 \mathrm{~K}$ noted for monoclinic $4 \mathrm{C} \mathrm{Fe}_{1-x} \mathrm{~S}$. In addition bulk $\mathrm{Fe}_{3} \mathrm{~S}_{4}$ has no low temperature magnetic transitions, unlike its oxide analogue $\mathrm{Fe}_{3} \mathrm{O}_{4}$ which famously displays the Verwey transition at $118 \mathrm{~K} .^{55}$ The absence of magnetic transitions is therefore in line with what is expected for the bulk phases; however it also demonstrates the absence of any size-related magnetic transitions (i.e. a ferrimagnetic to superparamagnetic transition).

The saturation magnetisation of bulk $\mathrm{Fe}_{3} \mathrm{~S}_{4}$ has been reported between 24 and 38 A m $\mathrm{kg}^{-1}$, hence at $14.3 \mathrm{~A} \mathrm{~m}^{2} \mathrm{~kg}^{-1}$ the saturation magnetisation of Sample VII is somewhat lower than bulk $\mathrm{Fe}_{3} \mathrm{~S}_{4}$. However XRD of Sample VII revealed the presence of significant amounts of $\mathrm{Fe}_{1-x} \mathrm{~S}$, which as evidenced by Sample $\mathrm{X}$ (pure $\mathrm{Fe}_{1-x} \mathrm{~S}$ ) has a very low saturation magnetisation - hence it is difficult to draw a direct comparison between Sample VII and reportedly pure $\mathrm{Fe}_{3} \mathrm{~S}_{4}$. Certainly the introduction of $\mathrm{Fe}_{3} \mathrm{~S}_{4}$ 
drastically increases the susceptibility of the sample when compared to pure $\mathrm{Fe}_{1-x} \mathrm{~S}$, and the observed decrease in saturation magnetisation with increasing temperature (from 16.9 $\mathrm{A} \mathrm{m}^{2} \mathrm{~kg}^{-1}$ at $10 \mathrm{~K}$ to $14.3 \mathrm{~A} \mathrm{~m}^{2} \mathrm{~kg}^{-1}$ at $300 \mathrm{~K}$ ) is in line with ferrimagnetic behaviour.

The coercivity of Sample VII at $15 \mathrm{kA} \mathrm{m}^{-1}$ at $300 \mathrm{~K}$ is far lower than pure $\mathrm{Fe}_{1-x} \mathrm{~S}$ (Sample X). It fits well within the range of coercivities observed for synthetic $\mathrm{Fe}_{3} \mathrm{~S}_{4}$ in the literature, namely $8-19 \mathrm{kA} \mathrm{m}^{-1} \cdot{ }^{20,55,56}$ However there is not much detail available in the literature to correlate the average crystallite size and morphology of the $\mathrm{Fe}_{3} \mathrm{~S}_{4}$ with the observed coercivity. Direct domain observations by Hoffmann ${ }^{57}$ placed the single-domain to multidomain boundary at 700-800 $\mathrm{nm}$. Given that the observed crystallite sizes in Sample VII fell well below this boundary, and also given that the measured retention of coercivity indicates that the sample was not superparamagnetic, the $\mathrm{Fe}_{3} \mathrm{~S}_{4}$ sheets in Sample VII should fall within the single-domain range. This matches predictions by Ricci et al. ${ }^{58}$ based on calculations of domain structure in $\mathrm{Fe}_{3} \mathrm{~S}_{4}$, that a square parallelepiped of $\mathrm{Fe}_{3} \mathrm{~S}_{4}$ of side lengths greater than $\sim 50 \mathrm{~nm}$ would be expected to fall within the single-domain region. Furthermore the $M_{\mathrm{r}} / M_{\mathrm{S}}$ ratio of 0.36 at $300 \mathrm{~K}$ matches well that of other samples of $\mathrm{Fe}_{3} \mathrm{~S}_{4}$ determined to be single-domain; ${ }^{.5}$ despite the fact that this figure is quite low compared to what is expected for a material with cubic magnetocrystalline anisotropy. ${ }^{59}$

Sample XII and XIII consisted of large $\mathrm{Fe}_{1-x} \mathrm{~S}$ crystallites with an increasing proportion of 5-6 nm Fe $\mathrm{S}_{4}$ nanocrystals. The ZFC/FCs plots of the samples indicated that Sample XII remained in a magnetically irreversible state between 10 and $270 \mathrm{~K}$, and transitioned to a magnetically reversible state above this temperature; whereas Sample XIII transitioned from a magnetically irreversible to reversible state above approximately $200 \mathrm{~K}$. In addition to this, discontinuities in the ZFC/FC plots around $100 \mathrm{~K}$ for Sample XII, and around $75 \mathrm{~K}$ for Sample XIII are indicative of magnetic ordering transitions occurring for populations of crystallites at these temperatures within the samples. Given that Sample X and Sample VII had confirmed a lack of magnetic ordering transitions for the bulk $\mathrm{Fe}_{1-x} \mathrm{~S}$ and $\mathrm{Fe}_{3} \mathrm{~S}_{4}$, respectively, which was synthesised during this project, this is suggestive that the magnetic ordering transitions are size-related. 
Field loops show a drop in coercivity from 92 and $52 \mathrm{kA} \mathrm{m}^{-1}$ for Sample XII and XIII, respectively, at $10 \mathrm{~K}$; to $4 \mathrm{kA} \mathrm{m}^{-1}$ for Sample XII and essentially no coercivity for Sample XIII, at $300 \mathrm{~K}$. Together with the evidence of magnetic ordering transition mentioned in the preceding paragraph, this suggests a transition to superparamagnetism at approximately 270 and $200 \mathrm{~K}$ for Samples XII and XIII, respectively.

However, the fluctuations in the ZFC/FC plot for Sample XII, and to a lesser extent Sample XIII; together with the fact that the field loops for these samples were wasp-waisted over some or all of the temperature measured, are evidence of significant inhomogeneity in the magnetic properties of the samples. This is obviously related to the mixture of phases of substantially differing coercivities and susceptibilities. As ferrimagnetic $\mathrm{Fe}_{3} \mathrm{~S}_{4}$ has a far higher initial susceptibility and saturation magnetisation, and is easier to saturate than high-coercivity $\mathrm{Fe}_{1-x} \mathrm{~S}$, it mostly overwhelms contributions from $\mathrm{Fe}_{1-x} \mathrm{~S}$ when present to any extent in the sample. However, the presence of substantial portions of $\mathrm{Fe}_{1-x} \mathrm{~S}$ makes it impossible to determine accurately the (mass-normalised) saturation magnetisation of $\mathrm{Fe}_{3} \mathrm{~S}_{4}$, and difficult to assess size-dependent transitions from ferrimagnetism to superparamagnetism in small $\mathrm{Fe}_{3} \mathrm{~S}_{4}$ nanocrystals. Given the desirability of phase-pure and monodisperse $\mathrm{Fe}_{3} \mathrm{~S}_{4}$ nanocrystal samples for the determination of these parameters, and given the difficulty producing phase-pure samples of $\mathrm{Fe}_{3} \mathrm{~S}_{4}$ using the methodology developed in this chapter, it was decided to pursue an alternative synthetic approach. This was namely the sulfidisation of iron nanocrystals to form $\mathrm{Fe}_{3} \mathrm{~S}_{4}$ nanocrystals (or alternatively oxidation of iron nanocrystals to produce structurally analogous $\mathrm{Fe}_{3} \mathrm{O}_{4}$ nanocrystals), and is detailed in the following chapter (Chapter 5). 


\subsection{Conclusions}

Reaction of $\mathrm{Fe}(\mathrm{acac})_{2}$ and elemental sulfur in oleylamine produced a number of iron sulfide phases and morphologies, depending primarily on reaction temperature and duration. Reactions at $300^{\circ} \mathrm{C}$ for 30 minutes produced solely submicron-sized faceted $\mathrm{Fe}_{1-x} \mathrm{~S}$ crystallites, whereas reaction at $200^{\circ} \mathrm{C}$ for 4 hours produced a mixture of $\mathrm{Fe}_{1-x} \mathrm{~S}$ crystallites and $\mathrm{Fe}_{3} \mathrm{~S}_{4}$ sheets. Rapidly inducing iron sulfide formation by injecting either precursor into a solution of the other at $280-290^{\circ} \mathrm{C}$, followed by rapid cooling produced a mixture of submicron-sized, faceted $\mathrm{Fe}_{1-x} \mathrm{~S}$ crystallites and $5 \mathrm{~nm} \mathrm{Fe} \mathrm{S}_{4}$ nanocrystals, which were produced by burst nucleation. Reaction at $>260^{\circ} \mathrm{C}$ for more than 10 minutes caused the absence of $\mathrm{Fe}_{3} \mathrm{~S}_{4}$, as $\mathrm{Fe}_{3} \mathrm{~S}_{4}$ is not stable above $\sim 200^{\circ} \mathrm{C}$.

Substituting $\mathrm{FeCl}_{2}$ for $\mathrm{Fe}(\mathrm{acac})_{2}$ increased the average crystallite size - when $\mathrm{FeCl}_{2}$ solution was injected into a sulfur solution at $280^{\circ} \mathrm{C}$, the $\mathrm{Fe}_{3} \mathrm{~S}_{4}$ nanocrystals produced had an average diameter of $20 \mathrm{~nm}$, and frequent examples of a twinned octahedral morphology were evident. Although this is probably due to the larger crystallite size making faceted morphologies evident, rather than as a direct effect of the iron source.

Altering the ratio of $\mathrm{Fe}(\mathrm{acac})_{2}: \mathrm{S}$ away from the ratio necessary for stoichiometric or near-stoichiometric $\mathrm{Fe}_{1-x} \mathrm{~S}$ or $\mathrm{Fe}_{3} \mathrm{~S}_{4}$ (1:1 and 1:1.3, respectively) did not alter the phases of the products produced, and unreacted excess elemental sulfur proved to be difficult to remove from the iron sulfide products.

Pure $\mathrm{Fe}_{1-x} \mathrm{~S}$ was an antiferromagnet, with a low susceptibility and high coercivity. The introduction of ferrimagnetic $\mathrm{Fe}_{3} \mathrm{~S}_{4}$ into the product mixture greatly increased the overall magnetic moment of the sample, overwhelming the contribution from $\mathrm{Fe}_{1-x} \mathrm{~S}$, although wasp-waisted field loops were still evident as a consequence of the high coercivity $\mathrm{Fe}_{1-x} \mathrm{~S}$ component. $\mathrm{Fe}_{3} \mathrm{~S}_{4}$ sheets remained in a ferrimagnetic state at room temperature, whereas samples containing $5 \mathrm{~nm}$ spherical $\mathrm{Fe}_{3} \mathrm{~S}_{4}$ nanocrystals exhibited transitions to superparamagnetism above 200 to $250 \mathrm{~K}$.

The difficulty producing phase-pure samples of $\mathrm{Fe}_{3} \mathrm{~S}_{4}$ using the methodology developed in this chapter, and the difficulty in establishing magnetic parameters from mixtures of phases led to the development of the synthetic methodology to be presented in the following chapter. 


\subsection{Method}

Iron(II) acetylacetonate (Fe(acac) $\left.)_{2}, 99.95 \%\right)$ and anhydrous iron(II) chloride $\left(\mathrm{FeCl}_{2}\right.$, 98\%) were purchased from Sigma-Aldrich and stored in a desiccator. Sulfur (sublimed) was purchased from BDH and used as received. Oleylamine (tech, $\geq 70 \%$ ), and hexadecylamine (HDA, tech., $\geq 90 \%$ ) were purchased from Fluka; and tetraethylene glycol dimethylether (tetraglyme, 99\%) was purchased from Sigma-Aldrich. Acetone, methanol, ethanol and toluene were of reagent grade. All solvents were used as received.

Oleylamine was degassed prior to use by vigorously bubbling nitrogen through for $15-$ 30 minutes.

\subsubsection{Reaction of $\mathrm{Fe}(\mathrm{acac})_{2}$ and sulfur in oleylamine}

In a typical experiment, $\mathrm{Fe}(\mathrm{acac}){ }_{2}\left(0.38 \mathrm{~g}, 1.5 \times 10^{-3} \mathrm{~mol}\right)$ and sulfur $\left(0.05 \mathrm{~g}, 1.5 \times 10^{-3}\right.$ mol) were loaded into a flask, and flushed with nitrogen for 10 minutes. Degassed oleylamine $\left(30 \mathrm{~cm}^{3}\right)$ was added, and the resulting red suspension was degassed for a further 15 minutes. The mixture was heated to a reaction temperature of between 140 and $330^{\circ} \mathrm{C}$, and held for between 30 and 240 minutes. The reaction mixture blackened at approximately $90^{\circ} \mathrm{C}$. The reaction mixture was then cooled, acetone $\left(20 \mathrm{~cm}^{3}\right)$ was added, and the mixture was centrifuged at 4,000 rpm for 5 minutes to form a black pellet and clear brown supernatant, which was discarded. The precipitate was resuspended in toluene $\left(15 \mathrm{~cm}^{3}\right)$ with the aid of sonication, acetone $\left(20 \mathrm{~cm}^{3}\right)$ was added and the mixture was centrifuged at 4,000 rpm for 5 minutes. The clear, colourless supernatant was discarded. This procedure was repeated once more, and the precipitate was dried in vacuo and stored as a powder.

Within the experimental method given above, the ratio of $\mathrm{Fe}(\mathrm{acac})_{2}$ to sulfur was varied between $1: 1$ and 4:11, and the concentration of the precursors was varied between 0.01 and $0.1 \mathrm{~mol} \mathrm{dm}^{3}$. 


\subsubsection{Reaction of $\mathrm{Fe}(\mathrm{acac})_{2}$ in oleylamine with injection of sulfur solution}

In a typical experiment, $\mathrm{Fe}(\mathrm{acac})_{2}\left(0.38 \mathrm{~g}, 1.5 \times 10^{-3} \mathrm{~mol}\right)$ was loaded into a flask and flushed with nitrogen for 10 minutes. Degassed oleylamine $\left(29 \mathrm{~cm}^{3}\right)$ was added, and the resulting red suspension was degassed for a further 15 minutes. The mixture was heated to a reaction temperature of $280^{\circ} \mathrm{C}$, and sulfur $\left(0.05 \mathrm{~g}, 1.5 \times 10^{-3} \mathrm{~mol}\right.$, dissolved in 3 $\mathrm{cm}^{3}$ oleylamine) was injected. The mixture of $\mathrm{Fe}(\mathrm{acac})_{2}$ in oleylamine had blackened between 90 and $130^{\circ} \mathrm{C}$, and no further colour change was observed, although slight bubbling was evident after sulfur was injected. The mixture was either cooled immediately after injection, or maintained at $280^{\circ} \mathrm{C}$ for 10 minutes before cooling. Methanol $\left(20 \mathrm{~cm}^{3}\right)$ was added to the cooled reaction mixture, which was centrifuged at 4,000 rpm for 10 - 15 minutes to form a black pellet and clear yellow supernatant, which was discarded. The pellet was resuspended in toluene $\left(15 \mathrm{~cm}^{3}\right)$ with the aid of sonication; methanol $\left(20 \mathrm{~cm}^{3}\right)$ was added, the mixture was centrifuged at $4,000 \mathrm{rpm}$ for 10 minutes; and the clear, colourless supernatant was discarded. This procedure was repeated once more, and the precipitate was dried in vacuo and stored as a powder.

Within this method the ratio of $\mathrm{Fe}(\mathrm{acac})_{2}: \mathrm{S}$ was varied between $1: 1$ and 1:2.75.

\subsubsection{Reaction of $\mathrm{FeCl}_{2}$ in oleylamine with injection of sulfur solution}

$\mathrm{FeCl}_{2}\left(0.19 \mathrm{~g}, 1.5 \times 10^{-3} \mathrm{~mol}\right)$ was loaded into a flask, and flushed with nitrogen for 15 minutes. Degassed oleylamine $\left(28 \mathrm{~cm}^{3}\right)$ was added, and the yellow suspension was degassed for a further 15 minutes. The suspension was heated to $280^{\circ} \mathrm{C}$, blackening above $100^{\circ} \mathrm{C}$, and sulfur $\left(0.050 \mathrm{~g}, 1.6 \times 10^{-3} \mathrm{~mol}\right.$, in $3 \mathrm{~cm}^{3}$ oleylamine $)$ was injected. The reaction mixture was immediately cooled. Acetone $\left(15 \mathrm{~cm}^{3}\right)$ was added, and the reaction mixture centrifuged at $4,000 \mathrm{rpm}$ for 5 minutes to give a black pellet and clear, brown supernatant, which was discarded. The pellet was resuspended in toluene (15 $\left.\mathrm{cm}^{3}\right)$ with the aid of sonication, acetone $\left(20 \mathrm{~cm}^{3}\right)$ was added, and the mixture was centrifuged at 4,000 rpm for 10 minutes. The clear, colourless supernatant was discarded. This procedure was repeated once more, and the precipitate was dried in vacuo and stored as a powder. 


\subsubsection{Reaction of $\mathrm{Fe}(\mathrm{acac})_{2}$ and sulfur in hexadecylamine}

In a typical experiment, $\mathrm{HDA}(17 \mathrm{~g})$ was loaded into a flask and heated to $100^{\circ} \mathrm{C}$ to melt. The solvent was degassed by bubbling nitrogen through for 15 minutes, then cooled to $65^{\circ} \mathrm{C}$. Sulfur $\left(0.040 \mathrm{~g}, 1.25 \times 10^{-3} \mathrm{~mol}\right)$, then Fe(acac) $)_{2}\left(0.32 \mathrm{~g}, 1.26 \times 10^{-3}\right)$ were added under nitrogen flow, to produce a red suspension, which soon blackened. The mixture was heated to a reaction temperature of between 200 and $300^{\circ} \mathrm{C}$, and held for between $30-120$ minutes. The reaction mixture was cooled to $70^{\circ} \mathrm{C}$, and toluene $\left(20 \mathrm{~cm}^{3}\right)$ was added to prevent solidification. Methanol $\left(20 \mathrm{~cm}^{3}\right)$ was added, and the mixture was centrifuged at 4,000 rpm for 5 minutes to produce a black pellet and clear, yellow supernatant, which was discarded. The pellet was resuspended in toluene (15 $\left.\mathrm{cm}^{3}\right)$ with the aid of sonication; methanol $\left(20 \mathrm{~cm}^{3}\right)$ was added, the mixture was centrifuged at 4,000 rpm for 5 minutes, and the clear colourless supernatant was discarded. This procedure was repeated once more, and the precipitate was dried in vacuo and stored as a powder.

\subsubsection{Addition of $\mathrm{FeCl}_{2}$ into solution of sulfur in oleylamine}

In a typical experiment, sulfur $\left(0.0052 \mathrm{~g}, 1.6 \times 10^{-4} \mathrm{~mol}\right)$ was loaded into a flask, and flushed with nitrogen for 15 minutes. Degassed oleylamine $\left(13.5 \mathrm{~cm}^{3}\right)$ was added to give a clear yellow solution, which was heated to between 200 and $260^{\circ} \mathrm{C} . \mathrm{FeCl}_{2}$ $\left(0.019 \mathrm{~g}, 1.5 \times 10^{-4} \mathrm{~mol}\right.$, in $1.5 \mathrm{~cm}^{3}$ tetraglyme $)$ was injected rapidly, whereupon the red solution blackened. The reaction was either immediately cooled, or aliquots were withdrawn over a period of 30 minutes, and quenched in chilled, degassed acetone. The resulting samples were centrifuged at 4,000 rpm for 5 minutes to produce precipitate pellets; and clear, brown supernatants, which were discarded. The pellets were resuspended in toluene $\left(5 \mathrm{~cm}^{3}\right)$ with the aid of sonication, acetone $\left(10 \mathrm{~cm}^{3}\right)$ was added, and the mixtures centrifuged at 4,000 rpm for 5 minutes, and the clear supernatant discarded. This procedure was repeated once more, and the samples were stored as suspensions in toluene. 


\subsection{References}

(1) R. A. Yund and H. T. Hall, Econ. Geol., 1969, 64, 420.

(2) H. Nakazawa and N. Morimoto, Mater. Res. Bull., 1971, 6, 345.

(3) S. A. Kissin and S. D. Scott, Econ. Geol., 1982, 77, 1739.

(4) E. J. Fasiska, Phys. Status Solidi A, 1972, 10, 169.

(5) M. Tokonami, K. Nishiguchi and N. Morimoto, Am. Mineral., 1972, 57, 1066.

(6) E. J. Schwarz and D. J. Vaughan, J. Geomagn. Geoelectr., 1972, 24, 441.

(7) A. F. Andresen and P. Torbo, Acta Chem. Scand., 1967, 21, 2841.

(8) T. Hirone, S. Maeda, S. Chiba and N. Tsuya, J. Phys. Soc. Jpn., 1954, 9, 500.

(9) H. Wang and I. Salveson, Phase Transitions, 2005, 78, 547.

(10) S. Hunger and L. G. Benning, Geochem. Trans., 2007, 8.

(11) M. Uda, Z. Anorg. Allg. Chem., 1967, 350, 105.

(12) M. S. Jagadeesh and M. S. Seehra, J. Phys. D: Appl. Phys., 1981, 14, 2153.

(13) E. F. Makarov, A. S. Marfunin, A. R. Mkrtchyan, G. N. Nadzharyan, V. A. Povitskii and R. A. Stukan, Sov. Phys. Solid State, 1969, 11, 391.

(14) J. P. Wilcoxon, P. P. Newcomer and G. A. Samara, Solid State Commun., 1996, 98, 581.

(15) S. Kar and S. Chaudhuri, Mater. Lett., 2005, 59, 289.

(16) X. Qian, X. Yi and Y. Qian, Mater. Lett., 2001, 48, 109.

(17) P. Chin, J. Ding, J. B. Yi and B. H. Liu, J. Alloys Compd., 2005, 309, 225.

(18) B. J. Skinner, R. C. Erd and F. S. Grimaldi, Am. Mineral., 1964, 49, 543.

(19) M. J. Dekkers, H. F. Passier and M. A. A. Schoonen, Geophys. J. Int., 2000, 141, 809.

(20) M. R. Spender, J. M. D. Coey and A. H. Morrish, Can. J. Phys., 1972, 50, 2313.

(21) X. Chen, X. Zhang, J. Wan, Z. Wang and Y. Qian, Chem. Phys. Lett., 2005, 403, 396.

(22) X. F. Qian, X. M. Zhang, C. Wang, Y. Xie, W. Z. Wang and Y. T. Qian, Mater. Sci. Eng., B, 1999, 64, 170.

(23) Z. He, S.-H. Yu, X. Zhou, X. Li and J. Qu, Adv. Funct. Mater., 2006, 16, 1105.

(24) S. Yamaguchi and H. Wada, Z. Anorg. Allg. Chem., 1970, 377, 339.

(25) P. V. Vanitha and P. O'Brien, J. Am. Chem. Soc., 2008, 130, 17256.

(26) M. Nath, A. Choudhury, A. Hundu and C. N. R. Rao, Adv. Mater., 2003, 15, 2098.

(27) W. Han and M. Gao, Cryst. Growth Des., 2008, 8, 1023.

(28) D. Rickard and G. W. I. Luther, Chem. Rev., 2007, 107, 514.

(29) J. Von Hoene, R. G. Charles and W. M. Hickam, J. Phys. Chem., 1958, 62, 1098.

(30) F. P. Daly and C. Brown, W., J. Phys. Chem., 1973, 77, 1859.

(31) A. K. Verma, T. Rauchfuss and S. R. Wilson, Inorg. Chem., 1995, 34, 3072.

(32) F. H. Chung, J. Appl. Crystallogr., 1975, 8, 17.

(33) H. P. Klug and L. E. Alexander, X-ray Diffraction Procedures, John Wiley \& Sons, New York, 1954.

(34) A. C. Bishop, An Outline of Crystal Morphology, Hutchinson \& Co., London, 1967.

(35) L. Smart and E. Moore, Solid State Chemistry: An Introduction, Chapman-Hall, Cheltenham, 1996.

(36) M. J. Dekkers, Phys. Earth Planet. Inter., 1988, 52, 376.

(37) J. Gjønnes and A. F. Moodie, Acta Crystallogr., 1965, 19, 65.

(38) M. Pósfai and I. Dódony, Eur. J. Mineral., 1990, 2, 525.

(39) M. Pósfai and I. Dódony, Eur. J. Mineral., 1990, 2, 529. 
(40) R. O'Handley, Modern Magnetic Material: Principles and Applications, John Wiley \& Sons, New York, 2000, 29-43.

(41) M. Uda, Am. Mineral., 1965, 50, 1487.

(42) A. Sugaki and H. Shima, Sci. Rep. Tohoku Univ. 3rd Ser. Mineral. Petro. Econ. Geol., 1977, 13, 147.

(43) H. T. J. Evans, Science, 1970, 167, 621.

(44) M. Pósfai, T. G. Sharp and A. Kontny, Am. Mineral., 2000, 85, 1406.

(45) S. Horiuchi, H. Wada and T. Moori, J. Cryst. Growth, 1974, 24/25, 624.

(46) H. Ohfuji and D. Rickard, Earth. Planet. Sci. Lett., 2006, 241, 227.

(47) A. J. Devey, R. Grau-Crespo and N. H. de Leeuw, J. Phys. Chem. C, 2008, 112, 10960.

(48) A. R. Lennie, S. A. T. Redfern, P. E. Champness, C. P. Stoddart, P. F. Schofield and D. J. Vaughan, Am. Mineral., 1997, 82, 302.

(49) M. J. Dekkers, J.-L. Mattéi, G. Fillion and P. Rochette, Geophys. Res. Lett., 1989, 16, 855.

(50) K. Sato, M. Yamada and T. Hirone, J. Phys. Soc. Jpn., 1964, 19, 1592.

(51) M. McElfresh, Quantum Design, San Diego, 1994.

(52) A. H. Morrish, The Physical Principles of Magnetism, The International Institute of Electrical and Electronics Engineers, New York, 2001, 450-453.

(53) H. Soffel, J. Geophys., 1977, 42, 351.

(54) A. P. Roberts, Y. Cui and K. L. Verosub, J. Geophys. Res., 1995, 100, 17.

(55) A. P. Roberts, Earth. Planet. Sci. Lett., 1995, 134, 227.

(56) I. F. Snowball, Phys. Earth Planet. Inter., 1991, 68, 32.

(57) V. Hoffmann, Phys. Earth Planet. Inter., 1992, 70, 288.

(58) J. C. D. Ricci and J. L. Kirschvink, J. Geophys. Res., 1992, 97, 17.

(59) E. P. Wohlfarth, J. Magn. Magn. Mater., 1983, 39, 39. 


\section{Chapter 5 Sulfidisation or oxidation of iron/iron oxide nanocrystals}

\subsection{Introduction}

In this chapter synthetic methodology was developed to produce nanocrystals of the isostructural iron sulfide spinel $\left(\mathrm{Fe}_{3} \mathrm{~S}_{4}\right)$ and iron oxide spinel $\left(\mathrm{Fe}_{3} \mathrm{O}_{4} / \gamma-\mathrm{Fe}_{2} \mathrm{O}_{3}\right)$ phases with similar average diameters and narrow size distributions. Magnetic measurements were performed to compare the magnetic behaviour of nanocrystals of the two phases. As described below (section 5.1.4), the reaction of $\mathrm{Fe}(\mathrm{acac})_{2}$ over a period of hours, followed by injection of sulfur proved to be a viable route to $\mathrm{Fe}_{3} \mathrm{~S}_{4}$ nanocrystals affording better phase purity and narrower size distribution than had been attained in Chapter 4. When sulfur was not introduced, the initial product of the decomposition reaction was observed to oxidise during work-up to produce nanocrystals of one or more of a variety of iron oxide phases.

In order to provide a point of reference for the iron oxide nanocrystals synthesised during this project, literature syntheses of iron and iron oxide nanocrystals are summarised below. Literature syntheses of $\mathrm{Fe}_{3} \mathrm{~S}_{4}$ were discussed previously in Chapter 4 section 4.1.3.2. The difficulties of distinguishing experimentally between the iron oxide spinel phases $\mathrm{Fe}_{3} \mathrm{O}_{4}$ and $\gamma-\mathrm{Fe}_{2} \mathrm{O}_{3}$ - especially in the case of nanocrystals will be discussed (section 5.1.3), followed by the motivation of the work undertaken in this project (section 5.1.4).

\subsubsection{Iron nanocrystals}

Iron nanocrystals have been synthesised by a variety of chemical and physical methods, ranging from decomposition of $\left[\mathrm{Fe}(\mathrm{CO})_{5}\right]$ - either thermally or sonochemically; reduction of iron salts in micelles; vapour phase deposition; and mechanochemical milling. ${ }^{1}$ Nanocrystals have been produced, ranging in size from 2 to $20 \mathrm{~nm}$, with varying degrees of shape and size uniformity. Thermal decomposition of $\left[\mathrm{Fe}(\mathrm{CO})_{5}\right]$, in particular has led to monodisperse, spherical nanocrystals with a variety of average diameters depending on the use of surfactant/solvent systems. 
Iron nanocrystals have been a target of interest due to the prospect of their use as active, and comparably cheap catalysts; or in a variety of applications exploiting their magnetic properties. Of the three metals which are ferromagnetic at room temperature, iron has the highest saturation magnetisation $\left(217,164\right.$, and $54 \mathrm{~A} \mathrm{~m}^{-2} \mathrm{~kg}^{-1}$ for iron, cobalt and nickel, respectively). ${ }^{2}$ In addition to this, iron is a soft ferromagnetic material, with low coercivity and low magnetocrystalline anisotropy. As a consequence of which, the superparamagnetic critical radius is approximately $10 \mathrm{~nm}$ at room temperature. Thus, low/zero coercivity materials can be prepared in either the multidomain or superparamagnetic regime, respectively. This combination of high potential magnetisation and low/zero coercivity is attractive for applications such as magnetic separation, MRI contrast reagents or magnetic hyperthermia treatment of tumours. ${ }^{1}$

By far the greatest problem encountered with iron nanocrystals is their extreme reactivity. Bare iron nanocrystals are pyrophoric, and great care must be taken during synthesis and handling to exclude oxygen and moisture. Capping of the iron surface with organic polymers, or small organic molecules in order to control the dispersity and size distribution often results in a significant reduction of saturation magnetisation (for example, the saturation magnetisation of capped 7-9 $\mathrm{nm}$ iron particles were found to decrease from 172 to $82 \mathrm{~A} \mathrm{~m}^{-2} \mathrm{~kg}^{-1}$ with increasing polymer polarity). Similarly, a reduction of saturation magnetisation occurs when an inorganic layer is used to encapsulate the iron nanocrystals, in order to prevent their oxidation. This is proposed to be due either to pinning of surface spins by strongly interacting surface species, or due to a spreading of the density of states allowing electrons to move from the spin majority to spin minority band. ${ }^{1}$ Regardless of this, passivated iron nanocrystals may still have a saturation magnetisation far in excess of that of iron oxide nanocrystals. Consequently iron nanocrystals passivated with gold, magnesium, and iron oxide have all been produced. ${ }^{1}$

\subsubsection{Iron oxide nanocrystals}

\section{$\alpha-\mathrm{Fe}_{2} \mathrm{O}_{3}$ (haematite)}

Nanocrystalline $\alpha-\mathrm{Fe}_{2} \mathrm{O}_{3}$ has been synthesised by a variety of methods, both in solution and in the solid state. High-energy milling of $\alpha$-FeOOH produced $\alpha-\mathrm{Fe}_{2} \mathrm{O}_{3}$ nanocrystals with an average diameter of $20 \mathrm{~nm}$ after 90 hours. However, as is often the case with milling methods, the particles were irregularly shaped with a wide size distribution. ${ }^{4}$ 
Nanowires of $\alpha-\mathrm{Fe}_{2} \mathrm{O}_{3}$ were grown on an iron substrate heated to $600^{\circ} \mathrm{C}$ under an oxidising atmosphere for several days. The wires were, on average, $40-50 \mathrm{~nm}$ in diameter, and up to $20 \mu \mathrm{m}$ long; and showed a high degree of crystal twinning along their lengths. ${ }^{4}$

Hierarchical dentritic $\alpha-\mathrm{Fe}_{2} \mathrm{O}_{3}$ assemblies were synthesised in solution by a microwave-assisted decomposition of $\mathrm{K}_{3}\left[\mathrm{Fe}(\mathrm{CN})_{6}\right]$ under hydrothermal conditions. The reaction was postulated to proceed via an initial precipitation of iron hydroxide and oxyhydroxide, which was dehydrated to $\alpha-\mathrm{Fe}_{2} \mathrm{O}_{3}$ as the reaction proceeded. ${ }^{5}$ Another solution-based synthesis used iron(III) acetylacetonate as a precursor in a solvothermal decomposition, which produced both spherical $\mathrm{Fe}_{3} \mathrm{O}_{4}$ and $\alpha-\mathrm{Fe}_{2} \mathrm{O}_{3}$ nanocrystals. Initial formation of $\alpha-\mathrm{Fe}_{2} \mathrm{O}_{3}$ was found to be assisted by the presence of water in the solvent (propanol). Upon extended reaction times at $300^{\circ} \mathrm{C}, \alpha-\mathrm{Fe}_{2} \mathrm{O}_{3}$ was converted to $\mathrm{Fe}_{3} \mathrm{O}_{4}$. The average diameter of the particles produced by this method was $11 \mathrm{~nm}^{6}$

As antiferromagnetic $\alpha-\mathrm{Fe}_{2} \mathrm{O}_{3}$ has a very low saturation magnetisation, most suggested applications for $\alpha-\mathrm{Fe}_{2} \mathrm{O}_{3}$ nanocrystals are based on the catalytic or photoelectric ${ }^{7}$ properties of $\alpha-\mathrm{Fe}_{2} \mathrm{O}_{3}$.

\section{$\mathrm{Fe}_{3} \mathrm{O}_{4}$ (magnetite) \& $\gamma-\mathrm{Fe}_{2} \mathrm{O}_{3}$ (maghemite)}

Nanocrystals of the two ferrimagnetic iron oxide spinel phases $\mathrm{Fe}_{3} \mathrm{O}_{4}$ and $\gamma-\mathrm{Fe}_{2} \mathrm{O}_{3}$ are of great interest for use in biomedical applications such as drug delivery, MRI contrast reagents, magnetic separation, and magnetic hyperthermia ${ }^{8}$; or applications in data storage. ${ }^{9}$

Nanocrystals which are superparamagnetic at room temperature are preferred for the biomedical applications, as they can be manipulated by an applied magnetic field, and upon removal of the field their magnetisation rapidly decays to zero, precluding aggregation due to magnetic dipole interactions. Conversely, ferro- or ferrimagnetism, with a large, stable magnetisation and high anisotropy is required for data storage applications. ${ }^{10}$ Although work in nanocrystals for this application has focused strongly in various FePt and CoPt alloys in recent years, ${ }^{10}$ currently traditional iron oxide media are still in use. ${ }^{9}$ 
Spherical $\mathrm{Fe}_{3} \mathrm{O}_{4}$ nanocrystals have been prepared by co-precipitation of $\mathrm{Fe}^{2+}$ and $\mathrm{Fe}^{3+}$ salts in a basic aqueous environment, followed by size-selective purification to yield samples with average diameters of $2-6 \mathrm{~nm}$ and tight size distributions. ${ }^{11}$ Alternatively, many high temperature syntheses for $\mathrm{Fe}_{3} \mathrm{O} 4$ and $\gamma-\mathrm{Fe}_{2} \mathrm{O}_{3}$ involving high boiling point organic solvents have been published, usually employing iron precursors such as $\left[\mathrm{Fe}(\mathrm{CO})_{5}\right], \mathrm{Fe}(\mathrm{acac})_{2}$ or $\mathrm{Fe}(\mathrm{acac})_{3}$ and mild oxidising agents such as hexane-1,2-diol or trimethylamine oxide. ${ }^{12-15}$

\section{$\mathrm{FeO}$ (wüstite)}

As $\mathrm{FeO}$ is both metastable and weakly paramagnetic at room temperature, it is not a target for applications, but has been used as an intermediary to iron and iron oxide nanostructures. Murray et al. ${ }^{16}$ synthesised spherical or cubic FeO nanocrystals by decomposition of $\left[\mathrm{Fe}(\mathrm{CO})_{5}\right], \mathrm{Fe}(\mathrm{OAc})_{2}, \mathrm{Fe}(\mathrm{acac})_{2}$ or $\mathrm{Fe}(\mathrm{acac})_{3}$ in trioctylamine or dioctylether. Upon heating above $300^{\circ} \mathrm{C}$ these were found to disproportionate to $\mathrm{Fe}_{3} \mathrm{O}_{4}$ core and $\alpha-F e$ shell, which would oxidise further upon exposure to air.

Sun et al. ${ }^{17}$ synthesised spherical or truncated octahedral nanocrystals with sizes ranging from 14 to $100 \mathrm{~nm}$ by thermal decomposition of $\mathrm{Fe}(\mathrm{acac})_{3}$ in oleylamine and oleic acid. These were then annealed in air to produce $\mathrm{Fe}_{3} \mathrm{O}_{4}, \gamma-\mathrm{Fe}_{2} \mathrm{O}_{3}$ or $\alpha-\mathrm{Fe}_{2} \mathrm{O}_{3}$ nanocrystals.

\subsubsection{Distinguishing experimentally between $\mathrm{Fe}_{3} \mathrm{O}_{4}$ and $\gamma-\mathrm{Fe}_{2} \mathrm{O}_{3}$}

As mentioned previously (Chapter 1 sections 1.3.3.3 and 1.3.3.4), the phases $\mathrm{Fe}_{3} \mathrm{O}_{4}$ and $\gamma-\mathrm{Fe}_{2} \mathrm{O}_{3}$ are both based upon the spinel structure. In moving from $\mathrm{Fe}_{3} \mathrm{O}_{4}$ to $\gamma-\mathrm{Fe}_{2} \mathrm{O}_{3}$, the oxidation of $\mathrm{Fe}^{2+}$ to smaller $\mathrm{Fe}^{3+}$ ions, and the inclusion of charge-balancing cation vacancies leads to a slight contraction of the lattice constant of $\gamma-\mathrm{Fe}_{2} \mathrm{O}_{3}$ compared to that of $\mathrm{Fe}_{3} \mathrm{O}_{4}\left(a=8.3960 \AA\right.$ for $\mathrm{Fe}_{3} \mathrm{O}_{4}, a=8.34 \AA$ for $\left.\gamma-\mathrm{Fe}_{2} \mathrm{O}_{3}\right){ }^{18}$ This slight alteration shifts diffraction peaks for $\gamma-\mathrm{Fe}_{2} \mathrm{O}_{3}$ to higher angles. However in this project small crystallite size broadened the diffraction peaks, making it essentially impossible to detect the smaller than $1 \%$ change in lattice constant that distinguishes $\gamma-\mathrm{Fe}_{2} \mathrm{O}_{3}$ and $\mathrm{Fe}_{3} \mathrm{O}_{4}$.

In addition to the shift in peak position, ordering of cation vacancies in $\gamma-\mathrm{Fe}_{2} \mathrm{O}_{3}$ may occur, altering the symmetry of the lattice and leading to weak superlattice reflections forbidden in the $F d \overline{3} m$ space group of $\mathrm{Fe}_{3} \mathrm{O}_{4}$. However, superlattice reflections are not 
observed for small crystallites, as the diameter of the particle approaches the repeat length of the supercell. Consequently superlattice reflections in $\gamma-\mathrm{Fe}_{2} \mathrm{O}_{3}$ have not been observed for particle sizes below $20 \mathrm{~nm}^{19}$

Given that $\gamma-\mathrm{Fe}_{2} \mathrm{O}_{3}$ and $\mathrm{Fe}_{3} \mathrm{O}_{4}$ may also form solid solutions, ${ }^{18}$ in small crystallites the primary distinction between the two phases must be made on the basis of compositional methods rather than diffractometry. Pure $\gamma-\mathrm{Fe}_{2} \mathrm{O}_{3}$ contains only $\mathrm{Fe}^{3+}$, whereas $\mathrm{Fe}_{3} \mathrm{O}_{4}$ contains $\mathrm{Fe}^{2+}$ and $\mathrm{Fe}^{3+}$. Spectroscopic techniques sensitive to oxidation state, such as X-ray Photoelectron Spectroscopy (XPS), have therefore been used previously to differentiate between the two phases in nanocrystals. ${ }^{20}$

As such oxidation state sensitive techniques were not used in this project, a definitive conclusion could not be made as to whether the nanocrystals in this project determined by SAED and XRD to be iron oxide with the spinel structure, where $\mathrm{Fe}_{3} \mathrm{O}_{4}, \gamma-\mathrm{Fe}_{2} \mathrm{O}_{3}$, or a solid solution of the two phases. However, the brown colour that samples possessed after exposure to the atmosphere suggests that the nanocrystals were at least partially oxidised from $\mathrm{Fe}_{3} \mathrm{O}_{4}$ (black) to $\gamma-\mathrm{Fe}_{2} \mathrm{O}_{3}$ (brown). For the sake of consistency, samples were referred to in this chapter as iron oxide spinel, and in diffraction experiments reflections were indexed to the $F d \overline{3} m$ space group of the spinel structure.

\subsubsection{Motivation}

Work conducted on the reaction of $\mathrm{Fe}(\mathrm{acac})_{2}$ and elemental sulfur above $200^{\circ} \mathrm{C}$ (see Chapter 4) led to the observation that small spherical nanocrystals of $\mathrm{Fe}_{3} \mathrm{~S}_{4}$ were formed if the reaction time was brief ( $<20$ minutes). These reactions produced large $\mathrm{Fe}_{1-x} \mathrm{~S}$ particles as the major product. The $\mathrm{Fe}_{3} \mathrm{~S}_{4}$ nanocrystals that were produced in these reactions had average diameters in the sub-10 nm range, but were considerably polydisperse. Attempts to improve phase selectivity and narrow the size distribution of $\mathrm{Fe}_{3} \mathrm{~S}_{4}$ nanocrystals by injecting sulfur into a hot solution of $\mathrm{Fe}(\mathrm{acac})_{2}$, and altering the cooling profile of the reaction were not successful in producing single-phase samples.

Work conducted during this project had indicated that the reaction of $\mathrm{Fe}(\mathrm{acac})_{2}$ in HDA at $300^{\circ} \mathrm{C}$ for approximately two hours produced small $(<10 \mathrm{~nm})$ spherical iron oxide nanocrystals with a relatively narrow size distribution. Observations of a distinct colour change in the reaction mixture from black to brown during purification in air, led to the 
hypothesis that the reaction initially produced nanocrystals of either iron metal (black) ${ }^{1}$ or $\mathrm{Fe}_{3} \mathrm{O}_{4}$ (black), ${ }^{21}$ which oxidised upon workup to an iron oxide with the spinel structure $\left(\gamma-\mathrm{Fe}_{2} \mathrm{O}_{3}[\right.$ brown $],{ }^{21}$ or a solid solution of $\mathrm{Fe}_{3} \mathrm{O}_{4}$ and $\left.\gamma-\mathrm{Fe}_{2} \mathrm{O}_{3}\right) .{ }^{20}$

Given that the size distribution of the iron oxide spinel nanocrystals produced in this reaction was far narrower than that of iron sulfide nanocrystals produced by the methodology used in Chapter 5, it was hypothesised that if the intermediate iron/iron oxide nanocrystals could be sulfidised, this would prove a better synthetic route to monodisperse iron sulfide nanocrystals than those investigated in Chapter 4. Initial results confirmed that $\mathrm{Fe}_{3} \mathrm{~S}_{4}$ nanocrystals could be produced by the introduction of elemental sulfur to the reaction mixture after several hours of reaction, and that the size distribution and phase purity of the nanocrystals produced was superior to those produced in Chapter 4. 


\subsection{Results}

Experiments were conducted to optimise the synthesis of iron oxide spinel nanocrystals; and to optimise the sulfidisation of the intermediate iron/iron oxide nanocrystals to produce $\mathrm{Fe}_{3} \mathrm{~S}_{4}$. Magnetic measurements were then performed on representative samples.

\subsubsection{Synthesis of iron oxide nanocrystals}

In order to optimise the size and size distribution of iron oxide nanocrystals, the effects of a variety of synthetic parameters were investigated. The effect of reaction time and temperature were investigated for the decomposition of $\mathrm{Fe}(\mathrm{acac})_{2}$ in $\mathrm{HDA}$, as outlined below (section 5.2.1.1). The effect of replenishing the iron precursor at some point during the reaction, in order to increase the average particle size while attempting to maintain a narrow size distribution, was also investigated (section 5.2.1.2).

\subsubsection{Effect of reaction time and temperature}

Iron oxide nanocrystals were synthesised as described in section 5.5.1, specific parameters are given below in Table 5-1. The effect of reaction time and temperature on the size, shape and size distribution was examined by varying the duration of the reaction from $1-4$ hours, and the temperature from $200-300^{\circ} \mathrm{C}$.

Micrographs and SAED patterns for Samples XX and XXI - produced by the decomposition of $\mathrm{Fe}(\mathrm{acac})_{2}$ in $\mathrm{HDA}$ at $200^{\circ} \mathrm{C}$ - are shown below in Figure 5-1; and histograms of the particle size distribution of the two samples (as determined from TEM measurements) are shown in Figure 5-2. The SAED patterns were analysed and indexed as described in A1-9, and an XRD pattern for Sample XXI is shown below in Figure 5-5. Samples XX and XXI were both identified as iron oxide spinel by SAED and XRD. 
Table 5-1 Synthesis of iron oxide nanocrystals in HDA (see 5.5.1).

\begin{tabular}{|c|c|c|c|c|c|c|}
\hline Sample & $\begin{array}{c}\text { Iron } \\
\text { source }\end{array}$ & Solvent & $\begin{array}{c}\text { Reaction } \\
\text { Temp. } \\
/{ }^{\circ} \mathrm{C}\end{array}$ & $\begin{array}{c}\text { Reaction } \\
\text { Duration } \\
/ \text { Hours }\end{array}$ & Result & $D_{h k l}$ \\
\hline XX & $\mathrm{Fe}(\mathrm{Tcac})_{2}$ & HDA size) & 200 & 1 & $\begin{array}{c}\text { Spherical iron oxide } \\
\text { spinel nanocrystals } \\
5.0 \pm 0.8 \mathrm{~nm}(\sigma 16 \%)\end{array}$ & $/$ \\
\hline XXI & $\mathrm{Fe}(\mathrm{acac})_{2}$ & HDA & 200 & 2 & $\begin{array}{c}\text { Spherical iron oxide } \\
\text { spinel nanocrystals } \\
6.1 \pm 0.7 \mathrm{~nm}(\sigma 11 \%)\end{array}$ & $7 \pm 1$ \\
\hline XXII & $\mathrm{Fe}(\mathrm{acac})_{2}$ & HDA & 300 & 1 & $\begin{array}{c}\text { Spherical iron oxide } \\
\text { spinel nanocrystals } \\
5.9 \pm 0.8 \text { nm }(\sigma 14 \%)\end{array}$ & $/$ \\
\hline XXIII & $\mathrm{Fe}(\mathrm{acac})_{2}$ & HDA & 300 & 2 & $\begin{array}{c}\text { Spherical iron oxide } \\
\text { spinel nanocrystals } \\
6.1 \pm 0.6 \mathrm{~nm}(\sigma 10 \%)\end{array}$ & $7 \pm 3$ \\
\hline XXIV & $\mathrm{Fe}(\mathrm{acac})_{2}$ & HDA & 300 & 4 & $\begin{array}{c}\text { Spherical iron oxide } \\
\text { spinel nanocrystals } \\
5.0 \pm 0.8 \text { nm }(\sigma 16 \%)\end{array}$ & $/$ \\
\hline
\end{tabular}

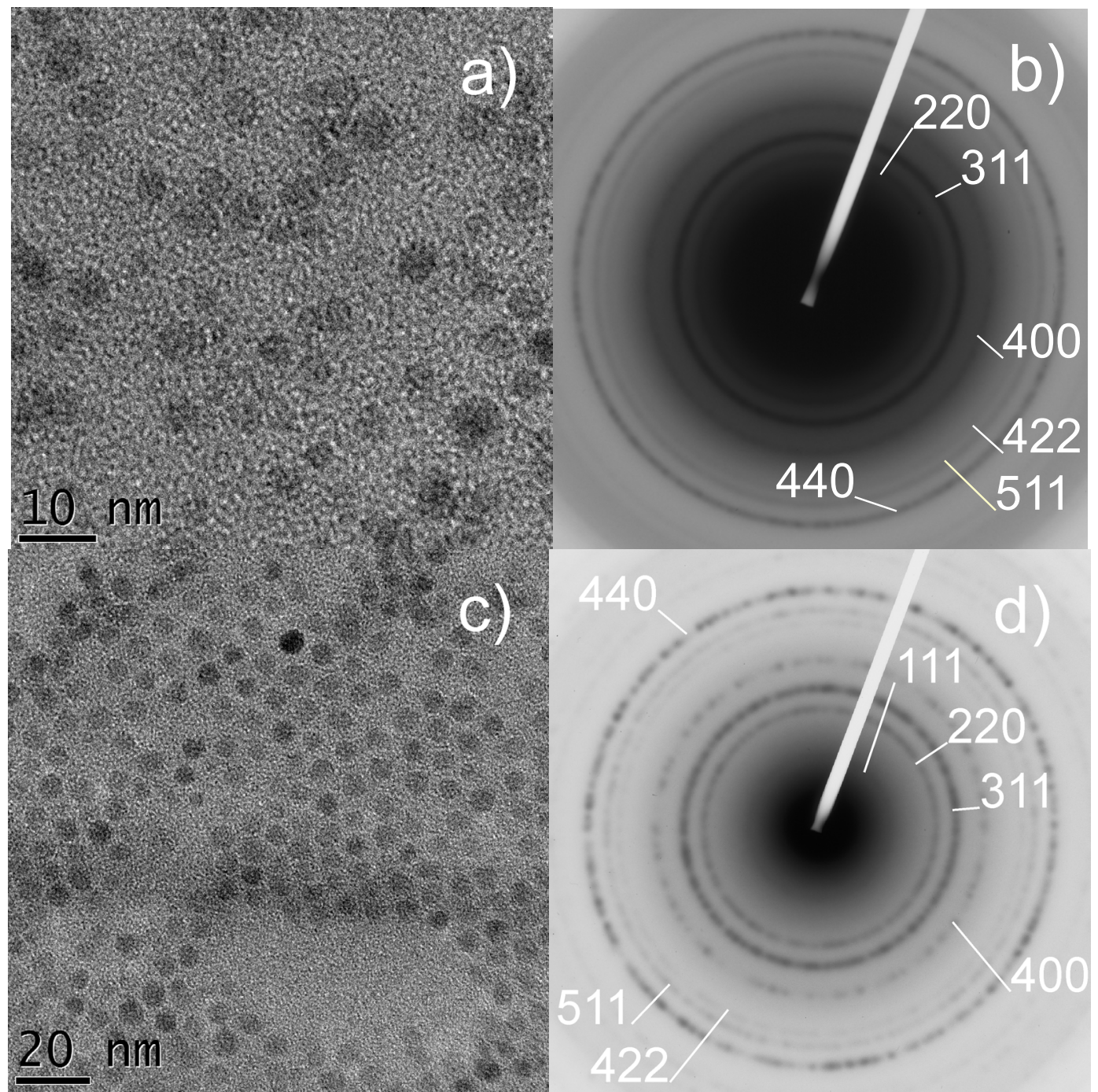

Figure 5-1 a) Micrograph of Sample XX, b) SAED pattern of Sample I, indexed to iron oxide spinel c) Micrograph of Sample XXI, d) SAED pattern of Sample II, indexed to iron oxide spinel. 


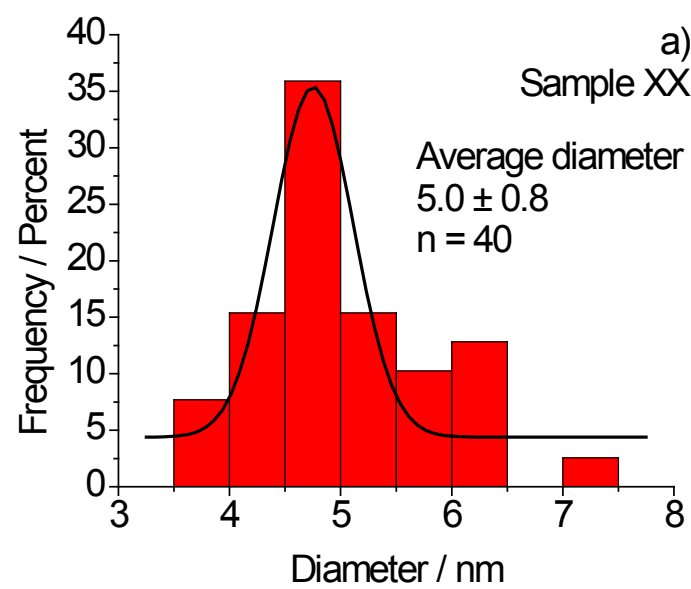

$\begin{array}{lll}\text { a) } & 40 \\ \text { Average diameter } & 6.1 \pm 0.7 \mathrm{~nm} & \text { b) } \\ \text { Sample } X X \mid\end{array}$

Diameter / nm

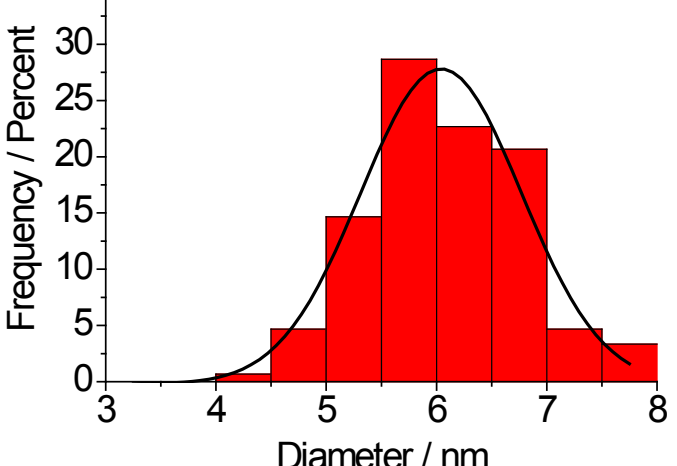

Figure 5-2 Histograms displaying the size distribution of a) Sample XX b) Sample XXI, as determined by TEM.

Both Sample XX and XXI consisted of spherical iron oxide spinel nanocrystals with relatively similar average diameters of 5.0 and $6.1 \mathrm{~nm}$, respectively. Both samples were highly monodisperse, with absolute standard deviations in nanocrystal diameter of 0.8 and $0.7 \mathrm{~nm}$, respectively - corresponding to relative standard deviations of $16 \%$ and $11 \%$ for Samples XX and XXI, respectively.

Micrographs and SAED patterns for Samples XXII-XXIV - produced by the decomposition of $\mathrm{Fe}(\mathrm{acac})_{2}$ in $\mathrm{HDA}$ at $300^{\circ} \mathrm{C}$ - are shown below in Figure 5-3; and histograms of the particle size distribution of the samples (as determined from TEM measurements) are shown in Figure 5-4. The SAED patterns were analysed and indexed as described in A1-9, and an XRD pattern for Sample XXIII is shown below in Figure 5-5. Samples XXII - XXIV were all identified as iron oxide spinel by SAED and XRD. XRD indicated the presence of an impurity - which was believed to be HDA - in Samples XXI and XXIII. 

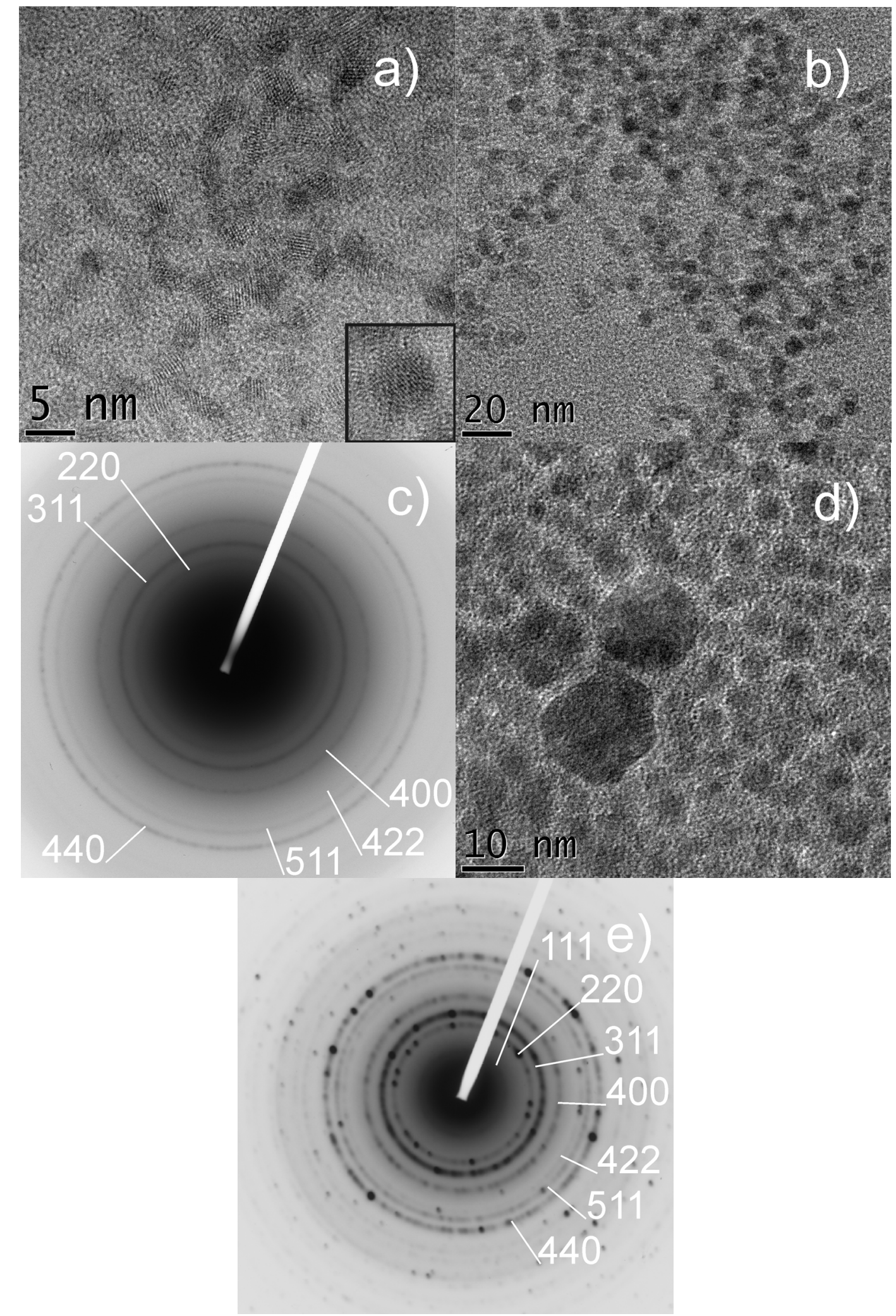

Figure 5-3 a) Micrograph of Sample XXII, inset high resolution image of single nanocrystal

b) Micrograph of Sample XXIII c) SAED pattern of Sample IV, indexed to iron oxide spinel.

d) Micrograph of Sample XXIV e) SAED pattern of Sample V, indexed to iron oxide spinel. 

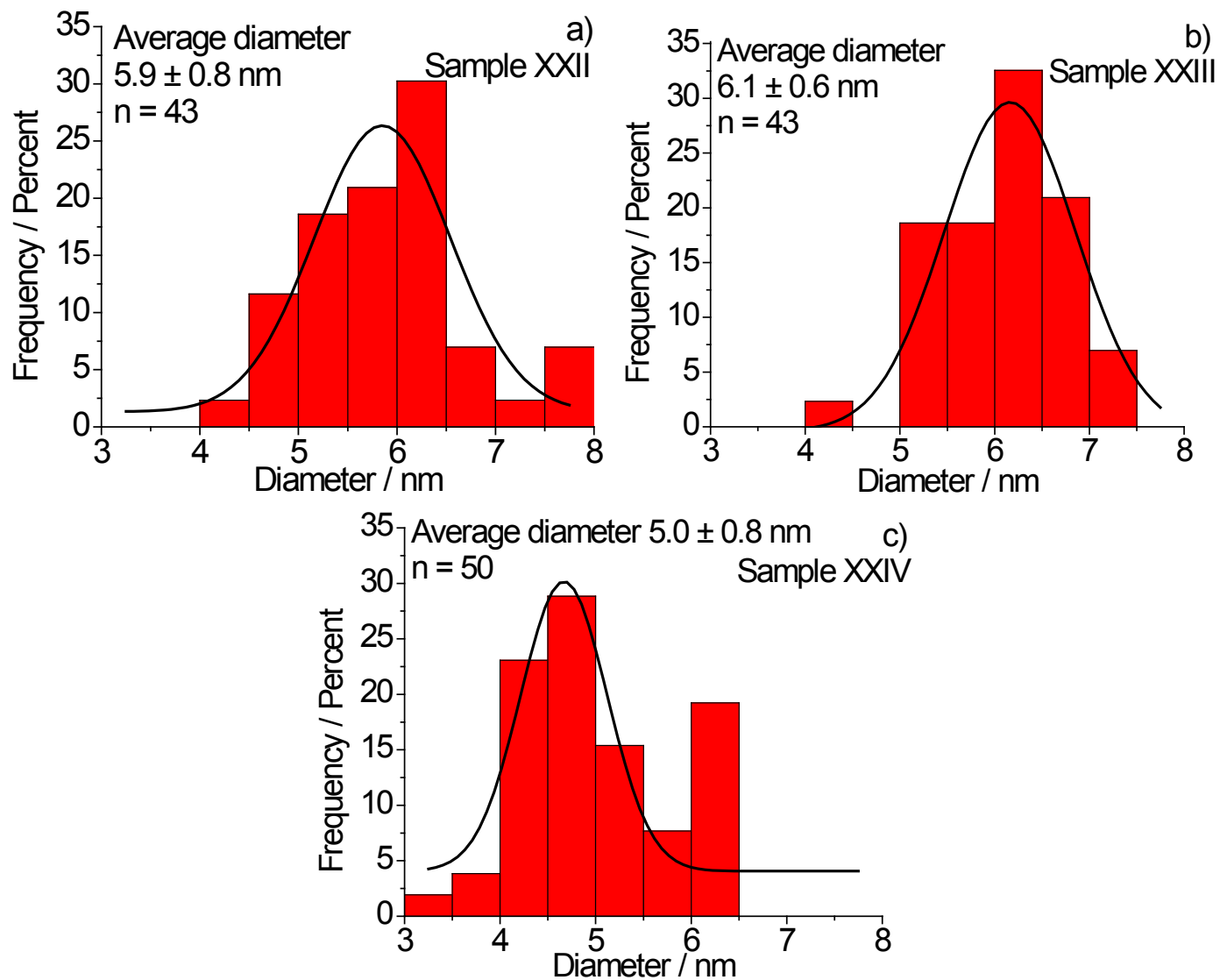

Figure 5-4 Histograms displaying the size distributions of a) Sample XXII b) Sample XXIII c) Sample XXIV.

Samples XXII - XXIV consisted of iron oxide spinel nanocrystals with similar average diameters of between $5-6 \mathrm{~nm}$. The absolute standard deviation in the nanocrystal diameters varied between 0.6 and $0.8 \mathrm{~nm}$, which corresponded to relative standard deviations of between 10 and 16\% (see Table 5-1). Scherrer X-ray broadening analysis on Samples XXI and XXIII gave average crystallite diameters of $7 \pm 1$ and $7 \pm 3 \mathrm{~nm}$, respectively (see A2-8) - in good accordance with the TEM measurements. 


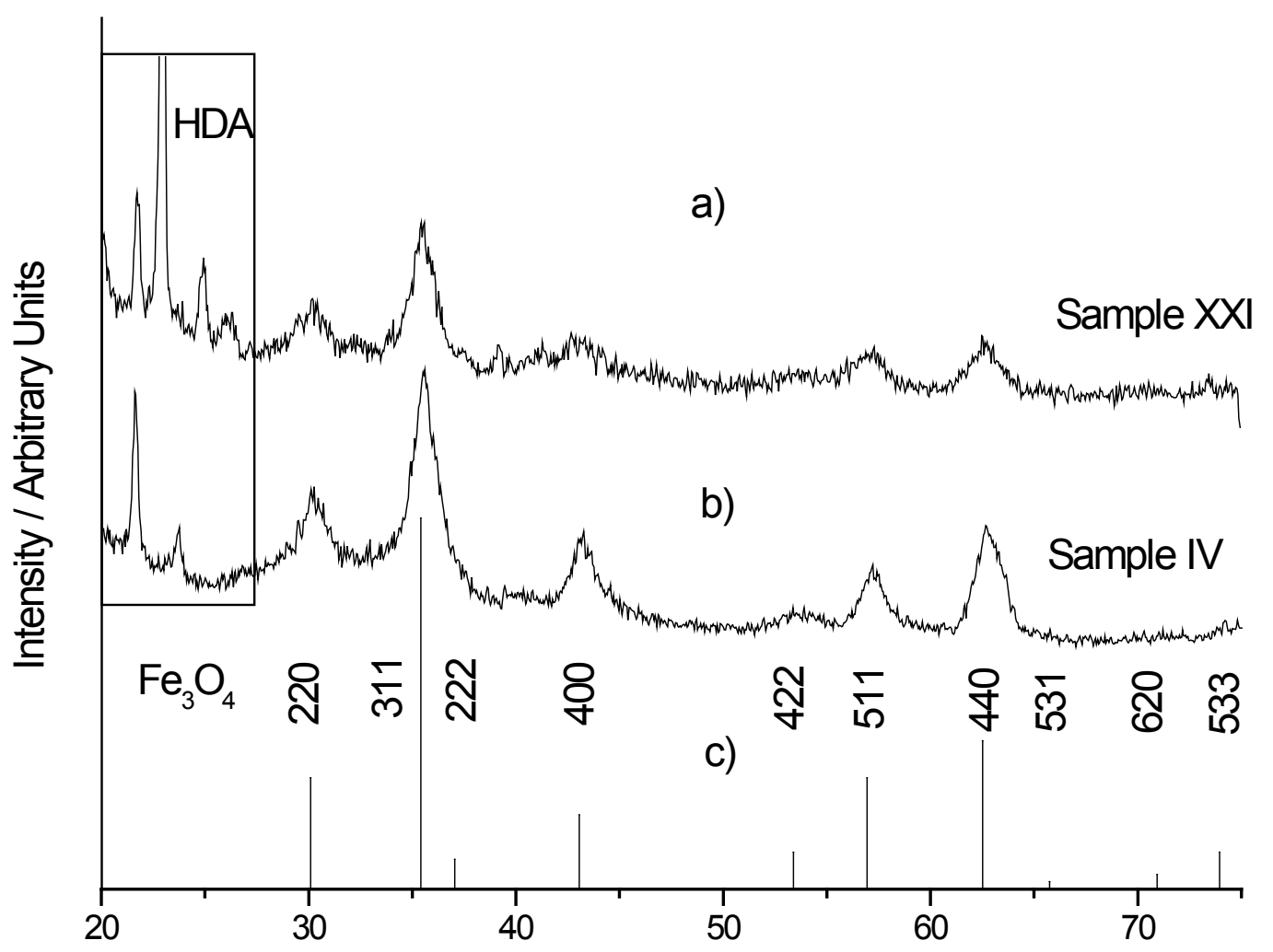

Figure 5-5 XRD patterns of a) Sample XXI b) Sample XXIII c) reference for $\mathrm{Fe}_{3} \mathrm{O}_{4}$ (PDF \#00-0190629).

The increase of reaction temperature from 200 to $300^{\circ} \mathrm{C}$ appeared to have no substantive effect on the average particle size, with the average diameter of samples synthesised at both temperatures varying between $5-6 \mathrm{~nm}$. Altering the duration of the reaction between one and four hours also did not appear to affect the average nanocrystal diameter substantively - there was no definite trend in average particle sizes evident within the range of reaction durations investigated. There did however appear to be a slight narrowing of the size distribution, with the narrowest size distribution obtained after two hours of reaction at either 200 or $300^{\circ} \mathrm{C}$ (Sample XXI and Sample XXIII, respectively). A reaction duration of two hours, with a reaction temperature within the range $200-300^{\circ} \mathrm{C}$ were therefore chosen as standard parameters for further experiments.

\subsubsection{Effect of replenishing iron precursor during reaction}

Experiments where $\mathrm{Fe}(\mathrm{acac})_{2}$ had been decomposed in $\mathrm{HDA}$ at $200-300^{\circ} \mathrm{C}$ for $1-4$ hours (section 5.2.1.1, Samples XX-XXIV) yielded spherical iron oxide nanocrystals of very similar average diameters. In order to attempt to increase the average diameter of the nanocrystals produced, while maintaining the narrow size distribution, iron precursor was replenished during the course of the reaction by addition of $\mathrm{Fe}(\mathrm{acac})_{2}$ powder under a flow of nitrogen (see section 5.5.2 for method). The purpose of this 
second addition was to attempt to replace iron precursor to allow continued growth without inducing a second round of nanocrystal nucleation. Experiments were performed as described in section 5.5.2, and relevant experimental parameters are tabulated below (Table 5-2).

Table 5-2 Experimental parameters for the synthesis of iron oxide nanocrystals with replenishment of the iron precursor (see section 5.5.2).

\begin{tabular}{|c|c|c|c|c|c|}
\hline Sample & $\begin{array}{l}\text { Reaction } \\
\text { duration } \\
\text { / Hours }\end{array}$ & $\begin{array}{l}\text { Iron } \\
\text { Source }\end{array}$ & Solvent & $\begin{array}{l}\text { Reaction } \\
\text { Temp. } \\
/{ }^{\circ} \mathrm{C} \\
\end{array}$ & $\begin{array}{c}\text { Result } \\
\text { (TEM size }) \\
\end{array}$ \\
\hline XXV & 1 & $\mathrm{Fe}(\mathrm{acac})_{2}$ & HDA & 200 & $\begin{array}{l}\text { Spherical iron oxide } \\
\text { spinel } \\
5.6 \pm 0.9 \mathrm{~nm}(\sigma 16 \%)\end{array}$ \\
\hline & 2 & & & & $\begin{array}{l}\text { Spherical iron oxide } \\
\text { spinel } \\
5.3 \pm 1.2 \mathrm{~nm}(\sigma 23 \%)\end{array}$ \\
\hline \multicolumn{6}{|c|}{ Replenishment of precursor } \\
\hline & 3 & & & & $\begin{array}{l}\text { Spherical iron oxide } \\
\text { spinel } \\
6.5 \pm 1.0 \mathrm{~nm}(\sigma 15 \%)\end{array}$ \\
\hline XXVI & 1 & $\mathrm{Fe}(\mathrm{acac})_{2}$ & HDA & 300 & $\begin{array}{l}\text { Spherical iron oxide } \\
\text { spinel } \\
8.0 \pm 4.4 \mathrm{~nm}(\sigma 55 \%)\end{array}$ \\
\hline & 2 & & & & $\begin{array}{l}\text { Spherical iron oxide } \\
\text { spinel } \\
8.8 \pm 5.0 \mathrm{~nm}(\sigma 57 \%)\end{array}$ \\
\hline \multicolumn{6}{|c|}{ Replenishment of precursor } \\
\hline & 3 & & & & $\begin{array}{l}\text { Spherical iron oxide } \\
\text { spinel } \\
9.8 \pm 3.4 \mathrm{~nm}(\sigma 35 \%)\end{array}$ \\
\hline
\end{tabular}

Sample XXV was synthesised by the decomposition of $\mathrm{Fe}(\mathrm{acac})_{2}$ in $\mathrm{HDA}$ at $200^{\circ} \mathrm{C}$, with replenishment of $\mathrm{Fe}(\mathrm{acac})_{2}$ after 2 hours. Micrographs of aliquots taken from Sample XXV at various times, and a representative SAED pattern are shown below (Figure 5-6). Histograms of the particle size distribution (as determined by TEM measurements) are shown in Figure 5-7. All aliquots were determined by SAED to be iron oxide spinel (see A1-10). 


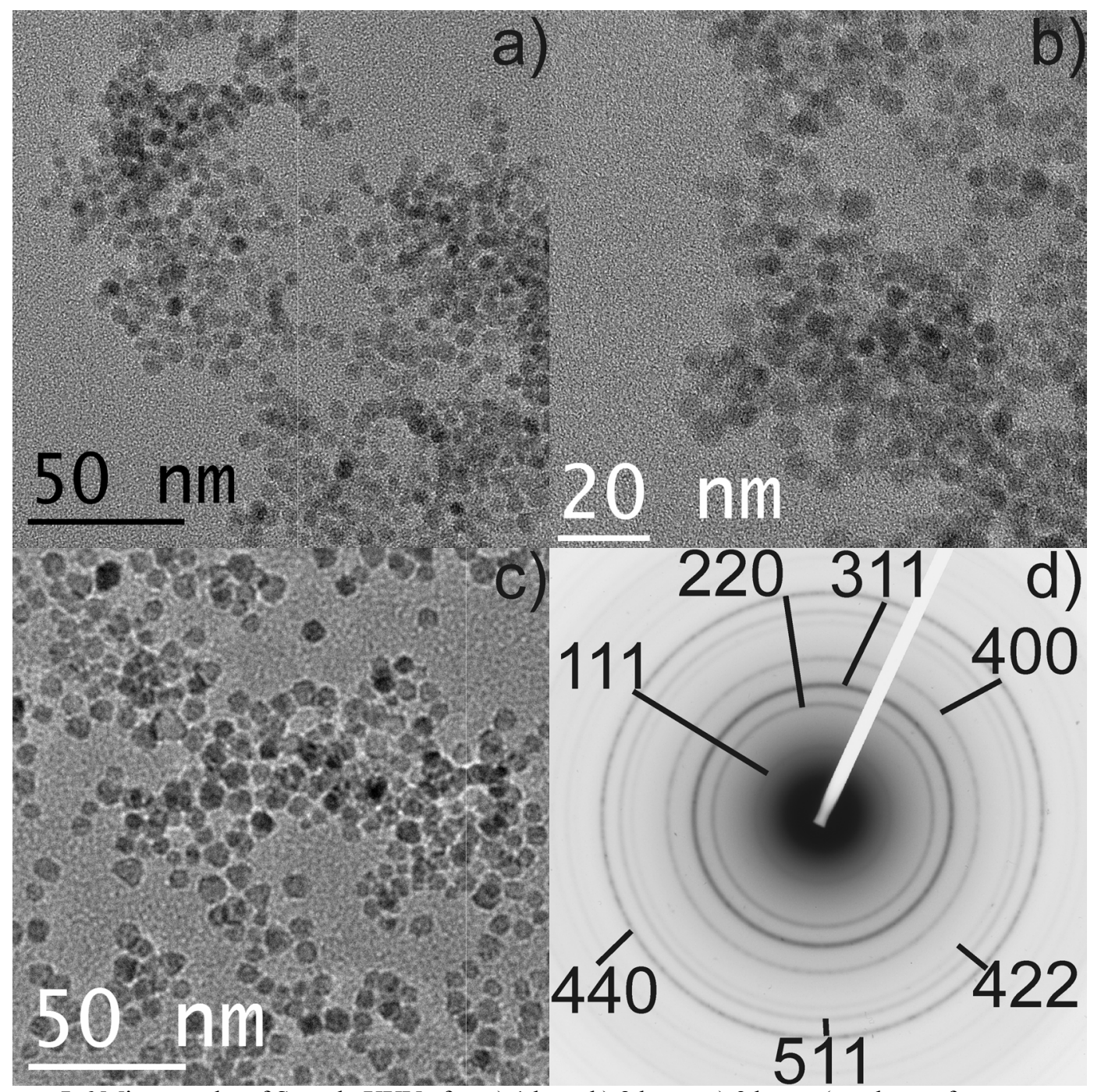

Figure 5-6 Micrographs of Sample XXV after a) 1 hour b) 2 hours c) 3 hours (one hour after replenishment of $\mathrm{Fe}(\mathrm{acac})_{2}$ ) d) SAED pattern representative of sample at all times sampled, indexed to iron oxide spinel.

The average diameter of iron oxide spinel nanocrystals sampled after 1 and 2 hours of reaction, were $5.6 \pm 0.9$ and $5.3 \pm 1.2 \mathrm{~nm}$, respectively. After 3 hours of reaction ( 1 hour after replenishment of $\left.\mathrm{Fe}(\mathrm{acac})_{2}\right)$ the average diameter had grown to $6.5 \pm 1.0 \mathrm{~nm}$. This represents only a very moderate increase in average diameter, given that previous experiments without replenishment of iron precursor had delivered samples with average diameters between $5-6 \mathrm{~nm}$ (see section 5.2.1.1). 

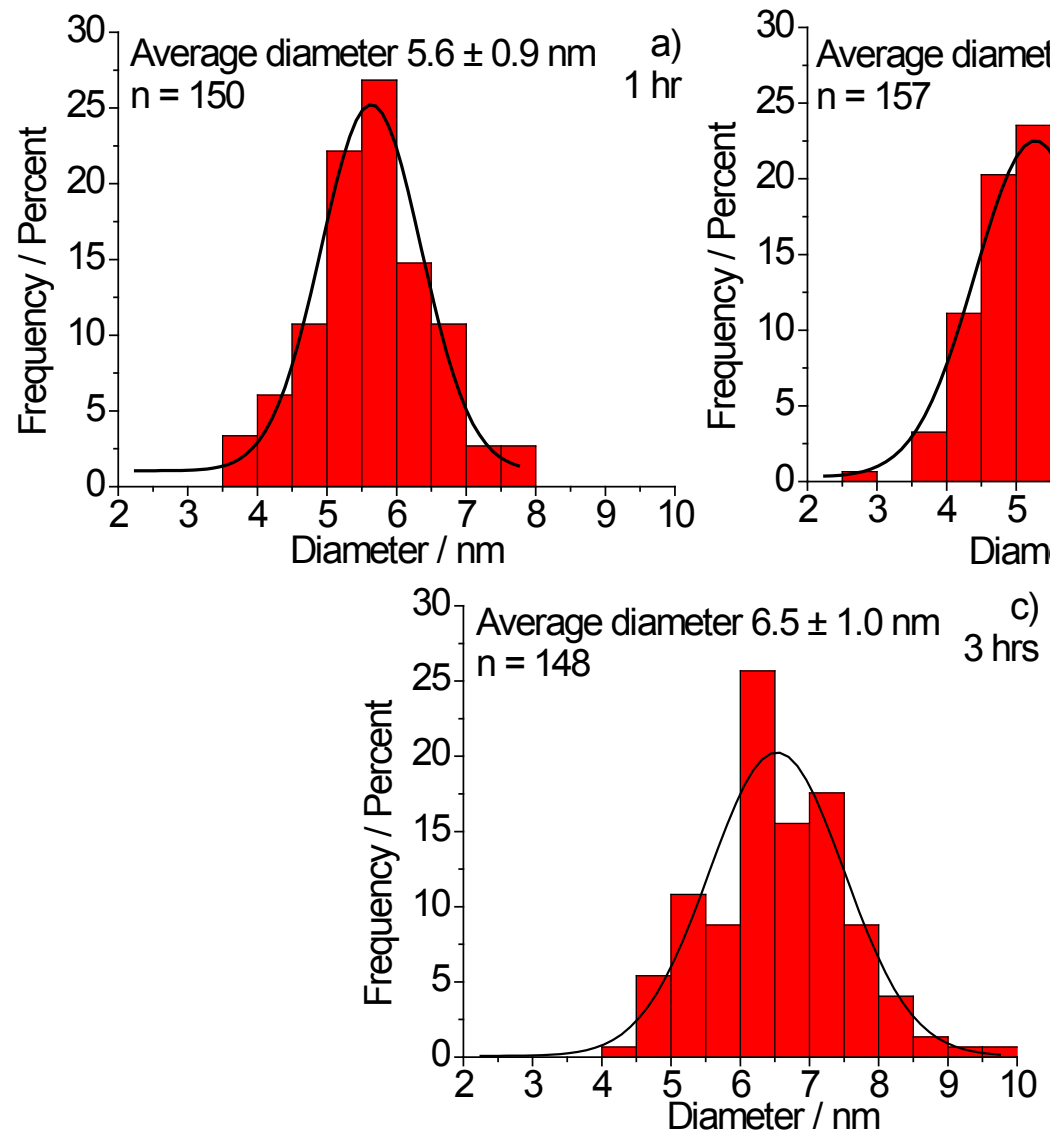

Figure 5-7 Histograms displaying the size distribution Sample XXV (iron oxide spinel nanocrystals) after a) 1 hour b) 2 hours c) 3 hours of reaction $-\mathrm{Fe}(\mathrm{acac})_{2}$ was replenished after initial 2 hours.

Sample XXVI was synthesised by the decomposition of $\mathrm{Fe}(\mathrm{acac})_{2}$ in $\mathrm{HDA}$ at $300^{\circ} \mathrm{C}$, with replenishment of $\mathrm{Fe}(\mathrm{acac})_{2}$ after 2 hours. Micrographs of aliquots taken from Sample XXVI at various times, and a representative SAED pattern are shown below (Figure 5-8). Histograms of the particle size distribution (as determined by TEM measurements) are shown in Figure 5-9. All aliquots were determined by SAED to be iron oxide spinel (see A1-10). 


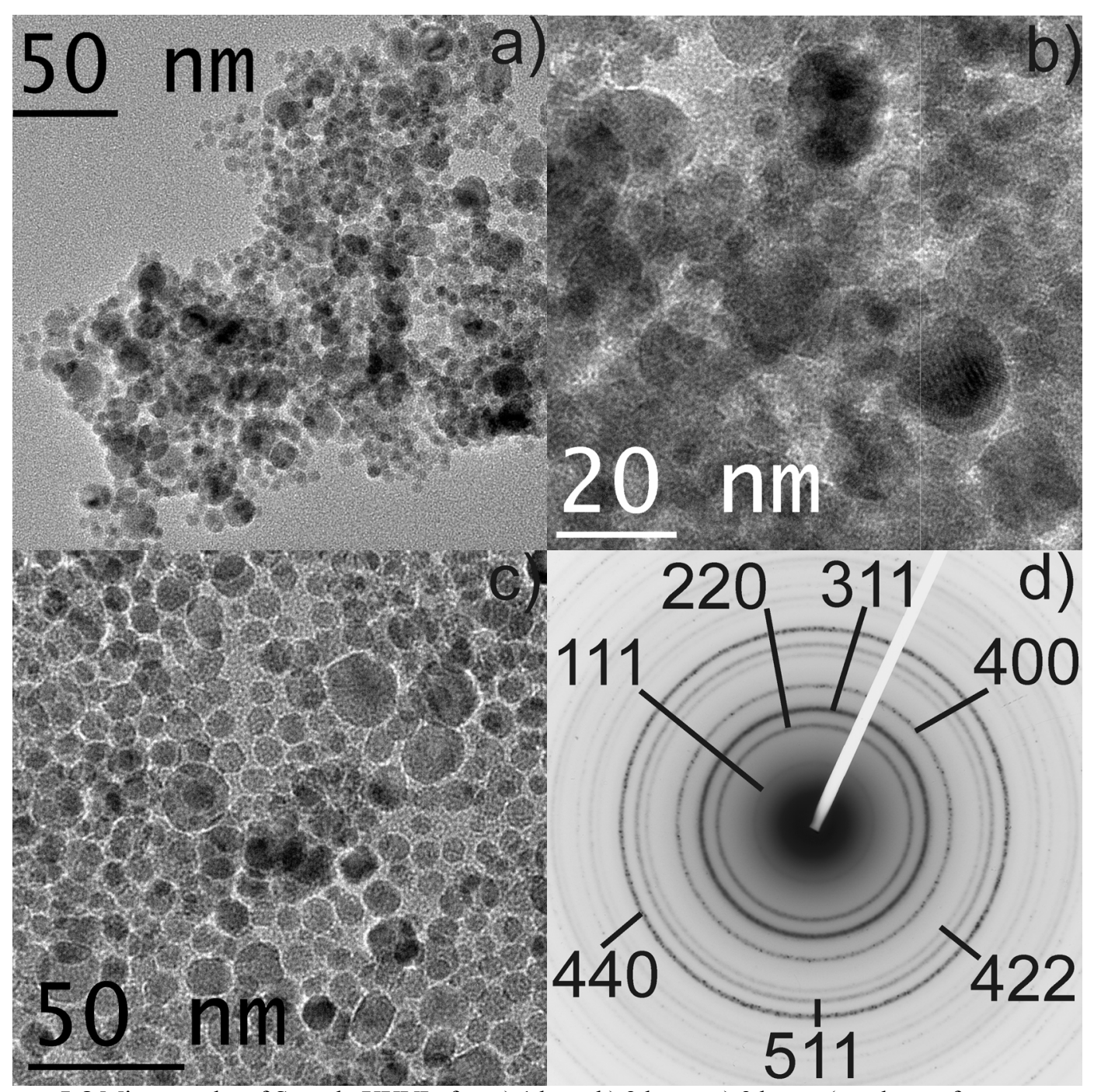

Figure 5-8 Micrographs of Sample XXVI after a) 1 hour b) 2 hours c) 3 hours (one hour after replenishment of $\left.\mathrm{Fe}(\mathrm{acac})_{2}\right)$ d) SAED pattern representative of sample at all times samples, indexed to iron oxide spinel.

Aliquots taken from Sample XXVI were distinctly bimodal at all times sampled. The average diameter increased from $8.0 \mathrm{~nm}$ after 1 hour to $9.8 \mathrm{~nm}$ after 3 hours. This increase did appear to be driven by the increase in the average diameter of the smaller diameter $(<10 \mathrm{~nm})$ ensemble of nanocrystals. However, the presence of a significant population of larger $(10-25 \mathrm{~nm})$ particles rendered the absolute and relative standard deviation of the average diameter much higher in all aliquots of Sample XXVI than previous experiments. 

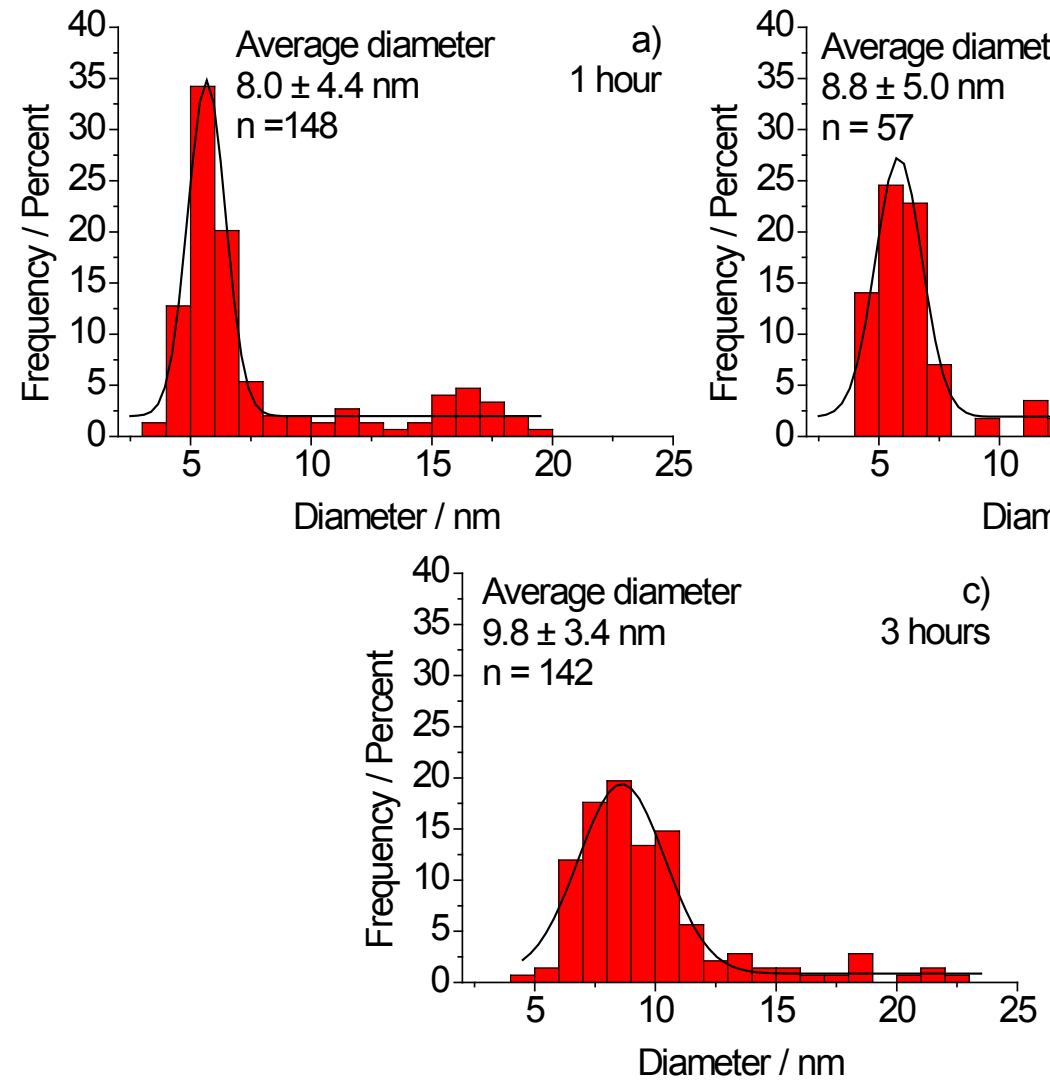

Figure 5-9 Histograms displaying the size distribution of Sample XXVI (iron oxide spinel nanocrystals) after a) 1 hour b) 2 hours c) 3 hours. 


\subsubsection{Synthesis of $\mathrm{Fe}_{3} \mathrm{~S}_{4}$ nanocrystals}

\subsubsection{Sulfidisation of iron/iron oxide nanocrystals}

After optimisation of the experimental parameters for the synthesis of monodisperse iron oxide spinel nanocrystals, sulfidisation of the postulated intermediate iron/iron oxide nanocrystals was attempted by the injection of a solution of sulfur in oleylamine, as detailed in 5.5.3. Specific parameters for the experiments are tabulated below in Table 5-3.

Table 5-3 Synthetic parameters for the sulfidisation of iron/iron oxide nanocrystals.

\begin{tabular}{|c|c|c|c|c|c|c|}
\hline Sample & $\begin{array}{c}\text { Iron } \\
\text { source }\end{array}$ & Solvent & $\begin{array}{c}\mathrm{S} \\
\text { eq. }\end{array}$ & $\begin{array}{c}\text { Reaction } \\
\text { Temp. } \\
/{ }^{\circ} \mathrm{C}\end{array}$ & $\begin{array}{c}\text { Reaction } \\
\text { Duration } \\
\text { / Hours }\end{array}$ & $\begin{array}{c}\text { Result } \\
\text { (TEM size) }\end{array}$ \\
\hline XXVII & $\mathrm{Fe}(\mathrm{acac})_{2}$ & HDA & 1 & 200 & 2 & $\begin{array}{c}\text { Amorphous } \\
\text { iron sulfide } \\
\text { material }\end{array}$ \\
\hline XXVIII & $\mathrm{Fe}(\mathrm{acac})_{2}$ & HDA & 1 & 300 & 2 & $\begin{array}{c}\text { Spherical } \\
\mathrm{Fe}_{3} \mathrm{~S}_{4} \\
\text { nanocrystals } \\
(7 \mathrm{~nm} \pm 1) \\
\text { and } \mathrm{Fe}_{1-x} \mathrm{~S}\end{array}$ \\
\hline XXIX & $\mathrm{Fe}(\mathrm{acac})_{2}$ & OAm & 1 & 200 & 2 & $\begin{array}{c}\text { Amorphous } \\
\text { iron sulphide } \\
\text { material and } \\
\alpha-\mathrm{Fe}_{2} \mathrm{O}_{3}\end{array}$ \\
\hline XXX & $\mathrm{Fe}(\mathrm{acac})_{2}$ & OAm & 1 & 300 & 2 & $\begin{array}{c}\text { Oblate } \mathrm{Fe}_{3} \mathrm{~S}_{4} \\
\text { nanocrystals } \\
(8 \mathrm{~nm} \pm 2) \\
\text { and } \mathrm{Fe}_{1-x} \mathrm{~S}\end{array}$ \\
\hline
\end{tabular}

Micrographs and SAED patterns for Samples XXVII - XXX are shown below in Figure 5-10 (see A1-11 for analysis). Histograms displaying the size distribution (as determined from TEM measurements) of Samples XXVIII and XXX are shown in Figure 5-12. Scherrer X-ray broadening analysis was not performed, as the peaks in the XRD patterns (see Figure 5-11) tended to be only poorly defined with respect to the amorphous contribution to the background. 


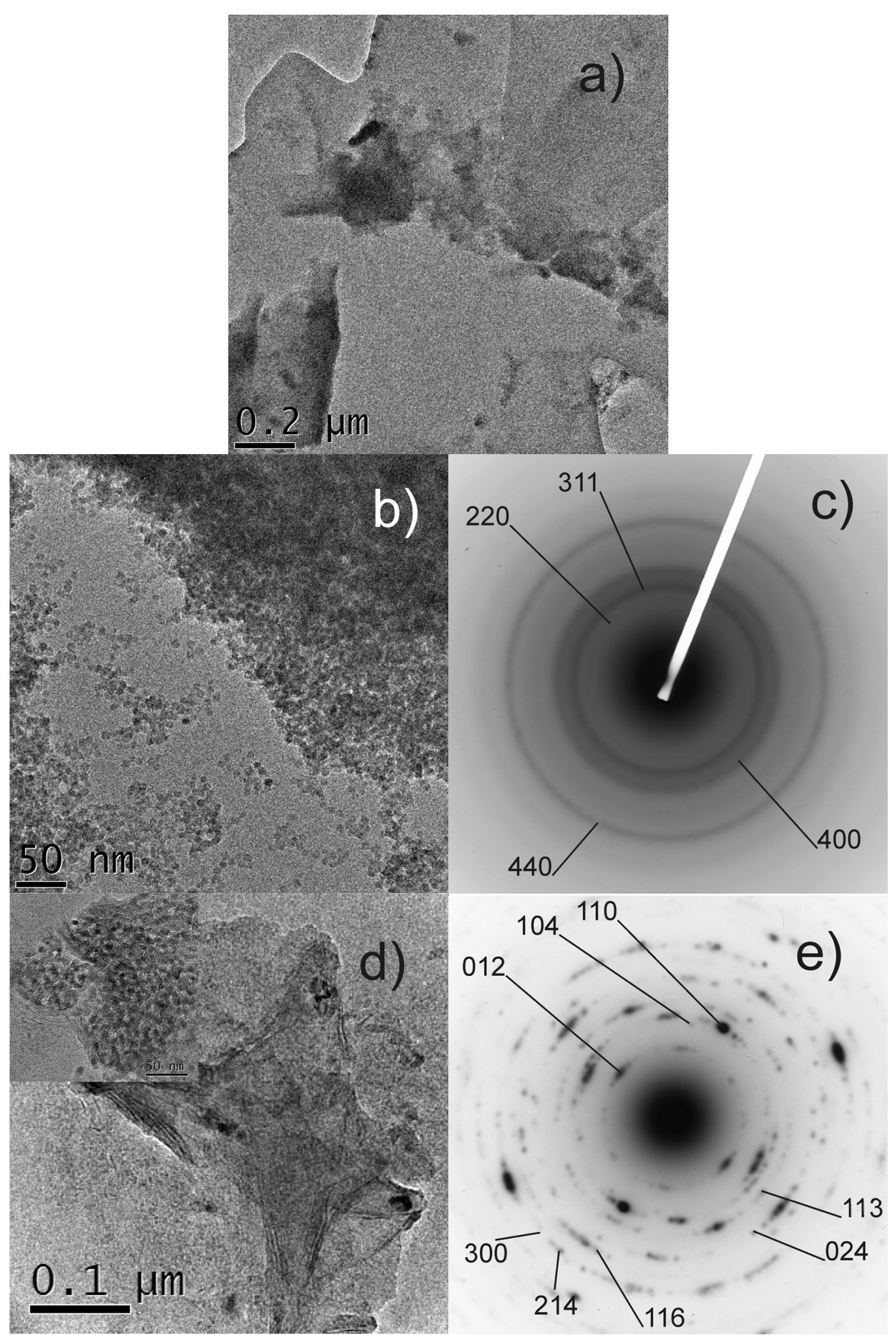

Figure 5-10 a) Micrograph of Sample XXVII b) Micrograph of Sample XXVIII c) SAED pattern of Sample XXVIII, indexed to $\mathrm{Fe}_{3} \mathrm{~S}_{4}$. d) Micrograph of Sample XXIX inset area leading to SAED e) SAED pattern of Sample XXIII, indexed to $\alpha-\mathrm{Fe}_{2} \mathrm{O}_{3}$ 


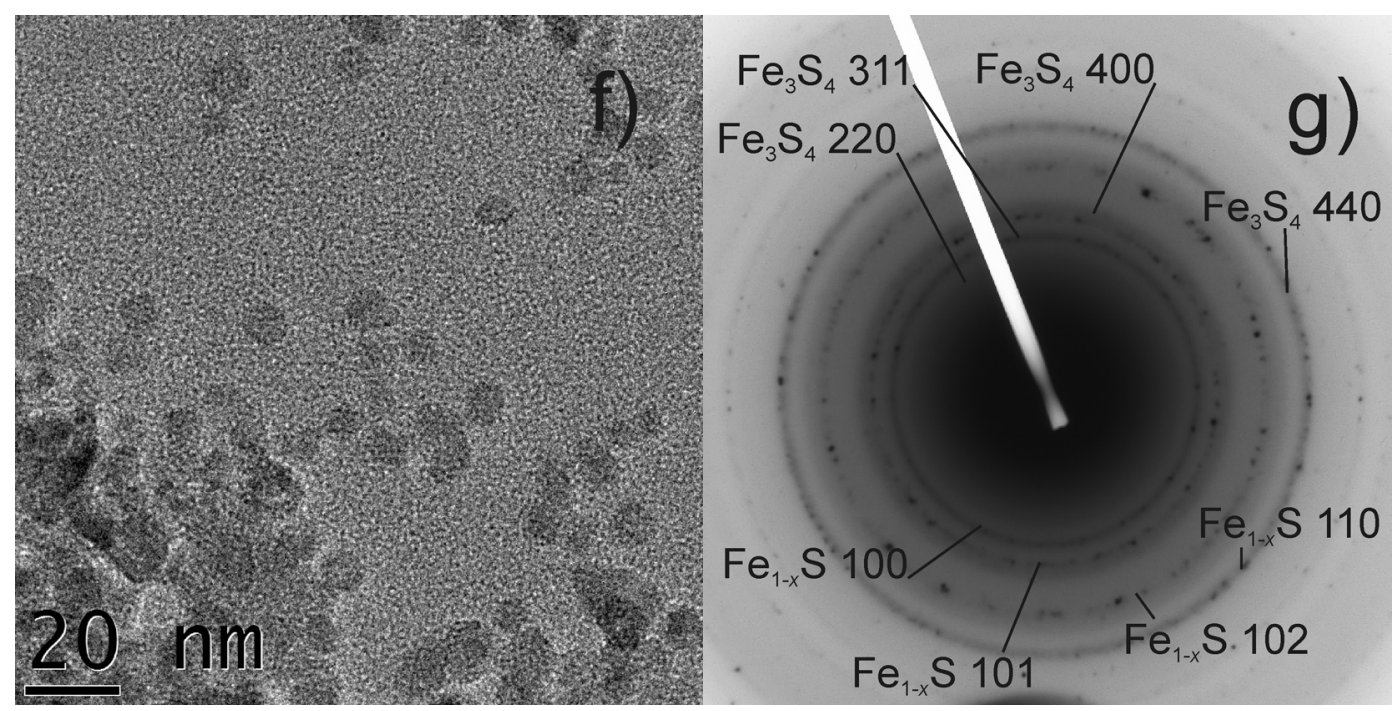

Figure 5-16 continued f) Micrograph of Sample XXX g) SAED pattern of Sample XXX, indexed to $\mathrm{Fe}_{3} \mathrm{~S}_{4}$ and $\mathrm{Fe}_{1-x} \mathrm{~S}$.

Sample XXVII was synthesised by the decomposition of $\mathrm{Fe}(\mathrm{acac})_{2}$ in $\mathrm{HDA}$ at $200^{\circ} \mathrm{C}$ for 2 hours, followed by injection of 1 equivalent of sulfur in oleylamine. The TEM micrograph (Figure 5-10 a)) shows only irregularly-shaped material, similar in appearance to the amorphous iron sulfide observed in Chapter 4. EDS confirmed the presence of iron and sulfur, however SAED did not reveal the presence of any crystalline material.

Sample XXVIII was synthesised by the decomposition of Fe(acac $)_{2}$ in $\mathrm{HDA}$ at $300^{\circ} \mathrm{C}$ for 2 hours, followed by injection of 1 equivalent of sulfur in oleylamine. Sample XXVIII was comprised of small, roughly spherical nanocrystals, which aggregated strongly on the TEM grid. SAED confirmed the sample as $\mathrm{Fe}_{3} \mathrm{~S}_{4}$, with the smooth, diffuse nature of the rings (see Figure 5-10) consistent with the small particle size. EDS indicated a composition of Fe:S of 1.2:1, and the presence of oxygen (see Table 5-4), indicating the probable presence of iron oxide in addition to $\mathrm{Fe}_{3} \mathrm{~S}_{4}$. The average diameter of the $\mathrm{Fe}_{3} \mathrm{~S}_{4}$ nanocrystals was $6.6 \pm 0.9 \mathrm{~nm}$. This average diameter is similar to that of iron oxide spinel nanocrystals described above (section 5.2.1), and the relative standard deviation in average diameter of $14 \%$ corresponds to a similar level of monodispersity.

Sample XXIX was synthesised by the decomposition of Fe(acac $)_{2}$ in oleylamine at $200^{\circ} \mathrm{C}$, followed by the injection of 1 equivalent of sulfur in oleylamine. Sample XXIX comprised of large, sheet-like material, which was judged to be amorphous by HRTEM imaging and electron diffraction. EDS confirmed the presence of iron, sulfur and oxygen - indicating that the material was an amorphous iron sulfide. In addition, there 
were areas of small, oblate nanocrystals, which were identified as $\alpha-\mathrm{Fe}_{2} \mathrm{O}_{3}$ by SAED (see Figure 5-10 e) for SAED pattern and A1-12 for indexing details).

Sample XXX was synthesised by the decomposition of Fe(acac $)_{2}$ in oleylamine at $300^{\circ} \mathrm{C}$ for 2 hours, followed by the injection of 1 equivalent of sulfur in oleylamine. The sample comprised of roughly oblate nanocrystals. SAED confirmed the presence of $\mathrm{Fe}_{3} \mathrm{~S}_{4}$ and $\mathrm{Fe}_{1-x} \mathrm{~S}$ (see Figure 5-10 g) for SAED pattern, and A1-13 for indexing details). The spotty nature of the $\mathrm{Fe}_{1-x} \mathrm{~S}$ reflections indicated a larger average crystallite size than for $\mathrm{Fe}_{3} \mathrm{~S}_{4}$. The average diameter of the slightly oblate nanocrystals (along the major axis) was $8.0 \pm 1.6 \mathrm{~nm}$. This is slightly larger than Sample XXVIII, which was synthesised in $\mathrm{HDA}$ at $300^{\circ} \mathrm{C}$, and the relative standard deviation in nanocrystal diameter of $20 \%$ is also larger than for Sample XXVIII.

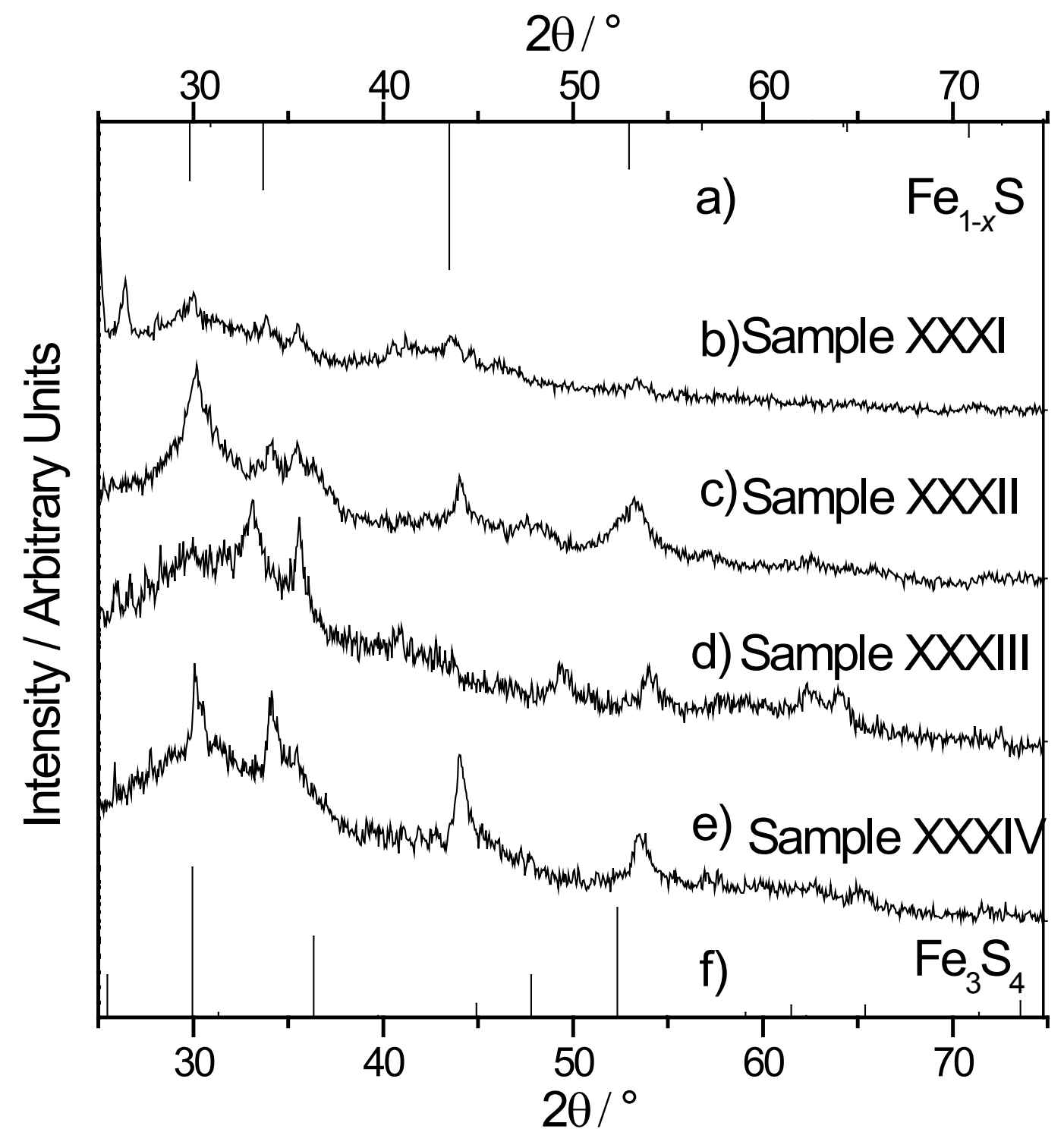

Figure 5-11 XRD patterns of a) $\mathrm{Fe}_{1-x} \mathrm{~S}$ reference b) Sample XXVII c) Sample XXVIII d) Sample XXIX e) Sample XXX f) $\mathrm{Fe}_{3} \mathrm{~S}_{4}$ reference. 


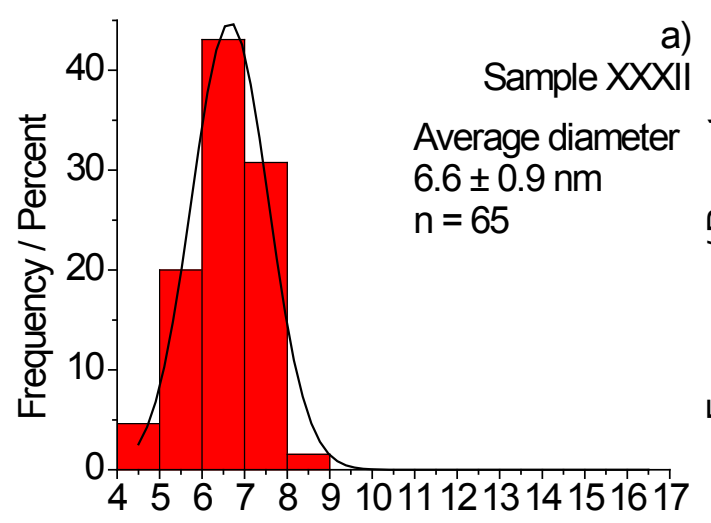

Diameter / nm

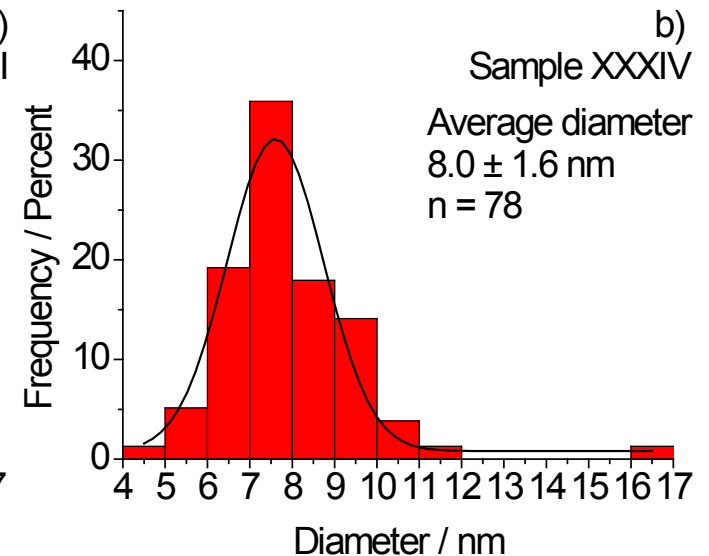

Diameter / nm

Figure 5-12 Histograms displaying the size distribution of $\mathrm{Fe}_{3} \mathrm{~S}_{4}$ nanocrystals in a) Sample XXVIII b) Sample XXX.

Table 5-4 Sample composition as quantified from EDS spectra.

\begin{tabular}{|l|l|l|l|}
\hline & $\mathrm{Fe} / \%$ (at.) & $\mathrm{S} / \%$ (at.) & $\mathrm{O} / \%$ (at.) \\
\hline Sample XXVIII & 45 & 37 & 18 \\
\hline Sample XXIX & 15 & 38 & 47 \\
\hline
\end{tabular}

5.2.2.2 Sulfidisation of iron/iron oxide nanocrystals followed by size-selective purification

The optimised synthetic parameters for iron oxide nanocrystals, as determined in section 5.2.1, were combined with the optimised conditions for sulfidisation as determined in section 5.2.2.1. The resulting experimental procedure is detailed in section 5.5.4, and specific experimental parameters are tabulated in Table 5-5. As the sulfidisation reactions investigated above (section 5.2.2.1) produced predominantly $\mathrm{Fe}_{3} \mathrm{~S}_{4}$, the amount of sulfur added was increased to 1.5 equivalents (in excess of the 1.33 equivalents required for $\mathrm{Fe}_{3} \mathrm{~S}_{4}$ ) in order to avoid the formation of iron oxides. Sulfur solution was injected at $280^{\circ} \mathrm{C}$, and the reaction was immediately cooled rapidly to avoid the formation of $\mathrm{Fe}_{1-x} \mathrm{~S}$. In addition, a size-selective purification procedure (section 5.5.5) was employed in order to remove any large $\mathrm{Fe}_{1-x} \mathrm{~S}$ crystallites which may have formed.

Table 5-5 Synthetic parameters for the sulfidisation of iron/iron oxide nanocrystals followed by size-selective purification.

\begin{tabular}{|c|c|c|c|c|c|c|}
\hline Sample & $\begin{array}{c}\text { Iron } \\
\text { source }\end{array}$ & Solvent & S eq. & $\begin{array}{c}\text { Reaction } \\
\text { Temp. } \\
/{ }^{\circ} \mathrm{C}\end{array}$ & $\begin{array}{c}\text { Reaction } \\
\text { Duration } \\
/ \text { Hours }\end{array}$ & $\begin{array}{c}\text { Result } \\
\text { (TEM size })\end{array}$ \\
\hline XXXI & $\mathrm{Fe}(\mathrm{acac})_{2}$ & HDA & 1.5 & 300 & 2 & $\begin{array}{c}\text { Spherical Fe } \mathrm{S}_{4} \\
\text { nanocrystals } \\
6.8 \pm 2.0 \mathrm{~nm}(\sigma=30 \%)\end{array}$ \\
\hline
\end{tabular}


Micrographs of Sample XXXI are shown below in Figure 5-13; along with an SAED pattern from the sample, indexed to $\mathrm{Fe}_{3} \mathrm{~S}_{4}$; and a histogram displaying the size distribution. An XRD pattern from Sample XXXI is shown in Figure 5-14. Sample XXXI comprised predominantly small, roughly spherical nanocrystals. Although some large crystallites and amorphous material was also present. The nanocrystals were often moderately aggregated, although dispersed enough for TEM measurements to determine that the average diameter was $6.8 \pm 2.0 \mathrm{~nm}$. The SAED pattern of Sample XXXI contained a number of broad rings characteristic of small diffracting domains, which were indexed to $\mathrm{Fe}_{3} \mathrm{~S}_{4}$ (see A1-16). There were in addition spots indicative of the presence of larger crystallites. The XRD of Sample XXXI was very broad, and the peaks were not fully resolved. The XRD pattern matched $\mathrm{Fe}_{3} \mathrm{~S}_{4}$, and corresponded well with the diffuse reflections observed in the SAED pattern. Scherrer X-ray broadening analysis was not performed, as the peaks of the XRD pattern were not properly resolved, and were difficult to distinguish from the amorphous contribution to the background. 

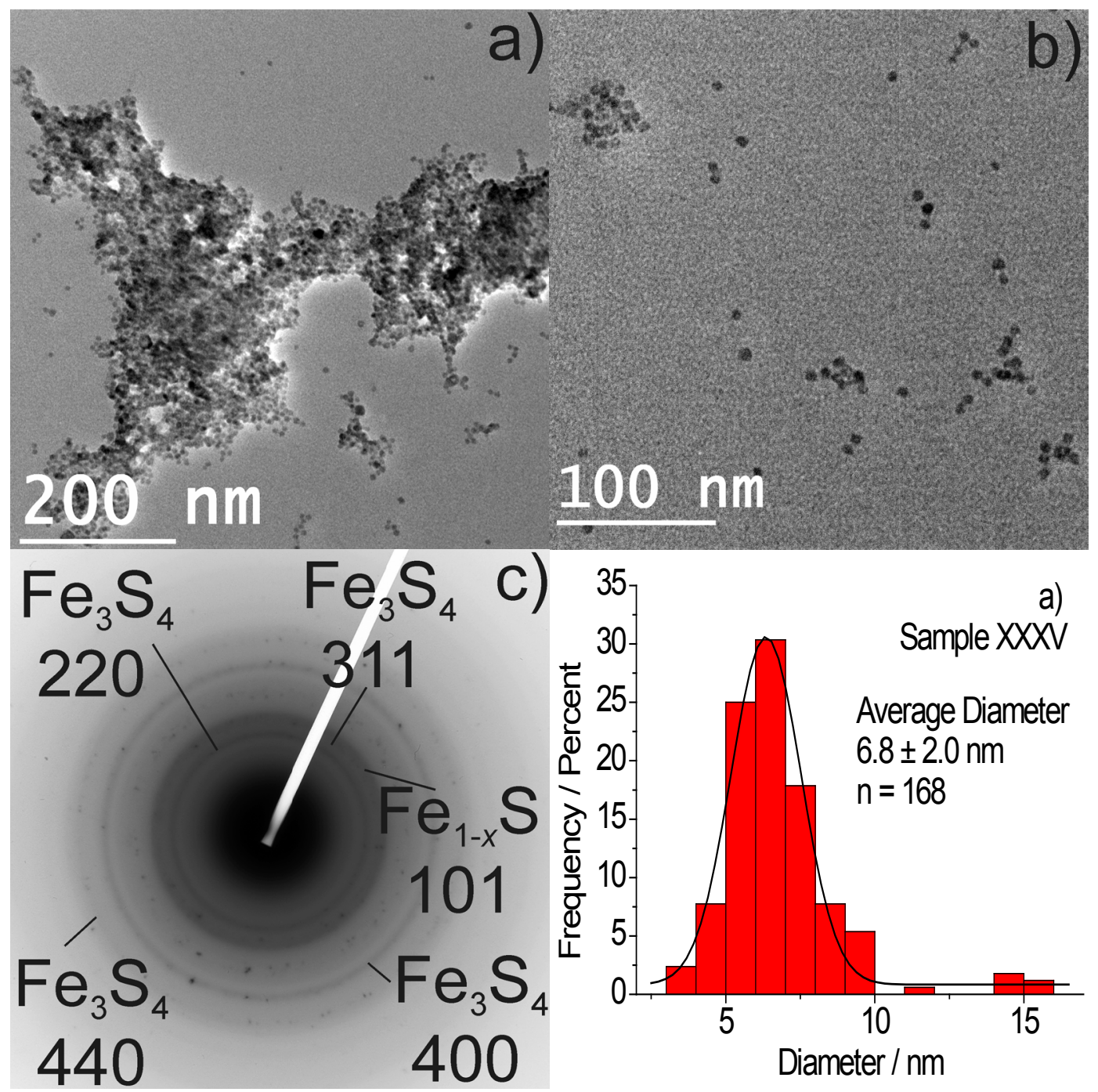

Figure 5-13 Micrographs of a) and b) Sample XXXI c) SAED pattern of Sample XXXI, indexed to $\mathrm{Fe}_{3} \mathrm{~S}_{4} \mathrm{~d}$ ) histogram displaying the size distribution of Sample XXXI.

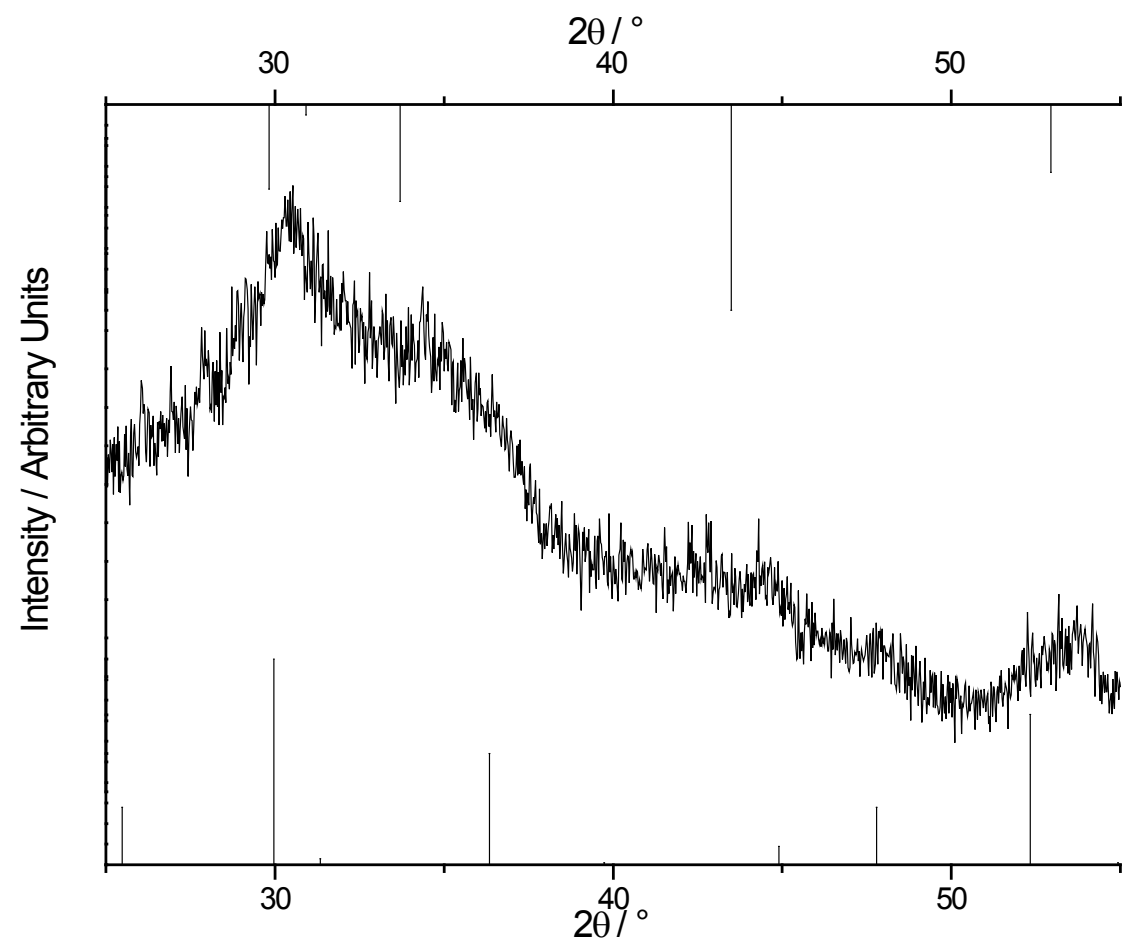

Figure 5-14 XRD pattern of a) $\mathrm{Fe}_{1-x} \mathrm{~S}$ reference b) Sample XXXI c) $\mathrm{Fe}_{3} \mathrm{~S}_{4}$ reference. 


\subsubsection{Magnetic measurements}

Sample XXIII and Sample XXXI were chosen as representative samples of $\mathrm{Fe}_{3} \mathrm{O}_{4}$ and $\mathrm{Fe}_{3} \mathrm{~S}_{4}$ nanocrystals, respectively. Magnetic measurements were performed on the samples, as detailed in Chapter 2 section 2.2.6.

\subsubsection{Iron oxide spinel nanocrystals (Sample XXIII)}

A ZFC/FC plot for Sample XXIII is shown below in Figure 5-15, and field loop plots recorded at 10 and $300 \mathrm{~K}$ are shown in Figure 5-16 a) and b), respectively. The ZFC/FC plot of Sample XXIII reveals a region of magnetic irreversibility at low temperatures, extending up to a clear magnetic ordering transition centred at $100 \mathrm{~K}$, after which the ZFC and FC plots coincide. Field loops at both $10 \mathrm{~K}$ and $300 \mathrm{~K}$ display a lack of coercivity and remanence. Field loops at both temperatures have a square profile, and display a clear approach to saturation, with saturation magnetisations of 50 and 43 $\mathrm{A} \mathrm{m}^{2} \mathrm{~kg}^{-1}$ at 10 and $300 \mathrm{~K}$, respectively.

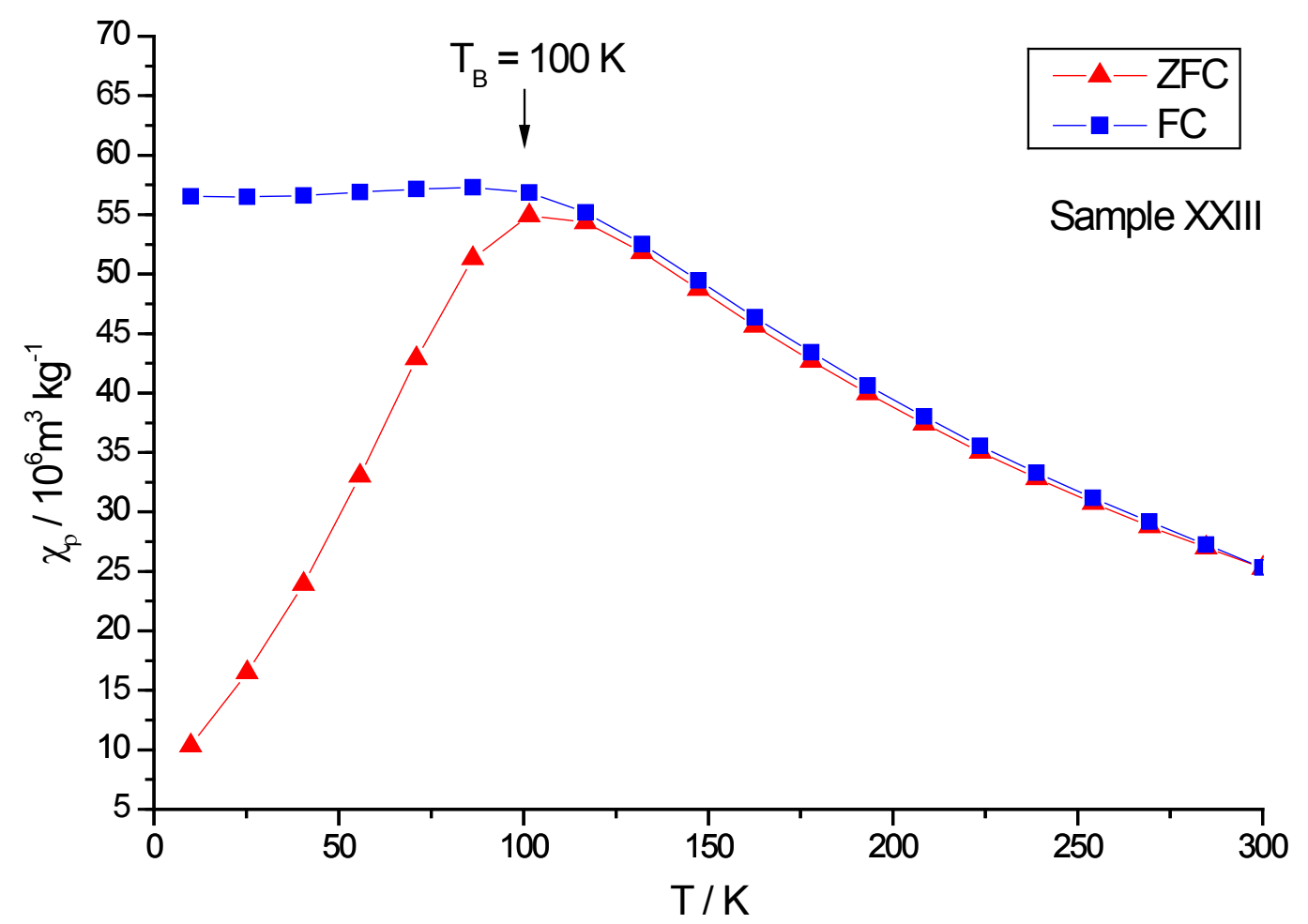

Figure 5-15 ZFC/FC plot of Sample XXIII (6 nm Fe $\mathrm{O}_{4}$ nanocrystals). $\mathrm{H}=1990 \mathrm{~A} \mathrm{~m}^{-1}$. 

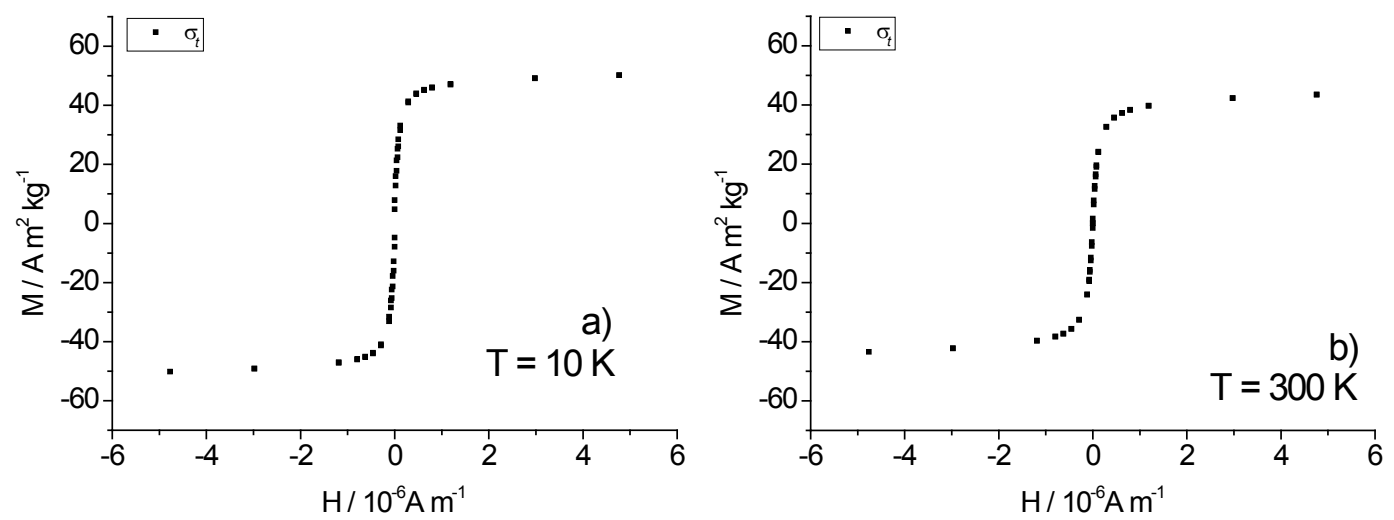

Figure 5-16 Field loop of Sample XXIII ( $6 \mathrm{~nm} \mathrm{Fe}_{3} \mathrm{O}_{4}$ nanocrystals) at a) $\left.\mathrm{T}=10 \mathrm{~K}, \mathrm{~b}\right) \mathrm{T}=300 \mathrm{~K}$.

\subsubsection{2 $\quad \mathrm{Fe}_{3} \mathrm{~S}_{4}$ nanocrystals (Sample XXXI)}

A ZFC/FC plot for Sample XXXI is shown below in Figure 5-17, and field loop plots recorded at 10 and $300 \mathrm{~K}$ are shown in Figure 5-18 a) and b), respectively. ZFC and FC plots for Sample XXXI are non-coincident over most of the temperature range $10-300$ $\mathrm{K}$. There appears a magnetic ordering transition centred around $50 \mathrm{~K}$, however the ZFC and FC plots remain non-coincident above this temperature. Field loops recorded at 10 and $300 \mathrm{~K}$ both display a lack of coercivity, although the field loop recorded at $10 \mathrm{~K}$ appears slightly wasp-waisted. Field loops at both temperatures have a rounded profile, and do not saturate fully at high field. Sample XXXI approaches an approximate saturation magnetisation of 12 and $9 \mathrm{~A} \mathrm{~m}^{2} \mathrm{~kg}^{-1}$ at 10 and $300 \mathrm{~K}$, respectively.

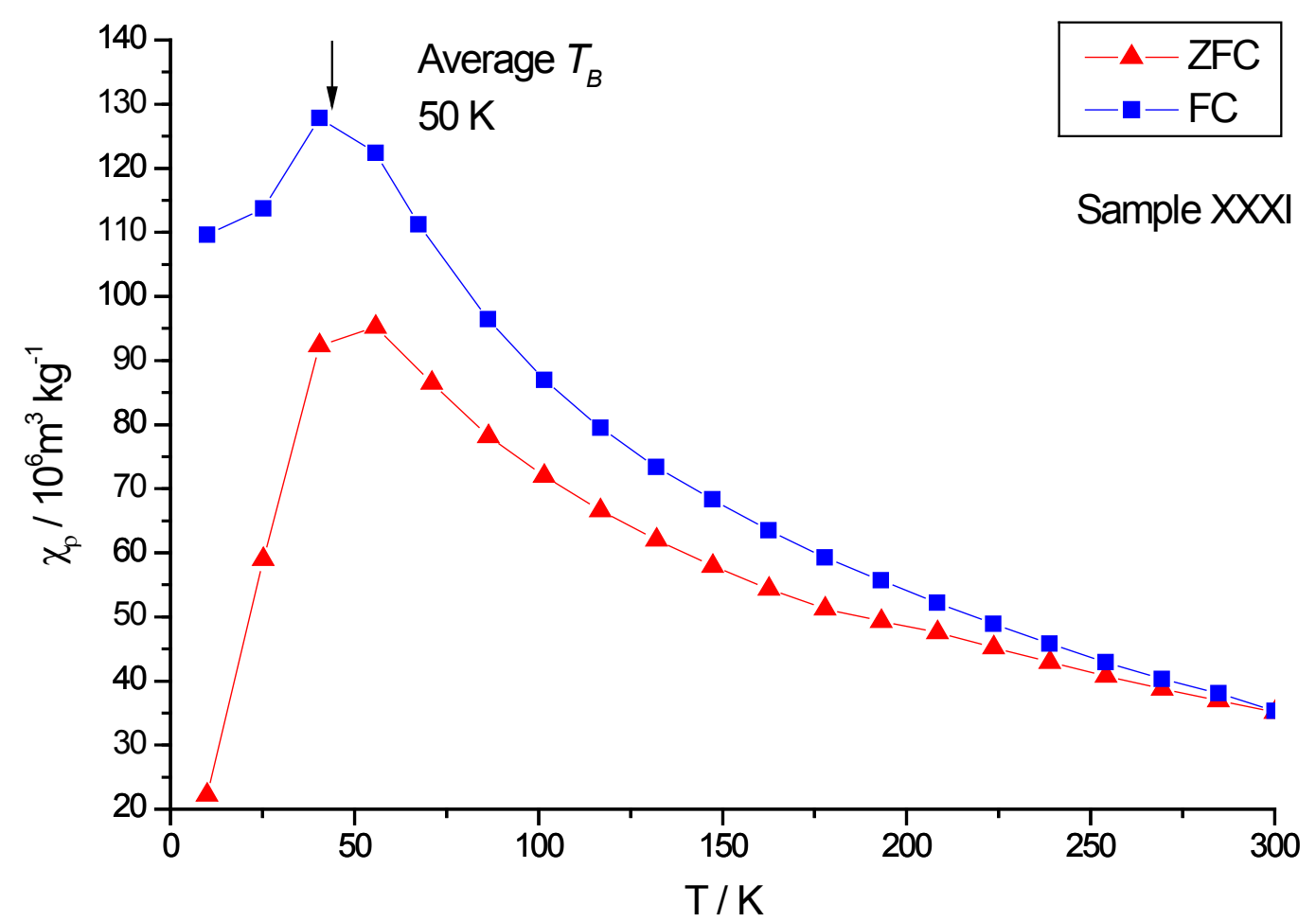

Figure 5-17 ZFC/FC plot of Sample XXXI ( $\mathrm{Fe}_{3} \mathrm{~S}_{4}$ nanocrystals). $\mathrm{H}=1990 \mathrm{~A} \mathrm{~m}^{-1}$. 

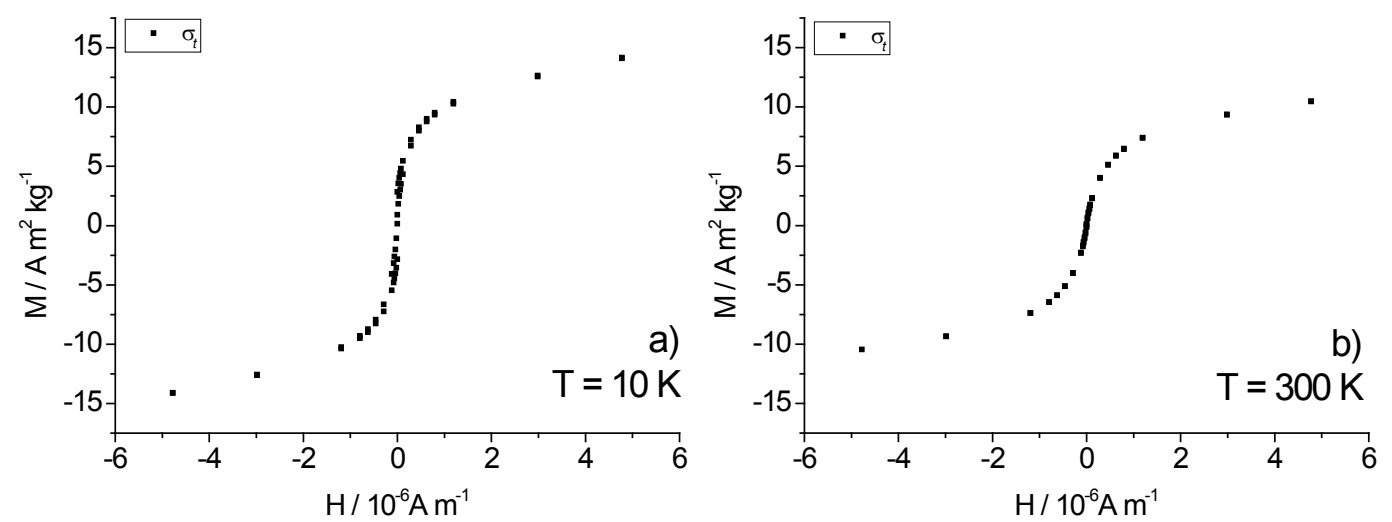

Figure 5-18 Field loop of Sample XXXI ( $\mathrm{Fe}_{3} \mathrm{~S}_{4}$ nanocrystals $)$ at a) $\left.\mathrm{T}=10 \mathrm{~K}, \mathrm{~b}\right) \mathrm{T}=300 \mathrm{~K}$. 


\subsection{Discussion}

The intention of the work presented in Chapter 5 was to build upon the initial work described in Chapter 4, to synthesise iron sulfide nanocrystals with control of crystal phase and size distribution. The initial work conducted in Chapter 4 had indicated the difficulty of achieving small crystallite size, as careful control of the nucleation conditions was necessary to produce $<10 \mathrm{~nm} \mathrm{Fe}_{3} \mathrm{~S}_{4}$ nanocrystals. In Chapter 4 small $\mathrm{Fe}_{3} \mathrm{~S}_{4}$ nanocrystals were always present in a mixture with either large aggregates of amorphous or poorly crystalline material, or large $\mathrm{Fe}_{1-x} \mathrm{~S}$ crystallites. It became apparent that it would be advantageous to separate the two halves of the problem - to be able to address the issues of average size and size distribution, and phase purity separately.

The issue of average size and size distribution was addressed by initially omitting sulfur from reactions, and instead synthesising monodisperse iron/iron oxides nanocrystals, which could then be sulfidised to produce $\mathrm{Fe}_{3} \mathrm{~S}_{4}$ and the reaction rapidly quenched to avoid further particle growth or formation of $\mathrm{Fe}_{1-x} \mathrm{~S}$. The postulated intermediate iron/iron oxide nanocrystals (see section 5.3.3 for further details) were stable during reaction under inert atmosphere, but oxidised upon work up in air to produce iron oxide nanocrystals.

Rather than attempt to rigorously preclude oxygen during work up, purification and characterisation; it was decided to deliberately allow oxidation to occur. Optimisation of the synthesis of the iron oxide nanocrystals was then used to provide a basis for the synthesis of $\mathrm{Fe}_{3} \mathrm{~S}_{4}$ nanocrystals, and the magnetic properties of the iron oxide spinel nanocrystals produced were used as a comparison for the magnetic properties of the isostructural $\mathrm{Fe}_{3} \mathrm{~S}_{4}$ nanocrystals.

\subsubsection{Synthesis of iron oxide nanocrystals}

Iron oxide nanocrystals were synthesised by the thermal decomposition of $\mathrm{Fe}(\mathrm{acac})_{2}$, in the high boiling point amine solvent HDA between $200-300^{\circ} \mathrm{C}$ for between 1-4 hours. Upon work-up in air, the intermediate nanocrystals - which were postulated to be elemental iron or an iron oxide - were observed to oxidise further to one of several iron oxide phases $\left(\gamma-\mathrm{Fe}_{2} \mathrm{O}_{3}, \alpha-\mathrm{Fe}_{2} \mathrm{O}_{3}, \mathrm{FeO}\right)$. 
Experiments in Chapter 5 were exclusively conducted by what has been termed the 'heating-up' method ${ }^{22}$ - the reactants were combined in solution at room temperature, and heated gradually to the designated reaction temperature. The 'hot injection' approach, whereby precursors are injected rapidly into pre-heated solution, was eschewed for the sake of experimental simplicity. Traditionally 'hot injection' was believed to be necessary to produce monodisperse samples, although empirically 'heating-up' approaches have frequently delivered equal or superior monodispersity. ${ }^{15}$ Recent investigations by Hyeon et $a .^{22}$ have shown that in some systems the 'heatingup' approach also proceeds via burst nucleation, and the nucleation and growth match well with the LaMer model.

Although the kinetic mechanism of the reactions conducted in this chapter was not determined, the high degree of monodispersity obtained in some experiments suggests that reactions proceeded via burst nucleation. An empirical approach was undertaken to identify which experimental parameters which affected the size distribution of the resulting iron oxide nanocrystals:

\section{Effect of reaction time and temperature}

In section 5.2.1.1, $\mathrm{Fe}(\mathrm{acac})_{2}$ was used as the iron precursor and HDA as the solvent, while the reaction temperature was varied between 200 and $300^{\circ} \mathrm{C}$, and the reaction duration was varied between 1 and 4 hours. The sole product obtained in all cases was spherical iron oxide spinel nanocrystals. There was little variation in the average nanocrystal diameter, which ranged from 5 to $6 \mathrm{~nm}$. For experiments conducted at both 200 and $300^{\circ} \mathrm{C}$, a minimum in the relative standard deviation of average nanocrystal diameter was observed after 2 hours of reaction. This minimum is congruent with the focusing/defocusing behaviour typically seen in the growth of nanocrystals - the size distribution of a growing ensemble of nanocrystals narrows while there remains a supply of monomer in solution (focusing regime), however once the monomer supply is exhausted Ostwald ripening leads to a broadening of the size distribution (defocusing regime).

\section{Effect of replenishing iron precursor during reaction}

The effect of replenishing the monomer supply on the average nanocrystal diameter and size distribution during decomposition of $\mathrm{Fe}(\mathrm{acac})_{2}$ in $\mathrm{HDA}$ between $200-300^{\circ} \mathrm{C}$ was investigated. When $\mathrm{Fe}(\mathrm{acac})_{2}$ was decomposed in $\mathrm{HDA}$ at $200^{\circ} \mathrm{C}$ (Sample XXV) - in 
contrast to previous experiments conducted in serial (section 5.2.1.1) - there was an apparent defocusing of the size distribution between 1 and 2 hours of reaction, with the relative standard deviation in nanocrystal diameter increasing from $16 \%$ to $23 \%$. 1 hour after the supply of $\mathrm{Fe}(\mathrm{acac})_{2}$ had been replenished, the size distribution refocused and the relative standard deviation in nanocrystal diameter decreased to $15 \%$. The average diameter increased from 5.6 to $6.5 \mathrm{~nm}$ during this period.

When $\mathrm{Fe}(\mathrm{acac})_{2}$ was decomposed in $\mathrm{HDA}$ at $300^{\circ} \mathrm{C}$ (Sample XXVI), the samples were distinctly bimodal at all times, and although there was a refocusing of the size distribution - with the standard deviation in nanocrystal diameter decreasing from 57\% to $35 \% 1$ hour after replenishment of $\mathrm{Fe}(\mathrm{acac})_{2}$ - the sample was much more polydisperse than other samples. It was decided that the modest increases in nanocrystal diameter achieved by replenishment of the iron precursor was outweighed by the wider size distribution in samples where replenishment had occurred, compared to reactions without replenishment (Samples XX - XXIV).

In this project spherical iron oxide nanocrystals were synthesised with average diameters of 5- $6 \mathrm{~nm}$, and relative standard deviations in diameter of as little as $10 \%$ without size selective purification. This is comparable with many published syntheses, and offers the benefit over some published syntheses of relatively cheap reactants and solvent; and a very simple experimental procedure. ${ }^{15}$

\subsubsection{Synthesis of iron sulfide nanocrystals}

Sulfidisation of the intermediate nanocrystals was achieved by the injection of sulfur dissolved in oleylamine. At $200^{\circ} \mathrm{C}$, in either HDA or oleylamine, sulfidisation produced a mixture of amorphous or poorly crystalline iron sulfide and $\alpha-\mathrm{Fe}_{2} \mathrm{O}_{3}$ (Samples XXVII and XXIX). The production of amorphous iron sulfide with a sheet-like appearance matches what was seen in Chapter 4 , where a reaction duration of 4 hours at $200^{\circ} \mathrm{C}$ was necessary to produce crystalline iron sulfide.

Increasing the reaction temperature to $300^{\circ} \mathrm{C}$ produced small, discrete, roughly spherical $\mathrm{Fe}_{3} \mathrm{~S}_{4}$ nanocrystals (Samples XXVIII and XXX). $\mathrm{Fe}_{1-x} \mathrm{~S}$ was detected by XRD, in addition to $\mathrm{Fe}_{3} \mathrm{~S}_{4}$; and $\sim 100 \mathrm{~nm}$ faceted crystallites with well-developed pinacoidal faces - whose appearance matched $\mathrm{Fe}_{1-x} \mathrm{~S}$ crystallites observed in Chapter 4 were observed by TEM. Quenching experiments conducted in Chapter 4 (section 4.2.7) 
indicated that large $\mathrm{Fe}_{1-x} \mathrm{~S}$ crystallites form within the 5 minutes of initiation of reaction at $250^{\circ} \mathrm{C}$ and above. Hence, although the reaction mixtures were allowed to cool immediately after the injection of sulfur, formation of some $\mathrm{Fe}_{1-x} \mathrm{~S}$ could not be avoided at this temperature.

Substituting oleylamine for HDA as the solvent had relatively little effect on the average diameter and size distribution of the $\mathrm{Fe}_{3} \mathrm{~S}_{4}$ nanocrysals produced (Sample XXVIII (HDA) 7 $71 \mathrm{~nm}$ cf Sample XXX (oleylamine) $8 \pm 2 \mathrm{~nm}$ ). However, $\mathrm{Fe}_{3} \mathrm{~S}_{4}$ nanocrystals synthesised in oleylamine had an oblate morphology, rather than the spheroid morphology of $\mathrm{Fe}_{3} \mathrm{~S}_{4}$ nanocrystals synthesised in HAD. This tendency towards elongation along one axis has been observed previously for nanocrystals from a number of phase systems synthesised in oleylamine. ${ }^{15}$ Although oleylamine is easier to remove during workup and purification than HDA, it was decided to proceed with HDA as the solvent due to the slightly narrower size distribution obtained.

Since sulfidisation to produce $\mathrm{Fe}_{3} \mathrm{~S}_{4}$ proceeded rapidly and completely at $300^{\circ} \mathrm{C}$, it was decided to retain this injection temperature. To minimise the formation of $\mathrm{Fe}_{1-x} \mathrm{~S}$ the reaction mixture of Sample XXXI was cooled in a water bath immediately after injection. In addition, size selective purification was used in order to enrich the sample in the smaller sized $\mathrm{Fe}_{3} \mathrm{~S}_{4}$ fraction. The resulting $\mathrm{Fe}_{3} \mathrm{~S}_{4}$ nanocrystals were largely free of $\mathrm{Fe}_{1-x} \mathrm{~S}$, as determined by XRD measurements.

The synthesis of spherical $\mathrm{Fe}_{3} \mathrm{~S}_{4}$ nanocrystals in this project represents one of only two syntheses of discrete, well-characterised $\mathrm{Fe}_{3} \mathrm{~S}_{4}$ nanocrystals known to us - the other being the recent synthesis of O'Brien, ${ }^{23}$ which involved the thermal decomposition of $\left[\mathrm{NBu}_{4}\right]_{2}\left[\mathrm{Fe}_{4} \mathrm{~S}_{4}(\mathrm{SPh})_{4}\right]$ for $12-16$ hours in dodecylamine at $200^{\circ} \mathrm{C}$. The synthesis of O'Brien produced samples with average diameters of 2.5 and $4.5 \mathrm{~nm}$, hence as will be shown below the synthesis in this project of $6.8 \pm 2.0 \mathrm{~nm}$ nanocrystals proves complementary in the investigation of the magnetic properties of small $\mathrm{Fe}_{3} \mathrm{~S}_{4}$ nanocrystals.

\subsubsection{Mechanism of reaction}

The mechanism whereby iron oxide spinel or $\mathrm{Fe}_{3} \mathrm{~S}_{4}$ was produced from the decomposition of $\mathrm{Fe}(\mathrm{acac})_{2}$ will now be considered. Direct identification of the phase 
and composition of the intermediate products of the solution-phase decomposition of the iron precursors was not possible due to their observed instability in air. In situ diffraction and compositional analysis techniques, such as synchrotron XRD and XAFS, may be suited to determining the phase of the intermediate nanocrystals, but were beyond of the scope of this project.

As there is no direct evidence of the nature of the intermediate, two alternative possible pathways will be suggested. The first proposed pathway involves the decomposition of $\mathrm{Fe}(\mathrm{acac})_{2}$ to $\mathrm{Fe}_{3} \mathrm{O}_{4}$, followed by oxidation to $\gamma-\mathrm{Fe}_{2} \mathrm{O}_{3}$ (and/or $\alpha-\mathrm{Fe}_{2} \mathrm{O}_{3}$ ) if exposed to the atmosphere; or sulfidisation to $\mathrm{Fe}_{3} \mathrm{~S}_{4}$ if a sulfur-containing solution is injected (Scheme $5-1)$. The second proposed pathway involves the decomposition of $\mathrm{Fe}(\mathrm{acac})_{2}$ to elemental iron, followed by oxidation to iron oxide spinel $\mathrm{Fe}_{3} \mathrm{O}_{4} / \gamma-\mathrm{Fe}_{2} \mathrm{O}_{3}$ (and/or $\alpha-\mathrm{Fe}_{2} \mathrm{O}_{3}$ ); or sulfidisation to $\mathrm{Fe}_{3} \mathrm{~S}_{4}$ if sulfur solution is injected (Scheme 5-2).

It should be noted that reaction mixtures of the thermal decomposition reactions were consistently black - a colour which is consistent with either finely divided elemental iron ${ }^{24}$ or $\mathrm{Fe}_{3} \mathrm{O}_{4}$. When exposed to air the reaction mixtures became brown within a few hours, indicating that the intermediate nanocrystals (either elemental iron or $\mathrm{Fe}_{3} \mathrm{O}_{4}$ ) oxidised to form $\gamma-\mathrm{Fe}_{2} \mathrm{O}_{3}$.

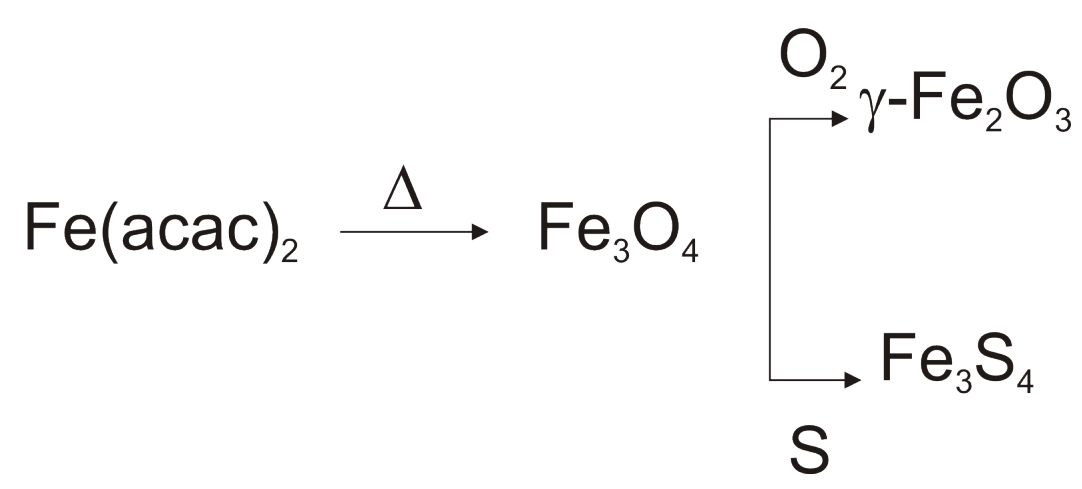

Scheme 5-1 First proposed pathway $-\mathrm{Fe}_{3} \mathrm{O}_{4}$ as an intermediate

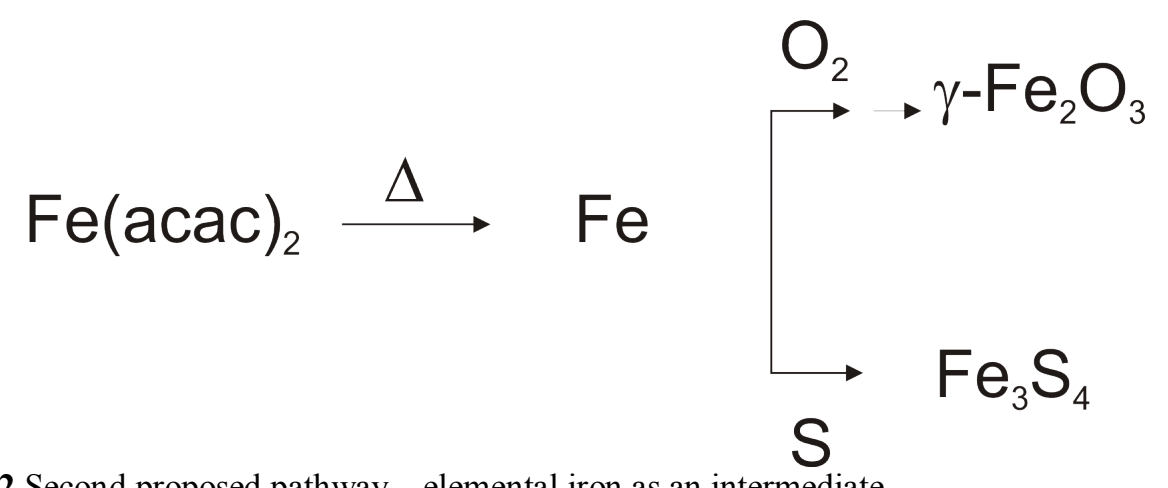

Scheme 5-2 Second proposed pathway - elemental iron as an intermediate. 


\subsubsection{First proposed pathway $-\mathrm{Fe}_{3} \mathrm{O}_{4}$ as the intermediate}

Studies of the solid-state thermal decomposition of metal acetylacetonates have only infrequently characterised the solid, as opposed to gaseous, decomposition products. However, in one study $\mathrm{Fe}_{3} \mathrm{O}_{4}$ was identified as the product of the thermal decomposition of $\mathrm{Fe}(\mathrm{acac})_{3}$ under helium. ${ }^{25} \mathrm{Fe}_{3} \mathrm{O}_{4}$ is a mixed metal oxide, and low temperature syntheses rely on the use of $\mathrm{Fe}^{2+}$ and $\mathrm{Fe}^{3+}$ salts in the appropriate stoichiometric ratio. ${ }^{11}$ However various high temperature syntheses have yielded $\mathrm{Fe}_{3} \mathrm{O}_{4}$ (or $\gamma-\mathrm{Fe}_{2} \mathrm{O}_{3}$ ) from solely zero-valent iron precursors (e.g. $\left[\mathrm{Fe}(\mathrm{CO})_{5}\right]$ ) or solely $\mathrm{Fe}^{3+}$ containing precursors (e.g. $\left.\mathrm{Fe}(\mathrm{acac})_{3}, \mathrm{FeCl}_{3} \cdot x \mathrm{H}_{2} \mathrm{O}\right) .{ }^{15}$ Evidently reduction or oxidation of the iron sources to the appropriate oxidation states proceeds facilely at elevated temperatures in the presence of dissolved oxygen and a variety of organic species. It therefore appears plausible that the high temperature decomposition of $\mathrm{Fe}(\mathrm{acac})_{2}$, like that of $\mathrm{Fe}(\mathrm{acac})_{3}$, could also lead to the formation of $\mathrm{Fe}_{3} \mathrm{O}_{4}$.

The second step of the proposed pathway involves the oxidation of $\mathrm{Fe}_{3} \mathrm{O}_{4}$ to $\gamma-\mathrm{Fe}_{2} \mathrm{O}_{3}$. In the bulk material, the complete oxidisation of $\mathrm{Fe}_{3} \mathrm{O}_{4}$ to $\gamma-\mathrm{Fe}_{2} \mathrm{O}_{3}$ in air at atmospheric pressure occurs above $200^{\circ} \mathrm{C}{ }^{26}$ However, the oxidation of small nanocrystals typically proceeds much more rapidly and under milder conditions than the bulk material, due to the very high relative surface area of nanocrystals. Hence the observed colour change of the reaction mixture from black to brown over the course of hours upon exposure to air is presumed to be the at least partial oxidation to $\gamma-\mathrm{Fe}_{2} \mathrm{O}_{3}$.

The proposed sulfidisation pathway is now considered: the sulfidisation of $\mathrm{Fe}_{3} \mathrm{O}_{4}$ has been observed in an aqueous solution saturated with hydrogen sulfide $\left(\mathrm{H}_{2} \mathrm{~S}\right)$, and aqueous solutions of sodium hydrogen sulfide (NaHS) and sodium sulfide $\left(\mathrm{Na}_{2} \mathrm{~S}\right)$ salts at $300^{\circ} \mathrm{C} .{ }^{27}$ In hydrogen sulfide and sodium hydrogen sulfide solutions, the sulfidisation product was $\mathrm{FeS}_{2}$ p; whereas in sodium sulfide solution the sulfidisation product was identified as colloidal $\mathrm{Fe}_{1-x} \mathrm{~S}$. In de novo syntheses of iron sulfides it was observed that acidic $\mathrm{pH}$ was necessary for the formation of $\mathrm{FeS}_{2} \mathrm{p}$, whereas $\mathrm{Fe}_{1-x} \mathrm{~S}$ was favoured at basic $\mathrm{pH} .{ }^{28}$ The observed preference in this project for the formation of $\mathrm{Fe}_{3} \mathrm{~S}_{4}$ and $\mathrm{Fe}_{1-x} \mathrm{~S}$ over $\mathrm{FeS}_{2} \mathrm{p}$ is therefore likely due to the basicity of solutions of sulfur in HDA and oleylamine. 
The dissolution of sulfur in primary amine solvents is believed to give rise to a number of dissolved sulfur species, including the radical anion $\mathrm{S}_{3} \cdot{ }^{-29}$ To the best of our knowledge, such studies have only been conducted with relatively short-chain amines such as ethylenediamine, rather than long-chain amines such as oleylamine and HDA, the deep red colour of sulfur solutions in oleylamine and HDA indicates the disproportionation of $\mathrm{S}_{8}$ molecules to smaller dissolved sulfur species. ${ }^{21}$ It is apparent that these dissolved sulfur species are capable of reacting in a manner analogous to the sulfide-containing solutions mentioned above.

\subsubsection{Second proposed pathway - elemental iron as the intermediate}

In the second proposed pathway, the thermal decomposition of $\mathrm{Fe}(\mathrm{acac})_{2}$ involves the reduction of $\mathrm{Fe}^{2+}$ to elemental iron nanocrystals. In this proposed pathway, when the iron nanocrystals are exposed to the atmosphere a series of oxidation reactions occur to produce $\gamma-\mathrm{Fe}_{2} \mathrm{O}_{3}$. This process may proceed through a number of intermediate oxides, as shown below in Scheme 5-3, which is based upon the known sequence of phases for the reduction of $\alpha-\mathrm{Fe}_{2} \mathrm{O}_{3}$ to elemental iron ${ }^{30}$ - however it is not proposed that the reaction necessarily proceeds through every intermediate.

\section{$\mathrm{Fe} \rightarrow \mathrm{FeO} \rightarrow \mathrm{Fe}_{3} \mathrm{O}_{4} \rightarrow \gamma-\mathrm{Fe}_{2} \mathrm{O}_{3} \rightarrow \alpha-\mathrm{Fe}_{2} \mathrm{O}_{3}$}

Scheme 5-3 Oxidation of elemental iron

The sulfidisation of iron metal is now discussed. The oxidation of metals - including iron - by elemental sulfur has been observed in various basic amine solvents such as $N$-substituted imidazoles. ${ }^{31}$ The reaction of dissolved sulfur species and metals in basic amine solvents are discussed more thoroughly in Chapter 6, in the context of the formation of stable amine-stabilised metal polysulfide complexes. The metal polysulfide complexes formed by these reactions can be thermally decomposed to binary metal sulfides, and therefore may represent intermediates in the reaction pathway between metal and elemental sulfur and metal sulfides. ${ }^{31}$

Although complex formation has not been demonstrated with less basic primary amines such as oleylamine and HDA, it is a possibility that at elevated temperatures the oxidation of elemental iron by dissolved sulfur species occurs in these solvents. Whether complex formation occurs or not, this postulated oxidation step would enable 
the subsequent formation of metal sulfide, and would account for the formation of metal sulfides from elemental sulfur, which is ostensibly a source of neutral octasulfur molecules $\left(\mathrm{S}_{8}\right)$, rather than sulfide ions $\left(\mathrm{S}^{2-}\right)$.

Although little experimental evidence is available on the mechanistic details of the reaction pathway from $\mathrm{Fe}(\mathrm{acac})_{2}$ to iron oxide spinel or iron sulfide spinel, the first proposed pathway - proceeding through $\mathrm{Fe}_{3} \mathrm{O}_{4}$ as an intermediate - appears the more likely of the two proposed pathways. This is due to the demonstrated conversion of $\mathrm{Fe}(\mathrm{acac})_{3}$ to $\mathrm{Fe}_{3} \mathrm{O}_{4}{ }^{(25)}$, and the lack of literature precedent for the formation of elemental iron from the thermal decomposition of iron acetylacetonates - although this may be due in fact to the tendency of iron to oxidise unless rigorous preventative measures are taken.

\subsubsection{Magnetic properties}

The magnetic properties of iron oxide spinel (Sample XXIII) and $\mathrm{Fe}_{3} \mathrm{~S}_{4}$ (Sample XXXI) nanocrystals of similar average diameters $\left(6.1 \pm 0.6\right.$ and $6.8 \pm 2 \mathrm{~nm}$ for $\mathrm{Fe}_{3} \mathrm{O}_{4}$ and $\mathrm{Fe}_{3} \mathrm{~S}_{4}$, respectively) were measured and contrasted. Both samples demonstrated a transition from ferrimagnetism at low temperatures to superparamagnetism, and were superparamagnetic at room temperature.

\subsubsection{Iron oxide spinel nanocrystals (Sample XXIII)}

The magnetic ordering transition observed in the ZFC/FC plot of Sample XXIII, centred at $100 \mathrm{~K}$, corresponds to the thermal 'unblocking' behaviour associated with a transition from ferrimagnetism to a superparamagnetic state. The $T_{B}$ of $100 \mathrm{~K}$ associated with the $6.1 \mathrm{~nm}$ iron oxide spinel nanocrystals fits well within the trend established for $\gamma-\mathrm{Fe}_{2} \mathrm{O}_{3}$ nanocrystals by Hyeon: ${ }^{12} 4 \mathrm{~nm}$ nanocrystals were reported to have a sharply defined unblocking temperature of approximately $25 \mathrm{~K}$, whereas $16 \mathrm{~nm}$ nanocrystals had a broad transition centred at $185 \mathrm{~K}$.

The lack of coercivity observed in the field loop recorded at $300 \mathrm{~K}$, supports the contention that the nanocrystal ensemble is in a superparamagnetic state, although even at $10 \mathrm{~K}$ - below the $T_{B}$ of $100 \mathrm{~K}$ - the coercivity of the sample is immeasurably small. This indicates that in the ferrimagnetic state, magnetisation occurs through highly reversible coherent domain rotations in single magnetic domain particles. 
The observed saturation magnetisation of 50 and $43 \mathrm{~A} \mathrm{~m}^{2} \mathrm{~kg}^{-1}$ at 10 and $300 \mathrm{~K}$, respectively, is somewhat lower compared to that of bulk $\mathrm{Fe}_{3} \mathrm{O}_{4}$ or $\gamma-\mathrm{Fe}_{2} \mathrm{O}_{3}(84$ and 74 $\mathrm{A} \mathrm{m}^{2} \mathrm{~kg}^{-1}$, respectively). However saturation magnetisations are typically smaller in nanocrystals than the bulk, due to the magnetic 'dead' layers which occur due to pinning of atomic magnetic moments at the surface. ${ }^{9}$ The saturation magnetisation of Sample XXIII is the same as previous reported saturation magnetisations of $6-7 \mathrm{~nm}$ iron spinel nanocrystals at $300 \mathrm{~K}\left(42 \mathrm{~A} \mathrm{~m}^{2} \mathrm{~kg}^{-1}\right) .{ }^{32}$

\subsubsection{2 $\quad \mathrm{Fe}_{3} \mathrm{~S}_{4}$ nanocrystals (Sample XXXI)}

The ZFC/FC plot of Sample XXXI also demonstrated a magnetic ordering transition centred around $50 \mathrm{~K}$ - from a ferrimagnetic to superparamagnetic state. The non-coincidence of the ZFC and FC plots after this transition, however, indicate the presence of sub-populations in the sample, which remain in a magnetically irreversible state even above the average transition temperature.

Like the iron oxide spinel nanocrystals of Sample XXIII, Sample XXXI also displays a lack of coercivity in field loop experiments conducted at 10 and $300 \mathrm{~K}$. The slight wasp-waistedness observed in the field loop recorded at $10 \mathrm{~K}$ supports the presence of a higher coercivity component in the sample - which may be due to $\mathrm{Fe}_{1-x} \mathrm{~S}$ impurities undetected by XRD, or may be due to size dispersity in the $\mathrm{Fe}_{3} \mathrm{~S}_{4}$ nanocrystals in the sample. The relative standard deviation in diameter for the $\mathrm{Fe}_{3} \mathrm{~S}_{4}$ nanocrystals was $30 \%$, indicating only a moderate degree of monodispersity in the sample.

The approximate saturation magnetisation of $6.8 \mathrm{~nm} \mathrm{Fe}_{3} \mathrm{~S}_{4}$ nanocrystals at 10 and $300 \mathrm{~K}$ is 12 and $9 \mathrm{~A} \mathrm{~m}^{2} \mathrm{~kg}^{-1}$, respectively. This is approximately double that of Sample XIII (Chapter 4), which comprised a mixture of large $\mathrm{Fe}_{1-x} \mathrm{~S}$ crystallites and $5-6 \mathrm{~nm} \mathrm{Fe}_{3} \mathrm{~S}_{4}$ nanocrystals $\left(5.4 \mathrm{~A} \mathrm{~m}^{2} \mathrm{~kg}^{-1}\right.$ at $\left.300 \mathrm{~K}\right)$. O'Brien ${ }^{23}$ reported the approximate saturation magnetisation of 4.5 and $2.5 \mathrm{~nm}$ spherical $\mathrm{Fe}_{3} \mathrm{~S}_{4}$ crystallites at $300 \mathrm{~K}$ as 2.5 and $1.5 \mathrm{~A}$ $\mathrm{m}^{2} \mathrm{~kg}^{-1}$, respectively. Hence the figure of $9 \mathrm{~A} \mathrm{~m}^{2} \mathrm{~kg}^{-1}$ for $6.8 \mathrm{~nm}$ nanocrystals derived in this project fits well with this trend. The figures from this project, and those quoted from O'Brien represent the only magnetic data on well-defined, spherical, monodisperse $\mathrm{Fe}_{3} \mathrm{~S}_{4}$ nanocrystals, of which we are aware. These figures are somewhat lower than the saturation magnetisation reported previously for $\mathrm{Fe}_{3} \mathrm{~S}_{4}$ by $\operatorname{Spender}^{33}\left(\sim 27 \mathrm{~A} \mathrm{~m}^{2} \mathrm{~kg}^{-1}\right.$ at $300 \mathrm{~K}$ for $9 \mathrm{~nm}$ crystallite size) - although in the aforementioned publication the 
average crystallite diameter was determined solely by Scherrer X-ray broadening analysis, and the crystallite morphology is uncharacterised.

\subsubsection{Comparision of $\mathrm{Fe}_{3} \mathrm{O}_{4}$ and $\mathrm{Fe}_{3} \mathrm{~S}_{4}$ nanocrystals}

Obvious differences in the magnetic behaviour of $\mathrm{Fe}_{3} \mathrm{O}_{4}$ and $\mathrm{Fe}_{3} \mathrm{~S}_{4}$ nanocrystals include the difference in average blocking temperature observed for the samples - $T_{B}$ for the 6.1 $\mathrm{nm} \mathrm{Fe} \mathrm{O}_{4}$ nanocrystals was $100 \mathrm{~K}$, compared to $50 \mathrm{~K}$ for $6.8 \mathrm{~nm} \mathrm{Fe} \mathrm{S}_{4}$ nanocrystals. The average blocking temperature, $T_{B}$, is controlled by the magnitude of the energy barrier that the magnetic moment of a magnetic domain must traverse to alter its spatial orientation. The origins of this energy barrier may lie in shape anisotropy or magnetocrystalline anisotropy. ${ }^{9}$ The morphology of both the $\mathrm{Fe}_{3} \mathrm{O}_{4}$ and $\mathrm{Fe}_{3} \mathrm{~S}_{4}$ nanocrystals was roughly spherical, indicating the origin of the energy barrier was likely magnetocrystalline anisotropy.

The first order magnetocrystalline anisotropy constants, $K_{l}$, of $\mathrm{Fe}_{3} \mathrm{O}_{4}{ }^{9}$ and $\gamma-\mathrm{Fe}_{2} \mathrm{O}_{3}{ }^{34}$ have been determined to be $-9 \times 10^{3}$ and $4.7 \times 10^{3} \mathrm{~J} \mathrm{~m}^{-3}$, respectively. To the best our knowledge, the magnetocrystalline anisotropy of $\mathrm{Fe}_{3} \mathrm{~S}_{4}$ has only ever been estimated ( $\left.K_{l} \sim 1 \times 10^{3} \mathrm{~J} \mathrm{~m}^{-3}\right){ }^{35}$ presumably as the difficulty of growing sufficiently large single crystals of $\mathrm{Fe}_{3} \mathrm{~S}_{4}$ precludes direct measurement. The fact that the $T_{B}$ of the spherical $\mathrm{Fe}_{3} \mathrm{~S}_{4}$ nanocrystals is lower than that of similarly sized iron oxide spinel nanocrystals provisionally supports the estimated magnetocrystalline anisotropy constant being lower than the established constants for $\mathrm{Fe}_{3} \mathrm{O}_{4}$ and $\gamma-\mathrm{Fe}_{2} \mathrm{O}_{3}$.

More in depth theoretical modelling is necessary to determine how the experimentally measured average blocking temperature of the $6.8 \mathrm{~nm} \mathrm{Fe}_{3} \mathrm{~S}_{4}$ nanocrystals correlates with the estimated magnetocrystalline anisotropy constant; and how the saturation magnetisation observed for this well-defined, and monodisperse sample $\left(9 \mathrm{~A} \mathrm{~m}^{2} \mathrm{~kg}^{-1}\right)$ compares with estimates of the magnetic moment per formula unit $\left(2.2 \mu_{\mathrm{B}}\right.$ /formula unit) ${ }^{33}$ of $\mathrm{Fe}_{3} \mathrm{~S}_{4}$ based on samples of 'bulk' $\mathrm{Fe}_{3} \mathrm{~S}_{4}$ published in the literature. However, it is hoped that the magnetic data measured in this project - the first for monodisperse $\mathrm{Fe}_{3} \mathrm{~S}_{4}$ nanocrystals of a well-defined size, and size distribution - will allow better understanding of the magnetic structure of the $\mathrm{Fe}_{3} \mathrm{~S}_{4}$ phase. 


\subsection{Conclusions}

The synthesis of monodisperse spherical iron oxide spinel nanocrystals was achieved by the thermal decomposition of $\mathrm{Fe}(\mathrm{acac})_{2}$ in $\mathrm{HDA}$ at $200-300^{\circ} \mathrm{C}$. The highest degree of monodispersity $(\sigma \sim 10 \%)$ was reached after 2 hours of reaction at either 200 or $300^{\circ} \mathrm{C}$, indicating the point where the nanocrystal ensemble moved from the focusing to defocusing growth regime. The average diameter of the iron oxide spinel nanocrystals at this point was $6 \mathrm{~nm}$. When decomposing $\mathrm{Fe}(\mathrm{acac})_{2}$ in HDA, replenishment of $\mathrm{Fe}(\mathrm{acac})_{2}$ after 2 hours led to a slight increase of the average iron oxide spinel nanocrystal diameter, but the relative standard deviation in nanocrystal diameter was higher than for experiments without replenishment.

Exposure to the atmosphere of the intermediate nanocrystals - believed to be either elemental iron or $\mathrm{Fe}_{3} \mathrm{O}_{4}$ - resulted in oxidation towards $\gamma-\mathrm{Fe}_{2} \mathrm{O}_{3}$. Sulfidisation of the intermediate nanocrystals to $\mathrm{Fe}_{3} \mathrm{~S}_{4}$ could be achieved by injecting 1.5 eq. of sulfur dissolved in oleylamine at $300^{\circ} \mathrm{C}$ before exposure to the atmosphere. Rapid cooling, and size selective purification was used to produce a sample of $7 \mathrm{~nm} \mathrm{Fe} \mathrm{S}_{4}$ nanocrystals $(\sigma=30 \%)$.

$6 \mathrm{~nm}$ iron oxide spinel nanocrystals had an average blocking temperature, $T_{B}$, of $100 \mathrm{~K}$, whereas the blocking temperature of $7 \mathrm{~nm} \mathrm{Fe}_{3} \mathrm{~S}_{4}$ nanocrystals was $50 \mathrm{~K}$ - an indication that the first order magnetocrystalline anisotropy constant of $\mathrm{Fe}_{3} \mathrm{~S}_{4}$ (which has only ever been estimated) may be lower than that of $\mathrm{Fe}_{3} \mathrm{O}_{4}$ and $\gamma-\mathrm{Fe}_{2} \mathrm{O}_{3}$. At $43 \mathrm{~A} \mathrm{~m}^{2} \mathrm{~kg}^{-1}$ at $300 \mathrm{~K}$, the saturation magnetisation measured for the iron oxide spinel nanocrystals matched literature values. The $\mathrm{Fe}_{3} \mathrm{~S}_{4}$ nanocrystals had an approximate saturation magnetisation of $9 \mathrm{~A} \mathrm{~m}^{2} \mathrm{~kg}^{-1}$ at $300 \mathrm{~K}$, which fits well within the trend set by the few saturation magnetisation values published for $\mathrm{Fe}_{3} \mathrm{~S}_{4}$ samples with well-characterised size distributions. It is hoped that the magnetic data determined in this project may assist with future theoretical studies of the magnetic properties of the $\mathrm{Fe}_{3} \mathrm{~S}_{4}$ phase, and of $\mathrm{Fe}_{3} \mathrm{~S}_{4}$ nanocrystals. 


\subsection{Method}

Iron(II) acetylacetonate ( $\left.\mathrm{Fe}(\mathrm{acac})_{2}, 99.95 \%\right)$ was purchased from Sigma Aldrich, and stored in a desiccator. Sulfur (sublimed) was purchased from BDH and used as received.

Oleylamine (tech., $\geq 70 \%$ ), and hexadecylamine (HDA, tech., $\geq 90 \%$ ) were purchased from Fluka. Ethanol and toluene were of reagent grade. All solvents were used as received.

A stock solution of sulfur in oleylamine was prepared by dissolving $\mathrm{S}(0.16 \mathrm{~g}, 5 \mathrm{mmol})$ in distilled oleylamine $\left(10 \mathrm{~cm}^{3}\right)$ to give a clear, bright red $0.5 \mathrm{M}$ solution, which was stored under nitrogen.

\subsubsection{General synthesis of iron oxide nanocrystals}

Fe(acac $)_{2}(0.2541 \mathrm{~g}, 1 \mathrm{mmol})$ and HDA $(13 \mathrm{~g})$ were loaded into a flask and flushed with nitrogen for 5 minutes. The flask was heated until the HDA melted to form a dark red solution with the $\mathrm{Fe}(\mathrm{acac})_{2}\left(\sim 50^{\circ} \mathrm{C}\right)$. This solution was degassed by bubbling nitrogen through for 30 minutes. This was heated to a reaction temperature of either 200 or $300^{\circ} \mathrm{C}$ for $1-4$ hours. The solution became black after reaching $90^{\circ} \mathrm{C}$. After cooling to $50^{\circ} \mathrm{C}$, ethanol $\left(15 \mathrm{~cm}^{3}\right)$ was added to prevent solidification of the solvent. See below for purification of samples containing HDA.

\subsubsection{Synthesis of iron oxide nanocrystals involving replenishment of precursor}

HDA ( $8 \mathrm{~g}$ ) was placed in a flask and heated to melt while the flask was flushed with nitrogen. Nitrogen was bubbled through the solvent for 15 minutes to degas. $\mathrm{Fe}(\mathrm{acac})_{2}$ $(0.13 \mathrm{~g}, 0.51 \mathrm{mmol})$ was added under nitrogen flow, and stirred to form a red suspension. This was heated to a reaction temperature of $200-300^{\circ} \mathrm{C}$. The suspension blackened at a temperature of $\sim 90^{\circ} \mathrm{C}$. Aliquots were withdrawn after 1 and 2 hours, and quenched in warm ethanol. After 2 hours, the reaction mixture was cooled in $90^{\circ} \mathrm{C}$, and further $\mathrm{Fe}(\mathrm{acac})_{2}(0.10 \mathrm{~g}, 0.39 \mathrm{mmol})$ was added under nitrogen flow. The reaction mixture was reheated to $300^{\circ} \mathrm{C}$, and the reaction continued for a further 2 hours. Further aliquots were taken at 1 and 2 hours after the second addition of $\mathrm{Fe}(\mathrm{acac})_{2}$, and were quenched in warm ethanol. The aliquots were purified as described below for samples containing HDA. 


\subsubsection{General synthesis of $\mathrm{Fe}_{3} \mathrm{~S}_{4}$ nanocrystals}

Synthesis with HDA

$\mathrm{Fe}(\mathrm{acac})_{2}(0.2541 \mathrm{~g}, 1 \mathrm{mmol})$ and HDA $(13 \mathrm{~g})$ were loaded into a flask and flushed with nitrogen for 5 minutes. The flask was heated until the HDA melted to form a dark red solution with the $\mathrm{Fe}(\mathrm{acac})_{2}\left(\sim 50^{\circ} \mathrm{C}\right)$. This solution was degassed by bubbling nitrogen through for 30 minutes. This was heated to a reaction temperature of either 200 or $300^{\circ} \mathrm{C}$ for 2 hours. The solution became black after reaching $90^{\circ} \mathrm{C}$. After 2 hours of reaction a solution of sulfur in oleylamine $\left(2-3 \mathrm{~cm}^{3}\right.$ of $0.5 \mathrm{M}$ solution, containing 1 $1.5 \mathrm{mmol}$ sulfur) was injected, and the reaction mixture cooled. After cooling to $50^{\circ} \mathrm{C}$, ethanol $\left(15 \mathrm{~cm}^{3}\right)$ was added to prevent solidification of the solvent. See below for purification of samples containing HDA.

\section{Synthesis with oleylamine}

$\mathrm{Fe}(\mathrm{acac})_{2}(0.2541 \mathrm{~g}, 1.00 \mathrm{mmol})$ was loaded into a flask, and flushed with nitrogen for 5 minutes. Oleylamine was degassed by bubbling nitrogen through vigorously for 30 minutes. Degassed oleylamine $\left(20 \mathrm{~cm}^{3}\right)$ was added to flask, via syringe, to produce a dark red solution. This was heated to a reaction temperature of either 200 or $300^{\circ} \mathrm{C}$ for 2 hours. After 2 hours of reaction a solution of sulfur in oleylamine $\left(2-3 \mathrm{~cm}^{3}\right.$ of $0.5 \mathrm{M}$ solution, containing $1-1.5 \mathrm{mmol}$ sulfur) was injected, and the reaction mixture cooled to room temperature. See below for purification of samples containing oleylamine.

\subsubsection{Synthesis of $\mathrm{Fe}_{3} \mathrm{~S}_{4}$ nanocrystals with rapid cooling}

HDA (15 g) was placed in a flask and heated to melt, while being flushed with nitrogen. Nitrogen was bubbled through the solvent for 30 minutes to degas. $\mathrm{Fe}(\mathrm{acac})_{2}(0.30 \mathrm{~g}$, $1.2 \mathrm{mmol}$ ) was added to the flask under nitrogen flow, and stirred to form a red suspension. The suspension was heated to $300^{\circ} \mathrm{C}$, and maintained at this temperature for 2 hours. The suspension had blackened by $90^{\circ} \mathrm{C}$. After 2 hours of reaction, the temperature of the flask was reduced to $280^{\circ} \mathrm{C}$, sulfur in oleylamine $\left(3.6 \mathrm{~cm}^{3}\right.$ of $0.5 \mathrm{M}$ solution, containing $1.8 \mathrm{mmol}$ sulfur $\equiv 1.5$ eq.) was injected. Immediately after injection, and a hot water bath was used to rapidly cool the flask, followed by a cool water bath. The temperature of the flask was reduced to below $200^{\circ} \mathrm{C}$ within 1 minute. The reaction mixture was purified as described below for samples containing HDA. 
After this, a toluene suspension of the sample was subjected to size selective purification, as described below.

\subsubsection{Purification}

The reaction mixture of experiments where no sulfur was added remained black as long as kept under nitrogen. During the process of purification, in air, the colour changed to brown - presumably due to oxidation of the sample. The product of reactions where sulfur was injected remained black - no oxidation was observed to occur.

\section{Purification of samples containing oleylamine}

Ethanol $\left(20 \mathrm{~cm}^{3}\right)$ was added to reaction mixture to destabilise the suspension. This was centrifuged at 4,000 rpm for $5-10$ minutes until the inorganic fraction had precipitated, and the supernatant was clear. The supernatant was discarded, and the pellet was resuspended in toluene $\left(10 \mathrm{~cm}^{3}\right)$ with the aid of sonication. Ethanol $\left(20 \mathrm{~cm}^{3}\right)$ was added to destabilise the suspension, and centrifugation was repeated. After $2-3$ cycles of purification the pellet was dried in vacuo, ground, and stored as a powder.

\section{Purification of samples containing $H D A$}

HDA is a solid at room temperature. Samples containing HDA tend to solidify slowly even if ethanol is added to the reaction mixture above the melting point. To remove excess HDA, boiling ethanol is added to dissolve the HDA. The reaction mixture is then quickly centrifuged at 4,000 rpm for 2 minutes to precipitate the inorganic fraction. If HDA recrystallised during centrifugation, the centrifuge tube was heated carefully with a heat gun until the HDA redissolved. The clear supernatant was then discarded. The inorganic was then resuspended in boiling ethanol with the aid of sonication, and centrifugation was repeated. After $2-3$ cycles of purification the resulting pellet was dried in vacuo, ground, and stored as a powder; or suspended in toluene for TEM.

\section{Size-selective purification}

The sample was suspended in toluene with the aid of sonication for 5 minutes. This toluene suspension was then centrifuged at 4,000 rpm for 1 minute, and the supernatant was decanted. This process was intended to leave the largest size fraction precipitated, 
while progressively enriching the supernatant with the smaller size fractions. This procedure was repeated on the precipitate $\sim 10$ times. The toluene was removed from the combined supernatant fractions by rotary evaporation. The resulting precipitate was suspended in hot ethanol, and centrifuged at 4,000 rpm for 1 hour, and let rest for a further 6 hours until all material had precipitated, and the supernatant was clear and colourless. The supernatant was discarded, and the precipitate was dried in vacuo, and ground into a powder. 


\subsection{References}

(1) D. Huber, Small, 2005, 1, 482.

(2) R. C. O'Handley, Modern Magnetic Materials: Principles and Applications, John Wiley and Sons, New York, 2001, 99.

(3) T. W. Smith and D. Wychick, J. Phys. Chem., 1980, 84, 1621.

(4) R. Wang, Y. Chen, Y. Fu, H. Zhang and C. Kisielowski, J. Phys. Chem. B, 2005, 109, 12245.

(5) X. Hu, J. C. Yu and J. Gong, J. Phys. Chem. C, 2007, 111, 11180.

(6) H. Kominami, S.-i. Onoue, K. Matsuo and Y. Kera, J. Am. Ceram. Soc., 1999, 82, 1937.

(7) T. Ohmori, H. Takahashi, H. Mametsuka and E. Suzuki, Phys. Chem. Chem. Phys., 2000, 2, 3519.

(8) K. A. Gupta and M. Gupta, Biomaterials, 2005, 26, 3995.

(9) R. O'Handley, Modern Magnetic Material: Principles and Applications, John Wiley \& Sons, New York, 2000, 29-43.

(10) X. Sun, Y. Huang and D. E. Nikles, Int. J. of Nanotechnology, 2004, 1, 328.

(11) T. Fried, G. Shemer and G. Markovich, Adv. Mater., 2001, 13, 1158.

(12) T. Hyeon, S. S. Lee, J. Park, Y. Chung and H. B. Na, J. Am. Chem. Soc., 2001, 123, 12798.

(13) T. Hyeon, Chem. Commun., 2003, 927.

(14) S. Sun and H. Zeng, J. Am. Chem. Soc., 2002, 124, 8204.

(15) J. Park, J. Joo, S. G. Kwon, Y. Jang and T. Hyeon, Angew. Chem. Int. Ed., 2007, 46, 4630.

(16) F. X. Redl, C. T. Black, G. C. Papaefthymiou, R. L. Sandstrom, M. Yin, H. Zeng, C. B. Murray and S. P. O'Brien, J. Am. Chem. Soc., 2004, 126, 14583.

(17) Y. Hou, Z. Xu and S. Sun, Angew. Chem. Int. Ed., 2007, 46, 6329.

(18) R. M. Cornell and U. Schwertmann, The Iron Oxides: Structure, Properties, Reactions, Occurences and Uses, Wiley, Weinheim, 2003, 9-36.

(19) C. Greaves, J. Solid State Chem., 1983, 49, 325.

(20) J. Park, K. An, Y. Hwang, J.-g. Park, H.-j. Noh, J.-y. Kim, J.-h. Park, N.-m. Hwang and T. Hyeon, Nature Mater., 2004, 3, 891.

(21) N. N. Greenwood and A. Earnshaw, Chemistry of the Elements, ButterworthHeinemann, Oxford, 1984.

(22) S. G. Kwon, Y. Piao, J. Park, S. Angappane, Y. Jo, N.-M. Hwang, J.-G. Park and T. Hyeon, J. Am. Chem. Soc., 2007, 129, 12571.

(23) P. V. Vanitha and P. O'Brien, J. Am. Chem. Soc., 2008, 130, 17256.

(24) D. Farrell, S. A. Majetich and J. P. Wilcoxon, J. Phys. Chem. B, 2003, 107, 11022.

(25) N. B. Morozova, V. N. Mit'kin, I. K. Igumenov, V. A. Varnek and O. G. Potapova, Zh. Neorg. Khim., 1988, 33, 2547.

(26) U. Colombo, F. Gazzarrini, G. Lanzavecchia and G. Sironi, Science, 1965, 147, 1033.

(27) J. L. Lindner and J. W. Gruner, Econ. Geol., 1939, 34, 537.

(28) R. Berner, J. Geol., 1964, 72, 293.

(29) F. P. Daly and C. Brown, W., J. Phys. Chem., 1973, 77, 1859.

(30) R. M. Cornell and U. Schwertmann, The Iron Oxides: Structure, Properties, Reactions, Occurrences and Uses Wiley, Weinheim, 2004.

(31) T. B. Rauchfuss, Inorg. Chem., 2004, 43, 14.

(32) D. K. Kim, Y. Zhang, W. Voit, K. V. Rao and M. Muhammed, J. Magn. Magn. Mater., 2001, 225, 30. 
(33) M. R. Spender, J. M. D. Coey and A. H. Morrish, Can. J. Phys., 1972, 50, 2313.

(34) G. Salazar-Alvarez, J. Qin, V. Šepelák, I. Bergmann, M. Vasilakaki, K. N.

Trohidou, J. D. Ardisson, W. A. A. Macedo, M. Mikhaylova, M. Muhammed, M. D. Baró and J. Nogués, J. Am. Chem. Soc., 2008, 130, 13234.

(35) J. C. D. Ricci and J. L. Kirschvink, J. Geophys. Res., 1992, 97, 17. 


\section{Chapter 6 Single-source metal sulfide precursors for nanocrystal synthesis}

\subsection{Introduction}

Single-source precursors have been used to synthesise nanocrystals of a wide variety of phases, including metal chalcogenides, as detailed below in section 6.1.1. However to the best of our knowledge, metal polysulfide complexes, such as the type described below in section 6.1.2, have not been previously used as precursors for the synthesis of nanocrystals. Hence, we synthesised a range of metal polysulfide complexes and investigated their use as precursors for nanocrystals of a variety of metal chalcogenide phases, including zinc sulfide, iron sulfides $\left(\mathrm{Fe}_{1-x} \mathrm{~S}\right.$ and $\left.\mathrm{Fe}_{3} \mathrm{~S}_{4}\right)$ and nickel sulfides $\left(\alpha-\mathrm{Ni}_{1-x} \mathrm{~S}\right.$ and $\left.\mathrm{NiS}_{2}\right)$. Literature uses of single-source precursors in general are outlined below, and the particular class of compound used in this project - metal polysulfide complexes - are discussed. This is followed by literature syntheses of zinc sulfide and nickel sulfide nanocrystals. The synthesis of iron sulfide nanocrystals was discussed previously (see Chapter 4 section 4.1.3).

\subsubsection{Single-source metal sulfide precursors}

Over the past decade, precursors which can provide both the metal and the non-metal component of a binary compound upon thermolysis, have been investigated for the synthesis of nanocrystals. The primary advantage that a single-source precursor offers over a multiple precursor approach is that intimate proximity of the metal and non-metal atoms is already established within the precursor. This performing of the bonding present in the desired structure has been credited with reducing the number of defects in the product, and simplifying concerns over the mixing and stoichiometry of multiple precursors. $^{1}$

Many of the single-source precursor metal complexes mentioned below offer further advantages: They are more stable than commonly used organometallic precursors, such as dialkyl metals (e.g. dimethyl cadmium), and highly reactive non-metal sources such as phosphines and alkylsilyl chalcogenides (e.g. bis(trimethylsilyl)selenium). Neutral single-source precursor complexes also avoid the use of non-volatile inorganic 
counterions such as halides or nitrate, which have been found to adversely affect the quality of the product. ${ }^{2,3}$

Single-source precursors have been synthesised for a wide range of metals, drawn from the main block ( $\mathrm{Ga}, \mathrm{In}, \mathrm{Pb})$ and the d-block block $(\mathrm{Cd}, \mathrm{Zn}, \mathrm{Hg}, \mathrm{Ni}, \mathrm{Cu}, \mathrm{Mn}) .{ }^{4}$ Of those metals, indium and cadmium have been prepared as metal diorganophosphides $\left(\operatorname{In}\left(\mathrm{PBu}_{2}^{i}\right)_{3}\right.$ and $\left.\left[\mathrm{MeCd}\left(\mathrm{PBu}_{2}^{t}\right)\right]_{3}\right)$ and decomposed to InP and $\mathrm{Cd}_{2} \mathrm{P}_{3}$ nanocrystals, respectively; ${ }^{5,6}$ while $\left(\mathrm{Et}_{3} \mathrm{~N}\right) \mathrm{Ga}\left(\mathrm{N}_{3}\right)_{3}$ was detonated in a controlled fashion to afford GaN nanocrystals. ${ }^{7}$ Lead and transition metal chalcogenide nanocrystals have been synthesised using a variety of single-source precursors, including: metal dithio- or diselenocarbamate complexes $\left(\mathrm{M}\left(\mathrm{E}_{2} \mathrm{CNRR}\right)_{2}, \mathrm{E}=\mathrm{S}, \mathrm{Se}\right){ }^{4,8}$ metal thio- or selenocarboxylates $\left(\mathrm{M}(\mathrm{EC}(\mathrm{O}) \mathrm{R})_{2} \mathrm{~L}_{2}, \mathrm{E}=\mathrm{S}, \mathrm{Se}\right) ;{ }^{9}$ metal alkylxanthates $\left(\mathrm{M}(\mathrm{SC}(\mathrm{S}) \mathrm{OR})_{2}\right) ;{ }^{4,10}$ and metal alkylthiourea $\left(\mathrm{M}\left(\mathrm{RR}{ }^{\prime} \mathrm{NC}(\mathrm{S}) \mathrm{NRR}{ }^{\prime}\right)_{2} \mathrm{X}_{2}\right)$ or dithiobiurea $\left.\mathrm{M}\left(\mathrm{H}_{2} \mathrm{NC}(\mathrm{S}) \mathrm{NHNHC}(\mathrm{S}) \mathrm{NH}_{2}\right) \mathrm{X}_{2}\right)$ complexes. ${ }^{4,}{ }^{10}$ Many of these syntheses appear to have yielded products of quality comparable to or better than that of more traditional, multiple precursor approaches. ${ }^{4}$

The theme common to all the metal chalcogenide precursors in the preceding paragraph, is that they are metal complexes with sulfur- or selenium-containing organic ligands. Coordination of the ligand to the metal via its thio- or seleno-moiety place the chalcogen atom in the coordination sphere of the metal, until thermolysis of the complex cleaves the hydrocarbon portion to produce the binary metal chalcogenide. Exceptions to this motif are the cluster molecules $\mathrm{Li}_{2}\left[\mathrm{Cd}_{10} \mathrm{Se}_{4}(\mathrm{SPh})_{16}\right]$, and $\left[\mathrm{NBu}_{4}\right]_{2}\left[\mathrm{Fe}_{4} \mathrm{~S}_{4}(\mathrm{SPh})_{4}\right]$, which were used as precursors for CdSe nanorods; ${ }^{11}$ and $\mathrm{Fe}_{7} \mathrm{~S}_{8}$ and $\mathrm{Fe}_{3} \mathrm{~S}_{4}$ nanocrystals, ${ }^{12}$ respectively. These clusters feature inorganic sulfido or seleno ligands, in addition to stabilising organic thiol ligands - and therefore resemble the metal polysulfide complexes used in this project, inasmuch as the chalcogen source is an inorganic rather than organic ligand.

\subsubsection{Metal polysulfide complexes}

The research group of T. Rauchfuss at the University of Illinois has published a number of papers on the subject of ligand-stabilised metal polysulfide complexes. ${ }^{13-18}$ Rauchfuss and coworkers demonstrated that highly basic, coordinating ligands (e.g. pyridine, ${ }^{14}$ substituted pyridines, ${ }^{14}$ substituted imidazoles, ${ }^{13,15,16}$ trisubstituted chelating diamines ${ }^{17}$ ) could assist the oxidation of metal powder by elemental sulfur (octasulfur, 
$\mathrm{S}_{8}$ ), to form neutral complexes containing a ligand-stabilised metal cation, and a polysulfide anion. Cases were demonstrated where the polysulfide anion directly ligated the metal ion (complexes of the type $\left[\mathrm{MS}_{\mathrm{y}} \mathrm{L}_{\mathrm{x}}\right] ;^{13}$ and where the polysulfide anion acted solely as a counterion located outside the coordination sphere of the metal ion (complexes of the type $\left.\left[\mathrm{M}(\mathrm{L})_{x}\right] \mathrm{S}_{\mathrm{y}}\right) .{ }^{15}$ To this day these reports remain one of two reported preparations of metal polysulfide complexes directly from elemental metal. ${ }^{19}$

$\mathrm{N}$-methylimidazole ( $\mathrm{N}$-MeIm) proved to be a particularly good stabilising ligand, due to its basicity ( $\sim 10$ times that of pyridine) and compact size. ${ }^{18}$ It was found to form octahedrally coordinated complexes with manganese, iron, nickel, and magnesium $\left(\left[\mathrm{M}(\mathrm{N}-\mathrm{MeIm})_{6}\right] \mathrm{S}_{8}, \mathrm{M}=\mathrm{Mn}, \mathrm{Fe}, \mathrm{Ni}, \mathrm{Mg}\right){ }^{15}$ as well as complexes with copper $\left(\mathrm{Cu}_{4}\left(\mathrm{~S}_{5}\right)_{2}(N-\mathrm{MeIm})_{4}\right),{ }^{14}$ and zinc $\left(\mathrm{ZnS}_{6}(N-\mathrm{MeIm})_{2}\right) .{ }^{13}$

Furthermore, reaction of a variety of thiophilic metals ( $\mathrm{Ag}, \mathrm{Sb}, \mathrm{Sn}, \mathrm{In}, \mathrm{Au}, \mathrm{Cd}$ and $\mathrm{Hg}$ ) with solutions of sulfur in $N$-MeIm was assisted by addition of magnesium metal; in which case the resulting product was a complex of the relevant metal polysulfide anion and the $\left[\mathrm{Mg}(N-\mathrm{MeIm})_{6}\right]^{2+}$ counterion (i.e. $\left[\mathrm{Mg}(N-\mathrm{MeIm})_{6}\right]\left[\mathrm{M}_{\mathrm{x}} \mathrm{S}_{\mathrm{y}}\right], \mathrm{M}=\mathrm{Ag}, \mathrm{Sb}, \mathrm{Sn}, \mathrm{In}$, $\mathrm{Au}, \mathrm{Cd}, \mathrm{Hg}){ }^{16}$

It was demonstrated that several of these complexes could be either thermally or chemically decomposed to the binary metal monosulfide; and that in several cases it was possible to regenerate the polysulfide complexes from freshly precipitated metal monosulfide by application of a solution of sulfur in neat $\mathrm{N}$-MeIm. ${ }^{13}$

\subsubsection{Motivation}

Given that it has been demonstrated by Rauchfuss et al. that several metal polysulfide compounds can be partially desulfidised to the metal monosulfide, such compounds appeared to us to be attractive candidates for use as single-source precursors for metal sulfide nanocrystals. As mentioned above, most single-source precursors used to date in the synthesis of metal chalcogenide nanocrystals have involved organic molecules with thio or seleno moieties, rather than an inorganic sulfido moiety as the chalcogenide source. The use of polysulfide complexes appeared to offer the benefit, over metal thio-organic complexes, of being produced from metal powder, elemental sulfur and a suitable stabilising ligand in a single synthetic step. To reiterate, this approach appeared to offer a combination of flexibility, synthetic simplicity and cost-effectiveness. 
$\mathrm{N}$-methylimidazolato metal polysulfide complexes appeared to be the obvious candidates, as it was demonstrated that they could be synthesised simply in moderate to good yield from reaction of metal powder and a solution of elemental sulfur in neat $N$-MeIm. ${ }^{13-15}$ In many cases reaction was very slow at room temperature, but could be completed in under two days at moderate reaction temperatures $\left(50-90^{\circ} \mathrm{C}\right) .^{15}$

Hence, the aim of this project was to synthesise a range of $N$-methylimidazolato metal polysulfide complexes, and investigate their use as single-source precursors for metal sulfide nanocrystals via thermolysis in a coordinating solvent.

\subsubsection{Iron sulfide nanocrystals $\left(\mathrm{Fe}_{1-x} \mathrm{~S}\right.$ and $\left.\mathrm{Fe}_{3} \mathrm{~S}_{4}\right)$}

Please refer to Chapter 4 section 4.1.3 for description of literature syntheses of $\mathrm{Fe}_{1-x} \mathrm{~S}$ and $\mathrm{Fe}_{3} \mathrm{~S}_{4}$ nanocrystals.

\subsubsection{Zinc sulfide nanocrystals (ZnS)}

Spherical $\mathrm{ZnS}$ nanocrystals, ${ }^{20} \mathrm{ZnS}$ nanorods, ${ }^{21,22}$ and $\mathrm{ZnS}$ nanowires have been synthesised. An early synthesis of $\mathrm{ZnS}$-capped $\mathrm{CdSe}$ nanocrystals used $\mathrm{Na}_{2} \mathrm{~S}$ and $\mathrm{Zn}\left(\mathrm{ClO}_{4}\right)_{2}$ in an aqueous inverse micelle medium; ${ }^{23}$ however later syntheses came to focus on the use of highly reactive organometallic precursors, such as dialkylzinc compounds and bis(alkylsilyl)sulfides in non-aqueous high boiling point solvents (TOPO, TOP, HDA). ${ }^{24-26}$ Subsequently, there has been a move towards the use of 'greener' reagents, that are less toxic and more stable than the aforementioned organometallic and silyl reagents; and spherical $\mathrm{ZnS}$ nanocrystals ${ }^{20}$ and $\mathrm{ZnS}$ nanorods ${ }^{22}$ have been synthesised using zinc carboxylate salts and elemental sulfur.

$\mathrm{ZnS}$ nanocrystals have also been synthesised from single-source precursors, such as zinc dithiocarbamates ${ }^{27}$ or zinc ethylxanthates. ${ }^{28}$ Both approaches reportedly resulted in nanocrystals of high quality.

\subsubsection{Nickel sulfide nanocrystals $\left(\mathrm{NiS}_{2}\right.$ and $\left.\alpha-\mathrm{Ni}_{1-x} \mathrm{~S}\right)$}

$\alpha-\mathrm{Ni}_{1-x} \mathrm{~S}$ nanocrystals have been synthesised from the high temperature reaction of $\mathrm{Ni}(\mathrm{acac})_{2}$ and sulfur in oleylamine, ${ }^{29}$ solvothermal reaction of $\mathrm{NiCl}_{2}$ and $\left(\mathrm{NH}_{4}\right)_{2} \mathrm{~S}$ in water/ethylenediamine; ${ }^{30}$ as well as the decomposition of the single-source precursors 
$\mathrm{Ni}(\mathrm{Mer})_{2}(\mathrm{Mer}=2$-mercaptobenzothiazole $)$ in oleylamine,${ }^{31}$ and

$(\mathrm{TMEDA}) \mathrm{Ni}\left(\mathrm{SC}\{\mathrm{O}\} \mathrm{C}_{6} \mathrm{H}_{5}\right)_{2}(\mathrm{TMEDA}=$ tetramethylethylenediamine) in oleic

acid/dodecanethiol mixtures. ${ }^{32}$ In contrast to the former two methods, which produced

ill-defined nanocrystalline material, the single-source precursor approaches were successful in producing dispersed nanocrystals.

$\mathrm{NiS}_{2}$ nanocrystals have been synthesised by the reaction of $\mathrm{NiSO}_{4}$ and $\mathrm{Na}_{2} \mathrm{~S}_{3}$ in ethylene diamine under solvothermal conditions, ${ }^{33}$ but to the best of our knowledge has not previously been synthesised from a single-source precursor. 


\subsection{Results}

\subsubsection{Iron sulfide}

$\left[\mathrm{Fe}(\mathrm{N} \text {-MeIm })_{6}\right] \mathrm{S}_{8}$ was synthesised in an $80 \%$ yield by the method published by Rauchfuss et al. ${ }^{15}$, as described in section 6.5.1.1. $\left[\mathrm{Fe}(N-\mathrm{MeIm})_{6}\right] \mathrm{S}_{8}$ was isolated as purple crystals and a fine purple powder. The results of elemental analysis, performed on the powder, matched well with the proposed molecular formula: $\mathrm{C}_{24} \mathrm{H}_{36} \mathrm{~N}_{12} \mathrm{~S}_{8} \mathrm{Fe}$.

\section{Solid state decomposition of $\left[\mathrm{Fe}(\mathrm{N}-\mathrm{MeIm})_{6}\right] \mathrm{S}_{8}$}

A thermogravimetric trace for the complex is shown below, in Figure 6-1, and in Table 6-1 are tabulated a number of mass losses with theoretical residual masses. These correlate well with the losses observed in the thermogravimetric trace, suggesting stepwise losses of all six $N$-MeIm ligands, either singly or pairwise, between 75 and $250^{\circ} \mathrm{C}$. It appears that at least three sulfur atoms are lost simultaneously with the $\mathrm{N}$-MeIm ligands. There appears to be some further loss of sulfur above $250^{\circ} \mathrm{C}$, though superimposed upon this is an artefact due to the Curie point of the nickel TGA pan. The total mass loss at $500^{\circ} \mathrm{C}$ is appropriate for the binary sulfide $\mathrm{FeS}_{2}(15 \%$ residual mass), whereas by $600^{\circ} \mathrm{C}$ the total mass loss matches FeS (11\% residual mass) or $\mathrm{Fe}_{2} \mathrm{O}_{3}(10 \%$ residual mass).

The final product of a TGA run to $600^{\circ} \mathrm{C}$ was a bright red powder (cf. purple $\left[\mathrm{Fe}\left(\mathrm{N}\right.\right.$-MeIm) $\left.\left.{ }_{6}\right] \mathrm{S}_{8}\right)$, which was confirmed as $\alpha-\mathrm{Fe}_{2} \mathrm{O}_{3}$ upon XRD analysis (XRD pattern not shown). When the TGA run was terminated below $300^{\circ} \mathrm{C}$ the product was a black powder, which did not diffract sufficiently to be identified by XRD. 


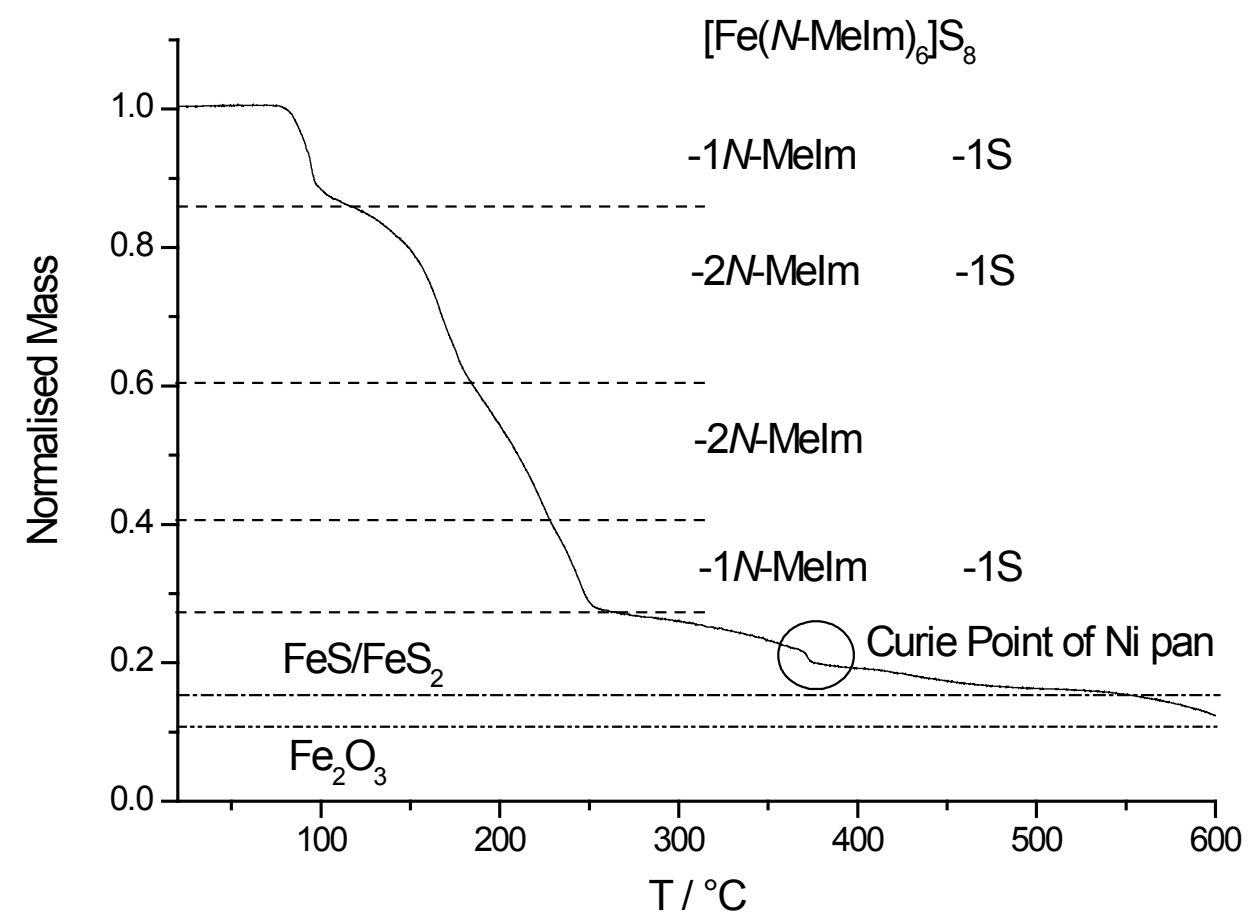

Figure 6-1 Thermogravimetric analysis trace for $\left[\mathrm{Fe}(N-\mathrm{MeIm})_{6}\right] \mathrm{S}_{8}$ under nitrogen flow, $5^{\circ}$ per minute heating rate.

Table 6-1 Theoretical residual masses for solid state decomposition of $\left[\mathrm{Fe}(N-\mathrm{MeIm})_{6}\right] \mathrm{S}_{8}$.

\begin{tabular}{|c|c|c|c|}
\hline & \multicolumn{2}{|c|}{ Cumulative Mass Losses } & $\begin{array}{c}\text { Theoretical residual } \\
\text { normalised mass }\end{array}$ \\
\hline$\left[\mathrm{Fe}(N-\mathrm{MeIm})_{6}\right] \mathrm{S}_{8}$ & $-1 N$-MeIm & $-1 \mathrm{~S}$ & 0.86 \\
\hline & $-3 N$-MeIm & $-2 \mathrm{~S}$ & 0.61 \\
\hline & $-5 N-\mathrm{MeIm}$ & $-2 \mathrm{~S}$ & 0.41 \\
\hline & $-6 N-\mathrm{MeIm}$ & $-3 \mathrm{~S}$ & 0.27 \\
\hline & & & 15 \\
\hline $\mathrm{FeS}_{2}$ & & 10 \\
\hline $\mathrm{Fe}_{2} \mathrm{O}_{3}$ & & & \\
\hline
\end{tabular}




\section{Rapid solution-phase decomposition of $\left[\mathrm{Fe}(\mathrm{N}-\mathrm{MeIm})_{6}\right] \mathrm{S}_{8}$}

$\left[\mathrm{Fe}(\mathrm{N} \text {-MeIm })_{6}\right] \mathrm{S}_{8}$ was dissolved in oleylamine and decomposed by injection into refluxing oleylamine, as described in section 6.5.1.2. A TEM micrograph of the product of this decomposition reaction is shown below in Figure 6-2 a), and an indexed SAED pattern is shown in Figure 6-2 b). An XRD pattern of the sample is shown in Figure 6-3 b).

The sample consisted of nanocrystals with a variety of faceted habits. The average crystallite size determined by Scherrer broadening analysis was $16 \pm 1 \mathrm{~nm}$ (see A2-9 for calculations), which correlated well with TEM observations. SAED and XRD both determined the sample to be pure $\mathrm{Fe}_{3} \mathrm{~S}_{4}$, with no indication of $\mathrm{Fe}_{1-x} \mathrm{~S}$ or elemental sulfur. The composition as determined by EDS was Fe:S 47:53, which is somewhat more iron rich than the composition of Fe:S 43:57 expected for stoichiometric $\mathrm{Fe}_{3} \mathrm{~S}_{4}$, however non-stochiometry has been observed previously in $\mathrm{Fe}_{3} \mathrm{~S}_{4}$ samples. ${ }^{34}$

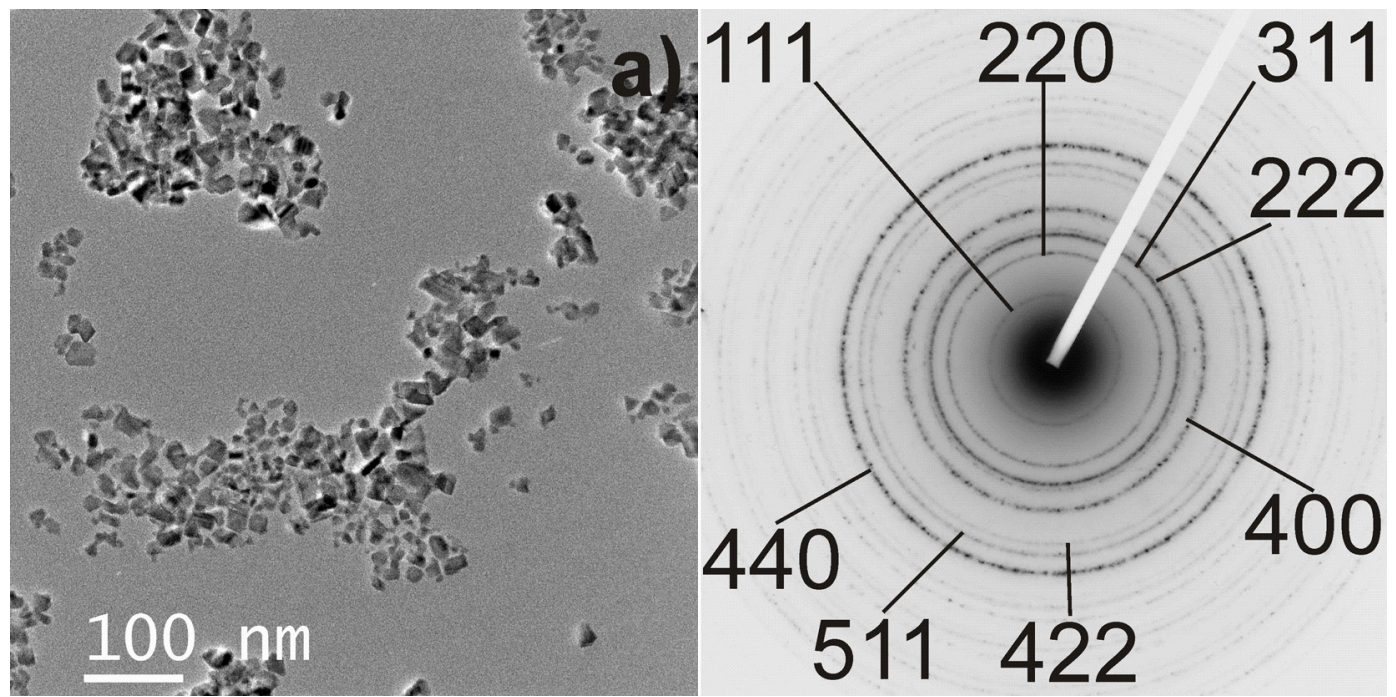

Figure 6-2 a) micrograph of iron sulfide nanocrystals resulting from rapid decomposition of $\left[\mathrm{Fe}(\mathrm{N} \text {-methylimidazole })_{6}\right] \mathrm{S}_{8}$ in oleylamine b) SAED from a) sample, indexed to $\mathrm{Fe}_{3} \mathrm{~S}_{4}$. 


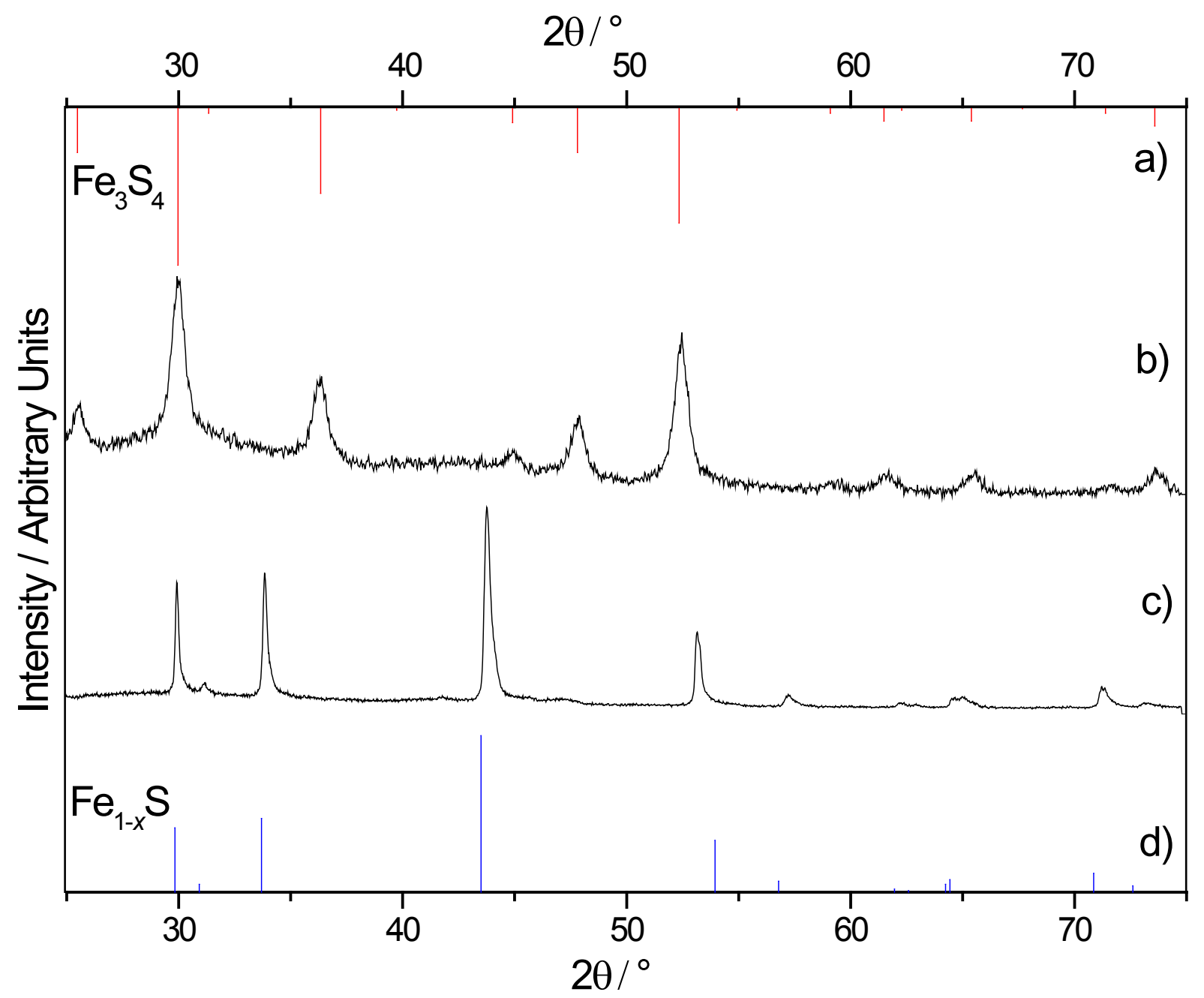

Figure 6-3 XRD pattern of a) product of rapid decomposition of $\left[\mathrm{Fe}(N-\mathrm{MeIm})_{6}\right] \mathrm{S}_{8}$ c) product of gradual decomposition of $\left[\mathrm{Fe}(N-\mathrm{MeIm})_{6}\right] \mathrm{S}_{8}$ d) reference for $\mathrm{Fe}_{1-x} \mathrm{~S}$. 


\section{Gradual solution-phase decomposition of $\left[\mathrm{Fe}(\mathrm{N}-\mathrm{MeIm})_{6}\right] \mathrm{S}_{8}$}

$\left[\mathrm{Fe}(\mathrm{N} \text {-MeIm })_{6}\right] \mathrm{S}_{8}$ was decomposed by heating to $300^{\circ} \mathrm{C}$ in oleylamine for 4 hours, as described in section 6.5.1.2. A TEM micrograph of the product of this decomposition reaction is shown below in Figure 6-4 a), with an EDS spectrum from the imaged areas shown in Figure 6-4 b). An XRD pattern of the sample is shown above in Figure 6-3 c).

The sample consisted of crystallites with lengths ranging from $10 \mathrm{~s}$ to $100 \mathrm{~s}$ of nanometres, and a variety of highly-faceted polyhedral crystal habits were observed, including truncated triangles, and rectangles. These were of the same type as observed for the $\mathrm{Fe}_{1-x} \mathrm{~S}$ nanocrystals synthesised in Chapter 4. EDS measurements determined the composition of the sample to be Fe:S 1:1. The XRD pattern (see Figure 6-3) matched $\mathrm{Fe}_{1-x} \mathrm{~S}$, with no indication of the presence of $\mathrm{Fe}_{3} \mathrm{~S}_{4}$ or elemental sulfur. Scherrer X-ray broadening analysis was performed (see A2-10 for calculations), and placed the average crystallite size at $73 \pm 51 \mathrm{~nm}$.

Reaction of $\left[\mathrm{Fe}(N\right.$-MeIm) $] \mathrm{S}_{8}$ at $300^{\circ} \mathrm{C}$ for durations less than 4 hours led to mixtures of $\mathrm{Fe}_{3} \mathrm{~S}_{4}$ and $\mathrm{Fe}_{1-x} \mathrm{~S}$, indicating that during the course of the reaction the initially nucleated $\mathrm{Fe}_{3} \mathrm{~S}_{4}$ phase was converted to the thermally more stable $\mathrm{Fe}_{1-x} \mathrm{~S}$ phase. ${ }^{35,36}$
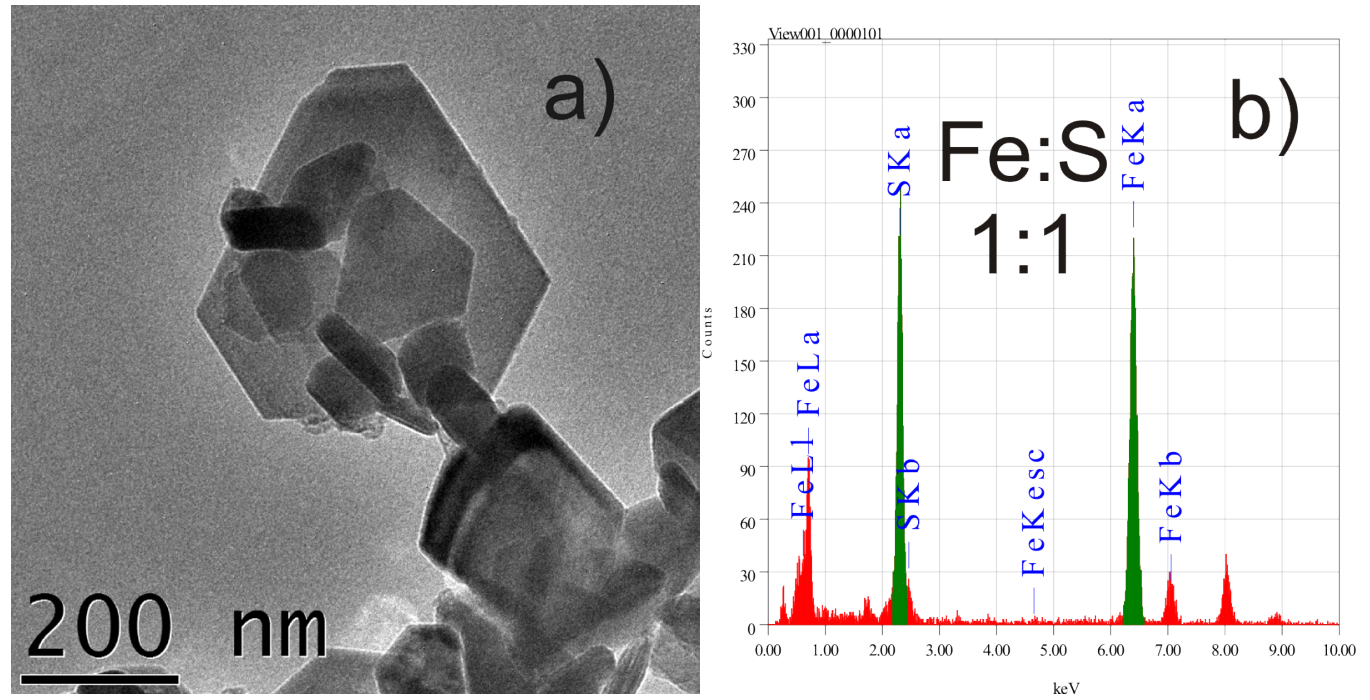

Figure 6-4 a) Micrograph of $\mathrm{Fe}_{1-x} \mathrm{~S}$ nanocrystals resulting from the gradual decomposition of $\left[\mathrm{Fe}(N-\mathrm{MeIm})_{6}\right] \mathrm{S}_{8}$ in oleylamine b) EDS spectrum from (a) 


\subsubsection{Zinc sulfide}

$\mathrm{ZnS}_{6}(N-\mathrm{MeIm})_{2}$ was synthesised in $62 \%$ yield by the method published by Rauchfuss et al., ${ }^{13}$ as described in section 6.5.2.1. The elemental analysis results for the course yellow-green powder matched well with the expected molecular formula: $\mathrm{C}_{8} \mathrm{H}_{12} \mathrm{~N}_{4} \mathrm{~S}_{6} \mathrm{Zn}$.

\section{Solid state decomposition of $\mathrm{ZnS}_{6}(\mathrm{~N}-\mathrm{MeIm})_{2}$}

A thermogravimetric trace for the complex is shown below in Figure 6-5 and theoretical residual masses are tabulated in Table 6-2. There appear between 120 and $210^{\circ} \mathrm{C}$ the stepwise losses of first 0.5 , then one sulfur atom (per formula unit). This is followed by the loss of one $N$-MeIm ligand between 215 and $225^{\circ} \mathrm{C}$, and the loss of the one remaining $N$-MeIm ligand and 1.5 further sulfur atoms between 225 and $290^{\circ} \mathrm{C}$. There appears to be a gradual further loss of sulfur above $290^{\circ} \mathrm{C}$ until a residual mass appropriate for $\mathrm{ZnS}$ is reached at $600^{\circ} \mathrm{C}$. The final product of the TGA run was a white powder (cf. green-yellow $\left.\mathrm{ZnS}_{6}(N \text {-MeIm) })_{2}\right)$, which was not analysed by XRD.

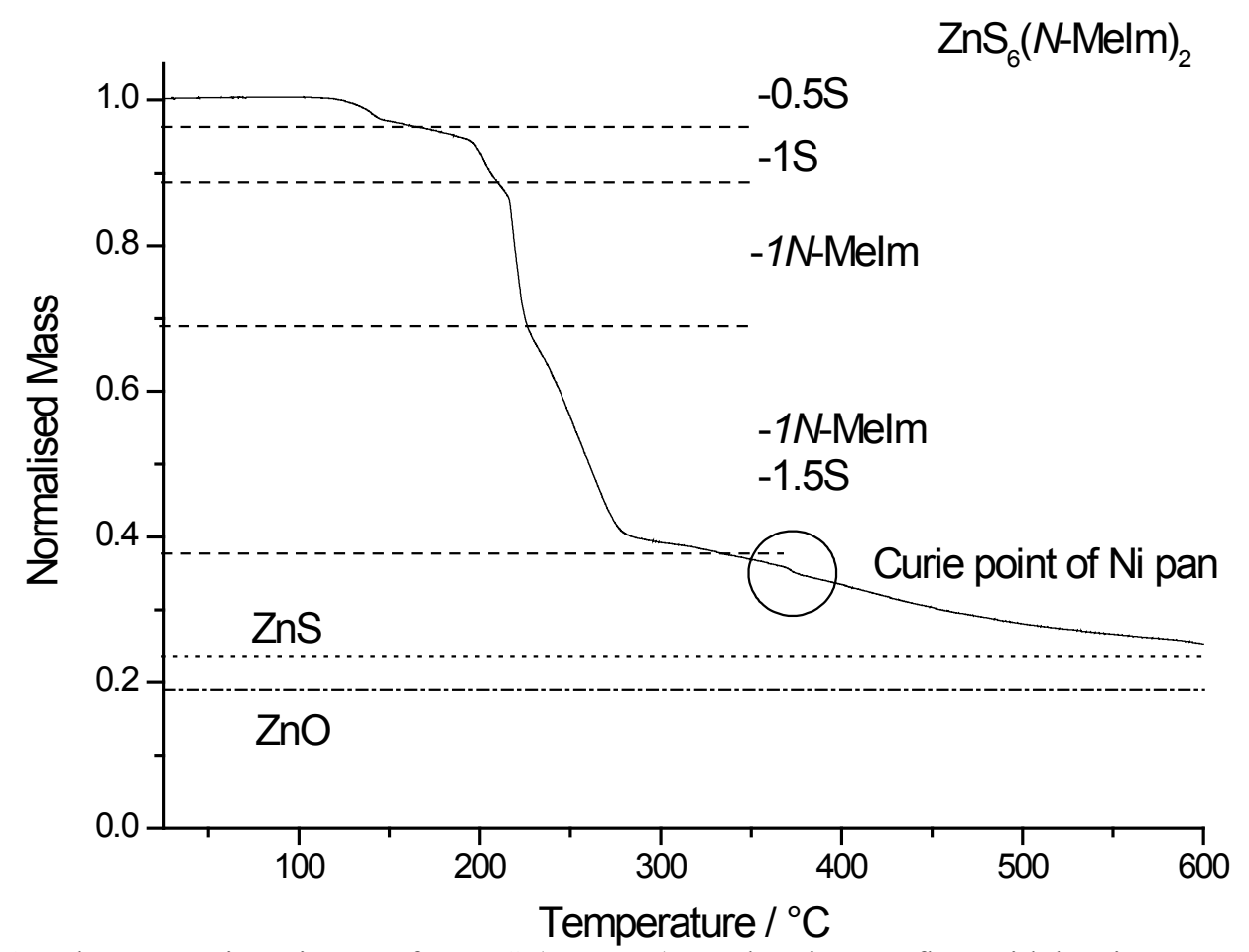

Figure 6-5 Thermogravimetric trace for $\mathrm{ZnS}_{6}(\mathrm{~N} \text {-MeIm })_{2}$, under nitrogen flow with heating rate of $5^{\circ}$ per minute. 
Table 6-2 Theoretical residual masses for solid state decomposition of $\mathrm{ZnS}_{6}(N-\mathrm{MeIm})_{2}$.

\begin{tabular}{|c|c|c|c|}
\hline & \multicolumn{2}{|c|}{ Cumulative Mass Losses } & $\begin{array}{c}\text { Theoretical residual } \\
\text { normalised mass }\end{array}$ \\
\hline $\mathrm{ZnS}_{6}(\mathrm{~N}-\mathrm{MeIm})_{2}$ & & $-0.5 \mathrm{~S}$ & 0.96 \\
\hline & & $-1.5 \mathrm{~S}$ & 0.89 \\
\hline & $-1 N-\mathrm{MeIm}$ & $-1.5 \mathrm{~S}$ & 0.69 \\
\hline & $-2 N-\mathrm{MeIm}$ & $-3 \mathrm{~S}$ & 0.38 \\
\hline $\mathrm{ZnS}$ & & & 0.23 \\
\hline $\mathrm{ZnO}$ & & & 0.19 \\
\hline
\end{tabular}




\section{Rapid solution-phase decomposition of $\mathrm{ZnS}_{6}(\mathrm{~N}-\mathrm{MeIm})_{2}$}

$\mathrm{ZnS}_{6}(N \text {-MeIm })_{2}$ was dissolved in oleylamine, and decomposed by injection into oleylamine at $300^{\circ} \mathrm{C}$, as described in section 6.5.2.2. A TEM micrograph of the product is shown below in Figure 6-6 a); an indexed SAED pattern is shown in Figure 6-6 b); and a densitometric trace of the SAED pattern is shown in Figure 6-6 c); and analysis of the pattern is tabulated in Table 6-3. An EDS spectrum of the product is shown in Figure 6-6 d).
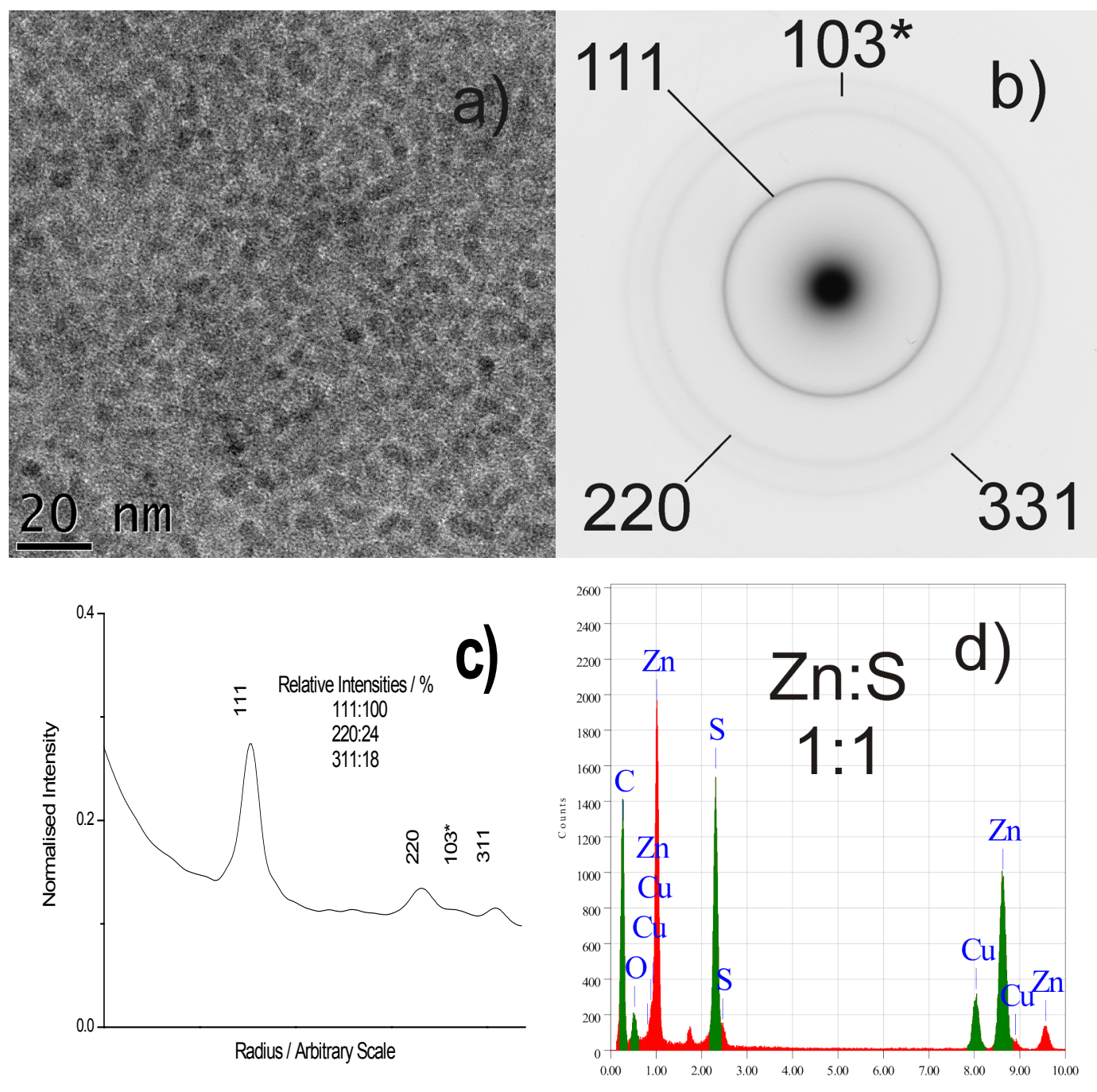

Figure 6-6 a) Micrograph of the product of rapid decomposition of $\mathrm{ZnS}_{6}(N-\mathrm{MeIm})_{2}$ in oleylamine b) SAED pattern of a), indexed to $\mathrm{ZnS}$ (zinc blende) - reflection marked with asterisk is indexed to $\mathrm{ZnS}$ (wurtzite) c)Densitometry trace of SAED pattern showing relative intensities of the reflections d) EDS spectrum of decomposition product. 
The product of the decomposition reaction was roughly spherical nanocrystals, with an average diameter of $5 \mathrm{~nm}$ (see Figure 6-7 for particle size determined from TEM measurements). EDS analysis returns a composition of $1: 1 \mathrm{Zn}: \mathrm{S}$, matching that of stoichiometric $\mathrm{ZnS}$.

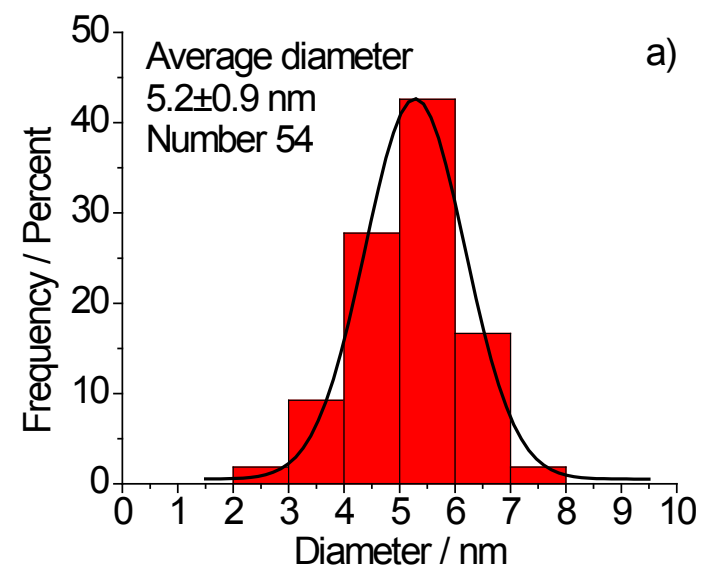

Figure 6-7 a) Histogram of diameter of $\mathrm{ZnS}$ nanocrytals

The SAED pattern showed characteristics of both the cubic zinc blende and hexagonal wurtzite polymorphs of $\mathrm{ZnS}$. The sample could be confirmed as crystalline $\mathrm{ZnS}$ by SAED, but the proportion of the two polymorphs in the sample could not be ascertained.

Table 6-3 Indexing of SAED patterns for the product of rapid decomposition of $\mathrm{ZnS}_{6}(N-\mathrm{MeIm})_{2}$ in oleylamine.

\begin{tabular}{|c|c|c|c|c|c|c|c|c|c|}
\hline & \multicolumn{3}{|c|}{ Observed } & \multicolumn{3}{c|}{$\begin{array}{c}\text { ZnS (zinc blende) } \\
\text { reference }\end{array}$} & \multicolumn{3}{c|}{$\begin{array}{c}\text { ZnS (wurtzite) } \\
\text { reference }\end{array}$} \\
\hline & $d / \AA$ & $\Delta d / \AA$ & $\mathrm{I} / \%$ & $h k l$ & $d / \AA$ & $\mathrm{I} / \%$ & $h k l$ & $d / \AA$ & $\mathrm{I} / \%$ \\
\hline $\mathrm{A}$ & 3.1 & \pm 0.2 & 100 & 111 & 3.12 & 100 & 002 & 3.13 & 61 \\
\hline $\mathrm{B}$ & $1.9_{3}$ & \pm 0.1 & 24 & 220 & 1.91 & 51 & 110 & 1.91 & 62 \\
\hline $\mathrm{C}$ & $1.8_{2}$ & \pm 0.1 & $/$ & $/$ & $/$ & $/$ & 103 & 1.76 & 59 \\
\hline $\mathrm{D}$ & $1.6_{9}$ & \pm 0.1 & 18 & 311 & 1.63 & 30 & 200 & 1.66 & 9 \\
\hline
\end{tabular}




\section{Gradual solution-phase decomposition of $\mathrm{ZnS}_{6}(\mathrm{~N}-\mathrm{MeIm})_{2}$}

$\mathrm{ZnS}_{6}(N \text {-MeIm })_{2}$ was decomposed by heating in oleylamine to $300^{\circ} \mathrm{C}$, as described in section 6.5.2.2. TEM micrographs of the decomposition product are shown below in Figure 6-8 (a) and (b); and an indexed SAED pattern is shown in Figure 6-8 (c). An EDS spectrum of the decomposition product is shown in Figure 6-8 (d).

The sample consisted of nanorods, with an average width of $5.1 \pm 1.1 \mathrm{~nm}$ and an average length of $14.6 \pm 2.7 \mathrm{~nm}$, based on TEM measurements (see Figure 6-9 for histograms of TEM measurements). The high resolution micrograph shown in Figure 6-8 (b) confirms that single-crystalline nature of the nanorod. Similarly to the SAED pattern of the spherical nanocrystals resulting from the rapid decomposition of $\mathrm{ZnS}_{6}(N-\mathrm{MeIm})_{2}$, the SAED pattern of an ensemble of nanorods shown in Figure 6-8 (c) contains contributions from both the zinc blende and wurtzite polymorphs of $\mathrm{ZnS}$. A densiometric trace (not shown) of the SAED pattern of the $\mathrm{ZnS}$ nanorods matches the densiometric trace shown for the SAED pattern of the spherical ZnS nanocrystals. EDS measurements on the sample give a composition of $\mathrm{Zn}: \mathrm{S} 1: 1$, appropriate for stoichiometric $\mathrm{ZnS}$.

An XRD pattern of the sample is shown in Figure 6-10, and is similar in appearance to the densiometric traces of the SAED patterns of both samples. In order to quantify the relative contributions of each of the two $\mathrm{ZnS}$ polymorphs to the sample, Rietveld refinement was performed on the XRD pattern, using the Rietica software package. The observed and calculated patterns, as well as a difference map, are shown below in Figure 6-10; and parameters relating to the refinement are tabulated in Figure 6-4. The Rietveld refinement $\mathrm{R}_{\mathrm{wp}}$ factor of 11.1, and indicated that the sample was composed primarily of the hexagonal wurtzite polymorph, with only trace amounts of the cubic zinc blende polymorph. There was no indication in the XRD pattern of additional phases. 

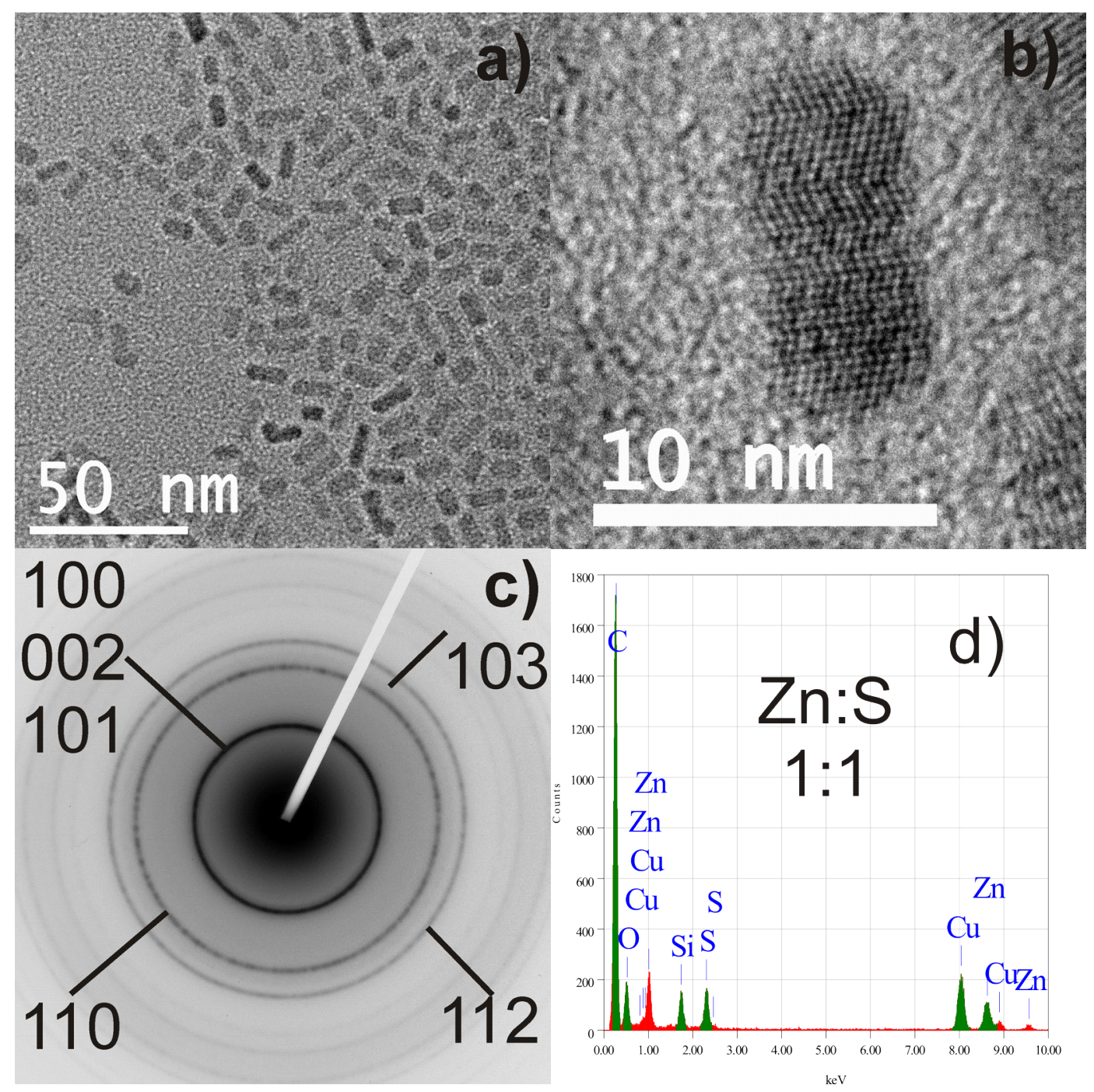

Figure 6-8 a) Micrograph of the product of gradual decomposition of $\mathrm{ZnS}_{6}(N-\mathrm{MeIm})_{2}$ in oleylamine b) High resolution micrograph of single $\mathrm{ZnS}$ nanorod. c) SAED pattern of a), indexed to ZnS d) EDS spectrum of decomposition product.

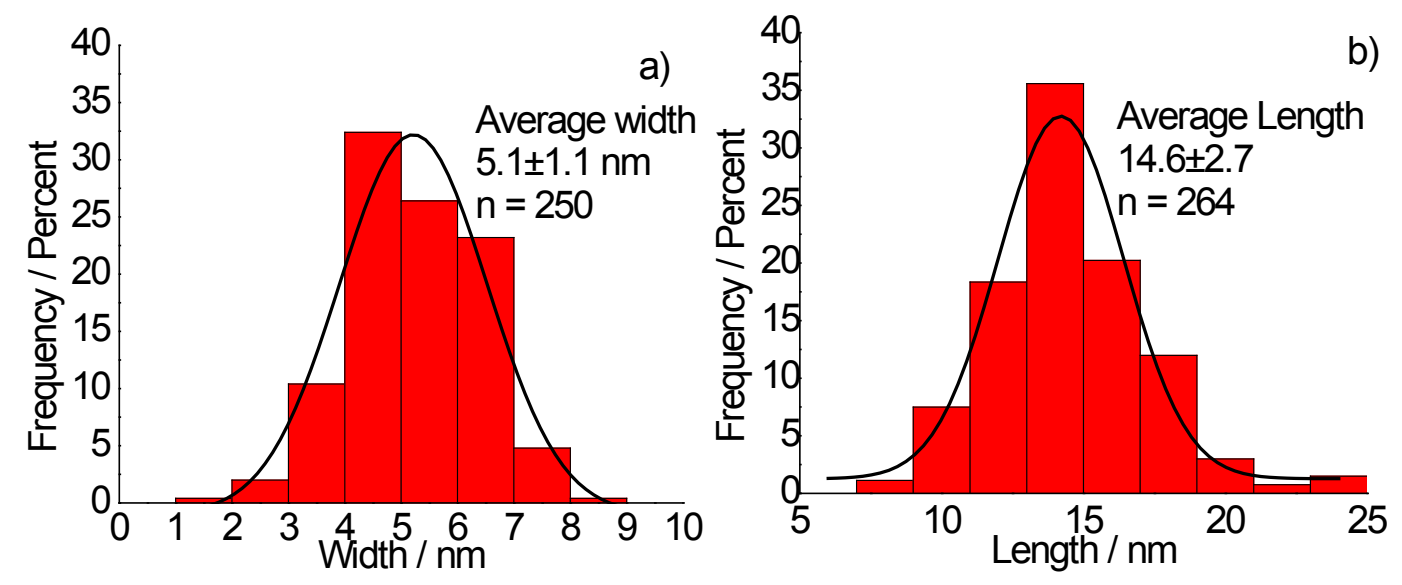

Figure 6-9 Histograms of a) width and b) length of $\mathrm{ZnS}$ nanorods 


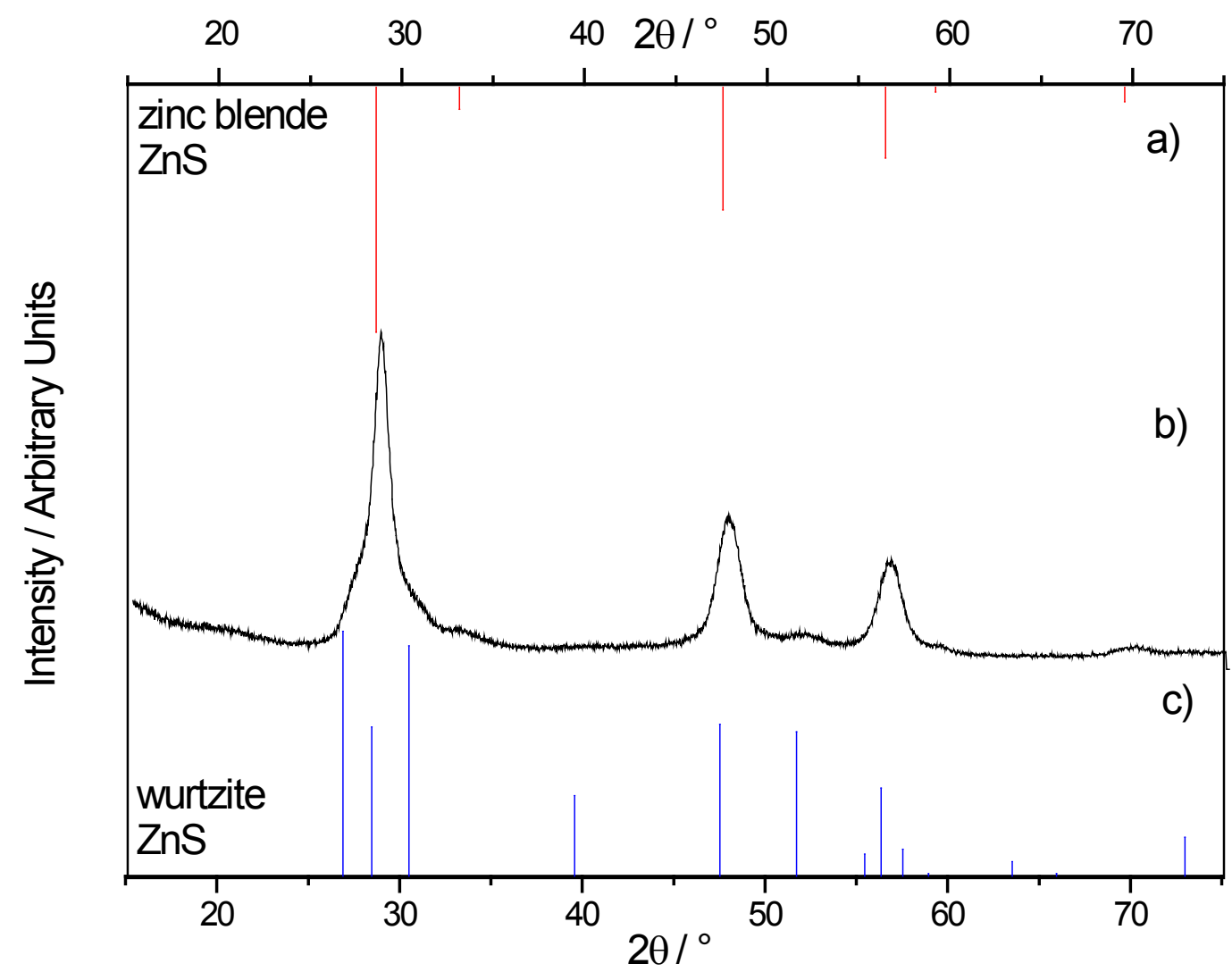

Figure 6-10 XRD patterns of a) reference for cubic zinc blende zinc sulfide b) product of gradual decomposition of $\mathrm{ZnS}_{6}(\mathrm{~N} \text {-MeIm })_{2} \mathrm{c}$ ) reference for hexagonal wurtzite zinc sulfide.

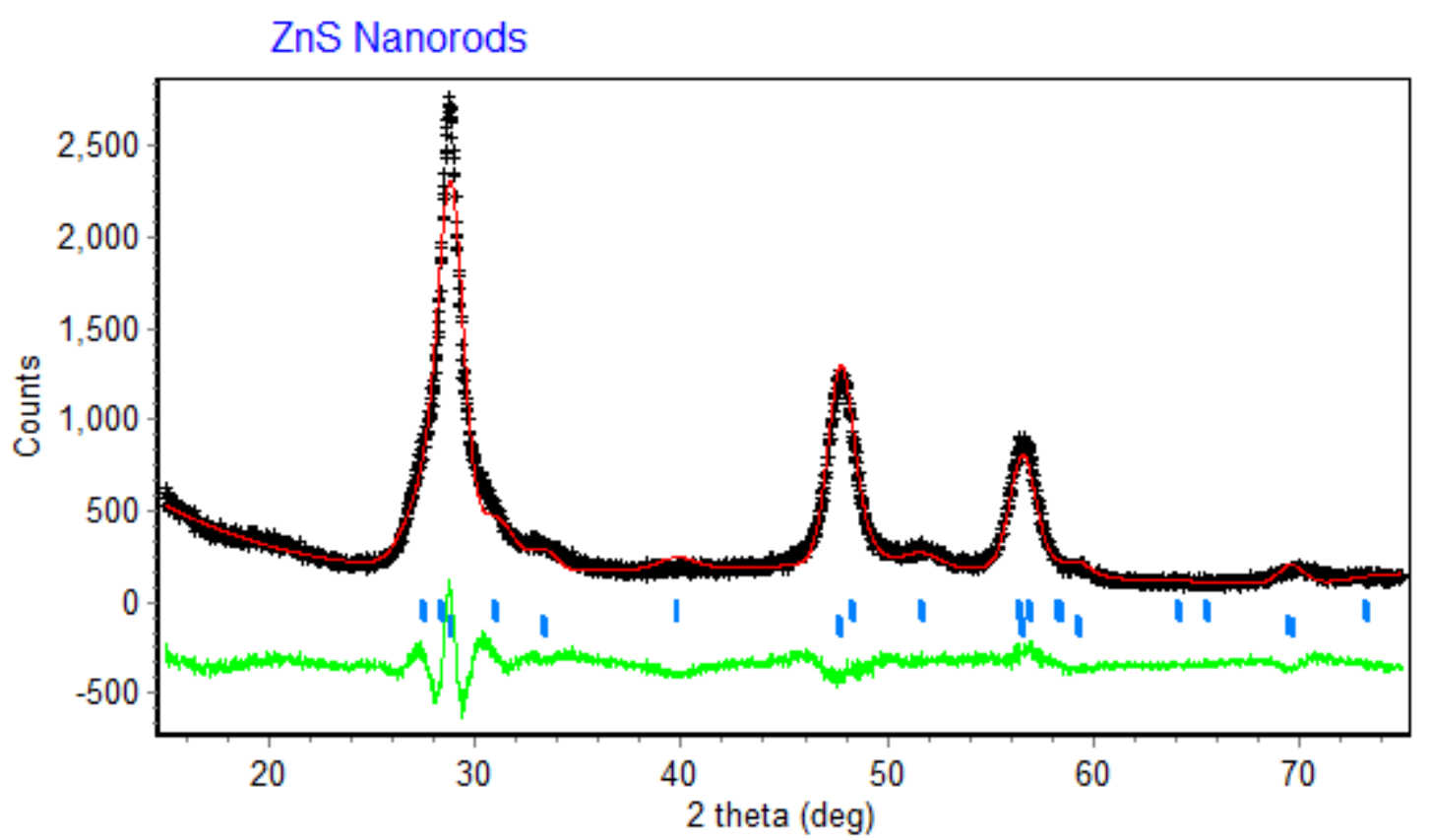

Figure 6-11 Observed (crosses), calculated (red) and difference (green) profiles for XRD pattern of ZnS nanorods using Rietveld refinement. 
Table 6-4 Rietveld refinement parameters for $\mathrm{ZnS}$ nanorods.

\begin{tabular}{|c|c|c|}
\hline & Zinc Blende & Wurtzite \\
\hline $2 \theta$ range $/^{\circ}$ & \multicolumn{2}{|c|}{$15-75$} \\
\hline Number of data points & \multicolumn{2}{|c|}{3000} \\
\hline Wavelength / $\AA$ & \multicolumn{2}{|c|}{1.5424} \\
\hline Refined parameters & \multicolumn{2}{|c|}{18} \\
\hline $\mathrm{R}_{\mathrm{p}}$ & \multicolumn{2}{|c|}{8.89} \\
\hline $\mathrm{R}_{\mathrm{wp}}$ & \multirow{2}{*}{\multicolumn{2}{|c|}{$\frac{11.1}{4.11}$}} \\
\hline$\chi^{2}$ & & \\
\hline Space Group & $F \overline{4} 3 m$ & $P 6_{3} m c$ \\
\hline Reflections & 6 & 13 \\
\hline$a / \AA$ & 5.426 & 3.788 \\
\hline$c / \AA$ & & 6.359 \\
\hline Volume / $\AA^{3}$ & 159.7 & 79.02 \\
\hline $\mathrm{Z}$ & 4 & 2 \\
\hline Abundance $/ \mathrm{wt} \%$ & 1.3 & 98.7 \\
\hline
\end{tabular}

$\mathrm{UV}$-vis and PL (photoluminescence) spectra of a sample of $\mathrm{ZnS}$ nanorods are shown in Figure 7. The UV-vis spectrum features a sharp absorption onset at $340 \mathrm{~nm}$ and an excitonic absorption band centred at $320 \mathrm{~nm}$, which is slightly blue-shifted from the band gap of bulk $\mathrm{ZnS}(330 \mathrm{~nm}){ }^{37}$ The PL spectrum for the $\mathrm{ZnS}$ nanorods (excited at $280 \mathrm{~nm}$ ) featured a sharp emission maximum at $325 \mathrm{~nm}$ and a broad emission peak centred around $400 \mathrm{~nm}$. PLE (Photoluminescence Excitation) spectra are shown in Figure 6-13. The PLE spectrum for an emission wavelength of $330 \mathrm{~nm}$ showed an excitation maximum at $310 \mathrm{~nm}$, which falls within the main UV-vis excitonic absorption band, whereas the PLE spectrum for an emission wavelength of $410 \mathrm{~nm}$ displayed a broad excitation band above $325 \mathrm{~nm}$. Hence, the sharp emission peak at $325 \mathrm{~nm}$ was assigned to band edge luminescence, while the lower energy peak at 400 $\mathrm{nm}$ was assigned to trap state emission. These optical features and assignments are in good agreement with previous studies on ZnS nanocrystals and nanorods. ${ }^{21,38}$ Given that trap state emission can be the dominant optical feature of $\mathrm{ZnS}$ containing sulfur vacancies, ${ }^{21}$ the relatively low ratio of trap state to band edge emission observed for samples can be taken as an indication of the high quality of the $\mathrm{ZnS}$ nanorods synthesized from $\mathrm{ZnS}_{6}(\mathrm{~N} \text {-MeIm })_{2}$. 


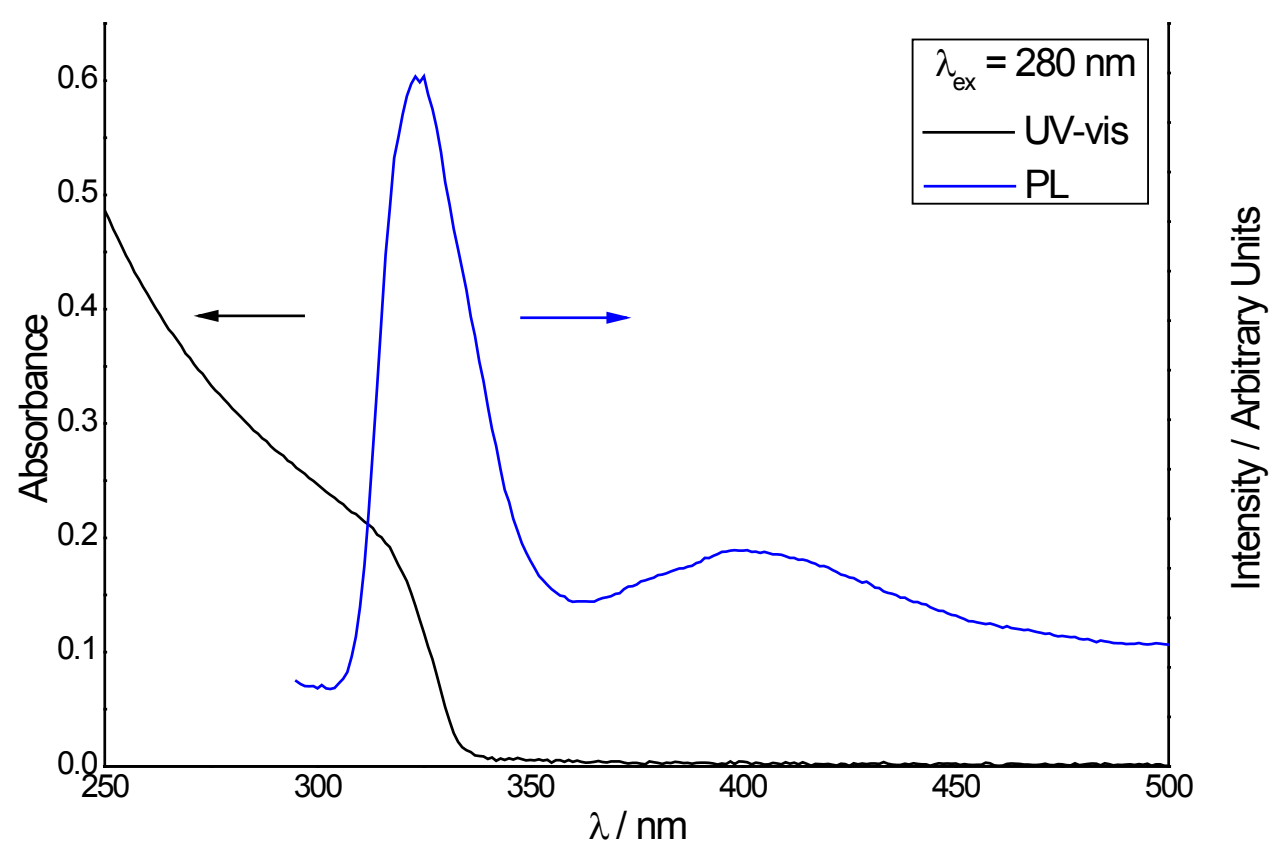

Figure 6-12 UV-vis absorption (black) and photoluminescence (PL) (blue) spectra of ZnS nanorods. Excitation wavelength $\left(\lambda_{e x}\right)$ of $280 \mathrm{~nm}$ for PL spectrum.

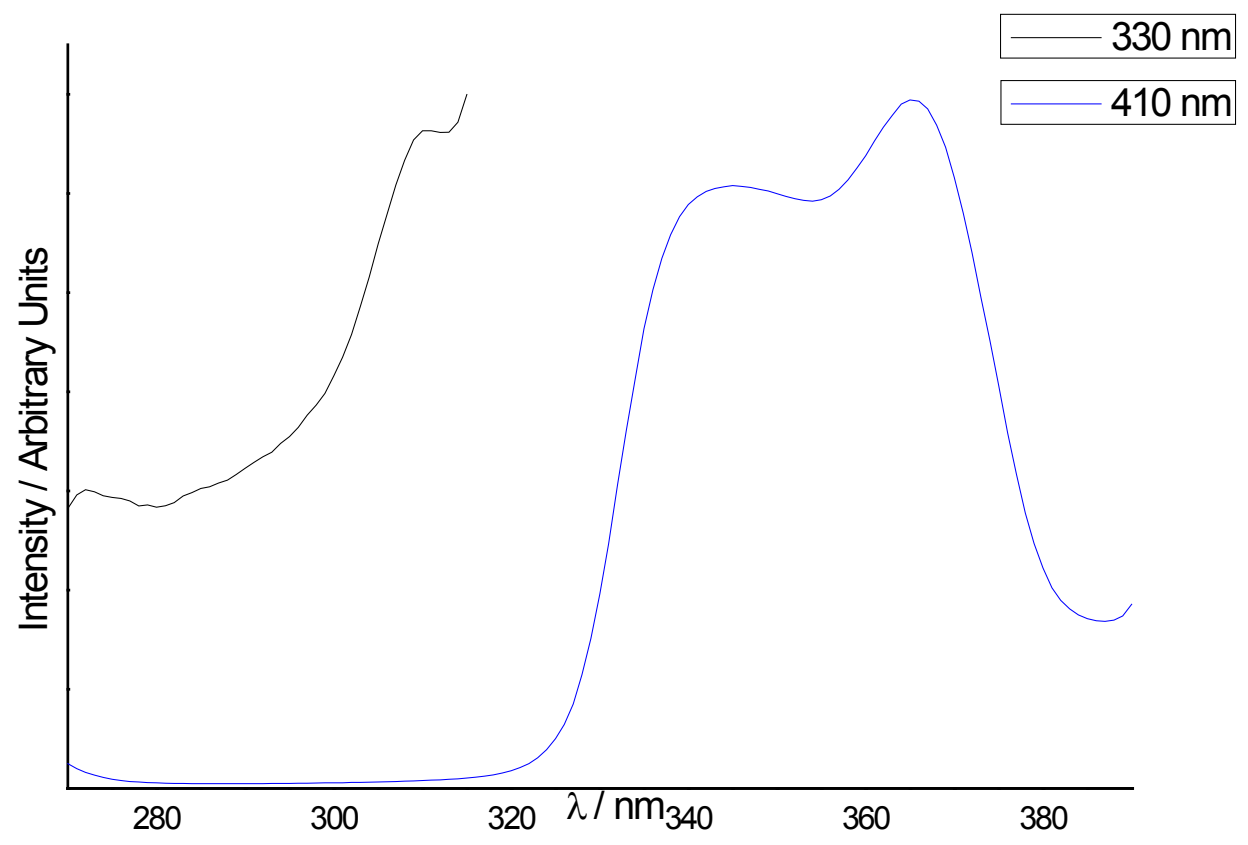

Figure 6-13 Photoluminescence excitation (PLE) spectrum of Zn S nanorods, for emission wavelengths of $330 \mathrm{~nm}$ (black) and $410 \mathrm{~nm}$ (blue). 


\subsubsection{Magnesium sulfide/Magnesium oxide}

$\left[\mathrm{Mg}(\mathrm{N}-\mathrm{MeIm})_{6}\right] \mathrm{S}_{8}$ was synthesised in $57 \%$ yield by the method published by Rauchfuss

et al., ${ }^{15}$ however because of the observed extreme hygroscopy of the complex, elemental analysis was not obtained to verify the proposed composition.

\section{Solid state decomposition of $\left[\mathrm{Mg}(\mathrm{N}-\mathrm{MeIm})_{6}\right] S_{8}$}

A thermogravimetric trace for the complex is shown below as Figure 6-14, and mass changes with their theoretical residual masses are tabulated in Table 6-5. There is a small, gradual mass loss from room temperature to $100^{\circ} \mathrm{C}$, which is presumed to be evaporation of the atmospheric moisture absorbed during the transfer of the complex to the TGA furnace. The mass at $100^{\circ} \mathrm{C}$ is assumed to be the dry weight of the complex, and all further mass losses are normalised to this value. There are two sequential mass losses, the first between 105 and $170^{\circ} \mathrm{C}$, and the second between 170 and $270^{\circ} \mathrm{C}$. These match the loss of three $\mathrm{N}$-MeIm ligands and four sulfur atoms; and the loss of a further three $N$-MeIm ligands, respectively. Between 270 and $350^{\circ} \mathrm{C}$ the mass plateaus, which is followed by an artefact due to the Curie point of the nickel TGA pan. The remaining region of the trace is marred by experimental noise, making any further loss of sulfur difficult to judge. The total mass loss to $900^{\circ} \mathrm{C}(13 \%$ residual mass $)$ is insufficient for the full desulfidisation of the proposed composition of the complex $\left(\left[\mathrm{Mg}(N-\mathrm{MeIm})_{6}\right] \mathrm{S}_{8}\right.$ $\rightarrow \mathrm{MgS}, 7 \%$ residual mass).

The final product of the TGA run a white powder, which was analysed by XRD (XRD pattern no shown), which confirmed the presence of $\mathrm{Mg}(\mathrm{OH})_{2}$ in addition to a second phase, which could not be identified. 


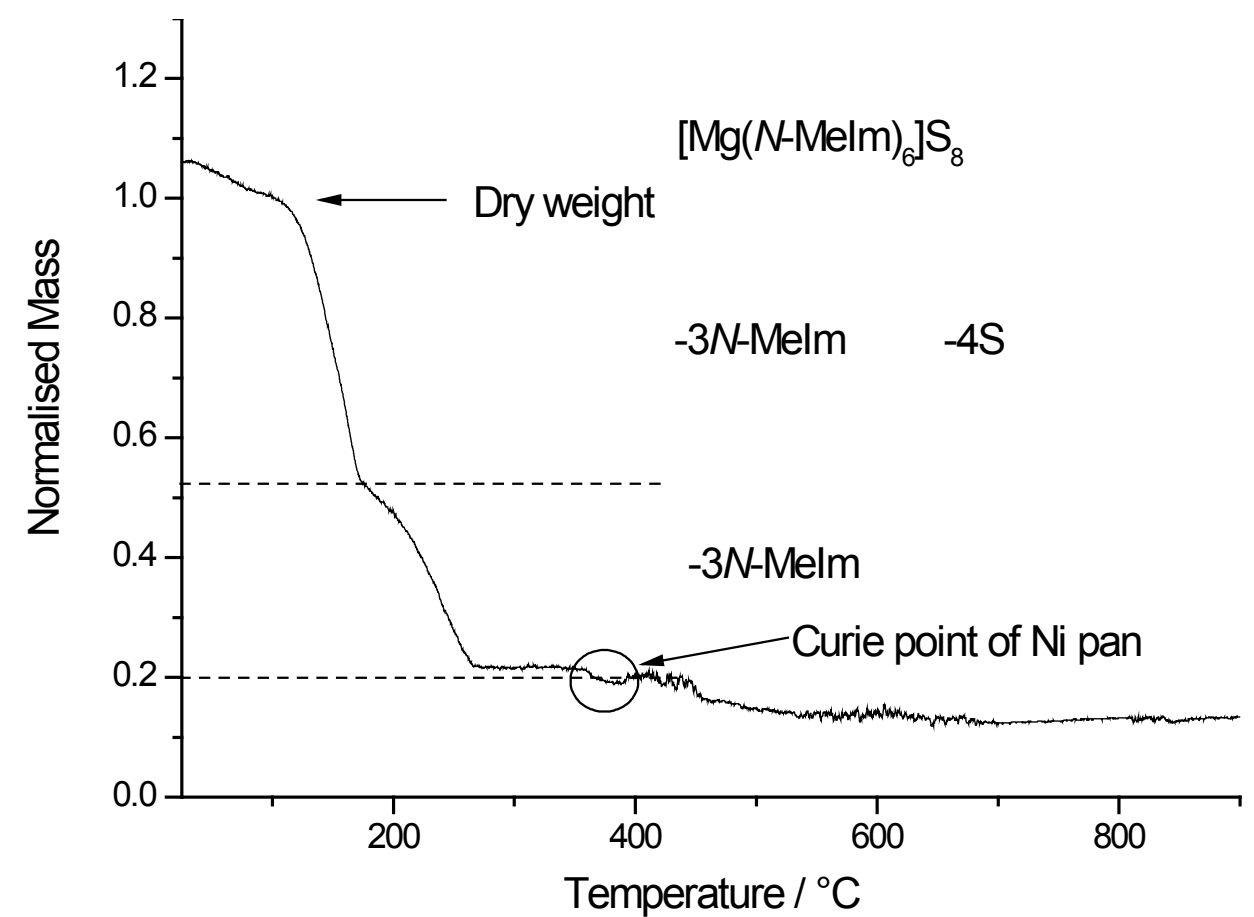

Figure 6-14 Thermogravimetric trace for decomposition of $\left[\mathrm{Mg}(N \text {-MeIm })_{6}\right] \mathrm{S}_{8}$ under nitrogen flow, with a heating rate of $5^{\circ} \mathrm{C}$ per minute.

Table 6-5 Theoretical residual masses for solid state decomposition of $\left[\mathrm{Mg}(N-\mathrm{MeIm})_{6}\right] \mathrm{S}_{8}$.

\begin{tabular}{|c|c|c|c|}
\hline & \multicolumn{2}{|c|}{ Cumulative Mass Losses } & $\begin{array}{c}\text { Theoretical residual } \\
\text { normalised mass }\end{array}$ \\
\hline$\left[\mathrm{Mg}(N-\mathrm{MeIm})_{6}\right] \mathrm{S}_{8}$ & $-3 N-\mathrm{MeIm}$ & $-4 \mathrm{~S}$ & 0.52 \\
\hline & $-6 N-\mathrm{MeIm}$ & $-4 \mathrm{~S}$ & 0.20 \\
\hline $\mathrm{MgS}$ & & & 0.07 \\
\hline $\mathrm{MgO}$ & & & 0.05 \\
\hline
\end{tabular}


$\left[\mathrm{Mg}(\mathrm{N} \text {-MeIm })_{6}\right] \mathrm{S}_{8}$ was decomposed at $310^{\circ} \mathrm{C}$ in oleylamine, as described in section 6.5.3.2. A TEM micrograph of the decomposition product is shown below as Figure 6-15 a); an EDS spectrum from the sample is shown in Figure 6-15 b); and an SAED pattern from the sample is shown in Figure 6-15 c).
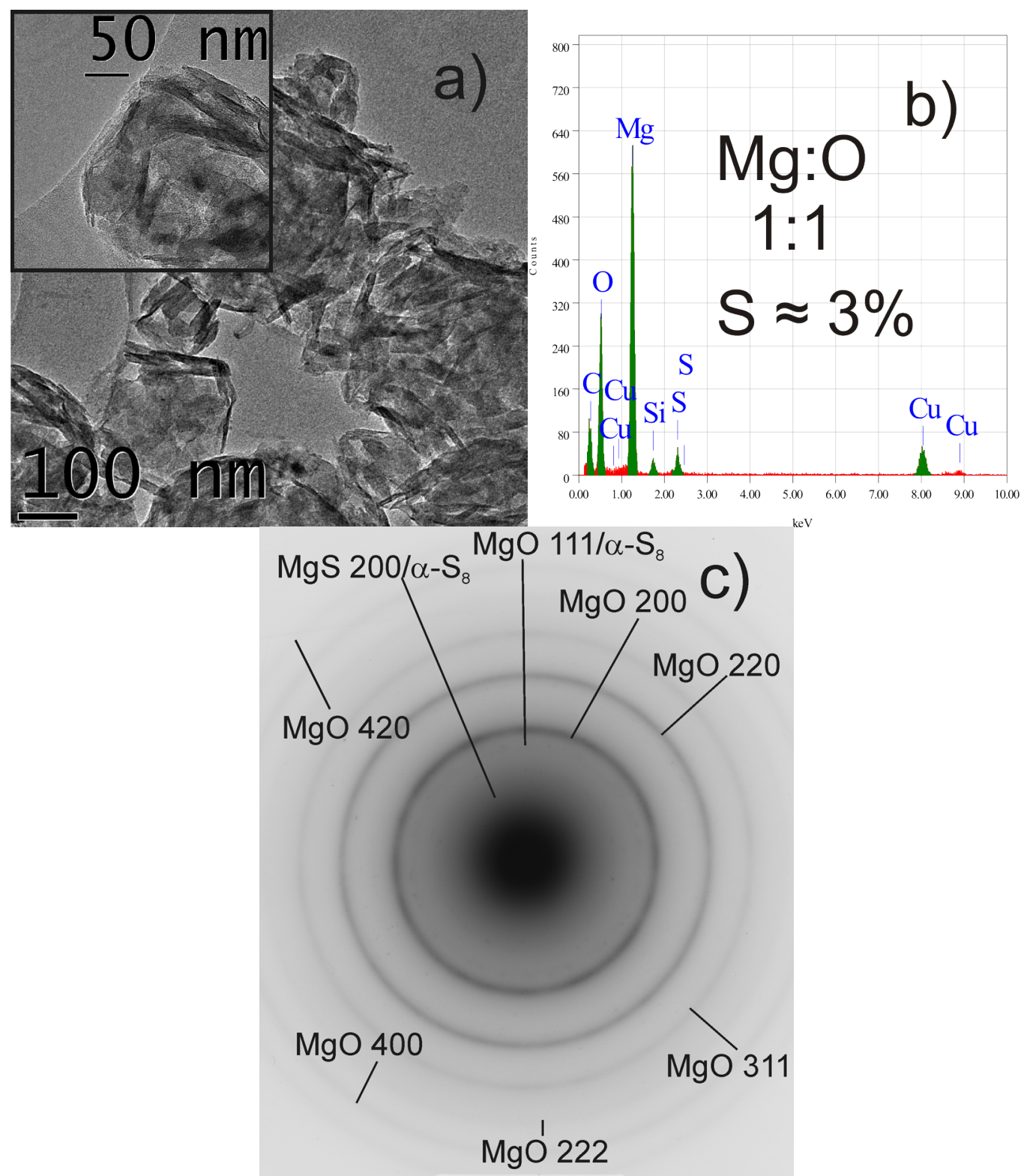

Figure 6-15 a) Micrograph of the product of decomposition of $\left[\mathrm{Mg}(N-\mathrm{MeIm})_{6}\right] \mathrm{S}_{8}$ in oleylamine c) EDS spectrum of decomposition product c) SAED pattern of decomposition product, indexed to $\mathrm{MgO}$ with additional weak reflections from $\mathrm{MgS}$ or $\alpha-\mathrm{S}_{8}$.

The decomposition product appeared as large aggregates of platy crystallites. The inset in Figure 6-14 a) shows a high magnification image of a portion of an aggregate - there does not appear to be any systematic orientation of the plates, and no lattice fringes are visible. 
EDS analysis of the decomposition product gives Mg:O 1:1, with only a trace of sulfur present (approximately 3\% by abundance). Analysis of the SAED pattern (A1-16)

determines the major phase to be $\mathrm{MgO}(\mathrm{NaCl}$ structure: $F m \overline{3} m$ ), rather than $\mathrm{MgS}$. There is an additional faint ring (corresponding to a $d$-spacing of $2.6 \AA$ ), which may correspond to either the $100 \%$ intensity 200 reflection of $\operatorname{MgS}(2.60 \AA)$, or $\alpha$-sulfur. 


\subsubsection{Nickel sulfide}

$\left[\mathrm{Ni}(\mathrm{N} \text {-MeIm })_{6}\right] \mathrm{S}_{8}$ was synthesised by the method of Rauchfuss et al. ${ }^{15}$ with a $9 \%$ isolated yield of relatively pure product, in addition to a further $\sim 70 \%$ yield contaminated by starting material, as described in section 6.5.4.1. Elemental analysis by Rauchfuss et al. established the molecular formula of the complex as $\left[\mathrm{Ni}(\mathrm{N}-\mathrm{MeIm})_{6}\right] \mathrm{S}_{8}$ $\left(\mathrm{C}_{24} \mathrm{H}_{36} \mathrm{~N}_{6} \mathrm{~S}_{8} \mathrm{Ni}\right)$, which was supported by crystal structure determination. Elemental analysis performed on the purer of the samples synthesised in this project, deviated from the values calculated on the basis of pure $\left[\mathrm{Ni}(\mathrm{N}-\mathrm{MeIm})_{6}\right] \mathrm{S}_{8}($ Calculated for $\mathrm{C}_{24} \mathrm{H}_{36} \mathrm{~N}_{12} \mathrm{~S}_{8} \mathrm{Ni}$ : C 35.68; H 4.49; N 20.81; S 31.76; Ni 7.26. Found: C 32.11; H 4.25; $18.57 ; 34.87$.). This deviation could be accounted for by assuming contamination of $\left[\mathrm{Ni}(\mathrm{N}-\mathrm{MeIm})_{6}\right] \mathrm{S}_{8}$ with $6 \%$ and $4 \%$ by weight of elemental sulfur and nickel, respectively. Given that unreacted nickel was observed in the reaction mixture, contamination is a possibility. No further attempts to purify the complex were made.

\section{Solid state decomposition of $\left[\mathrm{Ni}(\mathrm{N}-\mathrm{MeIm})_{6}\right] \mathrm{S}_{8}$}

A thermogravimetric trace for $\left[\mathrm{Ni}(\mathrm{N}-\mathrm{MeIm})_{6}\right] \mathrm{S}_{8}$ is shown below (Figure 6-16) and a table of mass changes with their corresponding theoretical residual masses are tabulated in Table 6-6. The mass of the sample at $100^{\circ} \mathrm{C}$ was assumed to be the dry weight, to which the following mass changes were normalised. There are three distinct mass losses observed, corresponding to loss of four $N$-MeIm ligands and one sulfur atom between $100-180^{\circ} \mathrm{C}$; the loss of a further two $N$-MeIm ligands and two sulfur atoms between $190-250^{\circ} \mathrm{C}$; and the apparent complete desulfidisation to $\mathrm{NiS}$ or $\mathrm{NiO}$ above $400^{\circ} \mathrm{C}$

The final product of the TGA run was analysed by XRD (XRD pattern not shown), and found to be a complex mixture of phases, including nickel oxide (NiO). The identity of every phase in the mixture was not confirmed. 


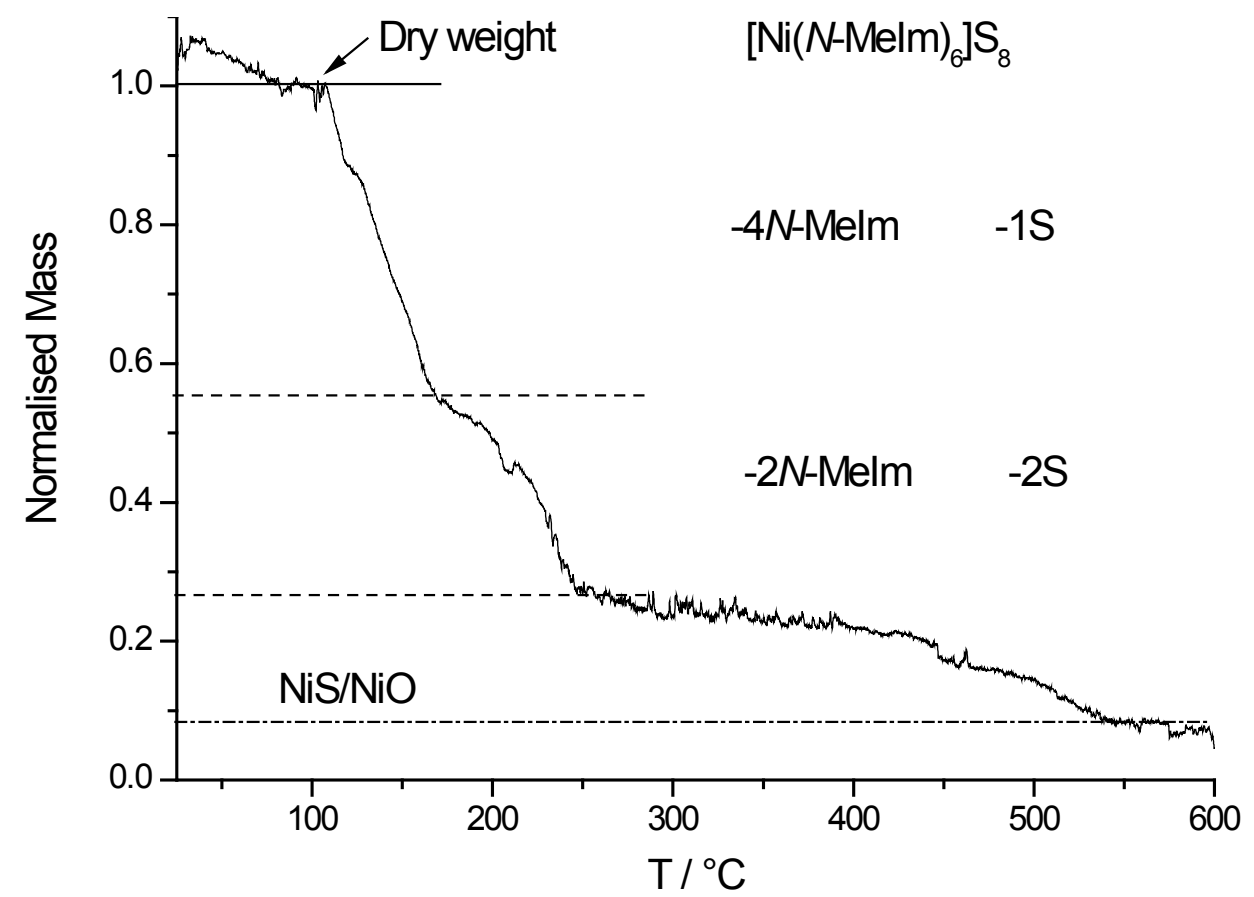

Figure 6-16 Thermogravimetric trace for $\left[\mathrm{Ni}(\mathrm{N}-\mathrm{MeIm})_{6}\right] \mathrm{S}_{8}$, under nitrogen flow with a heating rate of $5^{\circ}$ per minute.

Table 6-6 Theoretical residual masses for solid state decomposition of $\left[\mathrm{Ni}(\mathrm{N}-\mathrm{MeIm})_{6}\right] \mathrm{S}_{8}$.

\begin{tabular}{|c|c|c|c|}
\hline & \multicolumn{2}{|c|}{ Cumulative Mass Losses } & $\begin{array}{c}\text { Theoretical residual } \\
\text { normalised mass }\end{array}$ \\
\hline$\left[\mathrm{Ni}(N-\mathrm{MeIm})_{6}\right] \mathrm{S}_{8}$ & $-4 N-\mathrm{MeIm}$ & $-1 \mathrm{~S}$ & 0.55 \\
\hline & $-6 N-\mathrm{MeIm}$ & $-3 \mathrm{~S}$ & 0.27 \\
\hline $\mathrm{NiS}$ & & & 0.11 \\
\hline $\mathrm{NiO}$ & & & 0.09 \\
\hline
\end{tabular}




\section{Rapid solution-phase decomposition of $\left[\mathrm{Ni}(\mathrm{N}-\mathrm{MeIm})_{6}\right] \mathrm{S}_{8}$}

$\left[\mathrm{Ni}(\mathrm{N} \text {-MeIm })_{6}\right] \mathrm{S}_{8}$ was decomposed by rapid injection into oleylamine at $300^{\circ} \mathrm{C}$, as detailed in section 6.5.4.2. A micrograph of the decomposition product, an SAED pattern and an EDS spectrum of the area, are shown below (Figure 6-18 a), b) and c), respectively. An XRD pattern is shown in Figure 6-17 b).

The rapid decomposition product consisted of moderately monodisperse, roughly spherical 6.1 $\pm 1.2 \mathrm{~nm}$ particles. See Figure 6-18 d) for histogram of TEM measurements. The particles were significantly aggregated on the TEM grid. The SAED pattern (A118) was indexed to cubic $\mathrm{NiS}_{2}$. The smooth, well developed rings of the SAED pattern are consistent with a consistently small crystallite size, and high degree of crystallinity. EDS confirmed the composition of the sample as Ni:S 1:2, indicative of the stoichiometric nickel disulfide. The XRD pattern (see Figure 6-17) matched $\mathrm{NiS}_{2}$, with no indication of the presence of impurity phases. Scherrer X-ray broadening analysis was performed (see A2-11 for calculations) and gave an average crystallite diameter of $10 \pm 4$ $\mathrm{nm}$, which corresponds to the TEM observations.

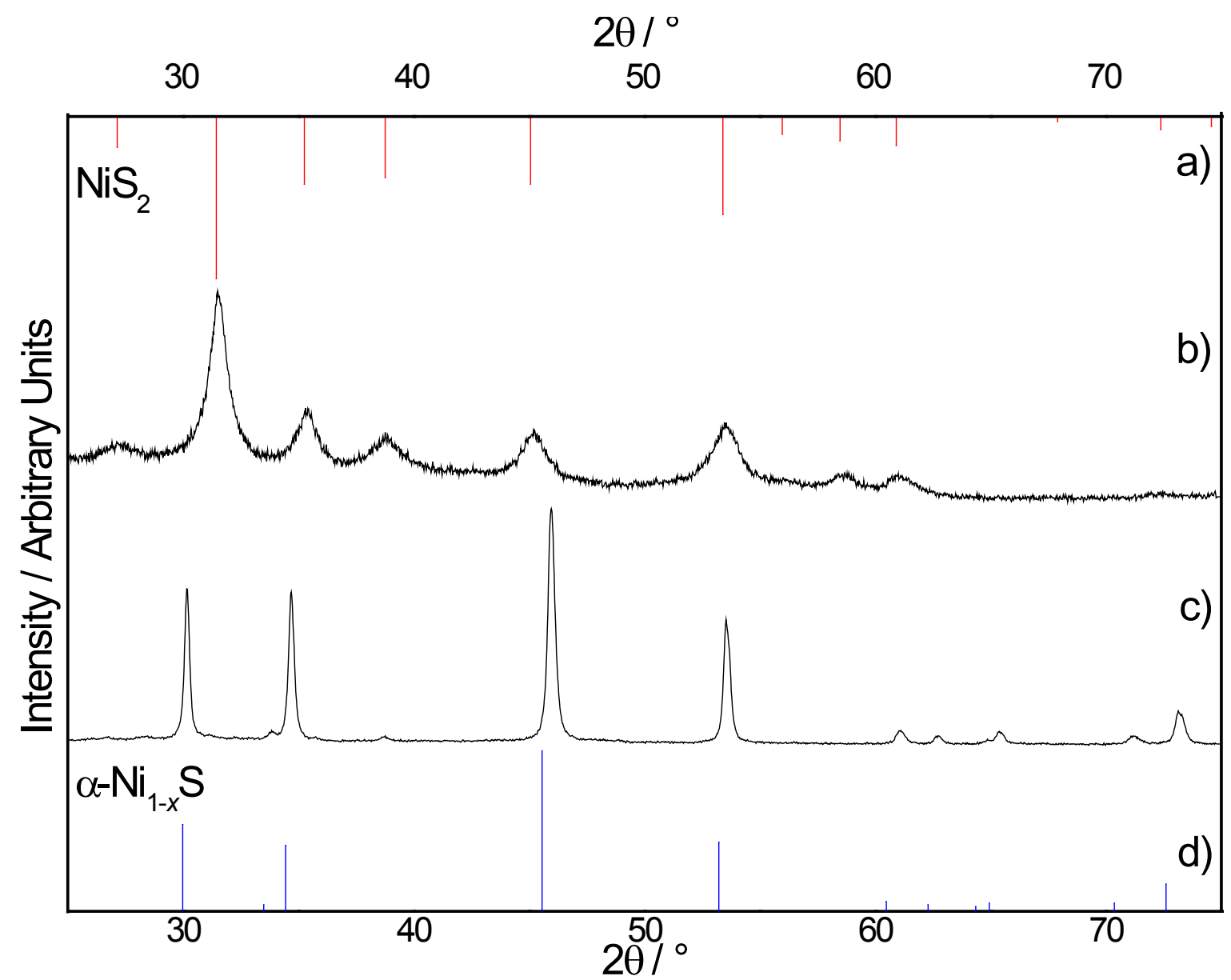

Figure 6-17 XRD patterns of a) product of rapid decomposition of $\left.\left[\mathrm{Ni}(\mathrm{N}-\mathrm{MeIm})_{6}\right] \mathrm{S}_{8} \mathrm{~b}\right)$ reference for $\mathrm{NiS}_{2}$ c) product of gradual decomposition of $\left[\mathrm{Ni}(N-\mathrm{MeIm})_{6}\right] \mathrm{S}_{8}$ d) reference for $\alpha-\mathrm{Ni}_{1-x} \mathrm{~S}$. 

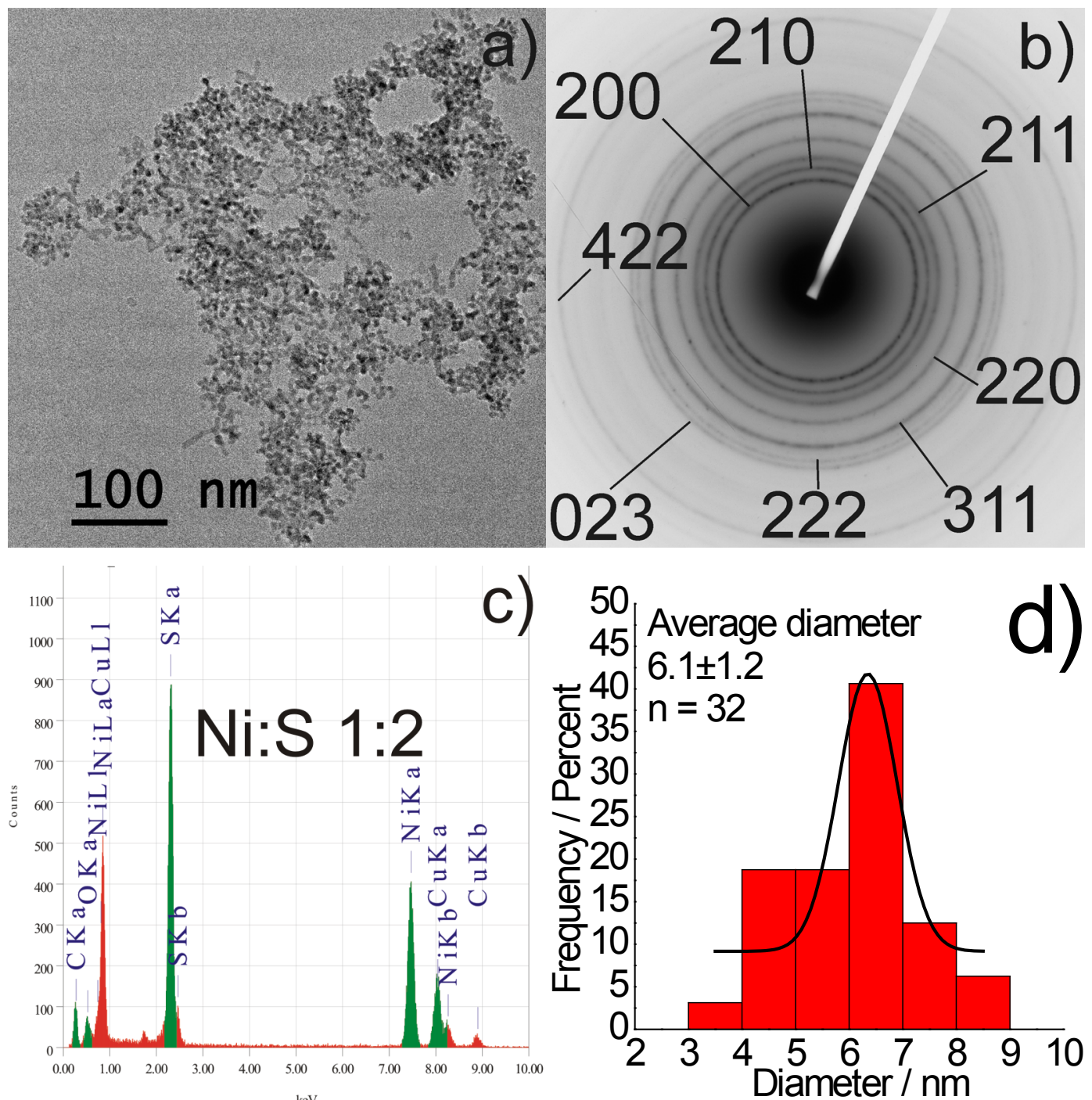

Figure 6-18 a) Micrograph of rapid decomposition product b) SAED pattern of rapid decomposition product, indexed to $\mathrm{NiS}_{2} \mathrm{c}$ ) EDS spectrum of rapid decomposition product d) histogram of particle diameter. 


\section{Gradual solution-phase decomposition of $\left[\mathrm{Ni}(\mathrm{N}-\mathrm{MeIm})_{6}\right] \mathrm{S}_{8}$}

$\left[\mathrm{Ni}(\mathrm{N} \text {-MeIm })_{6}\right] \mathrm{S}_{8}$ was decomposed by heating to $300^{\circ} \mathrm{C}$ in oleylamine, as detailed in section 6.5.4.2. A micrograph of the decomposition product is shown below, along with an SAED pattern and EDS spectrum of the area (Figure 6-19 a), b) and c), respectively). An XRD pattern is shown above in Figure 6-17 c).

The gradual decomposition product consisted of polydisperse, irregularly shaped particles, which were highly aggregated. The SAED pattern (A1-17) of the area was indexed to hexagonal NiAs-type $\alpha-\mathrm{Ni}_{1-x} \mathrm{~S}$, with several weak reflections from $\mathrm{NiS}_{2}$. The spotty nature of the SAED pattern was consistent with a large crystallite size. The XRD pattern of the sample matches $\alpha-\mathrm{Ni}_{1-x} \mathrm{~S}$, with only very weak traces of $\mathrm{NiS}_{2}$ reflections. Scherrer X-ray broadening analysis was performed (see A2-12 for calculations), and the average crystallite diameter was determined to be $45 \pm 15 \mathrm{~nm}$. The EDS spectrum confirmed an approximate Ni:S of 1:1. Repeated measurements at three locations yielded an average composition of $\mathrm{Ni}: \mathrm{S} 48: 52\left(\mathrm{Ni}_{12} \mathrm{~S}_{13}\right)$, in line with a moderately metal-deficient phase.

Both the gradual decomposition product $\left(\alpha-\mathrm{Ni}_{1-x} \mathrm{~S}\right)$ and rapid decomposition product $\left(\mathrm{NiS}_{2}\right)$ displayed featureless absorption between 210 and $800 \mathrm{~nm}$ (absorption spectra not shown). 


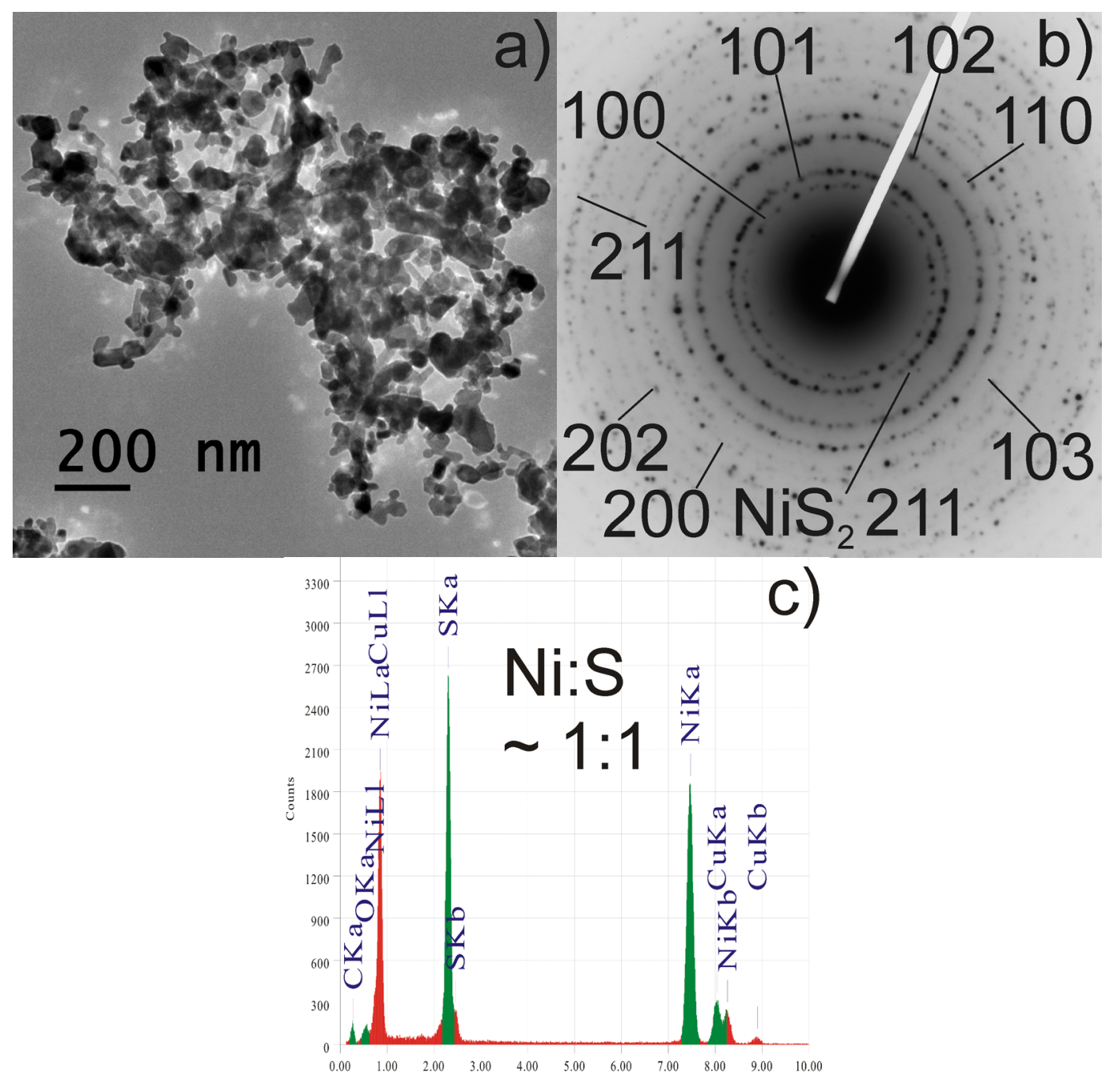

Figure 6-19 a) Micrograph of gradual decomposition product b) SAED pattern of gradual decomposition product, indexed to $\alpha-\mathrm{Ni}_{1-x} \mathrm{~S}$ with trace of $\mathrm{NiS}_{2} \mathrm{c}$ ) EDS spectrum of gradual decomposition product. 


\subsection{Discussion}

\subsubsection{Formation of $N$-methylimidazolato metal polysulfide complexes}

The metal polysulfide complexes $\left[\mathrm{M}(\mathrm{N} \text {-MeIm })_{6}\right] \mathrm{S}_{8}(\mathrm{M}=\mathrm{Mg}, \mathrm{Fe}, \mathrm{Ni})$ and $\mathrm{ZnS}_{6}(\mathrm{~N} \text {-MeIm })_{2}$ were synthesised according to literature procedure, ${ }^{13,15}$ by reaction of the metal powder with elemental sulfur in neat $N$-MeIm. All reactions proceeded well at moderate temperatures $\left(70-100^{\circ} \mathrm{C}\right)$ and, with the exception of $\left[\mathrm{Ni}(\mathrm{N} \text {-MeIm) })_{6}\right] \mathrm{S}_{8}$, all reactions went to completion within one day. The complexes were isolated as solids by layering with non-polar solvents, and purified by washing. Care is necessary to ensure the stoichiometry of the reaction is correct, and the reaction has gone to completion, in order to avoid contaminating the complex with unreacted starting materials. Yields ranged from $60-80 \%$, which combined with the simplicity of the experimental procedure, this gives scope for large scale preparation. The composition of $[\mathrm{Fe}(N-\mathrm{MeIm})] \mathrm{S}_{8},\left[\mathrm{Ni}(N \text {-MeIm })_{6}\right] \mathrm{S}_{8}$ and $\mathrm{ZnS}_{6}(N \text {-MeIm })_{2}$ were confirmed by elemental analysis.

$\left[\mathrm{Mg}(\mathrm{N} \text {-MeIm })_{6}\right] \mathrm{S}_{8}$ was observed to be highly hygroscopic, and deliquesced to form a paste upon exposure to the atmosphere. The transition metal complexes however were observed to be stable for months stored under nitrogen, and were stable enough to be transferred in air, although fine powders of the complexes were observed to tarnish overnight in air.

\subsubsection{Mechanism of complex formation}

Formation of the complexes involves a heterogeneous redox reaction at the metal surface, with oxidation of the metal and concomitant reduction of elemental sulfur to polysulfide anions. Molecular octasulfur $\left(\mathrm{S}_{8}\right)$ can be reduced in a two electron process to $\mathrm{S}_{8}{ }^{2-}$, which can disproportionate to $\mathrm{S}_{6}{ }^{2-}$ and $\mathrm{S}_{8} . \mathrm{S}_{6}{ }^{2-}$ itself is in equilibrium with $\mathrm{S}_{3}{ }^{-}$, as summarised in the scheme below. ${ }^{39}$

$$
\begin{aligned}
\mathrm{s}_{8}+2 \mathrm{e}^{-} & \rightleftharpoons \mathrm{s}_{8}{ }^{2-} \\
\mathrm{s}_{8}{ }^{2-} & \rightleftharpoons \mathrm{s}_{6}{ }^{2-}+1 / 4 \mathrm{~s}_{8} \\
\mathrm{~s}_{6}{ }^{2-} & \rightleftharpoons 2 \mathrm{~s}_{3}{ }^{-}
\end{aligned}
$$

Scheme 6-1 Reduction of octasulfur, and equilibration of polysulfide anions. 
The reduction potential of sulfur is $-1.4 \mathrm{~V}$ in acetonitrile, ${ }^{39}$ and $-0.7 \mathrm{~V}$ in DMSO. ${ }^{40}$ Basic solvents have been found to activate $\mathrm{S}_{8}$ towards reduction, ${ }^{39}$ and strongly Lewis basic ligands are noted for making metal surfaces more reductive. ${ }^{15}$ Given this, it is apparent that a strong Brønsted and Lewis base, such as $N$-MeIm, ${ }^{18}$ enables elemental sulfur to oxidise a variety of metals - although Rauchfuss and coworkers found no direct correlation between the reduction potential of a metal and its susceptibility to form $N$-MeIm stabilised polysulfide complexes. ${ }^{15}$

Once the metal has been oxidised by sulfur, the metal cations are solvated and stabilised by $\mathrm{N}$-MeIm ligands, presumably preventing their immediate deposition on the metal surface as insoluble binary metal sulfides. Hence, $N$-MeIm plays a further important role in the reaction by preventing passivation of the metal surface - thereby allowing the heterogeneous reaction to go to completion, and a stable, soluble intermediate of the reaction between metal and sulfur to be isolated. It was found in this project that no surface treatment of the metal powder was necessary prior to use, reinforcing that the robustness of the syntheses.

$\left[\mathrm{M}(\mathrm{N}-\mathrm{MeIm})_{6}\right] \mathrm{S}_{8}(\mathrm{M}=\mathrm{Mg}, \mathrm{Fe}, \mathrm{Ni})$ contain the $\mathrm{S}_{8}{ }^{2-}$ ion, which is formed directly upon reduction of $\mathrm{S}_{8}$, while the complex $\mathrm{ZnS}_{6}(N-\mathrm{MeIm})_{2}$ contains $\mathrm{S}_{6}{ }^{2-}$. The necessary rearrangement of $\mathrm{S}_{8}{ }^{2-}$ to $\mathrm{S}_{6}{ }^{2-}$ is a labile process, and it is therefore not believed to be a kinetic limiting step in the reaction. ${ }^{15}$ Rauchfuss postulated that selection of $\mathrm{S}_{8}{ }^{2-}$ or $\mathrm{S}_{6}{ }^{2-}$ as the counterion was determined by the stabilisation afforded by the crystal lattice. ${ }^{15}$

Rauchfuss reported that $N$-methylimidazolato metal polysulfide complexes do not form for the early transition row elements ( $\mathrm{Ti}, \mathrm{V}, \mathrm{Cr}$ ), and that complexes for the main block and lower transition metal elements ( $\mathrm{Sb}, \mathrm{Sn}, \mathrm{Pb}, \mathrm{Ag}, \mathrm{Au}, \mathrm{Cd}, \mathrm{Hg}$ ) only form with the addition of magnesium metal, whereby the product is of the type $\left[\mathrm{Mg}(N \text {-MeIm })_{6}\right]\left[\mathrm{M}_{\mathrm{x}} \mathrm{S}_{\mathrm{y}}\right] .{ }^{16}$ The presence of hexakis( $N$-methylimidazolato)magnesium ions might complicate the use of these polysulfide complexes as precursors for binary sulfides. 


\subsubsection{Decomposition of $N$-methylimidazolato metal polysulfide complexes}

Decomposition of $\mathrm{N}$-methylimidazolato metal polysulfide complexes was investigated both in the solid state, and in solution. Solid state decomposition of the complexes was monitored by TGA, and used to help confirm the structure of the synthesised complexes, and gauge their decomposition temperatures for the solution studies. Thermal decomposition of the complexes in solution was investigated as a synthesis route for binary metal sulfide nanocrystals.

\subsubsection{Solid state decomposition}

The thermogravimetric traces for all complexes displayed a number of distinct mass changes corresponding to the loss of $N$-MeIm ligands and sulfur, however there was substantial variation in the details of decomposition between complexes:

The temperature at which decomposition initiated varied between $80^{\circ} \mathrm{C}$ for $\left[\mathrm{Fe}(N-\mathrm{MeIm})_{6}\right] \mathrm{S}_{8}$ and $120^{\circ} \mathrm{C}$ for $\mathrm{ZnS}_{6}(N \text {-MeIm })_{2}$, while the temperature by which all $N$-MeIm ligands had been removed varied from $250^{\circ} \mathrm{C}$ for $\left[\mathrm{M}(N-\mathrm{MeIm})_{6}\right] \mathrm{S}_{8}(\mathrm{M}=\mathrm{Fe}$, $\mathrm{Ni})$ and $290^{\circ} \mathrm{C}$ for $\mathrm{ZnS}_{6}(N-\mathrm{MeIm})_{2}$.

The decomposition processes of $\left[\mathrm{M}(N-\mathrm{MeIm})_{6}\right] \mathrm{S}_{8}(\mathrm{M}=\mathrm{Mg}, \mathrm{Fe}, \mathrm{Ni})$ began with the loss of $N$-MeIm accompanied by the loss of sulfur, whereas the decomposition of $\mathrm{ZnS}_{6}(N \text {-MeIm })_{2}$ commenced with the stepwise loss of first one half, then one further sulfur atom (per formula unit).

The loss of $N$-MeIm ligands did not appear to follow any pattern for $\left.\mathrm{M}(N \text {-MeIm })_{6}\right] \mathrm{S}_{8}$ $(\mathrm{M}=\mathrm{Mg}, \mathrm{Fe}, \mathrm{Ni})$ : with $\left[\mathrm{Mg}(N-\mathrm{MeIm})_{6}\right] \mathrm{S}_{8}$ losing three $N$-MeIm ligands at a time in two steps; $\left[\mathrm{Ni}(\mathrm{N} \text {-MeIm })_{6}\right] \mathrm{S}_{8}$ losing first four then two $N$-MeIm ligands in sequential steps; and $\left[\mathrm{Fe}(N-\mathrm{MeIm})_{6}\right] \mathrm{S}_{8}$ losing one, then two, then two, then one $N$-MeIm in four sequential steps. Many, but not all losses of $N$-MeIm appeared to be accompanied by the loss of between one and four sulfur atoms.

After the loss of all $\mathrm{N}$-MeIm ligands, all complexes retained between two and five sulfur atoms, judging by their residual masses. In all cases, there then followed a period of gradual weight loss over several hundred degrees, which presumably corresponds to the evaporation of sulfur. Sulfur has a boiling point of $445^{\circ} \mathrm{C}$, but has an appreciable 
vapour pressure above $\sim 100^{\circ} \mathrm{C} .^{41}$ In a TGA trace of elemental sulfur (trace not shown) mass loss began at $160^{\circ} \mathrm{C}$, and $100 \%$ mass loss had occurred by $330^{\circ} \mathrm{C}$. The complete removal of sulfur from the metal polysulfide complexes appears to not proceed as smoothly, presumably because the sulfur is in an intimate mixture with non-volatile binary metal sulfide or oxide.

The final product of TGA runs of the metal polysulfide complexes to high temperatures $\left(600-900^{\circ} \mathrm{C}\right)$ were generally complex mixtures, which could only be partially characterised by XRD - although several were confirmed to contain metal oxides. The formation of oxides at such high temperatures is difficult to impede within the TGA apparatus, as it is not a closed system, but rather operates under a flow of nitrogen gas, which is unlikely to be rigorously oxygen-free. Therefore the formation of oxides was thought unlikely to indicate that binary metal sulfides would not form in oxygen-free solution at lower temperatures.

TGA analysis confirmed the composition by showing that all of the metal polysulfide complexes synthesised contained the number of $N$-MeIm indicated in the original publication, ${ }^{13,15}$ as well as confirming that all complexes displayed a complete loss of $\mathrm{N}$-MeIm ligands by $290^{\circ} \mathrm{C}$ (cf. the boiling point of liquid $\mathrm{N}$-MeIm is $198^{\circ} \mathrm{C}$ ). ${ }^{42}$ As this temperature was readily achievable in our solution-phase set up, this gave us confidence that thermal decomposition of the complexes in solution was feasible.

\subsubsection{Decomposition in solution}

Solution phase decomposition of the metal polysulfide complexes was conducted in two modes: a solution of the metal polysulfide complex was heated from room temperature to some reaction temperature (typically $\sim 300^{\circ} \mathrm{C}$ ), then cooled (termed gradual decomposition); or a concentrated solution of metal polysulfide complex was rapidly injected into solvent preheated to the reaction temperature (again typically $\sim 300^{\circ} \mathrm{C}$ ), before cooling (termed rapid decomposition). Both modes proved successful in producing binary metal sulfide nanocrystals in all cases except the decomposition of $\left[\mathrm{Mg}(\mathrm{N}-\mathrm{MeIm})_{6}\right] \mathrm{S}_{8}-$ which produced magnesium oxide. In the case of the other metal polysulfide complexes, dramatic differences were evident in the products produced by the two modes of decomposition - with variation in particle size, shape and crystal phase. 
The decomposition of the metal polysulfide complexes in solution was more difficult to directly monitor than in the solid state. In some cases colour changes were evident during the reaction, but reaction intermediates were not isolated - only the final products of the reactions. Metal polysulfide complexes have been noted to be susceptible to nucleophilic attack, ${ }^{17}$ and the oleylamine solvent may play a role in the decomposition as colour changes were observed during the dissolution of $\left[\mathrm{Mg}(N-\mathrm{MeIm})_{6}\right] \mathrm{S}_{8}$ and $\mathrm{ZnS}_{6}(N \text {-MeIm })_{2}$. However, even if decomposition of the metal polysulfide complex were initiated by the solvent, high temperatures would be necessary to obtain crystalline material. Thermolysis of the metal polysulfide complexes in solvent presumably produces free $N$-MeIm and sulfur species as by-products, both of which are miscible/soluble in oleylamine, and in most cases were easily removed during purification.

$\left[\mathrm{Fe}(\mathrm{N}-\mathrm{MeIm})_{6}\right] S_{8}$

Rapid decomposition of $\left[\mathrm{Fe}\left(N\right.\right.$-MeIm) $\left.{ }_{6}\right] \mathrm{S}_{8}$ yielded small $16 \mathrm{~nm}$, faceted $\mathrm{Fe}_{3} \mathrm{~S}_{4}$ nanocrystals; whereas gradual decomposition yielded larger $(70 \mathrm{~nm}) \mathrm{Fe}_{1-x} \mathrm{~S}$ crystallites with a variety of highly faceted crystal habits. This phase selectivity reflects that seen in Chapter 4, and appears to be a completely temperature controlled phenomenon prolonged reaction at temperatures in excess of $200^{\circ} \mathrm{C}$ produces $\mathrm{Fe}_{1-x} \mathrm{~S}$, whereas if the reaction temperature is either kept below this value, or reaction above $200^{\circ} \mathrm{C}$ is only very brief, then $\mathrm{Fe}_{3} \mathrm{~S}_{4}$ is favoured. As in Chapter 4, the size and morphology of $\mathrm{Fe}_{1-x} \mathrm{~S}$ appears thermodynamically driven, as the resulting anisotropic habits and crystallite size seem independent of reaction conditions, and establish themselves rapidly after initiation of reaction.

The only currently published synthesis of small, well-dispersed $\mathrm{Fe}_{3} \mathrm{~S}_{4}$ nanocrystals is achieved by the decomposition of $\left[\mathrm{NBu}_{4}\right]_{2}\left[\mathrm{Fe}_{4} \mathrm{~S}_{4}(\mathrm{SPh})_{4}\right]$ at $200^{\circ} \mathrm{C}$ in dodecylamine for $12-16$ hours, ${ }^{12}$ which produced $4.5 \mathrm{~nm}$ nanocrystals.

$\mathrm{Fe}_{1-x} \mathrm{~S}$ crystallites shown in the literature tend to display highly faceted habits, and have dimensions $>50 \mathrm{~nm},{ }^{43}$ similar to those seen in this project - both in this chapter and in Chapter 4. The synthesis of small spherical $\mathrm{Fe}_{7} \mathrm{~S}_{8}$ nanocrystals has been reported only recently, via the decomposition of $\left[\mathrm{NBu}_{4}\right]_{2}\left[\mathrm{Fe}_{4} \mathrm{~S}_{4}(\mathrm{SPh})_{4}\right]$ by injection into octylamine at $180^{\circ} \mathrm{C}$ followed by reaction at that temperature for $12-16$ hours. ${ }^{12}$ It is possible that the 
relatively low injection and reaction temperature, coupled with a very long reaction time restricts the growth of the nanocrystals while allowing the slow development of crystalline $\mathrm{Fe}_{7} \mathrm{~S}_{8}$.

\section{$\mathrm{ZnS}_{6}(\mathrm{~N}-\mathrm{MeIm})_{2}$}

Rapid decomposition of $\mathrm{ZnS}_{6}(N-\mathrm{MeIm})_{2}$ yielded monodisperse $5 \mathrm{~nm}$ spherical zinc sulfide nanocrystals; whereas gradual decomposition yielded $15 \times 5 \mathrm{~nm}$ zinc sulfide nanorods. In both cases, the samples were assigned as being predominantly the hexagonal wurtzite polymorph of zinc sulfide, with only a trace of the cubic zinc blende polymorph detected.

Growth of anisotropic structures, such as nanowires, can be driven by intrinsic or extrinsic factors. Extrinsically driven growth mechanisms include the use of mixed surfactant systems, where each surfactant is preferentially absorbed on different sets of facets, directing growth away from the faces stabilised with the least labile of the surfactants. However, this mechanism is a feature of anisotropic crystal systems, rather than the high symmetry cubic system - and hence does not appear to apply to the cubic zinc blende polymorph of zinc sulfide. ${ }^{21}$

Conversely, theories which are based on intrinsic factors focus on the reduction of surface energy when going from a collection of spherical nanocrystals to a nanorod of equal diameter and overall volume, as the driving force for nanorod formation. ${ }^{22}$ The orientated attachment mechanism is based upon the actual assembly of spherical nanocrystals into crystallographically-orientated chains, which is followed by fusion into a single-crystal and diffusion-based growth to fill the remaining indentations. ${ }^{21,44}$

The zinc sulfide nanorods synthesised in this project were grown in a single surfactant system and were found to be predominantly wurtzite polymorph. So although only a single surfactant system was used, may be due to extrinsic growth factors rather than an intrinsically driven orientated attachment mechanism.

Several syntheses have been published for zinc sulfide nanocrystals ${ }^{27}$ and nanorods - of both the zinc blende, ${ }^{21,22}$ and the wurtzite polymorph. ${ }^{45}$ Some of these have produced nanocrystals and nanorods with high degrees of monodispersity. The sizes and 
monodispersity of the spherical zinc sulfide nanocrystals $(\mathrm{r}=5 \mathrm{~nm}, \sigma=20 \%)$ and zinc sulfide nanorods $(5 \times 15 \mathrm{~nm}, \sigma=20 \%)$ produced in this project are comparable to some published syntheses, ${ }^{27}$ but the synthesis used in this project avoids the use of expensive and hazardous reagents such as dimethylzinc. In addition, the optical properties of the $\mathrm{ZnS}$ nanorods were demonstrated to be high quality, with a relatively low ratio of defect to band-edge emission.

$\left[\mathrm{Mg}(\mathrm{N}-\mathrm{MeIm})_{6}\right] S_{8}$

The decomposition of $\left[\mathrm{Mg}(N-\mathrm{MeIm})_{6}\right] \mathrm{S}_{8}$ yielded large aggregates of magnesium oxide platelets. It is unclear whether the decomposition yielded magnesium oxide directly, or whether magnesium sulfide was initially produced and later hydrolysed. Efforts were made to exclude water and oxygen during the synthesis - the precursor complex was stored under nitrogen, experimental apparatus were evacuated and filled with nitrogen, and dried distilled solvent was used. However, after synthesis no precautions were made to keep the sample dry or under an inert atmosphere during purification and sample storage. Given the complexities of performing the purification and sample preparation for TEM examination under a dry, inert atmosphere; and given the prospect that even if magnesium sulfide were to be produced it would be unstable when stored in air, it was decided not to proceed with further investigation of the decomposition products of $\left[\mathrm{Mg}(N-\mathrm{MeIm})_{6}\right] \mathrm{S}_{8}$.

$\left[\mathrm{Ni}(\mathrm{N}-\mathrm{MeIm})_{6}\right] S_{8}$

Rapid decomposition of $\left[\mathrm{Ni}(\mathrm{N} \text {-MeIm })_{6}\right] \mathrm{S}_{8}$ yielded $6 \mathrm{~nm}$ spherical $\mathrm{NiS}_{2}$ nanocrystals; whereas gradual decomposition yielded larger, polydisperse $\alpha-\mathrm{Ni}_{1-x} \mathrm{~S}$ nanocrystals. Analogous to the case of $\mathrm{Fe}_{3} \mathrm{~S}_{4}$ and $\mathrm{Fe}_{1-x} \mathrm{~S}$ synthesised from $\left[\mathrm{Fe}\left(N\right.\right.$-MeIm) $\left.{ }_{6}\right] \mathrm{S}_{8}$, phase selectivity appears to originate in temperature/reaction time: with $\mathrm{NiS}_{2}$ produced by short reaction times, and $\mathrm{Ni}_{1-x} \mathrm{~S}$ favoured by the longer reaction times entailed in the gradual decomposition procedure.

Phase selectivity within the Ni-S system is complex, however some patterns from the literature can be applied to this case. Nickel-rich phases such as $\mathrm{Ni}_{3} \mathrm{~S}_{2}$ are not favoured under sulfiding conditions, ${ }^{46}$ such as is the case for the thermolysis of $\left[\mathrm{Ni}(\mathrm{N}-\mathrm{MeIm})_{6}\right] \mathrm{S}_{8}$, where sulfur is in substantial excess. Pure $\mathrm{NiS}_{2}$ has been obtained at $130{ }^{\circ} \mathrm{C},{ }^{33}$ whereas 
temperatures $>140^{\circ} \mathrm{C}$ have been reported as favouring NiS polymorphs. ${ }^{29-31}$ This indicates that $\mathrm{NiS}$ is favoured over $\mathrm{NiS}_{2}$ at the reaction temperatures used for the gradual decomposition of $\left[\mathrm{Ni}(\mathrm{N} \text {-MeIm })_{6}\right] \mathrm{S}_{8}$. Whereas with only very short reaction times at elevated temperatures, as is the case with rapid decomposition of $\left[\mathrm{Ni}(\mathrm{N}-\mathrm{MeIm})_{6}\right] \mathrm{S}_{8}, \mathrm{NiS}_{2}$ remains favoured. The inversion temperature between $\beta$-NiS and $\alpha-\mathrm{Ni}_{1-x} \mathrm{~S}$ is $300^{\circ} \mathrm{C}$ for a composition of $\mathrm{Ni}_{48} \mathrm{~S}_{52}{ }^{47}$ (the composition of the gradual decomposition product as judged by EDS), meaning $\alpha-\mathrm{Ni}_{1-x} \mathrm{~S}$ is the thermodynamically favoured polymorph of NiS for the reaction conditions used in the gradual decomposition of $\left[\mathrm{Ni}(\mathrm{N}-\mathrm{MeIm})_{6}\right] \mathrm{S}_{8}$.

The small size and moderate monodispersity $(6 \mathrm{~nm}, \sigma=20 \%$ by TEM) of the spherical $\mathrm{NiS}_{2}$ nanocrystals produced by rapid decomposition of $\left[\mathrm{Ni}(\mathrm{N}-\mathrm{MeIm})_{6}\right] \mathrm{S}_{8}$, matches what one would expect from burst nucleation, followed by a relatively quick cessation of reaction before the system can enter the defocusing regime. Whereas the larger particle size, wide size distribution of the $\alpha-\mathrm{Ni}_{1-x} \mathrm{~S}$ particles produced by gradual decomposition is indicative of nucleation and growth throughout the reaction.

Although finely crystalline $\alpha-\mathrm{Ni}_{1-x} \mathrm{~S}$ has been synthesised by a variety of methods, ${ }^{29,} 30$, ${ }^{48}$ well-defined $\alpha-\mathrm{Ni}_{1-x} \mathrm{~S}$ nanocrystals have only been synthesised by the decomposition of single-source precursors: the decomposition of (TMEDA)Ni( $\left.\mathrm{SC}\{\mathrm{O}\} \mathrm{C}_{6} \mathrm{H}_{5}\right)_{2}$ in oleic acid at $180^{\circ} \mathrm{C}$ produced irregularly shaped $\sim 25 \mathrm{~nm}$ nanocrystals; ${ }^{32}$ whereas the decomposition of $\mathrm{Ni}(\mathrm{Mer})_{2}$ in oleylamine at $230^{\circ} \mathrm{C}$ for 6 hours produced moderately

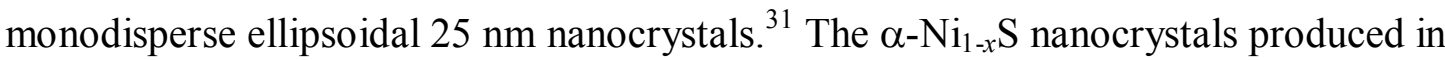
this project displayed irregular morphology, and a wider size distribution than the aforementioned examples. It appears extending the reaction time to several hours might lead to the attainment of a relatively uniform, equilibrium diameter and size distribution.

Nanocrystalline $\mathrm{NiS}_{2}$ has been synthesised via solvothermal methods, ${ }^{33}$ but to the best of our knowledge no synthesis of small, monodisperse $\mathrm{NiS}_{2}$ has been previously reported. The rapid decomposition of $\left[\mathrm{Ni}(\mathrm{N}-\mathrm{MeIm})_{6}\right] \mathrm{S}_{8}$ produces phase-pure, monodisperse $6 \mathrm{~nm} \mathrm{NiS}_{2}$ nanocrystals. 
Among the metal polysulfide complexes, two fundamental coordination modes between the metal cation and the polysulfide anion are evident. In the first, termed an inner sphere complex, there is direct ligation of the metal ion by the polysulfide anion through the terminal sulfur atoms of the polysulfide chain. In the second, termed an outer sphere complex, the polysulfide anion resides outside the first coordination sphere of the metal, and therefore serves only as a counterion and not as a ligand. Examples of both coordination modes are present among the metal polysulfide complexes featured in this project, with $\mathrm{ZnS}_{6}(\mathrm{~N} \text {-MeIm })_{2}$ being an example of an inner sphere complex, and the remainder $\left(\left[\mathrm{M}(\mathrm{N} \text {-MeIm })_{6}\right] \mathrm{S}_{8}, \mathrm{M}=\mathrm{Mg}, \mathrm{Fe}, \mathrm{Ni}\right)$ being examples of outer sphere complexes. In $\mathrm{ZnS}_{6}(\mathrm{~N} \text {-MeIm })_{2}$ in the solid state, the polysulfide anion $\mathrm{S}_{6}{ }^{2-}$ forms a 7-membered ring with $\mathrm{Zn}^{2+}$, which is also coordinated to the two $\mathrm{N}$-MeIm ligands. ${ }^{13} \mathrm{In}$ $\left[\mathrm{M}(\mathrm{N}-\mathrm{MeIm})_{6}\right] \mathrm{S}_{8}, \mathrm{M}=\mathrm{Mg}, \mathrm{Fe}, \mathrm{Ni}$ in the solid state, the metal ion forms an octahedral complex with the six $N$-MeIm ligands, and the polysulfide anion $\left(\mathrm{S}_{8}{ }^{2-}\right)$ exists as a disordered chain outside the first coordination sphere of the metal. ${ }^{15}$

Although the mode of coordination undoubtedly affects the chemistry of the metal polysulfide complexes, it is not apparent that this extends to affecting the nature of the thermal decomposition products. In all cases, the crystalline phase produced by the solution phase decomposition reactions was the thermodynamic product expected for the given reaction conditions, as discussed in specific details in the preceding sections. In addition, the morphologies of nanocrystals did not deviate from what was expected nanocrystals of less than $10 \mathrm{~nm}$ diameters were spherical or spheroid ( $\mathrm{ZnS}$ and $\left.\mathrm{NiS}_{2}\right)$ Whereas nanocrystals larger than $\sim 20 \mathrm{~nm}$ displayed a variety of faceted habits $\left(\mathrm{Fe}_{3} \mathrm{~S}_{4}\right.$, $\mathrm{Fe}_{1-x} \mathrm{~S}$ and $\left.\alpha-\mathrm{Ni}_{1-x} \mathrm{~S}\right)$, which in all cases conformed to the thermodynamically allowed forms for the crystal class (see Chapter 4 section 4.3.1) for a discussion of the morphologies expected for NiAs-type $\mathrm{Fe}_{1-x} \mathrm{~S}$, observations which are also applicable to NiAs-type $\left.\alpha-\mathrm{Ni}_{1-x} \mathrm{~S}\right)$. Gradual decomposition of $\mathrm{ZnS}_{6}(N-\mathrm{MeIm})_{2}$ led to wurtzite $\mathrm{ZnS}$ nanorods, a morphology which has been observed frequently for this polymorph of $\mathrm{ZnS}^{22}$

As only a single coordination mode exists for each $N$-methylimidazolato metal polysulfide complex, it was not possible to rigorously test the hypothesis that the mode of coordination of the metal does not affect the outcome of the decomposition reactions. 
Comparison across metal complexes with different coordination modes is also impractical, as radically different phase behaviour in each target metal sulfide system is likely to confound observation of any effect due to the polysulfide coordination mode. However, given that in no case did the resulting product differ from what could be predicted based on theory, or based on what had been observed previously for nanocrystals synthesised from unrelated precursors, it appears unlikely that the coordination mode of the polysulfide anion affects the outcome of decomposition reactions. 


\subsection{3 $\mathrm{N}$-methylimidazolato metal polysulfide precursors as single-source precursors}

To date $N$-methylimidazolato metal polysulfide complexes are the only known single-source precursors for metal sulfide nanocrystals that utilise a completely inorganic sulfur source. They have the benefit of being directly prepared - in a single synthetic step - from metal powder and elemental sulfur using only the inexpensive amine solvent $N$-MeIm. When thermally decomposed, excess sulfur is the only non-volatile by-product, and this can be removed effectively if a solvent is used in which elemental sulfur has appreciable solubility, such as oleylamine.

This excess of sulfur built into the precursor has some advantages: high sulfur to metal ratios during synthesis has been linked with the absence of sulfur vacancies and resulting high quality physical properties in nanocrystals in several cases. ${ }^{21,49,50}$ In addition, as demonstrated in this project, the excess of sulfur enables phases with a variety of metal-sulfur stoichiometries to be accessed from the same precursor by altering the reaction conditions (e.g. $\mathrm{Fe}_{1-x} \mathrm{~S}$ and $\mathrm{Fe}_{3} \mathrm{~S}_{4} ; \mathrm{Ni}_{1-x} \mathrm{~S}$ and $\mathrm{NiS}_{2}$ ).

$\mathrm{N}$-methylimidazolato metal polysulfide complexes may prove to be viable precursors only for mid-to-late transition row elements (Mn-Zn), as complexes for other metals have not been observed to form spontaneously in the absence of magnesium metal, ${ }^{16}$ however this still presents a significant avenue for research. There remains further scope in some cases for the optimisation of reaction conditions to improve the dispersity and dispersibility of nanocrystals produced by the decomposition of metal polysulfide complexes. 


\subsection{Conclusions}

In conclusion, the metal polysulfide complexes $\left[\mathrm{M}(N-\mathrm{MeIm})_{6}\right] \mathrm{S}_{8}(\mathrm{M}=\mathrm{Mg}, \mathrm{Fe}, \mathrm{Ni})$ and $\mathrm{ZnS}_{6}(\mathrm{~N} \text {-MeIm })_{2}$ were synthesised and decomposed in the solid state and solution phase. Solution phase decomposition of the complexes yielded $\mathrm{MgO}, \mathrm{Fe}_{3} \mathrm{~S}_{4}, \mathrm{Fe}_{1-x} \mathrm{~S}, \mathrm{Ni}_{1-x} \mathrm{~S}$, $\mathrm{NiS}_{2}$, and $\mathrm{ZnS}$ nanocrystals and $\mathrm{ZnS}$ nanorods, thereby demonstrating the viability of these complexes as single-source precursors for the synthesis of binary metal sulfide nanocrystals. These complexes have the advantages of being directly synthesised from the elements with addition of an inexpensive amine solvent; and of being relatively stable and soluble intermediates of the sulfidisation of metal, which can be reacted under moderate conditions to afford the desired products free of non-volatile by-products with the exception of excess elemental sulfur. 


\subsection{Method}

Synthesis and manipulations were conducted using standard airless techniques. Unless otherwise mentioned, the precursor complexes were assumed to be air-sensitive, and were stored under nitrogen. Samples dispatched for elemental analysis were dried under vacuum, and then packed under nitrogen.

$\mathrm{N}$-methylimidazole (N-MeIm) was purchased from Merck, and was distilled off $\mathrm{BaO}$ under reduced pressure (b.p. $43-44^{\circ} \mathrm{C}$ at $0.6 \mathrm{mmHg}$, colourless liquid) and stored under nitrogen. Sulfur (sublimed) was purchased from BDH and used as received. Iron powder was purchased from Scientific Supplies; magnesium, nickel and zinc powders were purchased from Merck; all metal powders were used as received.

Oleylamine (Tech. $\geq 70 \%$ ) was purchased from Fluka, and was distilled off $\mathrm{BaO}$ under reduced pressure (b.p. $148-176^{\circ} \mathrm{C}$ at $0.3 \mathrm{mmHg}$, colourless liquid) and stored under nitrogen.

Spectroscopic measurements were performed on dilute suspensions in spectroscopic grade cyclohexane. UV-vis spectroscopy was peformed on a sample in a matched two-sided $1 \mathrm{~cm}$ quartz cuvette against a solvent blank in a Perkin-Elmer Cary 100 double-beamed spectrometer. PL spectroscopy was performed on a sample in a four-sided $1 \mathrm{~cm}$ fused silica cuvette in a Horiba Jobin-Yvon Fluorolog FL-1057 fluorometer.

\subsubsection{Single-source iron sulfide precursor - $\left[\mathrm{Fe}(\mathrm{N} \text {-MeIm })_{6}\right] \mathrm{S}_{8}$}

\subsubsection{Synthesis of $\left[\mathrm{Fe}(N-\mathrm{MeIm})_{6}\right] \mathrm{S}_{8}$}

Iron powder $\left(0.142 \mathrm{~g}, 2.5 \times 10^{-3} \mathrm{~mol}\right)$ and sulfur $\left(0.646 \mathrm{~g}, 2.0 \times 10^{-2} \mathrm{~mol} \equiv 2.5 \times 10^{-3}\right.$ of $\left.\mathrm{S}_{8}\right)$ were placed in a Schlenk tube, which was evacuated and placed under nitrogen. $N$-MeIm $\left(10 \mathrm{~cm}^{3}\right)$ was added, forming a green solution with the sulfur. The reaction vessel was heated to $70^{\circ} \mathrm{C}$ for 4 hours. The iron powder dissolved over this period, and dark purple crystals formed $(1.52 \mathrm{~g})$. The dark purple mother liquor was decanted to another vessel, and layered with diethylether $\left(40 \mathrm{~cm}^{3}\right)$, to produce a crop of finely crystalline purple powder $(0.12 \mathrm{~g})$. Both crops were washed with diethyl ether, until the washings were clear $\left(\sim 60 \mathrm{~cm}^{3}\right)$, and were dried under vacuum. The complex appears 
stable under nitrogen, and when transferred in air, but tarnishes when exposed to the atmosphere overnight. Total yield $1.64 \mathrm{~g}, 80 \%$. Analysis. Calculated for $\mathrm{C}_{24} \mathrm{H}_{36} \mathrm{~N}_{12} \mathrm{~S}_{8} \mathrm{Fe}$ : C 35.80; H 4.51; N 20.88; S 31.87; Fe 6.94. Found: C 34.98; H 4.48; N 20.46; S 32.63.

\subsubsection{Decomposition of $\left[\mathrm{Fe}(N-\mathrm{MeIm})_{6}\right] \mathrm{S}_{8}$.}

Rapid decomposition of $\left[\mathrm{Fe}(\mathrm{N}-\mathrm{MeIm})_{6}\right] S_{8}$

$\left[\mathrm{Fe}(N \text {-MeIm })_{6}\right] \mathrm{S}_{8}\left(0.711 \mathrm{~g}, 8.8 \times 10^{-4} \mathrm{~mol}\right.$, purple crystals $)$ was dissolved in distilled oleylamine $\left(10 \mathrm{~cm}^{3}\right)$ to give a black solution. This solution was rapidly injected into a flask containing distilled oleylamine $\left(40 \mathrm{~cm}^{3}\right)$ preheated to $300^{\circ} \mathrm{C}$. The black reaction mixture was rapidly cooled immediately after injection by lifting the flask out of the heating mantle, and centrifuged to give a black precipitate and a clear yellow supernatant. The supernatant was discarded, and the precipitate was suspended in toluene $\left(20 \mathrm{~cm}^{3}\right)$, then centrifuged to give the precipitate and a clear, colourless supernatant. The supernatant was discarded and this procedure was repeated, this time with the addition of acetone $\left(80 \mathrm{~cm}^{3}\right)$ to destabilise the toluene suspension. The precipitate was dried in vacuo overnight, and then ground into a dark black powder.

\section{Gradual decomposition of $\left[\mathrm{Fe}(\mathrm{N}-\mathrm{MeIm})_{6}\right] \mathrm{S}_{8}$}

$\left[\mathrm{Fe}(N \text {-MeIm })_{6}\right] \mathrm{S}_{8}\left(0.406 \mathrm{~g}, 5.0 \times 10^{-4} \mathrm{~mol}\right.$, purple crystals $)$ was dissolved in distilled oleylamine $\left(50 \mathrm{~cm}^{3}\right)$ to give a black solution. The solution was heated to $300^{\circ} \mathrm{C}$ and held at this temperature for 4 hours, then allowed to cool. The black reaction mixture was centrifuged to give a black precipitate and a clear yellow supernatant. The supernatant was discarded and the precipitate was suspended in toluene $\left(20 \mathrm{~cm}^{3}\right)$, then centrifuged to give the precipitate and a clear, colourless supernatant. The supernatant was discarded and this procedure was repeated, this time with the addition of acetone $\left(80 \mathrm{~cm}^{3}\right)$ to destabilise the toluene suspension. The precipitate was dried in vacuo overnight, and then ground into a dark black powder. 


\subsubsection{Single-source zinc sulfide precursor $-\mathrm{ZnS}_{6}(\mathrm{~N} \text {-MeIm })_{2}$}

\subsubsection{Synthesis of $\mathrm{ZnS}_{6}(N-\mathrm{MeIm})_{2}$}

Zinc powder $\left(0.548 \mathrm{~g}, 8.4 \times 10^{-3} \mathrm{~mol}\right)$ and sulfur $\left(1.722 \mathrm{~g}, 0.054 \mathrm{~mol} \equiv 9 \times 10^{-3} \mathrm{~mol}\right.$ of $\mathrm{S}_{6}$ ) were placed in a Schlenk tube, which was evacuated and placed under nitrogen. $N$-MeIm $\left(10 \mathrm{~cm}^{3}\right)$ was added, and the reaction mixture was heated to $100^{\circ} \mathrm{C}$, and stirred overnight. The dark green reaction mixture was transferred to another vessel, and layered with toluene $\left(10 \mathrm{~cm}^{3}\right)$. A yellow-green powder or orange crystals crystallised after several days at low temperature. The dark red mother liquor was decanted and discarded, and the powder was washed repeatedly with toluene $\left(100 \mathrm{~cm}^{3}\right.$ total), until the washings were clear. The powder was dried under vacuum, and stored under nitrogen. The complex appears stable under nitrogen, and when transferred in air, but tarnishes when exposed to the atmosphere overnight. Yield $2.19 \mathrm{~g}, 62 \%$. Analysis. Calculated for $\mathrm{C}_{8} \mathrm{H}_{12} \mathrm{~N}_{4} \mathrm{~S}_{6} \mathrm{Zn}$ : C 22.78; H 2.87; N 13.28; S 45.61; Zn 15.50. Found: C 23.14; H 2.84; N $13.51 ; \mathrm{S} 44.58$.

\subsubsection{Decomposition of $\mathrm{ZnS}_{6}(N-\mathrm{MeIm})_{2}$}

Rapid decomposition of $\mathrm{ZnS}_{6}(\mathrm{~N}-\mathrm{MeIm})_{2}$

$\mathrm{ZnS}_{6}(N \text {-MeIm })_{2}(0.100 \mathrm{~g}, 0.24 \mathrm{~mol})$ was dissolved in distilled oleylamine $\left(3 \mathrm{~cm}^{3}\right)$ to give an orange solution, which was transferred to a syringe. A flask was flushed with nitrogen, then filled with distilled oleylamine $\left(12 \mathrm{~cm}^{3}\right)$. The colourless oleylamine was heated to $300^{\circ} \mathrm{C}$, whereupon the orange $\mathrm{ZnS}_{6}(N \text {-MeIm })_{2}$ solution was injected, immediately forming a yellow solution. Upon cooling of the reaction mixture, a cloudy white suspension formed. The reaction mixture was centrifuged at 4,000 rpm for $3 \mathrm{~min}$, to produce a white precipitate and a clear yellow supernatant, which was discarded. The precipitate was easily resuspended, with sonication, in toluene $\left(5 \mathrm{~cm}^{3}\right)$ to give a clear colourless liquid. Upon addition of acetone $\left(10 \mathrm{~cm}^{3}\right)$, a cloudy white suspension formed. Centrifugation at 4,000 rpm for 3 minutes produced a white precipitate and a clear colourless supernatant. After 2 cycles of purification the white precipitate was suspended in toluene. 
$\mathrm{ZnS}_{6}(N-\mathrm{MeIm})_{6}\left(0.425 \mathrm{~g}, 1.0 \times 10^{-3} \mathrm{~mol}\right.$, orange crystals $)$ was dissolved in distilled oleylamine $\left(50 \mathrm{~cm}^{3}\right)$ to give an orange solution. The solution was heated to $300^{\circ} \mathrm{C}$ and held at this temperature for $30 \mathrm{~min}$, then allowed to cool. A slight darkening in colour was observed at $140^{\circ} \mathrm{C}$, but the solution became light yellow by $300^{\circ} \mathrm{C}$. Upon cooling a cloudy white suspension formed. The reaction mixture was centrifuged to produce a white precipitate and a clear yellow supernatant. The supernatant was discarded and the precipitate was resuspended in toluene $\left(20 \mathrm{~cm}^{3}\right)$ to give a clear, colourless solution. Upon addition of acetone $\left(25 \mathrm{~cm}^{3}\right)$ a cloudy white suspension formed. Centrifugation produced a white precipitate and a clear colourless supernatant. The supernatant was discarded and this procedure was repeated twice. The precipitate was dried in vacuo overnight, and ground into a fine, off-white powder. 


\subsubsection{Single-source magnesium sulfide precursor - $\left[\mathrm{Mg}(\mathrm{N}-\mathrm{MeIm})_{6}\right] \mathrm{S}_{8}$}

\subsubsection{Synthesis of $\left[\mathrm{Mg}(N-\mathrm{MeIm})_{6}\right] \mathrm{S}_{8}$}

Magnesium powder $\left(0.0554 \mathrm{~g}, 2.28 \times 10^{-3} \mathrm{~mol}\right)$ and sulfur $(0.592 \mathrm{~g}, 0.0184 \mathrm{~g} \equiv 2.31 \times$ $10^{-3} \mathrm{~mol} \mathrm{~S}_{8}$ ) were placed in a Schlenk tube, which was evacuated and filled with nitrogen. $N$-MeIm $\left(10 \mathrm{~cm}^{3}\right)$ was added, whereupon partial dissolution of the sulfur produced a green solution. The reaction mixture was heated to $95^{\circ} \mathrm{C}$ with stirring for 24 hours, whereupon the solution had become dark red, and a purple precipitate was visible. The mother liquor was transferred to another flask and layered with toluene $\left(20 \mathrm{~cm}^{3}\right)$ and refrigerated overnight, whereupon a further crop of purple powder formed. Both crops of powder were washed with toluene $\left(100 \mathrm{~cm}^{3}\right)$ until the washings were clear, and were dried under vacuum and stored under nitrogen. The complex appears to be highly hygroscopic and forms a thick paste upon exposure to the atmosphere. Stored under nitrogen the complex tarnishes over a period of two months. Due to the apparent instability of the complex, no sample was submitted for elemental analysis. Total yield $1.09 \mathrm{~g}, 57 \%$.

\subsubsection{Decomposition of $\left[\mathrm{Mg}(N \text {-MeIm })_{6}\right] \mathrm{S}_{8}$}

$\left[\mathrm{Mg}(\mathrm{N}-\mathrm{MeIm})_{6}\right] \mathrm{S}_{8}\left(0.054 \mathrm{~g}, 6.4 \times 10^{-5} \mathrm{~mol}\right)$ was suspended in distilled oleylamine (13 $\mathrm{cm}^{3}$ ) and heated to $310^{\circ} \mathrm{C}$ with stirring. The red suspension darkened to purple at $140^{\circ} \mathrm{C}$; and at $200^{\circ} \mathrm{C}$ a clear red solution with flecks of purple solid formed. The flocks dissolved, and the colour of the solution changed to clear yellow by $270^{\circ} \mathrm{C}$. The colour of the solution remained yellow, and a cloudy white precipitate formed. The reaction mixture was centrifuged at $4,000 \mathrm{rpm}$ for 10 minutes to produce a white/grey precipitate and a clear yellow supernatant, which was discarded. The precipitate was resuspended in toluene with the aid of sonication. Upon centrifugation at 4,000 rpm for 10 minutes without the addition of a destabilising solvent, the precipitate reformed a pellet, and the colourless supernatant was discarded. The precipitate was suspended in toluene with the aid of sonication to give a clear, colourless solution. 


\subsubsection{Single-source nickel sulfide precursor - $\left[\mathrm{Ni}(\mathrm{N}-\mathrm{MeIm})_{6}\right] \mathrm{S}_{8}$}

\subsubsection{Synthesis of $\left[\mathrm{Ni}(N-\mathrm{MeIm})_{6}\right] \mathrm{S}_{8}$}

Nickel powder $\left(0.180 \mathrm{~g}, 3.07 \times 10^{-3} \mathrm{~mol}\right)$ and sulfur $\left(0.830 \mathrm{~g}, 2.59 \times 10^{-2} \mathrm{~mol} \equiv 3.24 \times\right.$ $10^{-3}$ mol of $\mathrm{S}_{8}$ ) were placed in a Schlenk tube, which was evacuated and filled with nitrogen. $\mathrm{N}$-MeIm $\left(10 \mathrm{~cm}^{3}\right)$ was added, and the reaction mixture was heated to $60^{\circ} \mathrm{C}$ with stirring for 21 hours. A dark red precipitate formed, and the solution turned dark red. The precipitate was isolated from the mother liquor, but was observed to be contaminated by unreacted nickel powder adhering to the magnetic stir bar. The precipitate was washed with diethylether $\left(5 \times 30 \mathrm{~cm}^{3}\right)$ until the washings were almost colourless. The crimson powder was dried under vacuum (1.575 g, 73\% of theory).

The dark red mother liquor, which had been removed by syringe to a nitrogen-filled Schlenk tube, was layered with diethylether $\left(25 \mathrm{~cm}^{3}\right)$ and refrigerated overnight to yield a further crop of crimson powder $(0.230 \mathrm{~g}, 9 \%$ of theory). This crop was used for characterisation and synthesis. Analysis. Calculated for $\mathrm{C}_{24} \mathrm{H}_{36} \mathrm{~N}_{12} \mathrm{~S}_{8} \mathrm{Ni}$ : C 35.68; $\mathrm{H}$ 4.49; N 20.81; S 31.76; Ni 7.26. Found: C 32.11; H 4.25; N 18.57; S 34.87.

\subsubsection{Decomposition of $\left[\mathrm{Ni}(N-\mathrm{MeIm})_{6}\right] \mathrm{S}_{8}$}

Rapid Decomposition of $\left[\mathrm{Ni}(\mathrm{N}-\mathrm{MeIm})_{6}\right] \mathrm{S}_{8}$

$\left[\mathrm{Ni}(\mathrm{N} \text {-MeIm) })_{6}\right] \mathrm{S}_{8}\left(0.284 \mathrm{~g}, 3.5 \times 10^{-4} \mathrm{~mol}\right.$, crimson powder $)$ was dissolved in distilled olyelamine $\left(11 \mathrm{~cm}^{3}\right)$ to give a crimson solution. This solution was injected rapidly into distilled oleylamine $\left(40 \mathrm{~cm}^{3}\right)$ preheated to $300^{\circ} \mathrm{C}$. The reaction mixture became black immediately after injection. The reaction mixture was cooled rapidly, diluted with acetone $\left(50 \mathrm{~cm}^{3}\right)$ and centrifuged to produce a black precipitate and clear yellow supernatant. The supernatant was discarded and the precipitate was resuspended in toluene $\left(20 \mathrm{~cm}^{3}\right)$. Acetone $\left(50 \mathrm{~cm}^{3}\right)$ was added and the suspension was centrifuged to give a black precipitate and a clear, colourless supernatant. This procedure was repeated twice and the precipitate was dried overnight in vacuo. 


\section{Gradual Decomposition of $\left[\mathrm{Ni}(\mathrm{N}-\mathrm{MeIm})_{6}\right] \mathrm{S}_{8}$}

$\left[\mathrm{Ni}(\mathrm{N} \text {-MeIm })_{6}\right] \mathrm{S}_{8}\left(0.275 \mathrm{~g}, 3.4 \times 10^{-4} \mathrm{~mol}\right.$, crimson powder $)$ was dissolved in distilled oleylamine $\left(50 \mathrm{~cm}^{3}\right)$ to give a crimson solution. This solution was heated to $300^{\circ} \mathrm{C}$. When this temperature was reached, the reaction mixture flocculated to produce black flocks and a clear, yellow supernatant. The reaction mixture was allowed to cool, and centrifuged to give a black precipitate and a clear yellow supernatant. The supernatant was discarded and the precipitate was resuspended in toluene $\left(20 \mathrm{~cm}^{3}\right)$, then centrifuged to give the precipitate and a clear, colourless supernatant. The supernatant was discarded and this procedure was repeated, this time with the addition of acetone $\left(80 \mathrm{~cm}^{3}\right)$ to destabilise the toluene suspension. The precipitate was dried in vacuo overnight, and then ground into a dark black powder. 


\subsection{References}

(1) D. Fan, M. Afzaal, M. A. Mallik, C. Q. Nguyen, P. O'Brien and P. J. Thomas, Coord. Chem. Rev., 2007, 251, 1878.

(2) L. Qu, Z. A. Peng and X. Peng, Nano Lett., 2001, 1, 333.

(3) Z. A. Peng and X. Peng, J. Am. Chem. Soc., 2001, 123, 183.

(4) N. Revaprasadu and S. N. Mlondo, Pure Appl. Chem., 2006, 78, 1691.

(5) M. Green and P. O'Brien, Chem. Commun., 1998, 2459.

(6) M. Green and P. O'Brien, J. Mater. Chem., 1999, 9, 243.

(7) A. C. Frank, F. Stowasser, H. Sussek, H. Pritzkow, C. R. Miskys, O. Ambacher, M. Giersig and R. A. Fischer, J. Am. Chem. Soc., 1998, 120, 3512.

(8) B. Rudolph, M. A. Malik, P. O'Brien and N. Revaprasadu, Chem. Commun., 1998, 1849.

(9) J. J. Vittal and M. T. Ng, Acc. Chem. Res., 2006, 39, 869.

(10) G. D. Scholes and S. P. Nair, J. Mater. Chem., 2006, 16, 467.

(11) S. G. Thoma, A. Sanchez, P. P. Provencio, B. L. Abrams and J. P. Wilcoxon, J. Am. Chem. Soc., 2005, 127, 7611.

(12) P. V. Vanitha and P. O'Brien, J. Am. Chem. Soc., 2008, 130, 17256.

(13) S. Dev, E. Ramli, T. B. Rauchfuss and C. L. Stern, J. Am. Chem. Soc., 1990, 112, 6385.

(14) E. Ramli, T. B. Rauchfuss and C. L. Stern, J. Am. Chem. Soc., 1990, 112, 4043.

(15) S. Dev, E. Ramli, T. B. Rauchfuss and S. R. Wilson, Inorg. Chem., 1991, 30, 2514.

(16) P. P. Paul, T. B. Rauchfuss and S. R. Wilson, J. Am. Chem. Soc., 1993, 115, 3316.

(17) A. K. Verma, T. Rauchfuss and S. R. Wilson, Inorg. Chem., 1995, 34, 3072.

(18) T. B. Rauchfuss, Inorg. Chem., 2004, 43, 14.

(19) N. Takeda, N. Tokitoh and R. Okazaki, Top. Curr. Chem., 2003, 231, 153.

(20) X. Zhong, S. Liu, Z. Zhang, L. Li, Z. Wei and W. Knoll, J. Mater. Chem., 2004, 14, 2790.

(21) H. J. Yu, J. Joo, H. M. Park, S.-I. Baik, Y. W. Kim, S. C. Kim and T. Hyeon, J. Am. Chem. Soc., 2005, 127, 5662.

(22) P. J. Thomas, P. Christian, S. Daniels, Y. Li, Y. S. Wang and P. O'Brien, Pure Appl. Chem., 2006, 78, 1651.

(23) A. R. Kortan, R. Hull, R. L. Opila, M. G. Bawendi, M. L. Steigerwald, P. J. Carroll and L. E. Brus, J. Am. Chem. Soc., 1990, 112, 1327.

(24) B. O. Dabbousi, J. Rodriguez-Viejo, F. V. Mikulec, J. R. Heine, H. Mattoussi, R. Ober, K. F. Jensen and M. G. Bawendi, J. Phys. Chem. B, 1997, 101, 9463.

(25) M. A. Hines and P. Guyot-Sionnest, J. Phys. Chem. B, 1996, 100, 468.

(26) D. V. Talapin, I. Mekis, S. Götzinger, A. Kornowski, O. Benson and H. Weller, J. Phys. Chem. B, 2004, 108, 18826.

(27) M. A. Malik, N. Revaprasadu and P. O'Brien, Chem. Mater., 2001, 13, 913.

(28) N. Pradhan and S. Efrima, J. Am. Chem. Soc., 2003, 125, 2050.

(29) H. T. Zhang, G. Wu and X. H. Chen, Mater. Lett., 2005, 59, 3728.

(30) Q. Wang, Z. Hu, H. Yin and Q. Nie, Mater. Chem. Phys., 2005, 90, 73.

(31) B. Geng, X. Liu, J. Ma and Q. Du, Mater. Sci. Eng., B, 2007, 145, 17.

(32) L. Tian, L. Y. Yep, T. T. Ong, J. Yi, J. Ding and J. J. Vittal, Cryst. Growth Des., 2009, 9, 353.

(33) X. Qian, Y. Li, X. Yi and Y. Qian, Mater. Chem. Phys., 2000, 66, 97.

(34) M. R. Spender, J. M. D. Coey and A. H. Morrish, Can. J. Phys., 1972, 50, 2313.

(35) M. Uda, Am. Mineral., 1965, 50, 1487. 
(36) H. Wang and I. Salveson, Phase Transitions, 2005, 78, 547.

(37) Z. Quan, Z. Wang, P. Yang, J. Lin and J. Fang, Inorg. Chem., 2007, 46, 1354.

(38) S. Wageh, Z. S. Ling and X. Xu-Rong, J. Cryst. Growth, 2003, 255, 332.

(39) T. Fujinaga, T. Kuwamoto, S. Okazaki and M. Hojo, Bull. Chem. Soc. Jpn., 1980, 53, 2851.

(40) R. Martin, P, W. H. J. Doub, J. L. J. Roberts and D. T. Sawyer, Inorg. Chem., 1973, 12, 1921.

(41) G. Nickless, ed., Inorganic Sulphur Chemistry, Elsevier Publishing Company, Amsterdam, 1968.

(42) M. L. Bender and B. W. Turnquest, J. Am. Chem. Soc., 1957, 79, 1656.

(43) W. Han and M. Gao, Cryst. Growth Des., 2008, 8, 1023.

(44) C. Pacholski, A. Kornowski and H. Weller, Angew. Chem. Int. Ed., 2002, 41, 1188.

(45) X. Chen, H. Xu, N. Xu, F. Zhao, W. Lin, G. Lin, Y. Fu, Z. Huang, H. Wang and M. Wu, Inorg. Chem., 2003, 42, 3100.

(46) I. Bezverkhyy, M. Danot and P. Afanasiev, Inorg. Chem., 2003, 42, 1764.

(47) G. Kullerud and R. A. Yund, J. Petrology, 1962, 3, 126.

(48) X. Liu, Mater. Sci. Eng., B, 2005, 119, 19.

(49) L. Qu and X. Peng, J. Am. Chem. Soc., 2002, 124, 2049.

(50) J. F. Suyver, S. F. Wuister, J. J. Kelly and A. Meijerink, Nano Lett., 2001, 1, 429. 


\section{Chapter 7 Conclusions and Future Work}

For the sake of convenience and ease of reference, and in order to place the work conducted in this project in the context of the current state of knowledge in the literature, the conclusions and suggested future work resulting from each of the four results chapters in this project are summarised and presented below.

\subsection{Chapter 3 Synthesis of cadmium selenide nanocrystals}

In Chapter 3 a modified literature procedure ${ }^{1}$ was followed to synthesise cadmium selenide nanocrystals by injection of $\mathrm{Se}(\mathrm{TOP})$ solution into a solution of $\mathrm{Cd}(\mathrm{OAc})_{2}$ in TOPO at $300^{\circ} \mathrm{C}$, in order to monitor the evolution of optical properties with reaction time. In an aliquot taken immediately after initiation of reaction, the positions of the absorption onset $(550 \mathrm{~nm}), 1 S_{3 / 2}-1 S_{e}$ excitonic transition $(500 \mathrm{~nm})$ and band-edge emission maximum (514 nm) were significantly blue-shifted compared to the optical absorption and emission features expected for bulk cadmium selenide with a band gap of $712 \mathrm{~nm}$. As the reaction proceeded, the absorption onset, position of the $1 S_{3 / 2}-1 S_{e}$ excitonic transition and the band-edge emission maximum progressively red-shifted and broadened to converge with the absorption and emission profiles expected for bulk cadmium selenide. This was correlated with the increase in average nanocrystal diameter from 2.8 to $6.0 \mathrm{~nm}$ over the course of the reaction, with an increase in the average standard deviation in diameter from 0.5 to $0.9 \mathrm{~nm}$. The trend observed in this project between the average diameter of cadmium selenide nanocrystals and the position and breadth of optical absorption and emission features matched well with previous observations in the literature, ${ }^{2}$ and is attributable to size-related quantum confinement effects.

The fluorescence quantum yield of a representative sample of cadmium selenide nanocrystals with an emission maximum at $630 \mathrm{~nm}$ was determined by comparison to two standard fluorescent dyes (fluorescein and coumarin 6). As the figure of $0.3 \%$ determined for the quantum yield of the cadmium selenide nanocrystals was less than the level of precision of the measurements, confidence cannot be taken in accuracy of the value. However, quantum yields of fewer than several percent are in not atypical for 
cadmium selenide nanocrystals in the spectral window above $600 \mathrm{~nm}^{2}$ and this indicates that the reaction conditions used in this project - especially the ratio of cadmium precursor to selenium precursor (1:3.5) were not optimised for the purpose of obtaining the fluorescent 'bright spot'.

Capping of cadmium selenide nanocrystals with the high band gap semiconductor zinc sulfide was attempted using literature procedures, ${ }^{3}$ however these attempts were not demonstrably successful in producing coatings of stoichiometric zinc sulfide. Modified literature procedures ${ }^{4}$ were followed to displace non-polar surfactant molecules (TOPO, TOP) from the surface of as-synthesised cadmium selenide nanocrystals and replace them with polar capping molecules - such as cysteine and mercaptopropionic acid rendering the nanocrystals dispersible in non-polar media, such as water and methanol.

The work conducted in Chapter 3 was based on established literature methodology. The results obtained in Chapter 3 were useful in that they confirmed results already published in the literature. The synthesis of cadmium selenide nanocrystals represents a highly researched area, and work conducted in this project was designed to develop skill and experience in synthesis and characterisation techniques before moving on to perform new work in more challenging areas. 


\subsection{Chapter 4 Synthesis of iron sulfide nanocrystals}

In Chapter 4, a new synthetic method was developed to produce iron sulfide nanocrystals. Much work has been conducted previously on characterising geological samples of the iron sulfides $;{ }^{5}$ and synthetic approaches have been developed to elucidate the phase diagram of the iron-sulfur system ${ }^{6-8}$ or produce bulk samples in order to investigate fundamental physical properties of iron sulfide phases. ${ }^{9}$ However, relatively little work has been conducted on syntheses where the size of the resulting iron sulfide particles is controlled. This limited work includes recent reports of the use of single-source precursors such as $\mathrm{Fe}(\mathrm{Dtdc})_{2}(\mathrm{Phen})^{10}$ and $\left[\mathrm{NBu}_{4}\right]_{2}\left[\mathrm{Fe}_{4} \mathrm{~S}_{4}(\mathrm{SPh})_{4}\right]^{11}$ to produce sub-micron $\mathrm{Fe}_{1-x} \mathrm{~S}$ crystallites and 2-4 $\mathrm{nm} \mathrm{Fe}_{3} \mathrm{~S}_{4}$ nanocrystals, respectively.

However the methodology developed in Chapter 4 of this project represents a relatively simple, flexible route to small iron sulfide crystallites using low cost precursors. The effect of various reaction parameters was examined - including reaction temperature, duration, order of addition, precursor, initial ratio of iron precursor to sulfur, solvent and the effect of rapid quenching:

Reaction of $\mathrm{Fe}(\mathrm{acac})_{2}$ and elemental sulfur in oleylamine was found to produce a number of iron sulfide phases and crystal morphologies, depending primarily on reaction temperature and duration. Reactions conducted at $300^{\circ} \mathrm{C}$ for 30 minutes produced solely submicron-sized faceted $\mathrm{Fe}_{1-x} \mathrm{~S}$ crystallites, whereas reactions conducted at $200^{\circ} \mathrm{C}$ for 4 hours produced a mixture of faceted $\mathrm{Fe}_{1-x} \mathrm{~S}$ crystallites and thin $\mathrm{Fe}_{3} \mathrm{~S}_{4}$ sheets. Rapidly inducing iron sulfide formation by injecting either precursor into a preheated solution of the other at $280-290^{\circ} \mathrm{C}$, followed by rapid cooling produced a mixture of submicron-sized, faceted $\mathrm{Fe}_{1-x} \mathrm{~S}$ crystallites and $5 \mathrm{~nm} \mathrm{Fe} \mathrm{S}_{4}$ nanocrystals, whose size is consistent with production by burst nucleation. In reactions conducted at $>260^{\circ} \mathrm{C}$ for more than 10 minutes, $\mathrm{Fe}_{3} \mathrm{~S}_{4}$ was absent as the phase is not stable above $\sim 200^{\circ} \mathrm{C}$, and decomposes to $\mathrm{Fe}_{1-x} \mathrm{~S}$ and elemental sulfur. ${ }^{12}$

Substituting $\mathrm{FeCl}_{2}$ for $\mathrm{Fe}(\mathrm{acac})_{2}$ as the iron source was found to give some control over the average crystallite size of $\mathrm{Fe}_{3} \mathrm{~S}_{4}$ - when $\mathrm{FeCl}_{2}$ solution was injected into a solution of sulfur in oleylamine at $280^{\circ} \mathrm{C}$, the $\mathrm{Fe}_{3} \mathrm{~S}_{4}$ nanocrystals produced had an average diameter of $20 \mathrm{~nm}$ (c.f. $5 \mathrm{~nm}$ with $\left.\mathrm{Fe}(\mathrm{acac})_{2}\right)$, and frequent examples of a twinned octahedral morphology were evident. Although this was determined to be due to the larger average 
crystallite size enabling faceted morphologies to emerge, rather than as a direct effect of the iron source.

Altering the ratio of $\mathrm{Fe}(\mathrm{acac})_{2}: \mathrm{S}$ away from the ratio necessary for stoichiometric or near-stoichiometric $\mathrm{Fe}_{1-x} \mathrm{~S}$ or $\mathrm{Fe}_{3} \mathrm{~S}_{4}$ (1:1 and 1:1.3, respectively) did not alter the phases of the products, and unreacted excess elemental sulfur proved to be difficult to remove from the iron sulfide products. The substitution of HDA for oleylamine as the solvent, did not substantively affect the size or crystal phase of the nanocrystal produced.

Experiments where aliquots of reaction mixture were rapidly quenched by injection into cooled solvent revealed that $\mathrm{Fe}_{1-x} \mathrm{~S}$ grew to sizes in excess of $100 \mathrm{~nm}$ within the first 5 minutes of reaction at reaction temperatures $>260^{\circ} \mathrm{C}$, and hence limited reaction times could not be used to decrease the average crystallite size of $\mathrm{Fe}_{1-x} \mathrm{~S}$.

Magnetic measurements were performed on various iron sulfide nanocrystal samples produced in Chapter 4. A pure sample of $\mathrm{Fe}_{1-x} \mathrm{~S}$ crystallites (Sample X) was found to be antiferromagnetic, with a low initial susceptibility and high coercivity $\left(112 \mathrm{kA} \mathrm{m}^{-1}\right.$ at $300 \mathrm{~K}$ ) - all properties consistent with bulk $\mathrm{Fe}_{1-x} \mathrm{~S} .{ }^{13}$ The presence of ferrimagnetic $\mathrm{Fe}_{3} \mathrm{~S}_{4}$ in product mixture greatly increased the overall magnetic moment of the sample, overwhelming the contribution from $\mathrm{Fe}_{1-x} \mathrm{~S}$ - although wasp-waisted field loops were still evident as a consequence of the high coercivity $\mathrm{Fe}_{1-x} \mathrm{~S}$ component. Large $\mathrm{Fe}_{3} \mathrm{~S}_{4}$ sheets (Sample VII) remained in a ferrimagnetic state at room temperature, whereas samples containing $5 \mathrm{~nm}$ spherical $\mathrm{Fe}_{3} \mathrm{~S}_{4}$ nanocrystals exhibited transitions to complete superparamagnetism above 200 to $250 \mathrm{~K}$ (Samples XII and XIII, respectively).

The work presented in Chapter 4 represents an advance in synthetic methodology, and the magnetic data points towards the significance of the contribution of the $\mathrm{Fe}_{3} \mathrm{~S}_{4}$ component to the overall magnetic properties of a mixed $\mathrm{Fe}_{1-x} \mathrm{~S} / \mathrm{Fe}_{3} \mathrm{~S}_{4}$ sample, especially with respect to the transition to superparamagnetic behaviour. However the synthetic methodology developed in Chapter 4 was unable to produce phase-pure samples of $\mathrm{Fe}_{3} \mathrm{~S}_{4}$, due to the tendency for $\mathrm{Fe}_{1-x} \mathrm{~S}$ to form above $200^{\circ} \mathrm{C}$. In addition the size distribution of samples was generally wide. The combination of phase mixtures and polydispersity in samples made it difficult to draw conclusions from the magnetic data. However no future work is suggested for Chapter 4 per se, as the synthetic methodology 
was developed further in Chapter 5, which circumvented several of the problems highlighted here. 


\subsection{Chapter 5 Sulfidisation or oxidation of iron/iron oxide nanocrystals}

In Chapter 5 new methodology was developed, which allowed the synthesis of iron oxide or iron sulfide spinel nanocrystals of almost identical average diameters and similar, monodisperse size distributions. While there are numerous previously published syntheses of monodisperse iron oxide spinel nanocrystals with a range of average diameters, ${ }^{14}$ there is only a single published synthesis of monodisperse iron sulfide spinel $\left(\mathrm{Fe}_{3} \mathrm{~S}_{4}\right)$ nanocrystals. ${ }^{11}$ The methodology developed in this chapter has the benefit of utilising relatively simply and cheap precursors compared to the single source precursor $\left[\mathrm{NBu}_{4}\right]_{2}\left[\mathrm{Fe}_{4} \mathrm{~S}_{4}(\mathrm{SPh})_{4}\right]$ used in the aforementioned published synthesis.

The methodology used in Chapter 5 was based upon the reaction of iron precursors such as $\mathrm{Fe}(\mathrm{acac})_{2}-$ at high temperature in a coordinating solvent for several hours, after which time it was inferred that a monodisperse population of nanocrystals had become established. If the reaction mixture was cooled and exposed to the atmosphere, a colour change from black to brown took place over several hours - which was associated with the oxidation of the intermediate nanocrystals (which were postulated to be either elemental iron, or $\mathrm{Fe}_{3} \mathrm{O}_{4}$ ) to either $\gamma-\mathrm{Fe}_{2} \mathrm{O}_{3}$ or a solid solution of $\mathrm{Fe}_{3} \mathrm{O}_{4}$ and $\gamma-\mathrm{Fe}_{2} \mathrm{O}_{3}$. If instead a solution containing dissolved elemental sulfur was injected at high temperature, followed by rapid cooling, the product of the reaction was monodisperse $\mathrm{Fe}_{3} \mathrm{~S}_{4}$ nanocrystals.

Various experimental parameters were investigated to optimise both the formation of iron oxide spinel nanocrystals, and the sulfidisation to $\mathrm{Fe}_{3} \mathrm{~S}_{4}$. These included reaction temperature, duration, solvent, iron precursor, replenishment of iron precursor during the reaction, temperature of injection of sulfur solution and iron precursor to sulfur ratio, as outlined below:

The synthesis of monodisperse spherical iron oxide spinel nanocrystals was achieved by the thermal decomposition of $\mathrm{Fe}(\mathrm{acac})_{2}$ in $\mathrm{HDA}$ at $200-300^{\circ} \mathrm{C}$. The highest degree of monodispersity $(\sigma \sim 10 \%)$ was found to be reached after 2 hours of reaction at either 200 or $300^{\circ} \mathrm{C}$. This indicated the point in time where the nanocrystal ensemble moved from the focusing to defocusing growth regime, and represented the optimum point at which to halt the reaction. The relative invariance of the time at which the reaction reached this 
minimum standard deviation in diameter with respect to reaction temperature was to prove beneficial for the subsequent sulfidisation step. The average diameter of all iron oxide spinel nanocrystal samples produced under these conditions was approximately 6 $\mathrm{nm}$.

When decomposing $\mathrm{Fe}(\mathrm{acac})_{2}$ in HDA, replenishment of $\mathrm{Fe}(\mathrm{acac})_{2}$ after 2 hours led to a slight increase of the average iron oxide spinel nanocrystal diameter, but the relative standard deviation in nanocrystal diameter was higher than for experiments without replenishment. Therefore the reaction of $\mathrm{Fe}(\mathrm{acac})_{2}$ in $\mathrm{HDA}$ between $200-300^{\circ} \mathrm{C}$ for 2 hours remained the optimum set of reaction conditions found for the production of monodisperse $6 \mathrm{~nm}$ iron oxide spinel nanocrystals.

Sulfidisation of the intermediate nanocrystals to $\mathrm{Fe}_{3} \mathrm{~S}_{4}$ could be achieved by injecting 1.5 eq. of sulfur dissolved in oleylamine at $300^{\circ} \mathrm{C}$ before exposure to the atmosphere. Rapid cooling, and size selective purification was used to produce a sample of $7 \mathrm{~nm}$ $\mathrm{Fe}_{3} \mathrm{~S}_{4}$ nanocrystals $(\sigma=30 \%)$. If the sulfidisation temperature was reduced to $200^{\circ} \mathrm{C}$, the product of the reaction was amorphous iron sulfide.

Magnetic measurements were performed on a representative sample of $6.1 \pm 0.6 \mathrm{~nm}$ iron oxide spinel nanocrystals, and on a sample of $6.8 \pm 2.0 \mathrm{~nm} \mathrm{Fe}{ }_{3} \mathrm{~S}_{4}$ nanocrystals. The iron oxide spinel nanocrystals had an average blocking temperature, $T_{B}$, of $100 \mathrm{~K}$, whereas the blocking temperature of the $\mathrm{Fe}_{3} \mathrm{~S}_{4}$ nanocrystals was $50 \mathrm{~K}$ - an indication that the first order magnetocrystalline anisotropy constant of $\mathrm{Fe}_{3} \mathrm{~S}_{4}$ (which has only ever been estimated) may be lower than those of $\mathrm{Fe}_{3} \mathrm{O}_{4}$ and $\gamma-\mathrm{Fe}_{2} \mathrm{O}_{3}\left(10^{4}-10^{5}\right.$ and $10^{5} \mathrm{~J} \mathrm{~m}^{-3}$, respectively). ${ }^{15}$ At $43 \mathrm{~A} \mathrm{~m}^{2} \mathrm{~kg}^{-1}$ at $300 \mathrm{~K}$, the saturation magnetisation measured for the iron oxide spinel nanocrystals matched literature values. ${ }^{16}$ The $\mathrm{Fe}_{3} \mathrm{~S}_{4}$ nanocrystals had an approximate saturation magnetisation of $9 \mathrm{~A} \mathrm{~m}^{2} \mathrm{~kg}^{-1}$ at $300 \mathrm{~K}$, which fits well within the trend set by the few saturation magnetisation values published for $\mathrm{Fe}_{3} \mathrm{~S}_{4}$ samples with well-characterised size distributions. ${ }^{11}$

The major achievement of Chapter 5 was the establishment of a synthetic methodology to produce monodisperse $\mathrm{Fe}_{3} \mathrm{~S}_{4}$ nanocrystals. This allowed the acquisition of magnetic data to add the scant amount of data from well-characterised, monodisperse samples of $\mathrm{Fe}_{3} \mathrm{~S}_{4}$ nanocrystals available in the literature. Suggested future work for this chapter centres around the use of this data, and that available from other literature sources ${ }^{11}$ to 
develop a firmer theoretical understanding of the magnetic structure of the $\mathrm{Fe}_{3} \mathrm{~S}_{4}$ phase. The difference in blocking temperature for samples of spherical iron oxide spinel $(100 \mathrm{~K})$ and $\mathrm{Fe}_{3} \mathrm{~S}_{4}$ nanocrystals $(50 \mathrm{~K})$ of essentially identical average diameter and similar size distribution $\left(6.1 \pm 0.6\right.$ and $6.8 \pm 2.0$ for iron oxide spinel and $\mathrm{Fe}_{3} \mathrm{~S}_{4}$, respectively) suggests a difference in the magnetocrystalline anisotropy of the two phases - which is significant, as the magnetocrystalline anisotropy constant of $\mathrm{Fe}_{3} \mathrm{~S}_{4}$ cannot be measured by conventional methods due to difficulties in growing large single crystals. This, and the significant difference in saturation magnetisation for the two phases will hopefully allow better understanding of the difference in the effective magnetic moment per formula unit for the two isostructural spinel phases. 


\subsection{Chapter 6 Single-source metal sulfide precursors for nanocrystal synthesis}

In Chapter 6, the amine-stabilised metal polysulfide complexes $\left[\mathrm{M}(N \text {-MeIm })_{6}\right] \mathrm{S}_{8}(\mathrm{M}=$ $\mathrm{Mg}, \mathrm{Fe}, \mathrm{Ni})$ and $\mathrm{ZnS}_{6}(\mathrm{~N} \text {-MeIm })_{2}$ were synthesised from literature methods. ${ }^{17}, 18$ The solid state and solution phases decomposition of the complexes was then studied representing the first known systematic study of the decomposition of these complexes, and the first known use of metal polysulfide complexes as single-source precursors for the synthesis of nanocrystals.

The solution phase decomposition of the complexes yielded magnesium oxide $(\mathrm{MgO})$, $\mathrm{Fe}_{3} \mathrm{~S}_{4}, \mathrm{Fe}_{1-x} \mathrm{~S}, \mathrm{NiS}_{2}, \alpha-\mathrm{Ni}_{1-x} \mathrm{~S}$, and spherical zinc sulfide nanocrystals and zinc sulfide nanorods. The method of reaction and temperature of reaction in each case exerting control over the phase, size and morphology produced:

Injection of a solution of $\left[\mathrm{Fe}(\mathrm{N}-\mathrm{MeIm})_{6}\right] \mathrm{S}_{8}$ in oleylamine into preheated oleylamine at $300^{\circ} \mathrm{C}$, followed by rapid cooling, produced $\sim 20 \mathrm{~nm} \mathrm{Fe}_{3} \mathrm{~S}_{4}$ nanocrystals. When $\left[\mathrm{Fe}(\mathrm{N} \text {-MeIm })_{6}\right] \mathrm{S}_{8}$ was dissolved in oleylamine and heated gradually to $300^{\circ} \mathrm{C}$ for 4 hours the product was faceted $\mathrm{Fe}_{1-x} \mathrm{~S}$ crystallites.

Injection of a solution of $\left[\mathrm{Ni}(\mathrm{N}-\mathrm{MeIm})_{6}\right] \mathrm{S}_{8}$ in oleylamine into preheated oleylamine at $300^{\circ} \mathrm{C}$, followed by rapid cooling, produced spherical, monodisperse $6 \mathrm{~nm} \mathrm{NiS} 2$ nanocrystals. When $\mathrm{Ni}\left(\mathrm{N}\right.$-MeIm) $\left.{ }_{6}\right] \mathrm{S}_{8}$ was dissolved in oleylamine and heated gradually to $300^{\circ} \mathrm{C}$ the product was irregularly shaped, polydisperse $\alpha-\mathrm{Ni}_{1-x} \mathrm{~S}$ crystallites.

Injection of a solution of $\mathrm{ZnS}_{6}(\mathrm{~N} \text {-MeIm })_{2}$ in oleylamine into preheated oleylamine at $300^{\circ} \mathrm{C}$, followed by rapid cooling, produced spherical, monodisperse $5 \mathrm{~nm}$ zinc sulfide nanocrystals. When $\mathrm{ZnS}_{6}(\mathrm{~N} \text {-MeIm })_{2}$ was dissolved in oleylamine and heated gradually to $300^{\circ} \mathrm{C}$ the product was highly crystalline $15 \times 5 \mathrm{~nm}$ zinc sulfide nanorods.

In Chapter 6, the viability of the use of amine-stabilised metal polysulfide complexes as precursors for binary metal sulfide nanocrystals was demonstrated. These complexes have the advantages of being directly synthesised from the elements - requiring the addition of only an inexpensive amine solvent. This allows an overall two step synthetic procedure from the elements to highly crystalline, size- and phase-controlled metal 
sulfide nanocrystals, which in several cases are highly monodisperse. Neither stage of the process requires extreme conditions, with the initial synthesis of the complexes accomplished in all cases within 1 day at $<100^{\circ} \mathrm{C}$, and the decomposition to the binary metal sulfide achieved in all cases $\leq 300^{\circ} \mathrm{C}$ with short to moderate reaction times. The only non-volatile by-product of the decomposition reactions is excess sulfur, which remains in solution and can be removed simply by washing the product.

Thus amine-stabilised metal polysulfide complexes offer a cheap, simple and elegant route to metal sulfide nanocrystals, and possess several advantages when compared to problems associated with other single-source precursors. Namely, the low cost of the complex precursors (metal powder, elemental sulfur, $N$-MeIm), the absence of any non-volatile by-product except excess sulfur, and the absence of halide counterions which can be detrimental to the quality of the metal sulfide product. ${ }^{19}$ 


\subsection{Chapter 6 Single-source metal sulfide precursors for nanocrystal synthesis - future work}

The decomposition reactions demonstrated above display a high degree of flexibility, and excellent phase-selectivity. However, in several cases there remains scope for further optimisation of reaction conditions to improve the dispersity and the uniformity of the crystal morphology.

The decomposition of $\left[\mathrm{Ni}(N-\mathrm{MeIm})_{6}\right] \mathrm{S}_{8}$ to $\alpha-\mathrm{Ni}_{1-x} \mathrm{~S}$ also requires further optimisation, as the size distribution is wide and the morphology irregular. Better control of the nucleation conditions by injection of the precursor followed by long reaction times are possible alterations to the methodology which may lead to improved dispersity, as suggested by a published synthesis of $\alpha-\mathrm{Ni}_{1-x} \mathrm{~S}$ nanocrystals from another single-source precursor. $^{20}$

There are several $\mathrm{N}$-MeIm-stabilised metal polysulfide complexes in addition to the four studied in this project $\left(\left[\mathrm{M}(N-\mathrm{MeIm})_{6}\right] \mathrm{S}_{8}(\mathrm{M}=\mathrm{Mg}, \mathrm{Fe}, \mathrm{Ni})\right.$ and $\left.\mathrm{ZnS}_{6}(N-\mathrm{MeIm})_{2}\right)$. These include $\left[\mathrm{Mn}(N-\mathrm{MeIm})_{6}\right] \mathrm{S}_{8}{ }^{(18)}$ and $\mathrm{Cu}_{4}\left(\mathrm{~S}_{5}\right)_{2}(N-\mathrm{MeIm})_{4}{ }^{(21)}$. The former was not chosen for investigation in this project, as manganese sulfide nanocrystals are unstable with respect to oxidation in air; while difficulty was encountered isolating the complex $\mathrm{Cu}_{4}\left(\mathrm{~S}_{5}\right)_{2}(\mathrm{~N} \text {-MeIm})_{4}$ following the synthesis of Rauchfuss et al. There are in addition to these aforementioned monometallic $N$-MeIm polysulfide complexes, complexes of a range of metals which only form in the presence of magnesium ${ }^{22}$ - these are of the type $\left[\mathrm{Mg}(\mathrm{N}-\mathrm{MeIm})_{6}\right]\left[\mathrm{M}_{\mathrm{x}} \mathrm{S}_{\mathrm{y}}\right]$ where $\mathrm{M}=\mathrm{Ag}, \mathrm{Sb}, \mathrm{Sn}, \mathrm{In}, \mathrm{Au}, \mathrm{Cd}, \mathrm{Hg}$. These complexes were not investigated as single-source precursors, as it was feared that they might lead to inseparable mixtures of metal sulfide and magnesium oxide. For the various reasons mentioned above, the use of $N$-MeIm complexes for the synthesis of the sulfides of manganese, copper, silver, antimony, tin, indium, gold, cadmium and mercury appear more problematic than for iron, zinc and nickel. However it is felt that they nonetheless represent an avenue for future investigation. 


\subsection{References}

(1) I. Mekis, D. V. Talapin, A. Kornowski, M. Haase and H. Weller, J. Phys. Chem. $B, 2003,107,7454$.

(2) L. Qu and X. Peng, J. Am. Chem. Soc., 2002, 124, 2049.

(3) X. Zhong, S. Liu, Z. Zhang, L. Li, Z. Wei and W. Knoll, J. Mater. Chem., 2004, 14, 2790.

(4) J. Li, Y. Bai, J. Li, X. Hong, D. Li, K. Zhao, H. Wang, Z. Du and T. Li, Chem. Commun., 2004, 15, 1740.

(5) H. Wang and I. Salveson, Phase Transitions, 2005, 78, 547.

(6) S. A. Kissin and S. D. Scott, Econ. Geol., 1982, 77, 1739.

(7) R. A. Yund and H. T. Hall, Econ. Geol., 1969, 64, 420.

(8) H. Nakazawa and N. Morimoto, Mater. Res. Bull., 1971, 6, 345.

(9) M. R. Spender, J. M. D. Coey and A. H. Morrish, Can. J. Phys., 1972, 50, 2313.

(10) W. Han and M. Gao, Cryst. Growth Des., 2008, 8, 1023.

(11) P. V. Vanitha and P. O'Brien, J. Am. Chem. Soc., 2008, 130, 17256.

(12) M. Uda, Z. Anorg. Allg. Chem., 1967, 350, 105.

(13) C. Peters and M. J. Dekkers, Physics and Chemistry of the Earth, 2003, 28, 659.

(14) J. Park, J. Joo, S. G. Kwon, Y. Jang and T. Hyeon, Angew. Chem. Int. Ed., 2007, 46, 4630.

(15) R. M. Cornell and U. Schwertmann, The Iron Oxides: Structure, Properties, Reactions, Occurrences and Uses Wiley, Weinheim, 2004.

(16) D. K. Kim, Y. Zhang, W. Voit, K. V. Rao and M. Muhammed, J. Magn. Magn. Mater., 2001, 225, 30.

(17) S. Dev, E. Ramli, T. B. Rauchfuss and C. L. Stern, J. Am. Chem. Soc., 1990, 112, 6385.

(18) S. Dev, E. Ramli, T. B. Rauchfuss and S. R. Wilson, Inorg. Chem., 1991, 30, 2514.

(19) L. Qu, Z. A. Peng and X. Peng, Nano Lett., 2001, 1, 333.

(20) B. Geng, X. Liu, J. Ma and Q. Du, Mater. Sci. Eng., B, 2007, 145, 17.

(21) E. Ramli, T. B. Rauchfuss and C. L. Stern, J. Am. Chem. Soc., 1990, 112, 4043.

(22) P. P. Paul, T. B. Rauchfuss and S. R. Wilson, J. Am. Chem. Soc., 1993, 115, 3316. 


\section{Appendix 1 Indexing of SAED patterns}

Chapter 4 Synthesis of iron sulfide nanocrystals

A1-1 Indexing of SAED pattern of polycrystalline area of Sample VII.

\begin{tabular}{|c||c||c|c|c|}
\hline \multicolumn{2}{|c||}{ Sample VII } & \multicolumn{3}{c|}{$\mathrm{Fe}_{3} \mathrm{~S}_{4}$ reference } \\
\hline$d / \AA$ & $\Delta d / \AA$ & $h k l$ & $d / \AA$ & $\mathrm{I} / \%$ \\
\hline$/$ & $/$ & 111 & 5.72 & 8 \\
\hline 3.5 & \pm 0.2 & 220 & 3.50 & 30 \\
\hline 2.9 & \pm 0.2 & 311 & 2.98 & 100 \\
\hline 2.4 & \pm 0.1 & 400 & 2.47 & 55 \\
\hline$/$ & $/$ & 422 & 2.02 & 10 \\
\hline 1.9 & \pm 0.1 & 511 & 1.90 & 30 \\
\hline 1.7 & \pm 0.1 & 440 & 1.75 & 75 \\
\hline 1.6 & \pm 0.1 & 620 & 1.56 & 10 \\
\hline$/$ & $/$ & 533 & 1.51 & 10 \\
\hline$/$ & $/$ & 444 & 1.43 & 8 \\
\hline 1.3 & \pm 0.1 & 731 & 1.29 & 12 \\
\hline 1.1 & \pm 0.1 & 800 & 1.23 & 10 \\
\hline
\end{tabular}

A1-2 Indexing of SAED pattern of single-crystal Fe3S4 sheet from Sample VII.

\begin{tabular}{|c||c||c|c|}
\hline \multicolumn{2}{|c||}{ Sample VII } & \multicolumn{2}{c|}{$\mathrm{Fe}_{3} \mathrm{~S}_{4}$ reference } \\
\hline$d / \AA$ & $\Delta d / \AA$ & $h k l$ & $d / \AA$ \\
\hline 2.4 & \pm 0.1 & 400 & 2.47 \\
\hline 2.9 & \pm 0.2 & 311 & 2.98 \\
\hline 3.4 & \pm 0.2 & 220 & 3.50 \\
\hline 1.9 & \pm 0.2 & 511 & 1.90 \\
\hline
\end{tabular}

A1-3 Indexing of SAED patterns for Samples VIII - X.

\begin{tabular}{|c||c||c||c||c|c|c|}
\hline \multicolumn{1}{|c||}{ Sample VIII } & Sample IX & Sample $\mathrm{X}$ & \multicolumn{3}{c||}{} & \multicolumn{3}{c|}{$\mathrm{Fe}_{1-\mathrm{x}} \mathrm{S}$ reference } \\
\hline$d / \AA$ & $d / \AA$ & $d / \AA$ & $\Delta d / \AA$ & $h k l$ & $d / \AA$ & $\mathrm{I} / \%$ \\
\hline 3.1 & 3.0 & 3.0 & \pm 0.2 & 100 & 2.99 & 41 \\
\hline$/$ & $/$ & $/$ & $/$ & 002 & 2.89 & 5 \\
\hline 2.7 & 2.6 & 2.6 & \pm 0.1 & 101 & 2.66 & 47 \\
\hline 2.1 & 2.1 & 2.0 & \pm 0.1 & 102 & 2.09 & 100 \\
\hline 2.0 & 1.7 & 1.7 & \pm 0.1 & 110 & 1.73 & 33 \\
\hline 1.5 & 1.6 & 1.6 & \pm 0.1 & 103 & 1.62 & 7 \\
\hline$/$ & $/$ & $/$ & $/$ & 200 & 1.50 & 2 \\
\hline$/$ & $/$ & $/$ & $/$ & 112 & 1.48 & 1 \\
\hline$/$ & 1.4 & $/$ & \pm 0.1 & 201 & 1.45 & 5 \\
\hline 1.3 & 1.3 & 1.3 & \pm 0.1 & 202 & 1.33 & 12 \\
\hline$/$ & $/$ & $/$ & $/$ & 104 & 1.30 & 4 \\
\hline$/$ & $/$ & 1.1 & \pm 0.1 & 203 & 1.18 & 2 \\
\hline$/$ & 1.0 & 1.1 & \pm 0.1 & 114 & 1.11 & 10 \\
\hline
\end{tabular}


A1-4 Indexing of SAED patterns for Samples XII and XIII.

\begin{tabular}{|c||c||c||c|c|c|c|c|c|}
\hline \multicolumn{1}{|c||}{ Sample XII } & \multicolumn{1}{c||}{ Sample XIII } & \multicolumn{1}{c||}{$\mathrm{Fe}_{1-x} \mathrm{~S}$ reference } & \multicolumn{3}{c|}{$\mathrm{Fe}_{3} \mathrm{~S}_{4}$ reference } \\
\hline$d / \AA$ & $d / \AA$ & $\Delta d / \AA$ & $d / \AA$ & $h k l$ & $\mathrm{I} / \%$ & $d / \AA$ & $h k l$ & $\mathrm{I} / \%$ \\
\hline 3.4 & 3.3 & \pm 0.2 & $/$ & $/$ & $/$ & 3.50 & 220 & 30 \\
\hline 3.0 & 2.9 & \pm 0.2 & 2.99 & 100 & 41 & 2.98 & 311 & 100 \\
\hline 2.6 & $/$ & \pm 0.1 & 2.66 & 101 & 47 & $/$ & $/$ & $/$ \\
\hline 2.5 & 2.5 & \pm 0.1 & $/$ & $/$ & $/$ & 2.47 & 400 & 55 \\
\hline 2.0 & $/$ & \pm 0.1 & 2.08 & 102 & 100 & 2.02 & 422 & 10 \\
\hline 1.9 & 1.9 & \pm 0.1 & $/$ & $/$ & $/$ & 1.90 & 511 & 30 \\
\hline 1.7 & 1.8 & \pm 0.1 & 1.73 & 110 & 33 & 1.74 & 440 & 75 \\
\hline 1.5 & $/$ & \pm 0.1 & $/$ & $/$ & $/$ & 1.56 & 620 & 10 \\
\hline 1.4 & $/$ & \pm 0.1 & 1.45 & 201 & 5 & $/$ & $/$ & $/$ \\
\hline 1.3 & $/$ & \pm 0.1 & 1.33 & 202 & 12 & $/$ & $/$ & $/$ \\
\hline 1.2 & $/$ & \pm 0.1 & 1.30 & 104 & 4 & $/$ & $/$ & $/$ \\
\hline
\end{tabular}

A1-5 Indexing of SAED pattern for Sample XV.

\begin{tabular}{|c||c||c|c|c|c|c|c|}
\hline \multicolumn{1}{|c||}{ Sample XV } & \multicolumn{1}{|c||}{} & \multicolumn{3}{c|}{$\mathrm{Fe}_{1-x} \mathrm{~S}$ reference } & \multicolumn{3}{c|}{$\mathrm{Fe}_{3} \mathrm{~S}_{4}$ reference } \\
\hline$d / \AA$ & $\Delta d / \AA$ & $d / \AA$ & $h k l$ & $\mathrm{I} / \%$ & $d / \AA$ & $h k l$ & $\mathrm{I} / \%$ \\
\hline 3.4 & \pm 0.2 & $/$ & $/$ & $/$ & 3.50 & 220 & 30 \\
\hline 2.9 & \pm 0.2 & 2.99 & 100 & 41 & 2.98 & 311 & 100 \\
\hline 2.8 & \pm 0.2 & 2.89 & 002 & 5 & $/$ & $/$ & $/$ \\
\hline 2.5 & \pm 0.1 & 2.66 & 101 & 47 & $/$ & $/$ & $/$ \\
\hline 2.4 & \pm 0.1 & $/$ & $/$ & $/$ & 2.47 & 400 & 55 \\
\hline 2.0 & \pm 0.1 & 2.08 & 102 & 100 & 2.02 & 422 & 10 \\
\hline 1.9 & \pm 0.1 & $/$ & $/$ & $/$ & 1.90 & 511 & 30 \\
\hline 1.7 & \pm 0.1 & 1.73 & 110 & 33 & 1.74 & 440 & 75 \\
\hline 1.6 & \pm 0.1 & $/$ & $/$ & $/$ & 1.56 & 620 & 10 \\
\hline 1.5 & \pm 0.1 & 1.45 & 201 & 5 & $/$ & $/$ & $/$ \\
\hline 1.4 & \pm 0.1 & 1.33 & 202 & 12 & $/$ & $/$ & $/$ \\
\hline
\end{tabular}

A1-6 Indexing of SAED pattern for Sample XVI

\begin{tabular}{|c||c||c|c|c|}
\hline \multicolumn{1}{|c||}{$\begin{array}{c}\text { Sample } \\
\text { XVI }\end{array}$} & \multicolumn{1}{|c||}{} & \multicolumn{3}{c|}{$\mathrm{Fe}_{3} \mathrm{~S}_{4}$ reference } \\
\hline$d / \AA$ & $\Delta d / \AA$ & $h k l$ & $d / \AA$ & $\mathrm{I} / \%$ \\
\hline$/$ & $/$ & 111 & 5.72 & 8 \\
\hline 3.4 & \pm 0.2 & 220 & 3.50 & 30 \\
\hline 2.9 & \pm 0.2 & 311 & 2.98 & 100 \\
\hline 2.5 & \pm 0.1 & 400 & 2.47 & 55 \\
\hline$/$ & $/$ & 422 & 2.02 & 10 \\
\hline 1.9 & \pm 0.1 & 511 & 1.90 & 30 \\
\hline 1.8 & \pm 0.1 & 440 & 1.75 & 75 \\
\hline$/$ & $/$ & 620 & 1.56 & 10 \\
\hline$/$ & $/$ & 533 & 1.51 & 10 \\
\hline$/$ & $/$ & 444 & 1.43 & 8 \\
\hline 1.3 & \pm 0.1 & 731 & 1.29 & 12 \\
\hline$/$ & $/$ & 800 & 1.23 & 10 \\
\hline 1.1 & \pm 0.1 & 840 & 1.11 & 16 \\
\hline
\end{tabular}


A1-7 Indexing of SAED pattern for Sample XIII, 0.5 minutes.

\begin{tabular}{|c||c||c|c|c|}
\hline $\begin{array}{c}\text { Sample XIII 0.5 } \\
\text { min }\end{array}$ & \multicolumn{3}{c|}{$\mathrm{FeS}_{2}$ reference } \\
\hline$d / \AA$ & $\Delta d / \AA$ & $d / \AA$ & $h k l$ & $\mathrm{I} / \%$ \\
\hline 3.1 & \pm 0.2 & 3.12 & 111 & 39 \\
\hline 2.7 & \pm 0.1 & 2.70 & 200 & 100 \\
\hline$/$ & $/$ & 2.42 & 210 & 67 \\
\hline 2.2 & \pm 0.1 & 2.21 & 211 & 53 \\
\hline 1.9 & \pm 0.1 & 1.91 & 220 & 46 \\
\hline
\end{tabular}

A1-8 Indexing of SAED pattern for Sample XIII, 5 minutes.

\begin{tabular}{|c||c||c|c|c|}
\hline \multicolumn{1}{|c||}{ Sample XIII 5 min } & \multicolumn{3}{c||}{$\mathrm{Fe}_{1-x} \mathrm{~S}$ reference } \\
\hline$d / \AA$ & $\Delta d / \AA$ & $d / \AA$ & $h k l$ & $\mathrm{I} / \%$ \\
\hline 2.9 & \pm 0.2 & 2.99 & 100 & 41 \\
\hline$/$ & \pm 0.2 & 2.89 & 002 & 5 \\
\hline 2.6 & \pm 0.1 & 2.66 & 101 & 47 \\
\hline 2.0 & \pm 0.1 & 2.08 & 102 & 100 \\
\hline 1.7 & \pm 0.1 & 1.73 & 110 & 33 \\
\hline 1.5 & \pm 0.1 & 1.62 & 103 & 7 \\
\hline
\end{tabular}




\section{Chapter 5 Sulfidisation or oxidation of iron/iron oxide nanocrystals}

A1-9 Indexing of SAED patterns for Samples XX, XXI, XXIII, and XXIV.

\begin{tabular}{|c|c|c|c||c||c|c|c|}
\hline $\begin{array}{c}\text { Sample } \\
\text { XX }\end{array}$ & $\begin{array}{c}\text { Sample } \\
\text { XXI }\end{array}$ & $\begin{array}{c}\text { Sample } \\
\text { XXIII }\end{array}$ & $\begin{array}{c}\text { Sample } \\
\text { XXIV }\end{array}$ & \multicolumn{3}{|c|}{$\mathrm{Fe}_{3} \mathrm{O}_{4}$ reference } \\
\hline$d / \AA$ & $d / \AA$ & $d / \AA$ & $d / \AA$ & $\Delta d / \AA$ & $h k l$ & $d / \AA$ & $\mathrm{I} / \%$ \\
\hline$/$ & 4.6 & $/$ & 4.6 & \pm 0.3 & 111 & 4.852 & 8 \\
\hline 3.0 & 2.8 & 2.9 & 3.0 & \pm 0.2 & 220 & 2.967 & 30 \\
\hline 2.6 & 2.5 & 2.6 & 2.6 & \pm 0.1 & 311 & 2.532 & 100 \\
\hline$/$ & $/$ & $/$ & $/$ & $/$ & 222 & 2.424 & 8 \\
\hline 2.1 & 2.1 & 2.1 & 2.2 & \pm 0.1 & 400 & 2.099 & 20 \\
\hline 1.8 & 1.7 & 1.7 & 1.8 & \pm 0.1 & 422 & 1.715 & 10 \\
\hline 1.6 & 1.6 & 1.7 & 1.7 & \pm 0.1 & 511 & 1.616 & 30 \\
\hline 1.5 & 1.5 & 1.5 & 1.6 & \pm 0.1 & 440 & 1.485 & 40 \\
\hline
\end{tabular}

A1-10 Indexing of SAED pattern of Samples XXIX and XXX

\begin{tabular}{|c||c||c||c|c|c|}
\hline \multicolumn{1}{|c||}{ Sample XXIX } & \multicolumn{1}{c||}{ Sample XXX } & \multicolumn{3}{c|}{$\mathrm{Fe}_{3} \mathrm{O}_{4}$ reference } \\
\hline$d / \AA$ & $d / \AA$ & $\Delta d / \AA$ & $h k l$ & $d / \AA$ & $\mathrm{I} / \%$ \\
\hline 4.9 & 4.8 & \pm 0.3 & 111 & 4.852 & 8 \\
\hline 3.0 & 3.0 & \pm 0.2 & 220 & 2.967 & 30 \\
\hline 2.6 & 2.6 & \pm 0.1 & 311 & 2.532 & 100 \\
\hline$/$ & $/$ & \pm 0.1 & 222 & 2.424 & 8 \\
\hline 2.1 & 2.2 & \pm 0.1 & 400 & 2.099 & 20 \\
\hline 1.8 & 1.8 & \pm 0.1 & 422 & 1.715 & 10 \\
\hline 1.7 & 1.7 & \pm 0.1 & 511 & 1.616 & 30 \\
\hline 1.5 & 1.5 & \pm 0.1 & 440 & 1.485 & 40 \\
\hline
\end{tabular}

A1-11 Indexing of SAED pattern for Sample XXXII.

\begin{tabular}{|c||c||c|c|c|}
\hline \multicolumn{2}{|c||}{ Sample XXXII } & \multicolumn{3}{c|}{$\mathrm{Fe}_{3} \mathrm{~S}_{4}$ reference } \\
\hline$d / \AA$ & $\Delta \mathrm{d} / \AA$ & $h k l$ & $d / \AA$ & $\mathrm{I} / \%$ \\
\hline$/$ & $/$ & 111 & 5.70 & 6 \\
\hline 3.3 & \pm 0.2 & 220 & 3.49 & 28 \\
\hline 3.0 & \pm 0.2 & 311 & 2.98 & 100 \\
\hline$/$ & $/$ & 222 & 2.85 & 3 \\
\hline 2.5 & \pm 0.1 & 400 & 2.47 & 54 \\
\hline$/$ & $/$ & 331 & 2.27 & 1 \\
\hline$/$ & $/$ & 422 & 2.02 & 9 \\
\hline$/$ & $/$ & 511 & 1.90 & 28 \\
\hline 1.8 & \pm 0.1 & 440 & 1.75 & 73 \\
\hline
\end{tabular}


A1-12 Indexing of SAED pattern for Sample XXXIII.

\begin{tabular}{|c||c||c|c|c|}
\hline \multicolumn{2}{|c||}{ Sample XXXIII } & \multicolumn{3}{c|}{$\alpha-\mathrm{Fe}_{2} \mathrm{O}_{3}$ reference } \\
\hline$d / \AA$ & $\Delta \mathrm{d} / \AA$ & $h k l$ & $d / \AA$ & $\mathrm{I} / \%$ \\
\hline 3.7 & \pm 0.2 & 012 & 3.69 & 31 \\
\hline 2.8 & \pm 0.2 & 104 & 2.70 & 100 \\
\hline 2.6 & \pm 0.1 & 110 & 2.52 & 70 \\
\hline$/$ & $/$ & 006 & 2.30 & 2 \\
\hline 2.3 & \pm 0.1 & 113 & 2.21 & 19 \\
\hline$/$ & $/$ & 202 & 2.08 & 2 \\
\hline 1.9 & \pm 0.1 & 024 & 1.84 & 34 \\
\hline 1.8 & \pm 0.1 & 116 & 1.70 & 41 \\
\hline$/$ & $/$ & 211 & 1.64 & 1 \\
\hline$/$ & $/$ & 018 & 1.60 & 8 \\
\hline 1.5 & \pm 0.1 & 214 & 1.49 & 25 \\
\hline \multicolumn{1}{|r|}{} & & 300 & 1.45 & 24 \\
\hline
\end{tabular}

A1-13 Indexing of SAED pattern for Sample XXXIV.

\begin{tabular}{|c||c||c|c|c||c|c|c|}
\hline \multicolumn{2}{|c|}{ Sample XXXIV } & \multicolumn{3}{c||}{$\mathrm{Fe}_{3} \mathrm{~S}_{4}$ reference } & \multicolumn{3}{c|}{$\mathrm{Fe}_{1-x} \mathrm{~S}$ reference } \\
\hline$d / \AA$ & $\Delta d / \AA$ & $h k l$ & $d / \AA$ & $\mathrm{I} / \%$ & $h k l$ & $d / \AA$ & $\mathrm{I} / \%$ \\
\hline 3.5 & \pm 0.2 & 220 & 3.49 & 28 & $/$ & $/$ & $/$ \\
\hline 3.1 & \pm 0.2 & 311 & 2.98 & 100 & $/$ & & \\
\hline & & $/$ & $/$ & $/$ & 100 & 2.99 & 41 \\
\hline 2.6 & \pm 0.1 & $/$ & $/$ & $/$ & 101 & 2.66 & 47 \\
\hline 2.6 & \pm 0.1 & 400 & 2.47 & 54 & $/$ & $/$ & $/$ \\
\hline 2.1 & \pm 0.1 & $/$ & $/$ & $/$ & 102 & 2.08 & 100 \\
\hline 1.8 & \pm 0.1 & 440 & 1.75 & 73 & $/$ & $/$ & $/$ \\
\hline
\end{tabular}

A1-14 Indexing of SAED pattern for Sample XXXV.

\begin{tabular}{|c||c||c|c|c||c|c|c|}
\hline \multicolumn{2}{|c||}{ Sample XXXV } & \multicolumn{3}{c||}{$\mathrm{Fe}_{3} \mathrm{~S}_{4}$ reference } & \multicolumn{3}{c|}{$\mathrm{Fe}_{1-x} \mathrm{~S}$ reference } \\
\hline$d / \AA$ & $\Delta d / \AA$ & $h k l$ & $d / \AA$ & $\mathrm{I} / \%$ & $h k l$ & $d / \AA$ & $\mathrm{I} / \%$ \\
\hline 3.5 & \pm 0.2 & 220 & 3.49 & 28 & $/$ & $/$ & $/$ \\
\hline 3.0 & \pm 0.2 & 311 & 2.98 & 100 & $/$ & & \\
\hline 2.6 & \pm 0.1 & $/$ & $/$ & $/$ & 100 & 2.99 & 41 \\
\hline 2.5 & \pm 0.1 & 400 & 2.47 & 54 & $/$ & $/$ & $/$ \\
\hline 1.8 & \pm 0.1 & 440 & 1.75 & 73 & $/$ & $/$ & $/$ \\
\hline
\end{tabular}


Chapter 6 Single-source metal sulfide precursors for nanocrystal synthesis

A1-15 Indexing of SAED pattern of iron sulfide nanocrystals from rapid decomposition of $\left[\mathrm{Fe}(N \text {-methylimidazole })_{6}\right] \mathrm{S}_{8}$ in oleylamine.

\begin{tabular}{|c|c||c|c|c||c|c|c|}
\hline \multicolumn{2}{|c|}{} & \multicolumn{3}{c||}{$\mathrm{Fe}_{3} \mathrm{~S}_{4}$ reference } & \multicolumn{3}{c|}{$\mathrm{Fe}_{1-x} \mathrm{~S}$ reference } \\
\hline$d / \AA$ & $\Delta d / \AA$ & $h k l$ & $d / \AA$ & $\mathrm{I} / \%$ & $h k l$ & $d / \AA$ & $\mathrm{I} / \%$ \\
\hline 3.5 & \pm 0.2 & 220 & 3.50 & 28 & $/$ & $/$ & $/$ \\
\hline 3.0 & \pm 0.2 & 311 & 2.98 & 100 & 100 & 2.99 & 41 \\
\hline 2.6 & \pm 0.1 & $/$ & $/$ & $/$ & 101 & 2.66 & 47 \\
\hline 2.5 & \pm 0.1 & 400 & 2.47 & 54 & $/$ & $/$ & $/$ \\
\hline 2.1 & \pm 0.1 & $/$ & $/$ & $/$ & 102 & 2.08 & 100 \\
\hline 1.9 & \pm 0.1 & 511 & 1.90 & 28 & $/$ & $/$ & $/$ \\
\hline 1.8 & \pm 0.1 & 440 & 1.75 & 73 & $/$ & $/$ & $/$ \\
\hline 1.5 & \pm 0.1 & 620 & 1.56 & 3 & $/$ & $/$ & $/$ \\
\hline 1.5 & \pm 0.1 & 444 & 1.51 & 8 & $/$ & $/$ & $/$ \\
\hline
\end{tabular}

A1-16 Analysis of SAED pattern of the decomposition product of $\left[\mathrm{Mg}(N-\mathrm{MeIm})_{6}\right] \mathrm{S}_{8}$.

\begin{tabular}{|c|c||c|c|c||c|c|c|}
\hline & \multicolumn{1}{|c||}{} & \multicolumn{3}{c|}{$\mathrm{MgO}$ reference } & \multicolumn{3}{c|}{ MgS reference } \\
\hline$d / \AA$ & $\Delta d / \AA$ & $h k l$ & $d / \AA$ & $\mathrm{I} / \%$ & $h k l$ & $d / \AA$ & $\mathrm{I} / \%$ \\
\hline 2.6 & \pm 0.1 & $/$ & $/$ & $/$ & 200 & 2.60 & 100 \\
\hline 2.4 & \pm 0.1 & 111 & 2.43 & 4 & $/$ & $/$ & $/$ \\
\hline 2.1 & \pm 0.1 & 200 & 2.11 & 100 & $/$ & $/$ & $/$ \\
\hline 1.6 & \pm 0.1 & 220 & 1.49 & 40 & $/$ & $/$ & $/$ \\
\hline 1.3 & \pm 0.1 & 311 & 1.27 & 5 & $/$ & $/$ & $/$ \\
\hline 1.1 & \pm 0.1 & 222 & 1.22 & 10 & $/$ & $/$ & $/$ \\
\hline 1.0 & \pm 0.1 & 400 & 1.05 & 8 & $/$ & $/$ & $/$ \\
\hline 0.9 & \pm 0.1 & 420 & 0.94 & 19 & $/$ & $/$ & $/$ \\
\hline
\end{tabular}

A1-17 Indexing parameters for gradual decomposition product of [Ni(N-Meim $\left.)_{6}\right] \mathrm{S}_{8}$.

\begin{tabular}{|c|c||c|c|c||c|c|c|}
\hline & \multicolumn{2}{|c|}{} & \multicolumn{3}{c|}{$\alpha-\mathrm{Ni}_{1-\mathrm{x}} \mathrm{S}$ reference } & \multicolumn{3}{c|}{$\mathrm{NiS}_{2}$ reference } \\
\hline$d / \AA$ & $\Delta d / \AA$ & $h k l$ & $d / \AA$ & $\mathrm{I} / \%$ & $h k l$ & $d / \AA$ & $\mathrm{I} / \%$ \\
\hline 2.94 & \pm 0.2 & 100 & 2.98 & 58 & $/$ & $/$ & $/$ \\
\hline$/$ & $/$ & 002 & 2.70 & 6 & $/$ & $/$ & $/$ \\
\hline 2.60 & \pm 0.1 & 101 & 2.61 & 43 & $/$ & $/$ & $/$ \\
\hline 2.33 & \pm 0.1 & $/$ & $/$ & $/$ & 211 & 2.32 & 60 \\
\hline 1.98 & \pm 0.1 & 102 & 2.00 & 100 & $/$ & $/$ & $/$ \\
\hline 1.75 & \pm 0.1 & 110 & 1.72 & 42 & $/$ & $/$ & $/$ \\
\hline 1.56 & \pm 0.1 & 103 & 1.54 & 5 & $/$ & $/$ & $/$ \\
\hline 1.48 & \pm 0.1 & 200 & 1.49 & 4 & $/$ & $/$ & $/$ \\
\hline 1.35 & \pm 0.1 & 202 & 1.31 & 15 & $/$ & $/$ & $/$ \\
\hline 1.09 & \pm 0.1 & 211 & 1.10 & 3 & $/$ & $/$ & $/$ \\
\hline
\end{tabular}


A1-18 Indexing parameters for rapid decomposition product of [ $\left.\mathrm{Ni}(\mathrm{N}-\mathrm{MeIm})_{6} \mathrm{~S}_{8}\right]$.

\begin{tabular}{|c|c||c|c|c|}
\hline \multicolumn{1}{|c||}{} & \multicolumn{1}{|c||}{} & \multicolumn{3}{c|}{$\mathrm{NiS}_{2}$ Reference } \\
\hline$d / \AA$ & $\Delta d / \AA$ & $h k l$ & $d / \AA$ & $\mathrm{I} / \%$ \\
\hline$/$ & $/$ & 111 & 3.29 & 18 \\
\hline 2.86 & \pm 0.1 & 200 & 2.84 & 100 \\
\hline 2.57 & \pm 0.1 & 210 & 2.54 & 41 \\
\hline 2.35 & \pm 0.1 & 211 & 2.32 & 37 \\
\hline 2.05 & \pm 0.1 & 220 & 2.01 & 41 \\
\hline 1.76 & \pm 0.1 & 311 & 1.74 & 60 \\
\hline 1.63 & \pm 0.1 & 222 & 1.64 & 10 \\
\hline 1.58 & \pm 0.1 & 023 & 1.58 & 14 \\
\hline$/$ & $/$ & 321 & 1.52 & 17 \\
\hline 1.16 & \pm 0.1 & 422 & 1.16 & 5 \\
\hline
\end{tabular}




\section{Appendix 2 Scherrer X-ray line broadening analysis}

\section{Chapter 4 Synthesis of iron sulfide nanocrystals}

A2-1 Scherrer analysis of crystallite sizes for Samples III - V.

\begin{tabular}{|c|c|c|c|c|c|}
\hline Sample & Phase & $h k l$ & $\begin{array}{c}\omega \\
/ 10^{3} \mathrm{rad}\end{array}$ & $\begin{array}{c}D_{h k l} \\
/ \mathrm{nm}\end{array}$ & Average \\
\hline III & $\mathrm{Fe}_{1-x} \mathrm{~S}$ & 101 & 3.87 & 37 & $\mathbf{3 7}$ \\
\hline & $\mathrm{Fe}_{3} \mathrm{~S}_{4}$ & 400 & 5.99 & 24 & \\
\hline & & 511 & 4.44 & 34 & \\
\hline & & 440 & 4.51 & 34 & $\mathbf{3 1} \pm \mathbf{7}$ \\
\hline $\mathrm{IV}$ & $\mathrm{Fe}_{1-\mathrm{x}} \mathrm{S}$ & 101 & 5.90 & 25 & \\
\hline & & 102 & 6.27 & 24 & $\mathbf{2 5} \pm \mathbf{1}$ \\
\hline & $\mathrm{Fe}_{3} \mathrm{~S}_{4}$ & 400 & 5.67 & 26 & \\
\hline & & 511 & 4.94 & 31 & \\
\hline & & 440 & 7.04 & 22 & $\mathbf{2 6} \pm \mathbf{5}$ \\
\hline $\mathrm{V}$ & $\mathrm{Fe}_{3} \mathrm{~S}_{4}$ & 311 & 2.75 & 52 & \\
\hline & & 400 & 4.00 & 36 & \\
\hline & & 511 & 2.70 & 56 & \\
\hline & & 440 & 2.70 & 58 & $\mathbf{5 1} \pm \mathbf{1 5}$ \\
\hline
\end{tabular}

A2-2 Scherrer analysis of crystallite size for Sample VII

\begin{tabular}{|c|c|c|c|c|c|}
\hline $\begin{array}{c}\text { Sample } \\
\text { VII }\end{array}$ & $\begin{array}{l}\text { Phase } \\
\mathrm{Fe}_{1-x} \mathrm{~S}\end{array}$ & $h k l$ & $\begin{array}{c}\omega \\
/ 10^{3} \mathrm{rad} \\
2.58\end{array}$ & $\begin{array}{c}D_{h k l} \\
/ \mathrm{nm} \\
56\end{array}$ & Average \\
\hline & & 102 & 5.34 & 28 & $42 \pm 14$ \\
\hline & $\mathrm{Fe}_{3} \mathrm{~S}_{4}$ & 400 & 9.09 & 16 & \\
\hline & & 440 & 7.62 & 20 & $18 \pm 2$ \\
\hline
\end{tabular}

A2-3 Scherrer analysis of crystallite size for Samples IX - X.

\begin{tabular}{|c|c|c|c|c|c|}
\hline Sample & Phase & $h k l$ & $\begin{array}{c}\omega \\
/ 10^{3} \mathrm{rad}\end{array}$ & $\begin{array}{c}D_{h k l} \\
/ \mathrm{nm}\end{array}$ & Average \\
\hline $\mathrm{IX}$ & $\mathrm{Fe}_{1-\mathrm{x}} \mathrm{S}$ & 100 & 0.954 & 150 & \\
\hline & & 101 & 1.43 & 100 & \\
\hline & & 102 & 2.86 & 52 & \\
\hline & & 110 & 1.93 & 80 & \\
\hline \hline $\mathrm{X}$ & & 103 & 4.36 & 36 & $\mathbf{8 4} \pm \mathbf{6 6}$ \\
\hline & $\mathrm{Fe}_{1-\mathrm{x}} \mathrm{S}$ & 100 & 1.54 & 93 & \\
\hline & & 101 & 2.11 & 69 & \\
\hline & & 102 & 3.89 & 38 & \\
\hline & & 110 & 3.11 & 50 & \\
\hline & & 103 & 6.08 & 26 & $\mathbf{5 5} \pm \mathbf{3 8}$ \\
\hline
\end{tabular}


A2-4 Scherrer analysis of crystallite size of Sample XI.

\begin{tabular}{|c|c|c|c|c|c|}
\hline Sample & Phase & $h k l$ & $\begin{array}{c}\omega \\
/ 10^{3} \mathrm{rad}\end{array}$ & $\begin{array}{c}D_{h k l} \\
/ \mathrm{nm}\end{array}$ & Average \\
\hline $\mathrm{XI}$ & $\mathrm{Fe}_{1-\mathrm{x}} \mathrm{S}$ & 101 & 1.76 & 83 & \\
\hline & & 102 & 3.83 & 39 & \\
\hline & & 110 & 2.84 & 55 & $\mathbf{5 9} \pm \mathbf{2 4}$ \\
\hline & $\mathrm{Fe}_{3} \mathrm{~S}_{4}$ & 400 & 8.71 & 17 & \\
\hline & & 511 & 10.0 & 15 & $\mathbf{1 6} \pm \mathbf{1}$ \\
\hline
\end{tabular}

A2-5 Scherrer analysis of crystallite size for Samples XII - XIV.

\begin{tabular}{|c|c|c|c|c|c|}
\hline Sample & Phase & $h k l$ & $\begin{array}{c}\omega \\
/ 10^{3} \mathrm{rad}\end{array}$ & $\begin{array}{c}D_{h k l} \\
/ \mathrm{nm}\end{array}$ & Average \\
\hline XII & $\mathrm{Fe}_{1-x} \mathrm{~S}$ & 101 & 2.49 & 58 & \\
\hline & & 102 & 4.26 & 35 & \\
\hline & & 110 & 4.34 & 36 & $\mathbf{4 3} \pm \mathbf{1 5}$ \\
\hline & $\mathrm{Fe}_{3} \mathrm{~S}_{4}$ & 400 & 30.7 & 5 & $\mathbf{5}$ \\
\hline XIII & $\mathrm{Fe}_{1-x} \mathrm{~S}$ & 101 & 5.03 & 29 & \\
\hline & & 102 & 4.26 & 35 & \\
\hline & & 110 & 11.3 & 14 & $\mathbf{2 6} \pm \mathbf{1 2}$ \\
\hline & $\mathrm{Fe}_{3} \mathrm{~S}_{4}$ & 400 & 43.2 & 3 & \\
\hline & & 511 & 19.4 & 8 & \\
\hline & & 440 & 22.0 & 7 & $\mathbf{6} \pm \mathbf{3}$ \\
\hline XIV & $\mathrm{Fe}_{1-x} \mathrm{~S}$ & 101 & 1.47 & 98 & $\mathbf{9 8}$ \\
\hline & $\mathrm{Fe}_{3} \mathrm{~S}_{4}$ & 400 & 47.1 & 3 & \\
\hline & & 511 & 25.0 & 6 & \\
\hline & & 440 & 17.3 & 9 & $\mathbf{6} \pm \mathbf{3}$ \\
\hline
\end{tabular}

A2-6 Scherrer analysis of crystallite size for Sample XV.

\begin{tabular}{|c|c|c|c|c|c|}
\hline Sample & Phase & $h k l$ & $\begin{array}{c}\omega \\
/ 10^{3} \mathrm{rad}\end{array}$ & $\begin{array}{c}D_{h k l} \\
/ \mathrm{nm}\end{array}$ & Average \\
\hline $\mathrm{XV}$ & $\mathrm{Fe}_{1-x} \mathrm{~S}$ & 101 & 1.95 & 74 & \\
\hline & & 102 & 3.74 & 40 & \\
\hline & & 110 & 3.92 & 40 & $\mathbf{5 1} \pm \mathbf{2 3}$ \\
\hline & $\mathrm{Fe}_{3} \mathrm{~S}_{4}$ & 400 & 6.68 & 22 & \\
\hline & & 511 & 7.64 & 20 & \\
\hline & & 440 & 9.32 & 17 & $\mathbf{2 0} \pm \mathbf{3}$ \\
\hline
\end{tabular}

A2-7 Scherrer analysis of crystallite size for Samples XVI - XVII.

\begin{tabular}{|c|c|c|c|c|c|}
\hline Sample & Phase & $h k l$ & $\begin{array}{c}\omega \\
/ 10^{3} \mathrm{rad}\end{array}$ & $\begin{array}{c}D_{h k l} \\
/ \mathrm{nm}\end{array}$ & Average \\
\hline XVI & $\mathrm{Fe}_{1-x} \mathrm{~S}$ & 102 & 3.13 & 48 & $\mathbf{4 8}$ \\
\hline & $\mathrm{Fe}_{3} \mathrm{~S}_{4}$ & 400 & 3.43 & 43 & \\
\hline & & 511 & 5.97 & 25 & \\
\hline & & 440 & 8.23 & 19 & $\mathbf{2 9} \pm \mathbf{1 4}$ \\
\hline \hline XVII & $\mathrm{Fe}_{1-x} \mathrm{~S}$ & 101 & 0.95 & 152 & \\
\hline & & 102 & 1.87 & 80 & \\
\hline & & 110 & 1.41 & 110 & $\mathbf{1 1 5} \pm \mathbf{3 7}$ \\
\hline
\end{tabular}


$\underline{\text { Chapter } 5 \text { Sulfidisation or oxidation of iron/iron oxide nanocrystals }}$

A2-8 Scherrer X-ray line broadening analysis for Samples XXI and XXIII

\begin{tabular}{|c||c|c||c|c|}
\hline \multicolumn{1}{|c||}{} & \multicolumn{2}{c||}{ Sample XXI } & \multicolumn{2}{c|}{ Sample XXIII } \\
\hline$h k l$ & $\omega / \mathrm{rad}$ & $\begin{array}{c}D_{h k l} \\
/ \mathrm{nm}\end{array}$ & $\omega / \mathrm{rad}$ & $\begin{array}{c}D_{h k l} \\
/ \mathrm{nm}\end{array}$ \\
\hline 220 & 0.0237 & 6 & 0.0356 & 4 \\
\hline 311 & 0.0173 & 8 & 0.0208 & 7 \\
\hline 400 & $/$ & $/$ & 0.0197 & 8 \\
\hline 511 & 0.0236 & 8 & 0.0175 & 9 \\
\hline 440 & 0.0239 & 7 & 0.0225 & 7 \\
\hline \multicolumn{2}{|c|}{ Average } & $7 \pm 1$ & & $7 \pm 3$ \\
\hline
\end{tabular}




\section{Chapter 6 Single-source metal sulfide precursors for nanocrystal synthesis}

A2-9 Scherrer X-ray line broadening analysis for product of rapid decomposition of $\left[\mathrm{Fe}(N-\mathrm{MeIm})_{6}\right] \mathrm{S}_{8}$ $\left(\mathrm{Fe}_{3} \mathrm{~S}_{4}\right.$ nanocrystals).

\begin{tabular}{|c||c|c|}
\hline$h k l$ & $\omega / \mathrm{rad}$ & $\mathrm{D}_{\mathrm{hkl}} / \mathrm{nm}$ \\
\hline 311 & 0.008787 & 16 \\
\hline 400 & 0.008961 & 16 \\
\hline 422 & 0.008692 & 17 \\
\hline 511 & 0.009564 & 16 \\
\hline 440 & 0.009215 & 17 \\
\hline 533 & 0.01292 & 13 \\
\hline 444 & 0.01075 & 15 \\
\hline 731 & 0.01093 & 16 \\
\hline \multicolumn{2}{|c|}{ Average } & $16 \pm 1$ \\
\hline
\end{tabular}

A2-10 Scherrer X-ray line broadening analysis for product of gradual decomposition of $\left[\mathrm{Fe}(N-\mathrm{MeIm})_{6}\right] \mathrm{S}_{8}$ ( $\mathrm{Fe}_{1-x} \mathrm{~S}$ crystallites).

\begin{tabular}{|c||c|c|}
\hline$h k l$ & $\omega / \mathrm{rad}$ & $D_{\mathrm{hkl}} / \mathrm{nm}$ \\
\hline 100 & 0.00116 & 124 \\
\hline 101 & 0.00198 & 73 \\
\hline 102 & 0.00363 & 41 \\
\hline 110 & 0.00298 & 52 \\
\hline \multicolumn{2}{|c|}{ Average } & $73 \pm 51$ \\
\hline
\end{tabular}

A2-11 Scherrer X-ray line broadening analysis for product of rapid decomposition of $\left[\mathrm{Ni}(N-\mathrm{Me} I m)_{6}\right] \mathrm{S}_{8}$ ( $\mathrm{NiS}_{2}$ nanocrystals).

\begin{tabular}{|c||c|c|}
\hline$h k l$ & $\omega / \mathrm{rad}$ & $D_{\mathrm{hkl}} / \mathrm{nm}$ \\
\hline 111 & 0.0224 & 6 \\
\hline 200 & 0.0128 & 11 \\
\hline 210 & 0.0123 & 12 \\
\hline 211 & 0.0196 & 8 \\
\hline 220 & 0.0167 & 9 \\
\hline 311 & 0.0180 & 9 \\
\hline 023 & 0.0141 & 11 \\
\hline 321 & 0.0157 & 10 \\
\hline \multicolumn{2}{|c|}{ Average } & $10 \pm 4$ \\
\hline
\end{tabular}

A2-12 Scherrer X-ray line broadening analysis for product of gradual decomposition of $\left.] \mathrm{Ni}(N-\mathrm{MeIm})_{6}\right] \mathrm{S}_{8}$ $\left(\alpha-\mathrm{Ni}_{1-x} \mathrm{~S}\right.$ nanaocrystals $)$.

\begin{tabular}{|c||c|c|}
\hline$h k l$ & $\omega / \mathrm{rad}$ & $D_{\mathrm{hkl}} / \mathrm{nm}$ \\
\hline 100 & 0.002329 & 62 \\
\hline 101 & 0.002678 & 54 \\
\hline 102 & 0.003805 & 40 \\
\hline 110 & 0.003804 & 40 \\
\hline 202 & 0.005517 & 31 \\
\hline \multicolumn{2}{|c|}{ Average } & $45 \pm 15$ \\
\hline
\end{tabular}

